\title{
Chiral Metal Salts as Ligands for Catalytic Asymmetric Mannich Reactions with Simple Amides
}

Yasuhiro Yamashita, ${ }^{1 *}$ Aika Noguchi, ${ }^{1}$ Seiya Fushimi, ${ }^{1}$ Miho Hatanaka, ${ }^{2}$ and Shū Kobayashi ${ }^{1 *}$

${ }^{1}$ Department of Chemistry, School of Science, The University of Tokyo, Hongo, Bunkyo-ku, Tokyo 113-0033, Japan

${ }^{2}$ Department of Chemistry, Faculty of Science and Technology, Keio University, Hiyoshi, Kohoku-ku, Yokohama, Kanagawa, 223-8522, Japan

\section{Supplementary Information}

\section{Contents}

1. Optimization of reaction conditions and mechanistic studies

2. NMR study

3. Calculation study

4. Experimental section

5. Cartesian coordinates and energies

6 . References

7. NMR spectra

8. HPLC charts 


\section{Optimization of reaction conditions and mechanistic studies}

Effect of chiral crown ethers was investigated in the catalytic asymmetric Mannich reactions of 1a with 2a (Table S1). Non-symmetric and symmetric chiral crown ethers (L01-06) were tested, and it was found that the reactions proceeded in moderate to good yields with good to high diastereoselectivities but poor enantioselectivities.

Table S1 Catalytic asymmetric Mannich reactions using KHMDS-chiral crown ether systems ${ }^{\mathrm{a}}$
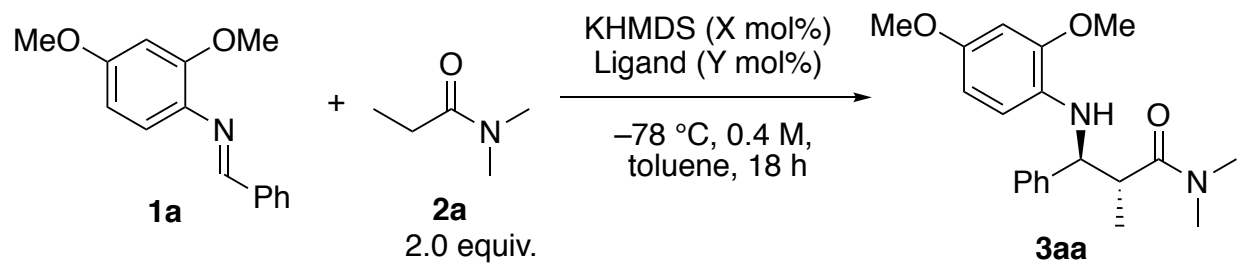

\begin{tabular}{ccccccc}
\hline Entry & Ligand & $\mathrm{X}$ & $\mathrm{Y}$ & ${\text { Yield }(\%)^{\mathrm{b}}}^{\text {Anti:syn }}$ & Ee (\%, anti) \\
\hline 1 & L01 & 10 & 11 & 81 & $78: 22$ & - \\
2 & L02 & 15 & 16.5 & 57 & $93: 7$ & 0 \\
3 & L03 & 15 & 16.5 & 63 & $78: 22$ & 24 \\
4 & L04 & 15 & 16.5 & 64 & $92: 8$ & -7 \\
5 & L05 & 15 & 16.5 & 64 & $91: 9$ & 0 \\
6 & L06 & 15 & 16.5 & 70 & $67: 33$ & 18 \\
\hline
\end{tabular}

${ }^{\text {a }}$ The reaction of $1 \mathrm{a}(0.5 \mathrm{mmol})$ with $2 \mathrm{a}(1.0 \mathrm{mmol})$ was conducted at $-78^{\circ} \mathrm{C}$ for $18 \mathrm{~h}$ in toluene $(0.4 \mathrm{M})$ under Ar atmosphere in the presence of a catalyst prepared from KHMDS (X mol\%) and LOX (Y mol\%) unless otherwise noted. ${ }^{b}$ Isolated yield. ${ }^{c}$ Determined by ${ }^{1} \mathrm{H}$ NMR analysis of a crude product mixture.<smiles>C1COCCOCCOCCOCCOCCO1</smiles>

L01

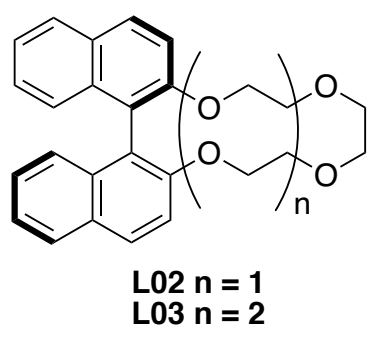

$\operatorname{L} 02 n=1$
$\operatorname{LO3} n=2$<smiles>CCOCC(C)(COCCOc1ccc2ccccc2c1-c1c(OCCOc2ccc3ccccc3c2-c2c(C)ccc3ccccc23)ccc2ccccc12)C(C)(C)OCCOc1ccc2ccccc2c1-c1cccc2ccccc12</smiles>

L04 $n=1$

L05 $n=2$

L06 $n=3$

We also investigated typical bisoxazoline (Box) ligands with disubstituted tethers; however, the obtained results were not promising (Scheme S1). When Box ligand L07 or L08 and KHMDS were used in 1:1 ratio, the reactions did not proceed at all. On the other hand, when they were used in 1:2 ratio, the reactions proceeded but almost racemic products were obtained.

Scheme S1 Catalytic asymmetric Mannich reactions using KHMDS and chiral Box ligands bearing disubstituted tethers 

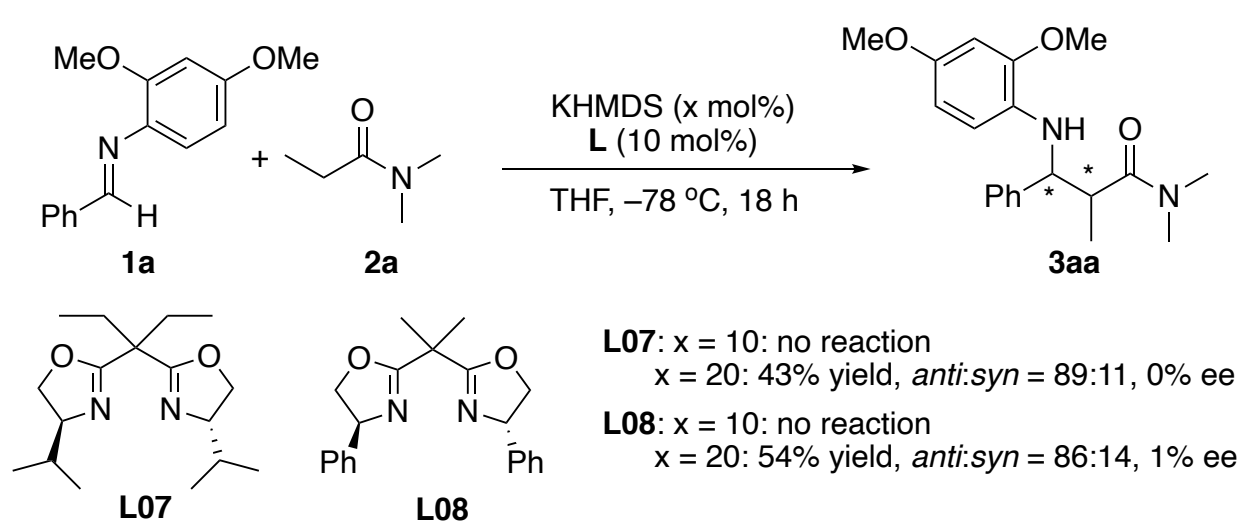

In our initial investigation, it was found that a chiral potassium base, which is consisting of KHMDS and a potassium salt of non-substituted methylene-tethered IndaBox Ligand L1 (K-L1), worked well as a catalyst in the catalytic asymmetric Mannich reaction in toluene, while K-L1 itself did not work at all due to its low Brønsted basicity (Table S2, entries 1, 2). Next, the amount of KHMDS to K-L1 was investigated. When the amount of KHMDS was a half of K-L1, the Mannich reaction proceeded in better enantioselectivity, but the yield was very low (entry 3). On the other hand, when the amount was two times to K-L1, the reaction proceeded in better yield but with lower enantioselectivity (entry 4).

Table S2 Effect of the ratio of KHMDS and K-L1

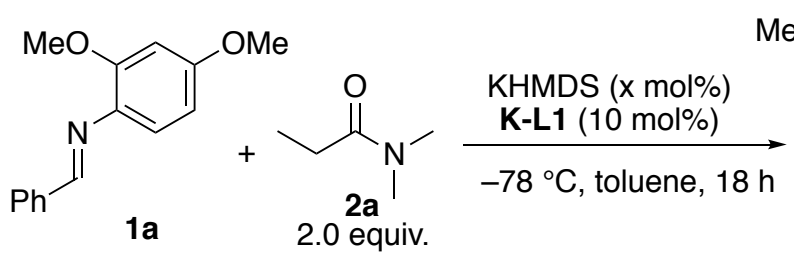<smiles>COc1ccc(N[C@@H](C)C(=O)N(C)C)c(OC)c1</smiles>

3aа

\begin{tabular}{|c|c|c|c|c|}
\hline Entry & $\mathrm{x}$ & Yield $(\%)^{\mathrm{b}}$ & Anti:syn ${ }^{\mathrm{c}}$ & $\operatorname{Ee}(\%, a n t i)$ \\
\hline 1 & 10 & 67 & $92: 8$ & 65 \\
\hline 2 & 0 & NR & - & - \\
\hline 3 & 5 & 7 & $92: 8$ & 71 \\
\hline 4 & 20 & 87 & $92: 8$ & 57 \\
\hline
\end{tabular}

${ }^{\text {a }}$ The reaction of $1 \mathrm{a}(0.5 \mathrm{mmol})$ with $2 \mathrm{a}(1.0 \mathrm{mmol})$ was conducted at $-78{ }^{\circ} \mathrm{C}$ for $18 \mathrm{~h}$ in toluene $(0.4 \mathrm{M})$ under Ar atmosphere in the presence of the catalyst prepared from KHMDS (2x mol\%) and L1 (10 mol\%). ${ }^{b}$ Isolated yield. ${ }^{c}$ Determined by ${ }^{1} \mathrm{H}$ NMR analysis of a crude mixture.

Optimization of the reaction conditions was conducted (Table S3). The effect of solvents on the reaction was first examined. While cyclopentyl methyl ether (CPME) and tert-butyl methyl ether (TBME) gave good 
enantioselectivities among the ether solvents, THF was found to be the best solvent. We then investigated potassium-Box salts K-L. While K-L2 and K-L4 were ineffective for this reaction, the other Box derivatives K-L3, K-L5-7 worked well, and good to high levels of enantioselectivities were obtained. Among them, the best K-Box was K-L1, which was selected for further investigations. Catalyst loading and the amount of $\mathbf{2 a}$ were optimized, and the use of 1.1 equivalents of $\mathbf{2 a}$ with 5 mol\% KHMDS and 5 mol\% K-L1 system was found to be the best condition. The use of excess KHMDS to K-L1 still gave good enantioselectivity. KHMDS itself also promoted the reaction, but the yield was very low.

Table S3 Optimization of reaction conditions
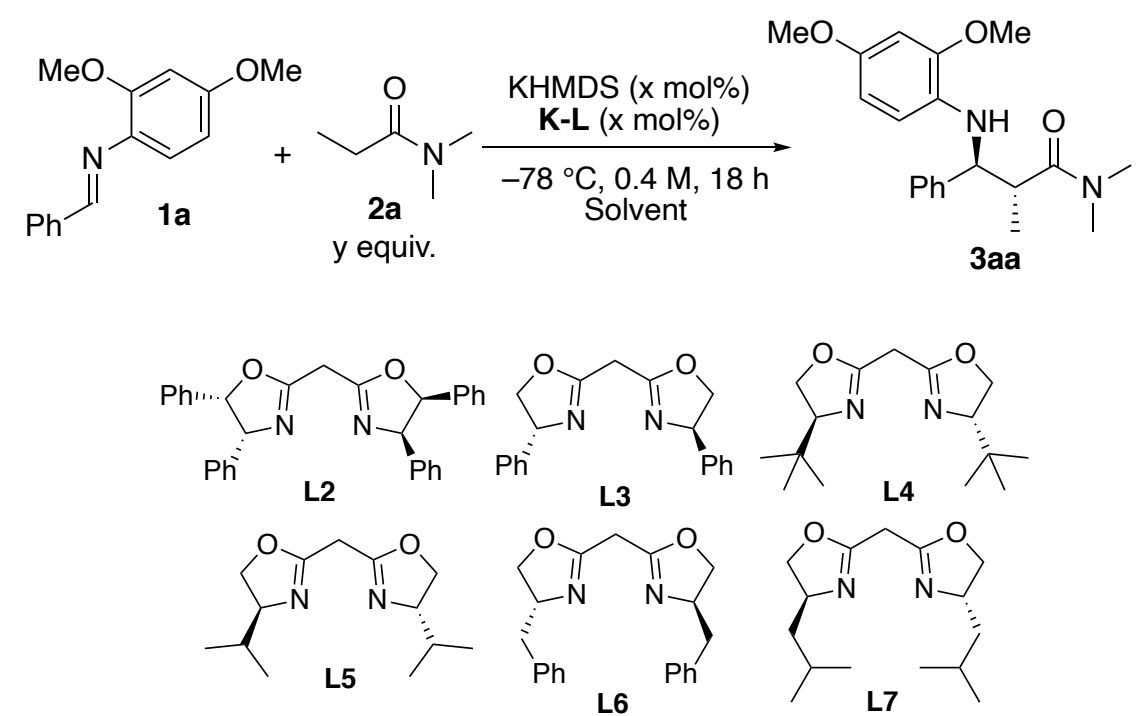

\begin{tabular}{llllllll}
\hline Entry & K-L & $\mathrm{x}$ & $\mathrm{y}$ & Solvent & Yield(\%) $^{\mathrm{a}}$ & Anti $_{\text {syn }}$ & Ee (\%, anti) \\
\hline 1 & K-L1 & 10 & 2.0 & THF & 98 & $99: 1$ & 91 \\
2 & K-L1 & 10 & 2.0 & toluene & 67 & $92: 8$ & 65 \\
3 & K-L1 & 10 & 2.0 & $\mathrm{Et}_{2} \mathrm{O}$ & 38 & $94: 6$ & 64 \\
4 & K-L1 & 10 & 2.0 & CPME & 21 & $93: 7$ & 82 \\
5 & K-L1 & 10 & 2.0 & TBME & 8 & $94: 6$ & 82 \\
6 & K-L2 & 10 & 2.0 & THF & 5 & $92: 8$ & 20 \\
7 & K-L3 & 10 & 2.0 & THF & quant. & $97: 3$ & 79 \\
8 & K-L4 & 10 & 2.0 & THF & 7 & $>99: 1$ & $73^{c}$ \\
9 & K-L5 & 10 & 2.0 & THF & 99 & $99: 1$ & $89^{c}$ \\
10 & K-L6 & 10 & 2.0 & THF & 94 & $98: 2$ & 87 \\
11 & K-L7 & 10 & 2.0 & THF & 96 & $98: 2$ & $82^{c}$ \\
12 & K-L1 & 5 & 2.0 & THF & 99 & $99: 1$ & 91 \\
13 & K-L1 & 5 & 1.5 & THF & 89 & $99: 1$ & 92 \\
14 & K-L1 & 5 & 1.1 & THF & 87 & $99: 1$ & 92 \\
$15^{\mathrm{d}}$ & K-L1 & 5 & 1.1 & THF & 96 & $99: 1$ & 92 \\
16 & K-L1 & $2^{\mathrm{e}}$ & 1.1 & THF & 83 & $99: 1$ & 89 \\
$17^{\mathrm{f}}$ & - & 10 & 2.0 & THF & 10 & $87: 13$ & - \\
\hline & & & & & & & \\
\hline
\end{tabular}


a Isolated yield. ${ }^{\mathrm{b}}$ Determined by ${ }^{1} \mathrm{H}$ NMR analysis of the crude mixture. ${ }^{\mathrm{c}}$ The opposite enantiomer was obtained. ${ }^{\mathrm{d}}$ For 24 h. ${ }^{\mathrm{e}}$ KHMDS ( 8 mol\%) and K-L1 ( 2 mol\%) were used. The catalyst was prepared from KHMDS (10 mol\%) and L1 (2 mol\%). ${ }^{\mathrm{f}}$ Without K-L.

The effect of other alkaline metal amides was examined (Table S4). When LiHMDS or NaHMDS was used, the reaction procceded sluggishly with very poor enantioselectivities (entries 2 and 3). On the other hand, the catalyst prepared from CsHMDS showed high reactivity; however, very poor enantioselectivity was obtained (entry 4). Those results indicated the KHMDS-K-Box system was the best catalyst for the reactions.

Table S4 Effect of other alkaline metals in the catalyst complex ${ }^{\text {a }}$
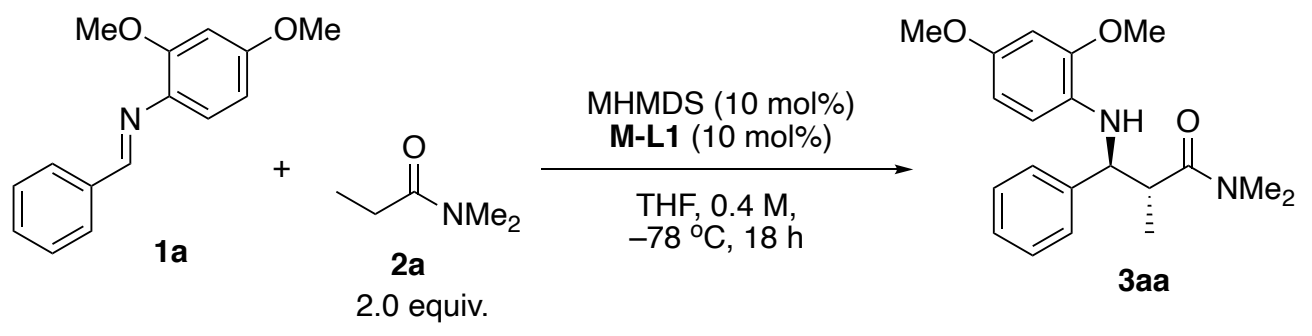

\begin{tabular}{ccccc}
\hline Entry & M & ${\text { Yield }(\%)^{\mathrm{b}}}^{\text {Anti:syn }}$ & Ee (\%, anti) \\
\hline 1 & $\mathrm{~K}$ & 98 & $99: 1$ & 91 \\
2 & $\mathrm{Li}$ & 3 & $67: 33$ & $3^{\mathrm{d}}$ \\
3 & $\mathrm{Na}$ & 4 & $94: 6$ & $3^{\mathrm{d}}$ \\
4 & $\mathrm{Cs}$ & 99 & $82: 18$ & 3 \\
\hline
\end{tabular}

a The reaction of $1 \mathbf{a}(0.6 \mathrm{mmol})$ with $\mathbf{2 a}(1.2 \mathrm{mmol})$ was conducted at $-78^{\circ} \mathrm{C}$ for $18 \mathrm{~h}$ in THF $(0.4 \mathrm{M})$ under Ar atmosphere in the presence of a catalyst consisting of MHMDS (20 mol\%) and L1 (10 mol\%) unless otherwise noted. The catalyst preparation conditions, $\mathrm{K}$ : at $0{ }^{\circ} \mathrm{C}$ for $0.5 \mathrm{~h}, \mathrm{Li}$ and $\mathrm{Na}$ : at $0{ }^{\circ} \mathrm{C}$ for $1.5 \mathrm{~h}, \mathrm{Cs}$ : at $-40{ }^{\circ} \mathrm{C}$ for $0.5 \mathrm{~h} .{ }^{\mathrm{b}}$ Isolated yield. ${ }^{\mathrm{c}}$ Determined by ${ }^{1} \mathrm{H}$ NMR analysis of a crude product mixture. ${ }^{\mathrm{d}}$ The opposite enantiomer was obtained.

Next, the reactions using hetero alkaline metal complexes were examined (Table S5). To avoid formation of the homo metal complexes, the catalysts were prepared by adding two metal amides separately following the scheme shown in Table S5. In fact, the mixed metal system did not work well, and lower reactivities and selectivities were obtained. It was found that the catalyst preparation conditions affected the selectivities. It might be considered that the metal exchange between $\mathbf{M}^{\mathbf{1}} \mathbf{- L 1}$ and $\mathrm{M}^{2} \mathrm{HMDS}$ occurred to form K-L1 and KHMDS for promotion of the reaction to some extent even when the metal amides were added separately.

Table S5 Effect of the complex prepared from different alkaline metals ${ }^{\mathrm{a}}$ 

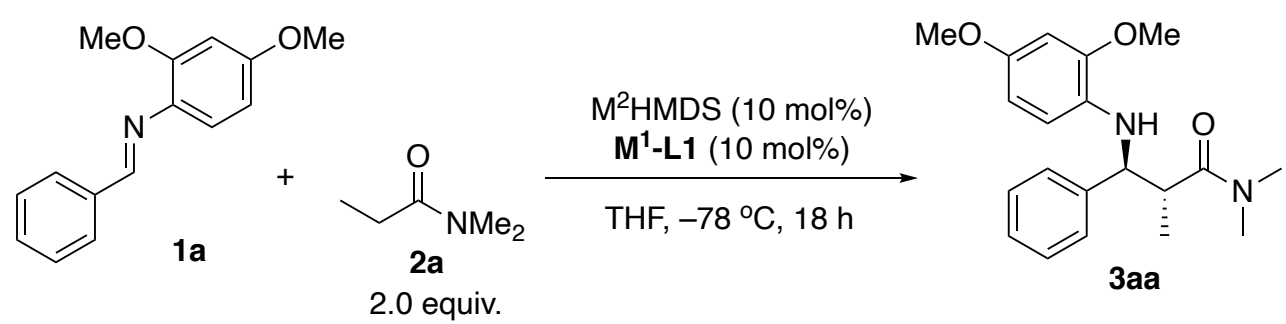

Catalyst preparation
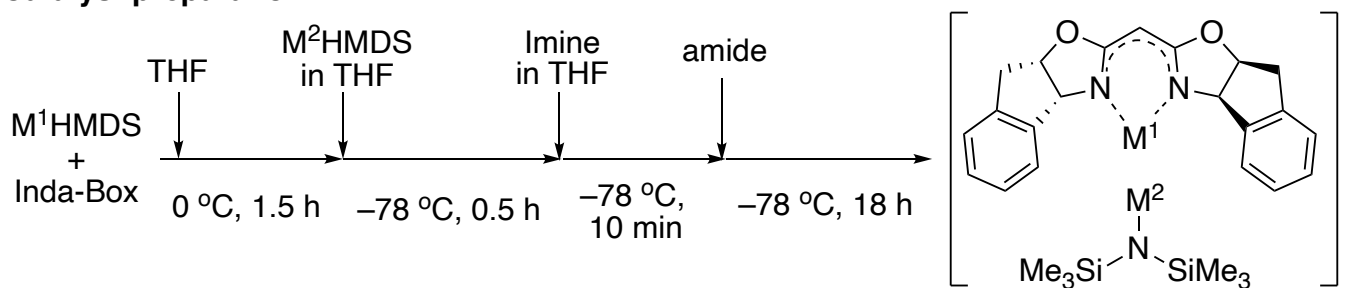

The target complex

\begin{tabular}{cccccc}
\hline Entry & $\mathrm{M}^{1}$ & $\mathrm{M}^{2}$ & ${\text { Yield }(\%)^{\mathrm{b}}}^{\text {Anti:syn }}$ & Ee (\%, anti) \\
\hline 1 & $\mathrm{~K}$ & $\mathrm{~K}$ & 95 & $99: 1$ & 91 \\
2 & $\mathrm{Li}$ & $\mathrm{K}$ & 16 & $93: 7$ & 76 \\
3 & $\mathrm{~K}$ & $\mathrm{Li}$ & 50 & $97: 3$ & 86 \\
4 & $\mathrm{Na}$ & $\mathrm{K}$ & 15 & $98: 2$ & 82 \\
5 & $\mathrm{~K}$ & $\mathrm{Na}$ & trace & - & - \\
6 & $\mathrm{Cs}$ & $\mathrm{K}$ & 40 & $83: 17$ & 23 \\
7 & $\mathrm{~K}$ & $\mathrm{Cs}$ & 83 & $87: 13$ & 54
\end{tabular}

a The reaction of $1 \mathbf{a}(0.6 \mathrm{mmol})$ with $\mathbf{2 a}(1.2 \mathrm{mmol})$ was conducted at $-78^{\circ} \mathrm{C}$ for $18 \mathrm{~h}$ in $\mathrm{THF}(0.4 \mathrm{M})$ under Ar atmosphere in the presence of a catalyst consisting of $\mathrm{M}^{1} \mathrm{HMDS}(10 \mathrm{~mol} \%)$ and $\mathrm{M}^{2} \mathrm{HMDS}(10 \mathrm{~mol} \%)$ and L1 (10 mol\%) unless otherwise noted. The catalyst was prepared by following the catalyst preparation method. ${ }^{b}$ Isolated yield. ${ }^{c}$ Determined by ${ }^{1} \mathrm{H}$ NMR analysis of a crude product mixture.

In the asymmetric Mannich reactions, N-2,4-dimethoxyphenyl (DMP) substituted imines showed high enantioselectivities. The reactions of other $N$-aryl substituted imines were also examined (Table S6). When $\mathrm{N}$-o-methoxypheny (OMP) substituted imine 1w was employed, good enantioselectivity was obtained but the yield decreased (entry 2). On the other hand, $N$ - $p$-methoxyphenyl substituted imine 1x was used, the yield and both the diastereo- and enantioselectivities decreased significantly (entry 3). Furthermore, when $\mathrm{N}$-o-ethylphenyl substituted imine $\mathbf{1 y}$ was employed, low enantioselectivity was obtained (entry 4). It was found that the reaction of $N$-phenyl substituted imine $\mathbf{1 z}$ proceeded but with low enantioselectivity (entry 5). $\mathrm{N}$-o-Chlorophenyl substituted imine 1za was employed, but low reactivity was obtained (entry 6). $\mathrm{N}$-o-hydroxyphenyl substituted imine 1zb did not work at all (entry 7). Those results suggested that the $o$-methoxy group on the phenyl ring was very important to form a good asymmetric environment.

Table S6 Effect of substituents on the $N$-aryl groups ${ }^{\text {a }}$ 


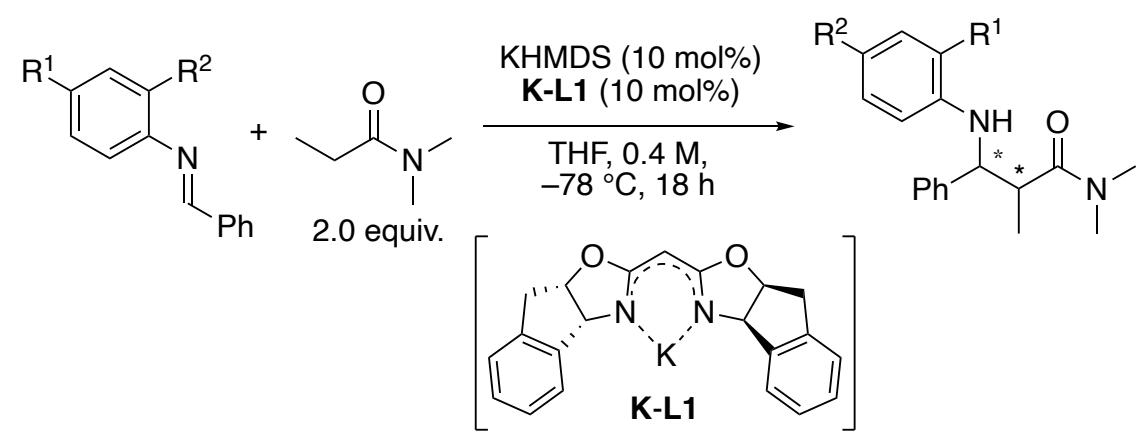

\begin{tabular}{|c|c|c|c|c|c|}
\hline Entry & $\mathrm{R}^{1}$ & $\mathrm{R}^{2}$ & Yield $(\%)^{b}$ & $\mathrm{Dr}^{\mathrm{c}}$ & Ee $(\%$, major $)$ \\
\hline 1 & $\mathrm{OMe}$ & $\mathrm{OMe}(\mathbf{1 a})$ & 99 & $99: 1$ & 91 \\
\hline 2 & $\mathrm{H}$ & $\mathrm{OMe}(\mathbf{1 w})$ & 41 & $96: 4$ & 87 \\
\hline 3 & $\mathrm{OMe}$ & $\mathrm{H}(\mathbf{1} \mathbf{x})$ & 53 & $80: 20$ & 35 \\
\hline 4 & $\mathrm{H}$ & $\operatorname{Et}(\mathbf{1 y})$ & 98 & $80: 20$ & 16 \\
\hline 5 & $\mathrm{H}$ & $\mathrm{H}(\mathbf{1 z})$ & 59 & $75: 25$ & 33 \\
\hline 6 & $\mathrm{H}$ & $\mathrm{Cl}$ (1za) & $<10$ & - & - \\
\hline 7 & $\mathrm{H}$ & $\mathrm{OH}(\mathbf{1 z b})$ & NR & - & - \\
\hline
\end{tabular}

${ }^{\text {a }}$ The reaction of $1(0.5 \mathrm{mmol})$ with $2 \mathrm{a}(1.0 \mathrm{mmol})$ was conducted at-78 ${ }^{\circ} \mathrm{C}$ for $18 \mathrm{~h}$ in $\mathrm{THF}(0.4 \mathrm{M})$ under Ar atmosphere in the presence of a catalyst consisting of KHMDS (20 mol\%) and L1 (10 mol\%) unless otherwise noted. ${ }^{b}$ Isolated yield. ${ }^{\mathrm{c}}$ Determined by ${ }^{1} \mathrm{H}$ NMR analysis of a crude product mixture.

In a catalytic cycle of the current reaction, there are two possible pathways to regenerate the active species, complex II (Main text, Chart 1). One is direct deprotonation of the next propionamide 2 by complex III to form desired product 3 and complex II (Chart 1, path A). The other is that protonation of complex III by H-HMDS to form KHMDS-K-Box complex I, then it deprotonates the next propionamide 2 to form complex II (Chart 1, path B). To investigate possibility of the path A, the asymmetric reaction was conducted using $\mathrm{KCH}_{2} \mathrm{SiMe}_{3}$ as a potassium base instead of KHMDS. The result was shown in Scheme S2. In fact, the result of the reaction using $\mathrm{KCH}_{2} \mathrm{SiMe}_{3}$ was almost the same to the reaction using KHMDS, which indicated that the path A was possible because acidity of the conjugate acid of $\mathrm{KCH}_{2} \mathrm{SiMe}_{3}\left(\mathrm{Me}_{4} \mathrm{Si}\right)$ should be very weak. However, we cannot exclude possibility of the path B when KHMDS was used. It is difficult to distinguish both pathways at the current stage.

Scheme S2 The reaction using $\mathrm{KCH}_{2} \mathrm{SiMe}_{3}$ 
<smiles>COc1ccc(/N=C/c2ccccc2)c(OC)c1</smiles><smiles>CCC(=O)N(C)C</smiles>

$\mathrm{K}$ base $(10 \mathrm{~mol} \%)$

K-L5 (10 mol\%)<smiles>COc1ccc(N[C@@H](c2ccccc2)C(C)C(=O)N(C)C)c(OC)c1</smiles>

3aa

2.0 equiv

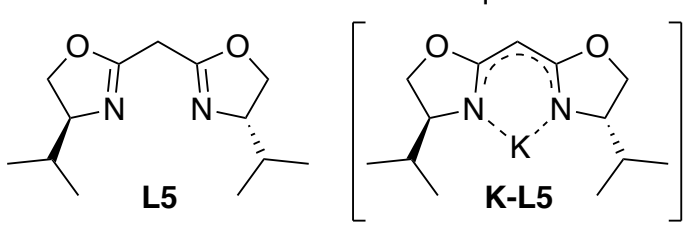

KHMDS: $99 \%$ yield, $d r=99: 1,89 \%$ ee

$\mathrm{KCH}_{2} \mathrm{SiMe}_{3}: 99 \%$ yield, $\mathrm{dr}=99: 1,90 \%$ ee 


\section{NMR studies}

\section{(1) Formation of K-Box species}

In this NMR study, ligand $\mathbf{L 5}$ was used instead of $\mathbf{L 1}$ due to instability of K-L1 at higher temperature ( $\sim \mathrm{rt})$ in the presence of a strong base. In the catalyst preparation, when KHMDS and ${ }^{i} \operatorname{PrBox}$ ligand (L5) were mixed, deprotonation of tethered methylene moiety of $\mathbf{L 5}$ should occur. In fact, it was supported by ${ }^{1} \mathrm{H}$ NMR experiments that the potassium salt of ${ }^{i} \operatorname{PrBox} \mathbf{L 5}$ (K-L5) formed in THF-d 8 (Chart S1 and S2). ${ }^{1} \mathrm{H}$ peaks of L5 changed after mixing with KHMDS, and newly appeared peaks were perfectly different from those of L5. Moreover, integration of the observed singlet peak around $3.2 \mathrm{ppm}$, which should be a hydrogen atom at the methylene tethered position, was about $1 \mathrm{H}$ when methyl peaks of ${ }^{i} \operatorname{Pr}$ groups were set in $6 \mathrm{H}$. Those observations indicated that deprotonation of $\mathbf{L 5}$ occurred to form K-L5. Furthermore, the catalyst solution was turned to be suspension when KHMDS and $\mathbf{L 5}$ were mixed in 2:1 ratio at $0{ }^{\circ} \mathrm{C}$ - $\mathrm{rt}$. The formed solid was collected and dried after centrifugation and removal of the solvent, and its ${ }^{1} \mathrm{H}$ NMR spectrum was collected. The observed peaks were almost the same to the peaks observed in the peaks in (b) but without any peak of $\mathrm{Me}_{3} \mathrm{Si}$ group of HMDS, which supported that the formed solid was K-L5. And it was also suggested that solubility of K-L5 in THF was low even at rt.

\section{Scheme S3}

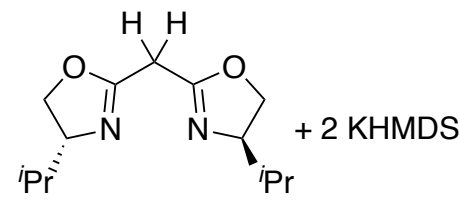

L5

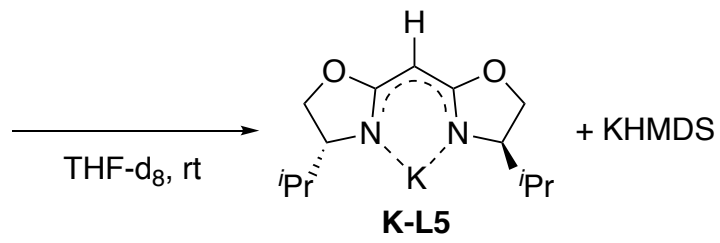




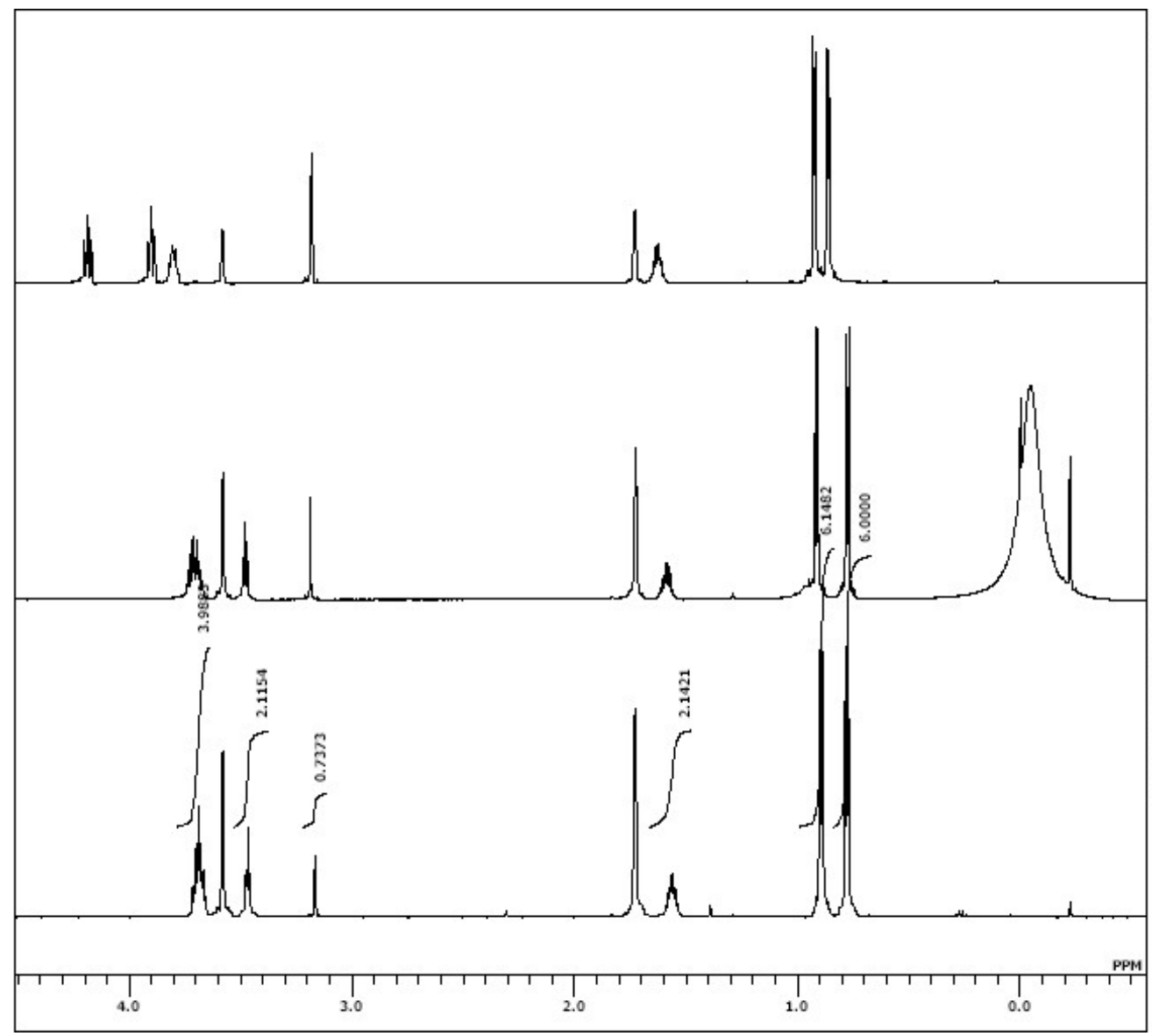

(a) ${ }^{i} \operatorname{PrBox}($ L5)

(b) L5-KHMDS (1:2)

(c) Solid collected from ${ }^{i}$ PrBox (L5)-KHMDS (1:2)

(K-i ${ }^{i}$ rBox complex, K-L5)

Chart S2 (in THF $-\mathrm{d}_{8}$ at $-78{ }^{\circ} \mathrm{C}$, concentration of $\mathbf{L 5}=0.03 \mathrm{M}$ )

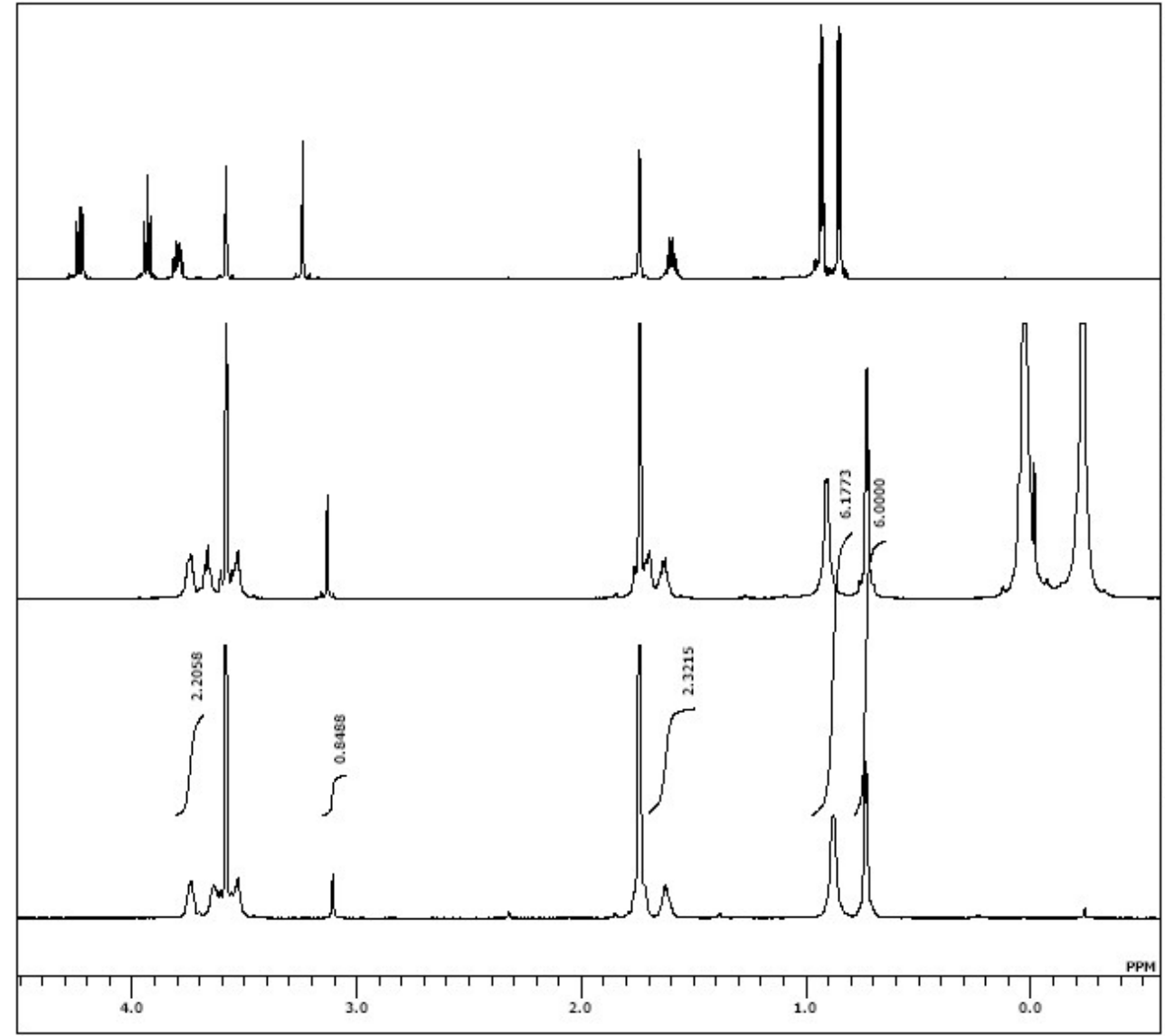

(a) ${ }^{i} \operatorname{PrBox}($ L5)

(b) L5-KHMDS (1:2)

(c) Solid collected from ${ }^{i} \operatorname{PrBox}($ L5)-KHMDS (1:2) (K- ${ }^{i}$ PrBox complex, K-L5) 


\section{(2) Interaction between KHMDS and K-Box species}

In the current catalyst system, one equivalent of KHMDS to the potassium salt of Box (K-Box) was required for the reaction. We assumed that the K-Box interacted with KHMDS to form a chiral Brønsted base complex. To investigate this possibility, the catalyst solutions in THF- $\mathrm{d}_{8}$ were prepared by mixing KHMDS and $\mathbf{L 5}$ in 2:1 - 10:1 ratio in the same scale, and their ${ }^{1} \mathrm{H}$ NMR spectra were collected at $-78{ }^{\circ} \mathrm{C}$ (Chart S3, spectra (b)-(d)). We observed one couple of the peaks derived from K-L5 on each chart, and gradual chemical shift changes of K-L5 peaks occurred when the amount of KHMDS was increased. Those results indicated that KHMDS and K-L5 interacted each other; however, the gradual and not significant chemical shift changes of the peaks suggested that the interaction was not significant and reversible under equilibrium conditions. Furthermore, the catalyst solution prepared by KHMDS and L5 in 2:1 ratio was suspension, in which the precipitate was K-L5 as mentioned before, but the solutions in 5:1 and 10:1 ratio were clear solutions at rt. This observation could also support that KHMDS and K-L5 complex interacted each other (Scheme S4).

\section{Scheme S4}

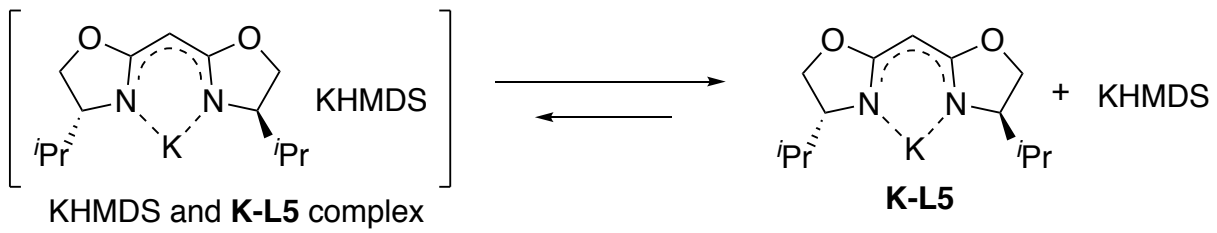

Chart S3 (in THF-d 8 at $-78^{\circ} \mathrm{C}$, concentration of $\mathbf{L 5}$ was $0.03 \mathrm{M}$ )

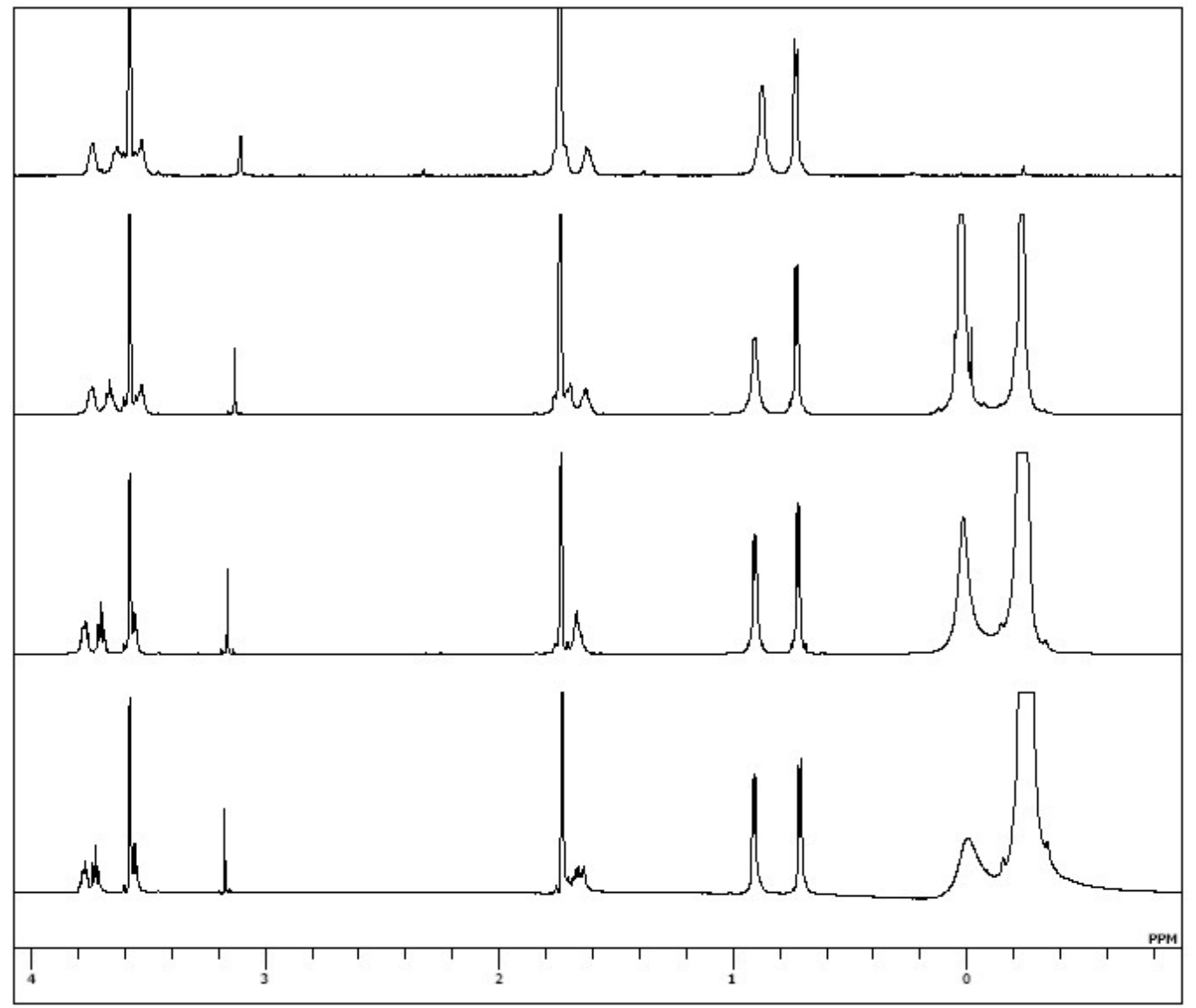

(a) Solid collected from ${ }^{i} \operatorname{PrBox}$ (L5)-KHMDS (1:2) (K-PrBox complex, K-L5)

(b) L5-KHMDS (1:2)

(c) L5-KHMDS (1:5)

(d) L5-KHMDS (1:10) 


\section{(3) Formation of potassium enolate of $N, N$-dimethylpropionamide}

Formation of a potassium enolate (K enolate) of $N, N$-dimethylpropionamide (2a) was investigated. 2a and KHMDS were mixed in 1:5 ratio in THF- $\mathrm{d}_{8}$, and ${ }^{1} \mathrm{H}$ NMR spectrum was collected at $-78{ }^{\circ} \mathrm{C}$ (Chart S4). However, no significant new peak was observed. On the other hand, new peaks were observed at higher temperature ( $\mathrm{rt}$ and $60{ }^{\circ} \mathrm{C}$ ), and the peaks were considered to be the corresponding $\mathrm{K}$ enolate. Those results indicated that the $\mathrm{K}$ enolate formed in the reaction system but the amount would be very small at $-78{ }^{\circ} \mathrm{C}$ (Scheme S5).

Scheme S5

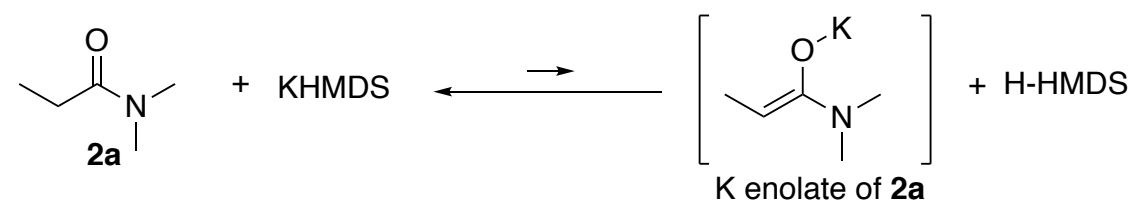

Chart S4 (in THF- $\mathrm{d}_{8}$, concentration of $2 \mathbf{a}=0.03 \mathrm{M}$ )

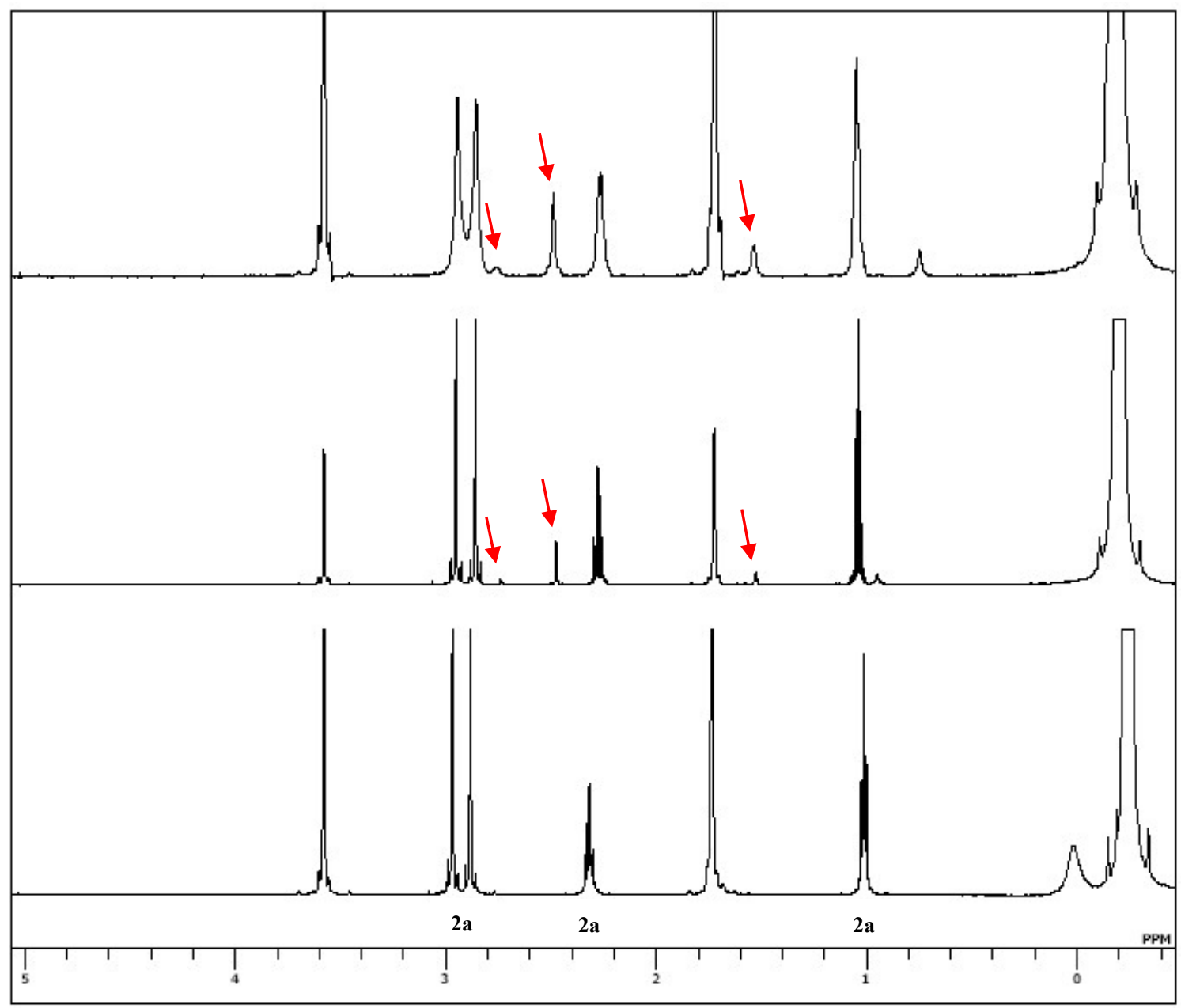

KHMDS+2a $(5: 1)$

At $60^{\circ} \mathrm{C}$

At rt

At $-78^{\circ} \mathrm{C}$

For further NMR experiments, the K enolate of 2a was prepared by using much stronger potassium bases, $\mathrm{KCH}_{2} \mathrm{Ph}$ and $\mathrm{KCH}_{2} \mathrm{SiMe}_{3}$. They were mixed with $\mathbf{2 a}$ in THF-d $\mathrm{d}_{8}$ in 1:1 ratio, and ${ }^{1} \mathrm{H}$ NMR spectra were collected at rt. As the result, a couple of new peaks were observed on the charts, which were corresponding to the pattern of $\mathrm{K} O$-enolate of 2 a (Chart S5). However, unreacted 2a was also observed. This might be due to instability of the $\mathrm{K}$ enolate at $\mathrm{rt}$ because integration of the peaks decreased after keeping the NMR samples at $\mathrm{rt}$. The ${ }^{1} \mathrm{H}$ NMR charts were also recorded at $-78{ }^{\circ} \mathrm{C}$. At $-78{ }^{\circ} \mathrm{C}$, the peaks 
turned to be broadened but could be identified (Chart S6).

Chart S5 (in THF-d 8 , concentration of $\mathbf{2 a}=0.03 \mathrm{M}$ )

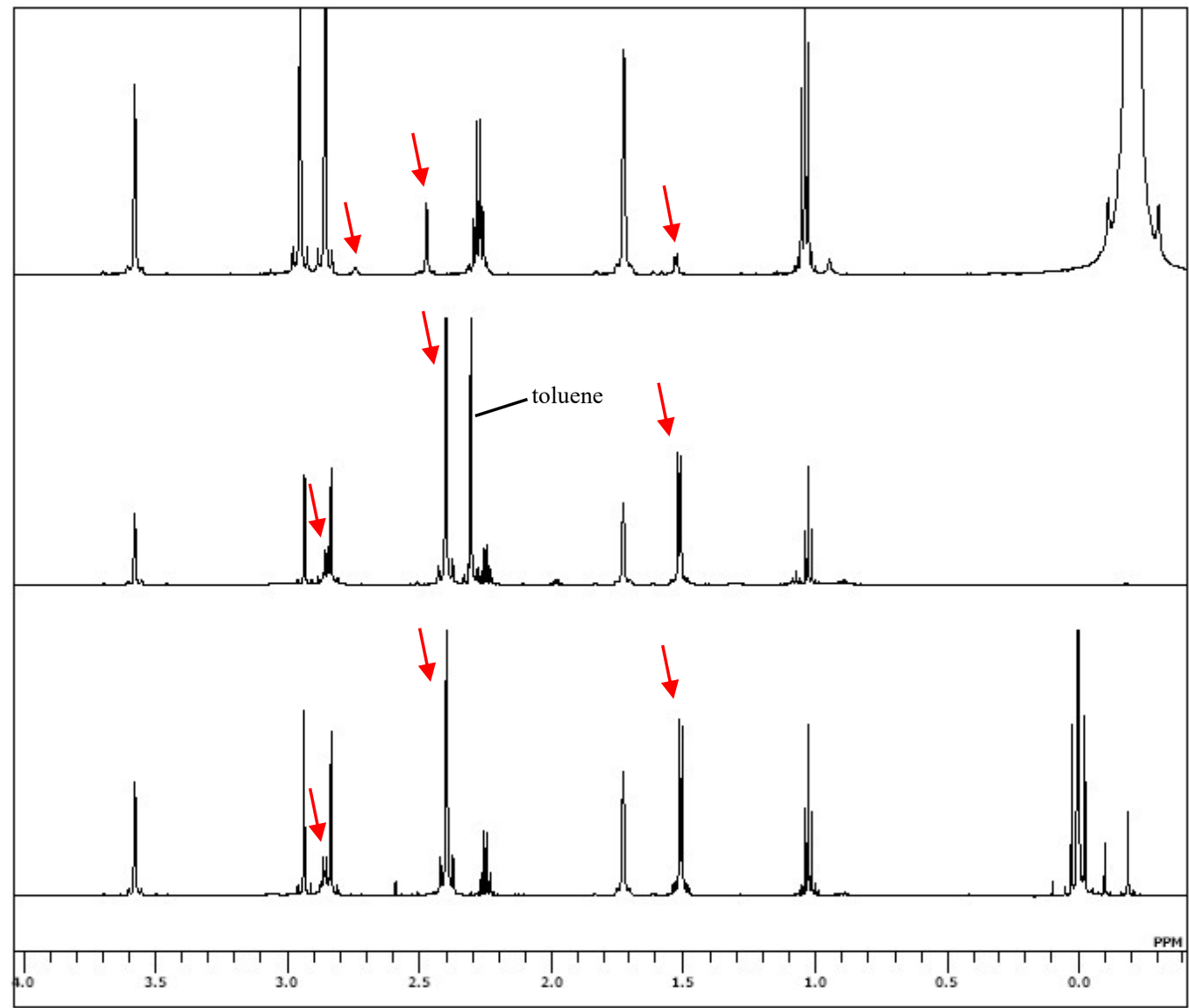

KHMDS $+\mathbf{2 a}$

$(5: 1)$ at $\mathrm{rt}$

$\mathrm{KCH}_{2} \mathrm{Ph}+\mathbf{2 a}$

$(1: 1)$ at $\mathrm{rt}$

$\mathrm{KCH}_{2} \mathrm{TMS}+\mathbf{2 a}$

$(1: 1)$ at $\mathrm{rt}$

Chart S6 (in THF- $\mathrm{d}_{8}$, concentration of $\mathbf{2 a}=0.03 \mathrm{M}$ )

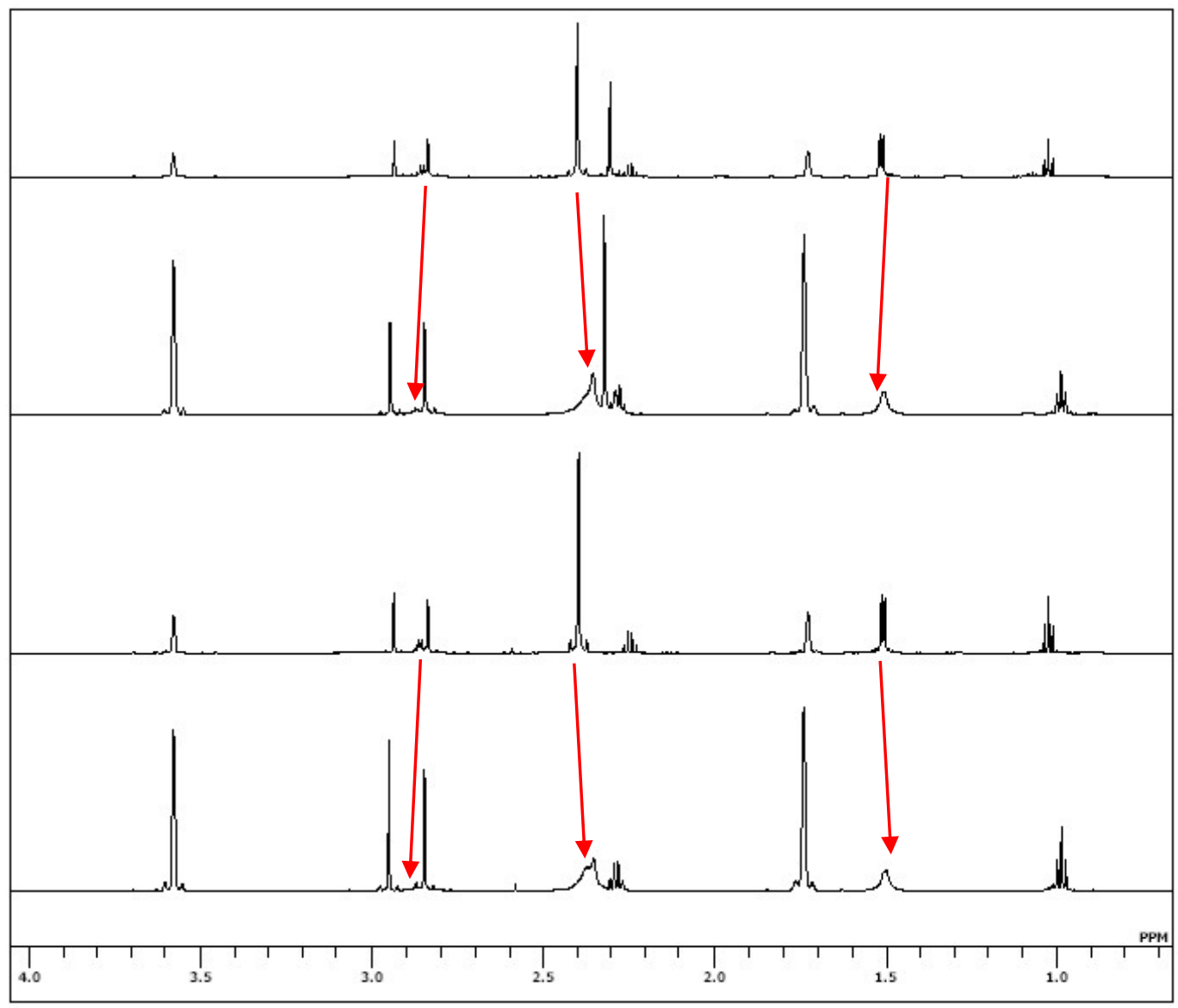

$\mathrm{KCH}_{2} \mathrm{Ph}+\mathbf{2 a}(1: 1)$ at $\mathrm{rt}$

$\mathrm{KCH}_{2} \mathrm{Ph}+\mathbf{2 a}(1: 1)$ at $-78^{\circ} \mathrm{C}$

$\mathrm{KCH}_{2} \mathrm{TMS}+\mathbf{2 a}$

(1:1) at rt

$\mathrm{KCH}_{2} \mathrm{TMS}+\mathbf{2 a}$

$(1: 1)$ at $-78^{\circ} \mathrm{C}$ 


\section{(4) Interaction between $K$ enolate and $K-{ }^{i} \operatorname{PrBox}$ (L5) species}

Next, interaction between the K enolate of $\mathbf{2 a}$ and K-L5 was investigated. $\mathrm{KCH}_{2} \mathrm{SiMe}_{3}$ and $\mathbf{~} \mathbf{5}$ and $\mathbf{2 a}$ were mixed in 2:1:1 ratio in THF- $\mathrm{d}_{8}$, and ${ }^{1} \mathrm{H}$ NMR spectra were recorded at $\mathrm{rt}$ and $-78{ }^{\circ} \mathrm{C}$ (Chart S7 and S8). It was found that peaks of the K enolate with K-L5 ( $\boldsymbol{\nabla})$ were clearly different from those of the free $\mathrm{K}$ enolate ( $\square$ ) and that the peaks of K-L5 (O) also changed a little. Those observations could suggest that the K enolate and K-L5 interacted each other to form a K enolate-K-L5 complex (Scheme S6). Furthermore, when $\mathrm{KCH}_{2} \mathrm{SiMe}_{3}$ and $\mathbf{L 5}$ and $\mathbf{2 a}$ were mixed in 3:1:2 ratio in THF- $\mathrm{d}_{8}$, which mean that the amount of the $\mathrm{K}$ enolate was more than that of the K-L5, peaks of the free $\mathrm{K}$ enolate was observed at $-78{ }^{\circ} \mathrm{C}$. This observation could support that the K-enolate-K-L5 complex was composed of equal amounts of the K-enolate and K-L5 (1:1 ratio). On the other hand, peaks of the free K enolate was not observed at rt. This result might be due to that the free $\mathrm{K}$ enolate and the K-enolate-K-L5 complex were under fast equilibrium at higher temperature.

\section{Scheme S6}
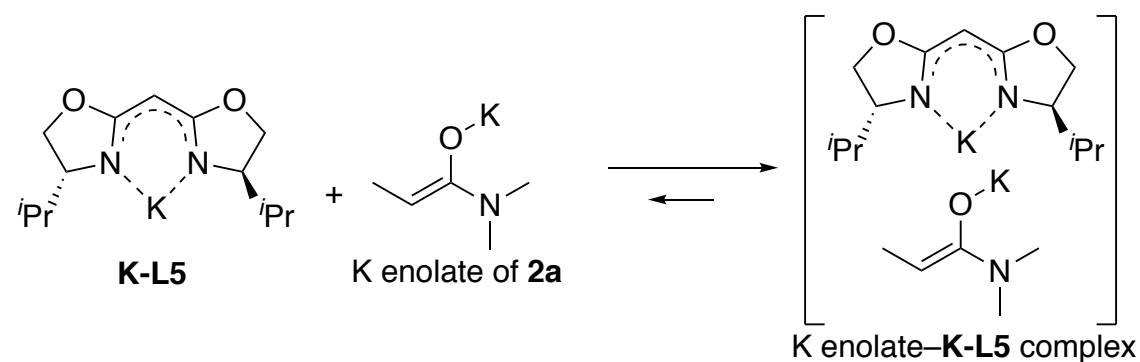

In summary, it was suggested that interaction between KHMDS and K-L5 existed in the catalyst solution but it was not significant. On the other hand, it was also suggested that significant interaction between the K enolate and K-L5 could exist in the reaction solution. Those results could support that a K-enolate-K-L5 complex formed and worked as a chiral active species. However, structure of the K-enolate-K-L5 complex is difficult to be predicted. Crystal formation of the complexe for X-ray single crystal structure analysis was not successful. To clarify the structure of the K-enolate-K-Box species, a calculation study was conducted (next section). 


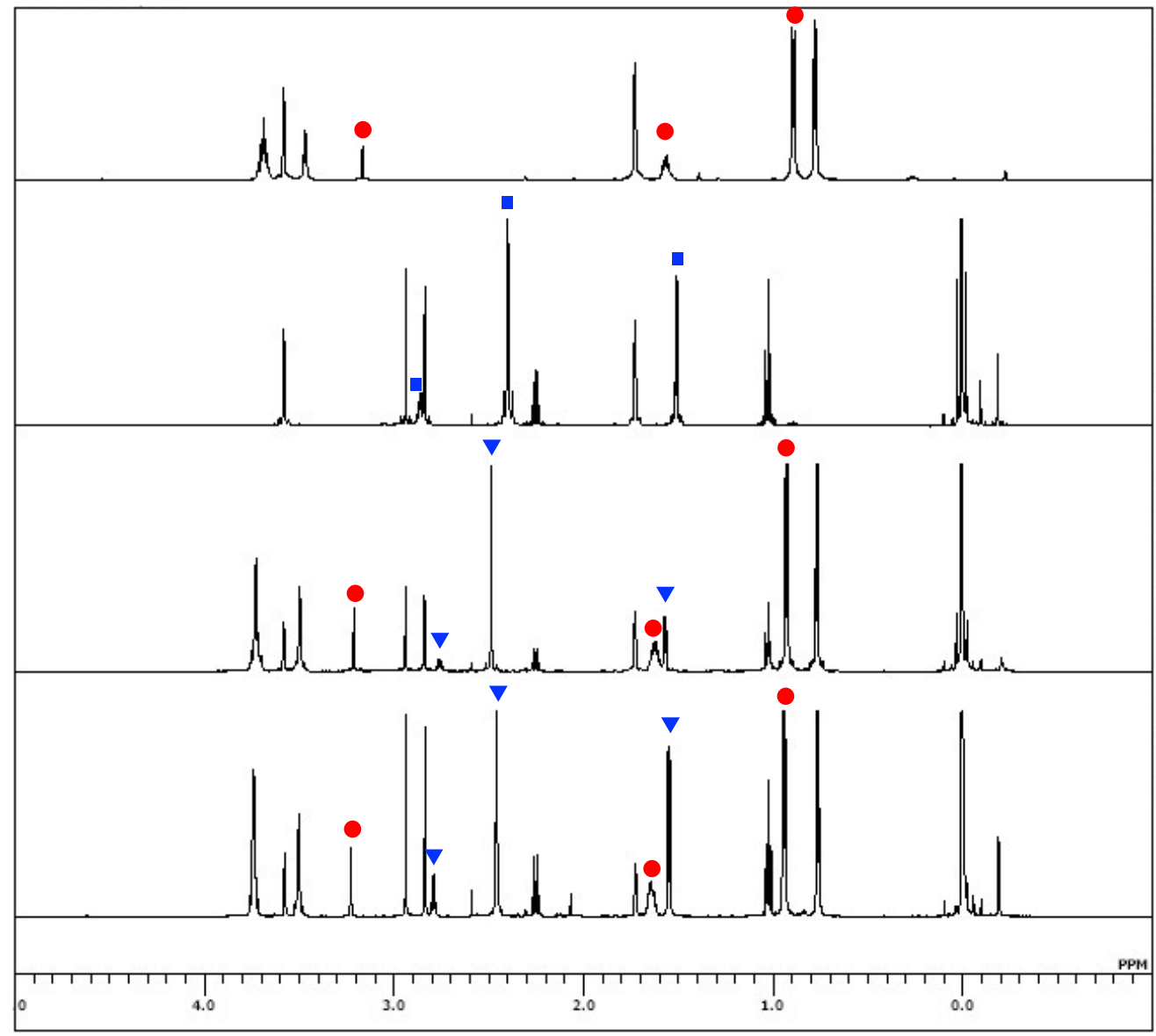

K-L5

$\mathrm{KCH}_{2} \mathrm{TMS}+\mathbf{2 a}$

$(1: 1)$

(K enolate of 2a)

$\mathrm{KCH}_{2} \mathrm{TMS}+\mathbf{L 5}+\mathbf{2 a}$

$(2: 1: 1)$

$(\mathrm{K}$ enolate $+\mathbf{K}-\mathbf{L 5})$

$\mathrm{KCH}_{2} \mathrm{TMS}+\mathbf{L 5}+\mathbf{2 a}$

$(3: 1: 2)$

$(\mathrm{K}$ enolate $+\mathbf{K}-\mathbf{L 5})$

Chart S8 (at $-78^{\circ} \mathrm{C}$ in THF- $\mathrm{d}_{8}$, concentration of $\mathbf{L 5}$ and $\mathbf{2 a}=$ both $0.03 \mathrm{M}$ )

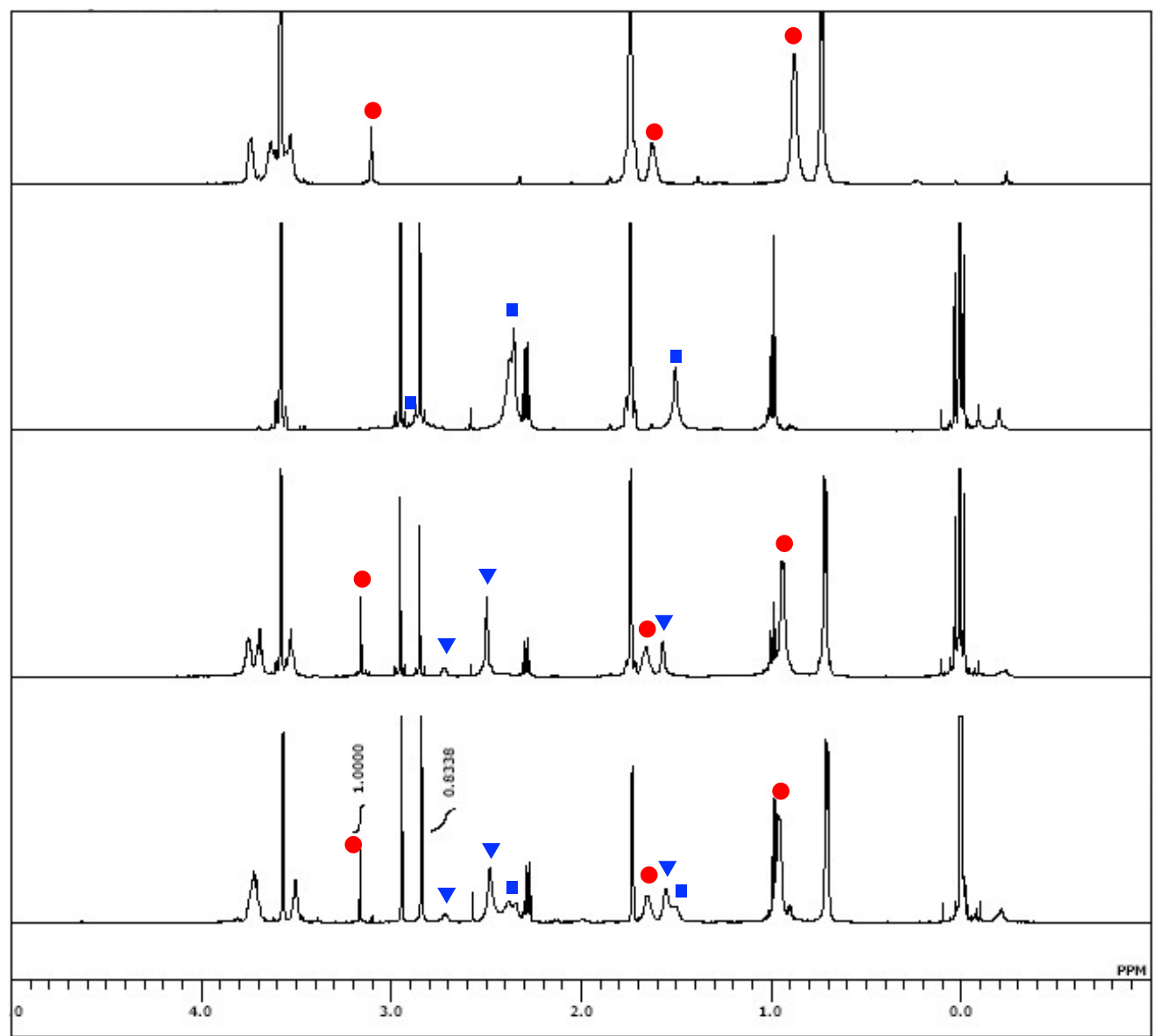

K-L5

$\mathrm{KCH}_{2} \mathrm{TMS}+\mathbf{2 a}$

(1:1)

(K enolate of 2a)

$\mathrm{KCH}_{2} \mathrm{TMS}+\mathbf{L 5}+\mathbf{2 a}$

$(2: 1: 1)$

$(\mathrm{K}$ enolate $+\mathbf{K}-\mathbf{L 5})$

$\mathrm{KCH}_{2} \mathrm{TMS}+\mathbf{L 5}+\mathbf{2 a}$

(3:1:2)

$(\mathrm{K}$ enolate $+\mathbf{K}$-L5) 


\section{Calculation study}

The structures of the active chiral Brønsted base species were searched using an automated reaction path search method called the multicomponent artificial force-induced reaction (MC-AFIR) method. We prepared 80 initial structures with the random orientations of $\mathrm{K}$ enolate and $\mathbf{K}-\mathbf{L} \mathbf{1}$, and applied the artificial forces between them. All the obtained structures were fully reoptimized without the artificial force. The MC-AFIR calculations and geometry optimizations were performed at the B3LYP-D3 level of theory ${ }^{1-3}$ with the basis set of $\mathrm{LanL} 2 \mathrm{DZ}{ }^{4}$ for $\mathrm{K}$ and $6-31 \mathrm{G}(\mathrm{d})^{5,6}$ for others. The Gibbs free energy correction terms were evaluated at $-78^{\circ} \mathrm{C}$ and $1 \mathrm{~atm}$. The solvation effects were included by the polarized continuum model (PCM) ${ }^{7}$ with a dielectric constant of 7.4257 (THF). All the MC-AFIR, reoptimization, and frequency calculations were performed by the GRRM program ${ }^{8,9}$ using energies and energy derivatives computed by the Gaussian09 program. ${ }^{10}$

The structures and the Gibbs free energy distribution of all the structures were shown in Figure S1. It was suggested that the two potassium ions were sited between the two nitrogen atoms of $\mathbf{L 1}$ symmetrically and that the enolate was sited between the potassium ions. This symmetric structure could be also supported by the
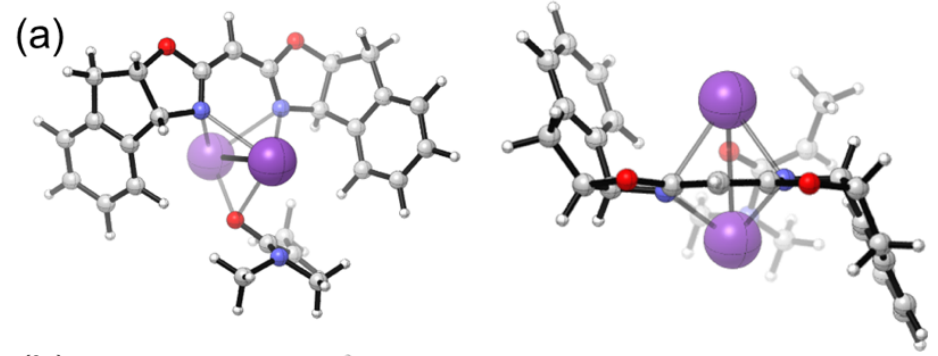

(b)
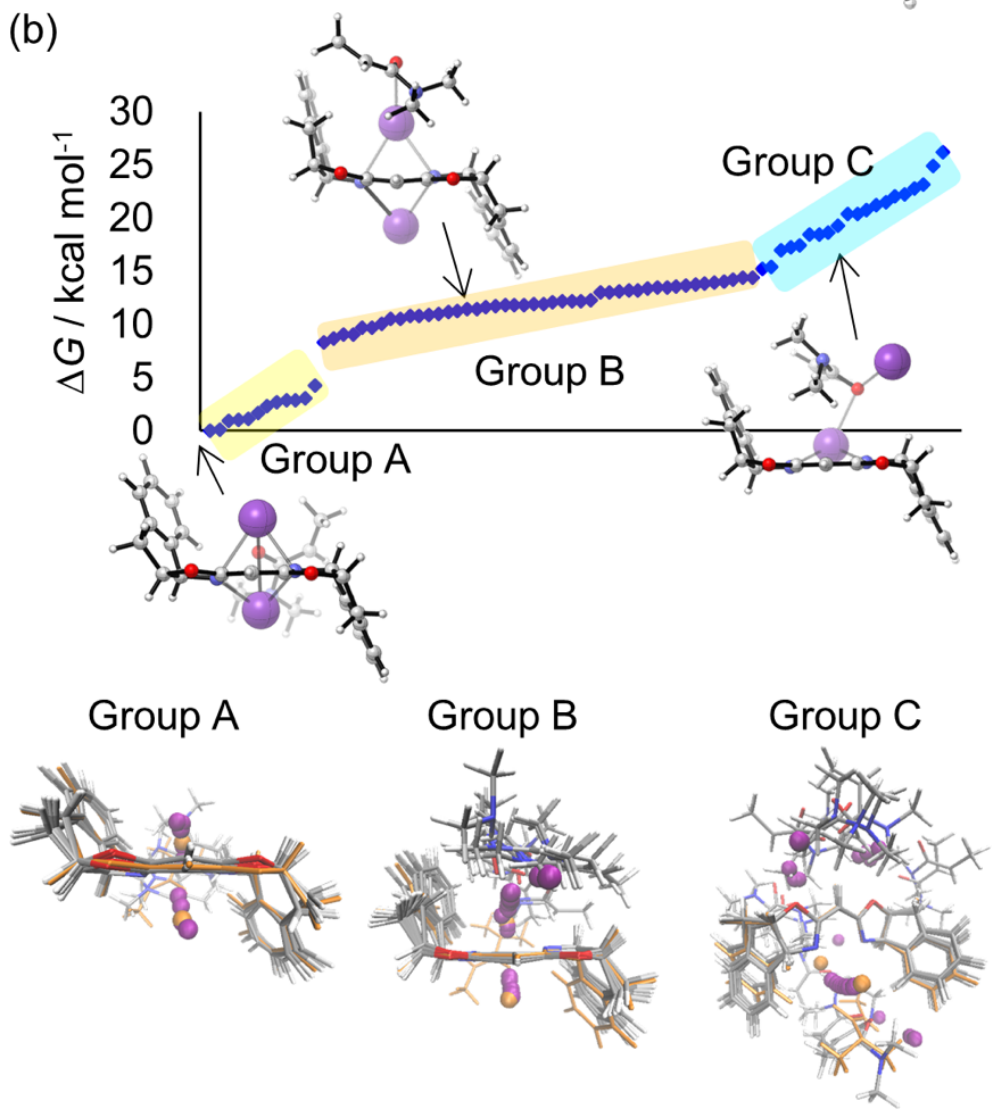

Figure S1 (a) Front and side views of the most stable structure of the K enolate-K-L1 complex and (b) the 
energy distribution of the other structures.

NMR study using L5, in which only one couple of the peaks of the K-L5 (ex. two kinds of Me peaks of the ${ }^{i} \mathrm{Pr}$ group) was observed in the presence of the K enolate. If the structure were not symmetric, two couples of the peaks of the K-L5 (ex. four kinds of Me peaks of the ${ }^{i} \operatorname{Pr}$ group) would be observed.

The KHMDS and K-L1 complex was also calculated under the same conditions, and a similar structure was observed (Figure S2). However, it was found that the KHMDS and K-L1 complex was $2.6 \mathrm{kcal} \mathrm{mol}^{-1}$ less stable than that of the K enolate-K-L1 complex due to steric repulsion between $\mathrm{Me}_{3} \mathrm{Si}$ groups of KHMDS and the aryl ring of $\mathbf{L 1}$. This tendency was also corresponding to results of the NMR study using $\mathbf{L 5}$, in which complexation of KHMDS and K-L5 was not perfect and under equilibrium.

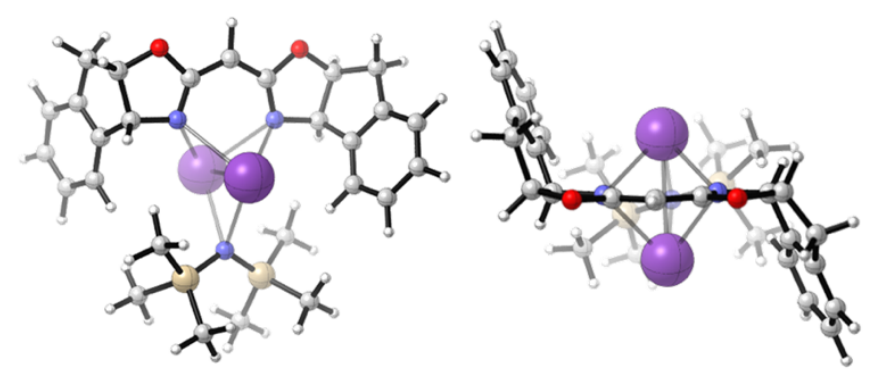

Figure S2 Front and side views of the most stable structure of the KHMDS and K-L1 complex

To understand the origin of the stability of the K enolate-K-L1 complex, the interaction energy between the K-L1 and enolate were decomposed using the natural energy decomposition analysis (NEDA). ${ }^{11-13}$ The total interaction energy $E$ (total) can be decomposed into three terms, which are the electrical term $E(\mathrm{el})$, the charge transfer term $E(\mathrm{ct})$, and the core term $E(\mathrm{cr})$. As shown in Table S7, the total interaction energy between the K-L1 and enolate mainly attributes to the electrical term, which means that the electrostatic interaction stabilized the $\mathrm{K}$ enolate-K-L1 complex. The strong electrostatic interaction between the K-L1 and enolate can be also confirmed from the highly positively charged $\mathrm{K}$ atoms and negatively charged enolate $\mathrm{O}$ atom (Figure S3).

Table S7. The interaction energy between the K-L1 and enolate and its components ( $\left.\mathrm{kcal} \mathrm{mol}^{-1}\right)$

\begin{tabular}{|c|c|c|c|}
\hline$E($ total $)$ & $E(\mathrm{ele})$ & $E(\mathrm{ct})$ & $E(\mathrm{cr})$ \\
\hline-130.1 & -150.5 & -25.9 & 46.3 \\
\hline
\end{tabular}




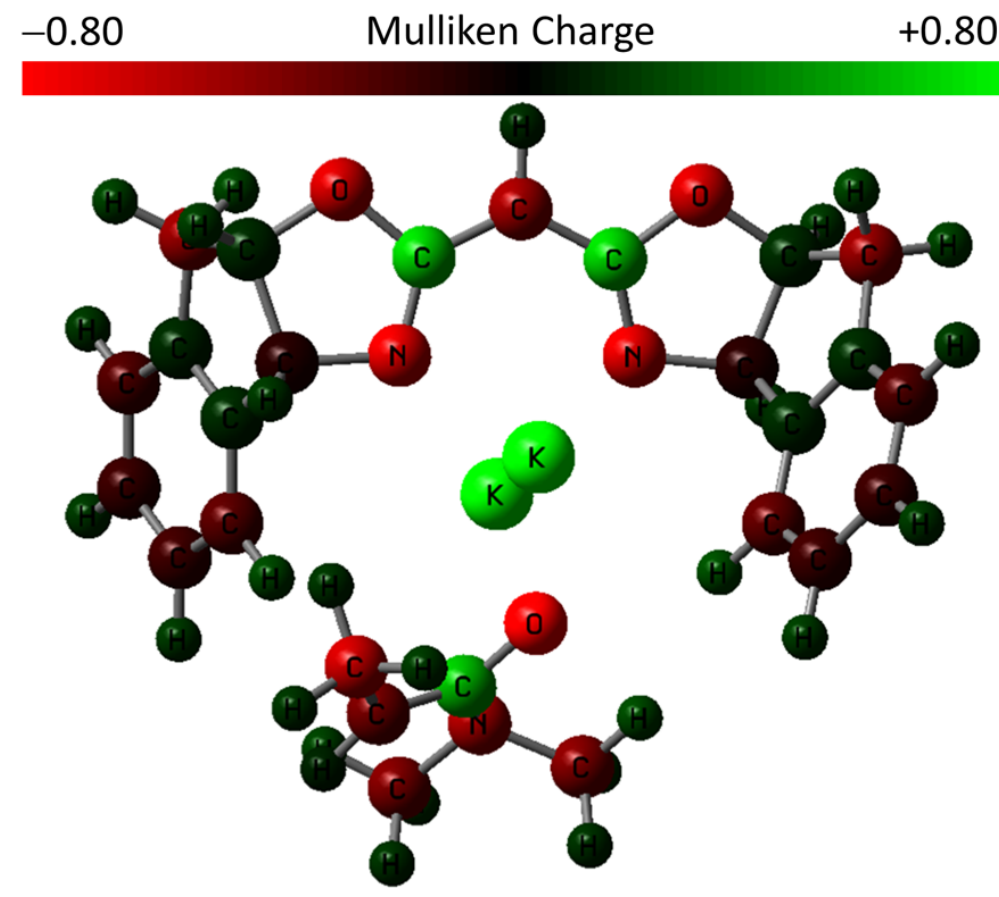

Figure S3 Mulliken charge distribution of the K enolate-K-L1 complex. Light green and red represent positively and negatively charged atoms, respectively. 


\section{Experimental Section}

\section{General}

Melting points were measured with a YAZAWA micro melting point BY-1 apparatus and are uncorrected. ${ }^{1} \mathrm{H}$ and ${ }^{13} \mathrm{C}$ NMR spectra were recorded on JEOL JNM-ECA500 and JNM-ECX600 spectrometers in $\mathrm{CDCl}_{3}$ unless otherwise noted. Tetramethylsilane (TMS) served as internal standard $(\delta=0)$ for ${ }^{1} \mathrm{H}$ NMR, and $\mathrm{CDCl}_{3}$ served as internal standard $(\delta=77.0)$ for ${ }^{13} \mathrm{C}$ NMR. Benzotrifluoride (BTF) served as internal standard $(\delta=-63.72)$ for ${ }^{19} \mathrm{~F}$ NMR. When $\mathrm{C}_{6} \mathrm{D}_{6}$ was used as a solvent in NMR analysis, $\mathrm{C}_{6} \mathrm{H}_{6}$ served as internal standard for ${ }^{1} \mathrm{H}$ NMR $(\delta=7.15)$ and ${ }^{13} \mathrm{C}$ NMR $(\delta=128.0)$. IR spectra were measured by JASCO FT/IR-4200 spectrometer. Preparative thin-layer chromatography (PTLC) was carried out using Wakogel B-5F. Potassium hexamethyldisilazide (KHMDS) sodium hexamethyldisilazide (NaHMDS), and lithium hexamethyldisilazide (LiHMDS) were purchased from Aldrich Co., Ltd. Benzylpotassium (KBn) ${ }^{14}$ and Trimethylsilylmethylpotassium ( $\left.\mathrm{KCH}_{2} \mathrm{TMS}\right){ }^{15}$ and cesium hexamethyldisilazide (CsHMDS) ${ }^{16}$ were prepared according to the literature. All imines were prepared from corresponding amines and corresponding aldehydes in $\mathrm{CH}_{2} \mathrm{Cl}_{2}$ (DCM) at room temperature in the presence of MS 4A. N,N-Dimethylpropionamide 2a, $N, N$-dimethyacetoamide $\mathbf{2 f}$, and $N, N$-diethylpropionamide $\mathbf{2 i}$ were purchased from Tokyo Chemical Industry Co., Ltd. The other amides (2b-j) were prepared from corresponding acid chlorides and dimethylamine hydrochloride salt following a typical procedure. Chiral Box ligands L1-L7 were prepared by following literature methods. ${ }^{17-22} \mathrm{THF}$, diethyl ether $\left(\mathrm{Et}_{2} \mathrm{O}\right)$ and toluene were purified by Ultimate Solvent System 3S-TSK (Nikko Hansen \& co., ltd.) and used directly. CPME and TBME were distilled before using in the presence of benzophenone and sodium. Relative and absolute configuration of $\mathbf{3 n a}$ was determined by $\mathrm{X}$-ray single crystal structure analysis. Relative and absolute configuration of other products were determined by analogy to 3 na.

\section{Typical experimental procedure for synthesis of 2,4-dimethoxyphenylimine 1a}

2,4-Dimethoxyaniline (3.06 g, $20.0 \mathrm{mmol})$ and benzaldehyde $(3.49 \mathrm{~mL}, 20.0 \mathrm{mmol})$ were added to DCM $(20 \mathrm{~mL})$ with MS 4A. The whole mixture was stirred for $18 \mathrm{~h}$ at room temperature, and then filtered through a Celite pad. The filtrate was concentrated under reduced pressure, and the crude oil obtained was purified by distillation to afford 1a as a yellow oil (4.94 g, 78\% yield).

\section{$\mathrm{N}$-(2,4-dimethoxyphenyl)-benzaldehyde imine (1a)}

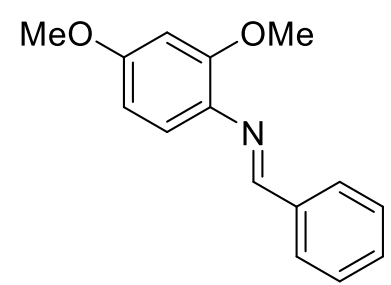

${ }^{1} \mathrm{H}$ NMR $\left(500 \mathrm{MHz}, \mathrm{CDCl}_{3}\right) \delta: 8.50(1 \mathrm{H}, \mathrm{s}), 7.90(2 \mathrm{H}, \mathrm{dd}, \mathrm{J}=6.88,2.88 \mathrm{~Hz})$, 7.45-7.44 (3H, m), $7.01(1 \mathrm{H}, \mathrm{d}, \mathrm{J}=8.59), 6.55(1 \mathrm{H}, \mathrm{d}, 2.28 \mathrm{~Hz}), 6.45(1 \mathrm{H}, \mathrm{dd}, \mathrm{J}=$ 8.59, $2.28 \mathrm{~Hz}), 3.88(3 \mathrm{H}, \mathrm{s}), 3.83(3 \mathrm{H}, \mathrm{s}) ;{ }^{13} \mathrm{C} \mathrm{NMR}\left(\mathrm{CDCl}_{3}, 100 \mathrm{MHz}\right) \delta: 159.5$, $159.1,153.7,136.6,135.1,130.9,128.7,128.6,120.5,104.3,99.5,55.9,55.5 ;$ IR (neat, $\mathrm{cm}^{-1}$ ) 1624, 1579, 1501, 1451, 1302, 1294, 1205, 1157, 1127, 1030; HRMS (Dart) calcd for $\mathrm{C}_{15} \mathrm{H}_{16} \mathrm{NO}_{2}[\mathrm{M}+\mathrm{H}]^{+}$242.1181, found 242.1175 .

\section{$N$-(2,4-dimethoxyphenyl)-p-tolaldehyde imine (1b)}

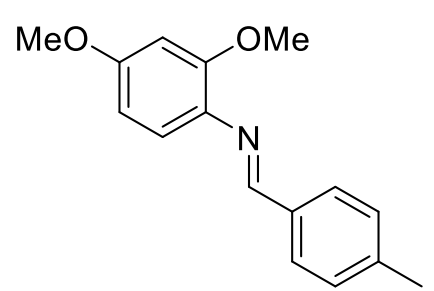

m.p. 57-58 ${ }^{\circ} \mathrm{C} ;{ }^{1} \mathrm{H}$ NMR $\left(500 \mathrm{MHz}, \mathrm{CDCl}_{3}\right) \delta: 8.46(1 \mathrm{H}, \mathrm{s}), 7.79(2 \mathrm{H}, \mathrm{d}, \mathrm{J}=7.94$ $\mathrm{Hz}), 7.25(2 \mathrm{H}, \mathrm{d}, \mathrm{J}=7.94 \mathrm{~Hz}), 7.00(1 \mathrm{H}, \mathrm{d}, \mathrm{J}=8.50 \mathrm{~Hz}), 6.54(1 \mathrm{H}, \mathrm{d}, \mathrm{J}=2.55$ $\mathrm{Hz}), 6.49(1 \mathrm{H}, \mathrm{dd}, \mathrm{J}=8.50,2.55 \mathrm{~Hz}), 3.87(3 \mathrm{H}, \mathrm{s}), 3.82(3 \mathrm{H}, \mathrm{s}), 2.40(3 \mathrm{H}, \mathrm{s}) ;{ }^{13} \mathrm{C}$ NMR $\left(\mathrm{CDCl}_{3}, 150 \mathrm{MHz}\right) \delta: 159.5,158.9,153.7,141.3,135.3,134.0,129.3$, 
128.7, 120.3, 104.2, 99.5, 55.9, 55.5, 21.6; IR (neat, $\mathrm{cm}^{-1}$ ) 1622, 1604, 1584, 1498, 1448, 1257, 1205, 1157, 1130, 1033; HRMS (Dart) calcd for $\mathrm{C}_{16} \mathrm{H}_{18} \mathrm{NO}_{2}[\mathrm{M}+\mathrm{H}]^{+} 256.1338$, found 256.1347.

\section{$N$-(2,4-dimethoxyphenyl)-m-tolaldehyde imine (1c)}

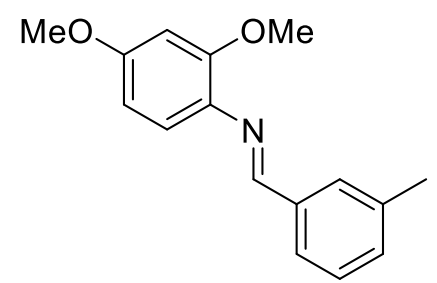

${ }^{1} \mathrm{H}$ NMR (500 MHz, $\left.\mathrm{CDCl}_{3}\right) \delta: 8.47(1 \mathrm{H}, \mathrm{s}), 7.79(1 \mathrm{H}, \mathrm{s}), 7.64(1 \mathrm{H}, \mathrm{d}, \mathrm{J}=7.30$ $\mathrm{Hz}), 7.33(1 \mathrm{H}, \mathrm{t}, \mathrm{J}=7.30 \mathrm{~Hz}), 7.25(1 \mathrm{H}, \mathrm{d}, \mathrm{J}=7.30 \mathrm{~Hz}), 7.00(1 \mathrm{H}, \mathrm{d}, \mathrm{J}=8.50$ $\mathrm{Hz}), 6.54(1 \mathrm{H}, \mathrm{d}, \mathrm{J}=2.27 \mathrm{~Hz}), 6.49(1 \mathrm{H}, \mathrm{dd}, \mathrm{J}=8.50,2.83 \mathrm{~Hz}), 3.88(3 \mathrm{H}, \mathrm{s})$, $3.82(3 \mathrm{H}, \mathrm{s}), 2.41(3 \mathrm{H}, \mathrm{s}) ;{ }^{13} \mathrm{C} \mathrm{NMR}\left(\mathrm{CDCl}_{3}, 150 \mathrm{MHz}\right) \delta: 159.8,159.0,153.7$, $138.4,136.5,135.1,131.8,128.7,128.5,126.4,120.4,104.3,99.5,55.9,55.5$, 21.2; IR (neat, $\mathrm{cm}^{-1}$ ) 1622, 1584, 1500, 1285, 1204, 1158, 1125, 1030; HRMS (Dart) calcd for $\mathrm{C}_{16} \mathrm{H}_{18} \mathrm{NO}_{2}$ $[\mathrm{M}+\mathrm{H}]^{+} 256.1338$, found 256.1348.

\section{$\mathrm{N}$-(2,4-dimethoxyphenyl)-o-tolaldehyde imine (1d)}

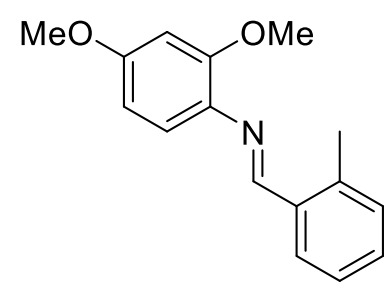

${ }^{1} \mathrm{H}$ NMR $\left(600 \mathrm{MHz}, \mathrm{CDCl}_{3}\right) \delta: 8.79(1 \mathrm{H}, \mathrm{s}), 8.11(1 \mathrm{H}, \mathrm{d}, \mathrm{J}=7.48 \mathrm{~Hz}), 7.32(1 \mathrm{H}, \mathrm{td}$, $\mathrm{J}=7.48,1.37 \mathrm{~Hz}), 7.27(1 \mathrm{H}, \mathrm{t}, \mathrm{J}=7.48 \mathrm{~Hz}), 7.20(1 \mathrm{H}, \mathrm{d}, \mathrm{J}=7.48 \mathrm{~Hz}), 6.98(1 \mathrm{H}, \mathrm{d}$, $\mathrm{J}=8.60 \mathrm{~Hz}), 6.55(1 \mathrm{H}, \mathrm{d}, \mathrm{J}=2.75 \mathrm{~Hz}), 6.50(1 \mathrm{H}, \mathrm{dd}, \mathrm{J}=8.60,2.75 \mathrm{~Hz}), 3.87(3 \mathrm{H}$, s), $3.83(3 \mathrm{H}, \mathrm{s}), 2.56(3 \mathrm{H}, \mathrm{s}) ;{ }^{13} \mathrm{C} \mathrm{NMR}\left(\mathrm{CDCl}_{3}, 150 \mathrm{MHz}\right) \delta: 159.0,158.2,153.6$, $138.2,135.8,134.5,130.7,130.6,127.6,126.2,120.5,104.2,99.5,55.9,55.5,19.3$; IR (neat, $\mathrm{cm}^{-1}$ ) 1618, 1602, 1501, 1454, 1284, 1205, 1157, 1030; HRMS (Dart) calcd for $\mathrm{C}_{16} \mathrm{H}_{18} \mathrm{NO}_{2}$ $[\mathrm{M}+\mathrm{H}]^{+} 256.1338$, found 256.1350 .

\section{$N$-(2,4-dimethoxyphenyl)-4-ethylbenzaldehyde imine (1e)}

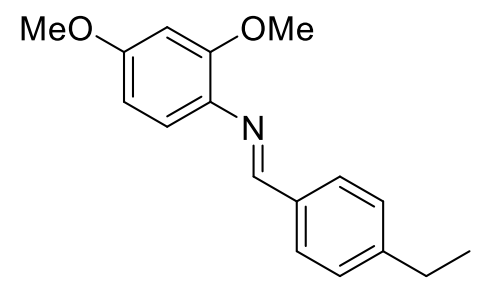

m.p. $47-48{ }^{\circ} \mathrm{C} ;{ }^{1} \mathrm{H}$ NMR (600 MHz, $\left.\mathrm{CDCl}_{3}\right) \delta: 8.44(1 \mathrm{H}, \mathrm{s}), 7.81(2 \mathrm{H}, \mathrm{d}, \mathrm{J}=$ $8.25 \mathrm{~Hz}), 7.25(2 \mathrm{H}, \mathrm{d}, \mathrm{J}=8.25 \mathrm{~Hz}), 6.97(1 \mathrm{H}, \mathrm{d}, \mathrm{J}=8.25 \mathrm{~Hz}), 6.52(1 \mathrm{H}, \mathrm{d}, \mathrm{J}=$ $2.41 \mathrm{~Hz}), 6.46(1 \mathrm{H}, \mathrm{dd}, \mathrm{J}=8.25,2.41 \mathrm{~Hz}), 3.84(3 \mathrm{H}, \mathrm{s}), 3.78(3 \mathrm{H}, \mathrm{s}), 2.67(2 \mathrm{H}$, $\mathrm{q}, \mathrm{J}=7.45 \mathrm{~Hz}), 1.24(3 \mathrm{H}, \mathrm{t}, \mathrm{J}=7.45 \mathrm{~Hz}) ;{ }^{13} \mathrm{C} \mathrm{NMR}\left(\mathrm{CDCl}_{3}, 150 \mathrm{MHz}\right) \delta$ : $159.3,158.7,153.5,135.2,134.1,128.6,128.0,120.2,104.1,99.3,55.7,55.3$,

28.7, 15.2; IR (neat, $\mathrm{cm}^{-1}$ ) 1608, 1581, 1500, 1437, 1415, 1298, 1258, 1207, 1160, 1122, 1031; HRMS (Dart) calcd for $\mathrm{C}_{17} \mathrm{H}_{20} \mathrm{NO}_{2}[\mathrm{M}+\mathrm{H}]^{+} 270.1494$, found 270.1490 .

\section{$N$-(2,4-dimethoxyphenyl)-4-phenylbenzaldehyde imine (1f)}

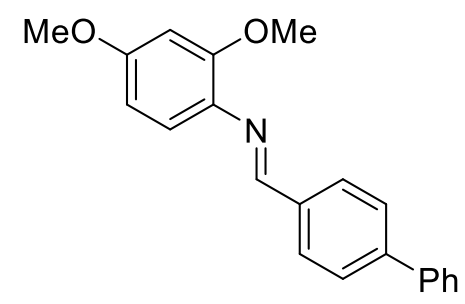

m.p. $128-130{ }^{\circ} \mathrm{C} ;{ }^{1} \mathrm{H}$ NMR $\left(600 \mathrm{MHz}, \mathrm{CDCl}_{3}\right) \delta: 8.55(1 \mathrm{H}, \mathrm{s}), 7.98(2 \mathrm{H}, \mathrm{d}, \mathrm{J}=$ $8.24 \mathrm{~Hz}), 7.69(2 \mathrm{H}, \mathrm{d}, \mathrm{J}=8.24 \mathrm{~Hz}), 7.65(2 \mathrm{H}, \mathrm{d}, \mathrm{J}=7.44 \mathrm{~Hz}), 7.46(2 \mathrm{H}, \mathrm{t}, \mathrm{J}=$ $7.44 \mathrm{~Hz}), 7.38(1 \mathrm{H}, \mathrm{t}, \mathrm{J}=7.44 \mathrm{~Hz}), 7.04(1 \mathrm{H}, \mathrm{d}, \mathrm{J}=8.60 \mathrm{~Hz}), 6.56(1 \mathrm{H}, \mathrm{d}, \mathrm{J}=$ $2.41 \mathrm{~Hz}), 6.51(1 \mathrm{H}, \mathrm{dd}, \mathrm{J}=8.60,2.41 \mathrm{~Hz}), 3.89(3 \mathrm{H}, \mathrm{s}), 3.83(3 \mathrm{H}, \mathrm{s}) ;{ }^{13} \mathrm{C} \mathrm{NMR}$

$\left(\mathrm{CDCl}_{3}, 150 \mathrm{MHz}\right) \delta: 159.1,159.0,153.8,143.6,140.3,135.6,135.1,129.1$,

$128.8,127.7,127.3,127.1,120.5,104.3,99.5,55.9,55.5$; IR (neat, $\mathrm{cm}^{-1}$ ) 1582, 1498, 1417, 1258, 1208, 1155, 1128, 1033; HRMS (Dart) calcd for $\mathrm{C}_{21} \mathrm{H}_{20} \mathrm{NO}_{2}[\mathrm{M}+\mathrm{H}]^{+} 318.1494$, found 370.1490.

\section{$\mathrm{N}$-(2,4-dimethoxyphenyl)-4-methoxybenzaldehyde imine (1g)}

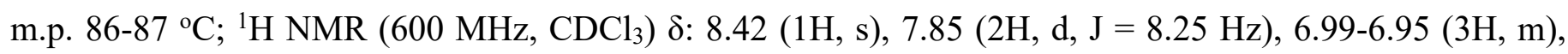
$6.54(1 \mathrm{H}, \mathrm{d}, \mathrm{J}=2.58 \mathrm{~Hz}), 6.49(1 \mathrm{H}, \mathrm{dd}, \mathrm{J}=8.59,2.58 \mathrm{~Hz}), 3.87(3 \mathrm{H}, \mathrm{s}), 3.86(3 \mathrm{H}, \mathrm{s}), 3.82(3 \mathrm{H}, \mathrm{s}) ;{ }^{13} \mathrm{C}$ NMR 


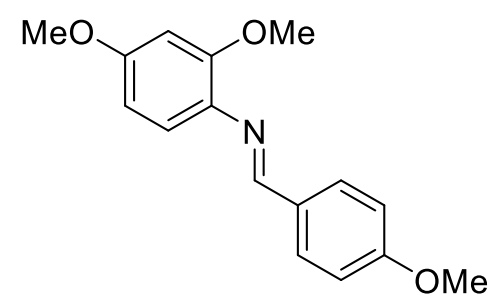

$\left(\mathrm{CDCl}_{3}, 150 \mathrm{MHz}\right) \delta: 161.9,158.9,158.7,153.6,135.4,130.4,129.7,120.2$, 114.0, 104.2, 99.4, 55.9, 55.5, 55.4; IR (neat, $\mathrm{cm}^{-1}$ ) 1607, 1592, 1514, 1305, 1254, 1167, 1157, 1130, 1028; HRMS (Dart) calcd for $\mathrm{C}_{16} \mathrm{H}_{18} \mathrm{NO}_{3}[\mathrm{M}+\mathrm{H}]^{+}$ 272.1287 , found 272.1281

\section{$\mathrm{N}$-(2,4-dimethoxyphenyl)-3-methoxybenzaldehyde imine (1h)}

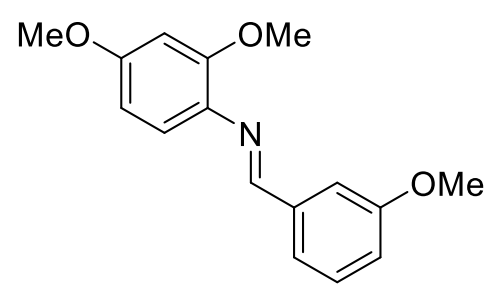

${ }^{1} \mathrm{H}$ NMR $\left(600 \mathrm{MHz}, \mathrm{CDCl}_{3}\right) \delta: 8.47(1 \mathrm{H}, \mathrm{s}), 7.52(1 \mathrm{H}, \mathrm{s}), 7.40(1 \mathrm{H}, \mathrm{d}, \mathrm{J}=$ $7.99 \mathrm{~Hz}), 7.34(1 \mathrm{H}, \mathrm{t}, \mathrm{J}=7.99 \mathrm{~Hz}), 7.02-6.99(2 \mathrm{H}, \mathrm{m}), 6.54(1 \mathrm{H}, \mathrm{d}, \mathrm{J}=2.55$ $\mathrm{Hz}), 6.49(1 \mathrm{H}, \mathrm{dd}, \mathrm{J}=8.50,2.55 \mathrm{~Hz}), 3.87(6 \mathrm{H}, \mathrm{s}), 3.82(3 \mathrm{H}, \mathrm{s}) ;{ }^{13} \mathrm{C} \mathrm{NMR}$ $\left(\mathrm{CDCl}_{3}, 125 \mathrm{MHz}\right) \delta: 159.9,159.5,159.1,153.6,138.0,134.9,129.5,122.2$, 120.6, 117.9, 111.7, 104.3, 99.5, 55.9, 55.5, 55.4; IR (neat, $\mathrm{cm}^{-1}$ ) 1621, 1579, 1501, 1454, 1262, 1204, 1157, 1030; HRMS (Dart) calcd for $\mathrm{C}_{16} \mathrm{H}_{18} \mathrm{NO}_{3}[\mathrm{M}+\mathrm{H}]^{+}$272.1287, found 272.1281 $N$-(2,4-dimethoxyphenyl)-2-methoxybenzaldehyde imine (1i)

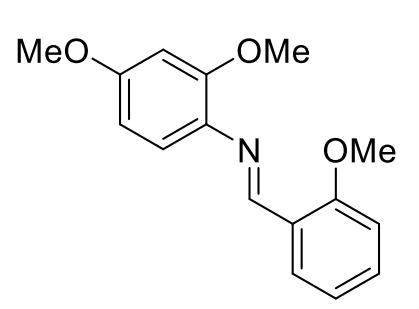

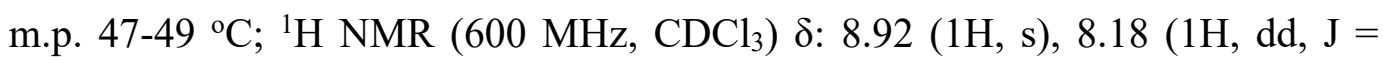
7.90, $1.90 \mathrm{~Hz}), 7.41-7.38(1 \mathrm{H}, \mathrm{td}, \mathrm{J}=7.77,1.90), 7.03-7.00(2 \mathrm{H}, \mathrm{m}), 6.92(1 \mathrm{H}, \mathrm{d}$, $\mathrm{J}=8.25 \mathrm{~Hz}), 6.53(1 \mathrm{H}, \mathrm{d}, \mathrm{J}=2.41 \mathrm{~Hz}), 6.48(1 \mathrm{H}, \mathrm{dd}, \mathrm{J}=8.25,2.41 \mathrm{~Hz}), 3.78(3 \mathrm{H}$, s), $3.78(3 \mathrm{H}, \mathrm{s}) 3.82(3 \mathrm{H}, \mathrm{s}) ;{ }^{13} \mathrm{C} \mathrm{NMR}\left(\mathrm{CDCl}_{3}, 150 \mathrm{MHz}\right) \delta: 159.3,158.9,155.4$, $153.8,136.0,132.2,127.7,125.1,120.8,120.3,110.9,104.2,99.4,55.9,55.5$, 55.5; IR (neat, $\mathrm{cm}^{-1}$ ) 1602, 1501, 1457, 1297, 1252, 1210, 1154, 1128, 1025; HRMS (Dart) calcd for $\mathrm{C}_{16} \mathrm{H}_{18} \mathrm{NO}_{3}[\mathrm{M}+\mathrm{H}]^{+} 272.1287$, found 272.1293.

\section{$\mathrm{N}$-(2,4-dimethoxyphenyl)-4-fluorobenzaldehyde imine (1j)}

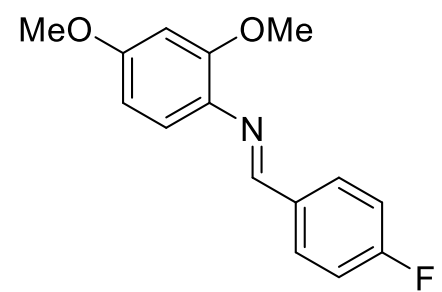

m.p. 88-89 ${ }^{\circ} \mathrm{C} ;{ }^{1} \mathrm{H}$ NMR $\left(500 \mathrm{MHz}, \mathrm{CDCl}_{3}\right) \delta: \quad 8.48(1 \mathrm{H}, \mathrm{s}), 7.90(2 \mathrm{H}, \mathrm{dd}, \mathrm{J}=$ 8.85, $5.45 \mathrm{~Hz}), 7.13(2 \mathrm{H}, \mathrm{t}, \mathrm{J}=8.50 \mathrm{~Hz}), 7.01(1 \mathrm{H}, \mathrm{d}, \mathrm{J}=8.50 \mathrm{~Hz}), 6.55(1 \mathrm{H}, \mathrm{d}$, $\mathrm{J}=2.27 \mathrm{~Hz}), 6.50(1 \mathrm{H}, \mathrm{dd}, \mathrm{J}=8.50,2.27 \mathrm{~Hz}), 3.88(3 \mathrm{H}, \mathrm{s}), 3.83(3 \mathrm{H}, \mathrm{s})$.

${ }^{13} \mathrm{C} \mathrm{NMR}\left(\mathrm{CDCl}_{3}, 150 \mathrm{MHz}\right) \delta: 164.4(\mathrm{~d}, \mathrm{~J}=251.4 \mathrm{~Hz}), 159.1,157.9,153.6$, 134.7, 132.9 (d, J = 4.33 Hz), 130.5 (d, J = 8.67 Hz), 120.5, 115.7 (d, J = 21.7 $\mathrm{Hz}$ ), 104.3, 99.5, 55.8, 55.4; ${ }^{19} \mathrm{~F}$ NMR (CDCl3, $565 \mathrm{MHz}$ ) $\delta:-112.2$; IR (neat, $\mathrm{cm}^{-1}$ ) 1587, 1498, 1455, 1261, 1207, 1184, 1160, 1128, 1043; HRMS (Dart) calcd for $\mathrm{C}_{15} \mathrm{H}_{15} \mathrm{FNO}_{2}[\mathrm{M}+\mathrm{H}]^{+}$260.1087, found 260.1092.

\section{$\mathrm{N}$-(2,4-dimethoxyphenyl)-4-chlorobenzaldehyde imine (1k)}

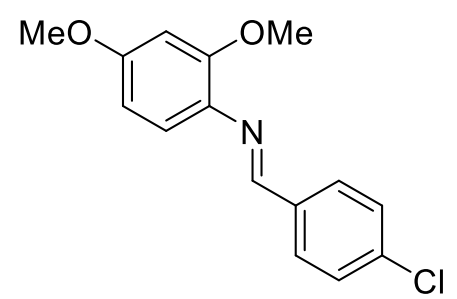

m.p. $60-61{ }^{\circ} \mathrm{C} ;{ }^{1} \mathrm{H}$ NMR $\left(500 \mathrm{MHz}, \mathrm{CDCl}_{3}\right) \delta: 8.48(1 \mathrm{H}, \mathrm{s}), 7.84(2 \mathrm{H}, \mathrm{d}, \mathrm{J}=$ $8.50 \mathrm{~Hz}), 7.42(2 \mathrm{H}, \mathrm{d}, \mathrm{J}=8.50 \mathrm{~Hz}), 7.02(1 \mathrm{H}, \mathrm{d}, \mathrm{J}=8.50 \mathrm{~Hz}), 6.54(1 \mathrm{H}, \mathrm{d}, \mathrm{J}=$ $2.27 \mathrm{~Hz}), 6.50(1 \mathrm{H}, \mathrm{dd}, \mathrm{J}=8.50,2.27 \mathrm{~Hz}), 3.88(3 \mathrm{H}, \mathrm{s}), 3.83(3 \mathrm{H}, \mathrm{s}) ;{ }^{13} \mathrm{C} \mathrm{NMR}$ $\left(\mathrm{CDCl}_{3}, 150 \mathrm{MHz}\right) \delta: 159.3,157.8,153.8,136.8,135.1,134.5,129.8,128.9$,

120.6, 104.3, 99.5, 55.9, 55.5; IR (neat, $\mathrm{cm}^{-1}$ ) 1581, 1501, 1464, 1418, 1261, 1208, 1157, 1127, 1030; HRMS (Dart) calcd for $\mathrm{C}_{15} \mathrm{H}_{15} \mathrm{ClNO}_{2}[\mathrm{M}+\mathrm{H}]^{+}$276.0791, found 276.0784.

\section{$\mathrm{N}$-(2,4-dimethoxyphenyl)-4-bromobenzaldehyde imine (11)}

m.p. 65-66 ${ }^{\circ} \mathrm{C} ;{ }^{1} \mathrm{H}$ NMR (600 MHz, $\left.\mathrm{CDCl}_{3}\right) \delta: 8.47(1 \mathrm{H}, \mathrm{s}), 7.77(2 \mathrm{H}, \mathrm{d}, \mathrm{J}=8.60 \mathrm{~Hz}), 7.58(2 \mathrm{H}, \mathrm{d}, \mathrm{J}=8.60$ $\mathrm{Hz}), 7.02(1 \mathrm{H}, \mathrm{d}, \mathrm{J}=8.42 \mathrm{~Hz}), 6.54(1 \mathrm{H}, \mathrm{d}, \mathrm{J}=2.58 \mathrm{~Hz}), 6.50(1 \mathrm{H}, \mathrm{dd}, \mathrm{J}=8.42,2.58 \mathrm{~Hz}), 3.88$ (3H, s), 3.83 


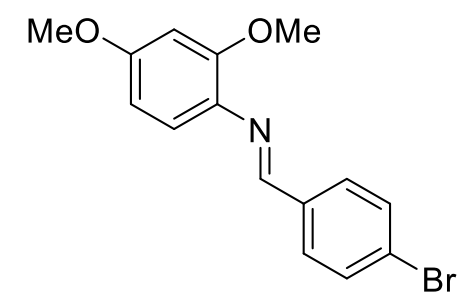

$(3 \mathrm{H}, \mathrm{s}) ;{ }^{13} \mathrm{C} \mathrm{NMR}\left(\mathrm{CDCl}_{3}, 150 \mathrm{MHz}\right) \delta: 159.3,157.9,153.8,135.6,134.5$, 131.9, 130.0, 125.3, 120.7, 104.4, 99.5, 55.9, 55.5; IR (neat, $\mathrm{cm}^{-1}$ ) 1622, 1585, 1501, 1258, 1207, 1158, 1130, 1032, 1008; HRMS (Dart) calcd for $\mathrm{C}_{15} \mathrm{H}_{15} \mathrm{BrNO}_{2}[\mathrm{M}+\mathrm{H}]^{+}$320.0286, found 320.0276.

\section{$N$-(2,4-dimethoxyphenyl)-3-bromobenzaldehyde imine (1m)}

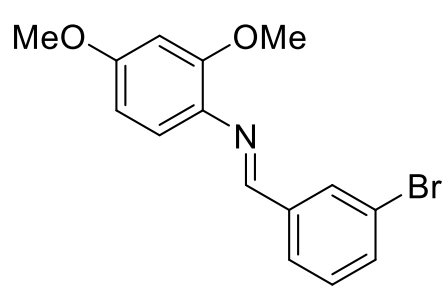

${ }^{1} \mathrm{H}$ NMR $\left(500 \mathrm{MHz}, \mathrm{CDCl}_{3}\right) \delta: 8.44(1 \mathrm{H}, \mathrm{s}), 8.08(1 \mathrm{H}, \mathrm{s}), 7.75(1 \mathrm{H}, \mathrm{d}, \mathrm{J}=8.24$ $\mathrm{Hz}), 7.53(1 \mathrm{H}, \mathrm{d}, \mathrm{J}=8.24 \mathrm{~Hz}), 7.28(1 \mathrm{H}, \mathrm{t}, \mathrm{J}=8.24 \mathrm{~Hz}), 7.01(1 \mathrm{H}, \mathrm{d}, \mathrm{J}=8.50$ $\mathrm{Hz}), 6.53(1 \mathrm{H}, \mathrm{d}, \mathrm{J}=2.27 \mathrm{~Hz}), 6.48(1 \mathrm{H}, \mathrm{dd}, \mathrm{J}=8.50,2.27 \mathrm{~Hz}), 3.86(3 \mathrm{H}, \mathrm{s})$, $3.80(3 \mathrm{H}, \mathrm{s}) ;{ }^{13} \mathrm{C} \mathrm{NMR}\left(\mathrm{CDCl}_{3}, 125 \mathrm{MHz}\right) \delta: 159.4,157.4,153.8,138.6,134.2$, 130.9, 130.0, 127.4, 122.9, 120.8, 104.4, 99.4, 55.8, 55.4; IR (neat, $\mathrm{cm}^{-1}$ ) 1618, 1581, 1500, 1454, 1304, 1260, 1284, 1205, 1182, 1157, 1030; HRMS (Dart) calcd for $\mathrm{C}_{15} \mathrm{H}_{15} \mathrm{BrNO}_{2}[\mathrm{M}+\mathrm{H}]^{+}$ 320.0286 , found 320.0275 .

\section{$N$-(2,4-dimethoxyphenyl)-2-bromobenzaldehyde imine (1n)}

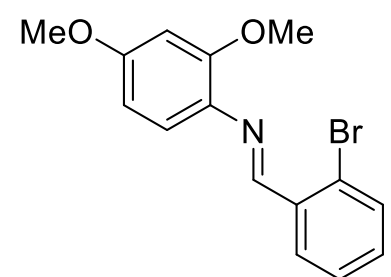

m.p. $37-39{ }^{\circ} \mathrm{C} ;{ }^{1} \mathrm{H}$ NMR $\left(500 \mathrm{MHz}, \mathrm{CDCl}_{3}\right) \delta: 8.44(1 \mathrm{H}, \mathrm{s}), 8.08(1 \mathrm{H}, \mathrm{s}), 7.75(1 \mathrm{H}$, $\mathrm{d}, \mathrm{J}=8.24 \mathrm{~Hz}), 7.53(1 \mathrm{H}, \mathrm{d}, \mathrm{J}=8.24 \mathrm{~Hz}), 7.28(1 \mathrm{H}, \mathrm{t}, \mathrm{J}=8.24 \mathrm{~Hz}), 7.01(1 \mathrm{H}, \mathrm{d}, \mathrm{J}=$ $8.50 \mathrm{~Hz}), 6.53(1 \mathrm{H}, \mathrm{d}, \mathrm{J}=2.27 \mathrm{~Hz}), 6.48(1 \mathrm{H}, \mathrm{dd}, \mathrm{J}=8.50,2.27 \mathrm{~Hz}), 3.86(3 \mathrm{H}, \mathrm{s})$, $3.80(3 \mathrm{H}, \mathrm{s}) ;{ }^{13} \mathrm{C} \mathrm{NMR}\left(\mathrm{CDCl}_{3}, 125 \mathrm{MHz}\right) \delta: 159.4,157.9,153.8,134.9,134.4$, $132.9,131.8,128.9,127.5,125.7,121.0,104.3,99.4,55.8,55.4$; IR (neat, $\mathrm{cm}^{-1}$ ) 1591, 1498, 1464, 1305, 1261, 1212, 1161, 1130, 1021; HRMS (Dart) calcd for $\mathrm{C}_{15} \mathrm{H}_{15} \mathrm{BrNO}_{2}[\mathrm{M}+\mathrm{H}]^{+}$ 320.0286 , found 320.0283 .

\section{$\mathrm{N}$-(2,4-dimethoxyphenyl)-3-trifluoromethylbenzaldehyde imine (10)}

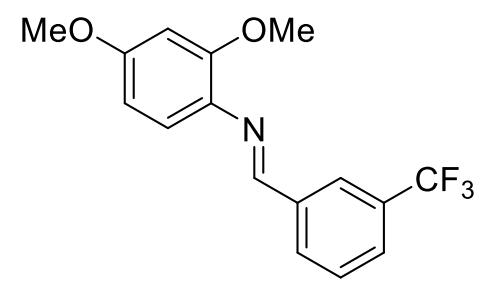

m.p. $33-34{ }^{\circ} \mathrm{C} ;{ }^{1} \mathrm{H}$ NMR $\left(600 \mathrm{MHz}, \mathrm{CDCl}_{3}\right) \delta: 8.59(1 \mathrm{H}, \mathrm{s}), 8.18(1 \mathrm{H}, \mathrm{s}), 8.07$ $(1 \mathrm{H}, \mathrm{d}, \mathrm{J}=7.90 \mathrm{~Hz}), 7.69(1 \mathrm{H}, \mathrm{d}, \mathrm{J}=7.90 \mathrm{~Hz}), 7.57(1 \mathrm{H}, \mathrm{t}, \mathrm{J}=7.90 \mathrm{~Hz}), 7.06$ $(1 \mathrm{H}, \mathrm{d}, \mathrm{J}=8.42 \mathrm{~Hz}), 6.56(1 \mathrm{H}, \mathrm{d}, \mathrm{J}=2.58 \mathrm{~Hz}), 6.51(1 \mathrm{H}, \mathrm{dd}, \mathrm{J}=8.42,2.58$ $\mathrm{Hz}), 3.89(3 \mathrm{H}, \mathrm{s}), 3.84(3 \mathrm{H}, \mathrm{s}) ;{ }^{13} \mathrm{C} \mathrm{NMR}\left(\mathrm{CDCl}_{3}, 150 \mathrm{MHz}\right) \delta: 159.5,157.4$, 153.8, 137.4, 134.1, 131.7, 131.1 (q, J = 32.3 Hz), 129.1, 127.2 (q, J = 3.9 Hz),

126.1 (q, J = 303.0), 125.2 (q, J = 3.9 Hz), 123.0, 121.1, 104.4, 99.5, 55.8, 55.5; IR (neat, $\mathrm{cm}^{-1}$ ) 1622, 1585, 1505, 1464, 1330, 1281, 1208, 1154, 1068, 1025; ${ }^{19} \mathrm{~F}$ NMR (CDCl3, $\left.465 \mathrm{MHz}\right)$ 8:-62.5; HRMS (Dart) calcd for $\mathrm{C}_{16} \mathrm{H}_{15} \mathrm{~F}_{3} \mathrm{NO}_{2}[\mathrm{M}+\mathrm{H}]^{+}$310.1055, found 310.1069 .

\section{$N$-(2,4-dimethoxyphenyl)-naphthylaldehyde imine (1p)}

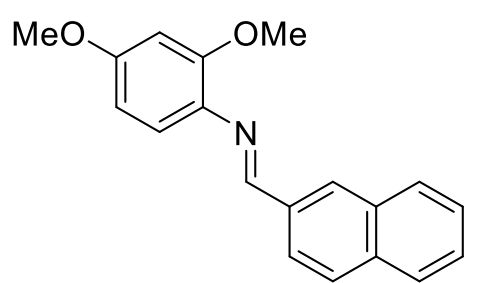

m.p. 100-101 ${ }^{\circ} \mathrm{C} ;{ }^{1} \mathrm{H}$ NMR (600 MHz, $\left.\mathrm{CDCl}_{3}\right) \delta: 8.67(1 \mathrm{H}, \mathrm{s}), 8.19-8.17(2 \mathrm{H}$, m), 7.92-7.85 (3H, m), 7.54-7.50 (2H, m), $7.08(1 \mathrm{H}, \mathrm{d}, \mathrm{J}=8.25 \mathrm{~Hz}), 6.57(1 \mathrm{H}$, $\mathrm{d}, \mathrm{J}=2.41 \mathrm{~Hz}), 6.52(1 \mathrm{H}, \mathrm{dd}, \mathrm{J}=8.25,2.41 \mathrm{~Hz}), 3.90(3 \mathrm{H}, \mathrm{s}), 3.84(3 \mathrm{H}, \mathrm{s}) ;{ }^{13} \mathrm{C}$ $\mathrm{NMR}\left(\mathrm{CDCl}_{3}, 150 \mathrm{MHz}\right) \delta: 159.3,159.1,153.8,135.0,134.8,134.4,133.1$, $130.7,128.7,128.4,127.9,127.2,126.4,124.2,120.5,104.3,99.5,55.9,55.5$; IR (neat, $\mathrm{cm}^{-1}$ ) 1615, 1597, 1498, 1454, 1311, 1257, 1205, 1158, 1127, 1047, 1025; HRMS (Dart) calcd for $\mathrm{C}_{19} \mathrm{H}_{18} \mathrm{NO}_{2}[\mathrm{M}+\mathrm{H}]^{+} 292.1338$, found 292.1352. 


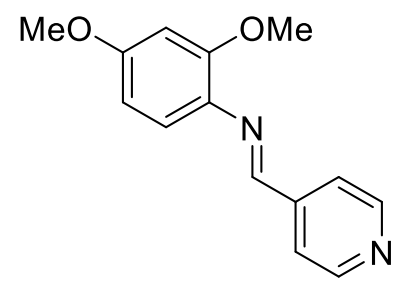

m.p. 79 80 ${ }^{\circ} \mathrm{C} ;{ }^{1} \mathrm{H}$ NMR $\left(600 \mathrm{MHz}, \mathrm{CDCl}_{3}\right) \delta: 8.72(2 \mathrm{H}, \mathrm{d}, \mathrm{J}=4.47 \mathrm{~Hz}), 8.57(1 \mathrm{H}$, s), $7.75(2 \mathrm{H}, \mathrm{d}, \mathrm{J}=4.47 \mathrm{~Hz}), 7.10(1 \mathrm{H}, \mathrm{d}, \mathrm{J}=8.59 \mathrm{~Hz}), 6.56(1 \mathrm{H}, \mathrm{d}, \mathrm{J}=2.38 \mathrm{~Hz})$, $6.52(1 \mathrm{H}, \mathrm{dd}, \mathrm{J}=8.59,2.38 \mathrm{~Hz}), 3.89(3 \mathrm{H}, \mathrm{s}), 3.84(3 \mathrm{H}, \mathrm{s}) ;{ }^{13} \mathrm{C} \mathrm{NMR}\left(\mathrm{CDCl}_{3}, 150\right.$ MHz) $\delta: 160.0,156.5,154.1,150.4,150.4,123.4,133.4,122.1,121.5,104.5,99.5$, 55.9, 55.5; IR (neat, $\mathrm{cm}^{-1}$ ) 1592, 1577, 1455, 1415, 1288, 1264, 1205, 1158, 1128, 1038, 1023; HRMS (Dart) calcd for $\mathrm{C}_{14} \mathrm{H}_{15} \mathrm{~N}_{2} \mathrm{O}_{2}[\mathrm{M}+\mathrm{H}]^{+}$243.1134, found 243.1130.

\section{$N$-(2,4-dimethoxyphenyl)-3-pyridylaldehyde imine (1r)}

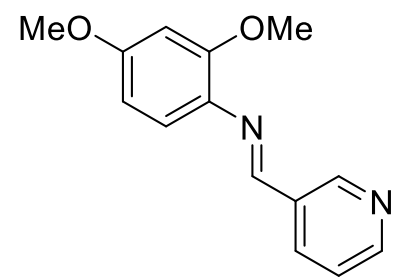

m.p. 64-65 ${ }^{\circ} \mathrm{C}$; ${ }^{1} \mathrm{H}$ NMR $\left(600 \mathrm{MHz}, \mathrm{CDCl}_{3}\right) \delta: 8.98(1 \mathrm{H}, \mathrm{d}, \mathrm{J}=1.37 \mathrm{~Hz}), 8.64(1 \mathrm{H}$, $\mathrm{d}, \mathrm{J}=4.81 \mathrm{~Hz}), 8.56(1 \mathrm{H}, \mathrm{s}), 8.27(1 \mathrm{H}, \mathrm{d}, \mathrm{J}=7.91 \mathrm{~Hz}), 7.34(1 \mathrm{H}, \mathrm{dd}, \mathrm{J}=7.91,4.81$ $\mathrm{Hz}), 7.05(1 \mathrm{H}, \mathrm{d}, \mathrm{J}=8.42 \mathrm{~Hz}), 6.53(1 \mathrm{H}, \mathrm{d}, \mathrm{J}=2.58 \mathrm{~Hz}), 6.49(1 \mathrm{H}, \mathrm{dd}, \mathrm{J}=8.42$, $2.58 \mathrm{~Hz}), 3.86(3 \mathrm{H}, \mathrm{s}), 3.80(3 \mathrm{H}, \mathrm{s}) ;{ }^{13} \mathrm{C} \mathrm{NMR}\left(\mathrm{CDCl}_{3}, 150 \mathrm{MHz}\right) \delta: 159.2,155.7$, $153.6,151.2,150.4,134.3,133.8,132.0,123.3,120.8,104.2,99.2,55.5,55.1 ; \mathrm{IR}$ (neat, $\mathrm{cm}^{-1}$ ) 1624, 1585, 1501, 1415, 1314, 1255, 1211, 1164, 1131, 1033; HRMS (Dart) calcd for $\mathrm{C}_{14} \mathrm{H}_{15} \mathrm{~N}_{2} \mathrm{O}_{2}[\mathrm{M}+\mathrm{H}]^{+}$243.1134, found 243.1142.

\section{$N$-(2,4-dimethoxyphenyl)-2-pyridylaldehyde imine (1s)}

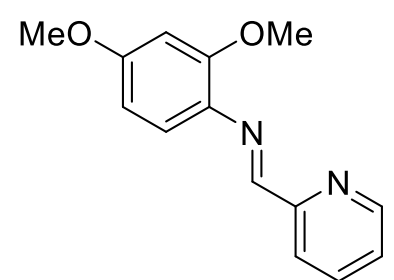

${ }^{1} \mathrm{H}$ NMR (600 MHz, $\left.\mathrm{CDCl}_{3}\right)$ $\delta: 8.72(1 \mathrm{H}, \mathrm{s}), 8.68(1 \mathrm{H}, \mathrm{d}, \mathrm{J}=4.12 \mathrm{~Hz}), 8.25(1 \mathrm{H}, \mathrm{d}$, $\mathrm{J}=8.94 \mathrm{~Hz}), 7.77(1 \mathrm{H}, \mathrm{t}, \mathrm{J}=7.90 \mathrm{~Hz}), 7.32-7.30(1 \mathrm{H}, \mathrm{m}), 7.16(1 \mathrm{H}, \mathrm{d}, \mathrm{J}=8.25 \mathrm{~Hz})$, $6.55(1 \mathrm{H}, \mathrm{d}, \mathrm{J}=2.75 \mathrm{~Hz}), 6.52(1 \mathrm{H}, \mathrm{dd}, \mathrm{J}=7.86,2.41 \mathrm{~Hz}), 3.89(3 \mathrm{H}, \mathrm{s}), 3.82(3 \mathrm{H}$, s), $3.82(3 \mathrm{H}, \mathrm{s}) ;{ }^{13} \mathrm{C} \mathrm{NMR}\left(\mathrm{CDCl}_{3}, 150 \mathrm{MHz}\right) \delta: 159.7,159.2,155.0,154.0,149.4$, 136.3, 133.4, 124.6, 121.4, 121.2, 104.4, 99.4, 55.8, 55.4; IR (neat, $\mathrm{cm}^{-1}$ ) 1578, 1567, 1500, 1464, 1435, 1305, 1288, 1261, 1205, 1158, 1127, 1028; HRMS (Dart) calcd for $\mathrm{C}_{14} \mathrm{H}_{15} \mathrm{~N}_{2} \mathrm{O}_{2}$ $[\mathrm{M}+\mathrm{H}]^{+} 243.1134$, found 243.1133 .

\section{$N$-(2,4-dimethoxyphenyl)-cyclopropanecarboxyaldehyde imine (1t)}

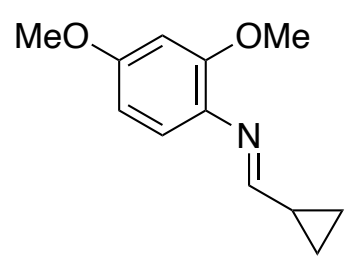

Mp 52-55 ${ }^{\circ} \mathrm{C} ;{ }^{1} \mathrm{H}$ NMR $\left(600 \mathrm{MHz}, \mathrm{C}_{6} \mathrm{D}_{6}\right) \delta: 7.20(1 \mathrm{H}, \mathrm{d}, \mathrm{J}=6.9 \mathrm{~Hz}), 6.82(1 \mathrm{H}, \mathrm{d}, \mathrm{J}=$ $8.9 \mathrm{~Hz}), 6.51(1 \mathrm{H}, \mathrm{d}, \mathrm{J}=2.0 \mathrm{~Hz}), 6.36-6.34(1 \mathrm{H}, \mathrm{dd}, \mathrm{J}=2.8 \mathrm{~Hz}, 8.3 \mathrm{~Hz}) .3 .36(3 \mathrm{H}, \mathrm{s})$, $3.32(3 \mathrm{H}, \mathrm{s}), 1.75-1.69(1 \mathrm{H}, \mathrm{m}), 0.63-0.60(2 \mathrm{H}, \mathrm{m}), 0.53-0.49(2 \mathrm{H}, \mathrm{m}) ;{ }^{13} \mathrm{C} \mathrm{NMR}$

$\left(\mathrm{C}_{6} \mathrm{D}_{6}, 150 \mathrm{MHz}\right) \delta: 166.8,158.6,153.5,136.8,121.4,104.5,100.4,55.3,55.0,17.6$, 7.0; IR (neat, $\mathrm{cm}^{-1}$ ): 3100, 3088, 1639, 1601, 1582, 1501, 1465, 1440, 1418, 1311, 1277, 1255, 1207, 1198, 1155, 1128, 1041, 1027; HRMS (Dart) calcd for $\mathrm{C}_{12} \mathrm{H}_{16} \mathrm{NO}_{2}[\mathrm{M}+\mathrm{H}]^{+}$206.1181, found 201.1175.

\section{$\mathrm{N}$-(2-methoxyphenyl)-2,2-dimethylpropionaldehyde imine (1u)}

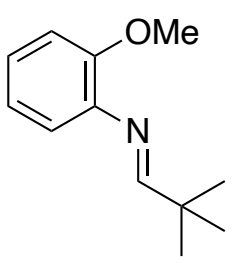

${ }^{1} \mathrm{H}$ NMR $\left(500 \mathrm{MHz}, \mathrm{C}_{6} \mathrm{D}_{6}\right) \delta: 7.56(1 \mathrm{H}, \mathrm{s}), 6.96-7.00(1 \mathrm{H}, \mathrm{m}), 6.80-6.86(2 \mathrm{H}, \mathrm{m}), 6.63(1 \mathrm{H}$, $\mathrm{d}, \mathrm{J}=8.1 \mathrm{~Hz}), 3.35(3 \mathrm{H}, \mathrm{s}), 1.09(9 \mathrm{H}, \mathrm{s}) ;{ }^{13} \mathrm{C}$ NMR $\left(\mathrm{C}_{6} \mathrm{D}_{6}, 125 \mathrm{MHz}\right) \delta: 173.1,152.0,143.4$, 125.6, 121.2, 121.1, 112.4, 55.5, 37.0, 26.8; IR (neat, $\mathrm{cm}^{-1}$ ) ; 1652, 1589, 1493, 1477, 1437 ,

1394, 1362, 1300, 1280, 1241, 1178, 1160, 1113, 1048, 1028; HRMS (Dart) calcd for $\mathrm{C}_{12} \mathrm{H}_{18} \mathrm{NO}[\mathrm{M}+\mathrm{H}]^{+}$192.1388, found 192.1381. 


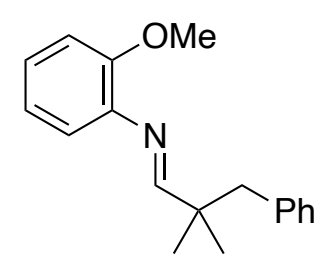

${ }^{1} \mathrm{H}$ NMR (500 MHz, $\left.\mathrm{C}_{6} \mathrm{D}_{6}\right)$ ): $7.62(1 \mathrm{H}, \mathrm{s}), 7.14-7.00(5 \mathrm{H}, \mathrm{m}), 6.98(1 \mathrm{H}, \mathrm{m}), 6.88-6.81$ $(2 \mathrm{H}, \mathrm{m}), 6.64(1 \mathrm{H}, \mathrm{d}, \mathrm{J}=8.6 \mathrm{~Hz}), 3.36(3 \mathrm{H}, \mathrm{s}), 2.73(2 \mathrm{H}, \mathrm{s}), 1.09(6 \mathrm{H}, \mathrm{s}) ;{ }^{13} \mathrm{C} \mathrm{NMR}$ $\left(\mathrm{C}_{6} \mathrm{D}_{6}, 125 \mathrm{MHz}\right) \delta: 172.7,152.0,143.3,138.6,131.0,128.1,126.4,125.7,121.2,121.0$,

112.4, 55.4, 46.4, 41.2, 24.8; IR (neat, $\mathrm{cm}^{-1}$ ) 1652, 1589, 1492, 1452, 1437, 1387, 1362, 1244, 1204, 1178, 1114, 1048, 1028; HRMS (Dart) calcd for $\mathrm{C}_{18} \mathrm{H}_{22} \mathrm{NO}[\mathrm{M}+\mathrm{H}]^{+}$268.1701, found 268.1688.

\section{Typical experimental procedure for preparation of $N, N$-dimethylalkylamides 2}

To a suspension of a corresponding acid chloride $(148 \mathrm{mmol})$ and dimethylamine hydrochloride $(222 \mathrm{mmol})$ in DCM $(250 \mathrm{~mL})$ cooled at $0{ }^{\circ} \mathrm{C}$ was added dropwise triethylamine (444 mmol) under Ar atmosphere. The whole mixture was stirred overnight at room temperature. After quenching by adding water, the organic phase was separated, and the aqueous phase was extracted with DCM. The organic layers were combined and washed successively with $1 \mathrm{M} \mathrm{HCl}$, water, sat. $\mathrm{NaHCO}_{3}$, and brine, and dried over anhydrous $\mathrm{Na}_{2} \mathrm{SO}_{4}$. After filtration and concentration under reduced pressure, the crude product obtained was purified by distillation under reduced pressure to afford the desired product.

\section{$N, N$-dimethylbutyramide (2b)}

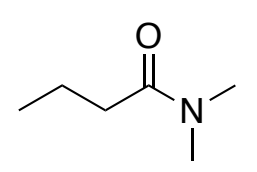

${ }^{1} \mathrm{H} \mathrm{NMR}\left(600 \mathrm{MHz}, \mathrm{CDCl}_{3}\right) \delta: 2.96(3 \mathrm{H}, \mathrm{s}), 2.90(3 \mathrm{H}, \mathrm{s}), 2.25(2 \mathrm{H}, \mathrm{t}, \mathrm{J}=7.6 \mathrm{~Hz}), 1.62(2 \mathrm{H}$, tq, $\mathrm{J}=7.6,7.5 \mathrm{~Hz}), 0.92(3 \mathrm{H}, \mathrm{t}, \mathrm{J}=7.6 \mathrm{~Hz}) ;{ }^{13} \mathrm{C} \mathrm{NMR}\left(\mathrm{CDCl}_{3}, 150 \mathrm{MHz}\right) \delta: 173.0,37.1$, 35.2, 18.5, 13.9; IR (neat, $\mathrm{cm}^{-1}$ ) 1637, 1495, 1463, 1456, 1410, 1395, 1346, 1331, 1264, 1152, 1093, 1068; HRMS (Dart) calcd for $\mathrm{C}_{6} \mathrm{H}_{14} \mathrm{NO}[\mathrm{M}+\mathrm{H}]^{+}$116.1075, found 116.1066; calcd for $\mathrm{C}_{12} \mathrm{H}_{27} \mathrm{~N}_{2} \mathrm{O}_{2}[2 \mathrm{M}+\mathrm{H}]^{+}$231.2073, found 231.2067.

\section{$N, N$-dimethylheptanamide (2c)}

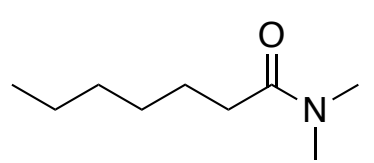

${ }^{1} \mathrm{H} \mathrm{NMR}\left(600 \mathrm{MHz}, \mathrm{CDCl}_{3}\right) \delta: 2.94(3 \mathrm{H}, \mathrm{s}), 2.87(3 \mathrm{H}, \mathrm{s}), 2.24(2 \mathrm{H}, \mathrm{t}, \mathrm{J}=7.6 \mathrm{~Hz})$, $1.56(2 \mathrm{H}, \mathrm{m}), 1.30-1.20(6 \mathrm{H}, \mathrm{m}), 0.82(3 \mathrm{H}, \mathrm{t}, \mathrm{J}=6.8 \mathrm{~Hz}) ;{ }^{13} \mathrm{C} \mathrm{NMR}\left(\mathrm{CDCl}_{3}, 150\right.$ MHz) $\delta: 173.1,37.1,35.2,33.3,31.5,29.0,25.0,22.4,13.9$; IR (neat, $\mathrm{cm}^{-1}$ ) 1644, 1495, 1467, 1457, 1410, 1395, 1265, 1148, 1102; HRMS (Dart) calcd for $\mathrm{C}_{9} \mathrm{H}_{20} \mathrm{NO}[\mathrm{M}+\mathrm{H}]^{+}$158.1545, found 158.1544; calcd for $\mathrm{C}_{18} \mathrm{H}_{39} \mathrm{~N}_{2} \mathrm{O}_{2}[2 \mathrm{M}+\mathrm{H}]^{+} 315.3012$, found 315.3010 .

\section{$N, N, 3-t r i m e t h y l b u t a n a m i d e ~(2 d)$}

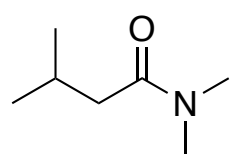

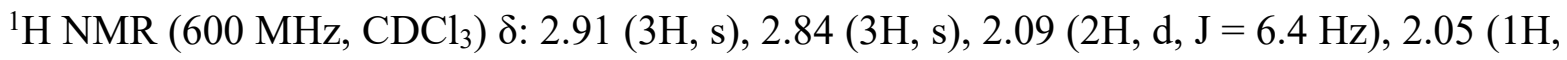
m), $0.86(6 \mathrm{H}, \mathrm{d}, \mathrm{J}=6.2 \mathrm{~Hz}) ;{ }^{13} \mathrm{C} \mathrm{NMR}\left(\mathrm{CDCl}_{3}, 150 \mathrm{MHz}\right) \delta: 172.3,41.9,37.2,35.1,25.4$, 22.5; IR (neat, $\mathrm{cm}^{-1}$ ) 1637, 1495, 1468, 1395, 1384, 1367, 1262, 1170, 1160, 1105, 1077, 1077, 1060; HRMS (Dart) calcd for $\mathrm{C}_{7} \mathrm{H}_{16} \mathrm{NO}[\mathrm{M}+\mathrm{H}]^{+}$130.1232, found 130.1224; calcd for $\mathrm{C}_{14} \mathrm{H}_{31} \mathrm{~N}_{2} \mathrm{O}_{2}$ $[2 \mathrm{M}+\mathrm{H}]^{+}$259.2386, found 259.2394.

\section{$\mathrm{N}, \mathrm{N}$-dimethyl-3-phenylpropanamide (2e)}

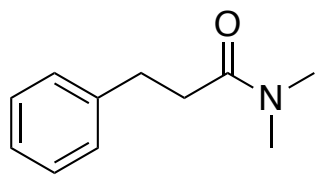

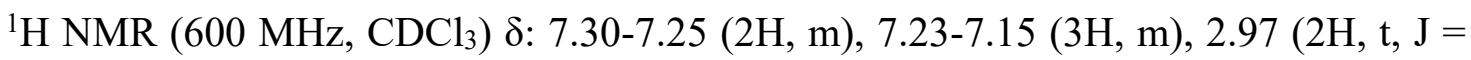
$7.6 \mathrm{~Hz}), 2.95(3 \mathrm{H}, \mathrm{s}), 2.93(3 \mathrm{H}, \mathrm{s}), 2.59(2 \mathrm{H}, \mathrm{t}, \mathrm{J}=7.6 \mathrm{~Hz}) ;{ }^{13} \mathrm{C} \mathrm{NMR}\left(\mathrm{CDCl}_{3}, 150\right.$ MHz) $\delta: 171.8,141.2,128.1,128.1,125.8,36.8,35.1,34.9,31.1$; IR (neat, $\mathrm{cm}^{-1}$ ) 1641, 1604, 1495, 1452, 1411, 1395, 1345, 1265, 1138, 1075; HRMS (Dart) calcd for $\mathrm{C}_{11} \mathrm{H}_{16} \mathrm{NO}[\mathrm{M}+\mathrm{H}]^{+}$ 
178.1232, found 178.1225; calcd for $\mathrm{C}_{22} \mathrm{H}_{31} \mathrm{~N}_{2} \mathrm{O}_{2}[2 \mathrm{M}+\mathrm{H}]^{+}$355.2386, found 355.2390.

\section{$N, N$-dimethyl-5-phenylpentanamide (2g)}

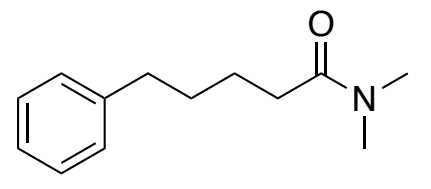

Synthesized following the literature ${ }^{8}$; Colorless oil; ${ }^{1} \mathrm{H}$ NMR $(600 \mathrm{MHz}, \mathrm{CDCl} 3)$

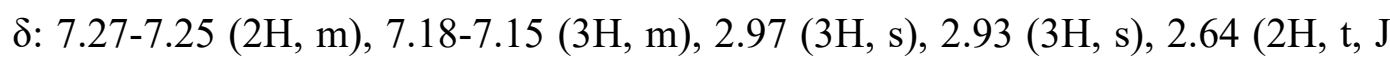
$=6.54 \mathrm{~Hz}), 2.32(2 \mathrm{H}, \mathrm{t}, \mathrm{J}=6.18 \mathrm{~Hz}), 1.69-1.67(4 \mathrm{H}, \mathrm{m}) ;{ }^{13} \mathrm{C} \mathrm{NMR}(\mathrm{CDCl} 3,150$

MHz) $\delta: 172.9,142.3,128.4,128.2,125.6,37.2,35.8,35.3,33.2,31.2,24.8$; IR (neat, $\mathrm{cm}^{-1}$ ) 1641, 1495, 1452, 1395; HRMS (Dart) calcd for $\mathrm{C}_{26} \mathrm{H}_{39} \mathrm{~N}_{2} \mathrm{O}_{2}[2 \mathrm{M}+\mathrm{H}]^{+} 411.3012$, found 411.3031 .

\section{$N$-propionoyl pyrrolidine (2h)}

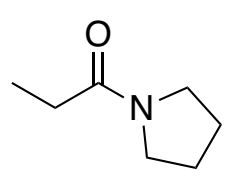

${ }^{1} \mathrm{H}$ NMR (500 MHz, $\left.\mathrm{CDCl}_{3}\right) \delta: 3.48-3.39(4 \mathrm{H}, \mathrm{m}), 2.28(2 \mathrm{H}, \mathrm{q}, \mathrm{J}=7.6 \mathrm{~Hz}), 1,96(2 \mathrm{H}, \mathrm{t}, \mathrm{J}=$ $6.5 \mathrm{~Hz}), 1,85(2 \mathrm{H}, \mathrm{t}, \mathrm{J}=6.5 \mathrm{~Hz}), 1,15(3 \mathrm{H}, \mathrm{t}, \mathrm{J}=7.6 \mathrm{~Hz}) ;{ }^{13} \mathrm{C} \mathrm{NMR}\left(\mathrm{CDCl}_{3}, 125 \mathrm{MHz}\right)$ $\delta: 171.7,45.9,45.1,27.3,25.6,23.9,8.5$; IR (neat, $\mathrm{cm}^{-1}$ ): 1641, 1461, 1430, 1375, 1341, 1310, 1252, 1227, 1194, 1171, 1081, 1035; HRMS (Dart) calcd for $\mathrm{C}_{14} \mathrm{H}_{27} \mathrm{~N}_{2} \mathrm{O}_{2}[2 \mathrm{M}+\mathrm{H}]^{+} 255.2073$, found 255.2074 .

\section{$N$-methyl- $N$-phenylpropionamide (2j)}<smiles>CCC(=O)N(C)c1ccccc1</smiles>

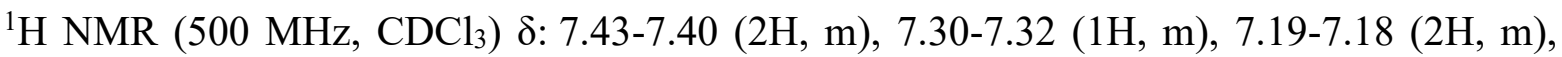
$3.27(3 \mathrm{H}, \mathrm{s}), 2.09(2 \mathrm{H}, \mathrm{q}, \mathrm{J}=7.2 \mathrm{~Hz}), 1.05(3 \mathrm{H}, \mathrm{t}, \mathrm{J}=7.4 \mathrm{~Hz}) ;{ }^{13} \mathrm{C} \mathrm{NMR}\left(\mathrm{CDCl}_{3}, 125 \mathrm{MHz}\right)$ $\delta: 173.9,144.3,129.7,127.7,127.3,37.3,27.5,9.7$; IR (neat, $\mathrm{cm}^{-1}$ ): 1652, 1597, 1494, 1461, 1452, 1420, 1387, 1377, 1285, 1122, 1035, 406; HRMS (Dart) calcd for $\mathrm{C}_{20} \mathrm{H}_{27} \mathrm{~N}_{2} \mathrm{O}_{2}[2 \mathrm{M}+\mathrm{H}]^{+} 327.2073$, found 327.2070 .

\section{Synthesis of Box ligand L7}

Malononitrile $(1.25 \mathrm{~g}, 19 \mathrm{mmol})$ was dissolved in $4 \mathrm{M} \mathrm{HCl}$ in dioxane $(25 \mathrm{~mL})$, subsequently dry EtOH $(2.4$ $\mathrm{mL}$ ) was added. The mixture was stirring at $\mathrm{rt}$ overnight. The resulting suspension was filtered and washed with dry $\mathrm{Et}_{2} \mathrm{O}$. The residue was dried under vacuum, and white solid was obtained. The white solid was used in the next step without further purification.

A solution of diethyl malonimidate $(1.03 \mathrm{~g}, 4.4 \mathrm{mmol})$ and $(S)$-2-amino-4-methylpentan-1-ol (1.04 g, 8.9 mmol) in $\mathrm{CH}_{2} \mathrm{Cl}_{2}(131 \mathrm{~mL})$ was refluxed overnight. After cooed to $\mathrm{rt}$, the reaction mixture was quenched with water and extracted with $\mathrm{CH}_{2} \mathrm{Cl}_{2}$. The combined organic layer was dried over anhydrous $\mathrm{Na}_{2} \mathrm{SO}_{4}$. After filtration and concentration under reduced pressure, the obtained crude product was purified by flash chromatography $\left(\mathrm{CHCl}_{3}-\mathrm{MeOH}-\mathrm{Et}_{3} \mathrm{~N}\right)$ and bulb-to-bulb distillation under vacuum to afford 1a as a colorless oil $(0.591 \mathrm{~g}, 50 \%$ yield $){ }^{17}$

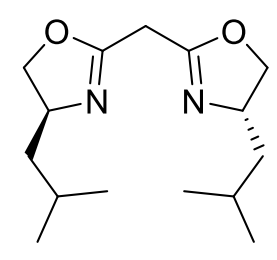

\section{bis((S)-4-isobutyl-4,5-dihydrooxazol-2-yl)methane (L7)}

Colorless oil; ${ }^{1} \mathrm{H}$ NMR (500 MHz, CDCl3) $\delta: ~ 4.37$ (2H, dd, J = 9.62, 8.25 Hz), 4.19-4.14 $(2 \mathrm{H}, \mathrm{m}), 3.86(2 \mathrm{H}, \mathrm{t}, \mathrm{J}=7.90 \mathrm{~Hz}), 3.32(2 \mathrm{H}, \mathrm{s}), 1.79-1.71(2 \mathrm{H}, \mathrm{m}), 1.61(2 \mathrm{H}, \mathrm{dt}, \mathrm{J}=13.02$ $\mathrm{Hz}), 1.29(2 \mathrm{H}, \mathrm{dt}, \mathrm{J}=13,74), 0.94(6 \mathrm{H}, \mathrm{d}, \mathrm{J}=7.68 \mathrm{~Hz}), 0.92(6 \mathrm{H}, \mathrm{dt}, \mathrm{J}=7.68 \mathrm{~Hz}) ;{ }^{13} \mathrm{C}$

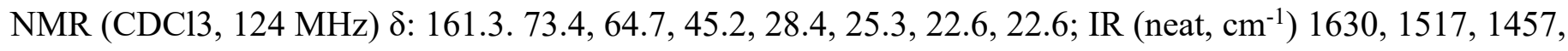
1241; HRMS (Dart) calcd for $\mathrm{C}_{15} \mathrm{H}_{27} \mathrm{~N}_{2} \mathrm{O}_{2}[\mathrm{M}+\mathrm{H}]^{+}$267.2073, found 267.2080. 
Experimental procedure of a catalytic asymmetric Mannich reaction of 1a with 2a (Table 2, Entry 15)

KHMDS (11.8 mg, $0.059 \mathrm{mmol})$, IndaBox (L1) $(9.8 \mathrm{mg}, 0.030 \mathrm{mmol})$ were placed in a flame-dried $10 \mathrm{~mL} 1$ neck round bottom flask with a thee ways cock inside a glove box fulfilled with argon. The flask was cooled to $0{ }^{\circ} \mathrm{C}$, then THF $(0.75 \mathrm{~mL})$ was added. The reaction mixture was stirred for $30 \mathrm{~min}$ at the same temperature and then cooled at $-78{ }^{\circ} \mathrm{C}$. To the reaction mixture, $N$-(2,4-dimethoxyphenyl)-benzaldehyde imine $1 \mathrm{a}(144.8 \mathrm{mg}, 0.600 \mathrm{mmol})$ that was placed in a flame-dried tube under argon atmosphere was added through cannula by using THF $(0.75 \mathrm{~mL}) . \quad N, N$-Dimethypropionamide $\mathbf{2 a}(71.8 \mu \mathrm{L}, 0.660 \mathrm{mmol})$ was then added to the mixture. The whole mixture was stirred at $-78^{\circ} \mathrm{C}$ for $24 \mathrm{~h}$. The reaction was quenched by a mixture of THF and water, and extracted with $\mathrm{CH}_{2} \mathrm{Cl}_{2}$ several times. The combined organic layers were dried over anhydrous $\mathrm{Na}_{2} \mathrm{SO}_{4}$. After filtration and concentration under reduced pressure, the crude product obtained was purified by silica-gel PTLC (hexane-ethyl acetate) to afford the desired adduct 3aa (197 mg, $96 \%$ yield).

\section{(2R,3S)-3-((2,4-dimethoxyphenyl)amino)- $N, N, 2$-trimethyl-3-phenylpropanamide (3aa)}

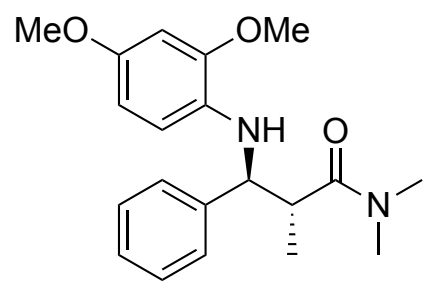

Mp $115-116^{\circ} \mathrm{C} ;[\alpha]_{\mathrm{D}}^{20}=-9.52\left(\mathrm{c} 0.66, \mathrm{CHCl}_{3}, 92 \%\right.$ ee); HPLC analysis using Daicel Chiralpak AD-3 column (Hex: ${ }^{2} \mathrm{PrOH}=95: 5,1.0 \mathrm{~mL} / \mathrm{min}, 254 \mathrm{~nm}, \mathrm{tR}=$ 20.9 min (Major), $32.3 \mathrm{~min}$ (miner)); ${ }^{1} \mathrm{H}$ NMR (600 MHz, $\left.\mathrm{CDCl}_{3}\right) \delta: 7.30(2 \mathrm{H}, \mathrm{d}$, $\mathrm{J}=7.44 \mathrm{~Hz}), 7.27(2 \mathrm{H}, \mathrm{t}, \mathrm{J}=7.44 \mathrm{~Hz}), 7.20(1 \mathrm{H}, \mathrm{t}, \mathrm{J}=7.44 \mathrm{~Hz}), 6.41(1 \mathrm{H}, \mathrm{d}, \mathrm{J}=$ $2.75 \mathrm{~Hz}), 6.20-6.15(2 \mathrm{H}, \mathrm{m}), 5.80(1 \mathrm{H}, \mathrm{s}), 4.46(1 \mathrm{H}, \mathrm{d}, \mathrm{J}=4.12 \mathrm{~Hz}), 3.87(3 \mathrm{H}, \mathrm{s})$, $3.67(3 \mathrm{H}, \mathrm{s}), 3.25-3.23(1 \mathrm{H}, \mathrm{m}), 2.83(3 \mathrm{H}, \mathrm{s}), 2.51(3 \mathrm{H}, \mathrm{s}), 1.30(3 \mathrm{H}, \mathrm{d}, \mathrm{J}=6.87 \mathrm{~Hz}) ;{ }^{13} \mathrm{C} \mathrm{NMR}(\mathrm{CDCl} 3,150$ MHz) $\delta: 174.4,51.1,147.9,143.0,131.8,128.4,127.1,126.6,110.2,103.5,99.1,61.8,55.7,41.2,37.1$, 35.5, 16.5; IR (neat, $\mathrm{cm}^{-1}$ ) 3379, 1632, 1515, 1455, 1204, 1154, 1033; HRMS (Dart) calcd for $\mathrm{C}_{20} \mathrm{H}_{27} \mathrm{~N}_{2} \mathrm{O}_{3}$ $[\mathrm{M}+\mathrm{H}]^{+}$343.2022, found 343.1989.

\section{(2R,3S)-3-((2,4-dimethoxyphenyl)amino)- $N, N, 2$-trimethyl-3-(p-tolyl)propanamide (3ba)}

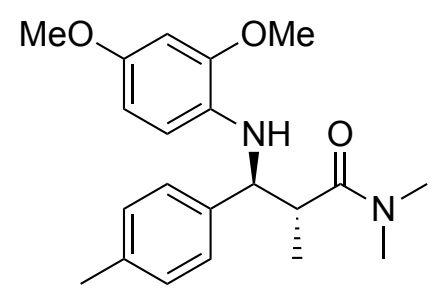

Mp 101-102 ${ }^{\circ} \mathrm{C} ;[\alpha]_{\mathrm{D}}{ }^{20}=-24.99$ (c $1.41, \mathrm{CHCl}_{3}, 95 \%$ ee); HPLC analysis using Daicel Chiralpak AD-3 column (Hex: ${ }^{i} \mathrm{PrOH}=95: 5,1.0 \mathrm{~mL} / \mathrm{min}, 254 \mathrm{~nm}$, tR = $18.3 \mathrm{~min}$ (Major), $32.8 \mathrm{~min}$ (miner)); ${ }^{1} \mathrm{H} \mathrm{NMR}\left(600 \mathrm{MHz}, \mathrm{CDCl}_{3}\right) \delta: 7.18(2 \mathrm{H}$, d, J = 7.91 Hz), $7.07(2 \mathrm{H}, \mathrm{d}, \mathrm{J}=7.91 \mathrm{~Hz}), 6.40(1 \mathrm{H}, \mathrm{d}, \mathrm{J}=2.75 \mathrm{~Hz}), 6.20-6.16$ $(2 \mathrm{H}, \mathrm{m}), 5.74(1 \mathrm{H}, \mathrm{s}), 4.42(1 \mathrm{H}, \mathrm{d}, \mathrm{J}=4.81 \mathrm{~Hz}), 3.86(3 \mathrm{H}, \mathrm{s}), 3.66(3 \mathrm{H}, \mathrm{s})$, 3.23-3.19 (1H, m), $2.84(3 \mathrm{H}, \mathrm{s}), 2.54(3 \mathrm{H}, \mathrm{s}), 2.29(3 \mathrm{H}, \mathrm{s}), 1.28(3 \mathrm{H}, \mathrm{d}, \mathrm{J}=6.87 \mathrm{~Hz}) ;{ }^{3} \mathrm{C} \mathrm{NMR}\left(\mathrm{CDCl}_{3}, 150\right.$ MHz) $\delta: 174.6,151.1,147.9,139.9,136.5,131.9,129.1,126.5,110.2,103.6,99.1,61.5,55.7,55.6,41.3$, 37.1, 35.6, 21.0, 16.5; IR (neat, $\mathrm{cm}^{-1}$ ) 3383, 1632, 1512, 1455, 1245, 1204, 1154, 1033; HRMS (Dart) calcd for $\mathrm{C}_{21} \mathrm{H}_{29} \mathrm{~N}_{2} \mathrm{O}_{3}[\mathrm{M}+\mathrm{H}]^{+}$357.2178, found 357.2171.

\section{(2R,3S)-3-((2,4-dimethoxyphenyl)amino)- $N, N, 2$-trimethyl-3-(m-tolyl)propanamide (3ca)}

Mp 80-81 ${ }^{\circ} \mathrm{C} ;[\alpha]_{\mathrm{D}}{ }^{20}=-7.51$ (c 1.56, $\mathrm{CHCl}_{3}, 90 \%$ ee); HPLC analysis using Daicel Chiralpak AD-3 column (Hex: ${ }^{\mathrm{PrOH}}=98: 2,1.2 \mathrm{~mL} / \mathrm{min}, 254 \mathrm{~nm}, \mathrm{tR}=27.8 \mathrm{~min}$ (Major), $41.0 \mathrm{~min}$ (miner)); ${ }^{1} \mathrm{H} \mathrm{NMR}(600 \mathrm{MHz}$, $\left.\mathrm{CDCl}_{3}\right) \delta: 7.13(3 \mathrm{H}, \mathrm{m}), 7.01(1 \mathrm{H}, \mathrm{d}, \mathrm{J}=7.56 \mathrm{~Hz}), 6.41(1 \mathrm{H}, \mathrm{d}, \mathrm{J}=2.58 \mathrm{~Hz}), 6.20(1 \mathrm{H}, \mathrm{dd}, \mathrm{J}=8.77,2.58$ 
Hz), $6.18(1 \mathrm{H}, \mathrm{d}, \mathrm{J}=8.77 \mathrm{~Hz}), 5.74(1 \mathrm{H}, \mathrm{s}), 4.39(1 \mathrm{H}, \mathrm{d}, \mathrm{J}=4.81 \mathrm{~Hz}), 3.87(3 \mathrm{H}, \mathrm{s}), 3.67$ (3H, s), $3.21(1 \mathrm{H}$, m), $2.83(3 \mathrm{H}, \mathrm{s}), 2.50(3 \mathrm{H}, \mathrm{s}), 2.30(3 \mathrm{H}, \mathrm{s}), 1.28(3 \mathrm{H}, \mathrm{d}, \mathrm{J}=6.87 \mathrm{~Hz}) ;{ }^{13} \mathrm{C} \mathrm{NMR}\left(\mathrm{CDCl}_{3}, 150 \mathrm{MHz}\right) \delta$ : $174.4,151.0,147.9,143.1,137.9,132.0,128.2$, 127.8, 127.1, 123.6, 110.0, 103.5, 99.0, 61.8, 55.7, 55.6, 41.3, 37.0, 35.5, 21.4, 16.6; IR (neat, $\mathrm{cm}^{-1}$ ) 3384, 1632, 1515, 1455, 1246, 1204, 1154, 1032; HRMS (Dart) calcd for $\mathrm{C}_{21} \mathrm{H}_{29} \mathrm{~N}_{2} \mathrm{O}_{3}[\mathrm{M}+\mathrm{H}]^{+}$357.2178, found 357.2164.

\section{(2R,3S)-3-((2,4-dimethoxyphenyl)amino)- $N, N, 2$-trimethyl-3-(o-tolyl)propanamide (3da)}

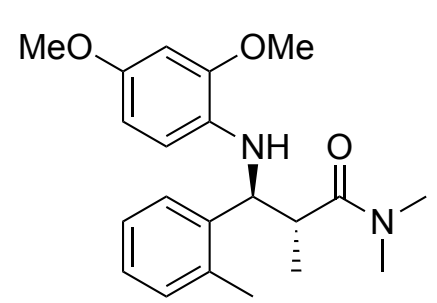

$[\alpha]_{\mathrm{D}}{ }^{20}=-8.63\left(\mathrm{c} 1.21 \mathrm{CHCl}_{3}, 91 \%\right.$ ee); HPLC analysis using Daicel Chiralpak AD-3 column (Hex: ${ }^{i} \mathrm{PrOH}=98: 2,1.2 \mathrm{~mL} / \mathrm{min}, 254 \mathrm{~nm}, \mathrm{tR}=23.3 \mathrm{~min}$ (Major), $25.2 \mathrm{~min}$ (miner)); ${ }^{1} \mathrm{H}$ NMR $\left(600 \mathrm{MHz}, \mathrm{CDCl}_{3}\right) \delta: 7.22(1 \mathrm{H}, \mathrm{d}, \mathrm{J}=7.56 \mathrm{~Hz})$, 7.13-7.05 (3H, m), $6.41(1 \mathrm{H}, \mathrm{d}, \mathrm{J}=2.58 \mathrm{~Hz}), 6.19(1 \mathrm{H}, \mathrm{dd}, \mathrm{J}=8.77,2.58 \mathrm{~Hz})$, $6.00(1 \mathrm{H}, \mathrm{d}, \mathrm{J}=8.77 \mathrm{~Hz}), 5.89(1 \mathrm{H}, \mathrm{s}), 4.65(1 \mathrm{H}, \mathrm{d}, \mathrm{J}=3.44 \mathrm{~Hz}), 3.88(3 \mathrm{H}, \mathrm{s})$, $3.67(3 \mathrm{H}, \mathrm{s}), 3.22-3.20(1 \mathrm{H}, \mathrm{m}), 2.80(3 \mathrm{H}, \mathrm{s}), 2.49(3 \mathrm{H}, \mathrm{s}), 2.32(3 \mathrm{H}, \mathrm{s}), 1.34(3 \mathrm{H}, \mathrm{d}, \mathrm{J}=6.87 \mathrm{~Hz}) ;{ }^{13} \mathrm{C}$ NMR $\left(\mathrm{CDCl}_{3}, 150 \mathrm{MHz}\right) \delta: 174.2,151.0,147.9,140.6,134.5,131.9,130.3,126.9,126.4,125.6,109.4,103.5$, 99.1, 57.6, 55.7, 55.7, 38.6, 36.7, 35.5, 19.0, 16.6; IR (neat, $\mathrm{cm}^{-1}$ ) 3380, 1632, 1517, 1457, 1244, 1204, 1155, 1033; HRMS (Dart) calcd for $\mathrm{C}_{21} \mathrm{H}_{29} \mathrm{~N}_{2} \mathrm{O}_{3}[\mathrm{M}+\mathrm{H}]^{+}$357.2178, found 357.2164.

(2R,3S)-3-((2,4-dimethoxyphenyl)amino)- $N, N, 2$-trimethyl-3-(4-ethylphenyl)propanamide (3ea)

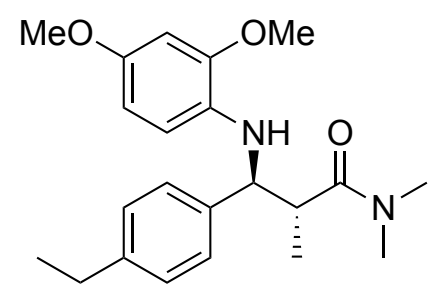

$[\alpha]_{\mathrm{D}}^{20}=-29.69$ (c 1.56, $\mathrm{CHCl}_{3}, 95 \%$ ee); HPLC analysis using Daicel Chiralpak AD-3 column (Hex: ${ }^{i} \mathrm{PrOH}=95: 5,1.0 \mathrm{~mL} / \mathrm{min}, 254 \mathrm{~nm}, \mathrm{tR}=15.0 \mathrm{~min}$ (Major), $26.3 \mathrm{~min}$ (miner)); ${ }^{1} \mathrm{H}$ NMR (600 $\left.\mathrm{MHz}, \mathrm{CDCl}_{3}\right) \delta: 7.20(2 \mathrm{H}, \mathrm{d}, \mathrm{J}=8.25 \mathrm{~Hz})$, $7.09(2 \mathrm{H}, \mathrm{d}, \mathrm{J}=8.25 \mathrm{~Hz}), 6.41(1 \mathrm{H}, \mathrm{d}, \mathrm{J}=2.06 \mathrm{~Hz}), 6.19(2 \mathrm{H}, \mathrm{m}), 5.72(1 \mathrm{H}, \mathrm{s})$, $4.43(1 \mathrm{H}, \mathrm{d}, \mathrm{J}=5.50 \mathrm{~Hz}), 3.86(3 \mathrm{H}, \mathrm{s}), 3.67(3 \mathrm{H}, \mathrm{s}), 3.23-3.19(1 \mathrm{H}, \mathrm{m}), 2.83(3 \mathrm{H}$, s), $2.59(2 \mathrm{H}, \mathrm{q}, \mathrm{J}=7.68 \mathrm{~Hz}), 2.52(3 \mathrm{H}, \mathrm{s}), 1.28(3 \mathrm{H}, \mathrm{d}, \mathrm{J}=6.87 \mathrm{~Hz}), 1.19(3 \mathrm{H}, \mathrm{t}, \mathrm{J}=7.68 \mathrm{~Hz}) ;{ }^{13} \mathrm{C} \mathrm{NMR}$ $\left(\mathrm{CDCl}_{3}, 150 \mathrm{MHz}\right) \delta: 174.5,151.1,147.9,143.0,140.2,132.0,127.9,126.5,110.1,103.5,99.1,61.5,55.7$, 55.7, 41.4, 37.1, 35.6, 28.4, 16.6, 15.6; IR (neat, $\mathrm{cm}^{-1}$ ) 3377, 1634, 1515, 1455, 1245, 1204, 1154, 1033; HRMS (Dart) calcd for $\mathrm{C}_{22} \mathrm{H}_{31} \mathrm{~N}_{2} \mathrm{O}_{3}[\mathrm{M}+\mathrm{H}]^{+} 371.2335$, found 371.2317 .

\section{(2R,3S)-3-([1,1'-biphenyl]-4-yl)-3-((2,4-dimethoxyphenyl)amino)- $N, N, 2$-trimethylpropanamide (3fa)}

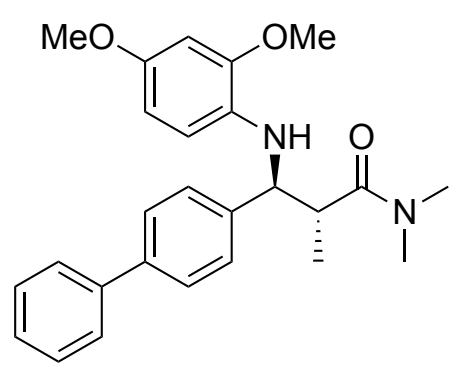

Mp $146-147{ }^{\circ} \mathrm{C} ;[\alpha]_{\mathrm{D}}{ }^{20}=-69.07$ (c $0.76, \mathrm{CHCl}_{3}, 94 \%$ ee); HPLC analysis using Daicel Chiralpak AD-3 column (Hex: ${ }^{i} \mathrm{PrOH}=95: 5,1.0 \mathrm{~mL} / \mathrm{min}, 254 \mathrm{~nm}$, $\mathrm{tR}=27.3 \mathrm{~min}$ (Major), $64.5 \mathrm{~min}$ (miner)) ${ }^{1} \mathrm{H} \mathrm{NMR}\left(600 \mathrm{MHz}, \mathrm{CDCl}_{3}\right) \delta: 7.56$ $(2 \mathrm{H}, \mathrm{d}, \mathrm{J}=7.56 \mathrm{~Hz}), 7.51(2 \mathrm{H}, \mathrm{d}, \mathrm{J}=7.78 \mathrm{~Hz}), 7.41(2 \mathrm{H}, \mathrm{t}, \mathrm{J}=7.78 \mathrm{~Hz}), 7.37$ $(2 \mathrm{H}, \mathrm{d}, \mathrm{J}=7.56 \mathrm{~Hz}), 7.32(1 \mathrm{H}, \mathrm{t}, \mathrm{J}=7.78 \mathrm{~Hz}), 6.43(1 \mathrm{H}, \mathrm{d}, \mathrm{J}=1.37 \mathrm{~Hz}), 6.21$ $(2 \mathrm{H}, \mathrm{s}), 5.82(1 \mathrm{H}, \mathrm{s}), 4.51(1 \mathrm{H}, \mathrm{d}, \mathrm{J}=4.81 \mathrm{~Hz}), 3.88(3 \mathrm{H}, \mathrm{s}), 3.67(3 \mathrm{H}, \mathrm{s})$, 3.28-3.26 (1H, m), $2.84(3 \mathrm{H}, \mathrm{s}), 2.57(3 \mathrm{H}, \mathrm{s}), 1.32(3 \mathrm{H}, \mathrm{d}, \mathrm{J}=6.87 \mathrm{~Hz}) ;{ }^{13} \mathrm{C} \mathrm{NMR}\left(\mathrm{CDCl}_{3}, 150 \mathrm{MHz}\right) \delta$ : 174.4, 151.2, 147.6, 142.2, 140.7, 139.8, 131.8, 128.7, 127.2, 127.1, 127.0, 126.9, 110.2, 103.5, 99.1, 61.5, 55.7, 41.3, 37.2, 35.6, 16.6; IR (neat, $\mathrm{cm}^{-1}$ ) 3367, 1628, 1512, 1455, 1265, 1198, 1117, 1028; HRMS (Dart) calcd for $\mathrm{C}_{26} \mathrm{H}_{31} \mathrm{~N}_{2} \mathrm{O}_{3}[\mathrm{M}+\mathrm{H}]^{+} 419.2335$, found 419.2318 


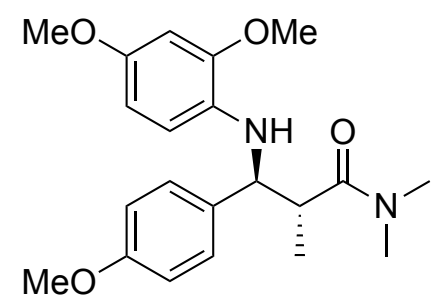

$[\alpha]_{\mathrm{D}}^{20}=-29.32\left(\mathrm{c} 1.19, \mathrm{CHCl}_{3}, 94 \%\right.$ ee); HPLC analysis using Daicel Chiralpak AD-3 column (Hex: ${ }^{i} \mathrm{PrOH}=95: 5,1.0 \mathrm{~mL} / \mathrm{min}, 254 \mathrm{~nm}, \mathrm{tR}=32.3 \mathrm{~min}$ (Major), $63.9 \mathrm{~min}($ miner $)) ;{ }^{1} \mathrm{H} \mathrm{NMR}\left(600 \mathrm{MHz}, \mathrm{CDCl}_{3}\right) \delta: 7.21(2 \mathrm{H}, \mathrm{d}, \mathrm{J}=8.60 \mathrm{~Hz}), 6.80$ $(2 \mathrm{H}, \mathrm{d}, \mathrm{J}=8.60 \mathrm{~Hz}), 6.41(1 \mathrm{H}, \mathrm{d}, \mathrm{J}=2.75 \mathrm{~Hz}), 6.20-6.16(2 \mathrm{H}, \mathrm{m}), 5.74(1 \mathrm{H}, \mathrm{s})$, $4.41(1 \mathrm{H}, \mathrm{d}, \mathrm{J}=4.81 \mathrm{~Hz}), 3.86(3 \mathrm{H}, \mathrm{s}), 3.76(3 \mathrm{H}, \mathrm{s}), 3.66(3 \mathrm{H}, \mathrm{s}), 3.22-3.18(1 \mathrm{H}$, m), $2.84(3 \mathrm{H}, \mathrm{s}), 2.57(3 \mathrm{H}, \mathrm{s}), 1.28(3 \mathrm{H}, \mathrm{d}, \mathrm{J}=6.87 \mathrm{~Hz}) ;{ }^{13} \mathrm{C} \mathrm{NMR}\left(\mathrm{CDCl}_{3}, 150 \mathrm{MHz}\right) \delta: 174.5,158.6,151.1$, 147.9, 135.0, 131.9, 127.5, 113.7, 110.1, 103.5, 99.0, 61.1, 55.6, 55.6, 55.1, 41.4, 37.1, 35.5, 16.5; IR (neat, $\mathrm{cm}^{-1}$ ) 3379, 1632, 1509, 1457, 1242, 1204, 1154, 1031; HRMS (Dart) calcd for $\mathrm{C}_{21} \mathrm{H}_{29} \mathrm{~N}_{2} \mathrm{O}_{3}[\mathrm{M}+\mathrm{H}]^{+}$ 373.2127 , found 373.2112 .

\section{(2R,3S)-3-((2,4-dimethoxyphenyl)amino)-3-(3-methoxyphenyl)- $N, N, 2$-trimethylpropanamide (3ha)}

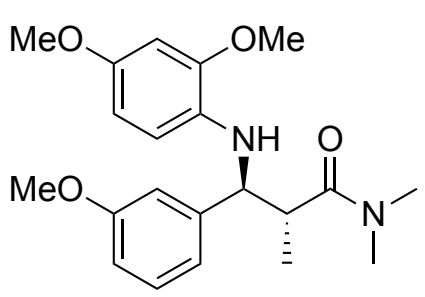

$[\alpha]_{\mathrm{D}^{20}}=-16.66\left(\mathrm{c} 1.17, \mathrm{CHCl}_{3}, 91 \%\right.$ ee); HPLC analysis using Daicel Chiralpak OD-3 column (Hex: ${ }^{i} \mathrm{PrOH}=95: 5,1.0 \mathrm{~mL} / \mathrm{min}, 254 \mathrm{~nm}$, tR = $20.9 \mathrm{~min}$ (miner), $31.1 \mathrm{~min}$ (Major) $)^{1} \mathrm{H}$ NMR (600 MHz, $\left.\mathrm{CDCl}_{3}\right) \delta: 7.19(1 \mathrm{H}, \mathrm{t}, \mathrm{J}=7.90 \mathrm{~Hz}), 6.91$ $(1 \mathrm{H}, \mathrm{d}, \mathrm{J}=7.90 \mathrm{~Hz}), 6.87(1 \mathrm{H}, \mathrm{d}, \mathrm{J}=1.37 \mathrm{~Hz}), 6.74(1 \mathrm{H}, \mathrm{dd}, \mathrm{J}=7.90,1.37$ $\mathrm{Hz}), 6.41(1 \mathrm{H}, \mathrm{d}, \mathrm{J}=1.37 \mathrm{~Hz}), 6.21-6.16(2 \mathrm{H}, \mathrm{m}), 5.74(1 \mathrm{H}, \mathrm{s}), 4.41(1 \mathrm{H}, \mathrm{d}, \mathrm{J}=$ $4.81 \mathrm{~Hz}), 3.87(3 \mathrm{H}, \mathrm{s}), 3.76(3 \mathrm{H}, \mathrm{s}), 3.67(3 \mathrm{H}, \mathrm{s}), 3.24-3.20(1 \mathrm{H}, \mathrm{m}), 2.85(3 \mathrm{H}, \mathrm{s}), 2.55(3 \mathrm{H}, \mathrm{s}), 1.29(3 \mathrm{H}, \mathrm{d}, \mathrm{J}$ $=6.87 \mathrm{~Hz}) ;{ }^{13} \mathrm{C} \mathrm{NMR}\left(\mathrm{CDCl}_{3}, 150 \mathrm{MHz}\right) \delta: 174.4,159.8,151.2,147.9,145.0,131.9,129.4,119.0,112.6$, 111.9, 110.2, 103.5, 99.1, 61.9, 55.7, 55.6, 55.2, 41.2, 37.1, 35.6, 16.6; IR (neat, $\mathrm{cm}^{-1}$ ) 3376, 1632, 1514, 1454, 1251, 1204, 1154, 1033; HRMS (Dart) calcd for $\mathrm{C}_{21} \mathrm{H}_{29} \mathrm{~N}_{2} \mathrm{O}_{4}[\mathrm{M}+\mathrm{H}]^{+}$373.2127, found 373.2116.

(2R,3S)-3-((2,4-dimethoxyphenyl)amino)-3-(2-methoxyphenyl)- $N, N, 2$-trimethylpropanamide (3ia)<smiles>COc1ccc(NC(C(=O)N(C)C)[C@H](C)c2ccccc2OC)c(OC)c1</smiles>

Mp 86-88 ${ }^{\circ} \mathrm{C} ;[\alpha]_{\mathrm{D}}{ }^{20}=-29.54$ (c $1.29, \mathrm{CHCl}_{3}, 92 \%$ ee); HPLC analysis using Daicel Chiralpak AD-3 column (Hex: ${ }^{2} \mathrm{PrOH}=98: 2,1.2 \mathrm{~mL} / \mathrm{min}, 254 \mathrm{~nm}$, tR = $42.9 \min$ (Major), $47.8 \mathrm{~min}$ (miner)) ${ }^{1} \mathrm{H}$ NMR (600 $\left.\mathrm{MHz}, \mathrm{CDCl}_{3}\right) \delta: \quad 7.10-7.08$ $(2 \mathrm{H}, \mathrm{m}), 6.79(1 \mathrm{H}, \mathrm{d}, \mathrm{J}=8.25 \mathrm{~Hz}), 6.73(1 \mathrm{H}, \mathrm{t}, \mathrm{J}=7.22 \mathrm{~Hz}), 6.33(1 \mathrm{H}, \mathrm{d}, \mathrm{J}=$ $2.58 \mathrm{~Hz}), 6.13(1 \mathrm{H}, \mathrm{dd}, \mathrm{J}=8.77,2.58 \mathrm{~Hz}), 6.09(1 \mathrm{H}, \mathrm{d}, \mathrm{J}=8.77 \mathrm{~Hz}), 5.82(1 \mathrm{H}, \mathrm{s})$, $4.76(1 \mathrm{H}, \mathrm{d}, \mathrm{J}=4.12 \mathrm{~Hz}), 3.85(3 \mathrm{H}, \mathrm{s}), 3.79(3 \mathrm{H}, \mathrm{s}), 3.59(3 \mathrm{H}, \mathrm{s}), 3.42-3.38(1 \mathrm{H}, \mathrm{m}), 2.70(3 \mathrm{H}, \mathrm{s}), 2.43(3 \mathrm{H}$, s), $1.22(3 \mathrm{H}, \mathrm{d}, \mathrm{J}=6.87 \mathrm{~Hz}) ;{ }^{13} \mathrm{C} \mathrm{NMR}\left(\mathrm{CDCl}_{3}, 150 \mathrm{MHz}\right) \delta: 174.2,151.0,147.9,140.6,134.5,131.9,130.3$, 126.9, 126.4, 125.6, 109.4, 103.5, 99.1, 57.6, 55.7, 55.7, 38.6, 36.7, 35.5, 19.0, 16.6; IR (neat) 3406, 1629, 1521, 1455, 1237, 1201, 1031; HRMS (Dart) calcd for $\mathrm{C}_{21} \mathrm{H}_{29} \mathrm{~N}_{2} \mathrm{O}_{3}[\mathrm{M}+\mathrm{H}]^{+}$373.2127, found 373.2139.

\section{(2R,3S)-3-((2,4-dimethoxyphenyl)amino)-3-(4-fluorophenyl)- $N, N, 2$-trimethylpropanamide (3ja)}

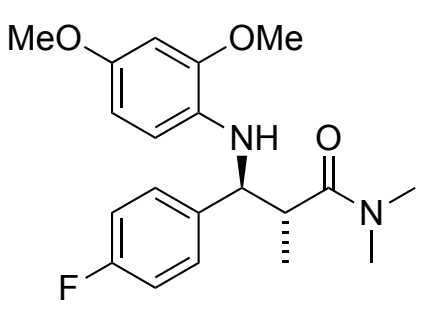

Mp $126-127{ }^{\circ} \mathrm{C} ;[\alpha]_{\mathrm{D}^{20}}=-7.65$ (c 1.13, $\mathrm{CHCl}_{3}, 94 \%$ ee); HPLC analysis using Daicel Chiralpak OD-3 column (Hex: ${ }^{i} \mathrm{PrOH}=95: 5,1.0 \mathrm{~mL} / \mathrm{min}, 254 \mathrm{~nm}$, $\mathrm{tR}=$ $28.0 \mathrm{~min}$ (miner), $31.3 \mathrm{~min}$ (Major)); ${ }^{1} \mathrm{H} \mathrm{NMR}\left(600 \mathrm{MHz}, \mathrm{CDCl}_{3}\right) \delta: 7.27$ (2H, dd, $\mathrm{J}=8.59,6.53 \mathrm{~Hz}), 6.96(2 \mathrm{H}, \mathrm{t}, \mathrm{J}=8.59 \mathrm{~Hz}), 6.42(1 \mathrm{H}, \mathrm{d}, \mathrm{J}=2.58 \mathrm{~Hz}), 6.19(1 \mathrm{H}$, $\mathrm{dd}, \mathrm{J}=8.77,2.58 \mathrm{~Hz}), 6.13(1 \mathrm{H}, \mathrm{d}, \mathrm{J}=8.77 \mathrm{~Hz}), 5.82(1 \mathrm{H}, \mathrm{s}), 4.45(1 \mathrm{H}, \mathrm{d}, \mathrm{J}=$ $4.81 \mathrm{~Hz}), 3.87(3 \mathrm{H}, \mathrm{s}), 3.67(3 \mathrm{H}, \mathrm{s}), 3.20(1 \mathrm{H}, \mathrm{m}), 2.84(3 \mathrm{H}, \mathrm{s}), 2.58(3 \mathrm{H}, \mathrm{s}), 1.29(3 \mathrm{H}, \mathrm{d}, \mathrm{J}=6.87 \mathrm{~Hz}) ;{ }^{13} \mathrm{C}$ NMR $\left(\mathrm{CDCl}_{3}, 150 \mathrm{MHz}\right) \delta: 174.3,161.8(\mathrm{~d}, \mathrm{~J}=245.6 \mathrm{~Hz}), 151.3,147.9,138.7,131.7,128.0$, (d, J = 8.67 
$\mathrm{Hz}), 115.2,(\mathrm{~d}, \mathrm{~J}=21.7), 110.1,103.5,99.1,61.0,55.6,55.6,41.3,37.1,35.5,16.4 ;{ }^{19} \mathrm{~F}$ NMR (CDCl3, 465 MHz) $\delta:-119.1$; IR (neat, $\mathrm{cm}^{-1}$ ) 3346, 1615, 1509, 1207, 1154, 1031; HRMS (Dart) calcd for $\mathrm{C}_{20} \mathrm{H}_{26} \mathrm{FN}_{2} \mathrm{O}_{3}$ $[\mathrm{M}+\mathrm{H}]^{+}$361.1928, found 361.1926.

(2R,3S)-3-(4-chlorophenyl)-3-((2,4-dimethoxyphenyl)amino)- $N, N, 2$-trimethylpropanamide (3ka)

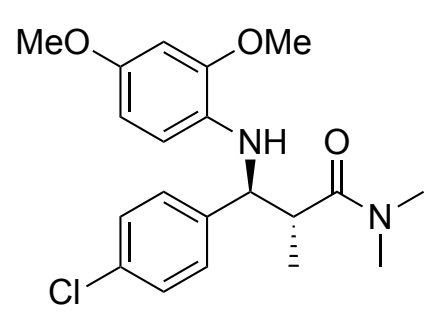

Mp 119-121 ${ }^{\circ} \mathrm{C} ;[\alpha]_{\mathrm{D}}{ }^{20}=-29.68$ (c $1.37, \mathrm{CHCl}_{3}, 95 \%$ ee); HPLC analysis using Daicel Chiralpak AD-3 column $\left(\mathrm{Hex}:{ }^{i} \mathrm{PrOH}=95: 5,1.0 \mathrm{~mL} / \mathrm{min}, 254 \mathrm{~nm}\right.$, tR = $20.0 \mathrm{~min}$ (Major), $40.0 \mathrm{~min}$ (miner)); ${ }^{1} \mathrm{H} \mathrm{NMR}\left(600 \mathrm{MHz}, \mathrm{CDCl}_{3}\right) \quad \delta: 7.25(4 \mathrm{H}$, m), $6.42(1 \mathrm{H}, \mathrm{d}, \mathrm{J}=2.58 \mathrm{~Hz}), 6.19(1 \mathrm{H}, \mathrm{dd}, \mathrm{J}=8.42,2.58 \mathrm{~Hz}), 6.11(1 \mathrm{H}, \mathrm{d}, \mathrm{J}=$ $8.42 \mathrm{~Hz}), 5.83(1 \mathrm{H}, \mathrm{s}), 4.44(1 \mathrm{H}, \mathrm{d}, \mathrm{J}=4.81 \mathrm{~Hz}), 3.87(3 \mathrm{H}, \mathrm{s}), 3.67(3 \mathrm{H}, \mathrm{s}), 3.21$

$(1 \mathrm{H}, \mathrm{m}), 2.84(3 \mathrm{H}, \mathrm{s}), 2.60(3 \mathrm{H}, \mathrm{s}), 1.30(3 \mathrm{H}, \mathrm{d}, \mathrm{J}=6.87 \mathrm{~Hz}) ;{ }^{13} \mathrm{C} \mathrm{NMR}\left(\mathrm{CDCl}_{3}, 150 \mathrm{MHz}\right) \delta: 174.2,151.3$, 148.0, 141.7, 132.6, 131.5, 128.6, 128.0, 110.1, 103.4, 99.1, 61.1, 55.7, 55.6, 41.1, 37.2, 35.6, 16.5; IR (neat, $\mathrm{cm}^{-1}$ ) 3379, 1632, 1515, 1455, 1204, 1154, 1031; HRMS (Dart) calcd for $\mathrm{C}_{20} \mathrm{H}_{26} \mathrm{ClN}_{2} \mathrm{O}_{3}[\mathrm{M}+\mathrm{H}]^{+} 377.1632$, found 377.1634 .

(2R,3S)-3-(4-bromophenyl)-3-((2,4-dimethoxyphenyl)amino)- $N, N, 2$-trimethylpropanamide (3la)<smiles>COc1ccc(NC(C(=O)N(C)C)[C@H](C)c2ccc(Br)cc2)c(OC)c1</smiles>

Mp 104-106 ${ }^{\circ} \mathrm{C} ;[\alpha]_{\mathrm{D}}{ }^{20}=-45.20\left(\mathrm{c} 1.07, \mathrm{CHCl}_{3}, 96 \%\right.$ ee); HPLC analysis using Daicel Chiralpak OD-3 column (Hex: ${ }^{\mathrm{PrOH}}=95: 5,1.0 \mathrm{~mL} / \mathrm{min}, 254 \mathrm{~nm}$, tR $=$ $26.8 \mathrm{~min}$ (miner), $30.2 \mathrm{~min}$ (Major)); ${ }^{1} \mathrm{H}$ NMR (600 MHz, CDCl3) $\delta: 7.39$ (2H, d, $\mathrm{J}=8.25 \mathrm{~Hz}), 7.20(2 \mathrm{H}, \mathrm{d}, \mathrm{J}=8.25 \mathrm{~Hz}), \quad 6.42(1 \mathrm{H}, \mathrm{d}, \mathrm{J}=2.75 \mathrm{~Hz}), 6.19(1 \mathrm{H}, \mathrm{dd}$, $\mathrm{J}=8.60,2.75 \mathrm{~Hz}), 6.11(1 \mathrm{H}, \mathrm{d}, \mathrm{J}=8.60 \mathrm{~Hz}), 5.84(1 \mathrm{H}, \mathrm{s}), 4.43(1 \mathrm{H}, \mathrm{d}, \mathrm{J}=5.50$ $\mathrm{Hz}), 3.87(3 \mathrm{H}, \mathrm{s}), 3.67(3 \mathrm{H}, \mathrm{s}), 3.20(1 \mathrm{H}, \mathrm{m}), 2.84(3 \mathrm{H}, \mathrm{s}), 2.60(3 \mathrm{H}, \mathrm{s}), 1.30(3 \mathrm{H}, \mathrm{d}, \mathrm{J}=6.87 \mathrm{~Hz}){ }^{13} \mathrm{C}$ NMR (CDCl3, $150 \mathrm{MHz}) \delta: 174.1,151.3,148.0,142.2$, 131.5, 131.5, 128.4, 120.7, 110.1, 103.4, 99.1, 61.2, 55.7, 55.6, 41.1, 37.2, 35.6, 16.5; IR (neat, $\mathrm{cm}^{-1}$ ) 3379, 1632, 1515, 1455, 1204, 1154, 1033; HRMS (Dart) calcd for $\mathrm{C}_{20} \mathrm{H}_{26} \mathrm{BrN}_{2} \mathrm{O}_{3}[\mathrm{M}+\mathrm{H}]^{+} 421.1127$, found 421.1131 .

\section{(2R,3S)-3-(3-bromophenyl)-3-((2,4-dimethoxyphenyl)amino)- $N, N, 2$-trimethylpropanamide (3ma)}

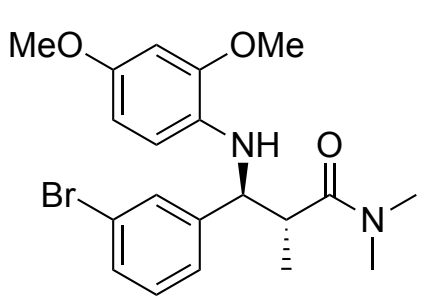

$[\alpha]_{\mathrm{D}}{ }^{20}=-18.35$ (c 1.13, $\mathrm{CHCl}_{3}, 86 \%$ ee); HPLC analysis using Daicel Chiralpak OD-3 column (Hex: ${ }^{i} \mathrm{PrOH}=95: 5,1.0 \mathrm{~mL} / \mathrm{min}, 254 \mathrm{~nm}, \mathrm{tR}=17.9 \mathrm{~min}$ (miner), $32.2 \mathrm{~min}$ (Major)); ${ }^{1} \mathrm{H}$ NMR (600 MHz, $\left.\mathrm{CDCl}_{3}\right) \delta: 7.49(1 \mathrm{H}, \mathrm{s}), 7.34(1 \mathrm{H}, \mathrm{d}, \mathrm{J}$ $=7.90 \mathrm{~Hz}), 7.24(1 \mathrm{H}, \mathrm{d}, \mathrm{J}=7.90 \mathrm{~Hz}), 7.14(1 \mathrm{H}, \mathrm{t}, \mathrm{J}=7.90 \mathrm{~Hz}), 6.42(1 \mathrm{H}, \mathrm{d}, \mathrm{J}=$ $2.58 \mathrm{~Hz}), 6.21(1 \mathrm{H}, \mathrm{dd}, \mathrm{J}=8.77,2.58 \mathrm{~Hz}), 6.15(1 \mathrm{H}, \mathrm{d}, \mathrm{J}=8.77 \mathrm{~Hz}), 5.79(1 \mathrm{H}, \mathrm{s})$, $4.41(1 \mathrm{H}, \mathrm{d}, \mathrm{J}=4.81 \mathrm{~Hz}), 3.87(3 \mathrm{H}, \mathrm{s}), 3.68(3 \mathrm{H}, \mathrm{s}), 3.22-3.18(1 \mathrm{H}, \mathrm{m}), 2.85(3 \mathrm{H}, \mathrm{s}), 2.58(3 \mathrm{H}, \mathrm{s}), 1.29(3 \mathrm{H}$, $\mathrm{d}, \mathrm{J}=6.19 \mathrm{~Hz}) ;{ }^{13} \mathrm{C} \mathrm{NMR}\left(\mathrm{CDCl}_{3}, 150 \mathrm{MHz}\right) \delta: 174.0,151.4,148.0,145.9,131.5,130.3,130.0,129.6$, 125.4, 122.7, 110.4, 103.5, 99.1, 61.5, 55.7, 55.6, 41.1, 37.2, 35.6, 16.5; IR (neat, $\mathrm{cm}^{-1}$ ) 3382, 1635, 1517, 1457, 1204, 1154, 1033; HRMS (Dart) calcd for $\mathrm{C}_{20} \mathrm{H}_{26} \mathrm{BrN}_{2} \mathrm{O}_{3}[\mathrm{M}+\mathrm{H}]^{+} 421.1127$, found 421.1113 .

\section{(2R,3S)-3-(2-bromophenyl)-3-((2,4-dimethoxyphenyl)amino)- $N, N, 2$-trimethylpropanamide (3na)}

Mp $150-152{ }^{\circ} \mathrm{C} ;[\alpha]_{\mathrm{D}}{ }^{20}=45.84\left(\mathrm{c} 0.98, \mathrm{CHCl}_{3}, 85 \%\right.$ ee); HPLC analysis using Daicel Chiralpak OD-3 column $\left(\mathrm{Hex}:{ }^{i} \mathrm{PrOH}=95: 5,1.0 \mathrm{~mL} / \mathrm{min}, 254 \mathrm{~nm}, \mathrm{tR}=10.7 \mathrm{~min}\right.$ (miner), $11.6 \mathrm{~min}$ (Major)); ${ }^{1} \mathrm{H}$ NMR (600 $\left.\mathrm{MHz} \mathrm{CDCl}_{3}\right) \delta: 7.52(1 \mathrm{H}, \mathrm{d}, \mathrm{J}=7.56 \mathrm{~Hz}), 7.27-7.26(1 \mathrm{H}, \mathrm{m}), 7.16(1 \mathrm{H}, \mathrm{t}, \mathrm{J}=7.56 \mathrm{~Hz}), 7.07(1 \mathrm{H}, \mathrm{t}, 7.56$ 
Hz), $6.43(1 \mathrm{H}, \mathrm{d}, \mathrm{J}=2.06 \mathrm{~Hz}), 6.25-6.19(2 \mathrm{H}, \mathrm{m}), 6.00(1 \mathrm{H}, \mathrm{d}, \mathrm{J}=7.56 \mathrm{~Hz}), 4.82(1 \mathrm{H}, \mathrm{s}), 3.90$ (3H, s), 3.67 $(3 \mathrm{H}, \mathrm{s}), 3.52-3.48(1 \mathrm{H}, \mathrm{m}), 2.77(3 \mathrm{H}, \mathrm{s}), 2.39(3 \mathrm{H}, \mathrm{s}), 1.41(3 \mathrm{H}, \mathrm{d}, \mathrm{J}=6.87 \mathrm{~Hz}) ;{ }^{13} \mathrm{C}$ NMR $(\mathrm{CDCl} 3,150$ MHz) $\delta: 174.1,151.1,147.9,141.0,132.6,131.4,128.8,128.2$, 127.7, 123.2, 109.3, 103.5, 99.1, 60.0, 55.7, 55.6, 37.2, 36.8, 35.4, 16.3; IR (neat, $\mathrm{cm}^{-1}$ ) 3419, 1634, 1521, 1457, 1247, 1201, 1164, 1034; HRMS (Dart) calcd for $\mathrm{C}_{20} \mathrm{H}_{26} \mathrm{BrN}_{2} \mathrm{O}_{3}[\mathrm{M}+\mathrm{H}]^{+}$421.1127, found 421.1129 .

(2R,3S)-3-((2,4-dimethoxyphenyl)amino)- $N, N, 2$-trimethyl-3-(3-(trifluoromethyl)phenyl)propanamide (30a)

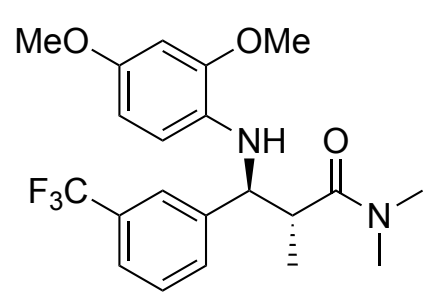

$[\alpha]_{\mathrm{D}}^{20}=-6.45\left(\mathrm{c} 1.42 \mathrm{CHCl}_{3}, 87 \%\right.$ ee); HPLC analysis using Daicel Chiralpak OD-3 column (Hex: ${ }^{i} \mathrm{PrOH}=95: 5,1.0 \mathrm{~mL} / \mathrm{min}, 254 \mathrm{~nm}, \mathrm{tR}=13.4 \mathrm{~min}$ (miner), $27.0 \mathrm{~min}$ (Major)); ${ }^{1} \mathrm{H}$ NMR (600 MHz, $\left.\mathrm{CDCl}_{3}\right) \delta: 7.60(1 \mathrm{H}, \mathrm{s}), 7.51(1 \mathrm{H}, \mathrm{d}, \mathrm{J}=$ $7.90 \mathrm{~Hz}), 7.48(1 \mathrm{H}, \mathrm{d}, \mathrm{J}=7.90 \mathrm{~Hz}), 7.39(1 \mathrm{H}, \mathrm{t}, \mathrm{J}=7.90 \mathrm{~Hz}), 6.43(1 \mathrm{H}, \mathrm{d}, \mathrm{J}=$ $2.24 \mathrm{~Hz}), 6.20(1 \mathrm{H}, \mathrm{dd}, \mathrm{J}=8.77,2.24 \mathrm{~Hz}), 6.14(1 \mathrm{H}, \mathrm{d}, \mathrm{J}=8.77 \mathrm{~Hz}), 5.88(1 \mathrm{H}, \mathrm{s})$, $4.52(1 \mathrm{H}, \mathrm{d}, \mathrm{J}=4.81 \mathrm{~Hz}), 3.88(3 \mathrm{H}, \mathrm{s}), 3.67(3 \mathrm{H}, \mathrm{s}), 3.24-3.22(1 \mathrm{H}, \mathrm{m}), 2.82(3 \mathrm{H}, \mathrm{s}), 2.53(3 \mathrm{H}, \mathrm{s}), 1.31(3 \mathrm{H}$, $\mathrm{d}, \mathrm{J}=6.87 \mathrm{~Hz}) ;{ }^{13} \mathrm{C} \mathrm{NMR}\left(\mathrm{CDCl}_{3}, 150 \mathrm{MHz}\right) \delta: 173.9,151.5,148.1,144.5,131.5,130.7$ (q, J = 31.8 Hz), $130.1,128.9,124.1$ (q, $\mathrm{J}=272.1 \mathrm{~Hz}), 124.0,123.4,110.2$, 103.5, 99.1, 61.7, 55.6, 41.1, 37.0, 35.5, 16.4; ${ }^{19} \mathrm{~F}$ NMR $\left(\mathrm{CDCl}_{3}, 565 \mathrm{MHz}\right) \delta$ :-62.3; IR (neat, $\left.\mathrm{cm}^{-1}\right)$ 3385, 1634, 1515, 1327, 1155, 1117, 1071, 1033; HRMS (Dart) calcd for $\mathrm{C}_{21} \mathrm{H}_{26} \mathrm{~F}_{3} \mathrm{~N}_{2} \mathrm{O}_{3}[\mathrm{M}+\mathrm{H}]^{+} 411.1896$, found 411.1905 .

\section{(2R,3S)-3-((2,4-dimethoxyphenyl)amino)- $N, N, 2$-trimethyl-3-(naphthalen-2-yl)propanamide (3pa)}

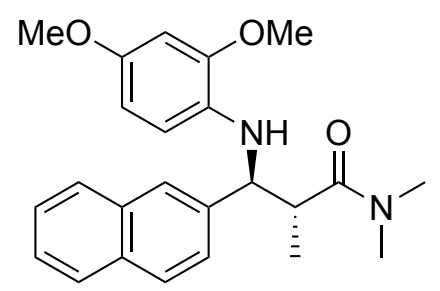

$[\alpha]_{\mathrm{D}^{20}}=-31.60\left(\mathrm{c} 1.11 \mathrm{CHCl}_{3}, 92 \%\right.$ ee); HPLC analysis using Daicel Chiralpak AD-3 column (Hex: ${ }^{i} \mathrm{PrOH}=95: 5,1.0 \mathrm{~mL} / \mathrm{min}, 254 \mathrm{~nm}$, tR = $26.2 \mathrm{~min}$ (Major), $53.4 \mathrm{~min}$ (miner)); ${ }^{1} \mathrm{H} \mathrm{NMR}\left(600 \mathrm{MHz}, \mathrm{CHCl}_{3}\right) \delta:$ 7.79-7.76 (4H, m), 7.46-7.41 $(3 \mathrm{H}, \mathrm{m}), 6.41(1 \mathrm{H}, \mathrm{d}, \mathrm{J}=2.75 \mathrm{~Hz}), 6.20(1 \mathrm{H}, \mathrm{d}, \mathrm{J}=8.25 \mathrm{~Hz}), 6.14(1 \mathrm{H}, \mathrm{d}, \mathrm{J}=$ $8.25 \mathrm{~Hz}), 5.91(1 \mathrm{H}, \mathrm{s}), 4.62(1 \mathrm{H}, \mathrm{d}, \mathrm{J}=3.44 \mathrm{~Hz}), 3.89(3 \mathrm{H}, \mathrm{s}), 3.63(3 \mathrm{H}, \mathrm{s})$, 3.38-3.28 (1H, m), $2.80(3 \mathrm{H}, \mathrm{s}), 2.46(3 \mathrm{H}, \mathrm{s}), 1.33(3 \mathrm{H}, \mathrm{d}, \mathrm{J}=6.87 \mathrm{~Hz}) ;{ }^{13} \mathrm{C} \mathrm{NMR}\left(\mathrm{CDCl}_{3}, 150 \mathrm{MHz}\right) \delta$ : $174.4,151.2,147.9,140.6,133.4,132.8,131.8,128.2,127.8,127.6,125.9,125.5,125.4,124.8,110.3,103.5$, 99.1, 62.1, 55.6, 55.6, 41.2, 37.1, 35.6, 16.6; HRMS (Dart) calcd for $\mathrm{C}_{24} \mathrm{H}_{29} \mathrm{~N}_{2} \mathrm{O}_{3}[\mathrm{M}+\mathrm{H}]^{+}$393.2178, found 393.2170 .

\section{(2R,3S)-3-((2,4-dimethoxyphenyl)amino)- $N, N, 2$-trimethyl-3-(pyridin-4-yl)propanamide (3qa)}

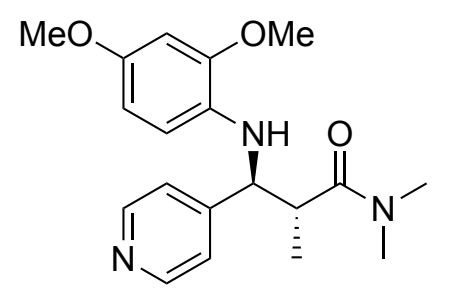

$[\alpha]_{\mathrm{D}^{20}}=-18.3\left(\mathrm{c} 0.48 \mathrm{CHCl}_{3}, 87 \%\right.$ ee); HPLC analysis using Daicel Chiralpak AD-3 column (Hex: ${ }^{i} \mathrm{PrOH}=80: 20,1.0 \mathrm{~mL} / \mathrm{min}, 254 \mathrm{~nm}, \mathrm{tR}=11.9 \mathrm{~min}$ (Major), $23.0 \mathrm{~min}($ miner $)) ;{ }^{1} \mathrm{H}$ NMR $\left(500 \mathrm{MHz}, \mathrm{CDCl}_{3}\right) \delta: 8.51(2 \mathrm{H}, \mathrm{d}, \mathrm{J}=6.24 \mathrm{~Hz})$, 7.28-7.27 (2H, m), $6.43(1 \mathrm{H}, \mathrm{d}, \mathrm{J}=2.69 \mathrm{~Hz}), 6.19(1 \mathrm{H}, \mathrm{dd}, \mathrm{J}=8.65,2.69 \mathrm{~Hz})$, $6.10(1 \mathrm{H}, \mathrm{d}, \mathrm{J}=8.65 \mathrm{~Hz}), 4.48(1 \mathrm{H}, \mathrm{d}, \mathrm{J}=4.53 \mathrm{~Hz}), 3.88(3 \mathrm{H}, \mathrm{s}), 3.68(3 \mathrm{H}, \mathrm{s})$, 3.28-3.23 (1H, m), $2.83(3 \mathrm{H}, \mathrm{s}), 2.67(3 \mathrm{H}, \mathrm{s}), 1.33(3 \mathrm{H}, \mathrm{d}, \mathrm{J}=6.80 \mathrm{~Hz}) ;{ }^{13} \mathrm{C} \mathrm{NMR}\left(\mathrm{CDCl}_{3}, 150 \mathrm{MHz}\right) \delta$ : $173.9,152.6,151.7,149.7,148.1,131.1,122.1,110.2,103.4,99.2,60.9$, 55.7, 55.6, 40.6, 37.3, 35.5, 16.4; IR (neat, $\mathrm{cm}^{-1}$ ) 3379, 1632, 1515, 1457, 1414, 1247, 1204, 1155, 1031; HRMS (Dart) calcd for $\mathrm{C}_{19} \mathrm{H}_{26} \mathrm{~N}_{3} \mathrm{O}_{3}$ $[\mathrm{M}+\mathrm{H}]^{+}$344.1974, found 344.1979. 


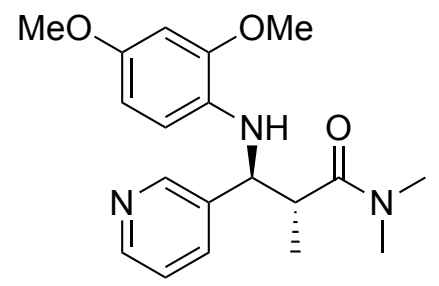

Mp 98-100 ${ }^{\circ} \mathrm{C} ;[\alpha]_{\mathrm{D}}{ }^{20}=-17.2\left(\mathrm{c} 0.54 \mathrm{CHCl}_{3}, 95 \%\right.$ ee); HPLC analysis using Daicel Chiralpak OD-3 column (Hex: ${ }^{i} \mathrm{PrOH}=80: 20,1.0 \mathrm{~mL} / \mathrm{min}, 254 \mathrm{~nm}$, $\mathrm{tR}=$ 11.2 min (major), 54.6 min (miner)); ${ }^{1} \mathrm{H} \mathrm{NMR}\left(500 \mathrm{MHz}, \mathrm{CDCl}_{3}\right) \delta: 8.62(1 \mathrm{H}, \mathrm{s})$, $8.47(1 \mathrm{H}, \mathrm{s}), 7.67(1 \mathrm{H}, \mathrm{d}, \mathrm{J}=7.94 \mathrm{~Hz}), 7.22(1 \mathrm{H}, \mathrm{dd}, \mathrm{J}=7.75,4.55 \mathrm{~Hz}), 6.42(1 \mathrm{H}$, $\mathrm{d}, \mathrm{J}=2.27 \mathrm{~Hz}), 6.21-6.17(2 \mathrm{H}, \mathrm{m}), 4.57(1 \mathrm{H}, \mathrm{d}, \mathrm{J}=5.10 \mathrm{~Hz}), 3.87(3 \mathrm{H}, \mathrm{s}), 3.67$ $(3 \mathrm{H}, \mathrm{s}), 3.30-3.25(1 \mathrm{H}, \mathrm{m}), 2.84(3 \mathrm{H}, \mathrm{s}), 2.69(3 \mathrm{H}, \mathrm{s}), 1.32(3 \mathrm{H}, \mathrm{d}, \mathrm{J}=6.80 \mathrm{~Hz}) ;{ }^{13} \mathrm{C} \mathrm{NMR}\left(\mathrm{CDCl}_{3}, 150\right.$ MHz) $\delta: 174.0,151.2,148.4,148.2,148.1,138.5,134.9,130.8,123.6,110.7,103.5,99.2,59.5,55.6,41.2$, 37.2, 35.5, 16.2; IR (neat, $\mathrm{cm}^{-1}$ ) 3350, 1615, 1517, 1208, 1154, 1027; HRMS (Dart) calcd for $\mathrm{C}_{19} \mathrm{H}_{26} \mathrm{~N}_{3} \mathrm{O}_{3}$ $[\mathrm{M}+\mathrm{H}]^{+}$344.1974, found 344.1936.

\section{(2R,3S)-3-((2,4-dimethoxyphenyl)amino)-N,N,2-trimethyl-3-(pyridin-2-yl)propanamide (3sa)}

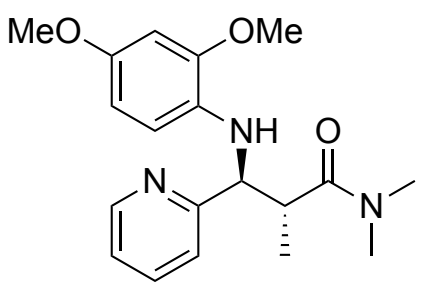

HPLC analysis using Daicel Chiralpak OD-3 column $\left(\mathrm{Hex}:{ }^{i} \mathrm{PrOH}=92.5: 7.5,1.0\right.$ $\mathrm{mL} / \mathrm{min}, 254 \mathrm{~nm}, \mathrm{tR}=14.3 \mathrm{~min}$ (Major), $15.5 \mathrm{~min}$ (miner)); ${ }^{1} \mathrm{H} \mathrm{NMR}(500 \mathrm{MHz}$, CDCl3) $\delta: ~ 8.58-8.57(1 \mathrm{H}, \mathrm{m}), 7.55(1 \mathrm{H}, \mathrm{td}, \mathrm{J}=7.51,1.70 \mathrm{~Hz}), 7.28(1 \mathrm{H}, \mathrm{d}, \mathrm{J}=$ $5.39 \mathrm{~Hz}), 7.13(1 \mathrm{H}, \mathrm{ddd}, \mathrm{J}=7.51,5.39,1.13 \mathrm{~Hz}), 6.43(1 \mathrm{H}, \mathrm{d}, \mathrm{J}=2.27 \mathrm{~Hz})$, 6.26-6.24 (2H, m), $4.62(1 \mathrm{H}, \mathrm{d}, \mathrm{J}=5.10 \mathrm{~Hz}), 3.86(3 \mathrm{H}, \mathrm{s}), 3.69-3.64(4 \mathrm{H}, \mathrm{m})$, $2.79(3 \mathrm{H}, \mathrm{s}), 2.67(3 \mathrm{H}, \mathrm{s}), 1.30(3 \mathrm{H}, \mathrm{d}, \mathrm{J}=6.80 \mathrm{~Hz}) ;{ }^{13} \mathrm{C} \mathrm{NMR}\left(\mathrm{CDCl}_{3}, 150 \mathrm{MHz}\right) \delta: 174.8,162.3,151.4$, 149.1, 148.0, 136.7, 131.8, 122.1, 121.5, 110.2, 103.4, 99.2, 63.0, 55.7, 55.6, 39.4, 37.2, 35.4, 16.1; IR (neat, $\mathrm{cm}^{-1}$ ) 3380, 1634, 1512, 1457, 1204, 1154, 1031; HRMS (Dart) calcd for $\mathrm{C}_{19} \mathrm{H}_{26} \mathrm{~N}_{3} \mathrm{O}_{3}[\mathrm{M}+\mathrm{H}]^{+} 344.1974$, found 344.1962 .

\section{(2S,3S)-3-((2,4-dimethoxyphenyl)amino)- $N, N$-dimethyl-3-cyclopropylpropanamide (3ta)}

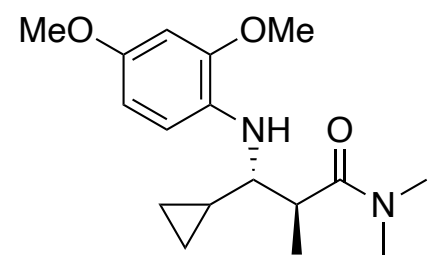

$[\alpha]_{\mathrm{D}}^{25}=+34.9$ (c 1.17, $\mathrm{CHCl}_{3}, 92 \%$ ee); HPLC analysis using Daicel Chiralpak AD-3 column (Hex: ${ }^{i} \mathrm{PrOH}=19: 1,1.0 \mathrm{~mL} / \mathrm{min}, 254 \mathrm{~nm}, \mathrm{tR}=20.0 \mathrm{~min}$ (major), 21.5 min (minor); ${ }^{1} \mathrm{H}$ NMR (600 MHz, $\left.\mathrm{CDCl}_{3}\right) \delta: 6.55(1 \mathrm{H}, \mathrm{d}, \mathrm{J}=8.9 \mathrm{~Hz}), 6.42$ $(1 \mathrm{H}, \mathrm{d}, \mathrm{J}=2.8 \mathrm{~Hz}), 6.36-6.34(1 \mathrm{H}, \mathrm{dd}, \mathrm{J}=8.9,2.8 \mathrm{~Hz}), 4.81(1 \mathrm{H}, \mathrm{br}, \mathrm{NH}), 3.83$ $(3 \mathrm{H}, \mathrm{s}), 3.73(3 \mathrm{H}, \mathrm{s}), 3.15-3.08(5 \mathrm{H}, \mathrm{m}), 2.93(3 \mathrm{H}, \mathrm{s}), 1.19(3 \mathrm{H}, \mathrm{d}, \mathrm{J}=6.8 \mathrm{~Hz}), 0.95-0.90(1 \mathrm{H}$, $\mathrm{m}), 0.46-0.42(1 \mathrm{H}, \mathrm{m}), 0.39-0.35(1 \mathrm{H}, \mathrm{m}), 0.32-0.28(1 \mathrm{H}, \mathrm{m}), 0.23-0.20(1 \mathrm{H}, \mathrm{m}) ;{ }^{13} \mathrm{C} \mathrm{NMR}\left(\mathrm{CDCl}_{3}, 150\right.$ MHz) $\delta: 175.7,151.3,148.0,133.3,111.2,103.9,99.2,60.6,55.8,55.7,40.1,37.7,35.7,15.2,15.0,3.2,1.9$; IR (neat, $\mathrm{cm}^{-1}$ ): 3373, 1634, 1594, 1514, 1460, 1414, 1397, 1204, 1154, 1033; HRMS (Dart) calcd for $\mathrm{C}_{17} \mathrm{H}_{27} \mathrm{~N}_{2} \mathrm{O}_{3}[\mathrm{M}+\mathrm{H}]^{+}$307.2022, found 307.2016.

\section{(2R,3S)-3-((2-methoxyphenyl)amino)- $N, N, 4,4$-tetramethylpentanamide (3ua)}

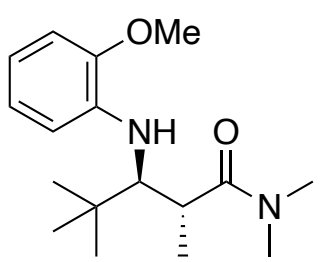

Mp 133-135 ${ }^{\circ} \mathrm{C} ;[\alpha]_{\mathrm{D}}{ }^{25}=+20.5\left(\mathrm{c} 0.35, \mathrm{CHCl}_{3}, 89 \%\right.$ ee); HPLC analysis using Daicel Chiralpak IBN-5 column $\left(\mathrm{Hex}:{ }^{i} \mathrm{PrOH}=50: 50,0.3 \mathrm{~mL} / \mathrm{min}, 254 \mathrm{~nm}, \mathrm{tR}=13.1 \mathrm{~min}\right.$ (minor), $22.5 \mathrm{~min}$ (major); ${ }^{1} \mathrm{H} \mathrm{NMR}\left(600 \mathrm{MHz}, \mathrm{CDCl}_{3}\right)$ 8: 6.80-6.74 (3H, m), 6.58 $(1 \mathrm{H}, \mathrm{d}, \mathrm{J}=7.6 \mathrm{~Hz}), 6.52(1 \mathrm{H}, \mathrm{m}), 3.90(3 \mathrm{H}, \mathrm{s}), 3.20(1 \mathrm{H}, \mathrm{d}, \mathrm{J}=2.8 \mathrm{~Hz}), 3.14(1 \mathrm{H}, \mathrm{m})$, $3.11(3 \mathrm{H}, \mathrm{s}), 2.96(3 \mathrm{H}, \mathrm{s}), 1.10(3 \mathrm{H}, \mathrm{d}, \mathrm{J}=6.9 \mathrm{~Hz}), 0.96(9 \mathrm{H}, \mathrm{s}) ;{ }^{13} \mathrm{C} \mathrm{NMR}\left(\mathrm{CDCl}_{3}, 150 \mathrm{MHz}\right) \delta: 177.1$, 146.7, 141.0, 120.9, 114.1, 109.7, 108.5, 66.2, 55.8, 37.5, 37.1, 35.7, 33.6, 27.9, 17.7; IR (neat, $\mathrm{cm}^{-1}$ ) 3339, 
$1641,1597,1517,1507,1457,1415,1392,1364,1292,1242,1221,1160,1122,1109,1051,1025$; HRMS (Dart) calcd for $\mathrm{C}_{17} \mathrm{H}_{29} \mathrm{~N}_{2} \mathrm{O}_{2}[\mathrm{M}+\mathrm{H}]^{+}$293.2229, found 293.2221.

\section{(2R,3S)-3-((2-methoxyphenyl)amino)- $N, N, 4,4-$ tetramethyl-5-phenylpentanamide (3va)}

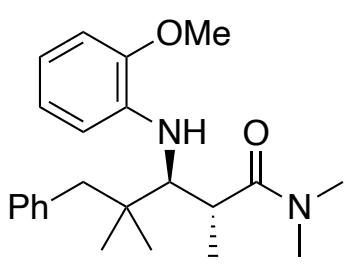

$[\alpha]_{\mathrm{D}}^{23}=+4.27$ (c 1.34, $\mathrm{CHCl}_{3}, 88 \%$ ee); HPLC analysis using Daicel Chiralpak IBN-5 column (Hex: ${ }^{i} \mathrm{PrOH}=67: 33,0.5 \mathrm{~mL} / \mathrm{min}, 254 \mathrm{~nm}$, tR = $10.3 \mathrm{~min}$ (minor), $24.1 \mathrm{~min}$ (major); ${ }^{1} \mathrm{H}$ NMR (600 MHz, $\left.\mathrm{CDCl}_{3}\right) \delta: 7.255-7.22(2 \mathrm{H}, \mathrm{m}), 7.18(1 \mathrm{H}, \mathrm{t}, \mathrm{J}$

$=7.6 \mathrm{~Hz}), 7.10(2 \mathrm{H}, \mathrm{d}, \mathrm{J}=7.6 \mathrm{~Hz}), 6.94(1 \mathrm{H}, \mathrm{br}), 6.79-6.75(2 \mathrm{H}, \mathrm{m}), 6.57-6.51(2 \mathrm{H}$, m), $3.92(3 \mathrm{H}, \mathrm{s}), 3.32(1 \mathrm{H}, \mathrm{br}), 3.25(1 \mathrm{H}, \mathrm{dq}, \mathrm{J}=6.9,2.8 \mathrm{~Hz}), 3.12(3 \mathrm{H}, \mathrm{s}), 2.96(3 \mathrm{H}, \mathrm{s}), 2.67-2.62(2 \mathrm{H}, \mathrm{m})$, $1.12(3 \mathrm{H}, \mathrm{d}, \mathrm{J}=6.8 \mathrm{~Hz}), 0.88(3 \mathrm{H}, \mathrm{s}), 0.85(3 \mathrm{H}, \mathrm{s}) ;{ }^{13} \mathrm{C} \mathrm{NMR}\left(\mathrm{CDCl}_{3}, 150 \mathrm{MHz}\right) \delta: 177.0,146.7,140.9$, 138.8, 130.8, 127.5, 125.7, 120.8, 114.3, 109.7, 108.6, 66.3, 55.8, 45.8, 40.9, 37.4, 35.7, 33.4, 24.3, 23.8, 17.7; IR (neat, $\mathrm{cm}^{-1}$ ) 3349, 1637, 1597, 1511, 1495, 1457, 1434, 1415, 1398, 1294, 1244, 1221, 1157, 1120 , 1053, 1028; HRMS (Dart) calcd for $\mathrm{C}_{23} \mathrm{H}_{33} \mathrm{~N}_{2} \mathrm{O}_{2}[\mathrm{M}+\mathrm{H}]^{+}$369.2542, found 369.2532.

(S)-2-((R)-((2,4-dimethoxyphenyl)amino)(phenyl)methyl)- $N, N$-dimethylbutanamide (3ab)

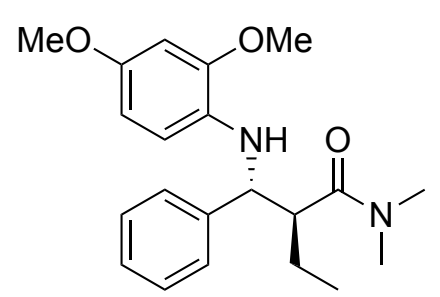

$[\alpha]_{\mathrm{D}^{20}}=+1.13\left(\mathrm{c} 1.41, \mathrm{CHCl}_{3}, 94 \%\right.$ ee); HPLC analysis using Daicel Chiralpak AD-3 column (Hex: ${ }^{i} \mathrm{PrOH}=95: 5,1.0 \mathrm{~mL} / \mathrm{min}, 254 \mathrm{~nm}$ ) $\mathrm{tR}=16.6 \mathrm{~min}$ (minor), 27.8 min (major); ${ }^{1} \mathrm{H}$ NMR (600 MHz, $\left.\mathrm{CDCl}_{3}\right) \delta: 7.3-7.2(4 \mathrm{H}, \mathrm{m}), 7.18(1 \mathrm{H}, \mathrm{t}, \mathrm{J}$ $=6.9 \mathrm{~Hz}), 6.41(1 \mathrm{H}, \mathrm{d}, \mathrm{J}=2.8 \mathrm{~Hz}), 6.17(1 \mathrm{H}, \mathrm{dd}, \mathrm{J}=8.9,2.8 \mathrm{~Hz}), 6.10(1 \mathrm{H}, \mathrm{d}, \mathrm{J}$ $=8.9 \mathrm{~Hz}), 6.06(1 \mathrm{H}, \mathrm{br}), 4.52(1 \mathrm{H}, \mathrm{d}, \mathrm{J}=4.1 \mathrm{~Hz}), 3.87(3 \mathrm{H}, \mathrm{s}), 3.65(3 \mathrm{H}, \mathrm{s}), 3.05$ $(1 \mathrm{H}, \mathrm{ddd}, \mathrm{J}=8.9,4.9,4.9 \mathrm{~Hz}), 2.79(3 \mathrm{H}, \mathrm{s}), 2.36(3 \mathrm{H}, \mathrm{s}), 2.05(1 \mathrm{H}, \mathrm{m}), 1.71(1 \mathrm{H}, \mathrm{m}), 0.91(3 \mathrm{H}, \mathrm{t}, \mathrm{J}=7.6$ $\mathrm{Hz}) ;{ }^{13} \mathrm{C} \mathrm{NMR}\left(\mathrm{CDCl}_{3}, 150 \mathrm{MHz}\right) \delta: 173.7,150.8,147.7,143.1,131.7,128.3,126.9,126.3,109.5,103.4$, 99.0, 59.8, 55.6, 55.5, 48.7, 36.9, 35.3, 24.7, 12.1; IR (neat, $\mathrm{cm}^{-1}$ ) 3377, 1629, 1515, 1495, 1437, 1414, 1398, 1285, 1244, 1202, 1154, 1135, 1031; HRMS (Dart) calcd for $\mathrm{C}_{21} \mathrm{H}_{29} \mathrm{~N}_{2} \mathrm{O}_{3}[\mathrm{M}+\mathrm{H}]^{+}$357.2178, found 357.2177 .

\section{$(S)-2-((R)-((2,4-d i m e t h o x y p h e n y l) a m i n o)($ phenyl)methyl)- $N, N$-dimethylheptanamide (3ac)}

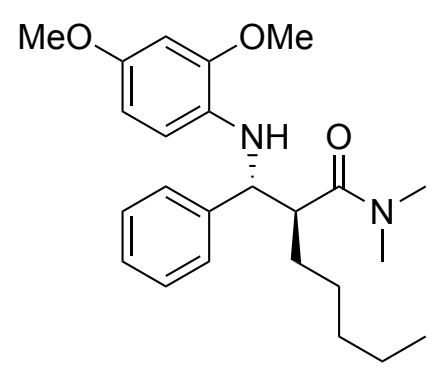

$[\alpha]_{\mathrm{D}}^{20}=-9.35$ (c 1.31, $\mathrm{CHCl}_{3}, 94 \%$ ee); HPLC analysis using Daicel Chiralcel OD-3 column $\left(\mathrm{Hex}:{ }^{i} \mathrm{PrOH}=97.5: 2.5,1.0 \mathrm{~mL} / \mathrm{min}, 254 \mathrm{~nm}\right) \mathrm{tR}=11.7 \mathrm{~min}$ (minor), $13.1 \mathrm{~min}$ (major); ${ }^{1} \mathrm{H}$ NMR (600 $\left.\mathrm{MHz}, \mathrm{CDCl}_{3}\right) \delta: 7.3-7.2(4 \mathrm{H}, \mathrm{m}), 7.17$ $(1 \mathrm{H}, \mathrm{t}, \mathrm{J}=6.8 \mathrm{~Hz}), 6.41(1 \mathrm{H}, \mathrm{d}, \mathrm{J}=2.1 \mathrm{~Hz}), 6.17(1 \mathrm{H}, \mathrm{dd}, \mathrm{J}=8.3,2.8 \mathrm{~Hz}), 6.10$ $(1 \mathrm{H}, \mathrm{br}), 6.09(1 \mathrm{H}, \mathrm{d}, \mathrm{J}=8.2 \mathrm{~Hz}), 4.51(1 \mathrm{H}, \mathrm{d}, \mathrm{J}=4.1 \mathrm{~Hz}), 3.87(3 \mathrm{H}, \mathrm{s}), 3.64(3 \mathrm{H}$, s), $3.11(1 \mathrm{H}, \mathrm{ddd}, \mathrm{J}=8.9,4.8,4.8 \mathrm{~Hz}), 2.78(3 \mathrm{H}, \mathrm{s}), 2.34(3 \mathrm{H}, \mathrm{s}), 2.04(1 \mathrm{H}, \mathrm{m})$, $1.66(1 \mathrm{H}, \mathrm{m}), 1.35-1.20(6 \mathrm{H}, \mathrm{m}), 0.85(3 \mathrm{H}, \mathrm{t}, \mathrm{J}=6.9 \mathrm{~Hz}) ;{ }^{13} \mathrm{C} \mathrm{NMR}\left(\mathrm{CDCl}_{3}, 150 \mathrm{MHz}\right) \delta: 173.8,150.8$, $147.7,143.1,131.7,128.3,126.9$, 126.3, 109.5, 103.4, 99.0, 60.2, 55.6, 55.5, 47.0, 36.8, 35.3, 31.7, 31.6, 27.4, 22.4, 13.9; IR (neat, $\mathrm{cm}^{-1}$ ) 3377, 1632, 1517, 1495, 1492, 1455, 1437, 1414, 1398, 1287, 1245, 1204, 1154, 1137, 1111, 1034; HRMS (Dart) calcd for $\mathrm{C}_{24} \mathrm{H}_{35} \mathrm{~N}_{2} \mathrm{O}_{3}[\mathrm{M}+\mathrm{H}]^{+} 399.2648$, found 399.2636.

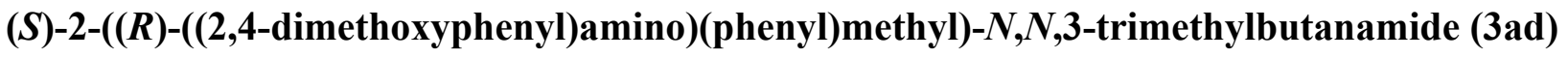

Mp $106{ }^{\circ} \mathrm{C} ;[\alpha]_{\mathrm{D}}{ }^{20}=-15.2$ (c 1.36, $\mathrm{CHCl}_{3}, 91 \%$ ee); HPLC analysis using two Daicel Chiralcel OD-3 columns (Hex: ${ }^{i} \mathrm{PrOH}=80: 20,0.5 \mathrm{~mL} / \mathrm{min}, 254 \mathrm{~nm}$ ) $\mathrm{tR}=17.0 \mathrm{~min}$ (minor), $17.9 \mathrm{~min}$ (major); ${ }^{1} \mathrm{H}$ NMR (600 


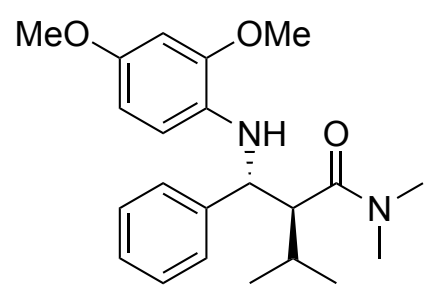

$\left.\mathrm{MHz}, \mathrm{CDCl}_{3}\right) \delta: 7.3-7.2(4 \mathrm{H}, \mathrm{m}), 7.16(1 \mathrm{H}, \mathrm{t}, \mathrm{J}=6.9 \mathrm{~Hz}), 6.42(1 \mathrm{H}, \mathrm{d}, \mathrm{J}=2.8$ Hz), $6.34(1 \mathrm{H}, \mathrm{br}), 6.16(1 \mathrm{H}, \mathrm{dd}, \mathrm{J}=8.3,2.8 \mathrm{~Hz}), 6.07(1 \mathrm{H}, \mathrm{d}, \mathrm{J}=8.2 \mathrm{~Hz}), 4.67$ (1H, br), $3.88(3 \mathrm{H}, \mathrm{s}), 3.65(3 \mathrm{H}, \mathrm{s}), 2.73(3 \mathrm{H}, \mathrm{s}), 2.71(1 \mathrm{H}, \mathrm{m}), 2.48$ (1H. m), 2.23 $(3 \mathrm{H}, \mathrm{s}), 1.06(3 \mathrm{H}, \mathrm{d}, \mathrm{J}=6.8 \mathrm{~Hz}), 0.88(3 \mathrm{H}, \mathrm{d}, \mathrm{J}=6.8 \mathrm{~Hz}) ;{ }^{13} \mathrm{C} \mathrm{NMR}\left(\mathrm{CDCl}_{3}, 150\right.$

MHz) $\delta: 173.9,150.6,147.7,143.0,131.6,128.2,126.9,126.4,108.8,103.3$, 99.0, 56.6, 55.6, 55.6, 54.3, 36.9, 35.1, 28.6, 21.4, 20.2; IR (neat, $\mathrm{cm}^{-1}$ ) 3369, 1624, 1494, 1464, 1457, 1452, 1438, 1412, 1398, 1290, 1284, 1265, 1245, 1220, 1198, 1182, 1161, 1095, 1045, 1030; HRMS (Dart) calcd for $\mathrm{C}_{22} \mathrm{H}_{31} \mathrm{~N}_{2} \mathrm{O}_{3}[\mathrm{M}+\mathrm{H}]^{+}$371.2335, found 371.2326.

\section{(2S,3R)-2-benzyl-3-((2,4-dimethoxyphenyl)amino)- $N, N$-dimethyl-3-phenylpropanamide (3ae)}

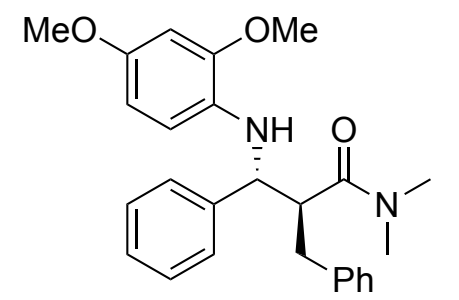

$[\alpha]_{\mathrm{D}}{ }^{20}=-34.2\left(\mathrm{c} 1.21, \mathrm{CHCl}_{3}, 97 \%\right.$ ee); HPLC analysis using Daicel Chiralcel OD-3 column $\left(\mathrm{Hex}:{ }^{i} \mathrm{PrOH}=97.5: 2.5,1.0 \mathrm{~mL} / \mathrm{min}, 254 \mathrm{~nm}\right) \mathrm{tR}=21.4 \mathrm{~min}$ (major), $25.9 \mathrm{~min}$ (minor); ${ }^{1} \mathrm{H} \mathrm{NMR}\left(600 \mathrm{MHz}, \mathrm{CDCl}_{3}\right) \delta: 7.25-7.10(10 \mathrm{H}, \mathrm{m})$, $6.44(1 \mathrm{H}, \mathrm{d}, \mathrm{J}=2.0 \mathrm{~Hz}), 6.24(1 \mathrm{H}, \mathrm{br}), 6.17(1 \mathrm{H}, \mathrm{dd}, \mathrm{J}=8.9,2.8 \mathrm{~Hz}), 6.08(1 \mathrm{H}$, $\mathrm{d}, \mathrm{J}=8.2 \mathrm{~Hz}), 4.54(1 \mathrm{H}, \mathrm{d}, \mathrm{J}=4.1 \mathrm{~Hz}), 3.91(3 \mathrm{H}, \mathrm{s}), 3.66(3 \mathrm{H}, \mathrm{s}), 3.33(1 \mathrm{H}, \mathrm{m})$, $3.23(1 \mathrm{H}, \mathrm{dd}, \mathrm{J}=13,8.2 \mathrm{~Hz}), 3.04(1 \mathrm{H}, \mathrm{dd}, \mathrm{J}=13,6.2 \mathrm{~Hz}), 2.69(3 \mathrm{H}, \mathrm{s}), 2.12(3 \mathrm{H}, \mathrm{s}) ;{ }^{13} \mathrm{C} \mathrm{NMR}\left(\mathrm{CDCl}_{3}\right.$, $150 \mathrm{MHz}) \delta: 173.1,150.9,147.9,142.7,139.6,131.5,128.9,128.3,128.3,127.0,126.4,126.2,109.8,103.5$, 99.0, 59.2, 55.6, 55.6, 49.3, 37.6, 36.7, 35.3; IR (neat, $\mathrm{cm}^{-1}$ ) 3357, 1622, 1520, 1494, 1471, 1461, 1452, 1415, 1404, 1292, 1257, 1244, 1204, 1151, 1135, 1047, 1034; HRMS (Dart) calcd for $\mathrm{C}_{26} \mathrm{H}_{31} \mathrm{~N}_{2} \mathrm{O}_{3}[\mathrm{M}+\mathrm{H}]^{+}$ 419.2335, found 419.2323.

\section{(S)-3-((2,4-dimethoxyphenyl)amino)- $N, N, 3-$ dimethyl-3-phenylpropanamide (3af)}

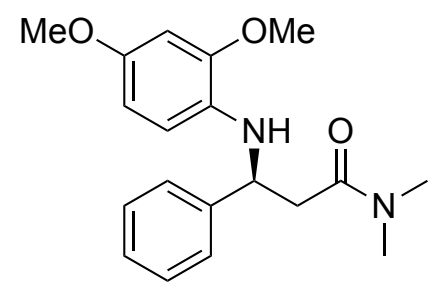

$[\alpha]_{\mathrm{D}}^{20}=+20.9\left(\mathrm{c} 1.28, \mathrm{CHCl}_{3}, 82 \%\right.$ ee); HPLC analysis using Daicel Chiralpak AD-3 column (Hex: $\left.{ }^{i} \mathrm{PrOH}=90: 10,1.0 \mathrm{~mL} / \mathrm{min}, 254 \mathrm{~nm}\right) \mathrm{tR}=25.8 \mathrm{~min}$ (major), $31.9 \min$ (minor); ${ }^{1} \mathrm{H}$ NMR $\left(600 \mathrm{MHz}, \mathrm{CDCl}_{3}\right) \delta: 7.40 \quad(2 \mathrm{H}, \mathrm{d}, \mathrm{J}=7.6 \mathrm{~Hz}), 7.29$ $(2 \mathrm{H}, \mathrm{t}, \mathrm{J}=7.6 \mathrm{~Hz}), 7.21(1 \mathrm{H}, \mathrm{t}, 7.6 \mathrm{~Hz}), 6.42(1 \mathrm{H}, \mathrm{d}, \mathrm{J}=2.8 \mathrm{~Hz}), 6.30(1 \mathrm{H}, \mathrm{d}, \mathrm{J}=$ $8.2 \mathrm{~Hz}), 6.21(1 \mathrm{H}, \mathrm{dd}, \mathrm{J}=8.2,2.8 \mathrm{~Hz}), 5.05(1 \mathrm{H}, \mathrm{br}), 4.78(1 \mathrm{H}, \mathrm{t}, \mathrm{J}=6.2 \mathrm{~Hz})$, $3.84(3 \mathrm{H}, \mathrm{s}), 3.66(3 \mathrm{H}, \mathrm{s}), 2.87(1 \mathrm{H}, \mathrm{dd}, \mathrm{J}=14,7.6 \mathrm{~Hz}), 2.87(3 \mathrm{H}, \mathrm{s}), 2.80(1 \mathrm{H}, \mathrm{dd}, \mathrm{J}=15,5.5 \mathrm{~Hz}), 2.77(3 \mathrm{H}$, s); ${ }^{13} \mathrm{C} \mathrm{NMR}\left(\mathrm{CDCl}_{3}, 150 \mathrm{MHz}\right) \delta: 170.4,151.8,148.1,143.3,131.1,128.5,127.0,126.3,111.6,103.4,98.9$, 55.7, 55.5, 55.4, 41.2, 37.2, 35.3; IR (neat, $\mathrm{cm}^{-1}$ ) 3379, 1634, 1452, 1438, 1413, 1411, 1397, 1285, 1251, 1238, 1204, 1152, 1132, 1031; HRMS (Dart) calcd for $\mathrm{C}_{19} \mathrm{H}_{25} \mathrm{~N}_{2} \mathrm{O}_{3}[\mathrm{M}+\mathrm{H}]^{+} 329.1865$, found 329.1860.

\section{(2R,3S)- $N$-[3-((2,4-dimethoxyphenyl)amino)-2-methyl-3-phenylpropionoyl]pyrrolidine (3ah)}

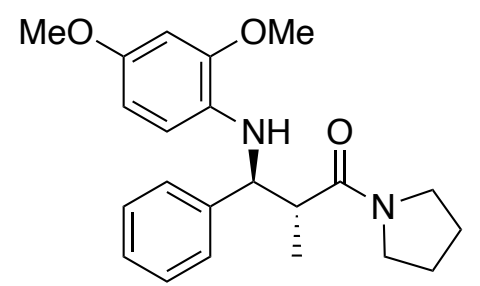

Mp $135-138{ }^{\circ} \mathrm{C} ;[\alpha]_{\mathrm{D}}^{25}=-5.99\left(\mathrm{c} 0.81, \mathrm{CHCl}_{3}, 89 \%\right.$ ee); HPLC analysis using Daicel Chiralpak AD-3 column (Hex: ${ }^{i} \mathrm{PrOH}=19: 1,1.0 \mathrm{~mL} / \mathrm{min}, 254 \mathrm{~nm}, \mathrm{tR}=$ 24.6 min (major), $31.8 \mathrm{~min}$ (minor); ${ }^{1} \mathrm{H}$ NMR (600 $\left.\mathrm{MHz}, \mathrm{CDCl}_{3}\right)$ $\delta: 7.30-7.29$ $(2 \mathrm{H}, \mathrm{m}), 7.25-7.22(2 \mathrm{H}, \mathrm{m}), 7.17-7.15(1 \mathrm{H}, \mathrm{m}), 6.39(1 \mathrm{H}, \mathrm{d}, \mathrm{J}=2.0 \mathrm{~Hz}), 6.16$ $(1 \mathrm{H}, \mathrm{dd}, \mathrm{J}=2.8 \mathrm{~Hz}, 8.3 \mathrm{~Hz}), 6.12(1 \mathrm{H}, \mathrm{d}, \mathrm{J}=8.3 \mathrm{~Hz}) 5.93(1 \mathrm{H}, \mathrm{s}), 4.42(1 \mathrm{H}, \mathrm{d}$, $\mathrm{J}=4.8 \mathrm{~Hz}), 3.85(3 \mathrm{H}, \mathrm{s}), 3.64(3 \mathrm{H}, \mathrm{s}), 3.38-3.34(1 \mathrm{H}, \mathrm{m}), 3.31-3.27(1 \mathrm{H}, \mathrm{m}), 3.12-3.08(1 \mathrm{H}, \mathrm{m}), 3.00-2.97$ $(1 \mathrm{H}, \mathrm{m}), 2.44-2.40(1 \mathrm{H}, \mathrm{m}), 1.69-1.64(2 \mathrm{H}, \mathrm{m}), 1.59-1.56(1 \mathrm{H}, \mathrm{m}), 1.47-1.45(1 \mathrm{H}, \mathrm{m}), 1.32(3 \mathrm{H}, \mathrm{d}, \mathrm{J}=6.9$ 
$\mathrm{Hz}) ;{ }^{13} \mathrm{C} \mathrm{NMR}\left(\mathrm{CDCl}_{3}, 150 \mathrm{MHz}\right) \delta: 172.7,151.0,147.7,143.2,131.9,128.3,127.0,126.48$ 109.9, 103.5, 99.1, 61.7, 55.7, 55.6, 46.4, 45.4, 43.9, 25.8, 24.1, 16.3; IR (neat, $\mathrm{cm}^{-1}$ ): 3342, 1615, 1517, 1452, 1204, 1147 , 1033; HRMS (Dart) calcd for $\mathrm{C}_{22} \mathrm{H}_{29} \mathrm{~N}_{2} \mathrm{O}_{3}[\mathrm{M}+\mathrm{H}]^{+} 369.2178$, found 369.2181.

\section{(2R,3S)-3-((2-methoxyphenyl)amino)- $N, N$-diethyl-3-phenylpropanamide (3ai)}

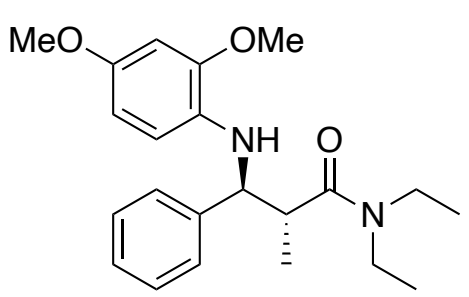

$[\alpha]_{\mathrm{D}^{25}}=-24.12$ (c $0.61, \mathrm{CHCl}_{3}, 94 \%$ ee); HPLC analysis using Daicel Chiralpak AD-3 column (Hex: ${ }^{i} \mathrm{PrOH}=19: 1,1.0 \mathrm{~mL} / \mathrm{min}, 254 \mathrm{~nm}$, tR = 13.9 $\min$ (major), $36.4 \mathrm{~min}$ (minor); ${ }^{1} \mathrm{H} \mathrm{NMR}\left(600 \mathrm{MHz}, \mathrm{CDCl}_{3}\right) \delta$ : 7.31-7.30 (2H, m), 7.25-7.22 (2H, m), 7.17-7.15 (1H, m), $6.40(1 \mathrm{H}, \mathrm{d}, \mathrm{J}=2.8 \mathrm{~Hz}), 6.17-6.13$ $(2 \mathrm{H}, \mathrm{m}), 5.96(1 \mathrm{H}, \mathrm{br}, \mathrm{NH}), 4.42(1 \mathrm{H}, \mathrm{d}, \mathrm{J}=4.8 \mathrm{~Hz}), 3.85(3 \mathrm{H}, \mathrm{s}), 3.64(3 \mathrm{H}, \mathrm{s})$, 3.49-3.43 (2H, m), 2.89-2.79 (2H, m), $1.30(3 \mathrm{H}, \mathrm{d}, \mathrm{J}=6.8 \mathrm{~Hz}), 0.97(3 \mathrm{H}, \mathrm{t}, \mathrm{J}=7.2 \mathrm{~Hz}), 0.88(3 \mathrm{H}, \mathrm{t}, \mathrm{J}=7.2$ $\mathrm{Hz}) ;{ }^{13} \mathrm{C} \mathrm{NMR}\left(\mathrm{CDCl}_{3}, 150 \mathrm{MHz}\right) \delta: 173.8,151.0,147.9,143.1,132.0,128.3,127.0,126.8,109.9,103.5$, 99.1, 61.9, 55.7, 55.6, 41.7, 41.4, 40.4, 17.0, 14.4, 12.8; IR (neat, cm-1): 3379, 1628, 1592, 1521, 1458, 1252, 1202, 1044, 1033; HRMS (Dart) calcd for $\mathrm{C}_{22} \mathrm{H}_{31} \mathrm{~N}_{2} \mathrm{O}_{3}[\mathrm{M}+\mathrm{H}]^{+}$371.2335, found 371.2322.

\section{(2R,3S)-3-((2-methoxyphenyl)amino)- $N$-methyl- $N, 3$-diphenylpropanamide (3aj)}

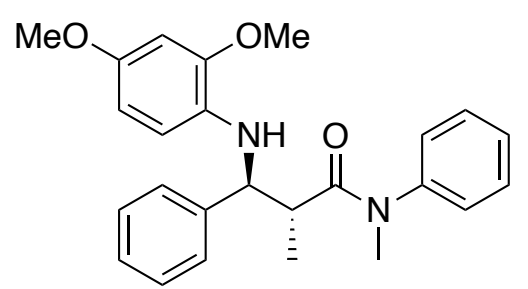

Mp $146-149{ }^{\circ} \mathrm{C} ;[\alpha]_{\mathrm{D}}^{25}=-2.23\left(\mathrm{c} 1.34, \mathrm{CHCl}_{3}, 78 \%\right.$ ee); HPLC analysis using Daicel Chiralpak AD-3 and AD-3 column (two column were connected.) (Hex:iPrOH $=19: 1,1.0 \mathrm{~mL} / \mathrm{min}, 254 \mathrm{~nm}, \mathrm{tR}=56.0 \mathrm{~min}$ (minor), $59.7 \mathrm{~min}$ (major); ${ }^{1} \mathrm{H} \mathrm{NMR}\left(600 \mathrm{MHz}, \mathrm{CDCl}_{3}\right) \delta: 7.28-7.20(6 \mathrm{H}$, m), 7.12-7.11 (2H, m), $6.59(2 \mathrm{H}$, br), $6.41(1 \mathrm{H}, \mathrm{d}, \mathrm{J}=2.0 \mathrm{~Hz}), 6.17-6.15$ $(1 \mathrm{H}, \mathrm{dd}, \mathrm{J}=2.8 \mathrm{~Hz}, 8.2 \mathrm{~Hz}), 6.12(1 \mathrm{H}, \mathrm{d}, \mathrm{J}=8.3 \mathrm{~Hz}), 5.93(1 \mathrm{H}, \mathrm{s}), 4.32(1 \mathrm{H}, \mathrm{d}, \mathrm{J}=4.8 \mathrm{~Hz}), 3.90$ (3H, s), $3.64(3 \mathrm{H}, \mathrm{s}), 3.13(3 \mathrm{H}, \mathrm{s}), 2.78(1 \mathrm{H}, \mathrm{q}, \mathrm{J}=4.1 \mathrm{~Hz}), 1.22(3 \mathrm{H}, \mathrm{d}, \mathrm{J}=6.9 \mathrm{~Hz}) ;{ }^{13} \mathrm{C} \mathrm{NMR}\left(\mathrm{CDCl}_{3}, 150 \mathrm{MHz}\right)$ $\delta: 174.7,151.0,147.8,143.6,142.8,131.7,129.5,128.3,127.7,127.1,126.9,126.8,110.0,103.5,99.1,61.5$, 55.6, 55.6, 42.3, 37.1, 16.7; IR (neat, $\mathrm{cm}^{-1}$ ): 3327, 1647, 1595, 1517, 1270, 1248, 1200, 1031; HRMS (Dart) calcd for $\mathrm{C}_{25} \mathrm{H}_{29} \mathrm{~N}_{2} \mathrm{O}_{3}[\mathrm{M}+\mathrm{H}]^{+} 405.2178$, found 405.2169.

\section{tert-butyl 3-((2,4-dimethoxyphenyl)amino)-2-methyl-3-phenylpropanoate (3ak, diastereomer mixture)}

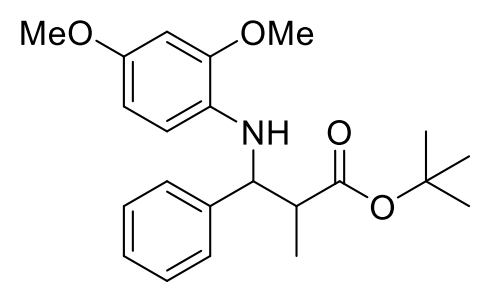

HPLC analysis using Daicel Chiralpak OD-3 column and OD-3 column (two column were connected.) (Hex: ${ }^{i} \mathrm{PrOH}=80: 1,1.0 \mathrm{~mL} / \mathrm{min}, 254 \mathrm{~nm}, \mathrm{tR}=14.5$ $\min$ (miner), $18.6 \mathrm{~min}$ (major)); ${ }^{1} \mathrm{H}$ NMR $\left(600 \mathrm{MHz}, \mathrm{CDCl}_{3}\right)$ major isomer $\delta$ : 7.32-7.26 (4H, m), 7.21-7.19 (1H, m), 6.41-6.40 (1H, m), 6.27-6.24 (1H, m), 6.22-6.17 (1H, m), $4.92(1 \mathrm{H}, \mathrm{s}), 4.35(1 \mathrm{H}, \mathrm{d}, \mathrm{J}=8.25 \mathrm{~Hz}), 3.84(3 \mathrm{H}, \mathrm{s}), 3.67$ $(3 \mathrm{H}, \mathrm{s}), 2.72-2.70(1 \mathrm{H}, \mathrm{m}), 1.40(9 \mathrm{H}, \mathrm{s}), 1.08(3 \mathrm{H}, \mathrm{d}, \mathrm{J}=6.87 \mathrm{~Hz})$; minor isomer $\delta: 7.32-7.26(4 \mathrm{H}, \mathrm{m})$, 7.21-7.19 (1H, m), 6.41-6.40 (1H, m), 6.27-6.24 (1H, m), 6.22-6.17 $(1 \mathrm{H}, \mathrm{m}), 4.92(1 \mathrm{H}, \mathrm{s}), 4.58(1 \mathrm{H}, \mathrm{d}, \mathrm{J}=$ $6.19 \mathrm{~Hz}), 3.84(3 \mathrm{H}, \mathrm{s}), 3.67(3 \mathrm{H}, \mathrm{s}), 2.87-2.85(1 \mathrm{H}, \mathrm{m}), 1.34(9 \mathrm{H}, \mathrm{s}), 1.15(3 \mathrm{H}, \mathrm{d}, \mathrm{J}=7.56 \mathrm{~Hz}) ;{ }^{13} \mathrm{C}$ NMR (CDCl3, $150 \mathrm{MHz}$ ) detectable peaks $\delta: 174.1,173.3,151.4,147.8,147.6,141.8,131.4,128.3,128.2,127.1$, 127.0, 111.1, 110.7, 103.6, 99.1, 99.0, 80.8, 80.7, 61.4, 60.5, 55.6, 55.6, 55.5, 55.4, 47.8, 47.3, 27.9, 27.8, 15.1, 12.5; IR (neat, $\mathrm{cm}^{-1}$ ) 3420, 1724, 1562, 1150, 1033; HRMS (Dart) calcd for $\mathrm{C}_{22} \mathrm{H}_{30} \mathrm{~N}_{1} \mathrm{O}_{4}[\mathrm{M}+\mathrm{H}]^{+}$ 372.2175 , found 372.2167 . 


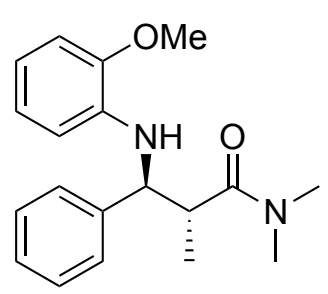

$[\alpha]_{\mathrm{D}^{20}}=-3.53$ (c $0.73 \mathrm{CHCl}_{3}, 86 \%$ ee); HPLC analysis using Daicel Chiralpak AD-3 column (Hex: ${ }^{i} \mathrm{PrOH}=95: 5,1.0 \mathrm{~mL} / \mathrm{min}, 254 \mathrm{~nm}, \mathrm{tR}=15.2 \mathrm{~min}$ (major), $21.2 \mathrm{~min}$ (miner) $) ;{ }^{1} \mathrm{H}$ NMR $\left(500 \mathrm{MHz}, \mathrm{CDCl}_{3}\right) \delta: 7.31(2 \mathrm{H}, \mathrm{d}, \mathrm{J}=7.37 \mathrm{~Hz}), 7.26(2 \mathrm{H}, \mathrm{t}, \mathrm{J}=$ $7.37 \mathrm{~Hz}), 7.19(1 \mathrm{H}, \mathrm{t}, \mathrm{J}=7.37 \mathrm{~Hz}), 6.73(1 \mathrm{H}, \mathrm{d}, \mathrm{J}=7.77 \mathrm{~Hz}), 6.66-6.62(1 \mathrm{H}, \mathrm{t}, \mathrm{J}=$ $7.77 \mathrm{~Hz}), 6.53(1 \mathrm{H}, \mathrm{t}, \mathrm{J}=7.77 \mathrm{~Hz}), 6.28-6.24(2 \mathrm{H}, \mathrm{m}), 4.50(1 \mathrm{H}, \mathrm{d}, \mathrm{J}=3.97 \mathrm{~Hz}), 3.90$ $(3 \mathrm{H}, \mathrm{s}), 3.28-3.25(1 \mathrm{H}, \mathrm{m}), 2.81(3 \mathrm{H}, \mathrm{s}), 2.46(3 \mathrm{H}, \mathrm{s}), 1.33(3 \mathrm{H}, \mathrm{d}, \mathrm{J}=6.80 \mathrm{~Hz}) ;{ }^{13} \mathrm{C} \mathrm{NMR}\left(\mathrm{CDCl}_{3}, 150\right.$ MHz) $\delta: 174.4,147.0,142.8,137.5,128.5,127.1,126.4,120.9,115.5,109.9,109.3,61.0,55.6,41.0,37.0$, 35.5, 16.6; IR (neat, $\mathrm{cm}^{-1}$ ) 3377, 1632, 1599, 1511, 1455, 1221, 1025; HRMS (Dart) calcd for $\mathrm{C}_{19} \mathrm{H}_{25} \mathrm{~N}_{2} \mathrm{O}_{2}$ $[\mathrm{M}+\mathrm{H}]^{+}$313.1916, found 313.1910.

\section{3-((4-methoxyphenyl)amino)- $N, N, 2$-trimethyl-3-phenylpropanamide (3xa)}

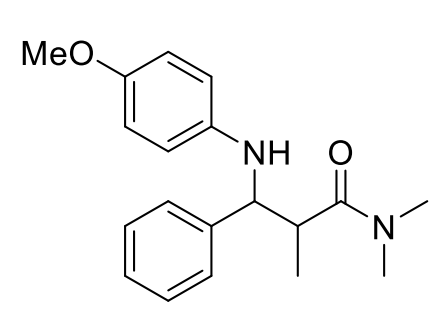

Mp 127-129 ${ }^{\circ} \mathrm{C} ;[\alpha]_{\mathrm{D}^{20}}=-10.8\left(\mathrm{c} 0.90 \mathrm{CHCl}_{3}, 35 \%\right.$ ee); HPLC analysis using Daicel Chiralpak AD-3 column (Hex: ${ }^{\mathrm{PrOH}}=95: 5,1.0 \mathrm{~mL} / \mathrm{min}, 254 \mathrm{~nm}, \mathrm{tR}=$ $32.3 \mathrm{~min}$ (miner), $41.6 \mathrm{~min}$ (Major)); ${ }^{1} \mathrm{H}$ NMR (500 MHz, $\left.\mathrm{CDCl}_{3}\right) \delta: 7.30-7.25$ $(4 \mathrm{H}, \mathrm{m}), 7.20(1 \mathrm{H}, \mathrm{t}, \mathrm{J}=6.80 \mathrm{~Hz}), 6.65(2 \mathrm{H}, \mathrm{d}, \mathrm{J}=8.50 \mathrm{~Hz}), 6.45(2 \mathrm{H}, \mathrm{d}, \mathrm{J}=$ $8.50 \mathrm{~Hz}), 5.70(1 \mathrm{H}, \mathrm{s}), 4.44(1 \mathrm{H}, \mathrm{d}, \mathrm{J}=4.53 \mathrm{~Hz}), 3.66(3 \mathrm{H}, \mathrm{s}), 3.28-3.23(1 \mathrm{H}, \mathrm{m})$, $2.79(3 \mathrm{H}, \mathrm{s}), 2.39(3 \mathrm{H}, \mathrm{s}), 1.34(3 \mathrm{H}, \mathrm{d}, \mathrm{J}=7.37 \mathrm{~Hz}) ;{ }^{13} \mathrm{C} \mathrm{NMR}\left(\mathrm{CDCl}_{3}, 150 \mathrm{MHz}\right) \delta: 174.4,151.3,142.9$, 141.5, 128.5, 127.2, 126.4, 114.7, 113.9, 61.9, 55.7, 41.0, 36.9, 35.4, 16.7; IR (neat, $\mathrm{cm}^{-1}$ ) 3379, 1625, 1515, 1245, 1031; HRMS (Dart) calcd for $\mathrm{C}_{19} \mathrm{H}_{24} \mathrm{~N}_{2} \mathrm{O}_{2}[\mathrm{M}+\mathrm{H}]^{+} 313.1916$, found 313.1906 .

\section{3-((2-ethylphenyl)amino)-N,N,2-trimethyl-3-phenylpropanamide (3ya, diastereomer mixture)}

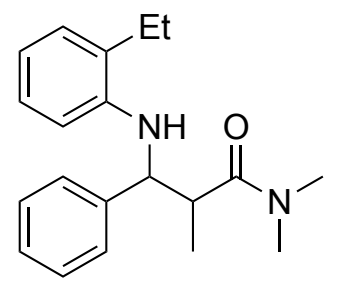

HPLC analysis using Daicel Chiralpak AD-3 column (Hex: ${ }^{\mathrm{PrOH}}=98: 2,0.5 \mathrm{~mL} / \mathrm{min}$, $254 \mathrm{~nm}$ ) major diastereomer: $\mathrm{tR}=14.5 \mathrm{~min}$ (major), $20.7 \mathrm{~min}$ (minor); minor diastereomer: $\mathrm{tR}=19.4 \mathrm{~min}$ (minor), $22.3 \mathrm{~min}$ (major); ${ }^{1} \mathrm{H} \mathrm{NMR}\left(600 \mathrm{MHz}, \mathrm{CDCl}_{3}\right)$ major isomer $\delta: 7.4-7.15(\mathrm{~m} 5 \mathrm{H}), 7.05(1 \mathrm{H}, \mathrm{d}, \mathrm{J}=6.9 \mathrm{~Hz}), 6.87(1 \mathrm{H}, \mathrm{t}, \mathrm{J}=8.3 \mathrm{~Hz})$, $6.55(1 \mathrm{H}, \mathrm{t}, \mathrm{J}=7.6 \mathrm{~Hz}), 6.31(1 \mathrm{H}, \mathrm{br}), 6.19(1 \mathrm{H}, \mathrm{d}, \mathrm{J}=8.2 \mathrm{~Hz}), 3.28(1 \mathrm{H}, \mathrm{m}), 2.76(3 \mathrm{H}$, s), $2.72(2 \mathrm{H}, \mathrm{m}), 2.31(3 \mathrm{H}, \mathrm{s}), 1.40(3 \mathrm{H}, \mathrm{d}, \mathrm{J}=6.9 \mathrm{~Hz}), 1.38(3 \mathrm{H}, \mathrm{t}, \mathrm{J}=7.6 \mathrm{~Hz})$; detectable peaks of minor isomer $\delta$ : $6.62(1 \mathrm{H}, \mathrm{t}, 7.6 \mathrm{~Hz}), 3.17(1 \mathrm{H}, \mathrm{m}), 2.98(3 \mathrm{H}, \mathrm{s}), 2.86(3 \mathrm{H}, \mathrm{s}), 2.64(2 \mathrm{H}, \mathrm{m}), 1.34(3 \mathrm{H}, \mathrm{t}, \mathrm{J}=7.6 \mathrm{~Hz})$, $1.15(3 \mathrm{H}, \mathrm{d}, \mathrm{J}=6.9 \mathrm{~Hz}) ;{ }^{13} \mathrm{C}$ NMR $\left(\mathrm{CDCl}_{3}, 150 \mathrm{MHz}\right)$ detectable peaks $\delta: 174.5,174.3,144.8,144.6,142.9$, 141.6, 128.6, 128.4, 128.4, 128.2, 127.5, 127.1, 127.1, 126.6, 126.5, 126.4, 126.1, 117.0, 115.8, 111.6, 109.5, 60.8, 59.2, 41.8, 40.7, 37.2, 36.9, 35.6, 35.3, 24.1, 24.0, 16.9, 12.9, 12.8, 12.0; IR (neat, $\mathrm{cm}^{-1}$ ) 3376, 1625, 1605, 1581, 1511, 1507, 1495, 1450, 1437, 1417, 1397, 1375, 1352, 1330, 1302, 1285, 1261, 1174, 1158, 1078, 1061, 1052; HRMS (Dart) calcd for $\mathrm{C}_{20} \mathrm{H}_{27} \mathrm{~N}_{2} \mathrm{O}_{1}[\mathrm{M}+\mathrm{H}]^{+} 311.2123$, found 311.2117.

\section{3-(phenylamino)- $N, N, 2$-trimethyl-3-phenylpropanamide (3za)}

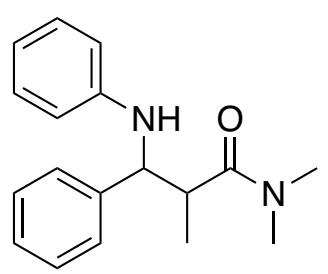

Mp $147-152^{\circ} \mathrm{C} ;[\alpha]_{\mathrm{D}}^{25}=-12.80$ (c $0.80, \mathrm{CHCl}_{3}, 33 \%$ ee); HPLC analysis using Daicel Chiralpak AD-3 column (Hex: ${ }^{\mathrm{PrOH}}=19: 1,1.0 \mathrm{~mL} / \mathrm{min}, 254 \mathrm{~nm}$, tR $=12.1 \mathrm{~min}$ (minor), $12.9 \mathrm{~min}$ (major); ${ }^{1} \mathrm{H}$ NMR $\left(600 \mathrm{MHz}, \mathrm{CDCl}_{3}\right)$ 8: 7.31-7.26 (4H, m), 
7.21-7.20 (1H, m), 7.06-7.03 (2H, m), 6.57-6.54 (1H, m), 6.50-6.48 (2H, m), $6.07(1 \mathrm{H}, \mathrm{s}, \mathrm{NH}), 4.49(1 \mathrm{H}, \mathrm{d}$, $\mathrm{J}=3.4 \mathrm{~Hz}), 3.26(1 \mathrm{H}, \mathrm{q}, \mathrm{J}=3.7 \mathrm{~Hz}), 2.77(3 \mathrm{H}, \mathrm{s}), 2.34(3 \mathrm{H}, \mathrm{s}), 1.38(3 \mathrm{H}, \mathrm{d}, \mathrm{J}=6.8 \mathrm{~Hz}) ;{ }^{13} \mathrm{C} \mathrm{NMR}\left(\mathrm{CDCl}_{3}\right.$, $150 \mathrm{MHz}) \delta: 174.4,147.3,142.8,129.0,128.5,127.2,126.3,116.2,112.7,60.9,40.8,36.9,35.4,16.8$; IR (neat, $\mathrm{cm}^{-1}$ ): 3340, 1622, 1605, 1498, 1317; HRMS (Dart) calcd for $\mathrm{C}_{18} \mathrm{H}_{23} \mathrm{~N}_{2} \mathrm{O}[\mathrm{M}+\mathrm{H}]^{+} 283.1810$, found 283.1797 .

\section{Determination of absolute configuration of $3 \mathrm{na}$}

X-ray single crystal structure analysis was conducted using a crystal of 3na obtained in the Mannich reaction using ligand L1. The configuration of major diastereomer of $3 \mathbf{n a}$ is $2 R, 3 S$, (anti isomer). The analytical data was available on website of The Cambridge Crystallographic Data Centre (CCDC 1956655).

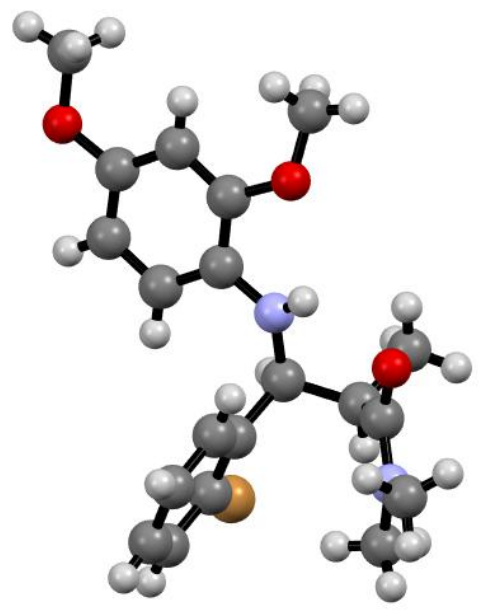<smiles>COc1ccc(NC(C(=O)N(C)C)[C@H](C)c2ccccc2Br)c(OC)c1</smiles>

$(2 R, 3 S)-3$ na

\section{Assignment of relative configuration of the Mannich adduct}

Relative configuration of the Mannich adducts was determined based on NMR analysis of the obtained products by analogy to 3na. ${ }^{13} \mathrm{C}$ NMR chemical shifts of the methyl groups at the 2-positions of the products have similar tendency. This tendency is quite similar to the related aldol adducts. ${ }^{23}$ However, the product bearing cyclopropyl group (3ta) has a different tendency. We assigned its relative configuration based on consideration of similarity of the reaction conditions.

Table S8 ${ }^{13} \mathrm{C}$ NMR chemical shifts table of 2-methyl groups of the Mannich adducts ${ }^{\mathrm{a}}$

\begin{tabular}{|c|c|c|}
\hline & anti-form & \\
\hline Mannich adduct 3 & $\begin{array}{l}{ }^{13} \mathrm{C} \text { NMR chemical shift of } \\
\text { 2-methyl group of the } \\
\text { major isomer (anti) (ppm) }\end{array}$ & $\begin{array}{l}{ }^{13} \mathrm{C} \text { NMR chemical shift of } \\
\text { 2-methyl group of the } \\
\text { minor isomer (syn) (ppm) }\end{array}$ \\
\hline $\mathrm{R}=\mathrm{Ph}(\mathbf{3 a a})$ & 16.6 & 14.0 \\
\hline
\end{tabular}




$\begin{array}{lll}\mathrm{R}=o-\mathrm{MeC}_{6} \mathrm{H}_{4} \text { (3da) } & 16.5 & 13.9 \\ \mathrm{R}=o-\mathrm{MeOC}_{6} \mathrm{H}_{4} \text { (3ia) } & 16.2 & 12.1 \\ \mathrm{R}=p-\mathrm{BrC}_{6} \mathrm{H}_{4} \text { (3la) } & 16.4 & 14.0 \\ \mathrm{R}=m-\mathrm{BrC}_{6} \mathrm{H}_{4} \text { (3ma) } & 16.3 & 13.8 \\ \mathrm{R}=o-\mathrm{BrC}_{6} \mathrm{H}_{4} \text { (3na) } & 16.3 & 11.8 \\ \mathrm{R}=m-\mathrm{CF}_{3} \mathrm{C}_{6} \mathrm{H}_{4} \text { (3oa) } & 16.4 & 14.2 \\ \mathrm{R}=\text { cyclopropyl (3ta) }^{\text {(3) }} & 15.0 & 15.2 \\ \mathrm{R}=\text { tert }-\mathrm{Bu}_{\text {(3ua) }} & 17.5 & 15.7 \\ \mathrm{R}=\mathrm{PhCH}_{2} \mathrm{CMe}_{2} \text { (3va) } & 17.7 & 15.7\end{array}$

${ }^{a}$ The ${ }^{13} \mathrm{C}$ NMR analysis was conducted using a mixture of the anti- and syn-isomers.

\section{Deprotection of 2,4-dimethoxyphenyl group and protection by benzoyl chloride}

3aa (218 mg, $0.637 \mathrm{mmol}, 91 \%$ ee) was dissolved in $\mathrm{CH}_{3} \mathrm{CN}(6.7 \mathrm{~mL})$. A solution of CAN (1.047 g, 1.911 $\mathrm{mmol}$ ) in water was added to the solution of 3aa at $0{ }^{\circ} \mathrm{C}$. The reaction mixture was stirred for $2.5 \mathrm{~h}$ at the same temperature, and it was diluted with water. The mixture was washed with $\mathrm{Et}_{2} \mathrm{O}$, and the aqueous phase was basified by $15 \%$ aq. $\mathrm{NaOH}$ and extracted by EtOAc. The organic layers were combined and dried over anhydrous $\mathrm{Na}_{2} \mathrm{SO}_{4}$. After filtration and concentration under reduced pressure, the crude product 4aa (115 mg, $88 \%$ crude yield) was obtained. The crude product was directly used in the next step without further purification. ${ }^{24}$

4aa $(115 \mathrm{mg}, 0.559 \mathrm{mmol})$ in $\mathrm{CH}_{2} \mathrm{Cl}_{2}(6.7 \mathrm{~mL})$ were placed in a round bottom flask. Benzoyl chloride $(80$ $\mu \mathrm{L}, 0.671 \mathrm{mmol})$ and $\mathrm{Et}_{3} \mathrm{~N}(390 \mu \mathrm{L}, 2.795 \mathrm{mmol})$ were added successively to the flask at room temperature. The reaction mixture was stirred for $13 \mathrm{~h}$ the at same temperature. Then reaction was quenched by sat. $\mathrm{NaHCO}_{3}$ and extracted by $\mathrm{CH}_{2} \mathrm{Cl}_{2}$. After filtration and concentration under reduced pressure, the crude product obtained was purified by silica-gel PTLC (chloroform-methanol) to afford the desired product 5aa (165 mg, $83 \%$ yield in 2 steps, $91 \%$ ee).

\section{(2R,3S)-N-(3-(dimethylamino)-2-methyl-3-oxo-1-phenylpropyl)benzamide (5aa)}

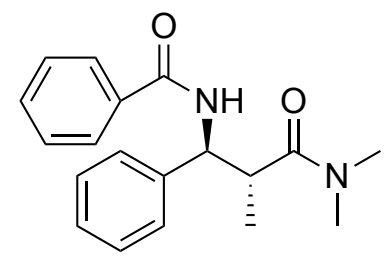

Mp 126-128 ${ }^{\circ} \mathrm{C} ;[\alpha]_{\mathrm{D}}{ }^{20}=+46.90\left(\right.$ c $1.53 \mathrm{CHCl}_{3}, 91 \%$ ee); HPLC analysis using Daicel Chiralpak AD-3 column $\left(\mathrm{Hex} ;{ }^{i} \mathrm{PrOH}=80: 20,1.0 \mathrm{~mL} / \mathrm{min}, 254 \mathrm{~nm}, \mathrm{tR}=\right.$ 14.7 min (miner), $24.8 \mathrm{~min}$ (major)); ${ }^{1} \mathrm{H}$ NMR (500 MHz, CDCl3) $\delta: 9.16(1 \mathrm{H}, \mathrm{d}, \mathrm{J}$ $=7.69 \mathrm{~Hz}), 7.97-7.95(2 \mathrm{H}, \mathrm{m}), 7.52-7.44(3 \mathrm{H}, \mathrm{m}), 7.29-7.28(3 \mathrm{H}, \mathrm{m}), 7.24-7.20(1 \mathrm{H}$, m), $5.30(1 \mathrm{H}, \mathrm{dd}, \mathrm{J}=7.69,3.43 \mathrm{~Hz}), 3.36-3.31(1 \mathrm{H}, \mathrm{m}), 2.82(3 \mathrm{H}, \mathrm{s}), 2.54(3 \mathrm{H}, \mathrm{s}), 1.39(3 \mathrm{H}, \mathrm{d}, \mathrm{J}=6.80 \mathrm{~Hz})$; ${ }^{13} \mathrm{C}$ NMR (CDCl3, $\left.150 \mathrm{MHz}\right) \delta: 175.4,166.7,141.7,134.3,131.4,128.5,128.5,127.3,127.1,125.9,56.6$, 39.8, 37.3, 35.5, 16.5; IR (neat, $\mathrm{cm}^{-1}$ ) 3289, 1657, 1622, 1507, 1481, 1324, 1258; HRMS (Dart) calcd for $\mathrm{C}_{19} \mathrm{H}_{23} \mathrm{~N}_{2} \mathrm{O}_{2}[\mathrm{M}+\mathrm{H}]^{+}$311.1760, found 311.1746.

\section{Hydrolysis of the Mannich adduct and conversion to ester}

Mannich product 3aa $(216 \mathrm{mg}, 0.630 \mathrm{mmol})$ was placed in a flask and $6 \mathrm{M} \mathrm{HCl}(30.4 \mathrm{~mL})$ was added. The 
reaction mixture was heated to $90{ }^{\circ} \mathrm{C}$ and stirred for $72 \mathrm{~h}$. After cooling to $\mathrm{rt}$, the mixture was diluted with water and extracted with $\mathrm{CH}_{2} \mathrm{Cl}_{2}$. The organic layers were combined, and the solvent was removed by using an evaporator. The residue was dissolved in water and $15 \%$ aq. $\mathrm{NaOH}$ was added. The aqueous phase was washed with $\mathrm{Et}_{2} \mathrm{O}$. The washed aqueous phase was acidified by $2 \mathrm{M} \mathrm{HCl}$ and extracted with $\mathrm{CH}_{2} \mathrm{Cl}_{2}$. The organic layers were combined and dried over anhydrous $\mathrm{Na}_{2} \mathrm{SO}_{4}$. After filtration and concentration under reduced pressure, the crude product (161 $\mathrm{mg}, 81 \%$ crude yield) was obtained. The crude product was directly used in next step without further purification. ${ }^{25}$

The crude carboxylic acid was dissolved in dry $\mathrm{MeOH}(20 \mathrm{~mL})$, and conc. $\mathrm{H}_{2} \mathrm{SO}_{4}(1.3 \mathrm{~mL})$ was added. The mixture was heated to reflux for $12 \mathrm{~h}$. After cooling to $\mathrm{rt}$, the reaction mixture was diluted with water and basified by $15 \% \mathrm{NaOH}$. It was then extracted with $\mathrm{CH}_{2} \mathrm{Cl}_{2}$. The organic layers were combined and dried over anhydrous $\mathrm{Na}_{2} \mathrm{SO}_{4}$. After filtration and concentration under reduced pressure, the crude product obtained was purified by silica-gel PTLC (hexane-EtOAc) to afford the desired product 6aa (138 $\mathrm{mg}, 65 \%$ yield in 2 steps). ${ }^{26}$

\section{(2R,3S)-Methyl 3-((2,4-dimethoxyphenyl)amino)-2-methyl-3-phenylpropanoate (6aa)}

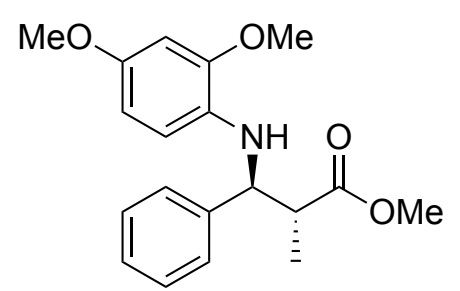

Purple oil; $[\alpha]_{\mathrm{D}}{ }^{20}=-27.52$ (c $1.90 \mathrm{CHCl}_{3}, 92 \%$ ee); HPLC analysis using Daicel Chiralpak OJ-3 column (Hex: ${ }^{\mathrm{PrOH}}=80: 20,1.0 \mathrm{~mL} / \mathrm{min}, 254 \mathrm{~nm}$, tR $=14.5$

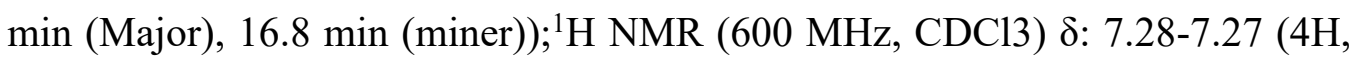
$\mathrm{m}), 7.20(1 \mathrm{H}, \mathrm{td}, \mathrm{J}=8.42,4.35 \mathrm{~Hz}), 6.39(1 \mathrm{H}, \mathrm{d}, \mathrm{J}=2.75 \mathrm{~Hz}), 6.33(1 \mathrm{H}, \mathrm{d}, \mathrm{J}=$ $8.60 \mathrm{~Hz}), 6.22(1 \mathrm{H}, \mathrm{dd}, \mathrm{J}=8.60,2.75 \mathrm{~Hz}), 4.88(1 \mathrm{H}, \mathrm{s}), 4.45(1 \mathrm{H}, \mathrm{d}, \mathrm{J}=8.25$ $\mathrm{Hz}), 3.81(3 \mathrm{H}, \mathrm{s}), 3.65-3.64(6 \mathrm{H}, \mathrm{m}), 2.88-2.83(1 \mathrm{H}, \mathrm{m}), 1.10(3 \mathrm{H}, \mathrm{d}, \mathrm{J}=6.87 \mathrm{~Hz}) ;{ }^{13} \mathrm{C} \mathrm{NMR}(\mathrm{CDCl}, 150$ MHz) $\delta: 175.3,151.8,147.9,141.4,131.1,128.4,127.3,126.9,111.5,103.7,99.1,61.3,55.6,55.5,51.7$, 46.7, 14.9; IR (neat, $\mathrm{cm}^{-1}$ ) 3416, 1732, 1515, 1454, 1227, 1203, 1154, 1030; HRMS (Dart) calcd for $\mathrm{C}_{19} \mathrm{H}_{24} \mathrm{NO}_{4}[\mathrm{M}+\mathrm{H}]^{+}$330.1705, found 330.1710.

\section{Gram scale reaction of a catalytic asymmetric Mannich reaction of 1a with 2a}

KHMDS (81.8 mg, $41.0 \mathrm{mmol})$, IndaBox (L1) $(68.7 \mathrm{mg}, 20.8 \mathrm{mmol})$ were placed in a flame-dried $50 \mathrm{~mL} 1$ neck round bottom flask with a three ways cock inside a glove box fulfilled with argon. The flask was cooled to $0{ }^{\circ} \mathrm{C}$, then THF $(5.2 \mathrm{~mL})$ was added. The reaction mixture was stirred for $30 \mathrm{~min}$ at the same temperature, and then cooled at $-78{ }^{\circ} \mathrm{C}$. After stirring, the solution of 2,4-dimethoxyphenylimine 1a (1.00 $\mathrm{g}, 4.14 \mathrm{mmol})$ in THF $(2.59 \mathrm{ml})$ cooled at $-78^{\circ} \mathrm{C}$ was added to the reaction mixture through a cannula. The solution of $N, N$-dimethypropionamide $2 \mathrm{a}(461 \mathrm{mg}, 4.56 \mathrm{mmol})$ in $\mathrm{THF}(2.0 \mathrm{~mL})$ cooled to $-78{ }^{\circ} \mathrm{C}$ was then added to the reaction mixture. The whole mixture was stirred at $-78{ }^{\circ} \mathrm{C}$ for $24 \mathrm{~h}$. The reaction was quenched by a mixture of THF and water, and extracted with $\mathrm{CH}_{2} \mathrm{Cl}_{2}$ several times. The combined organic layers were dried over anhydrous $\mathrm{Na}_{2} \mathrm{SO}_{4}$. After filtration and concentration under reduced pressure, the crude product obtained was purified by silica-gel column chromatography (hexane-ethyl acetate) to afford the desired adduct 3aa $(1.39 \mathrm{~g}, 97 \%$ yield$)$. 


\section{Synthetic procedures for SCH-48462}

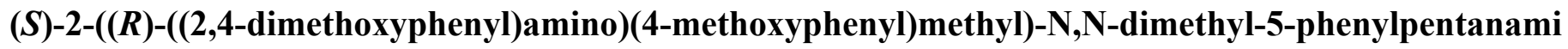
de (3gg)

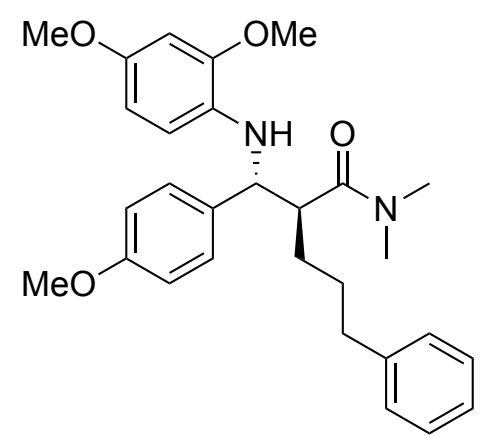

Mp 109-110 ${ }^{\circ} \mathrm{C} ;[\alpha]_{\mathrm{D}}^{20}=-3.64$ (c $2.16 \mathrm{CHCl}_{3}, 94 \%$ ee); HPLC analysis using Daicel Chiralpak OD-3 column (Hex: ${ }^{i} \mathrm{PrOH}=85: 15,1.0 \mathrm{~mL} / \mathrm{min}, 254 \mathrm{~nm}$, tR $=10.1 \mathrm{~min}$ (miner), $12.1 \mathrm{~min}$ (Major)); ${ }^{1} \mathrm{H} \mathrm{NMR}(600 \mathrm{MHz}, \mathrm{CDCl} 3) \delta: 7.25$ $(2 \mathrm{H}, \mathrm{t}, \mathrm{J}=7.56 \mathrm{~Hz}), 7.17-7.13(5 \mathrm{H}, \mathrm{m}), 6.78(2 \mathrm{H}, \mathrm{d}, \mathrm{J}=8.25 \mathrm{~Hz}), 6.42(1 \mathrm{H}, \mathrm{d}$, $\mathrm{J}=2.41 \mathrm{~Hz}), 6.17(1 \mathrm{H}, \mathrm{dd}, \mathrm{J}=8.25,2.41 \mathrm{~Hz}), 6.09-6.03(2 \mathrm{H}, \mathrm{m}), 4.46(1 \mathrm{H}, \mathrm{s})$, $3.88(3 \mathrm{H}, \mathrm{s}), 3.74(3 \mathrm{H}, \mathrm{s}), 3.66(3 \mathrm{H}, \mathrm{s}), 3.10-3.08(1 \mathrm{H}, \mathrm{m}), 2.79(3 \mathrm{H}, \mathrm{s})$, 2.64-2.53 (2H, m), $2.36(3 \mathrm{H}, \mathrm{s}), 2.14-2.12(1 \mathrm{H}, \mathrm{m}), 1.74-1.69(1 \mathrm{H}, \mathrm{m})$, 1.64-1.53 (2H, m); ${ }^{13} \mathrm{C} \mathrm{NMR}(\mathrm{CDCl} 3,150 \mathrm{MHz}) \delta: 173.8,158.6,150.9,147.8,142.2,135.0,131.7,128.3$, 128.2, 127.3, 125.7, 113.7, 109.6, 103.4, 99.0, 59.5, 55.6, 55.6, 55.1, 47.1, 37.1, 36.0, 35.5, 31.5, 29.9; IR (neat, $\mathrm{cm}^{-1}$ ) 3377, 1631, 1511, 1455, 1241, 1198, 1178, 1030; HRMS (Dart) calcd for $\mathrm{C}_{29} \mathrm{H}_{36} \mathrm{~N}_{2} \mathrm{O}_{4}[\mathrm{M}+\mathrm{H}]^{+}$ 477.2753, found 477.2743.

\section{$\beta$-lactam formation}

Mannich adduct $\mathbf{3 g g}(115 \mathrm{mg}, 0.240 \mathrm{mmol})$ was dissolved in dry $\mathrm{CH}_{2} \mathrm{Cl}_{2}(12 \mathrm{~mL})$, and $N, N$-dimethyl-o-toluidine $(52.8 \mu \mathrm{L}, 0.361 \mathrm{mmol})$ was then added. The reaction mixture was cooled to $0{ }^{\circ} \mathrm{C}$, and $\mathrm{Tf}_{2} \mathrm{O}(59.2 \mu \mathrm{L}, 0.361 \mathrm{mmol})$ was added. The reaction mixture was then stirred at $\mathrm{rt}$ for $2.5 \mathrm{~h}$. sat. $\mathrm{NaHCO}_{3}$ was added to the reaction mixture, and it was stirred for $30 \mathrm{~min}$. The reaction mixture was extracted with $\mathrm{CH}_{2} \mathrm{Cl}_{2}$. The organic layers were combined and dried over anhydrous $\mathrm{Na}_{2} \mathrm{SO}_{4}$. After filtration and concentration under reduced pressure, the crude product was obtained. The obtained crude product was dissolved in a mixture of $\mathrm{MeCN}(5.6 \mathrm{~mL})$ and ${ }^{i} \mathrm{PrOH}(5.6 \mathrm{~mL})$. It was then cooled to $0{ }^{\circ} \mathrm{C}$, and $1 \mathrm{M}$ aq. $\mathrm{NaOH}\left(11.3 \mathrm{~mL}\right.$, cooled at $\left.0{ }^{\circ} \mathrm{C}\right)$ was added. After stirring for $30 \mathrm{~min}$, the reaction mixture was diluted with water and extracted with $\mathrm{CH}_{2} \mathrm{Cl}_{2}$. The organic layers were combined and dried over anhydrous $\mathrm{Na}_{2} \mathrm{SO}_{4}$. After filtration and concentration under reduced pressure, the crude product obtained was purified by silica-gel PTLC (hexane-EtOAc) to afford the desired product $\mathbf{7 f g}\left(66.8 \mathrm{mg}, 65 \%\right.$ yield). ${ }^{27}$

\section{(3S,4R)-1-(2,4-dimethoxyphenyl)-4-(4-methoxyphenyl)-3-phenethylazetidin-2-one (7gg)}

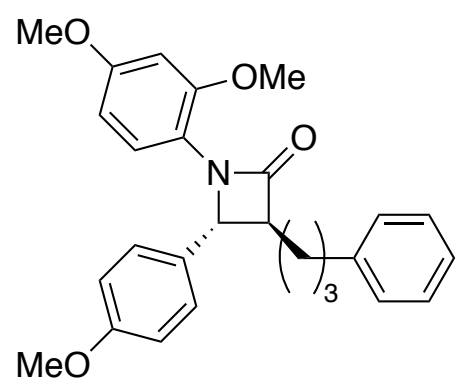

Colorless oil; HPLC analysis using Daicel Chiralpak OD-3 column $\left(\mathrm{Hex} ;{ }^{i} \mathrm{PrOH}=85: 15,1.0 \mathrm{~mL} / \mathrm{min}, 254 \mathrm{~nm}, \mathrm{tR}=12.4 \mathrm{~min}\right.$ (miner), $91.9 \mathrm{~min}$ (Major)); ${ }^{1} \mathrm{H}$ NMR (500 MHz, CDCl3) $\delta: 7.53(1 \mathrm{H}, \mathrm{d}, \mathrm{J}=8.50 \mathrm{~Hz}), 7.29-7.25$ $(3 \mathrm{H}, \mathrm{m}), 7.22-7.15(5 \mathrm{H}, \mathrm{m}), 6.82(2 \mathrm{H}, \mathrm{d}, \mathrm{J}=8.65 \mathrm{~Hz}), 6.40(1 \mathrm{H}, \mathrm{dd}, \mathrm{J}=8.65$, $2.69 \mathrm{~Hz}), 6.34(1 \mathrm{H}, \mathrm{d}, \mathrm{J}=2.69 \mathrm{~Hz}), 4.85(1 \mathrm{H}, \mathrm{d}, \mathrm{J}=2.27 \mathrm{~Hz}), 3.76(3 \mathrm{H}, \mathrm{s})$, $3.73(3 \mathrm{H}, \mathrm{s}), 3.64(3 \mathrm{H}, \mathrm{s}), 3.10-3.07(1 \mathrm{H}, \mathrm{m}), 2.67-2.64(2 \mathrm{H}, \mathrm{m}), 2.00-1.96(1 \mathrm{H}$, m), 1.90-1.80 (3H, m); $\left.{ }^{13} \mathrm{C} \mathrm{NMR} \mathrm{(CDCl3,} 150 \mathrm{MHz}\right) \delta: 169.0,159.2,158.3,153.1,141.9,131.5,128.4$, 128.3, 127.3, 125.8, 125.2, 118.6, 114.1, 104.3, 99.6, 63.9, 60.6, 55.4, 55.4, 55.2, 35.8, 28.9, 28.6; IR (neat, 
$\mathrm{cm}^{-1}$ ) 1732, 1511, 1285, 1247, 1207, 1028; HRMS (Dart) calcd for $\mathrm{C}_{27} \mathrm{H}_{29} \mathrm{NO}_{4}[\mathrm{M}+\mathrm{H}]^{+} 432.2175$, found 432.2164 .

\section{Deprotection of 2,4-dimethoxyphenyl group from $\beta$-lactam}

$7 \mathrm{gg}$ (553 mg, $1.28 \mathrm{mmol}$, trans:cis $=96: 4$ ) was dissolved in $\mathrm{MeCN}\left(51.3 \mathrm{~mL}\right.$ ) and cooled at $0{ }^{\circ} \mathrm{C}$. Ammonium Cerium(IV) Nitrate $(2.12 \mathrm{~g}, 3.84 \mathrm{mmol})$ dissolved in water $(17.0 \mathrm{~mL})$ was added to the reaction mixture, and it was stirred at $0{ }^{\circ} \mathrm{C}$ for $1 \mathrm{~h}$. The reaction mixture was diluted with water and extracted with EtOAc. The organic layers were combined and washed with $5 \% \mathrm{Na}_{2} \mathrm{~S}_{2} \mathrm{O}_{3}$ until the aqueous layer turned to be clear. The organic layer were further washed with sat. $\mathrm{NaHCO}_{3}$. The organic layer was then dried over anhydrous $\mathrm{Na}_{2} \mathrm{SO}_{4}$. After filtration and concentration under reduced pressure, the obtained crude product was purified by flash chromatography (hexane-EtOAc) to afford the desired product $\mathbf{8 g g}$ in $94 \%$ ee as a single diastereomer (286 mg, 76\% yield, the cis-isomer was separated). Further recrystallization (hexane-EtOAc) afforded the desired product in $>99 \%$ ee (145 mg, 51\% yield (recrystallization step)).

(3S,4R)-4-(4-methoxyphenyl)-3-phenethylazetidin-2-one (8gg)

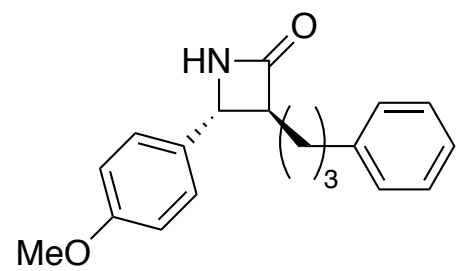

Mp 89-91 ${ }^{\circ} \mathrm{C} ;[\alpha]_{\mathrm{D}}^{27}=-15.70\left(\mathrm{c} 1.454 \mathrm{CHCl}_{3},>99 \%\right.$ ee); HPLC analysis using Daicel Chiralpak OD-3 column (Hex; ${ }^{i} \mathrm{PrOH}=85: 15,1.0 \mathrm{~mL} / \mathrm{min}, 210 \mathrm{~nm}, \mathrm{tR}=$ 15.8 min (miner), 22.2 min (Major)); ${ }^{1} \mathrm{H}$ NMR (500 MHz, CDCl3) $\delta: 7.28-7.25$ (4H, m), 7.19-7.14 (3H, m), $6.89(2 \mathrm{H}, \mathrm{d}, \mathrm{J}=9.07 \mathrm{~Hz}), 6.04(1 \mathrm{H}, \mathrm{s}), 4.31(1 \mathrm{H}, \mathrm{d}$, $\mathrm{J}=2.27 \mathrm{~Hz}), 3.81(3 \mathrm{H}, \mathrm{s}), 3.02-3.00(1 \mathrm{H}, \mathrm{m}), 2.64-2.61(2 \mathrm{H}, \mathrm{m}), 1.94-1.74(4 \mathrm{H}, \mathrm{m}) ;{ }^{13} \mathrm{C} \mathrm{NMR}(\mathrm{CDCl} 3,150$ MHz) $\delta: 171.2,159.5,141.7,131.9,128.4,128.3,126.8,125.8,114.2,61.6,57.2,55.3,35.7,28.9,28.2$; IR (neat, $\mathrm{cm}^{-1}$ ) 3245, 1739, 1512, 1245, 1174, 1031; HRMS (Dart) calcd for $\mathrm{C}_{19} \mathrm{H}_{21} \mathrm{NO}_{2}[\mathrm{M}+\mathrm{H}]^{+}$296.1651, found 296.1649 .

\section{Coupling reaction of the $\beta$-lactam and $p$-bromoanisole to synthesize SCH-48462}

8fg (69.3 mg, $0.235 \mathrm{mmol}), \mathrm{CuI}(4.7 \mathrm{mg}, 0.025 \mathrm{mmol}), \mathrm{K}_{2} \mathrm{CO}_{3}(48.9 \mathrm{mg}, 0.354 \mathrm{mmol})$ and dioxane $(1.9 \mathrm{~mL})$ were placed in a flask. trans- $N, N^{\prime}$-Dimethylcyclohexane-1,2-diamine $(8.2 \mu \mathrm{L}, 0.052 \mathrm{mmol})$ and $p$-bromoanisole $(32.2 \mu \mathrm{L}, 0.258 \mathrm{mmol})$ were then added to the flask. The reaction mixture was degassed by bubbling with argon under sonication for $20 \mathrm{~min}$. After degassing, the reaction mixture was stirred at $100{ }^{\circ} \mathrm{C}$ for $20 \mathrm{~h}$. The reaction mixture was cooled to $\mathrm{rt}$ and filtered through a pad of silica gel eluting with ethyl acetate. The filtrate was concentrated under reduced pressure, and the crude product obtained was purified by silica-gel PTLC (hexane-EtOAc) to afford the desired product SCH-48462 in $>99 \%$ ee (96.6 mg, $95 \%$ yield). ${ }^{28}$

\section{(3S,4R)-1,4-bis(4-methoxyphenyl)-3-(3-phenylpropyl)azetidin-2-one (SCH-48462) ${ }^{29}$}

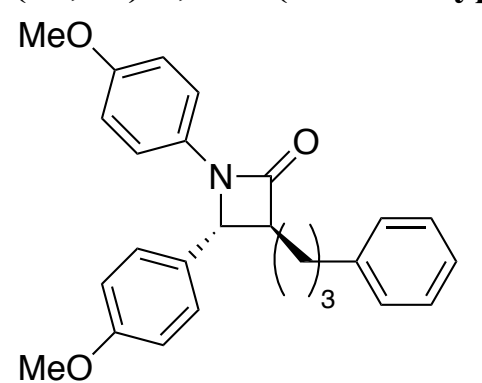

SCH 48462

Colorless oil; $[\alpha]_{\mathrm{D}}{ }^{28}=+17.78$ (c $0.966 \mathrm{CHCl}_{3},>99 \%$ ee); HPLC analysis using Daicel Chiralpak OD-3 column $\left(\mathrm{Hex} ;{ }^{i} \mathrm{PrOH}=85: 15,1.0 \mathrm{~mL} / \mathrm{min}, 254 \mathrm{~nm}, \mathrm{tR}=\right.$ $10.1 \mathrm{~min}$ (miner), $12.3 \mathrm{~min}$ (Major)); ${ }^{1} \mathrm{H}$ NMR (500 MHz, $\mathrm{CDCl}_{3}$ ) $\delta: 7.28-7.24$ 
$(4 \mathrm{H}, \mathrm{m}), 7.22-7.16(5 \mathrm{H}, \mathrm{m}), 6.88(2 \mathrm{H}, \mathrm{d}, \mathrm{J}=9.07 \mathrm{~Hz}), 6.76(2 \mathrm{H}, \mathrm{d}, \mathrm{J}=9.07 \mathrm{~Hz}), 4.55(1 \mathrm{H}, \mathrm{d}, \mathrm{J}=2.27 \mathrm{~Hz})$, $3.79(3 \mathrm{H}, \mathrm{s}), 3.73(3 \mathrm{H}, \mathrm{s}), 3.08-3.05(1 \mathrm{H}, \mathrm{m}), 2.65-2.63(2 \mathrm{H}, \mathrm{m}), 2.00-1.93(1 \mathrm{H}, \mathrm{m}), 1.88-1.78(3 \mathrm{H}, \mathrm{m}) ;{ }^{13} \mathrm{C}$ NMR $\left(\mathrm{CDCl}_{3}, 150 \mathrm{MHz}\right) \delta: 167.2,159.6,155.8,141.7,131.4,130.0,128.4,128.3,127.1,125.9,118.2$, 114.5, 114.2, 60.8, 60.4, 55.4, 55.2, 35.7, 28.9, 28.4; IR (neat, $\mathrm{cm}^{-1}$ ) 1737, 1508, 1242, 1030; MS (Dart) $[\mathrm{M}+\mathrm{H}]^{+} 402.18$.

\section{Cartesian coordinates and energies}

The most stable K enolate-K-L1 complex

Electronic energy: -1453.442511128192 (a.u.) / Gibbs free energy: -1452.994419106994

\begin{tabular}{|c|c|c|c|}
\hline K & 1.088365866202 & -0.777602070186 & 0.614889043111 \\
\hline $\mathrm{N}$ & 1.859271225783 & -0.439977121193 & -2.060172408319 \\
\hline $\mathrm{C}$ & 2.155013463892 & 0.704505786795 & -2.635363257264 \\
\hline $\mathrm{O}$ & 1.958966063276 & 0.746688877313 & -4.010518779629 \\
\hline $\mathrm{C}$ & 1.418189623021 & -0.521211845405 & -4.424188413661 \\
\hline $\mathrm{H}$ & 0.405359872173 & -0.347411747253 & -4.801074038183 \\
\hline $\mathrm{C}$ & 1.468648600152 & -1.375852987221 & -3.118941618686 \\
\hline $\mathrm{H}$ & 0.502812180615 & -1.843320497418 & -2.881511674951 \\
\hline $\mathrm{C}$ & 2.325110258573 & -1.207169704986 & -5.470718286053 \\
\hline $\mathrm{H}$ & 3.008601795594 & -0.481382481051 & -5.925677338369 \\
\hline $\mathrm{H}$ & 1.723809168044 & -1.633675312132 & -6.284051263235 \\
\hline $\mathrm{C}$ & 3.041230407254 & -2.295541688623 & -4.698855659506 \\
\hline $\mathrm{C}$ & 2.526433688324 & -2.426778010546 & -3.404032197528 \\
\hline $\mathrm{C}$ & 3.029046534146 & -3.392336112585 & -2.527210486117 \\
\hline $\mathrm{H}$ & 2.659504340414 & -3.467393170301 & -1.505956244787 \\
\hline $\mathrm{C}$ & 4.066313559953 & -4.225554452171 & -2.963294793035 \\
\hline $\mathrm{H}$ & 4.470735709791 & -4.979059349742 & -2.291897577649 \\
\hline $\mathrm{C}$ & 4.586913380181 & -4.093019066308 & -4.258143703006 \\
\hline $\mathrm{H}$ & 5.391397489443 & -4.746286204864 & -4.586260593645 \\
\hline $\mathrm{C}$ & 4.075820545782 & -3.127525381609 & -5.132292680294 \\
\hline $\mathrm{H}$ & 4.481265164170 & -3.028224766485 & -6.136606143854 \\
\hline $\mathrm{C}$ & 2.635355405350 & 1.908000817376 & -2.080971220617 \\
\hline $\mathrm{H}$ & 2.690544347424 & 2.768411415626 & -2.735423403114 \\
\hline $\mathrm{N}$ & 3.152757027688 & 1.223226258063 & 0.240358132511 \\
\hline $\mathrm{C}$ & 3.034597260838 & 2.090927729040 & -0.741840114222 \\
\hline $\mathrm{O}$ & 3.389220346905 & 3.397898713144 & -0.424888362859 \\
\hline $\mathrm{C}$ & 3.626845128606 & 3.446091886760 & 0.990343026832 \\
\hline $\mathrm{H}$ & 4.575525331006 & 3.964717951851 & 1.149243350328 \\
\hline $\mathrm{C}$ & 3.648759054029 & 1.947489511714 & 1.419183283250 \\
\hline $\mathrm{H}$ & 4.653380233718 & 1.588924602342 & 1.691631991403 \\
\hline $\mathrm{C}$ & 2.461126345969 & 4.123905465688 & 1.761767652021 \\
\hline $\mathrm{H}$ & 1.661164029727 & 4.383949034083 & 1.057603844033 \\
\hline $\mathrm{H}$ & 2.775993903419 & 5.055833820827 & 2.245966188039 \\
\hline $\mathrm{C}$ & 2.014418440151 & 3.084632696603 & 2.767589748984 \\
\hline $\mathrm{C}$ & 2.706273370554 & 1.878557143047 & 2.601850857181 \\
\hline $\mathrm{C}$ & 2.459410500002 & 0.787674066484 & 3.438618981082 \\
\hline $\mathrm{H}$ & 2.995618769899 & -0.154548554964 & 3.313231697314 \\
\hline $\mathrm{C}$ & 1.486051327149 & 0.913049558375 & 4.437535663124 \\
\hline $\mathrm{H}$ & 1.281614234091 & 0.074151800546 & 5.098657043847 \\
\hline $\mathrm{C}$ & 0.785824721847 & 2.115483237588 & 4.601829717932 \\
\hline $\mathrm{H}$ & 0.039475081380 & 2.203611482629 & 5.387344383970 \\
\hline $\mathrm{C}$ & 1.051259064758 & 3.209677884110 & 3.770175671943 \\
\hline $\mathrm{H}$ & 0.514816838767 & 4.145664193581 & 3.908513745513 \\
\hline $\mathrm{K}$ & 4.591800311880 & -1.058705747000 & -0.772279682865 \\
\hline $\mathrm{C}$ & 2.984043914329 & -2.768046953183 & 2.066157193038 \\
\hline $\mathrm{O}$ & 3.199546457361 & -2.636116992199 & 0.796093134868 \\
\hline $\mathrm{N}$ & 1.618964849587 & -3.161035738953 & 2.399287589990 \\
\hline $\mathrm{C}$ & 1.260862386706 & -3.231438399380 & 3.807904691083 \\
\hline $\mathrm{H}$ & 1.791911185387 & -4.033735719245 & 4.352606277847 \\
\hline $\mathrm{H}$ & 0.184283476198 & -3.419945090906 & 3.896974042579 \\
\hline
\end{tabular}




$\begin{array}{ll}\mathrm{H} & 1.486311587715 \\ \mathrm{C} & 1.169704696471 \\ \mathrm{H} & 0.075580903780 \\ \mathrm{H} & 1.586727270987 \\ \mathrm{H} & 1.491601296372 \\ \mathrm{C} & 3.896052527441 \\ \mathrm{H} & 3.662712021013 \\ \mathrm{C} & 5.258464267118 \\ \mathrm{H} & 5.789662278790 \\ \mathrm{H} & 5.901511945103 \\ \mathrm{H} & 5.228789638473\end{array}$

-2.279831728547
-4.351982256521
-4.424663641977
-5.282692671229
-4.278802995609
-2.499435355738
-2.672601735782
-1.943810875939
-2.532680850473
-1.926617059531
-0.902631769110

4.297134689617 1.675497502412 1.710227818892 2.104897996068 0.636069364272 3.057311216306 4.100848037005 2.731067457571 1.965534927049 3.620174079873 2.356054291502

The most stable KHMDS and K-L1 complex

Electronic energy: --2000.258703036918 (a.u.) / Gibbs free energy: -1999.731599749192

\begin{tabular}{|c|c|c|c|}
\hline K & -2.522934498987 & -2.991234114080 & 1.165843666181 \\
\hline $\mathrm{N}$ & -1.398352223836 & -0.280836425733 & 1.388198038157 \\
\hline $\mathrm{C}$ & -0.604679456115 & 0.118619726297 & 0.414024233165 \\
\hline $\mathrm{O}$ & 0.469783479951 & 0.903366037877 & 0.805468101025 \\
\hline $\mathrm{C}$ & 0.456229204946 & 1.006690842948 & 2.237323095067 \\
\hline $\mathrm{H}$ & 1.340689287681 & 0.488529484531 & 2.621860869253 \\
\hline $\mathrm{C}$ & -0.892114431834 & 0.327411944952 & 2.631796819751 \\
\hline $\mathrm{H}$ & -0.765116103226 & -0.439651548736 & 3.406479585467 \\
\hline $\mathrm{C}$ & 0.397256353311 & 2.483606563378 & 2.681299075157 \\
\hline $\mathrm{H}$ & 0.711961006082 & 3.144177197247 & 1.865868151995 \\
\hline $\mathrm{H}$ & 1.079031133541 & 2.663951228742 & 3.522413667016 \\
\hline $\mathrm{C}$ & -1.039813255181 & 2.692415557812 & 3.103731313577 \\
\hline $\mathrm{C}$ & -1.740346363655 & 1.481079146217 & 3.142789007804 \\
\hline $\mathrm{C}$ & -3.060775767022 & 1.444040282063 & 3.594497982220 \\
\hline $\mathrm{H}$ & -3.591332405137 & 0.500729227088 & 3.666993975998 \\
\hline $\mathrm{C}$ & -3.691756210304 & 2.635018427836 & 3.968197195347 \\
\hline $\mathrm{H}$ & -4.719181991871 & 2.612967805297 & 4.323110828142 \\
\hline $\mathrm{C}$ & -2.999740531030 & 3.851351162440 & 3.900041011677 \\
\hline $\mathrm{H}$ & -3.495902142921 & 4.772949241918 & 4.192839519274 \\
\hline $\mathrm{C}$ & -1.667129408128 & 3.883187108379 & 3.475483736582 \\
\hline $\mathrm{H}$ & -1.125182002315 & 4.825453094044 & 3.443329990089 \\
\hline $\mathrm{C}$ & -0.636026405319 & -0.110626858330 & -0.974386222082 \\
\hline $\mathrm{H}$ & 0.180510495704 & 0.314429756650 & -1.543920087101 \\
\hline $\mathrm{N}$ & -2.635732349987 & -1.544957657351 & -1.330012682266 \\
\hline $\mathrm{C}$ & -1.556393095282 & -0.886367944245 & -1.702465690899 \\
\hline $\mathrm{O}$ & -1.256022905890 & -0.956988102734 & -3.057579185211 \\
\hline $\mathrm{C}$ & -2.115378664693 & -1.945029526144 & -3.637401476579 \\
\hline $\mathrm{H}$ & -2.536190440136 & -1.524784713811 & -4.553993555226 \\
\hline $\mathrm{C}$ & -3.175872931243 & -2.217166360945 & -2.527534786718 \\
\hline $\mathrm{H}$ & -4.168887769844 & -1.816628980800 & -2.775313329437 \\
\hline $\mathrm{C}$ & -1.369486937585 & -3.281338188622 & -3.901159352786 \\
\hline $\mathrm{H}$ & -0.337127670075 & -3.200189055259 & -3.539473995089 \\
\hline $\mathrm{H}$ & -1.315550988224 & -3.517569977275 & -4.970357702652 \\
\hline $\mathrm{C}$ & -2.162484049702 & -4.316297608504 & -3.135367243805 \\
\hline $\mathrm{C}$ & -3.204794006457 & -3.728300737343 & -2.407688372604 \\
\hline $\mathrm{C}$ & -4.087705520893 & -4.519170291521 & -1.670452878517 \\
\hline $\mathrm{H}$ & -4.917322139958 & -4.069410525503 & -1.135931123295 \\
\hline $\mathrm{C}$ & -3.897278325144 & -5.905198197304 & -1.633810137162 \\
\hline $\mathrm{H}$ & -4.581276911150 & -6.528019884203 & -1.062833923222 \\
\hline $\mathrm{C}$ & -2.841618928700 & -6.491411407581 & -2.343622267405 \\
\hline $\mathrm{H}$ & -2.704622006837 & -7.569288027921 & -2.316688705035 \\
\hline $\mathrm{C}$ & -1.975198014058 & -5.699163742188 & -3.105645563416 \\
\hline $\mathrm{H}$ & -1.170619256771 & -6.159401895147 & -3.674439316498 \\
\hline $\mathrm{K}$ & -4.187587178375 & 0.146349419484 & 0.557474636374 \\
\hline $\mathrm{N}$ & -5.216663443304 & -2.172320652928 & 2.088175378300 \\
\hline $\mathrm{Si}$ & -5.147860720965 & -2.236932611816 & 3.786445319941 \\
\hline $\mathrm{Si}$ & -6.594806030557 & -2.667299503475 & 1.224003221484 \\
\hline $\mathrm{C}$ & -3.338618828078 & -2.259518449437 & 4.419270644175 \\
\hline $\mathrm{H}$ & -2.697445562584 & -1.516764805608 & 3.931303907188 \\
\hline $\mathrm{H}$ & -3.300684985212 & -2.061649983031 & 5.498433829181 \\
\hline $\mathrm{H}$ & -2.886650954933 & -3.250274265098 & 4.262128424932 \\
\hline
\end{tabular}




$\begin{array}{ll}\mathrm{C} & -5.910658419545 \\ \mathrm{H} & -5.442791562929 \\ \mathrm{H} & -5.766736055984 \\ \mathrm{H} & -6.988445099352 \\ \mathrm{C} & -6.014935457364 \\ \mathrm{H} & -5.845749850839 \\ \mathrm{H} & -5.682149280172 \\ \mathrm{H} & -7.098032926385 \\ \mathrm{C} & -8.282292860563 \\ \mathrm{H} & -8.456349989499 \\ \mathrm{H} & -8.334965581709 \\ \mathrm{H} & -9.113603138748 \\ \mathrm{C} & -6.750043215953 \\ \mathrm{H} & -5.816930550676 \\ \mathrm{H} & -6.980024985270 \\ \mathrm{H} & -7.548794167953 \\ \mathrm{C} & -6.614858455891 \\ \mathrm{H} & -6.913444538825 \\ \mathrm{H} & -5.645343089406 \\ \mathrm{H} & -7.345762412109\end{array}$

$-3.792059955206$ $-4.707280078445$ $-3.779521031258$ $-3.870407345970$ $-0.769671910404$ $-0.776414790338$ 0.203781193660 $-0.827857454080$ $-2.121819140406$ $-2.543810598461$ $-1.028686810587$ $-2.441212314115$ $-4.566381731355$ $-5.023373397004$ $-5.018876641684$ $-4.852109448363$ $-1.929085585795$ $-0.870372849328$ $-1.992647098985$ $-2.447388143625$
4.602274358660

4.215250045602

5.691628236335

4.411629082273

4.660082425152

5.745595473215

4.277709500151

4.490220950138

1.944878624578

2.942885475227

2.038725849058

1.300995619535

1.031209869952

0.677729825209

2.004699267776

0.333060018873

$-0.545451844231$

$-0.520731900432$

$-1.052638829358$

$-1.179702627100$ 


\section{References}

1) Becke, A. D. Phys. Rev. A 1988, 38, 3098-3100.

2) Lee, C.; Yang, W.; Parr, R. G. Phys. Rev. B 1998, 37, 785-789.

3) Grimme, S.; Antony, J.; Ehrlich, S.; Krieg, H. J. Chem. Phys. 2010, 132, 154104-154122.

4) Wadt, W. R.; Hay, P. J. J. Chem. Phys. 1985, 82, 284-298.

5) Hehre, W. J.; Ditchfield, R.; Pople, J. A. J. Chem. Phys. 1972, 56, 2257-2261.

6) Hariharan, P. C.; Pople, J. A. Theor. Chim. Acta, 1973, 28, 213-222.

7) Tomasi, J.; Mennucci, B.; Cammi, R. Chem. Rev. 2005, 105, 2999-3093.

8) GRRM17, Maeda, S.; Harabuchi, Y.; Sumiya, Y.; Takagi, M.; Suzuki, K.; Hatanaka, M.; Osada, Y.; Taketsugu, T.; Morokuma, K.; Ohno, K. http://iqce.jp/GRRM/index_e.shtml.

9) Maeda, S.; Harabuchi, Y.; Takagi, M.; Saita, K.; Suzuki, K.; Ichino, T.; Sumiya, Y.; Sugiyama, K.; Ono, Y. J. Comput. Chem. 2018, 39, 233-251.

10) Gaussian 16, Revision C.01, Frisch, M. J.; Trucks, G. W.; Schlegel, H. B.; Scuseria, G. E.; Robb, M. A.; Cheeseman, J. R.; Scalmani, G.; Barone, V.;Mennucci, B.; Petersson, G. A.; Nakatsuji, H.; Caricato, M.; Li, X.; Hratchian, H. P.; Izmaylov, A. F.; Bloino, J.; Zheng, G.; Sonnenberg, J. L.; Hada, M.; Ehara, M.; Toyota, K.; Fukuda, R.; Hasegawa, J.; Ishida, M.; Nakajima, T.; Honda, Y.; Kitao, O.; Nakai, H.; Vreven, T.; Montgomery, J. A. Jr.; Peralta, J. E.; Ogliaro, F.; Bearpark, M.; Heyd, J. J.; Brothers, E.; Kudin, K. N.; Staroverov, V. N.; Keith, T.; Kobayashi, R.; Normand, J.; Raghavachari, K.; Rendell, A.; Burant, J. C.; Iyengar, S. S.; Tomasi, J.; Cossi, M.; Rega, N.; Millam, J. M.; Klene, M.; Knox, J. E.; Cross, J. B.; Bakken, V.; Adamo, C.; Jaramillo, J.; Gomperts, R.; Stratmann, R. E.; Yazyev, O.; Austin, A. J.; Cammi, R.; Pomelli, C.; Ochterski, J. W.; Martin, R. L.; Morokuma, K.; Zakrzewski, V. G.; Voth, G. A.; Salvador, P.; Dannenberg, J. J.; Dapprich, S.; Daniels, A. D.; Farkas, O.; Foresman, J. B.; Ortiz, J. V.; Cioslowski, J.; Fox, D. J. Gaussian, Inc., Wallingford CT, 2016.

11) Glendening, E. D.; Streitwieser Jr., A.; J. Chem. Phys. 1994, 100, 2900.

12) Glendening, E. D. J. Am. Chem. Soc. 1996, 118, 2473-2482.

13) Schenter, G. K.; Glendening, E. D. J. Phys. Chem. 1996, 100, 17152-17156.

14) Bailey, P. J.; Coxall, R. A.; Dick, C. M.; Fabre, S.; Henderson, L. C.; Herber, C.; Liddle, S. T.; Loroño-González, D.; Parkin, A. Parson, S. Chem. Eur. J. 2003, 9, 4820-4828.

15) Clegg, W.; Conway, B.; Graham, D. V.; Hevia, E.; Kennedy, A. R.; Mulvey, R. E.; Russo, L.; Wright, D. S. Chem. Eur. J. 2009, 15, 7074-7082.

16) Ojeda-Amador, A. I.; Martínez-Martínez, A. J.; Kennedy, A. R.; O’Hara, C. T., Inorg. Chem. 2016, 55, 5719-5728.

17) Chollet, G.; Didier, D.; Schulz, E. Catalysis Communications, 2010, 11, 351-355.

18) Evans, A. D.; Woerpel, A. K.; Hinman, M. M.; Faul, M. M. J. Am. Chem. Soc. 1991, 113, 726-728.

19) Liu, F.; Zhong, J.; Zhou, Y.; Gao, Z.; Walsh, J. P.; Wang, X.; Ma, S.; Hou, S.; Liu, S.; Wang, M.; Wang, M.; Bian, Q. Chem. Eur. J. 2018, 24, 2059-2064. 
20) Crowley, C. D.; Lynch, D.; Maruire, R. A. J. Org. Chem. 2018, 83, 3794-3805.

21) Mou, Q. X.; Rong, M. F.; Zhang, H.; Chen, G.; He, G. Org. Lett. 2019, 21, 4657-4661.

22) Chan, G. H.; Ong, D. Y.; Yen, Z.; Chiba, S. Helv. Chim. Acta. 2018, 101, e1800049.

23) Heathcock, C. H., in Asymmetric Synthesis (Ed.: Morrison, J. D.), Academic Press, Inc., 1984, vol. 3, Chap. 2, pp 111-212.

24) Kronenthal. D. R, Han. C. Y, Taylor. M. K, J. Org. Chem. 1982, 47, 2765-2768.

25) Vicario, J. L.; Badía, D.; Carrillo, L. J. Org. Chem. 2001, 66, 9030-9032.

26) Suzuki, H.; Igarashi, R.; Yamashita, Y.; Kobayashi, S. Angew. Chem. Int. Ed. 2017, 56, 4520-4524.

27) Montgomery, T. D. and Smith III, A. B. Org. Lett. 2017, 19, 6216-6219.

28) Klapars, A.; Huang, X.; Buchwald, S. L. J. Am. Chem. Soc. 2002, 124, 7421-7428.

29) Burnett, D. A.; Caplen, M. A.; Davis, H. R.; Jr., Burrier, R. E.; Clader, J. W. J. Med. Chem. 1994, 37, 1733-1736. 
7. NMR charts of the products

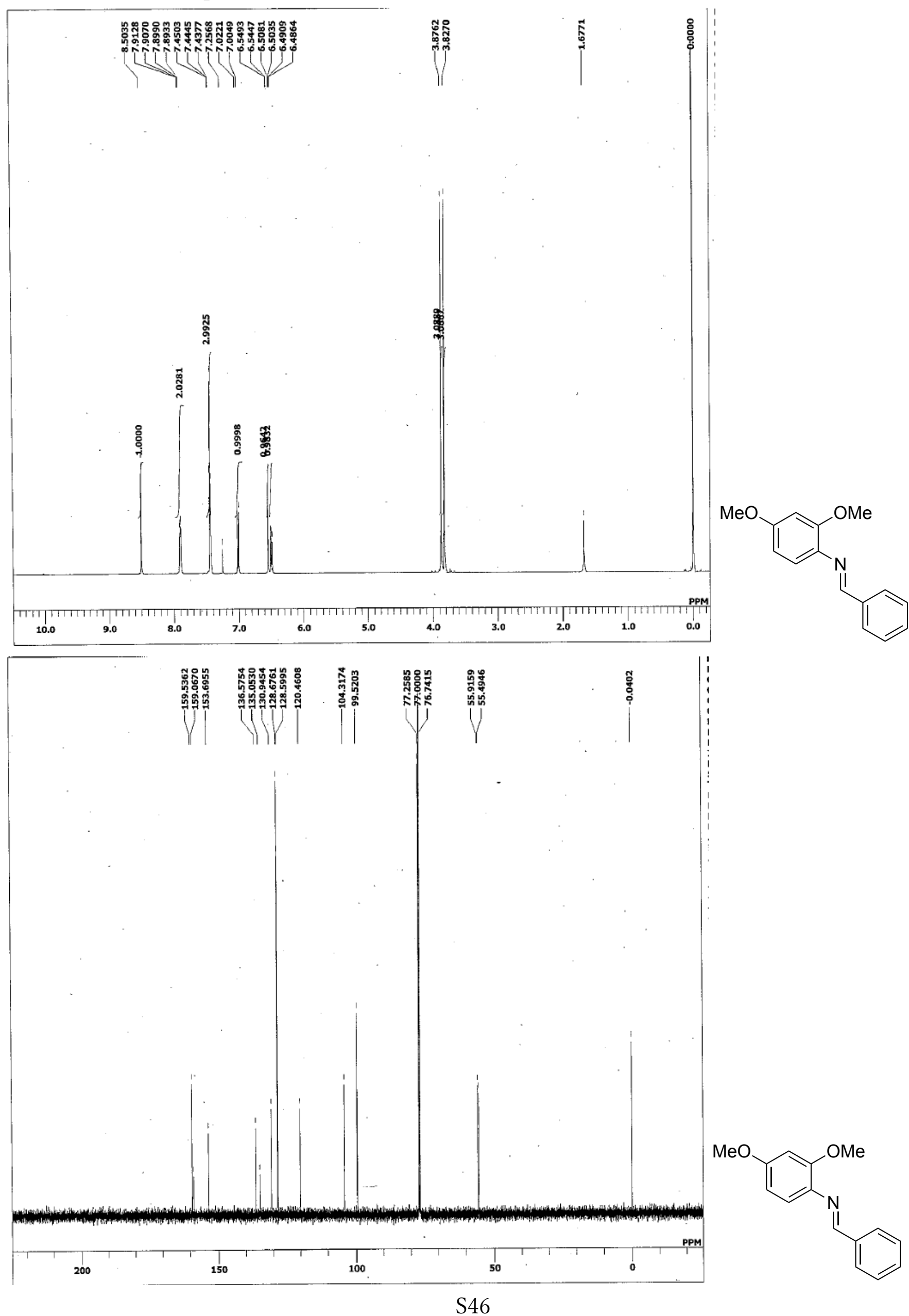



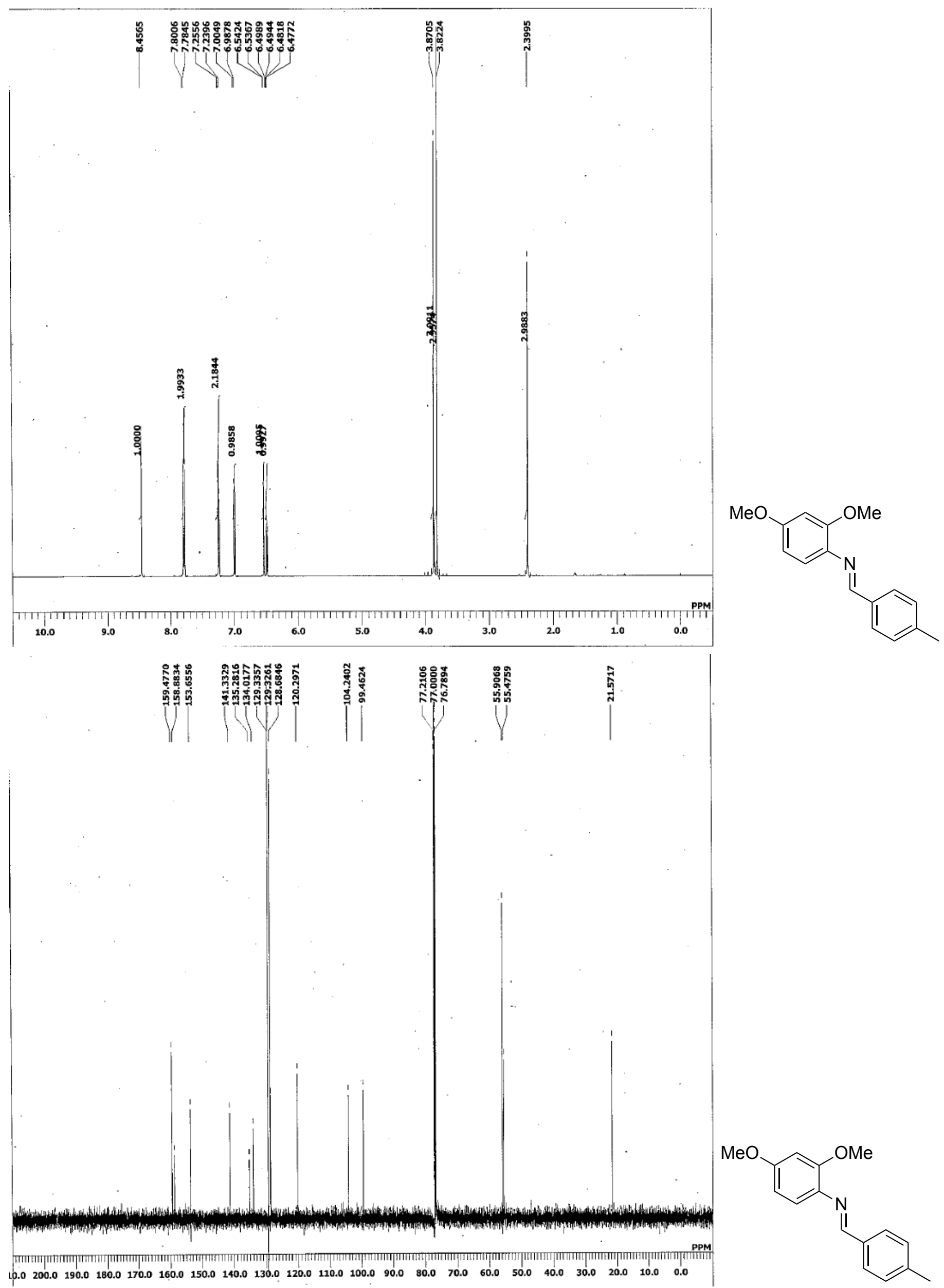

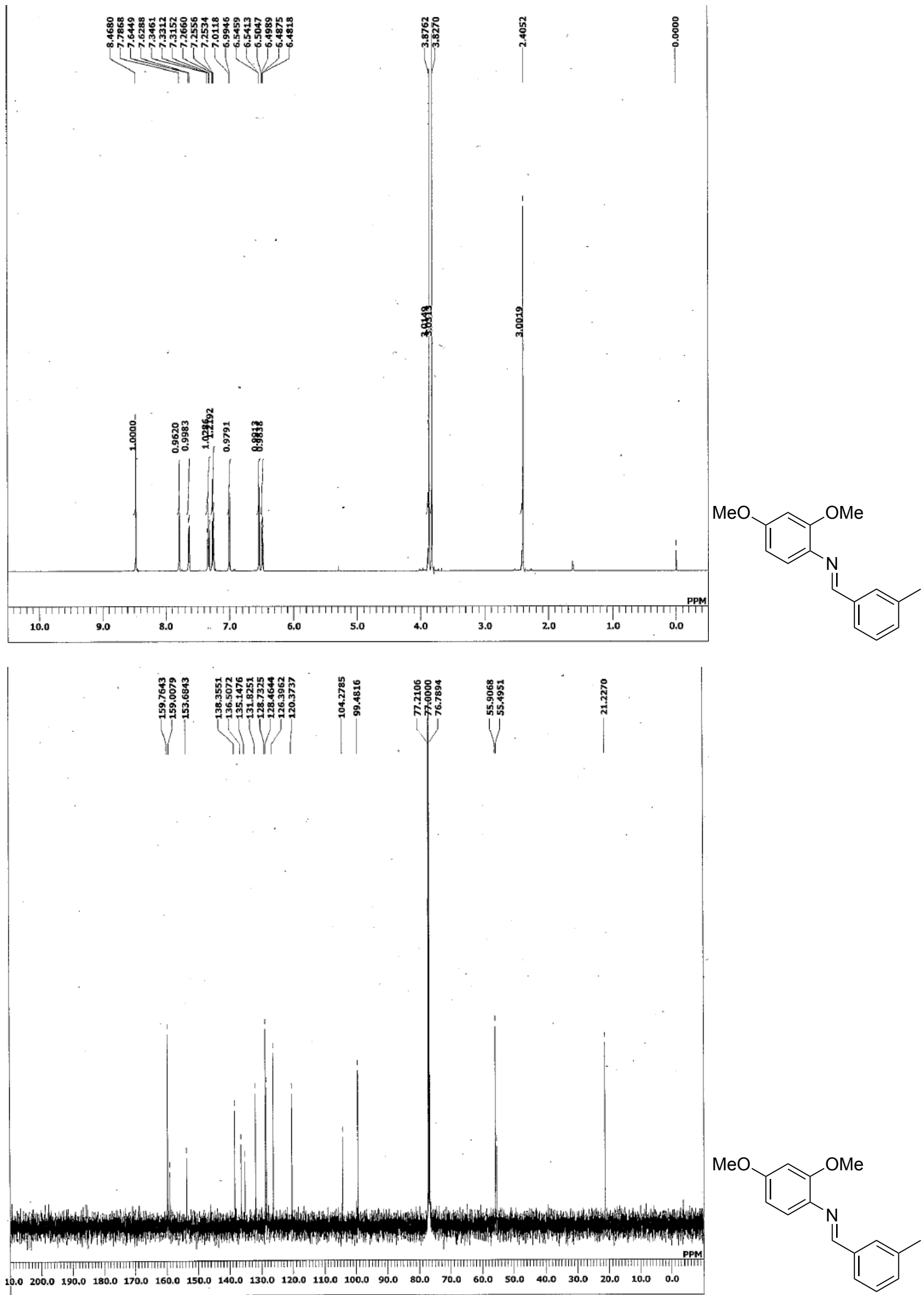


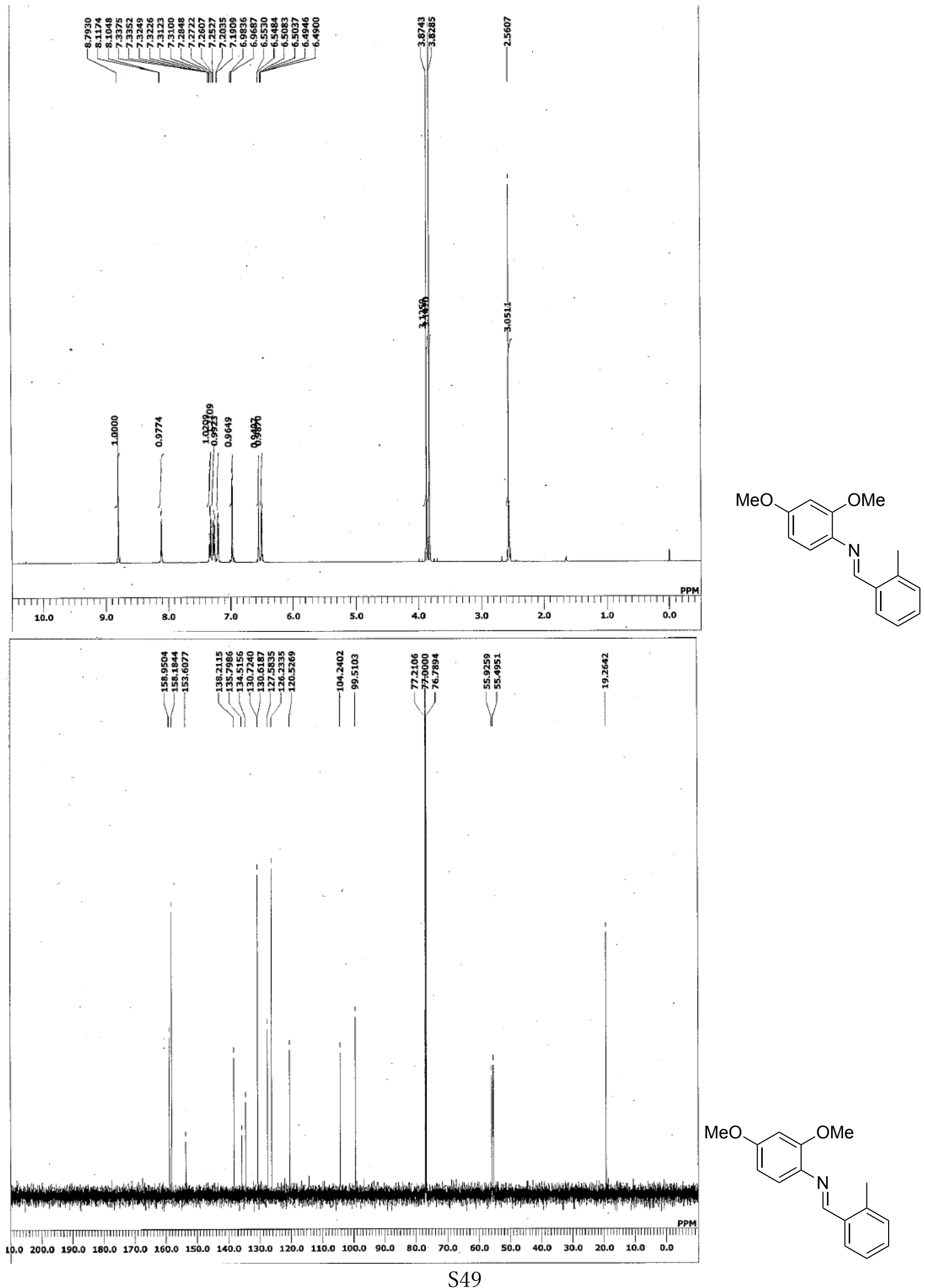




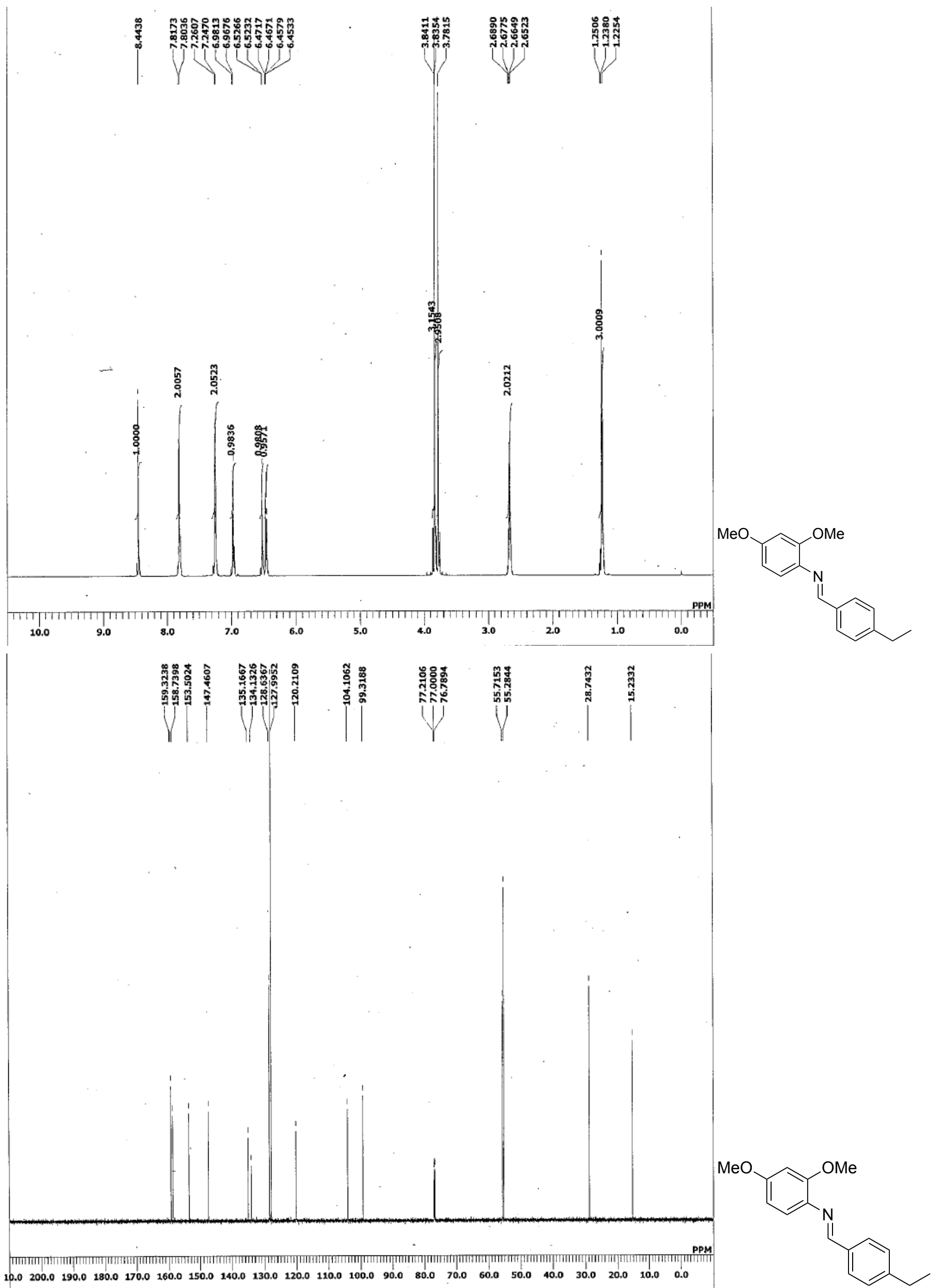



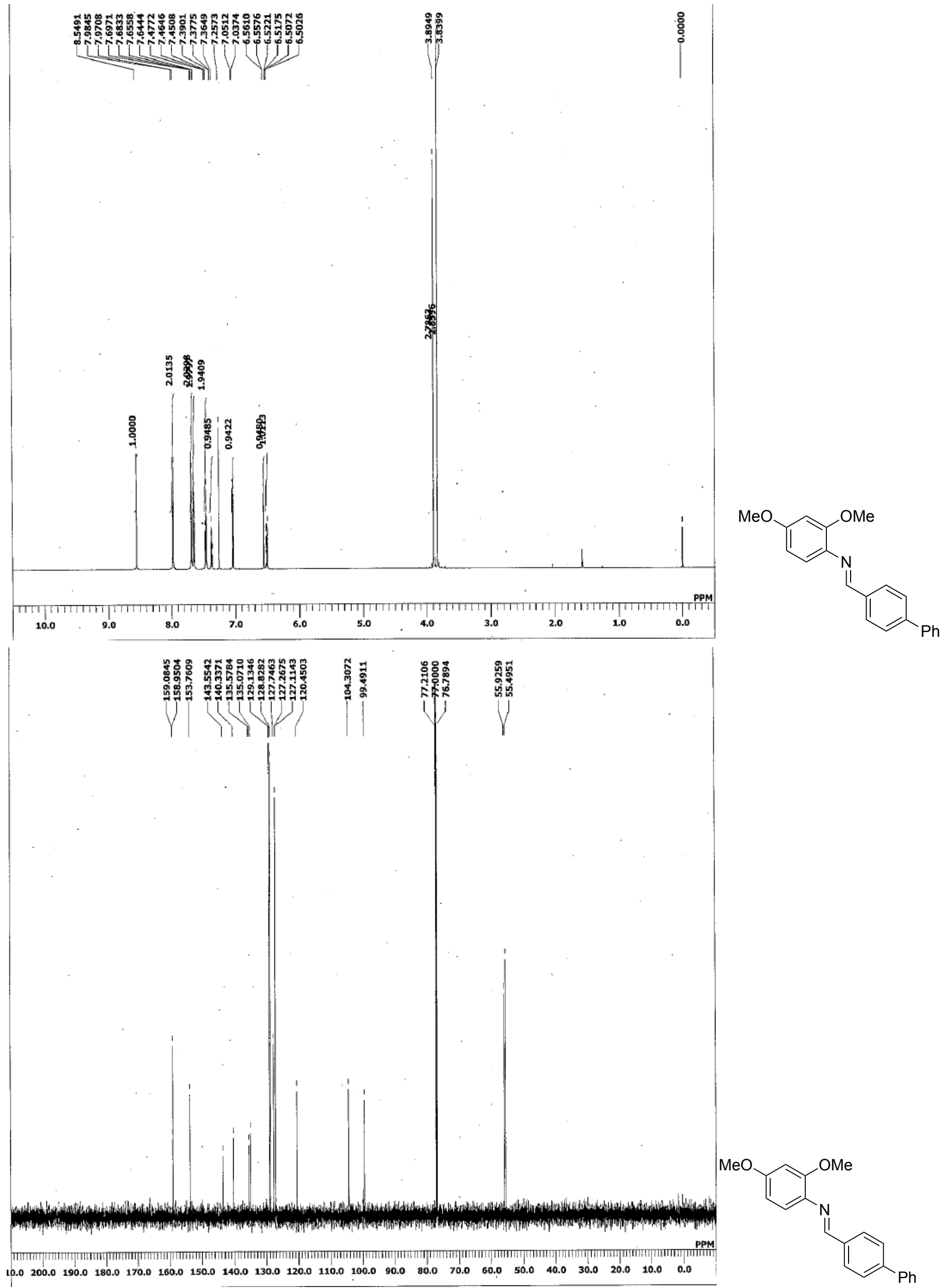

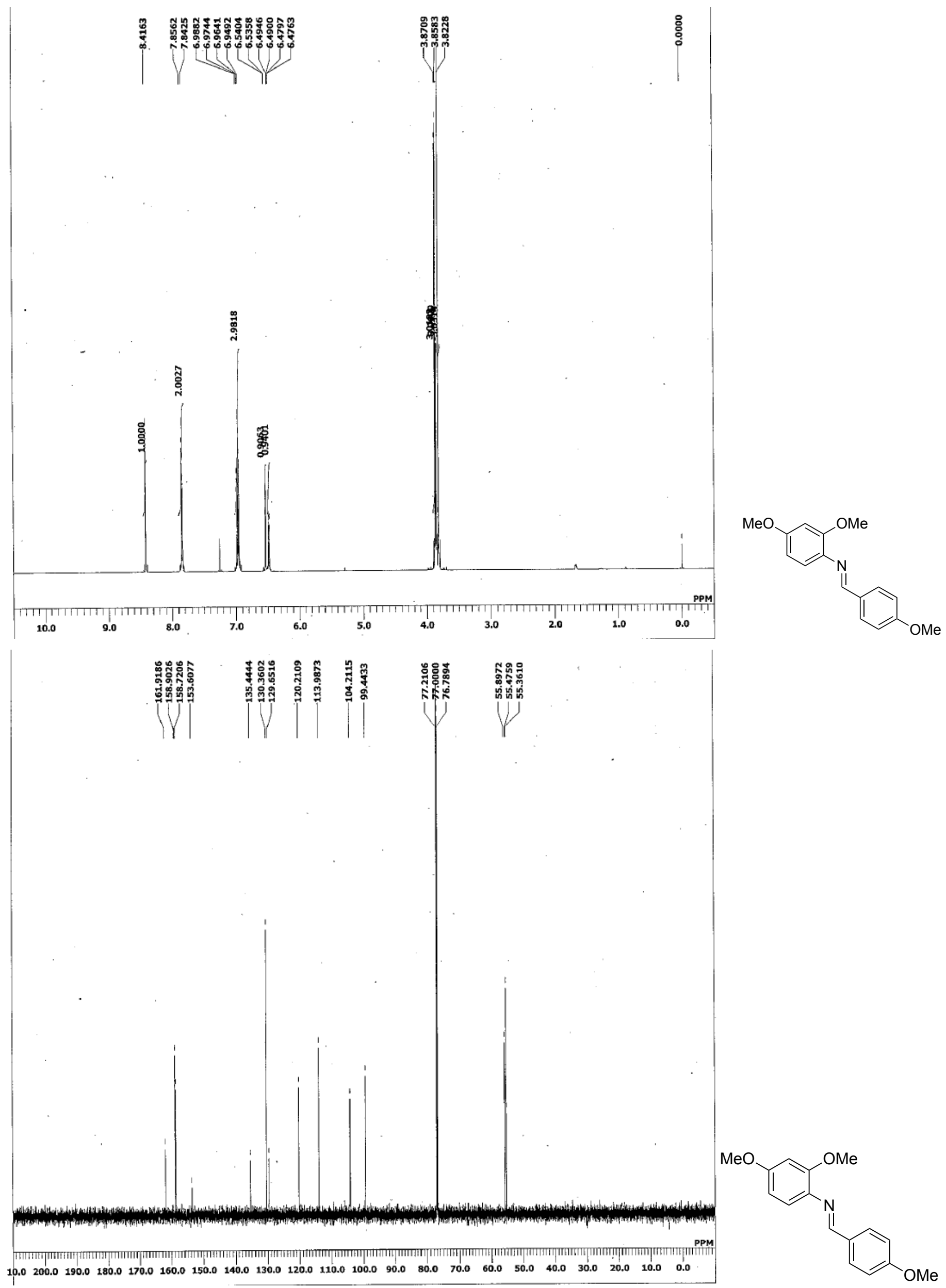


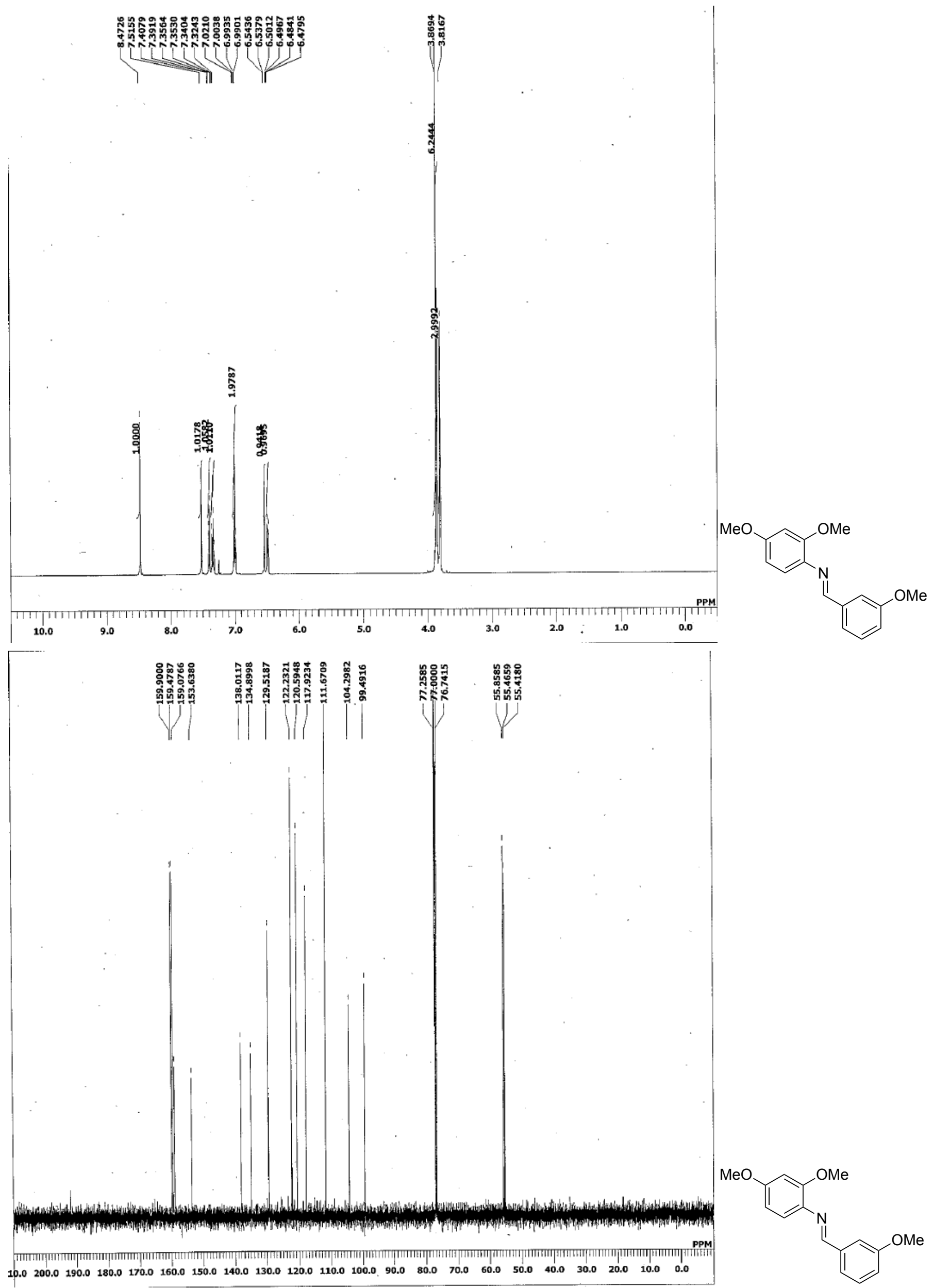




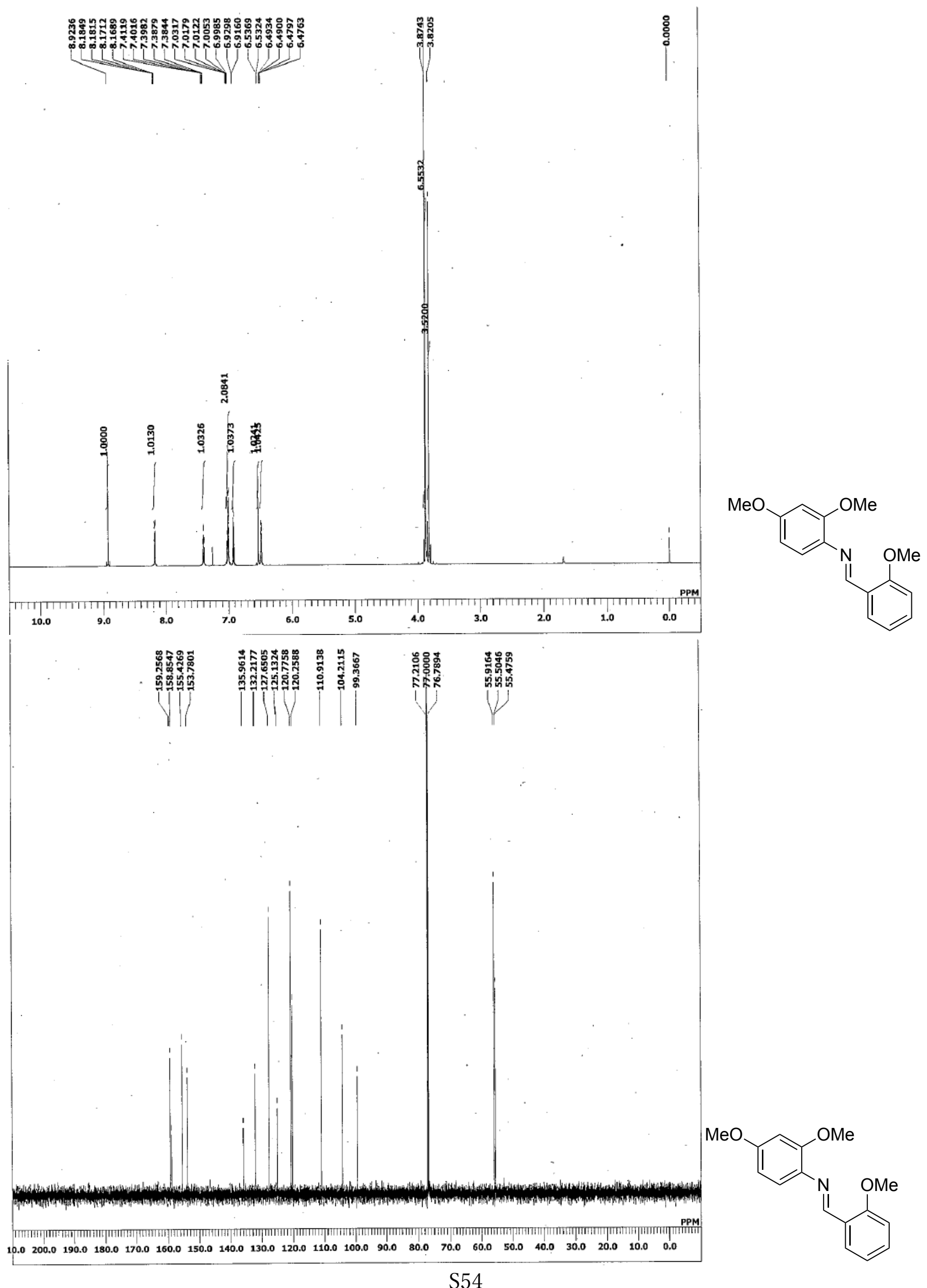



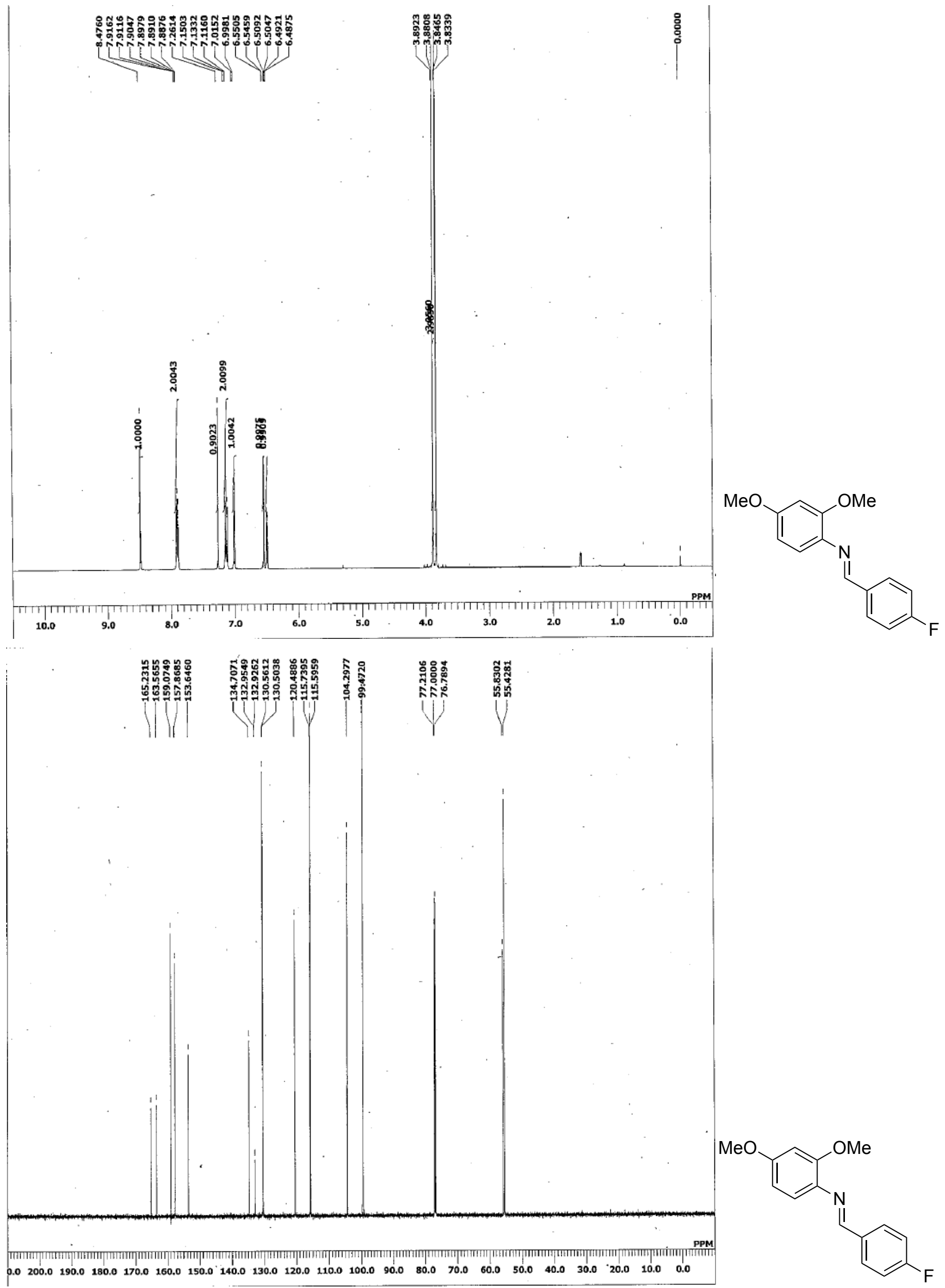


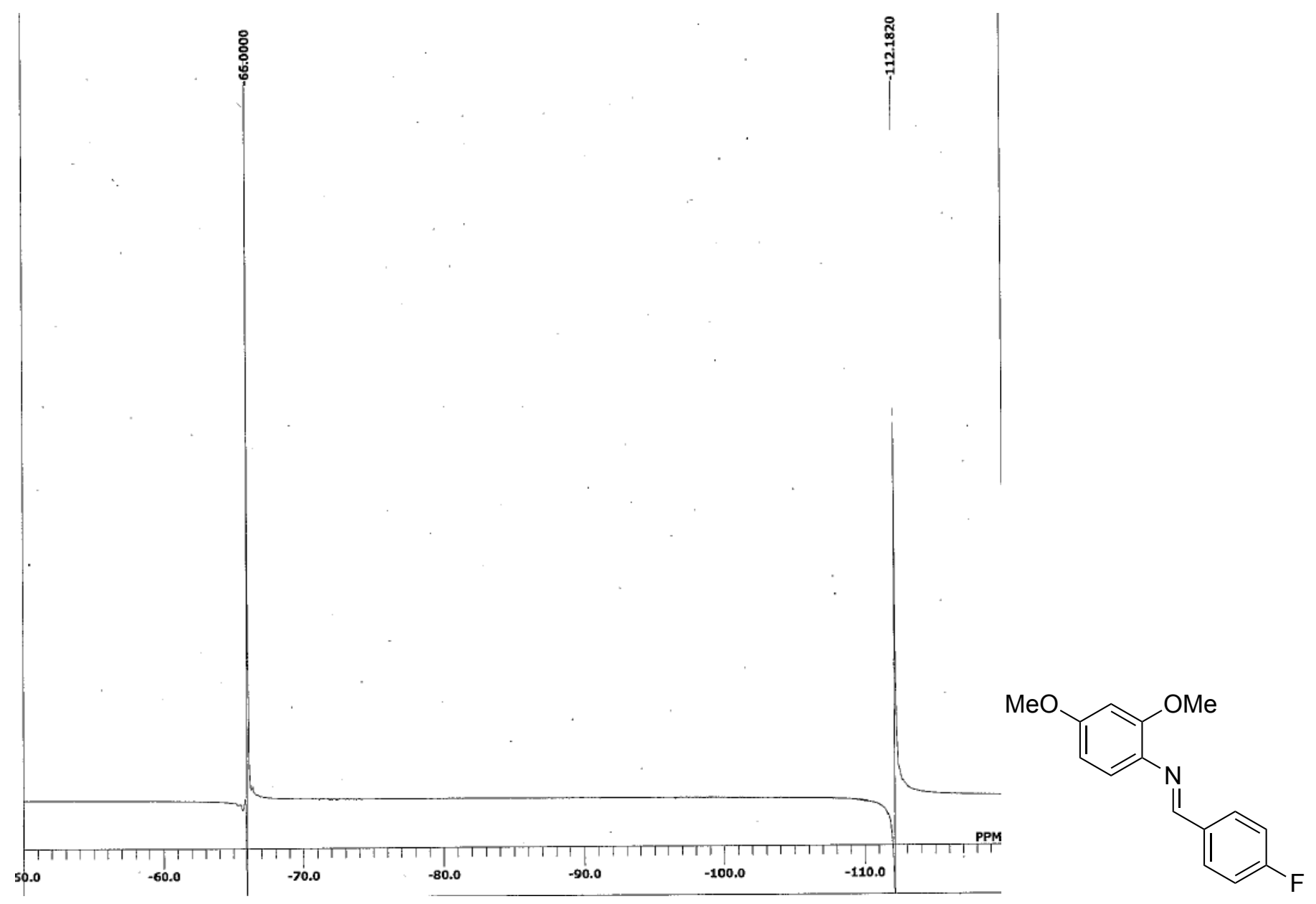



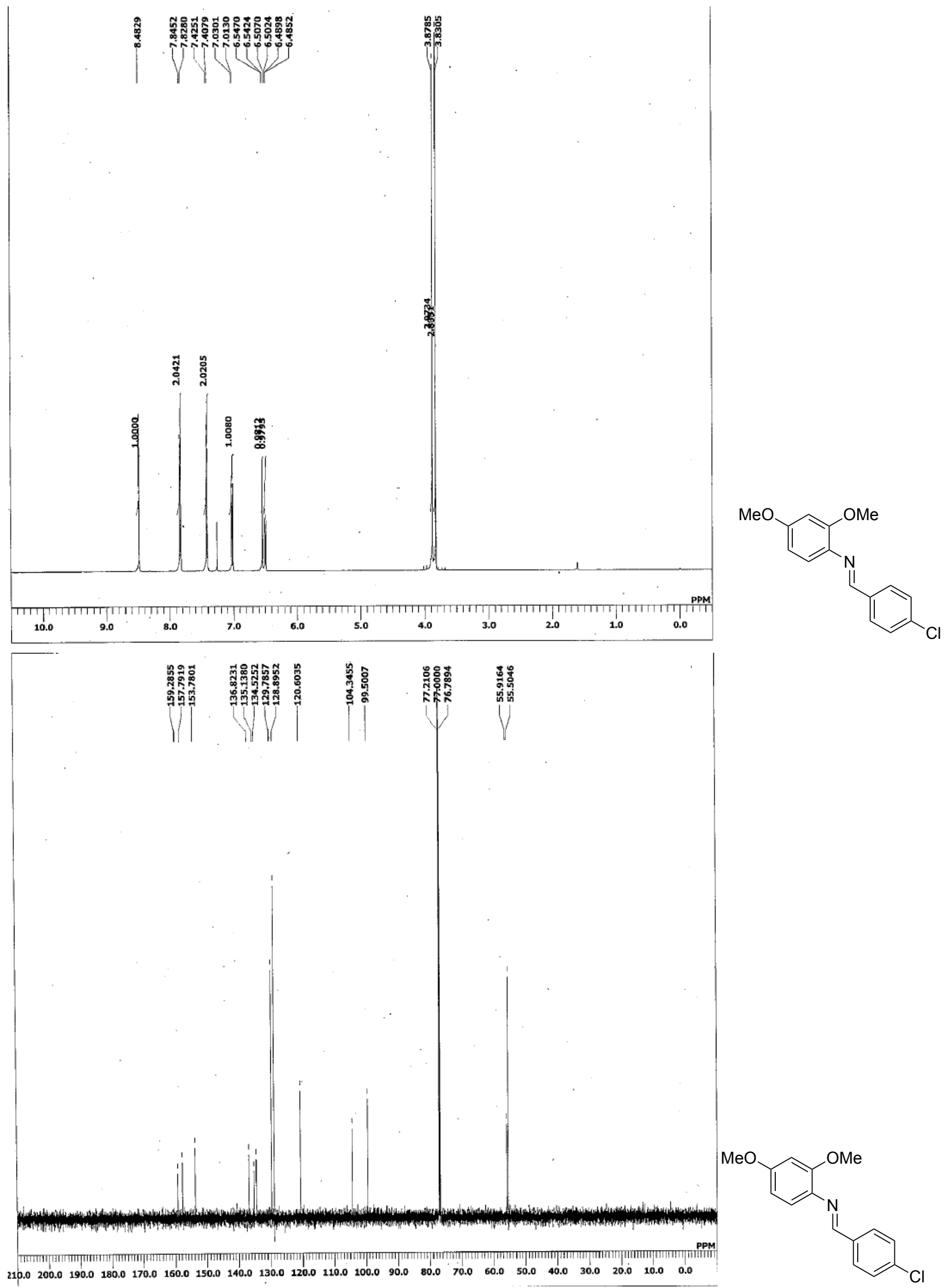


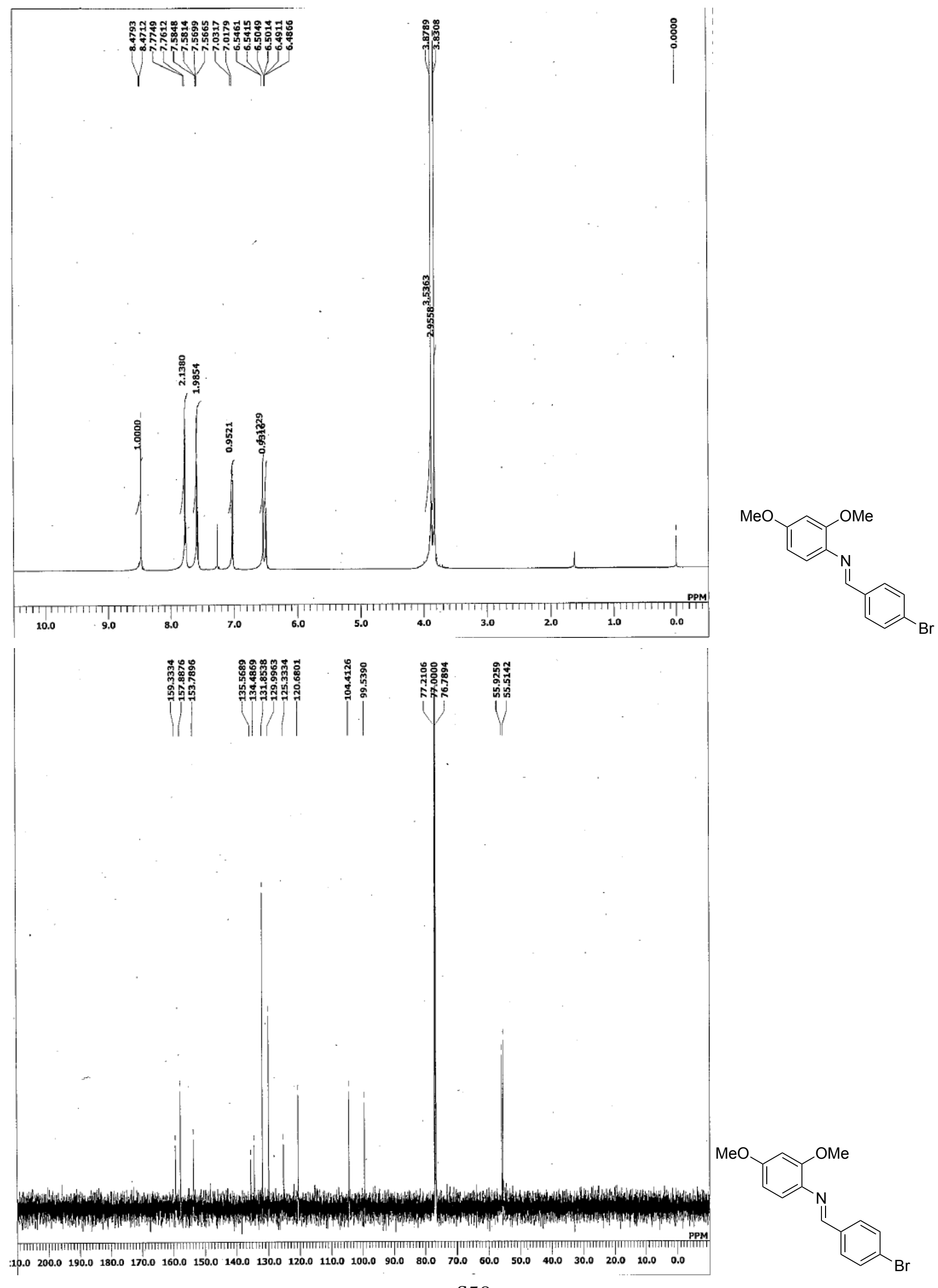

S58 

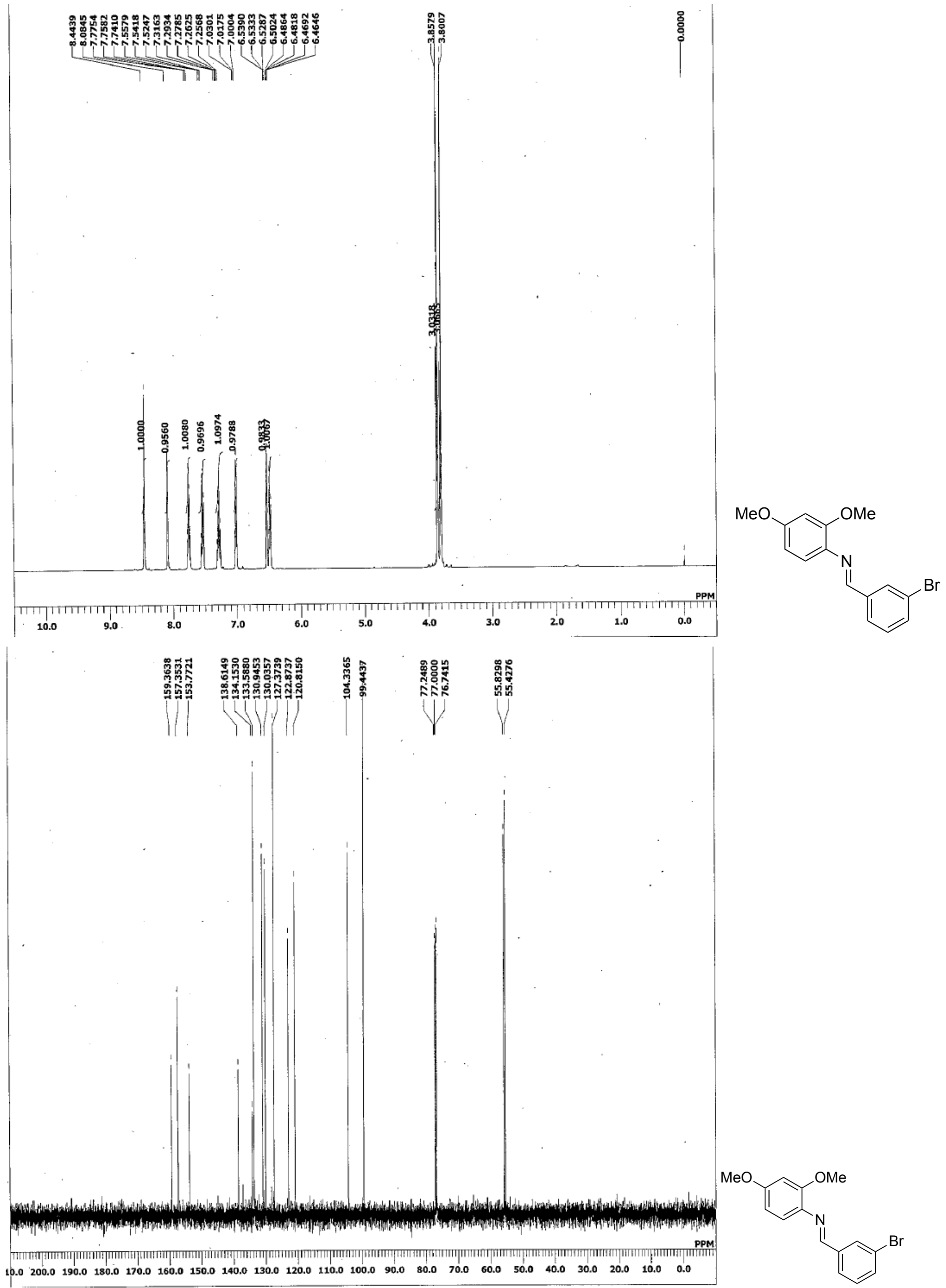


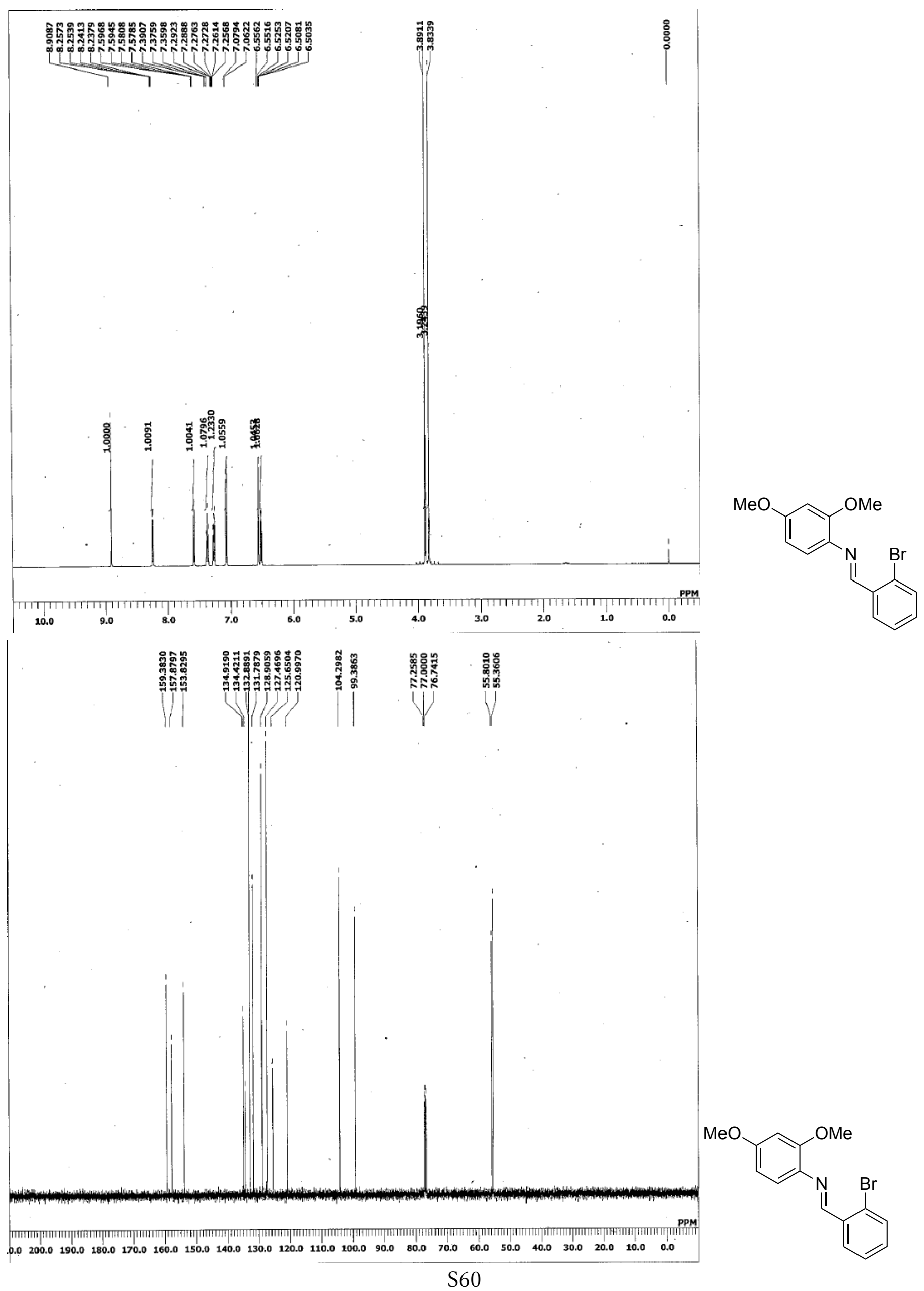




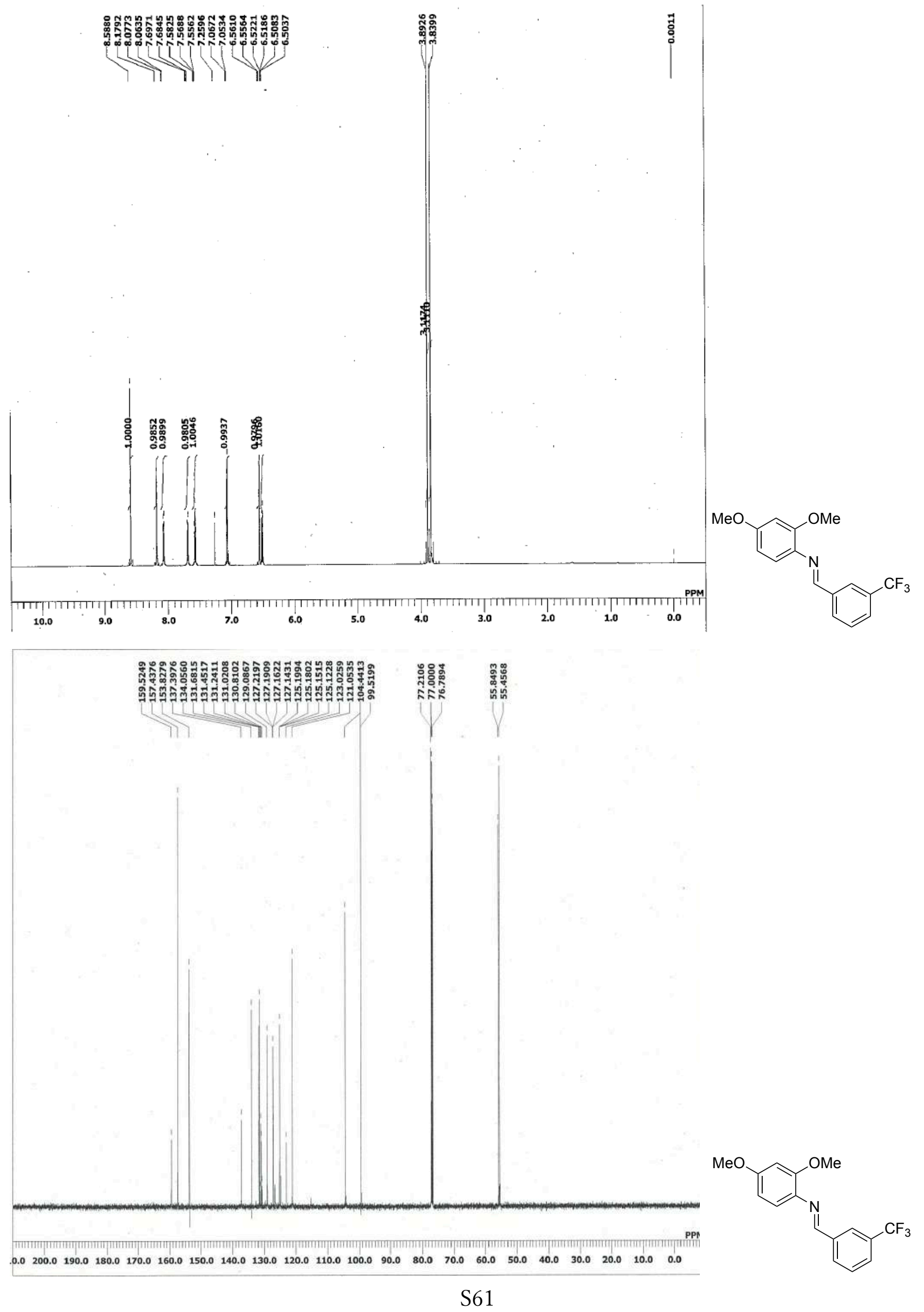




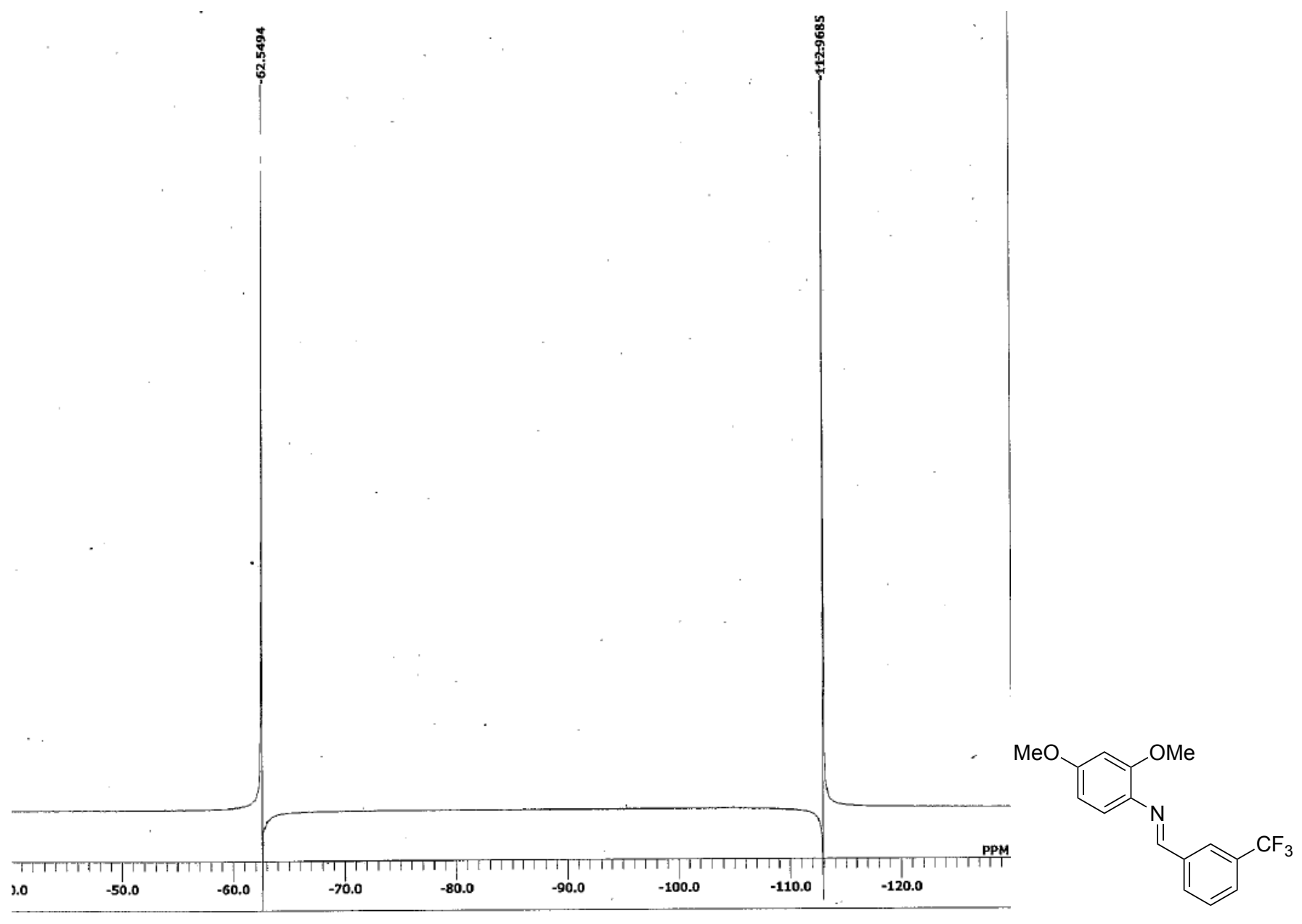



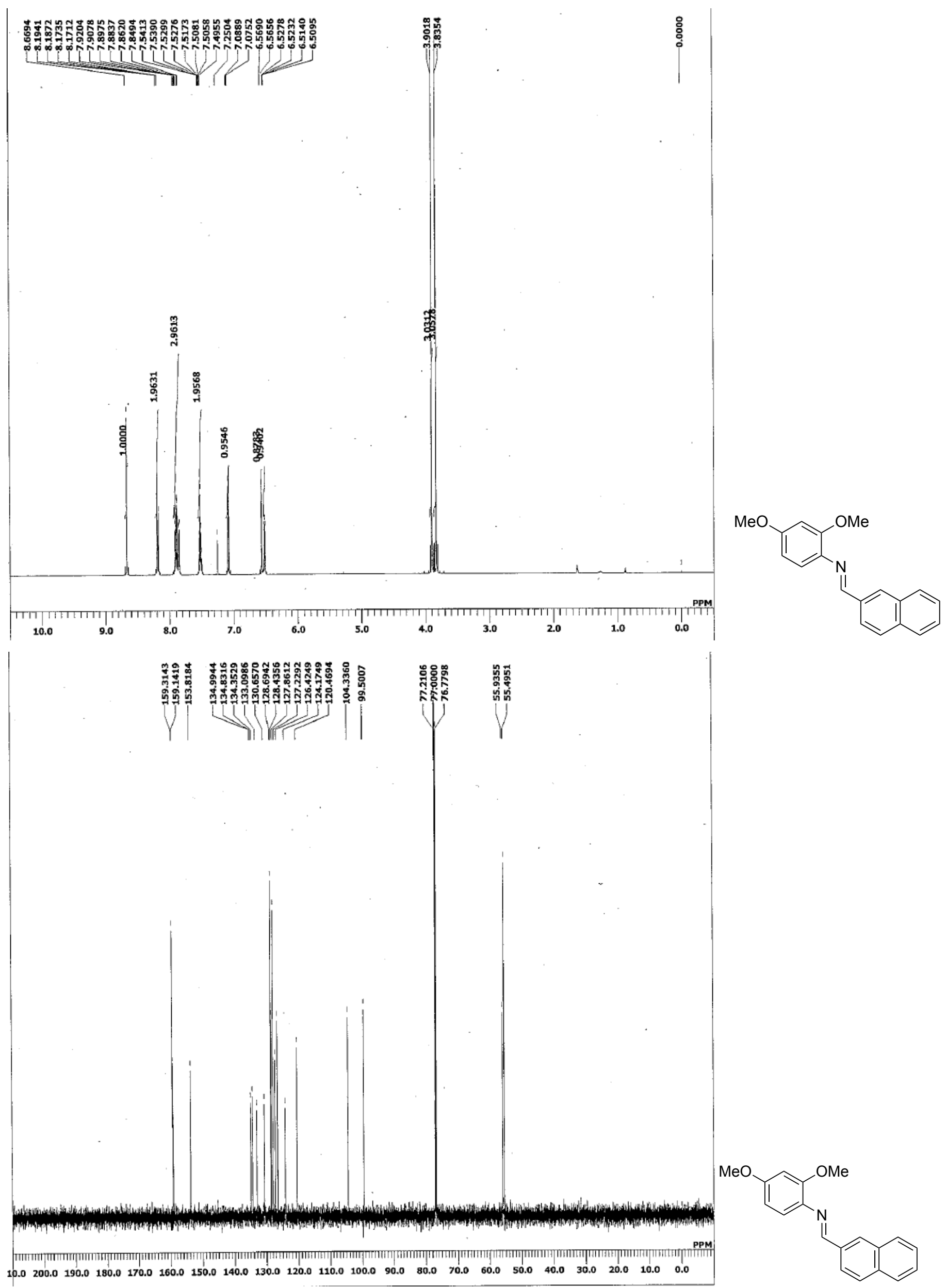

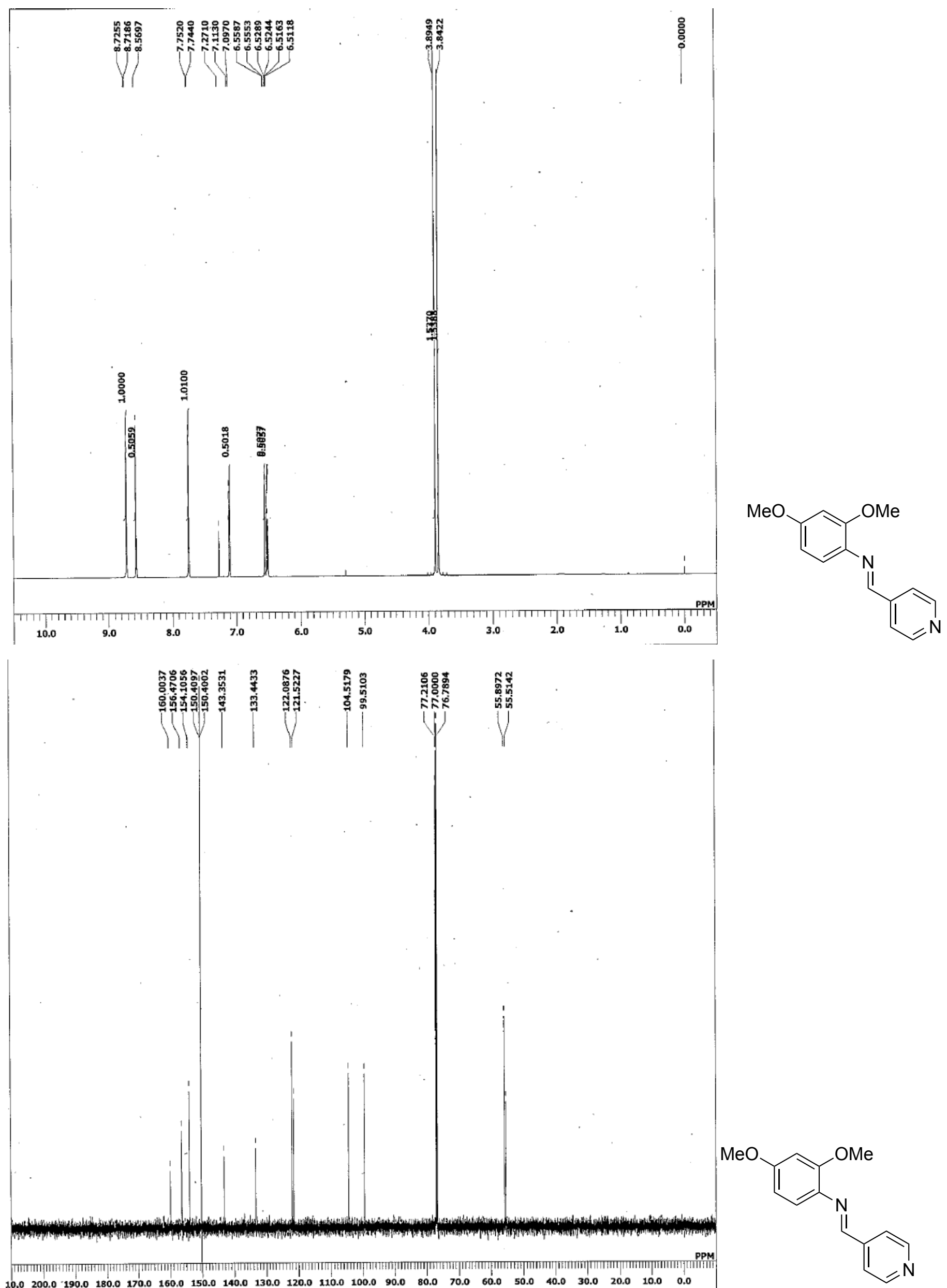


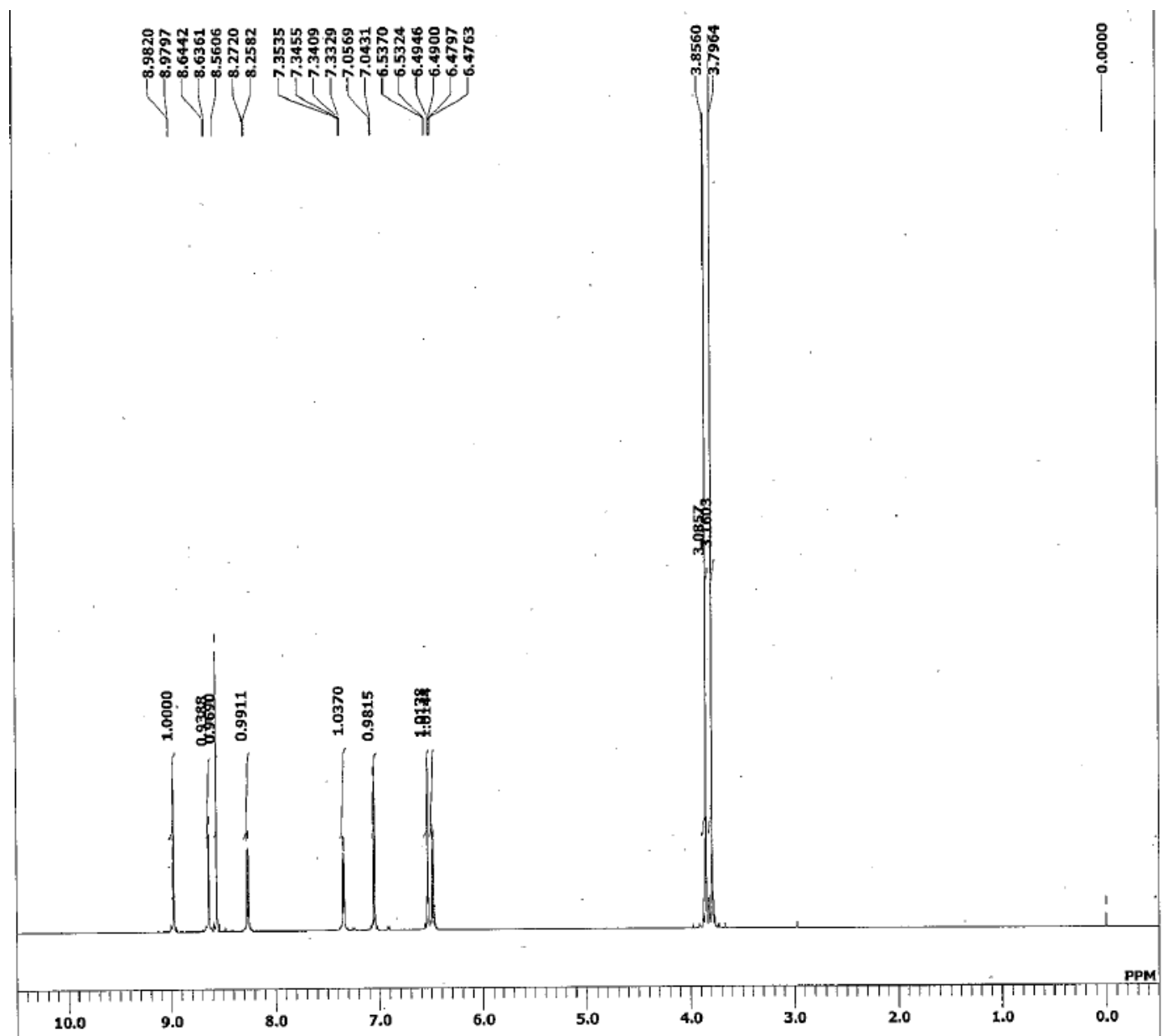<smiles>COc1ccc(/N=C/c2cccnc2)c(OC)c1</smiles>

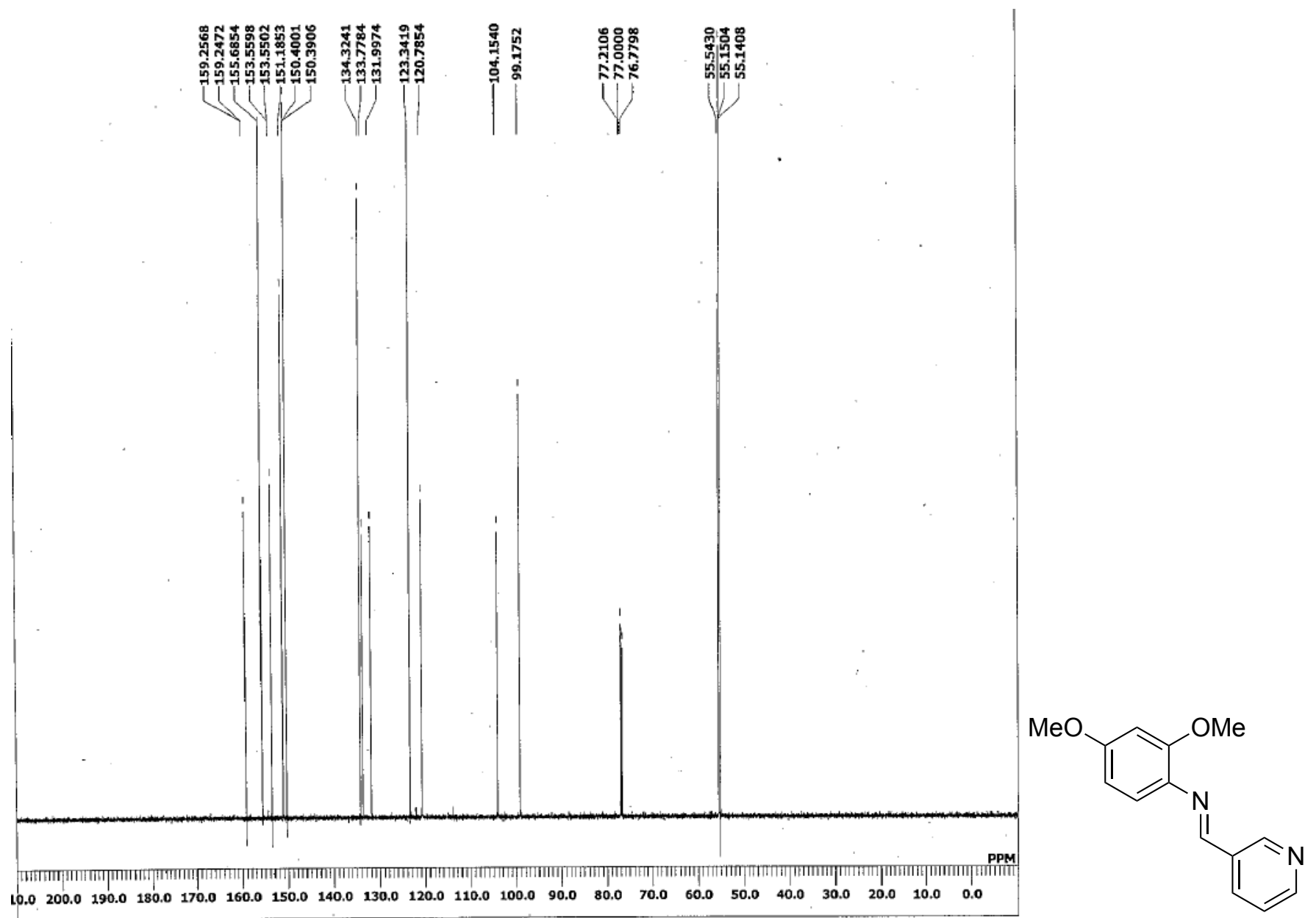




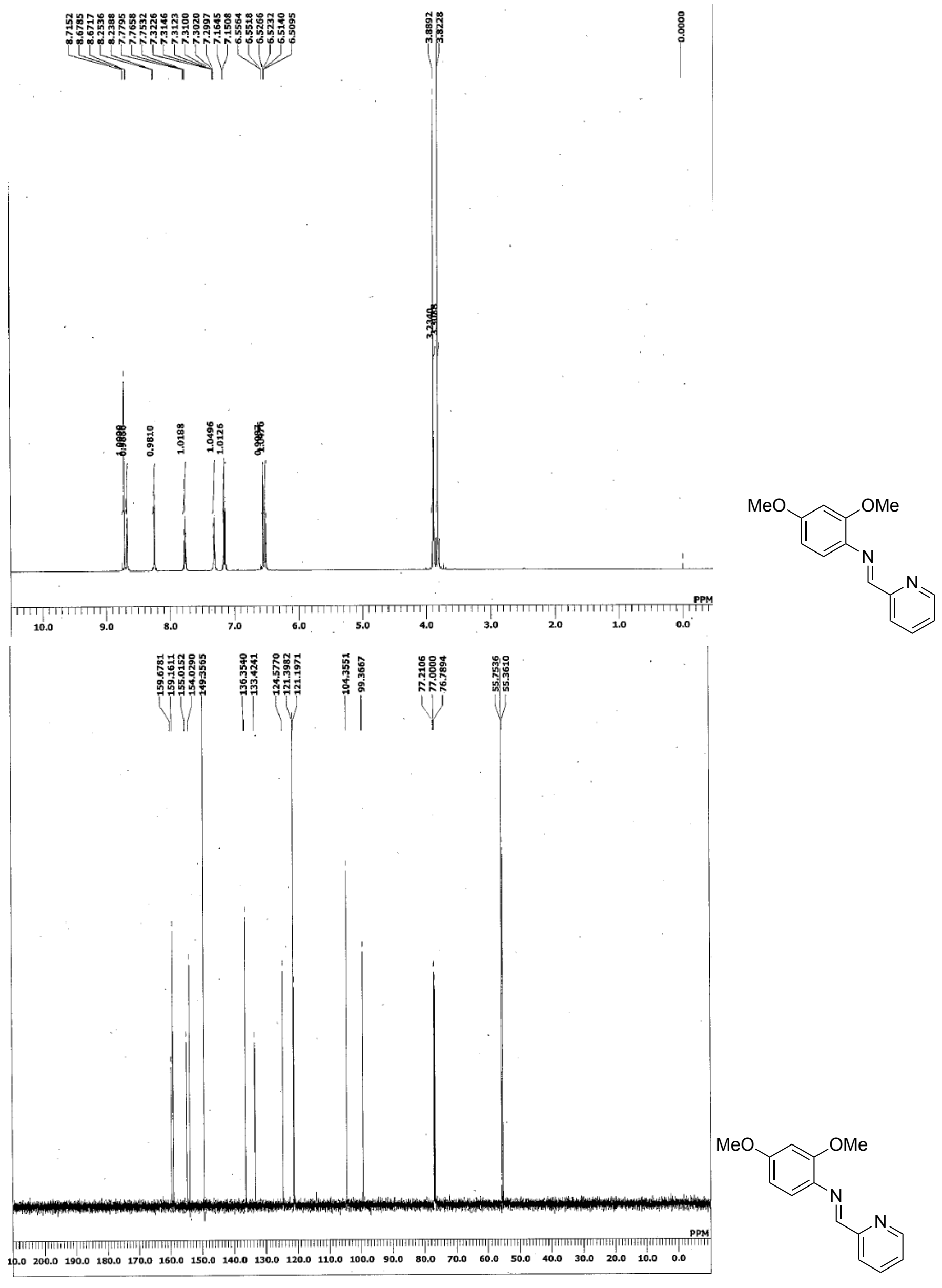




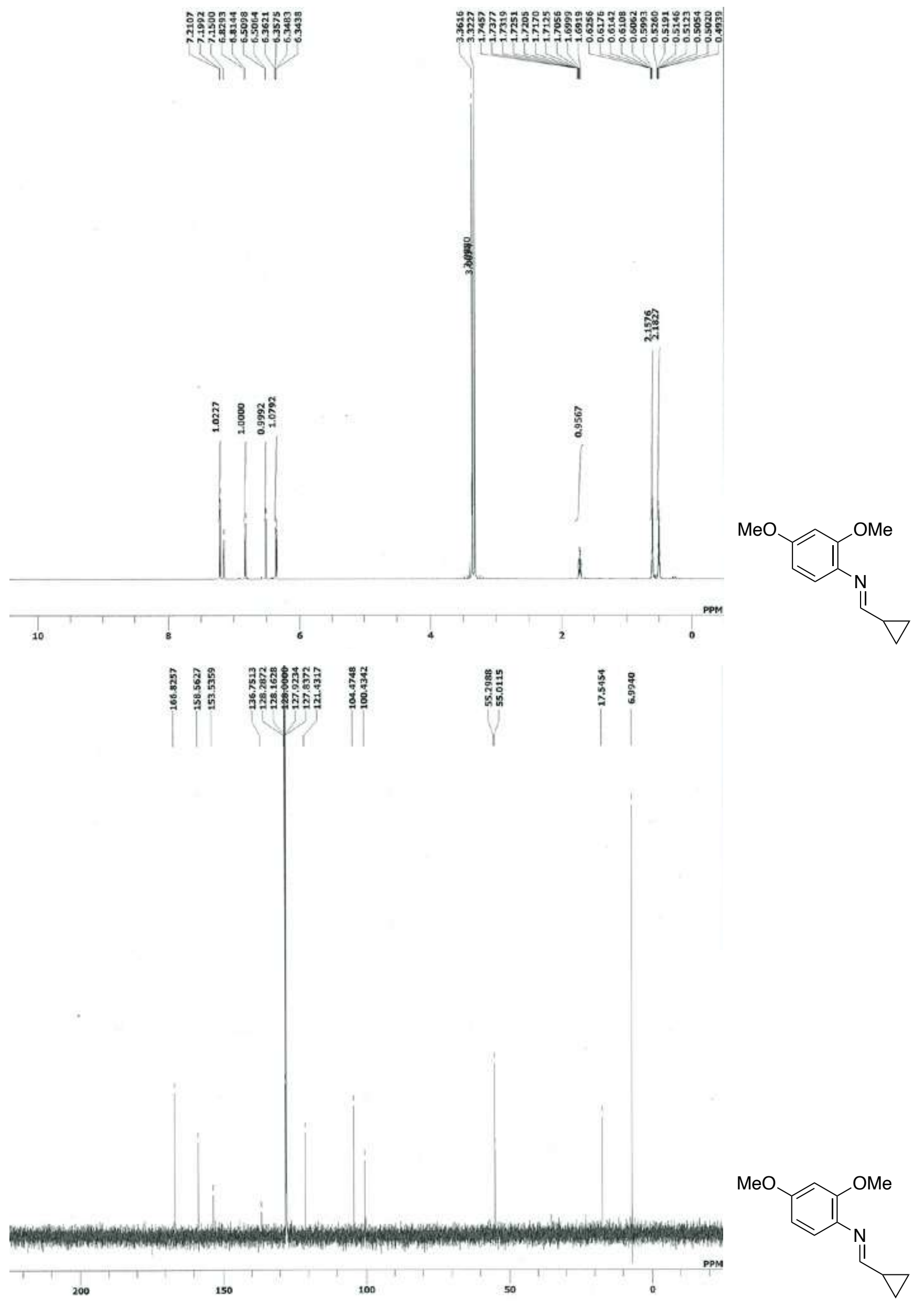




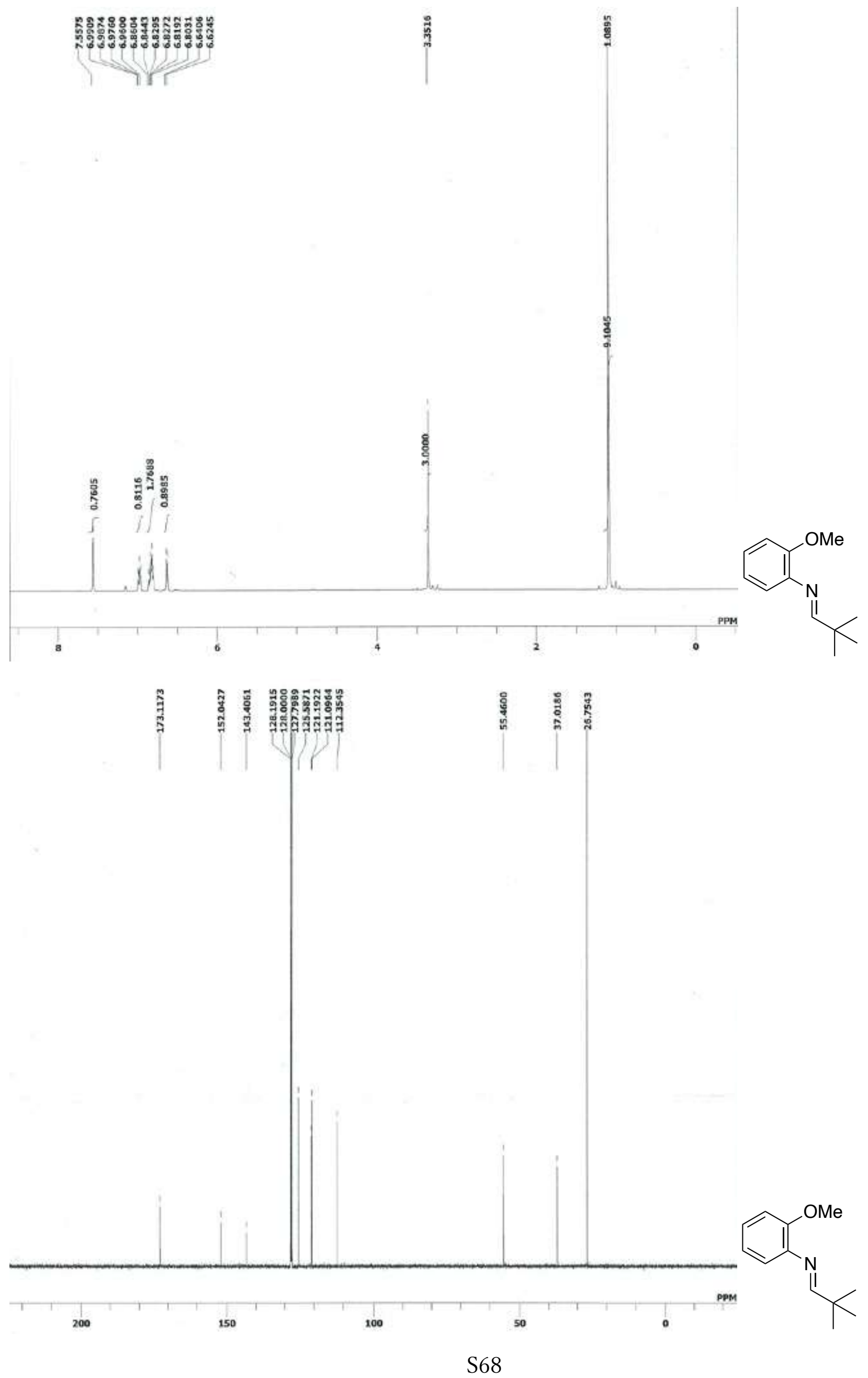




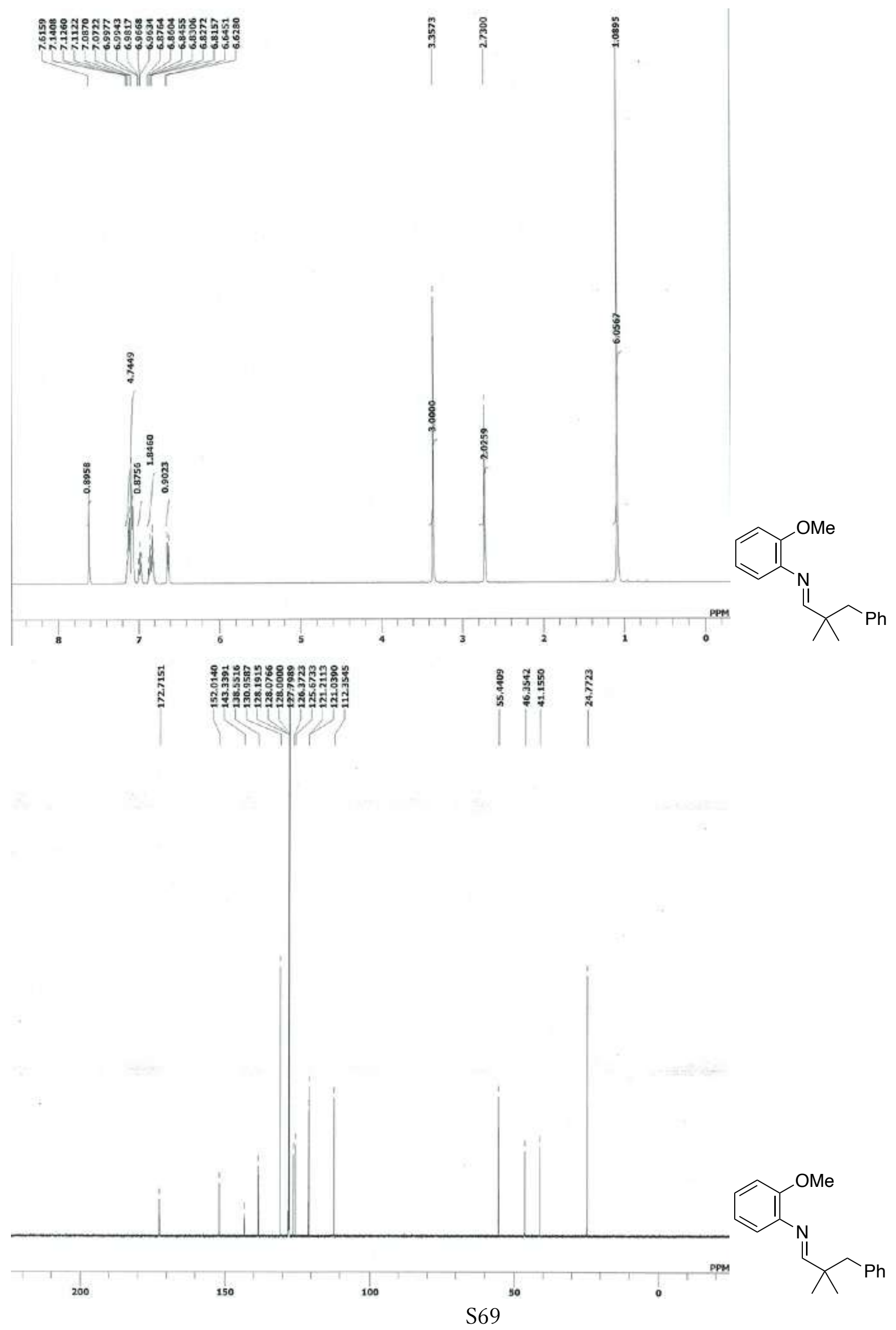




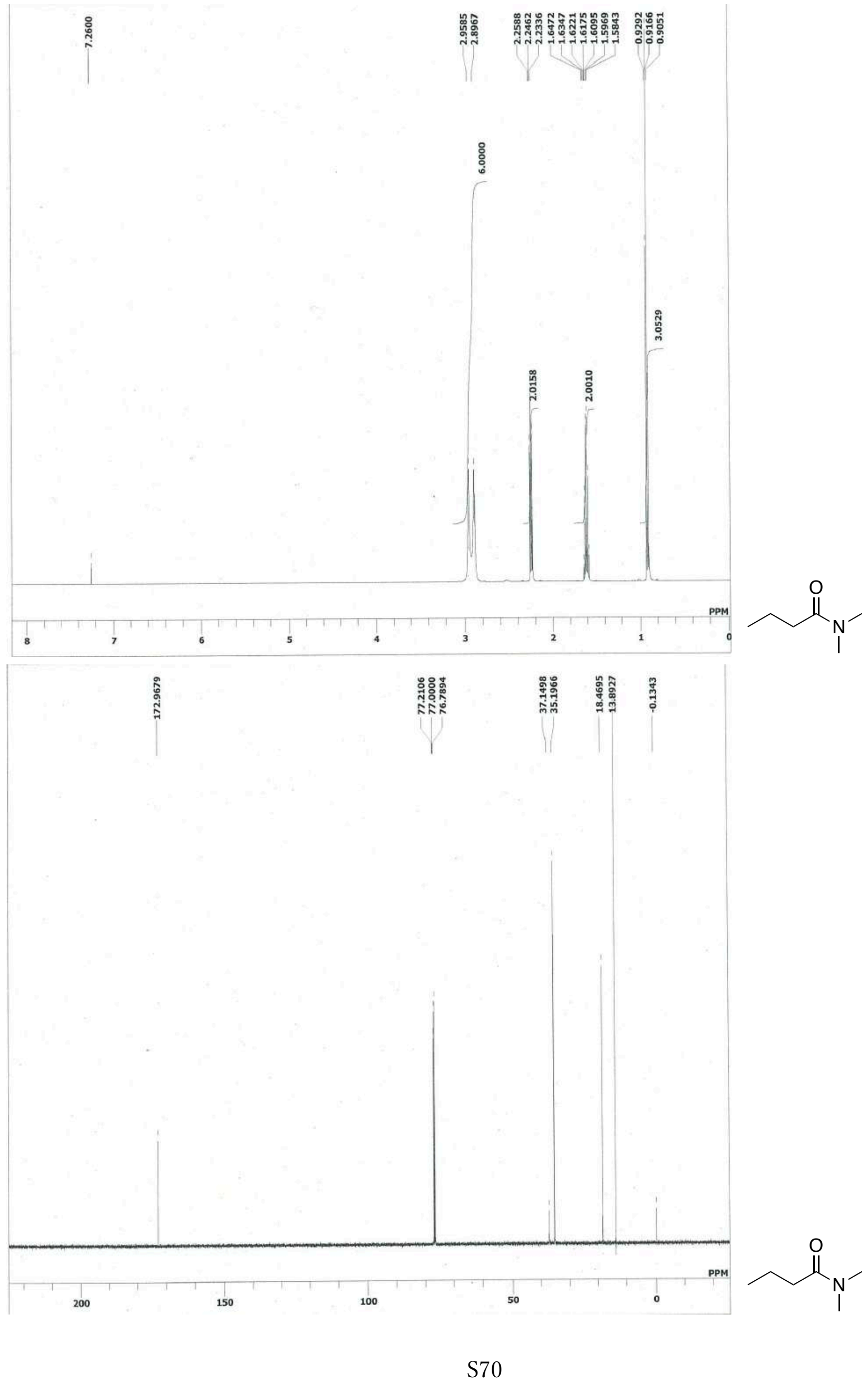




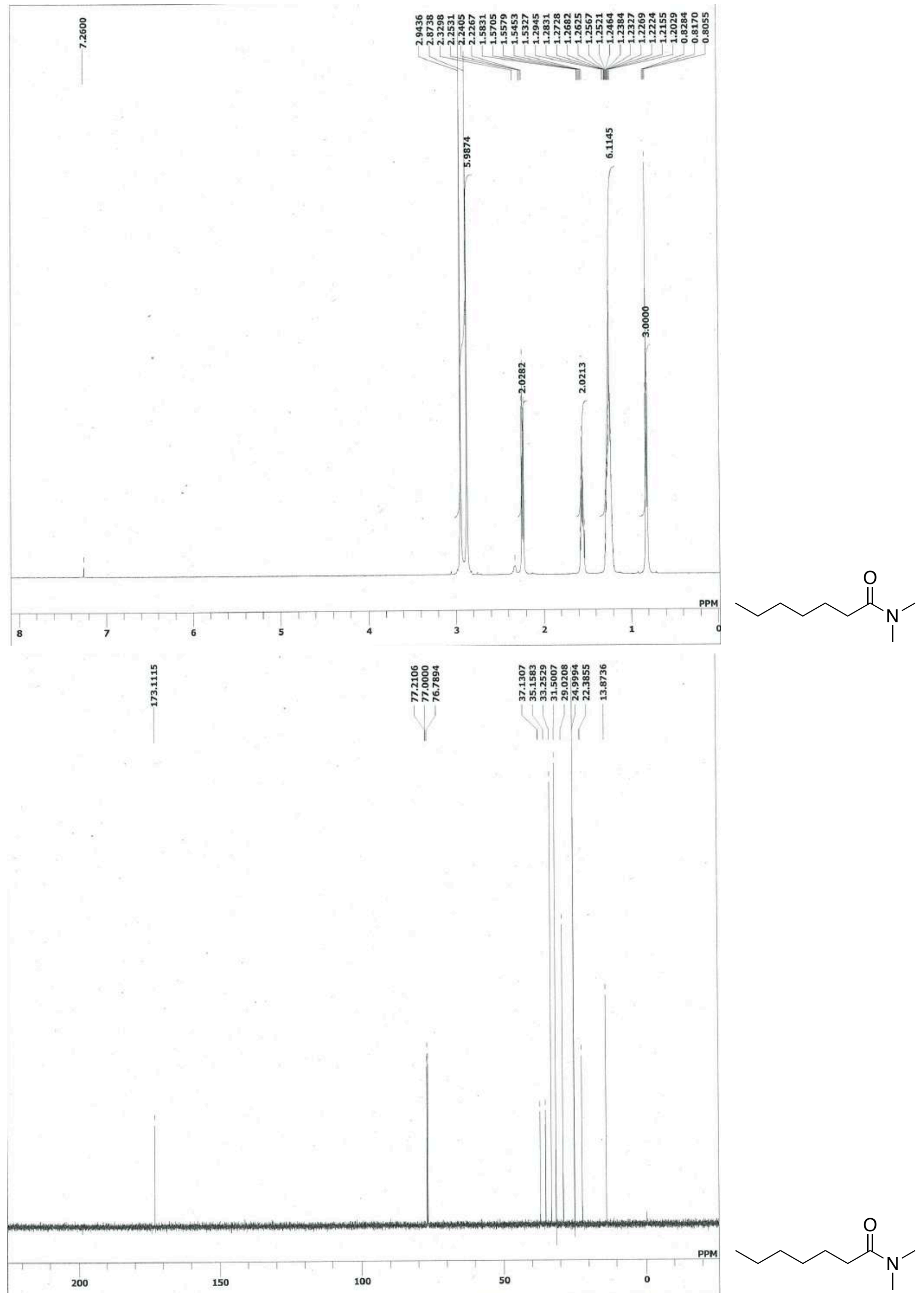




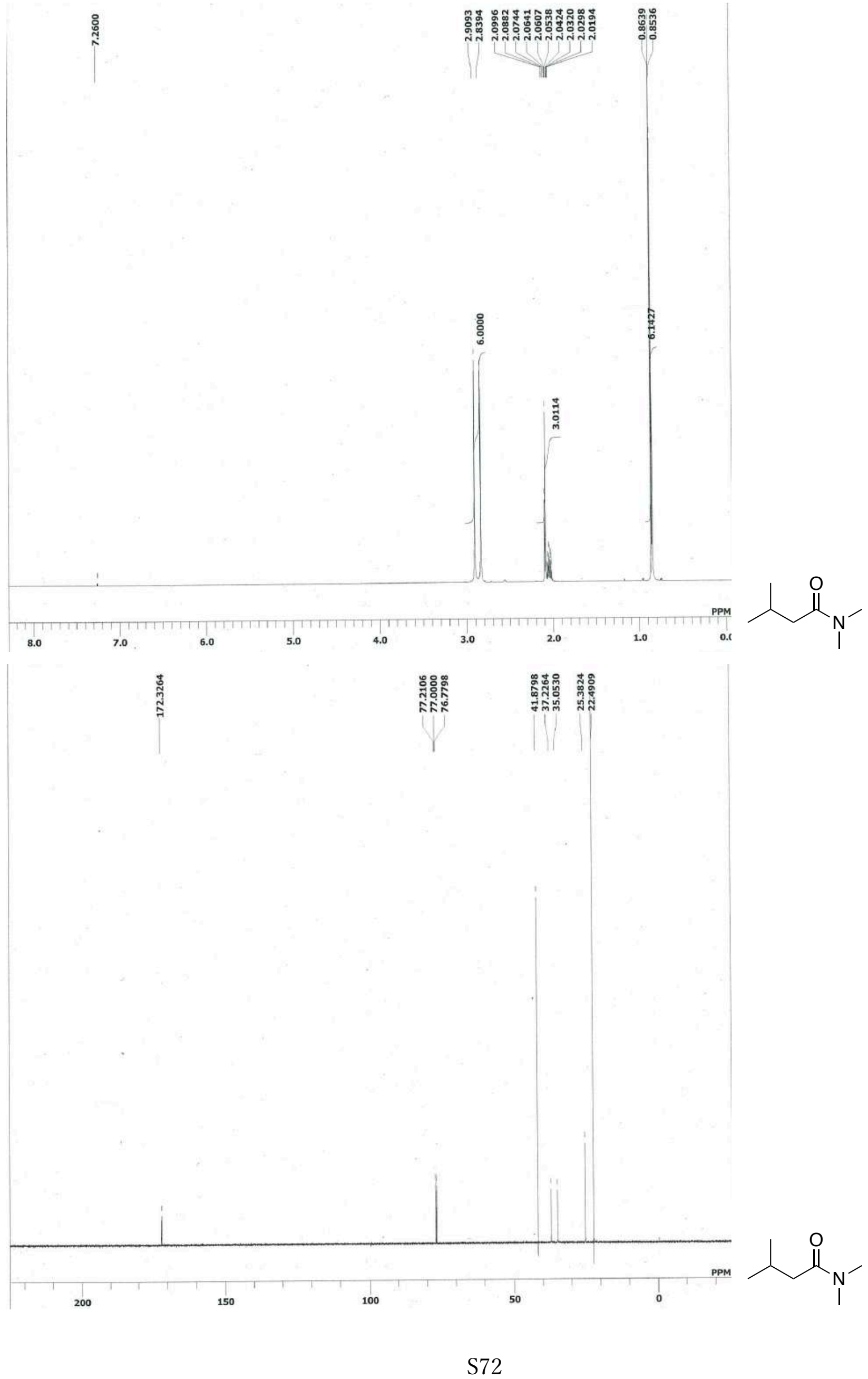




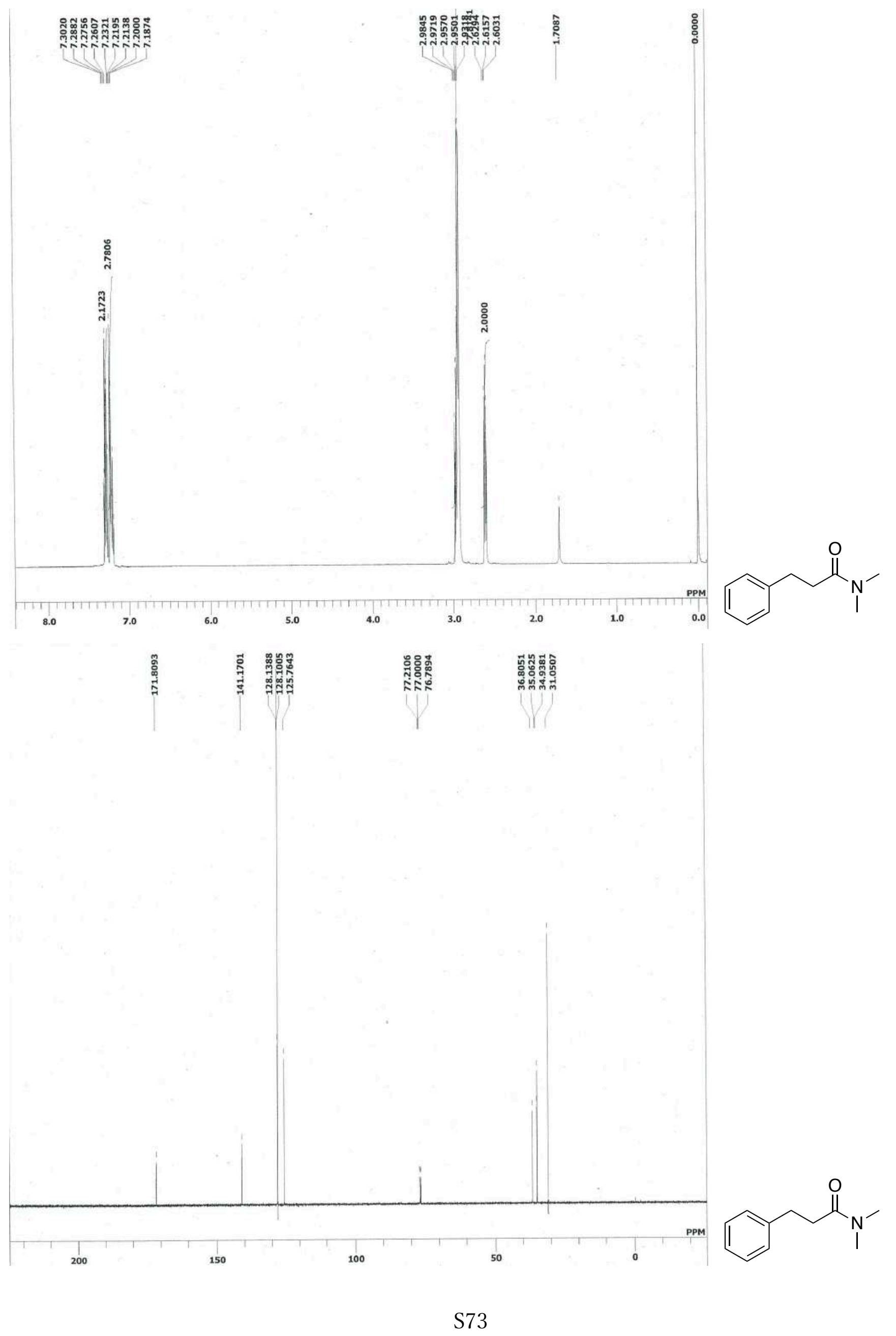




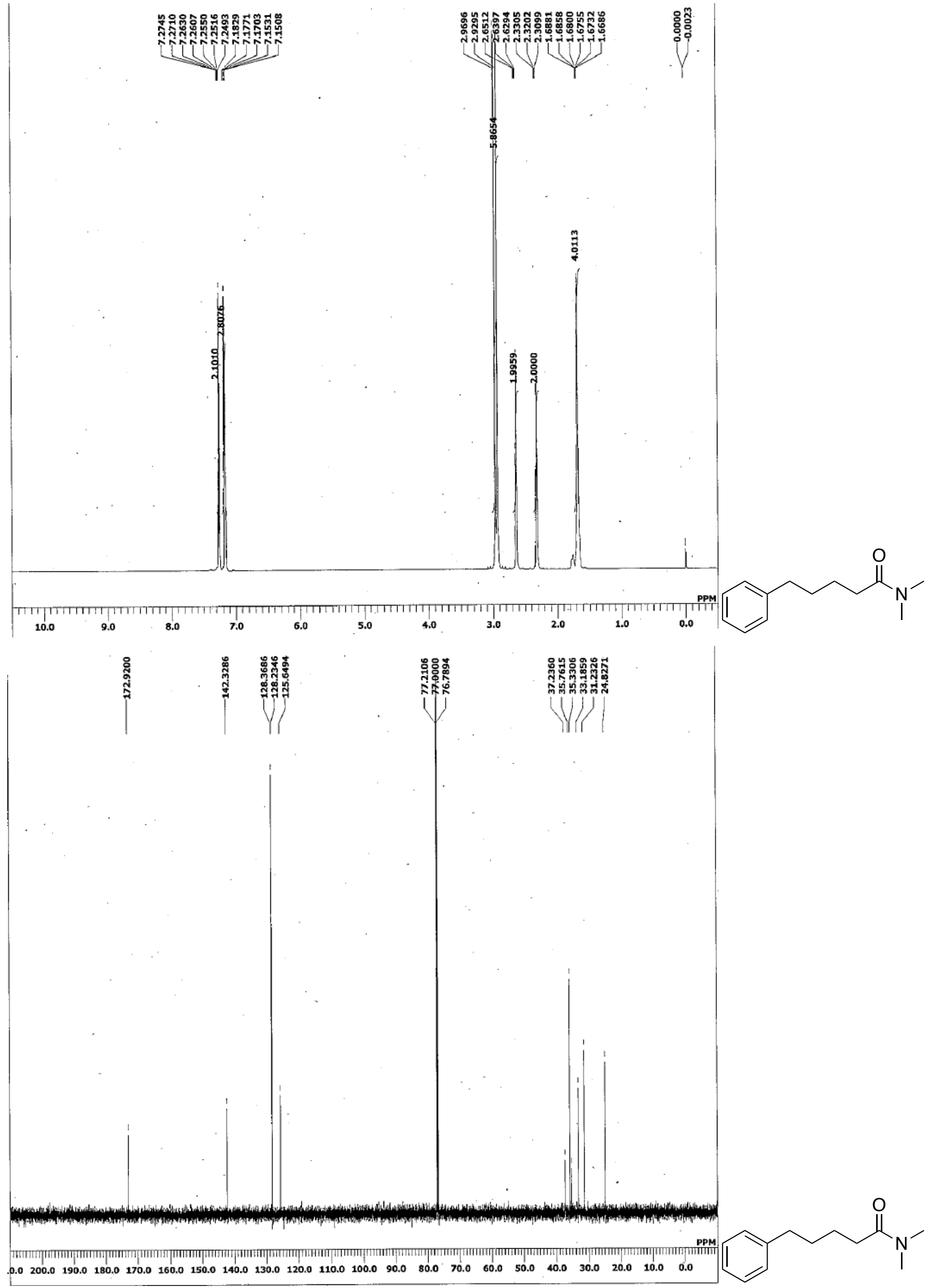




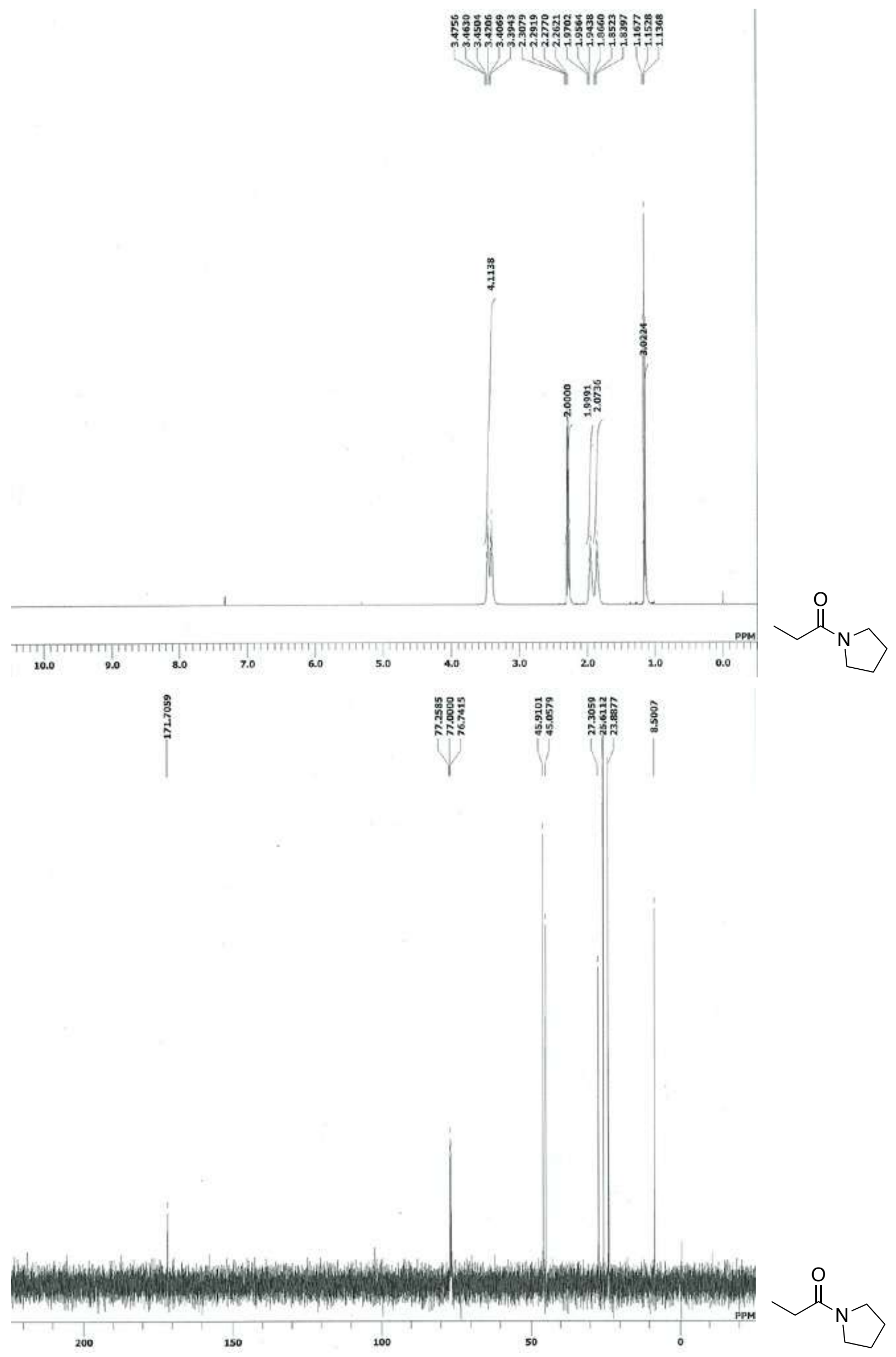




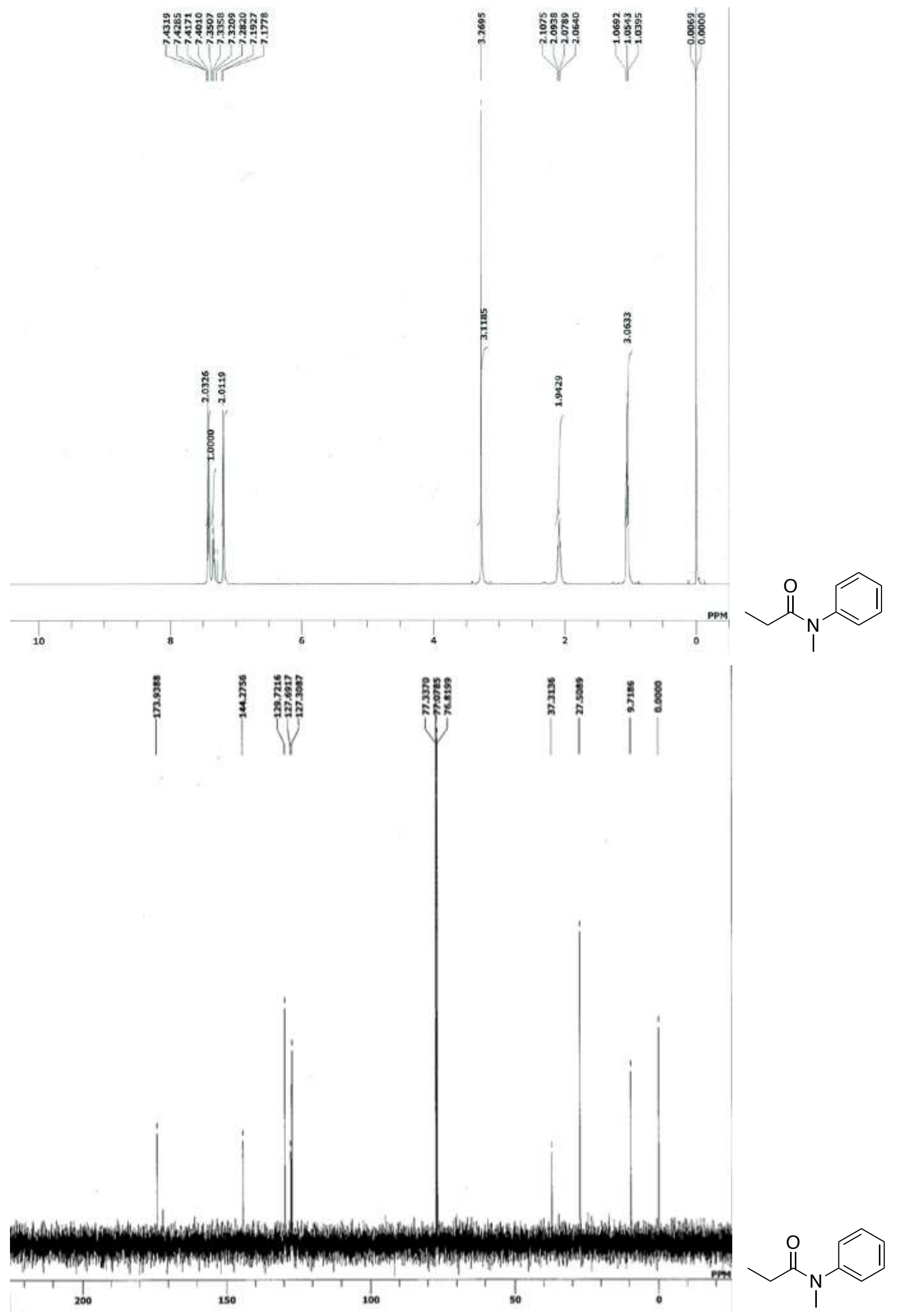




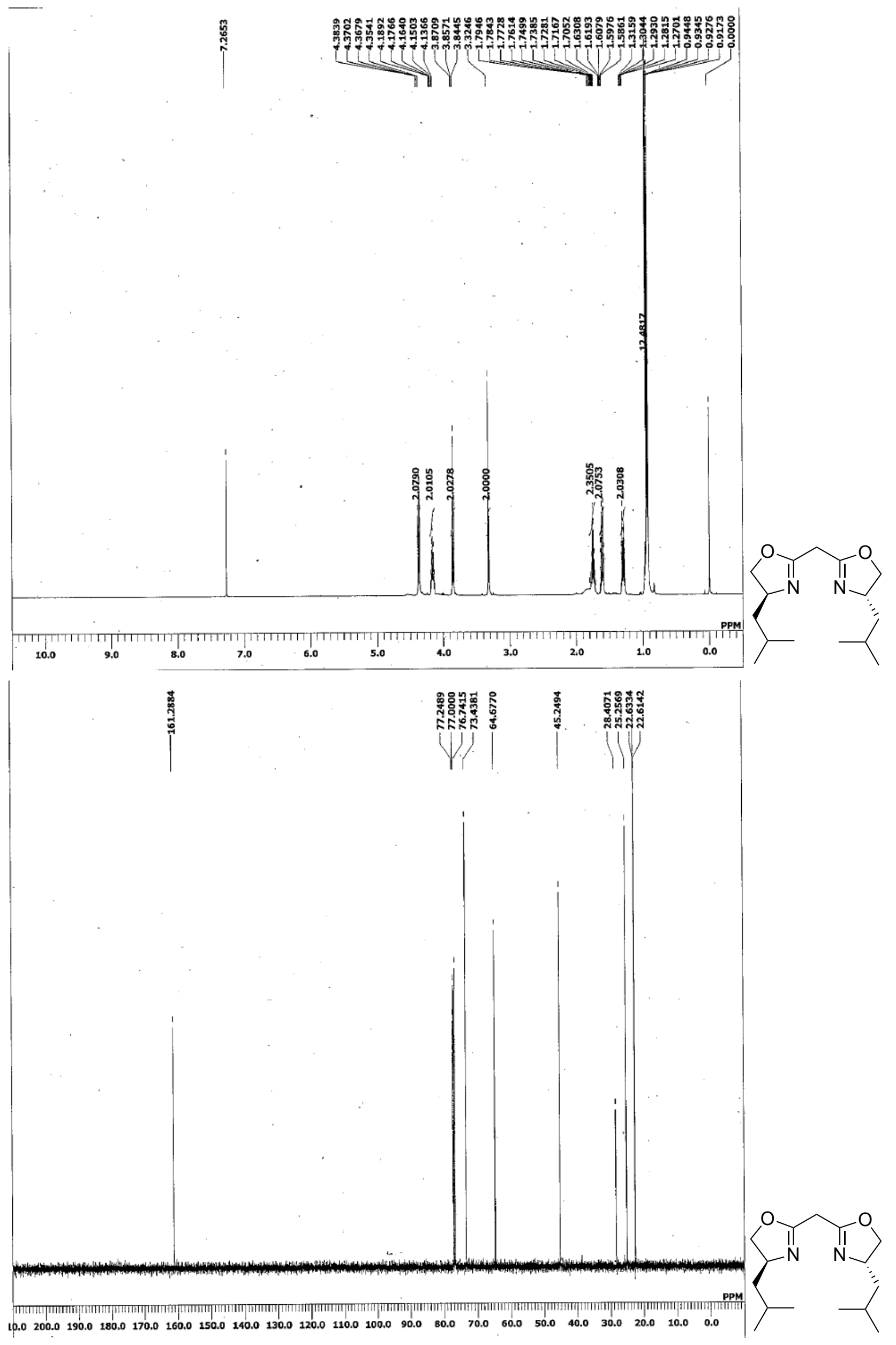




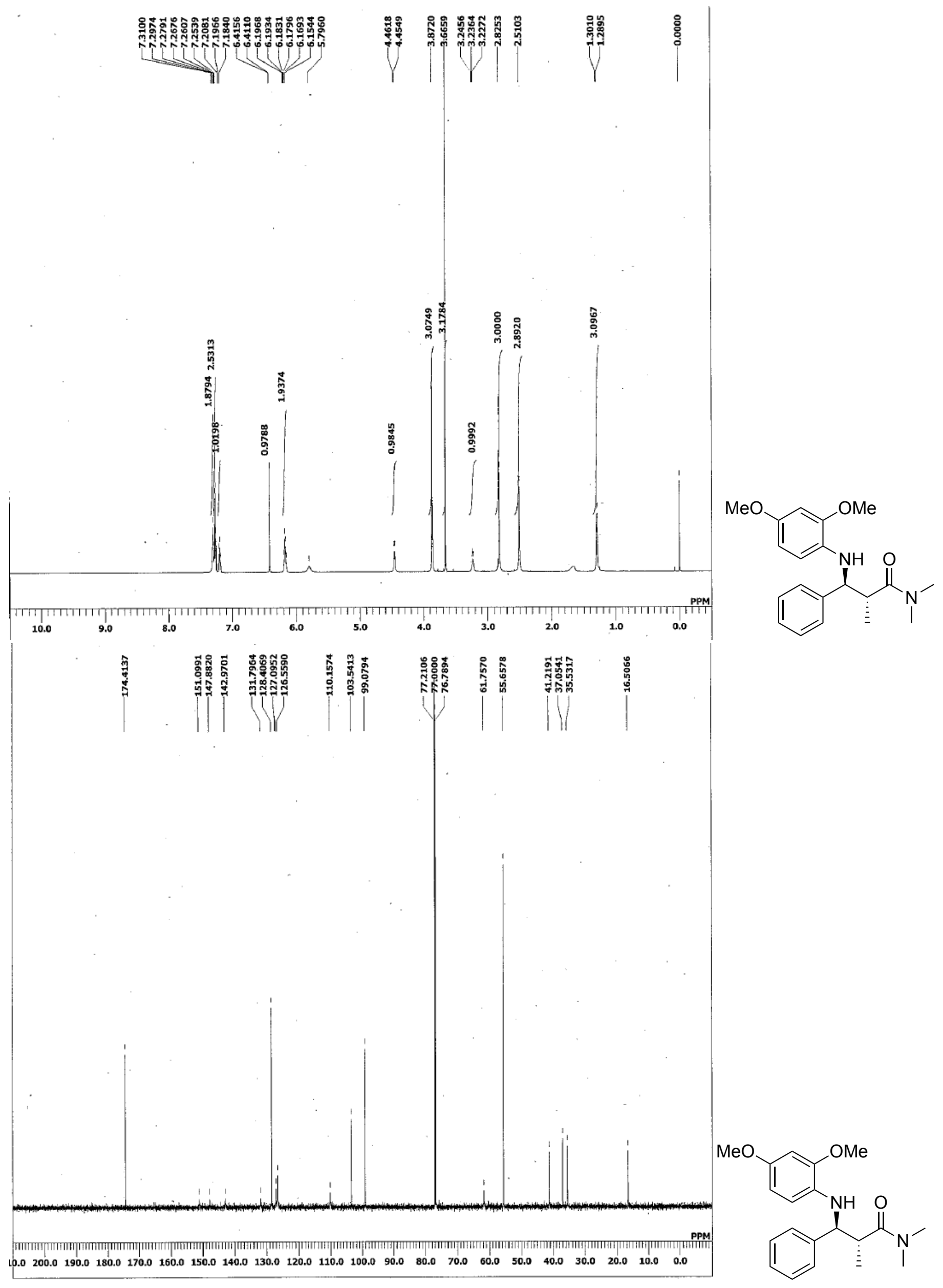




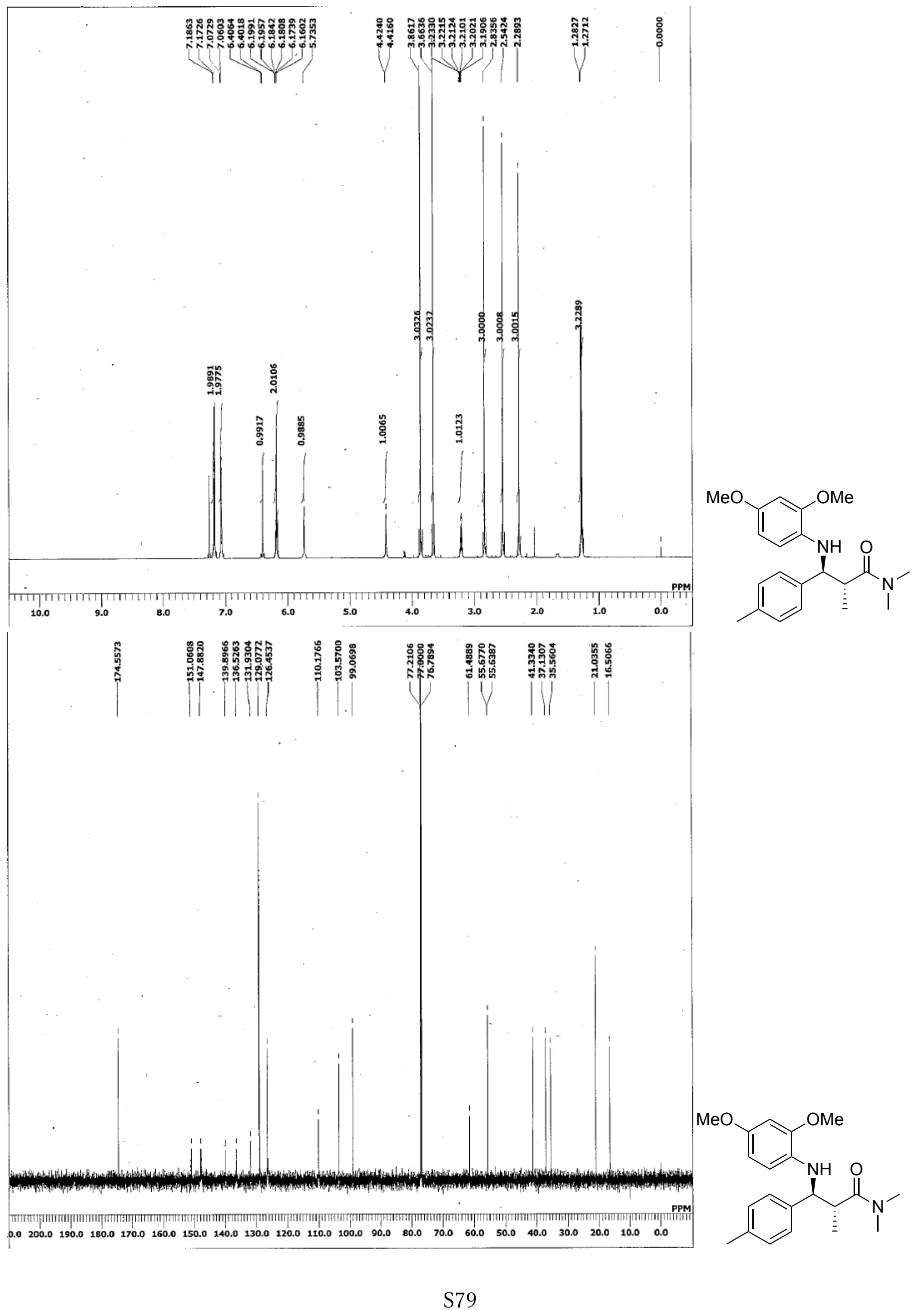




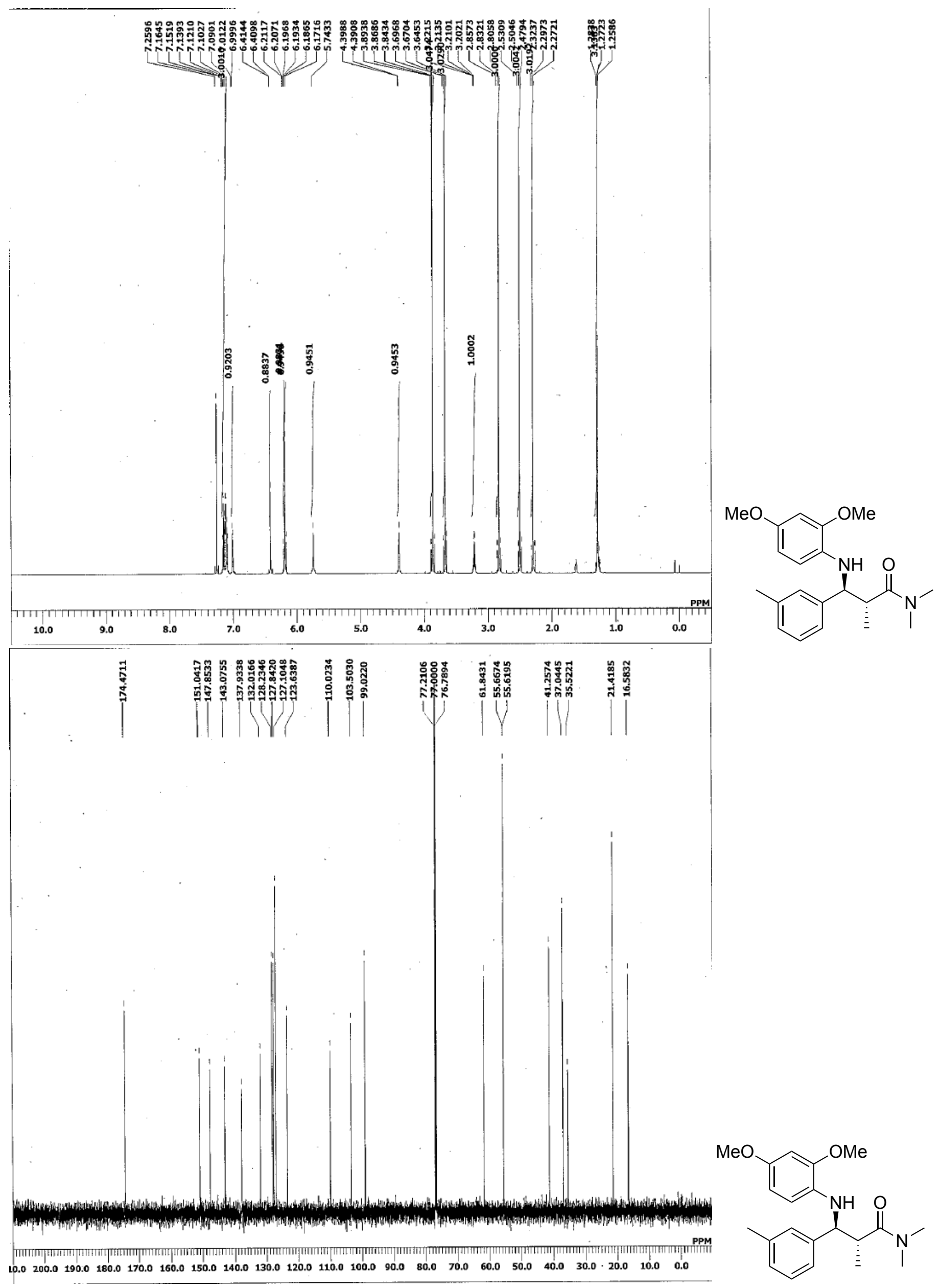




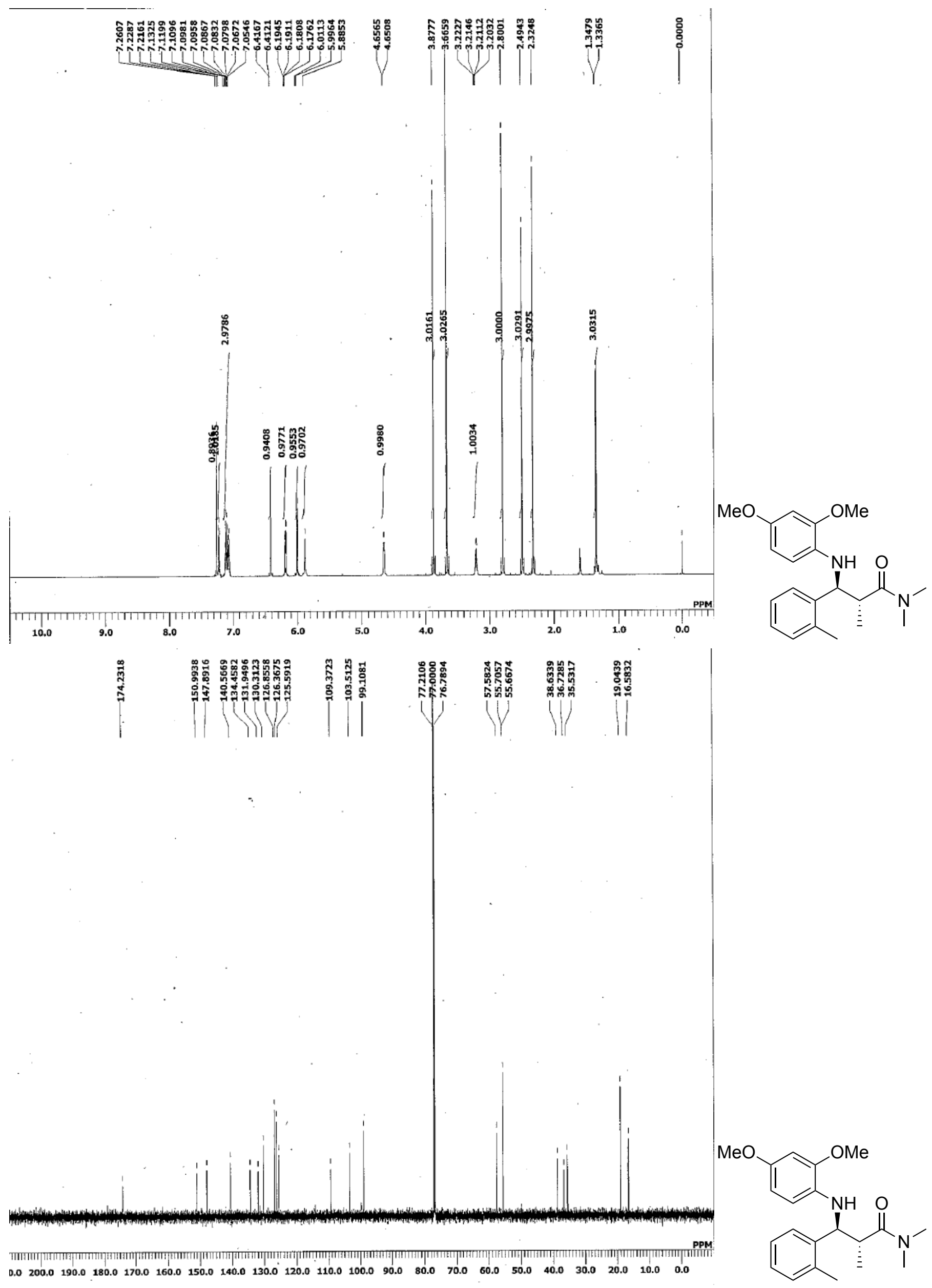




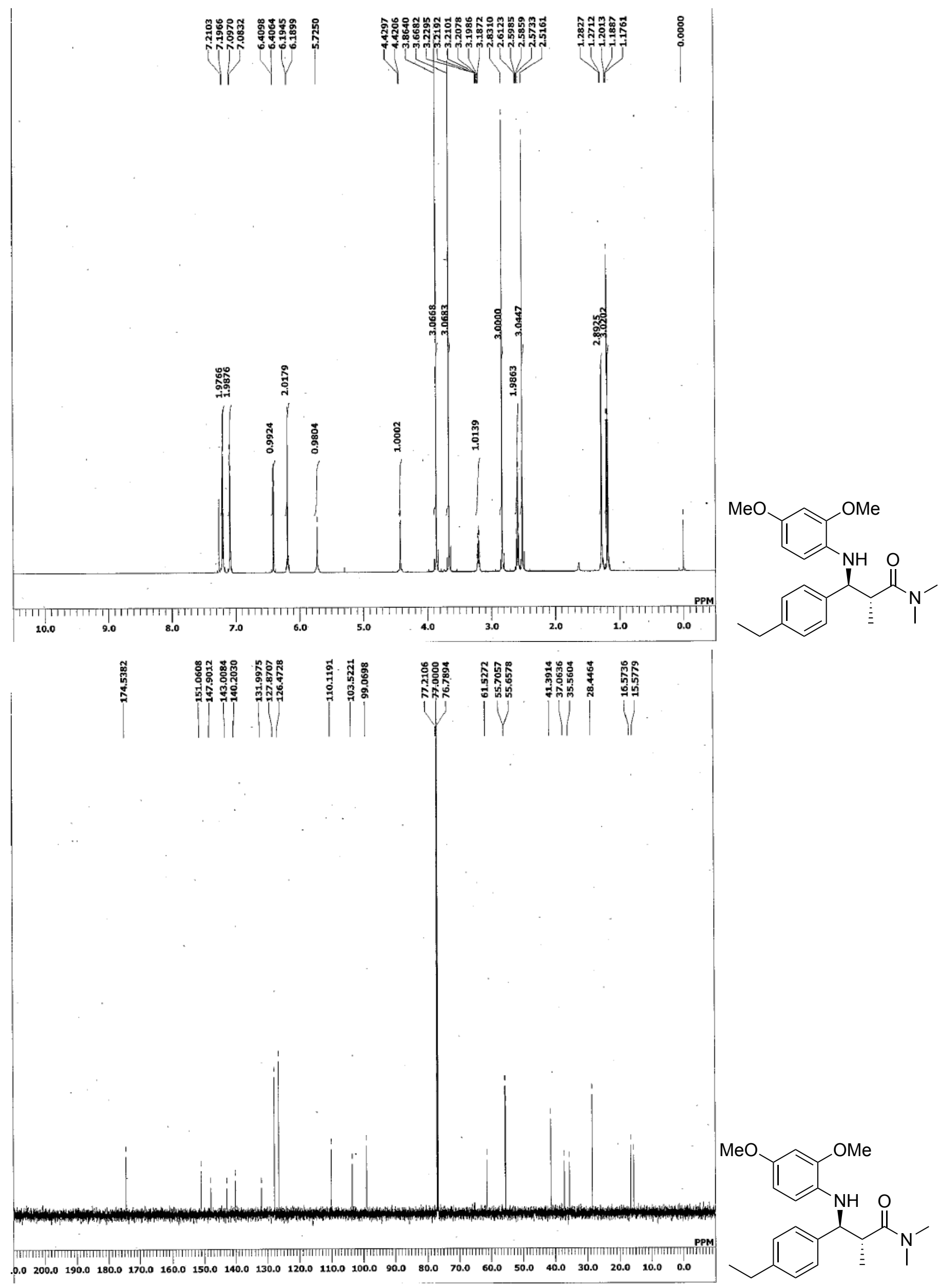




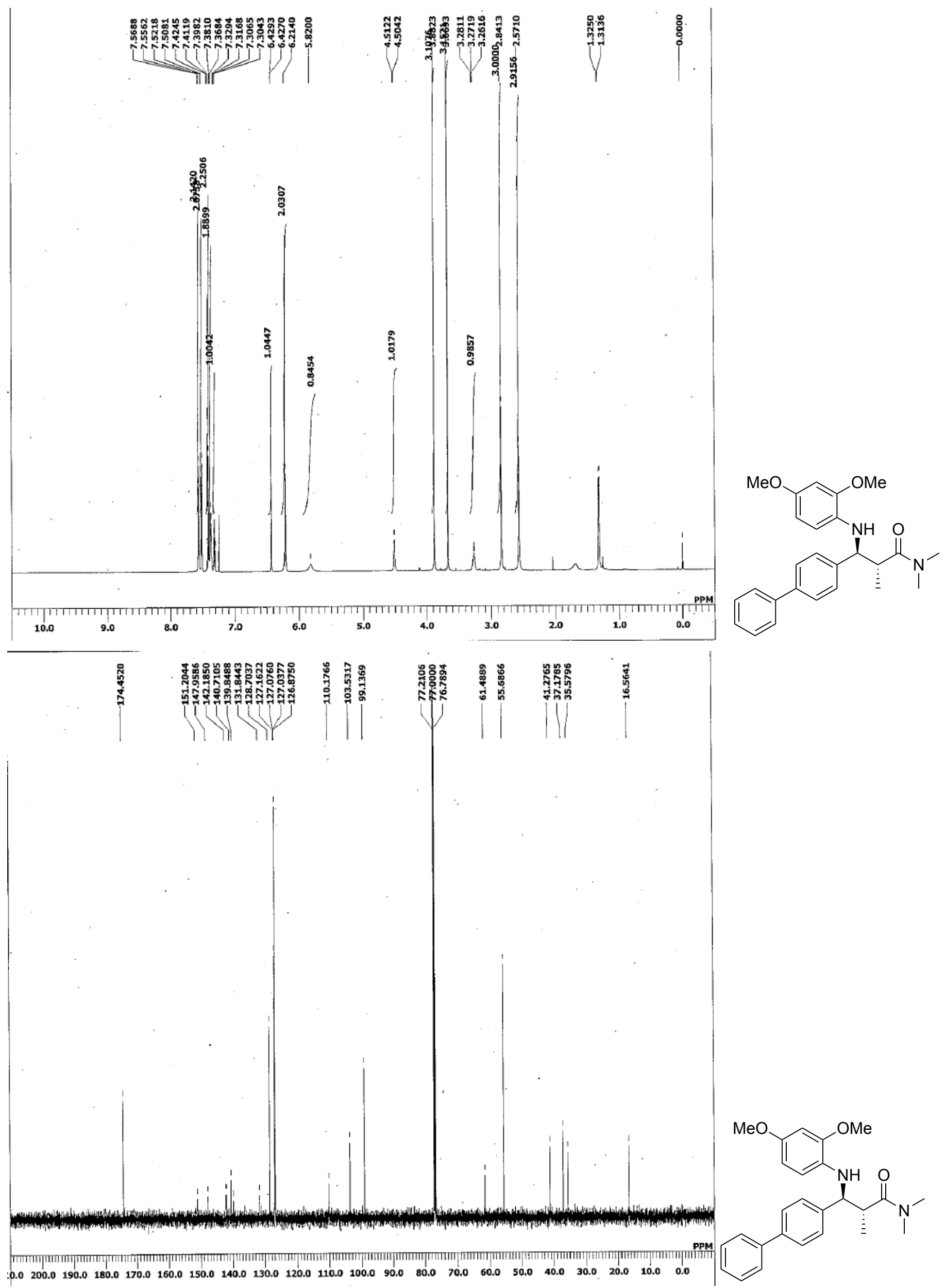




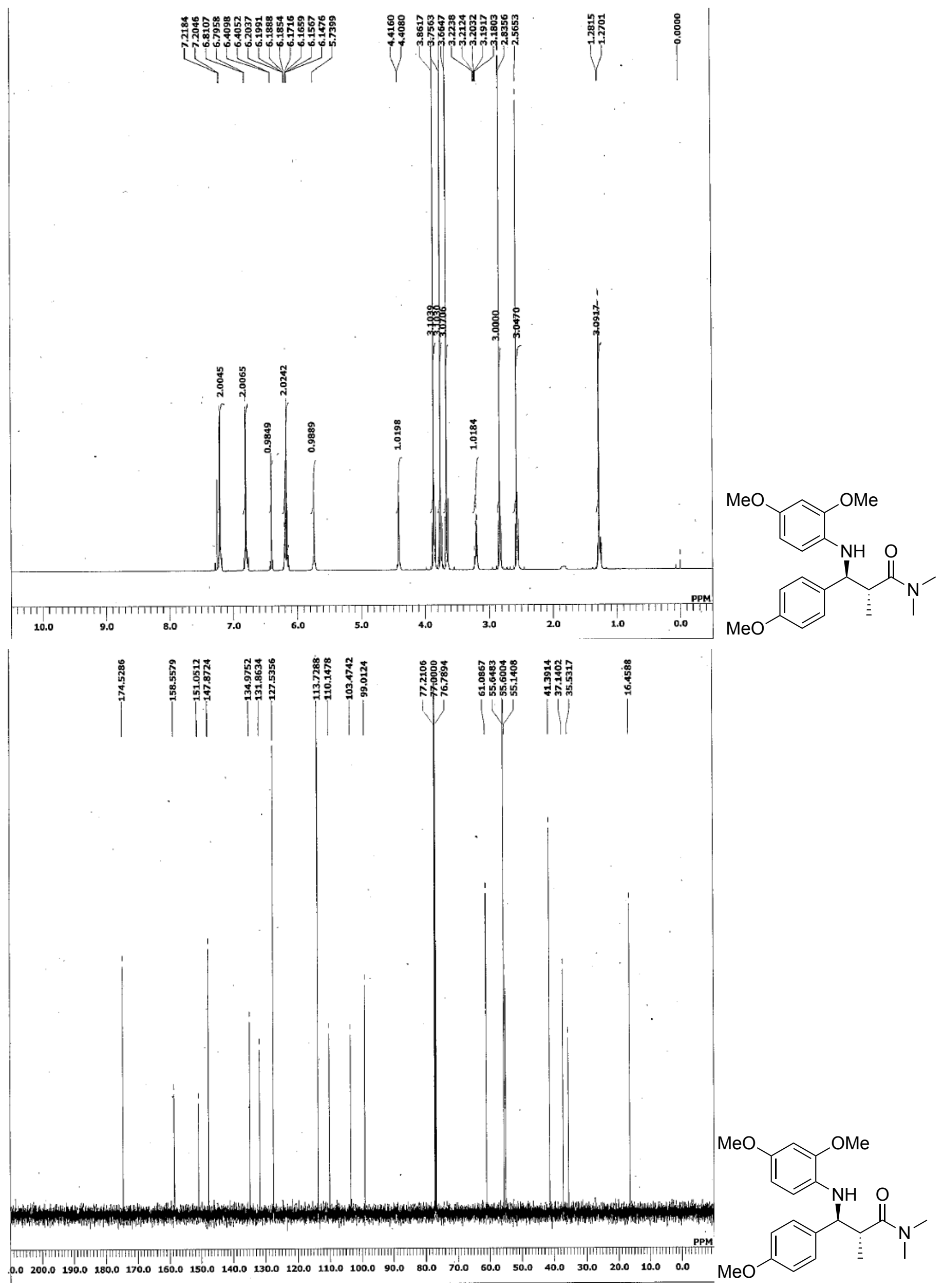




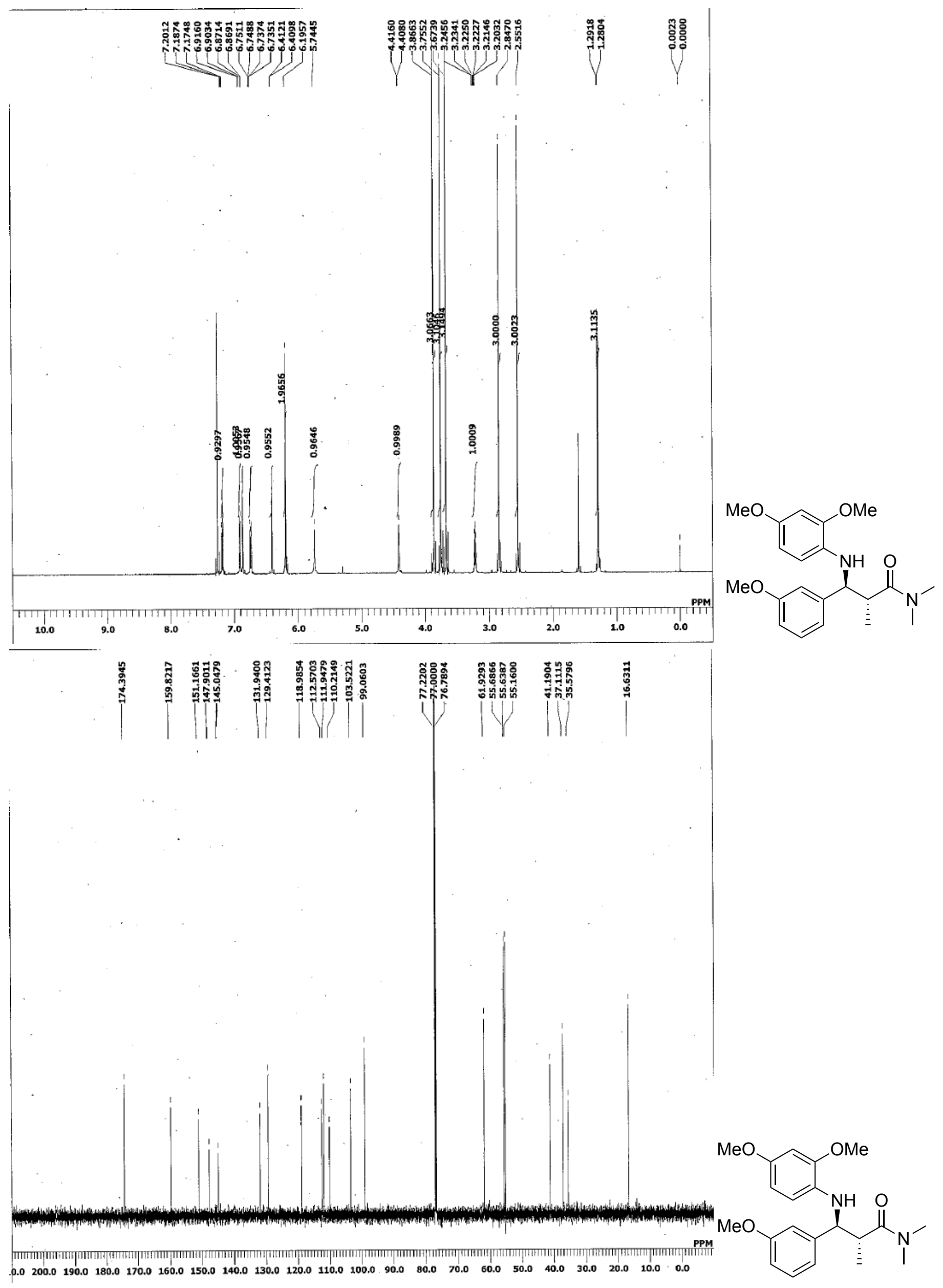




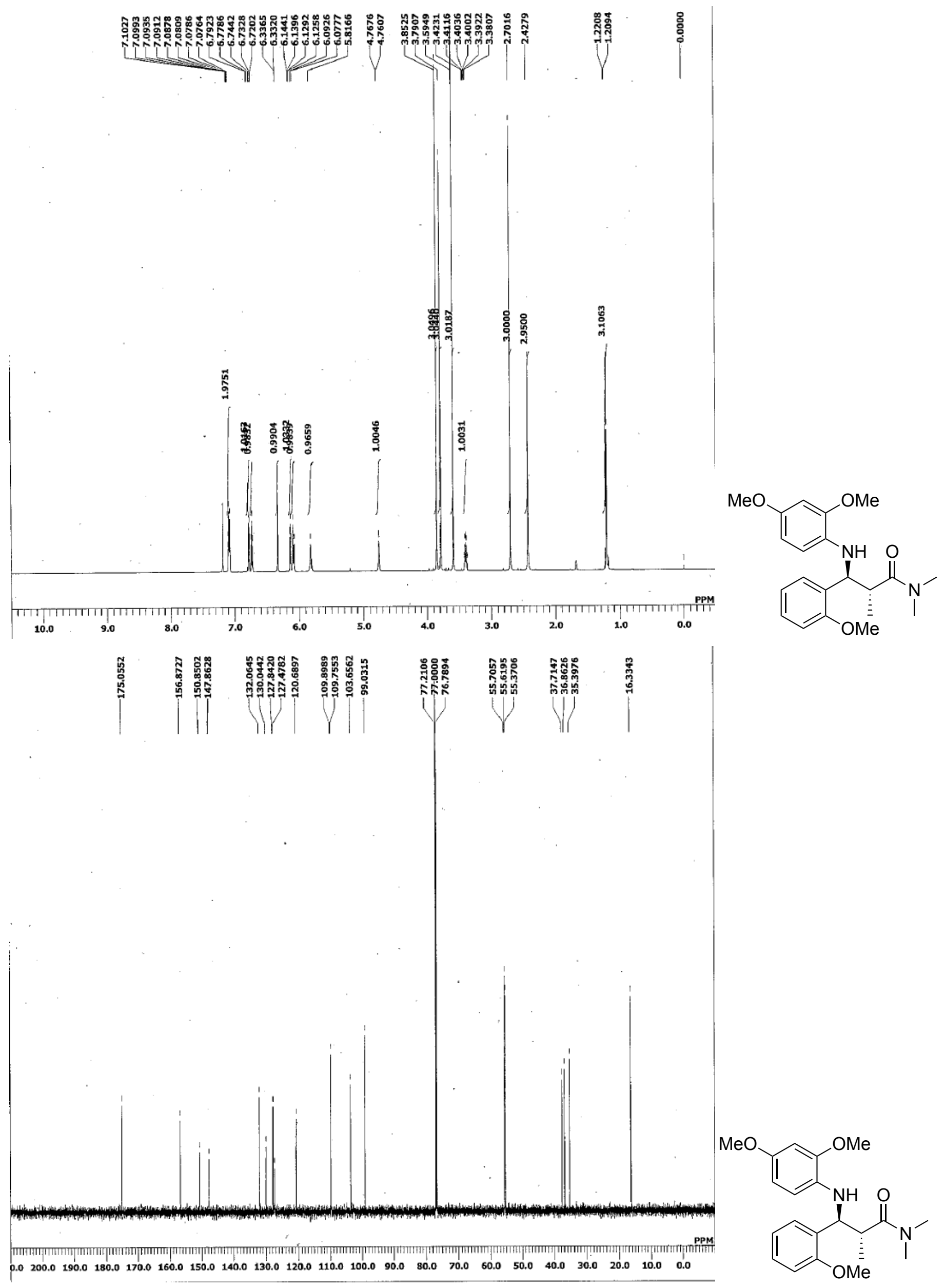




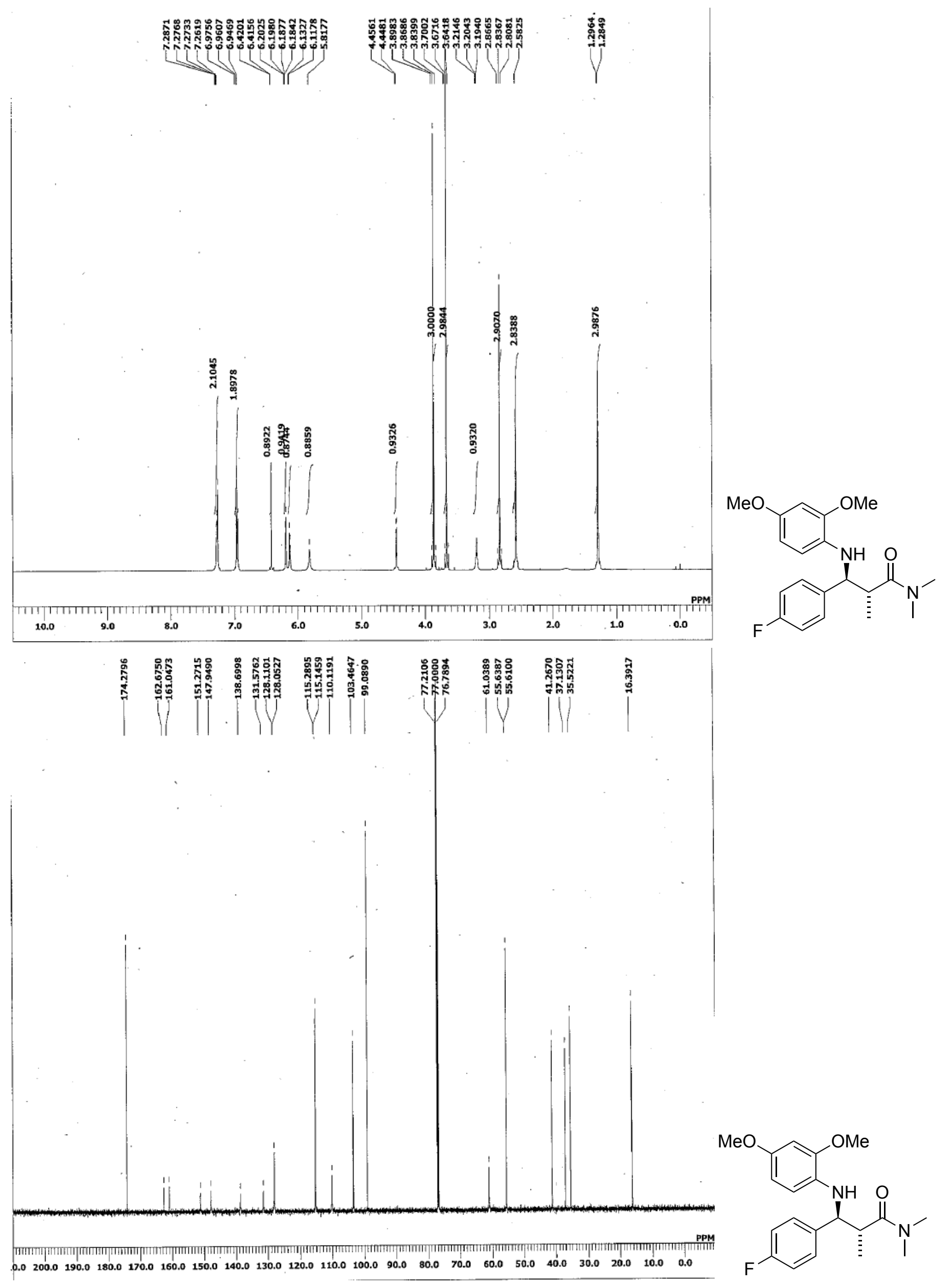




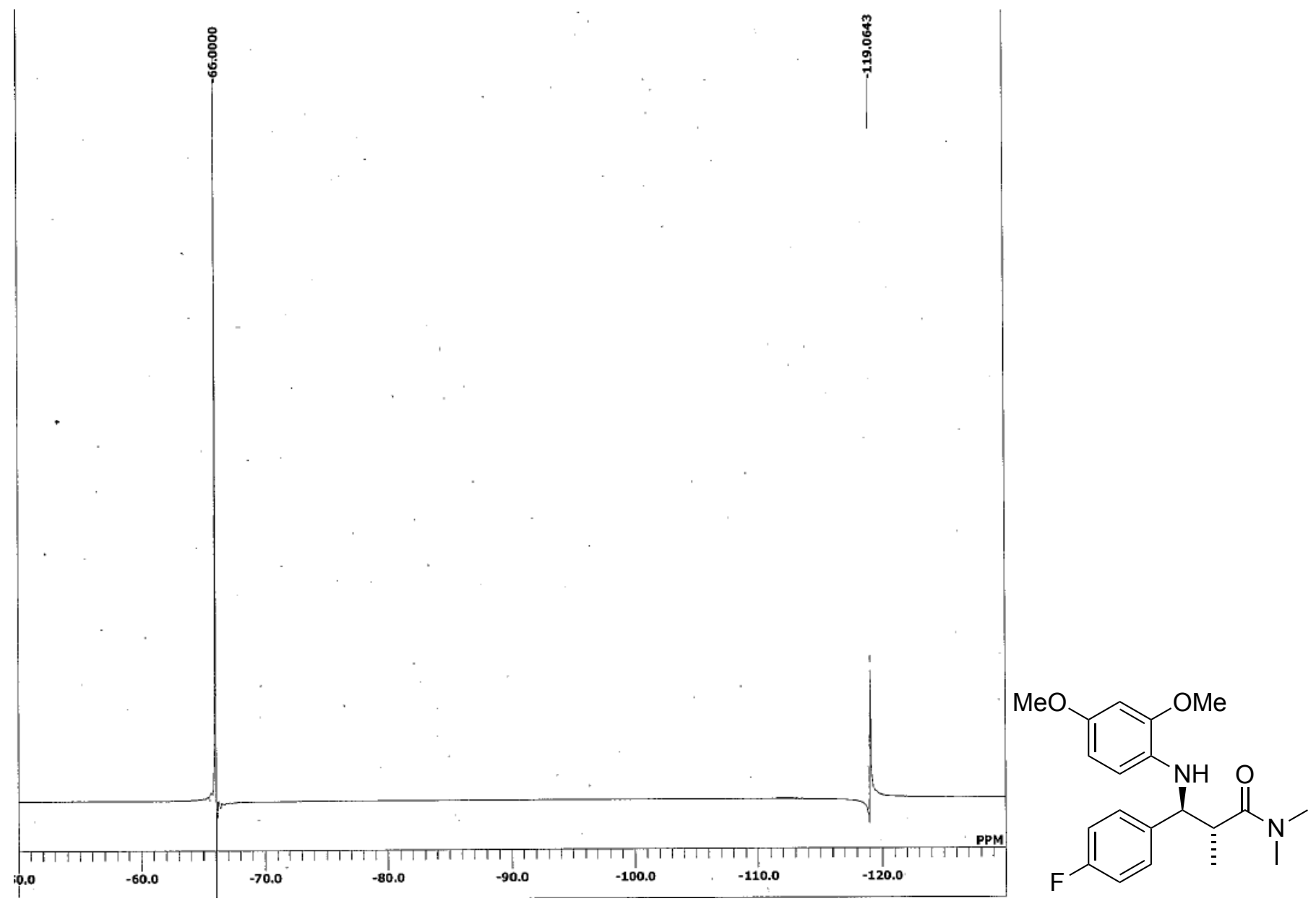



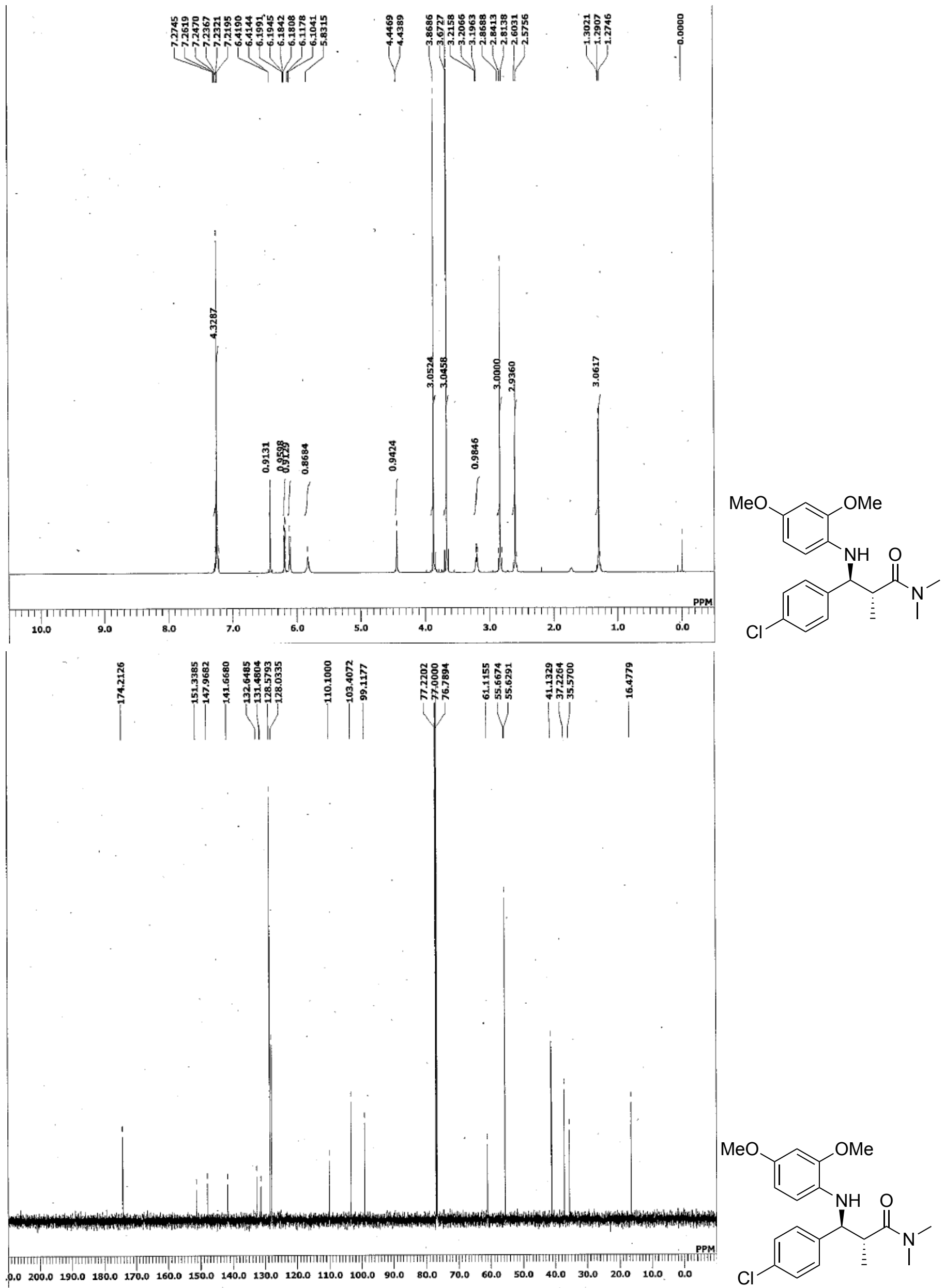


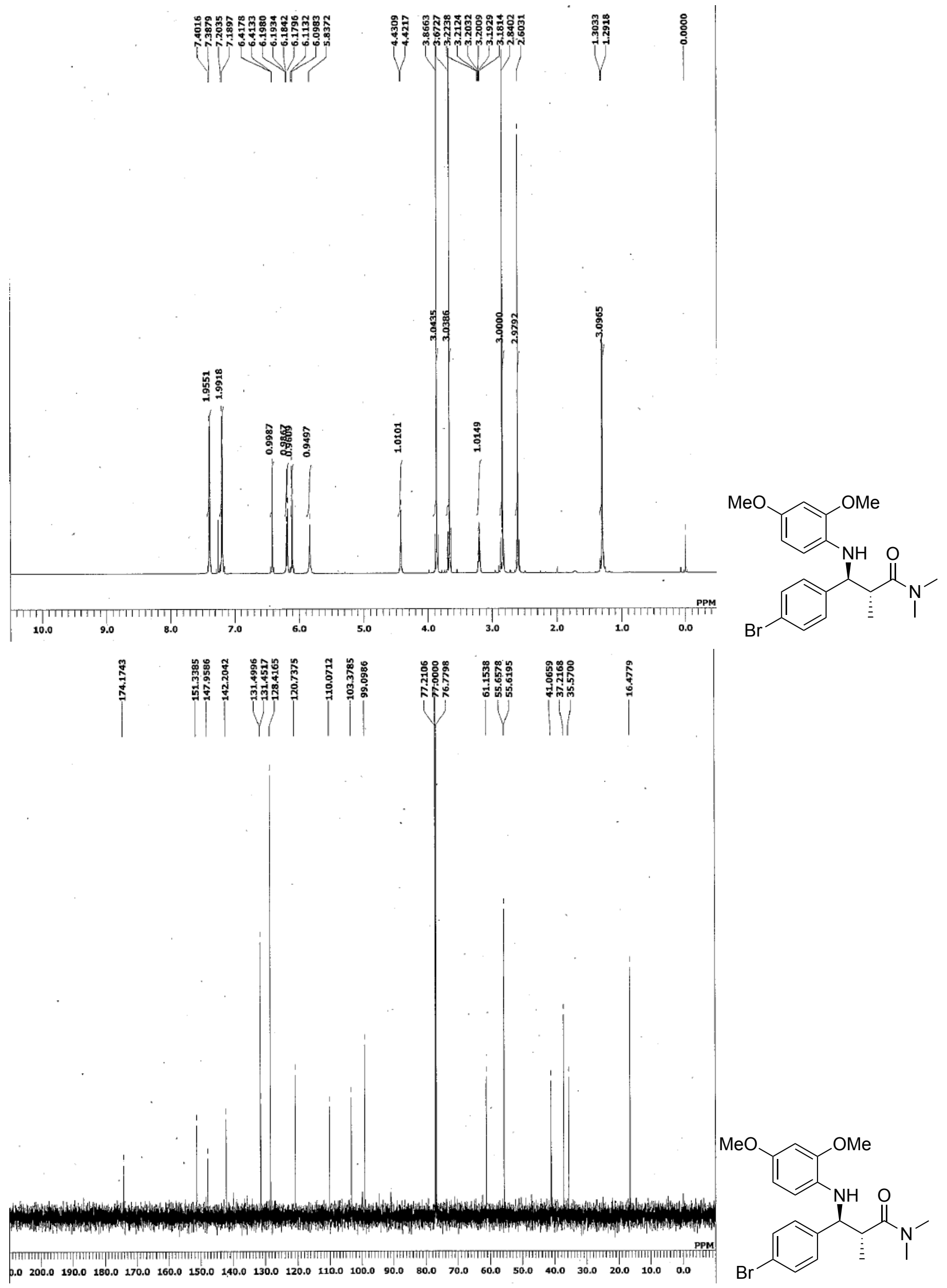



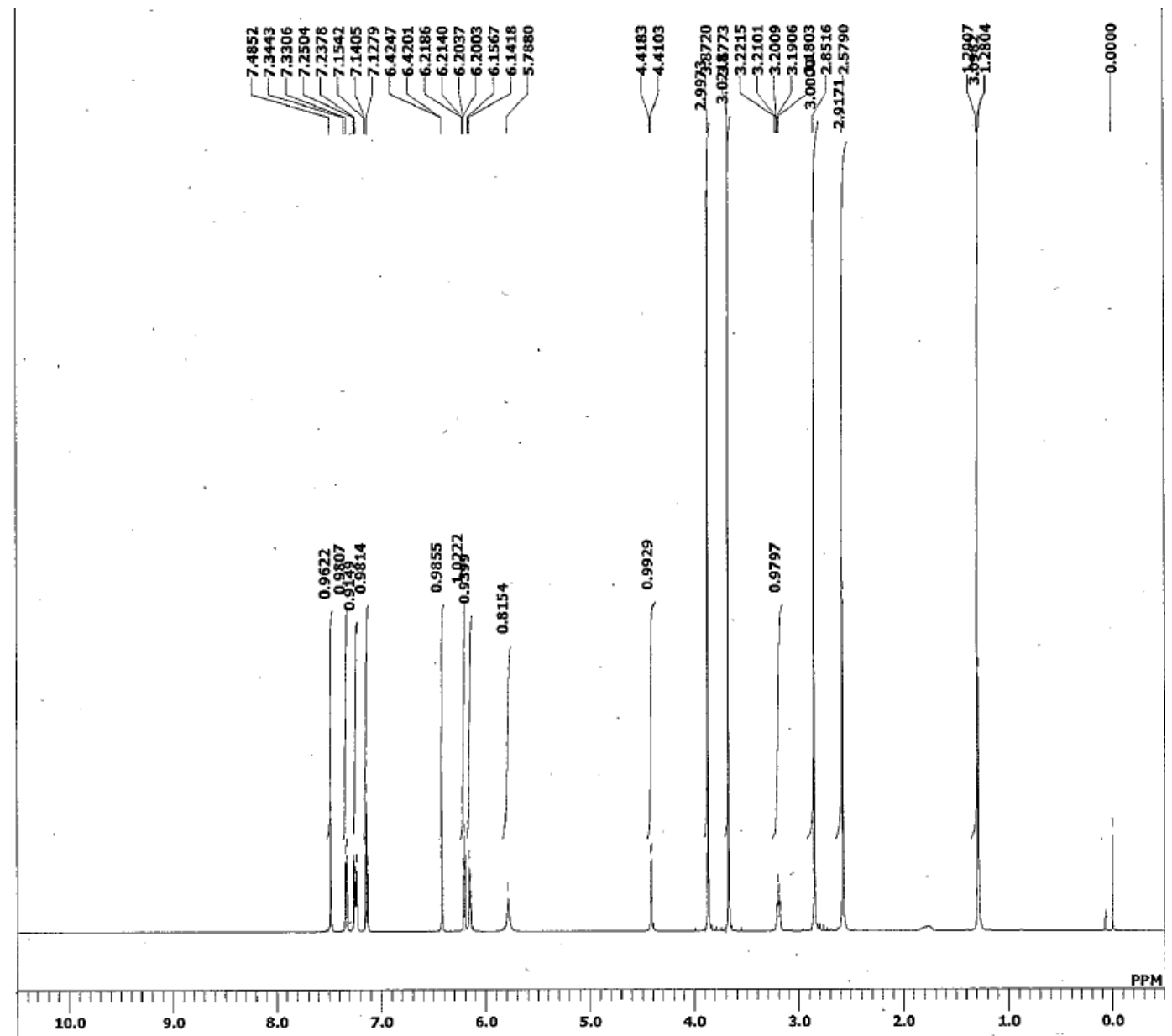<smiles>COc1ccc(N[C@@H](c2cccc(Br)c2)[C@H](C)C(=O)N(C)C)c(OC)c1</smiles>
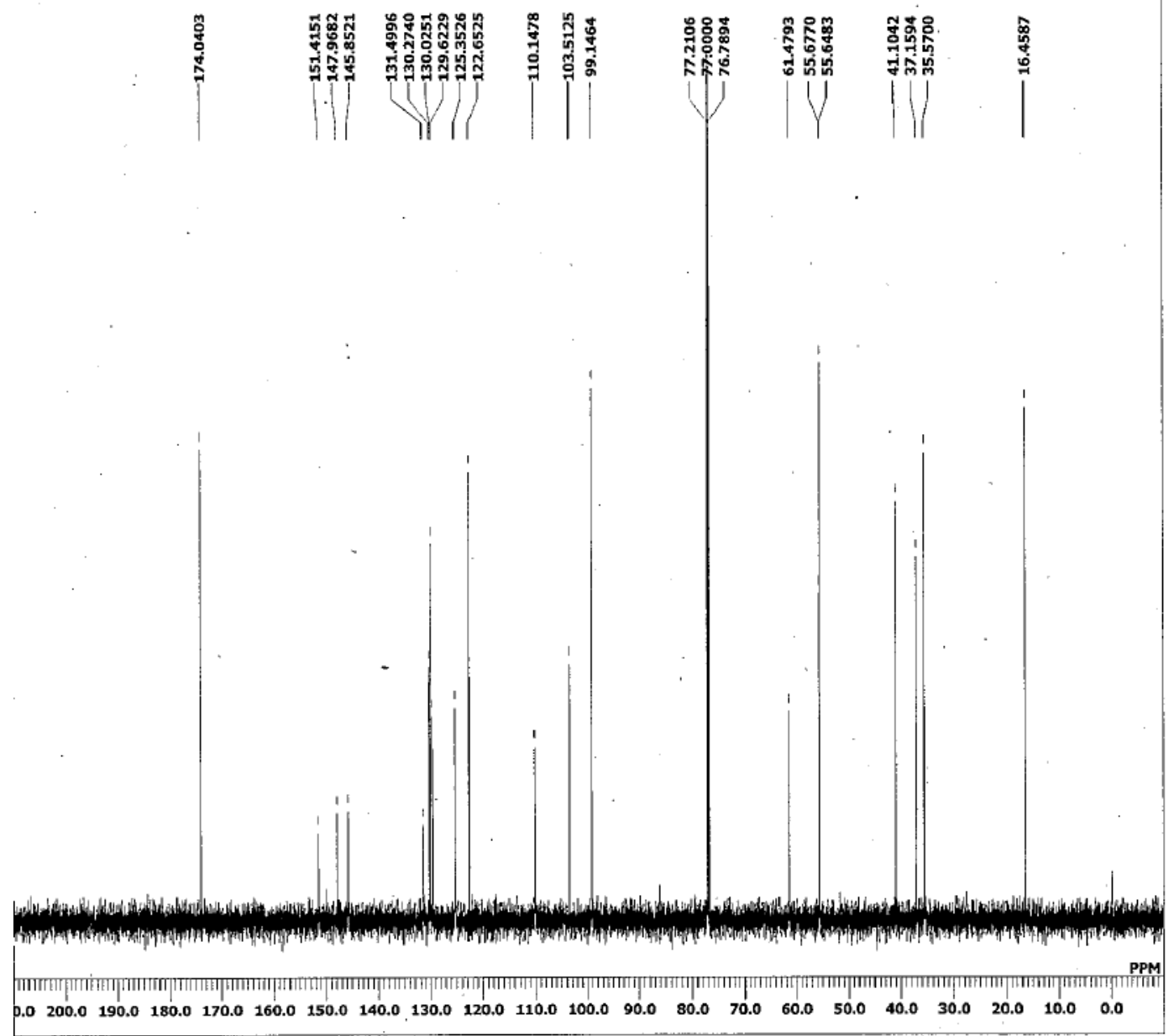<smiles>COc1ccc(N[C@H](c2cccc(Br)c2)[C@H](C)C(=O)N(C)C)c(OC)c1</smiles> 

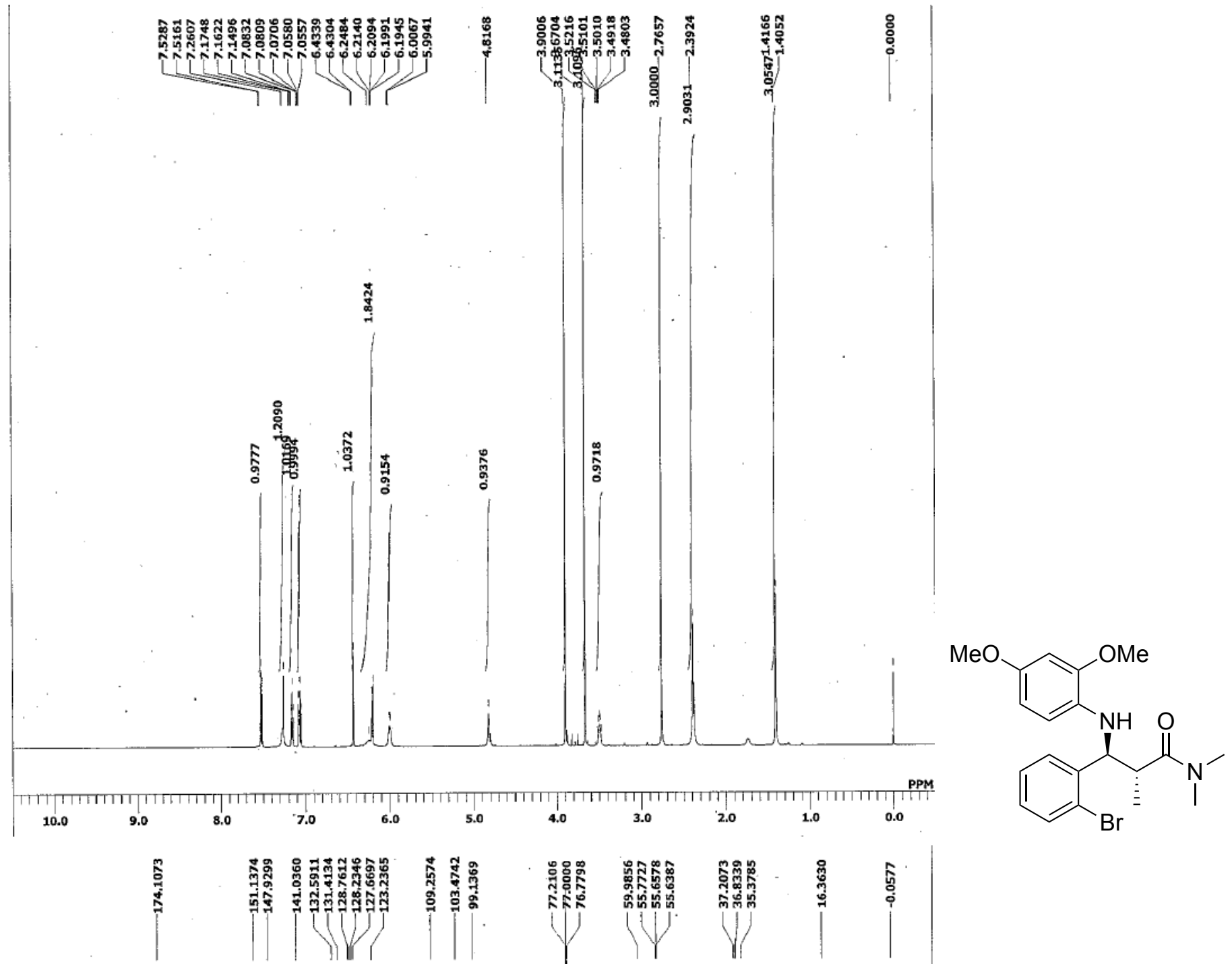

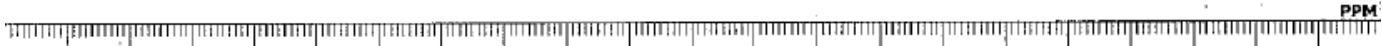

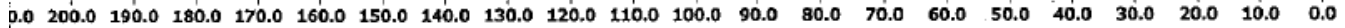

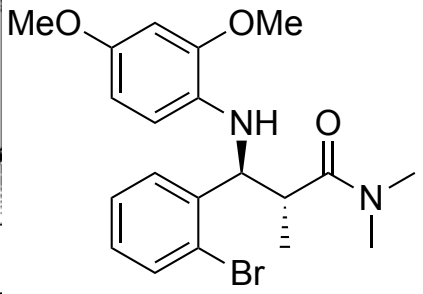



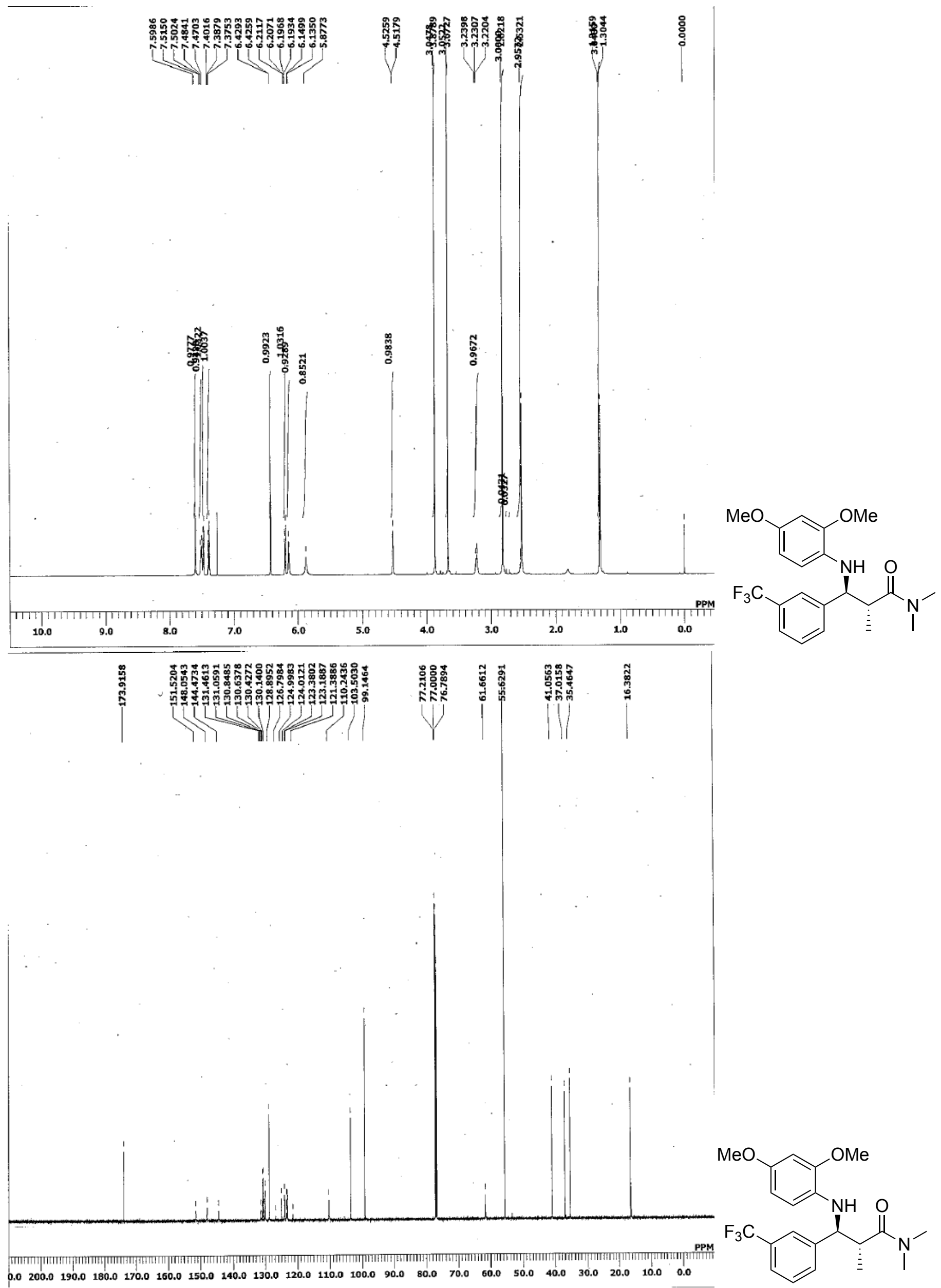


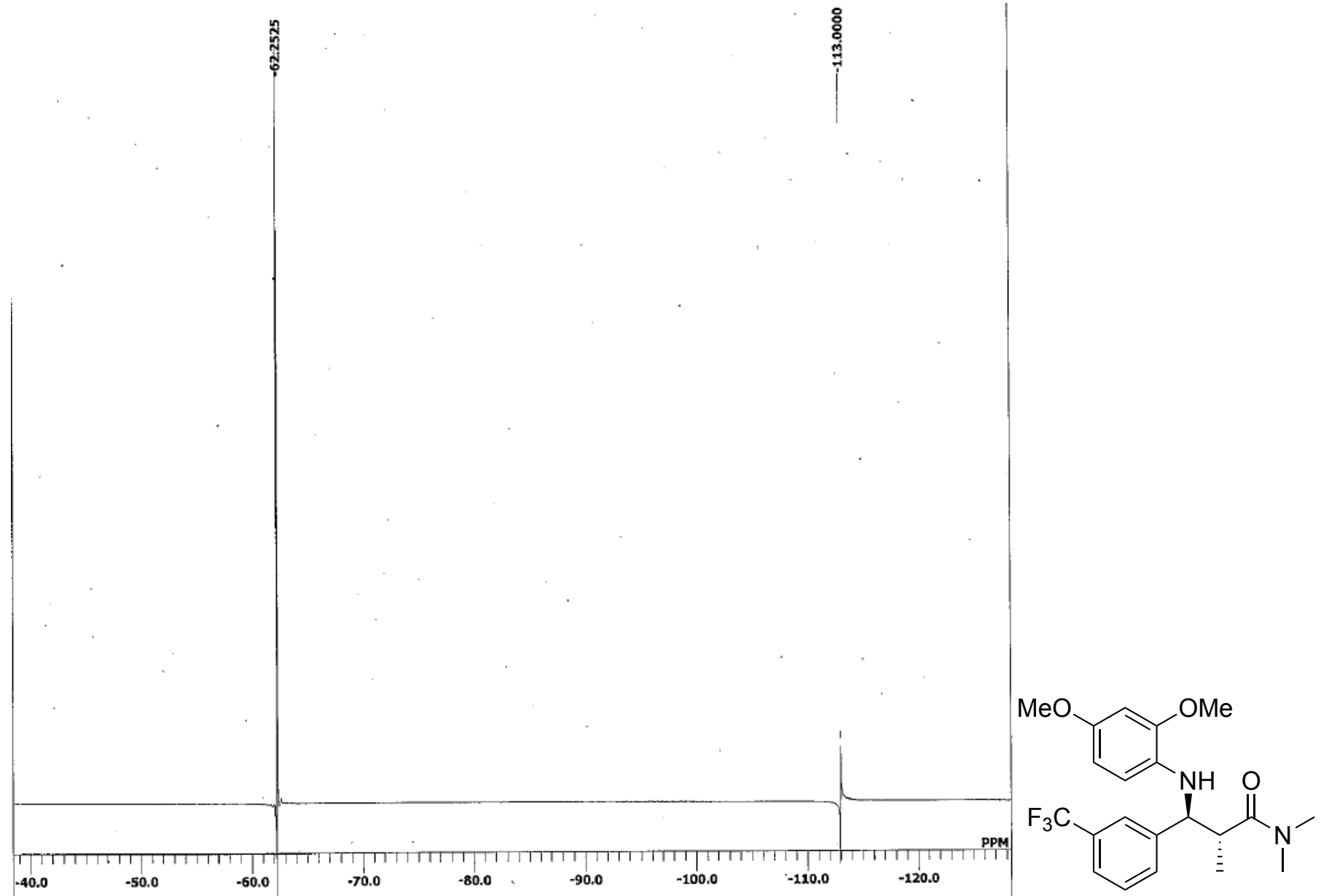




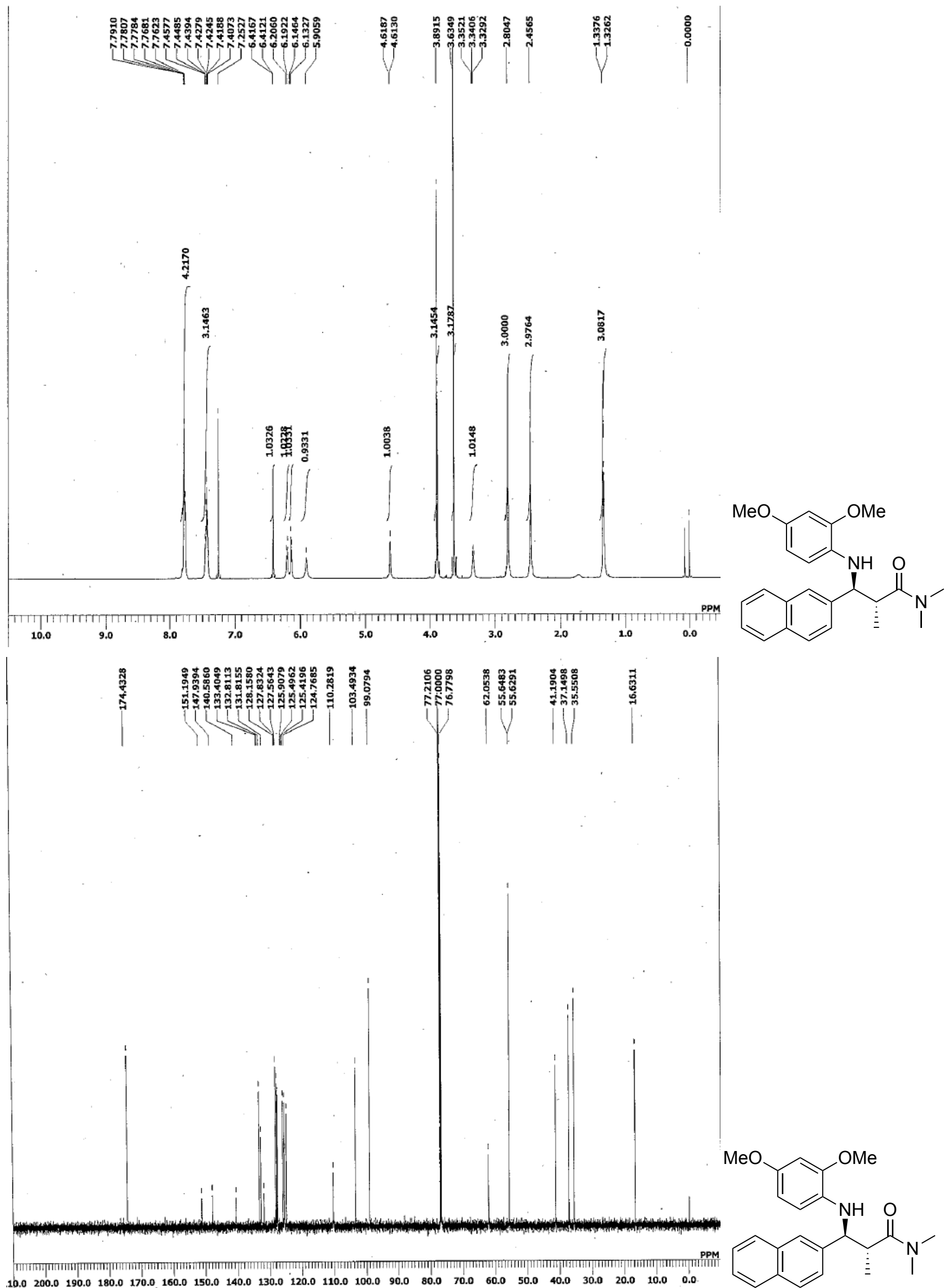



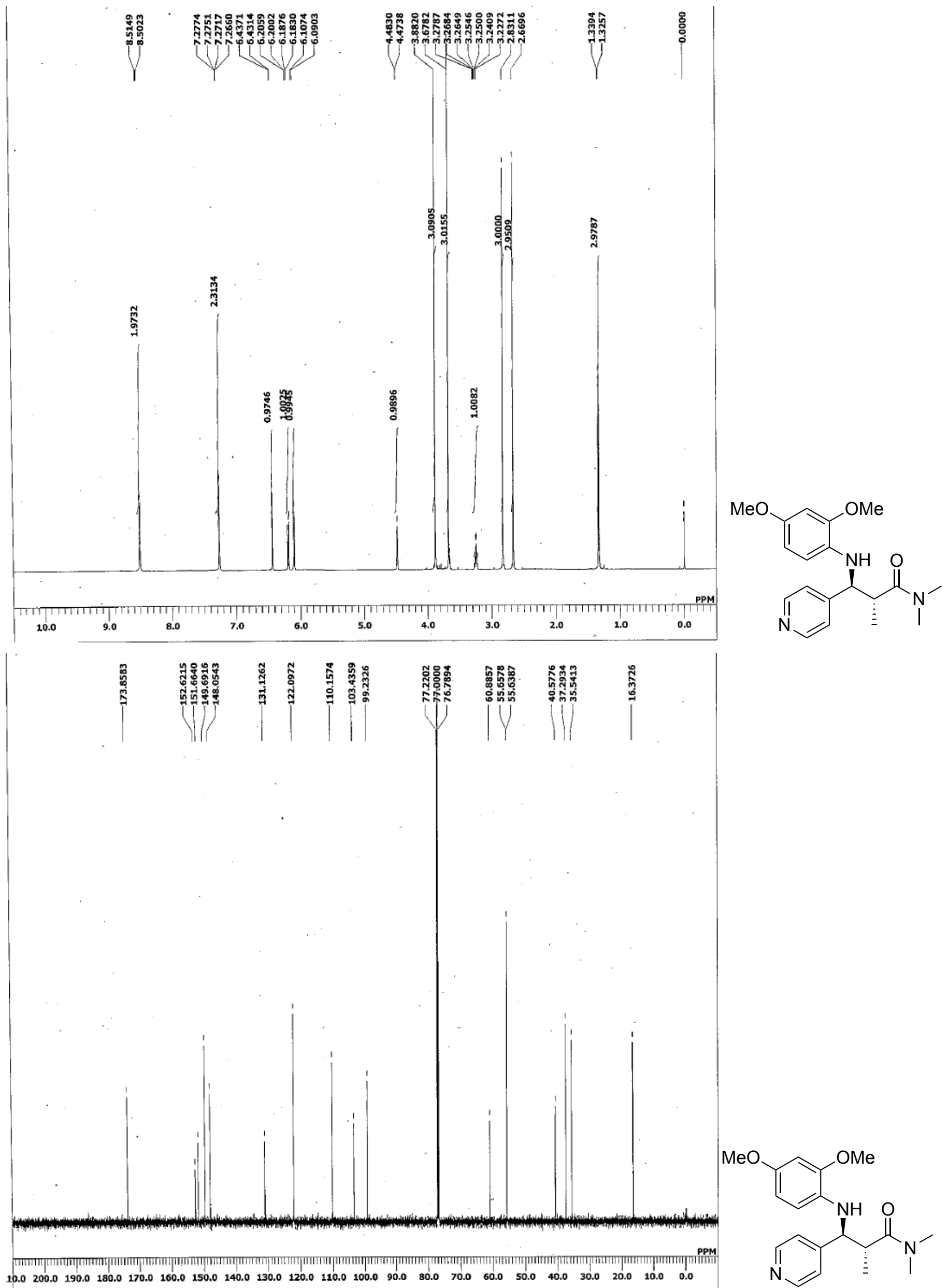


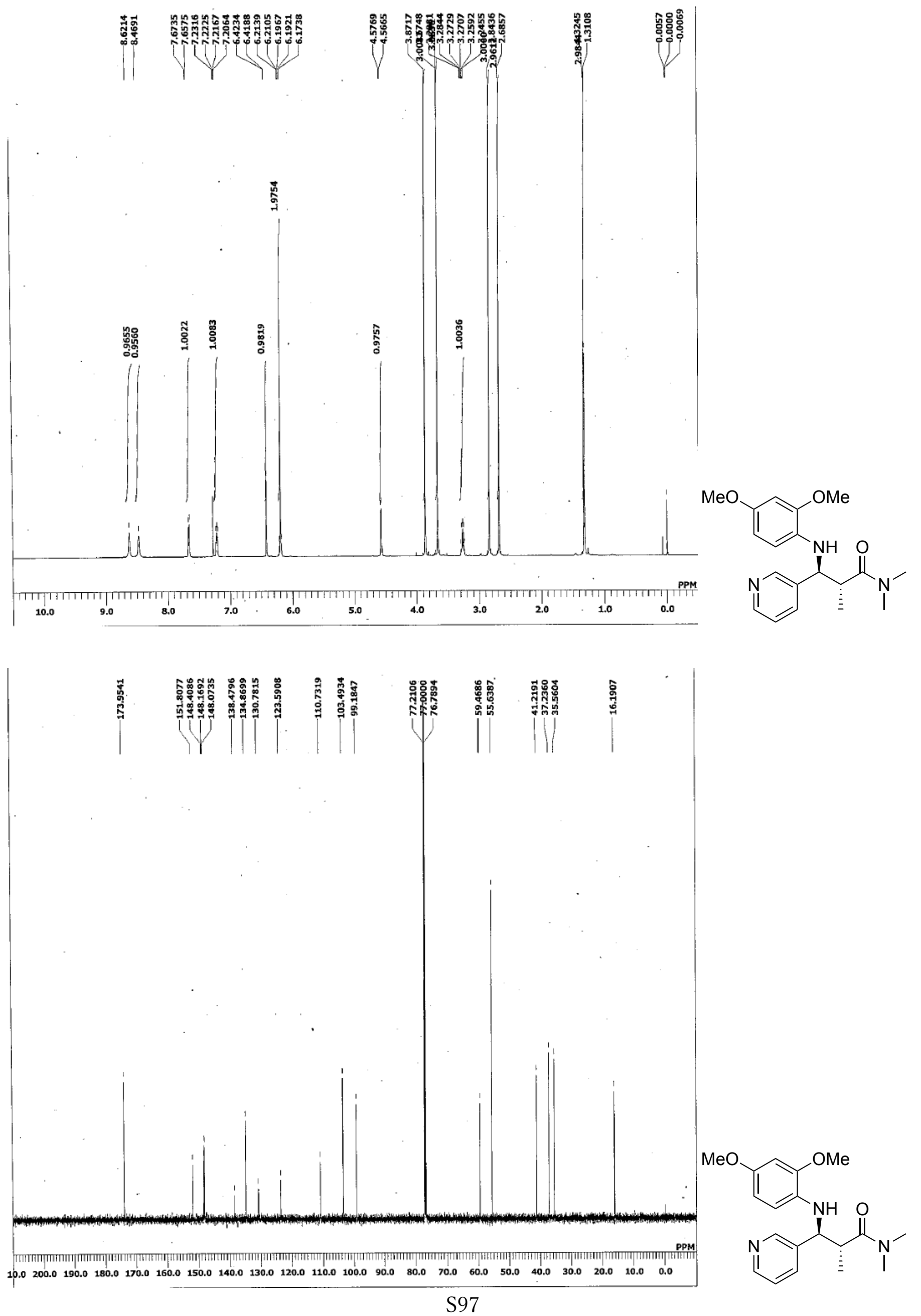




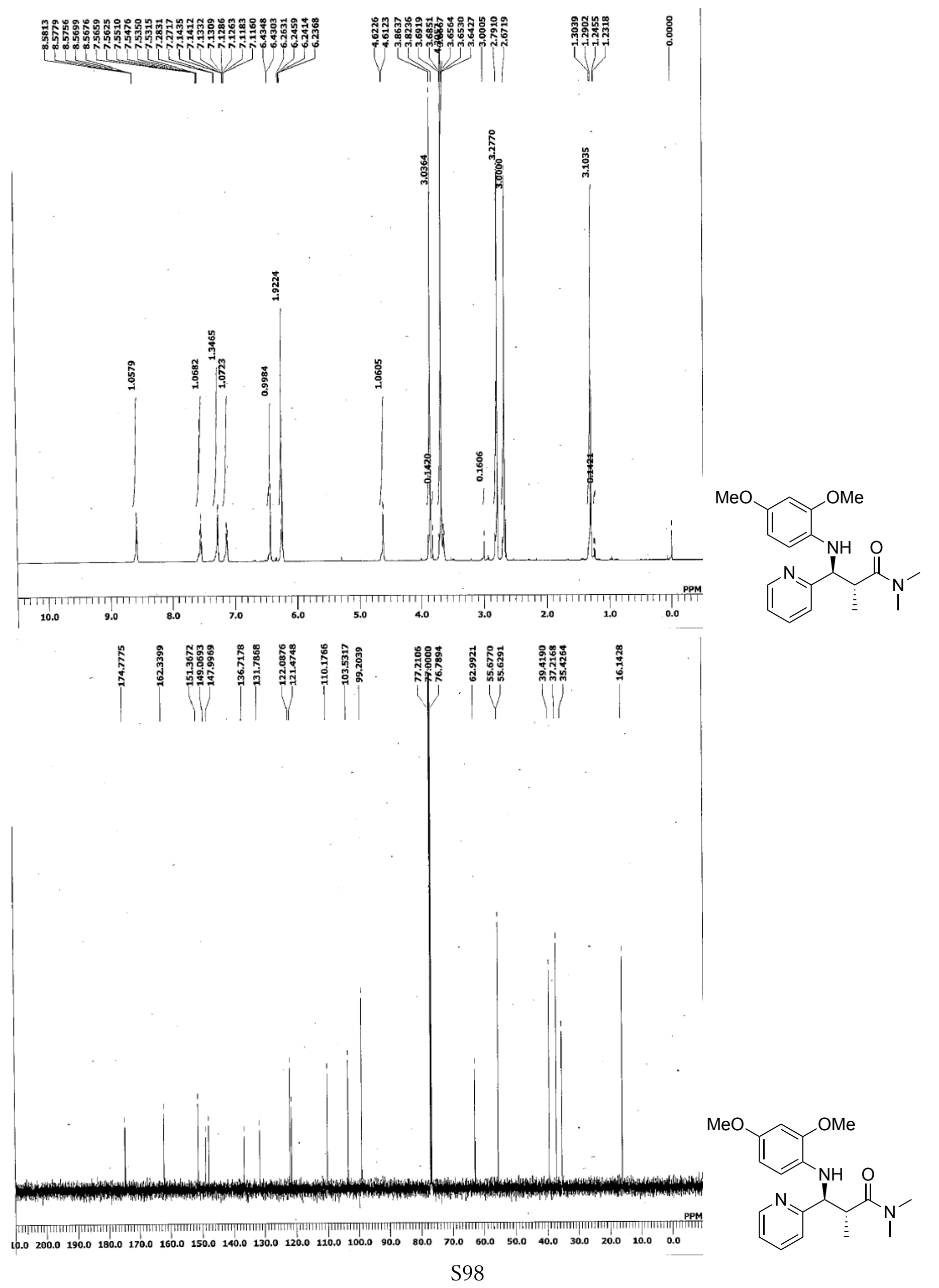




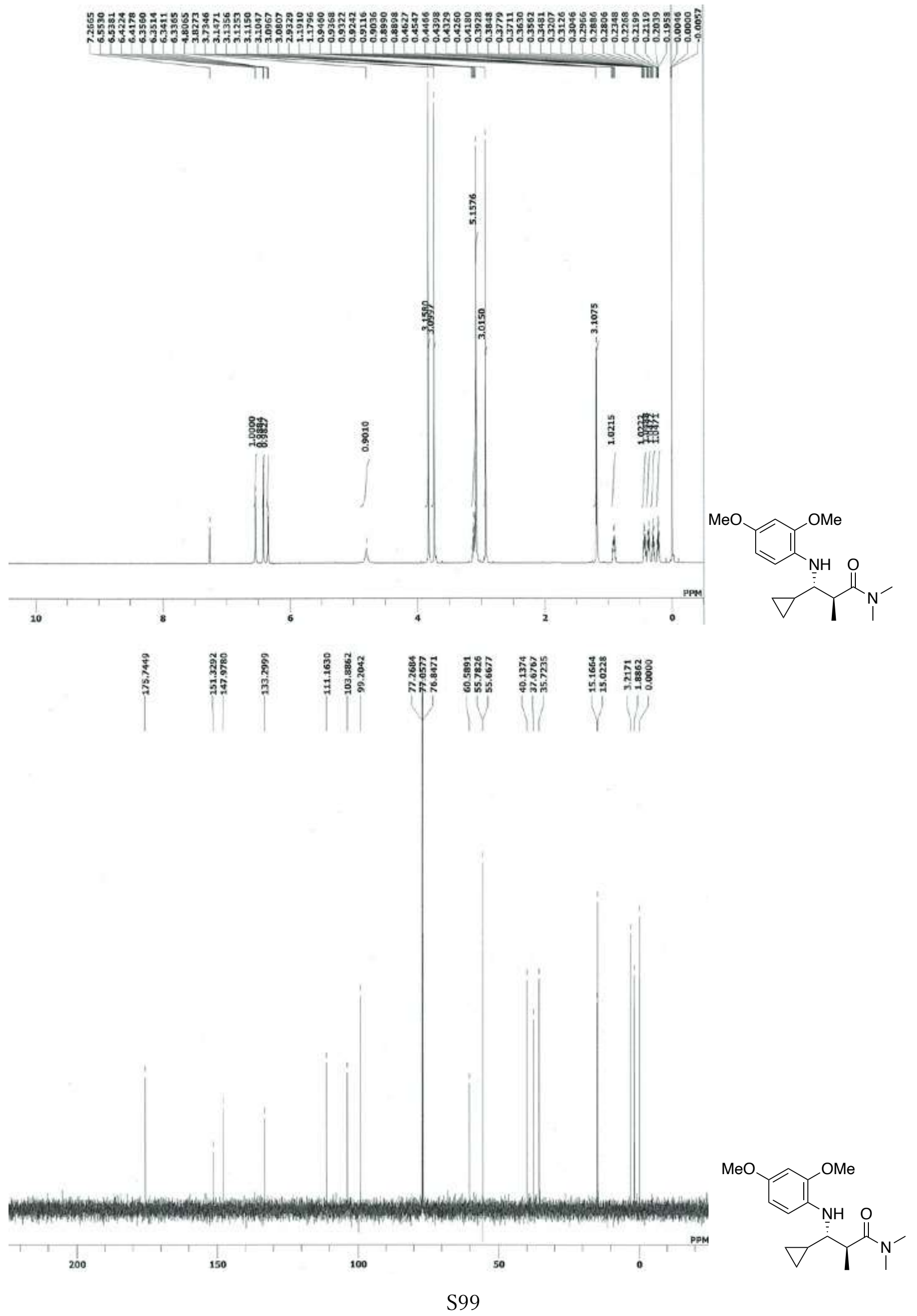




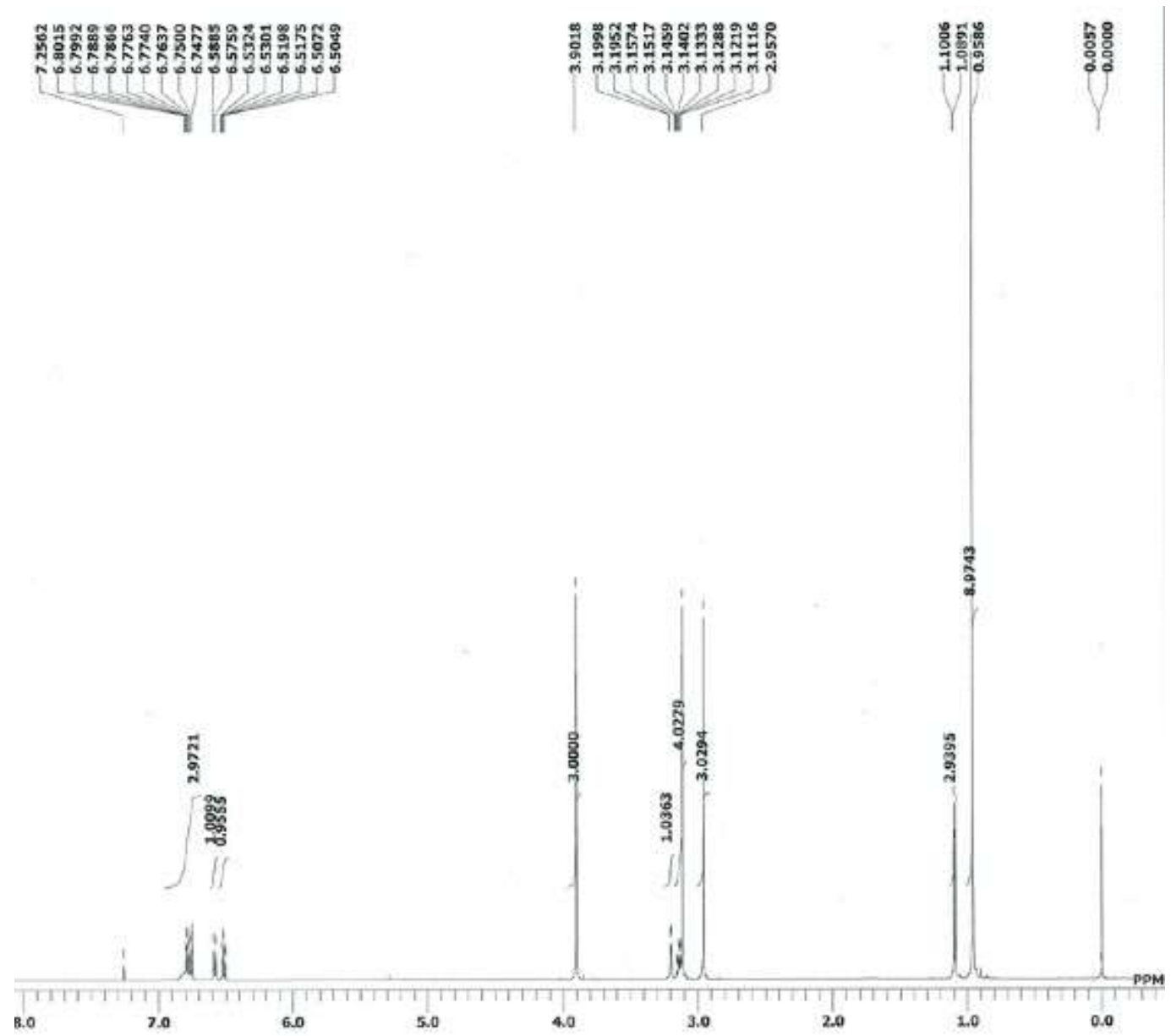

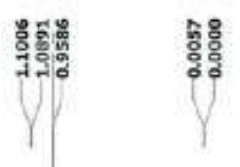

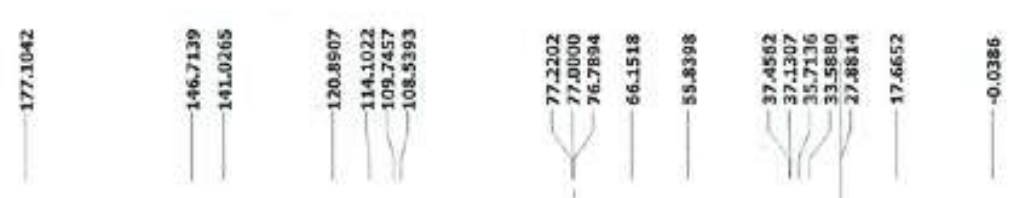

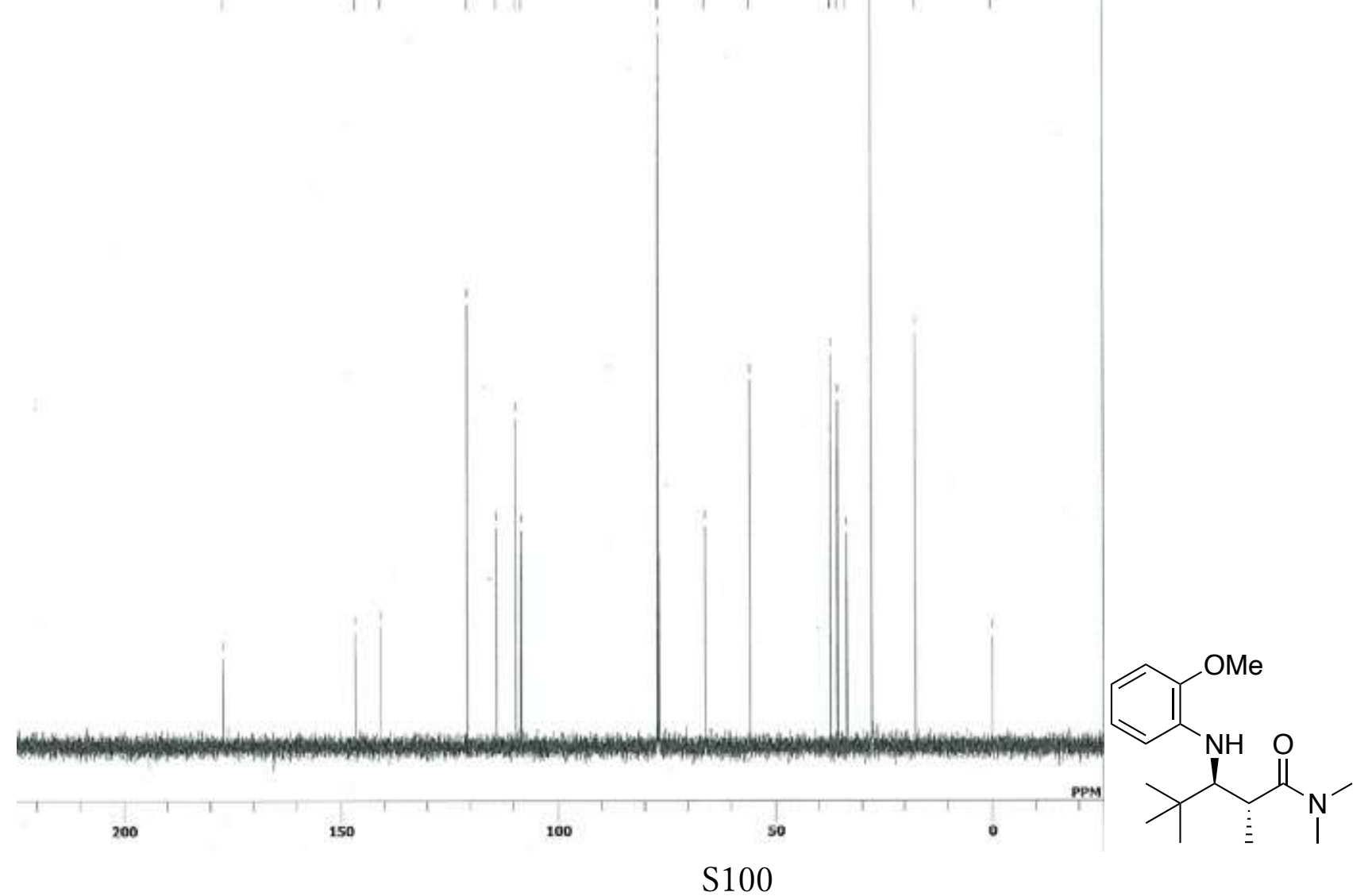




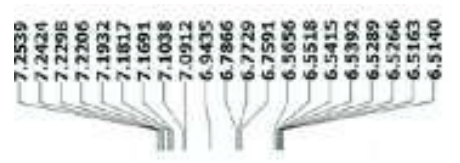

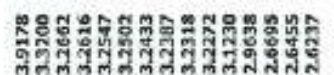

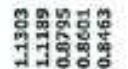

?

vi

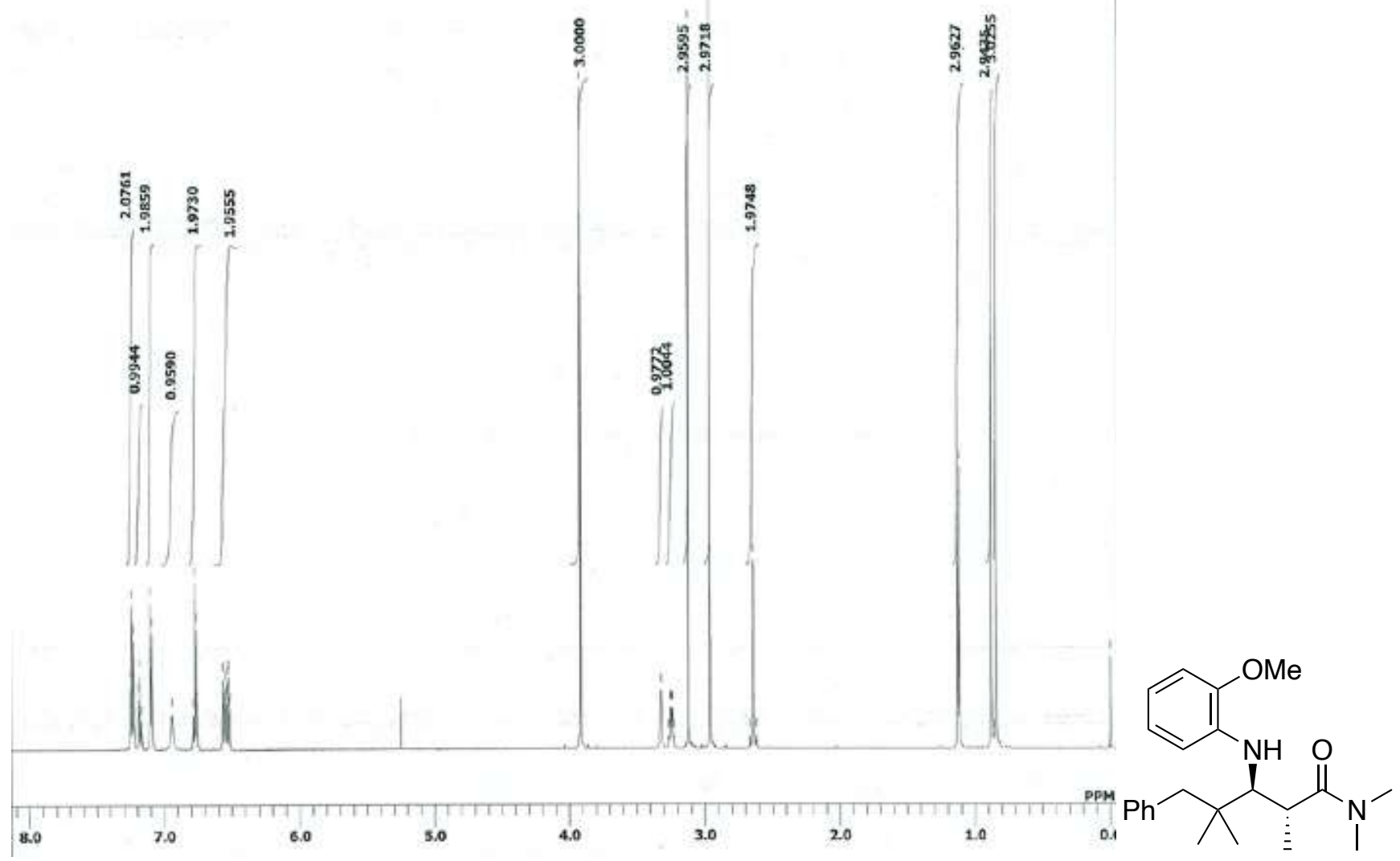

| In

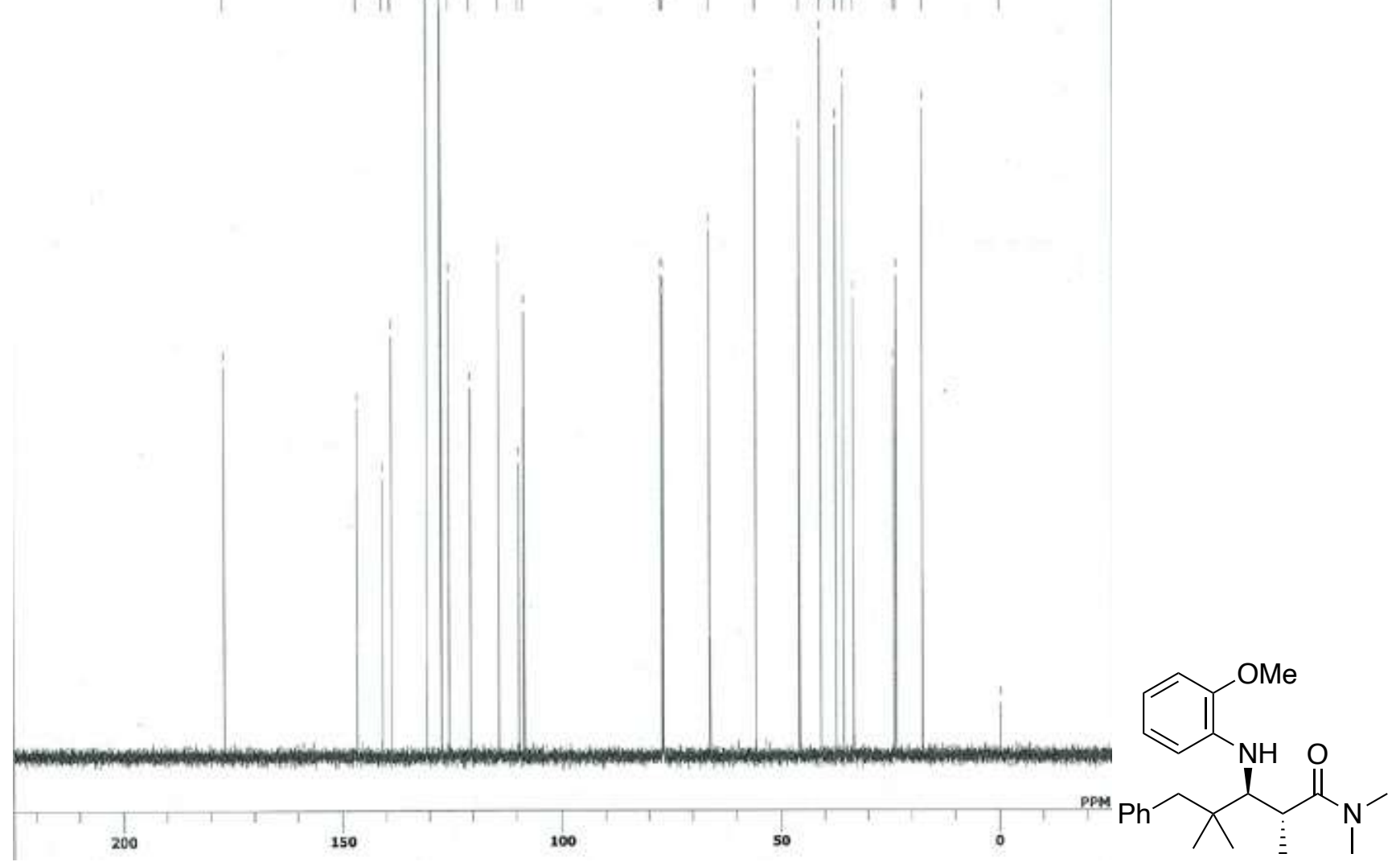




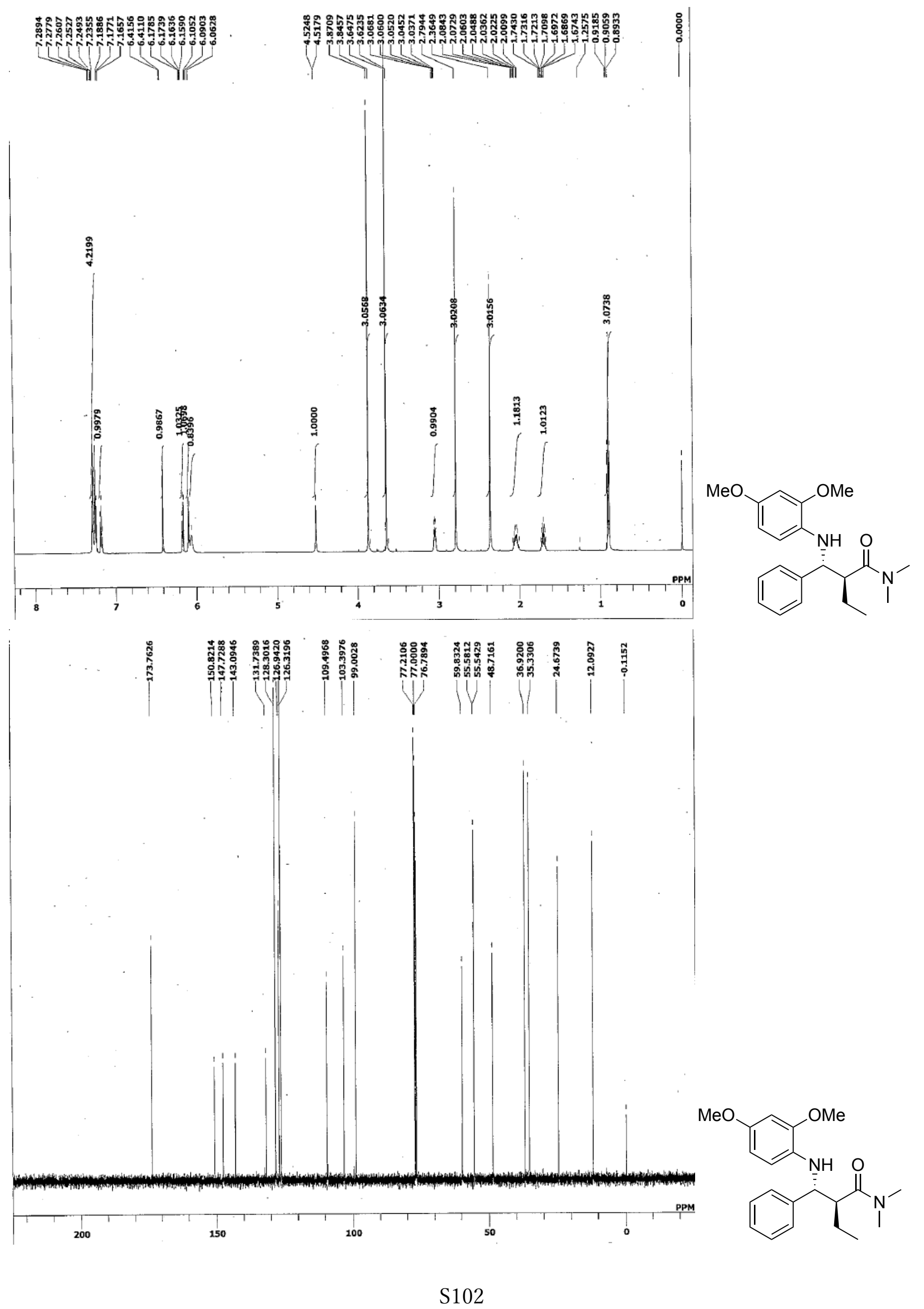




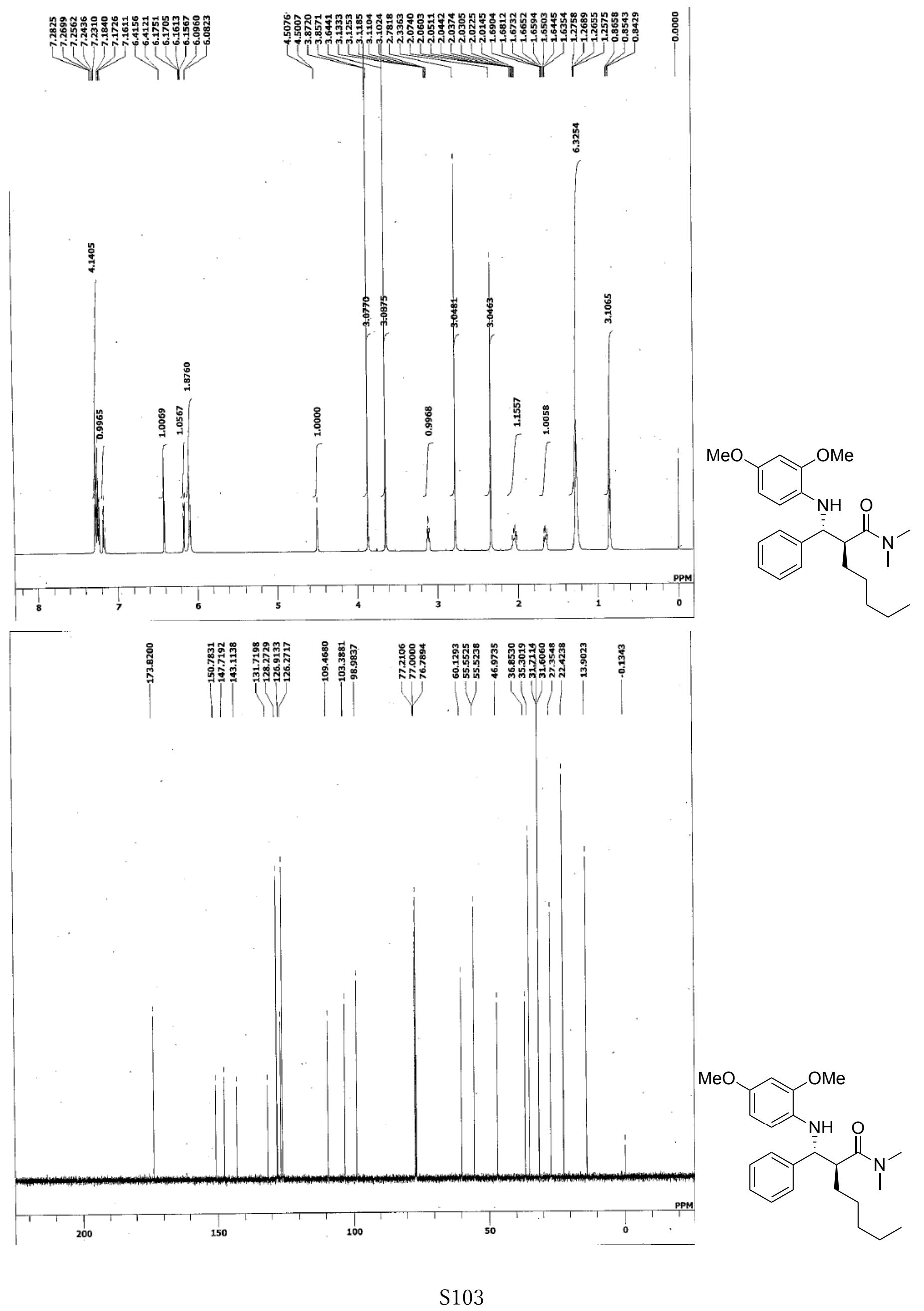




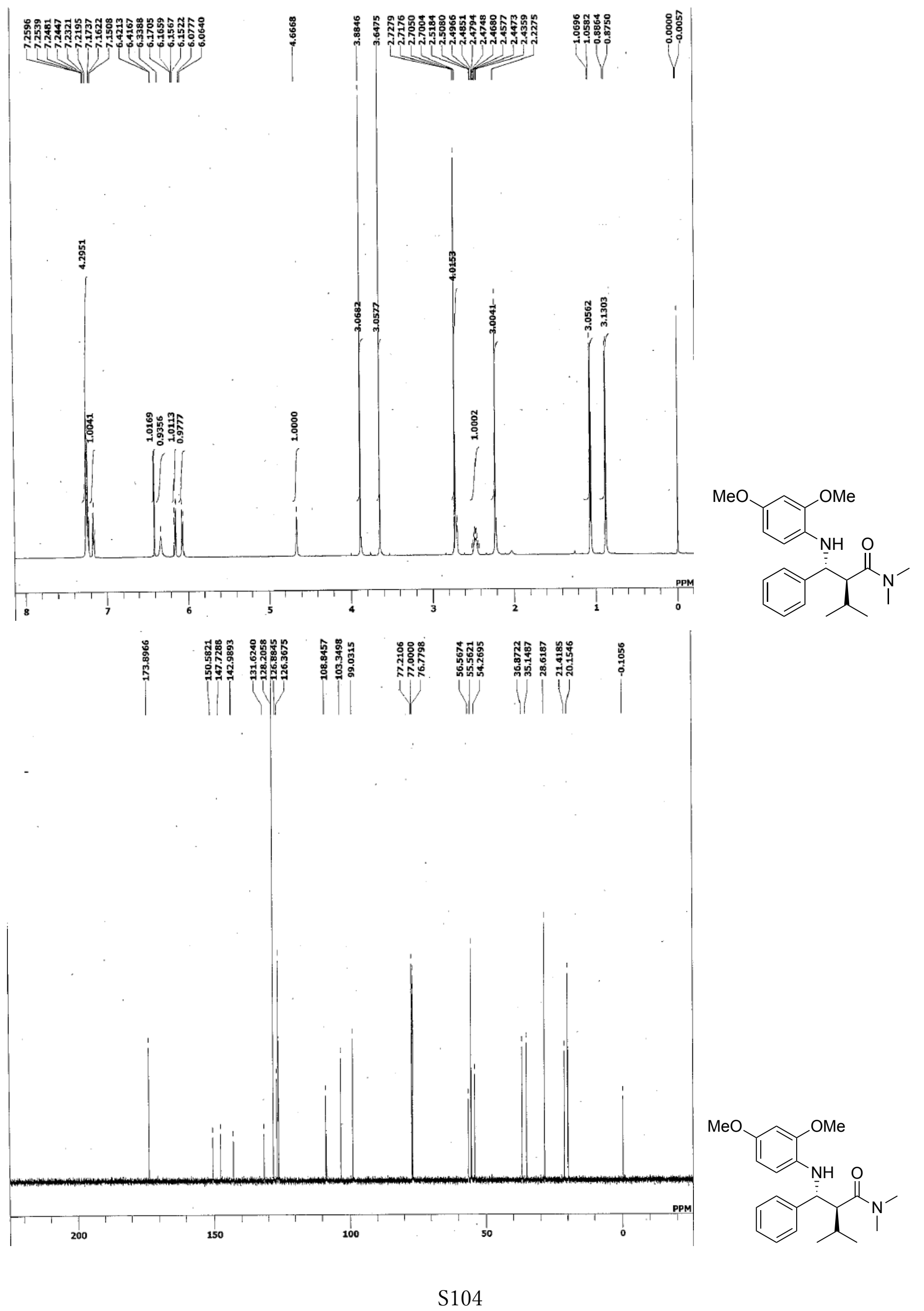



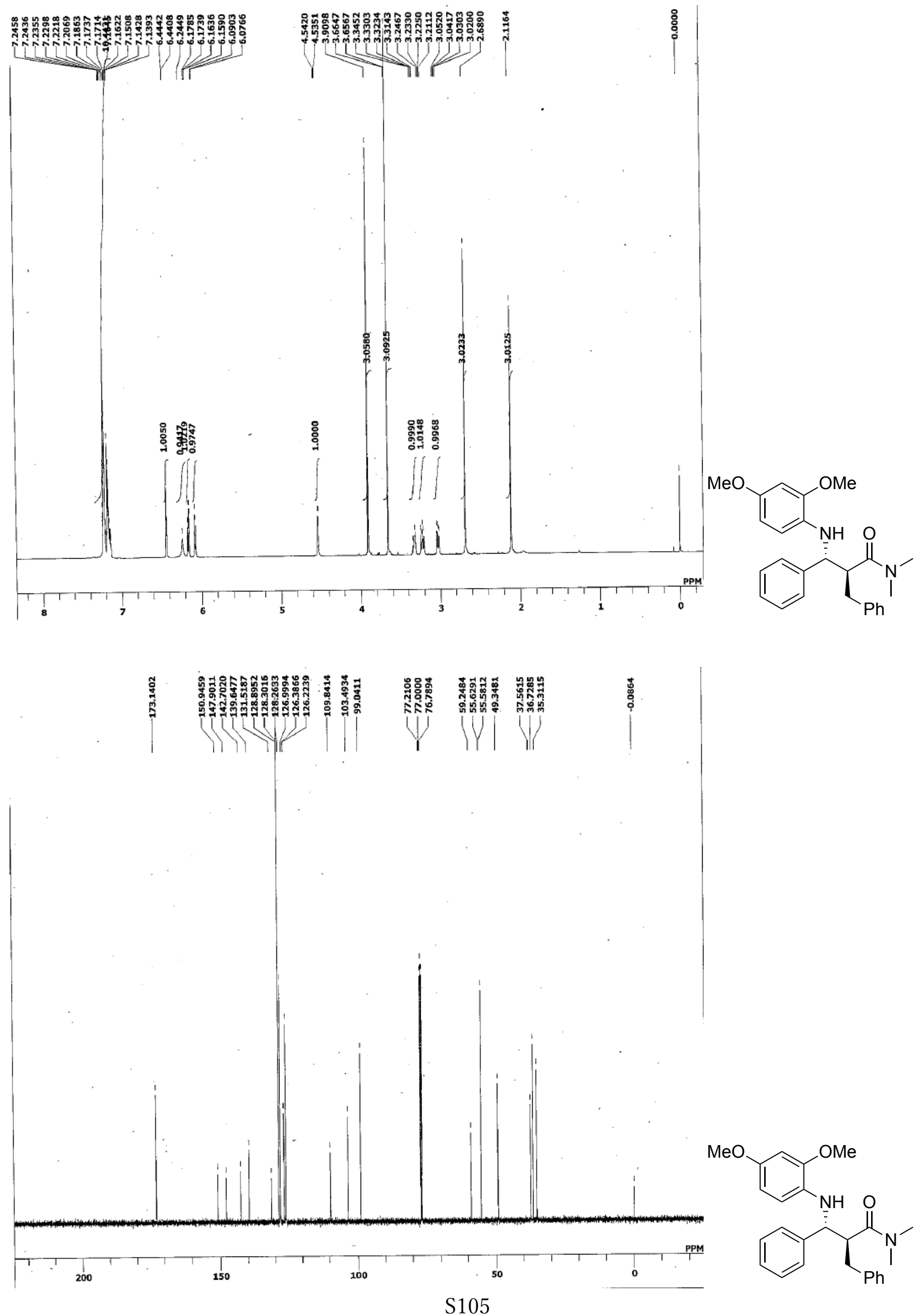


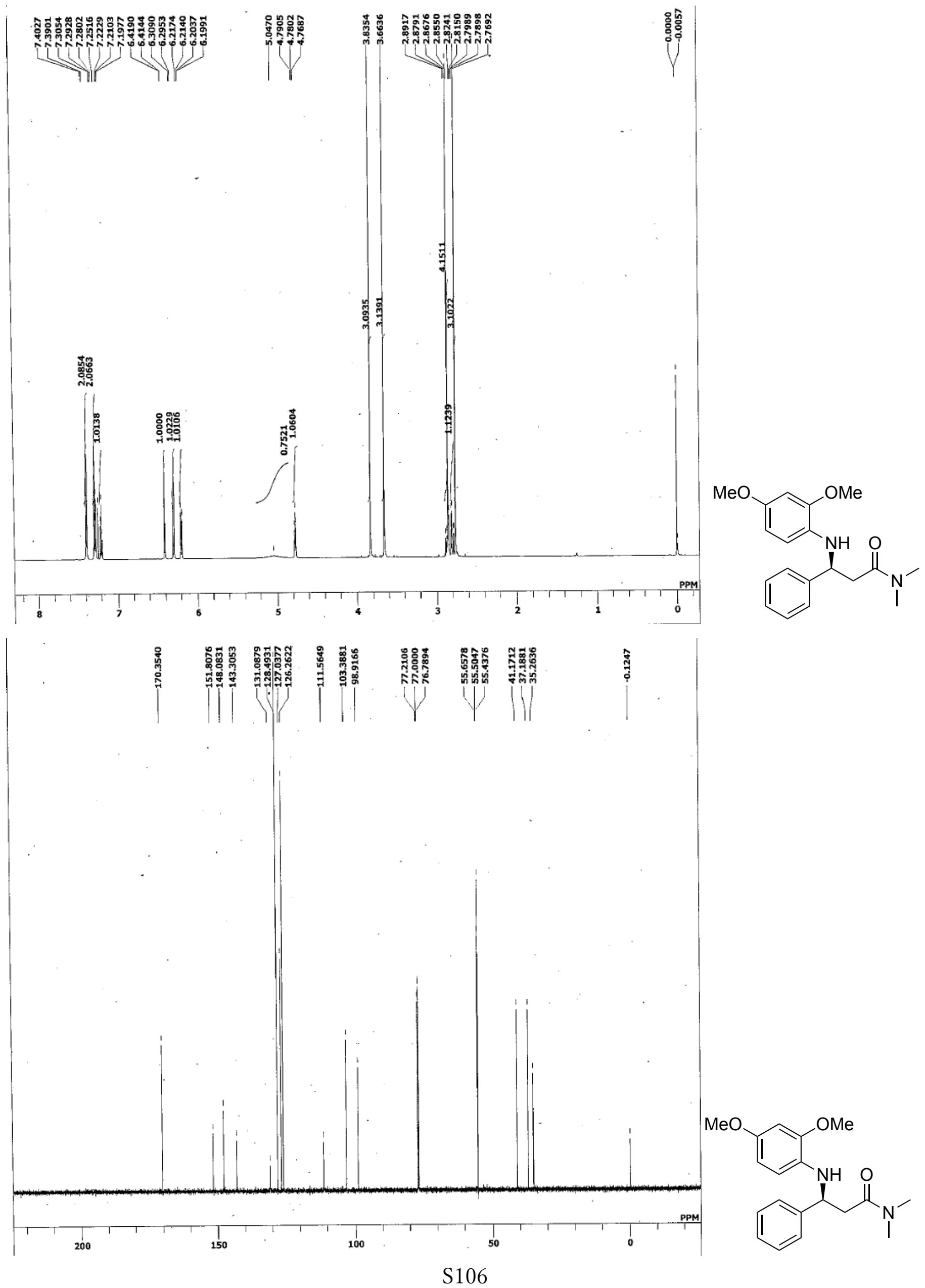



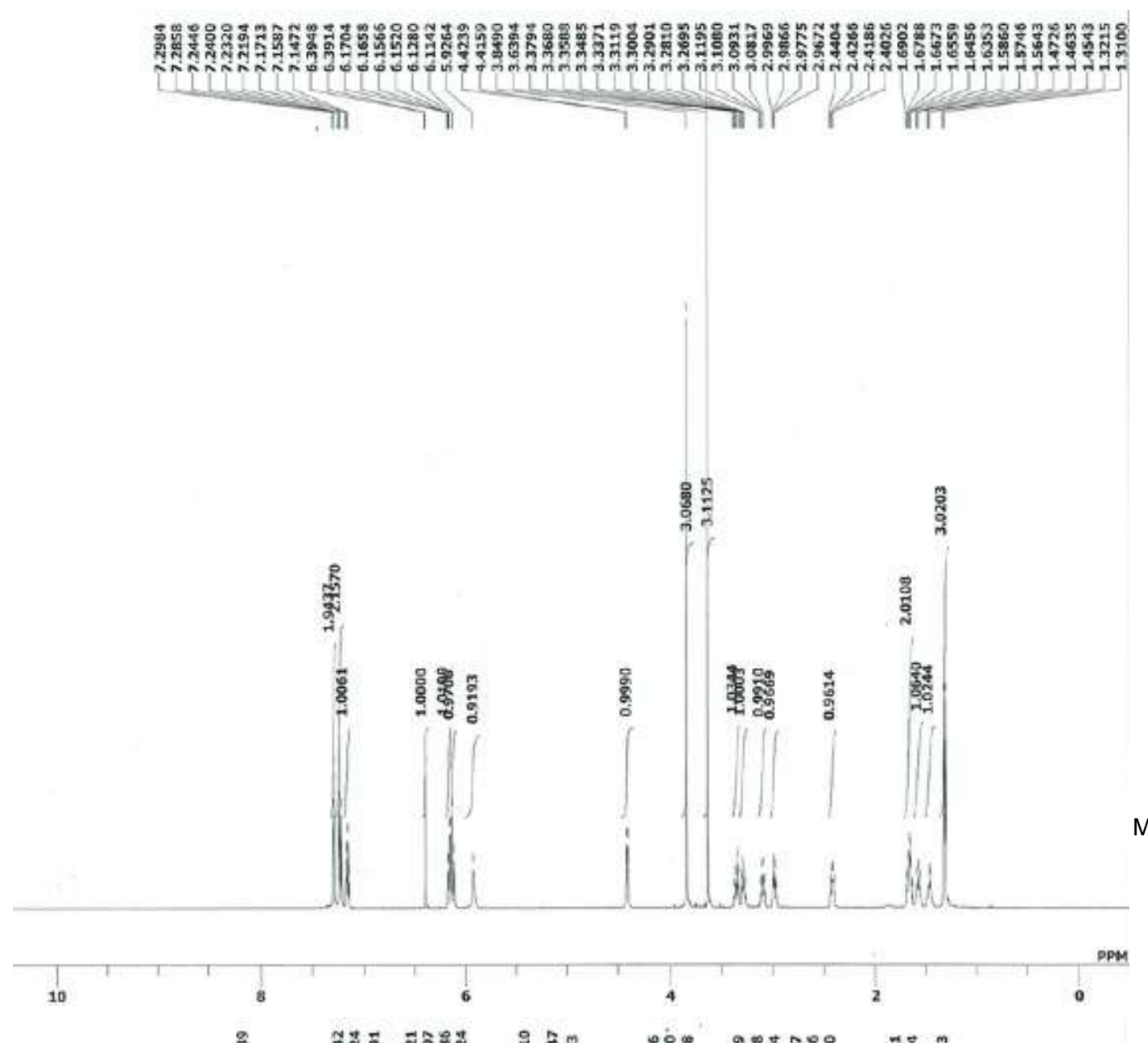<smiles>COc1ccc(N[C@H](c2ccccc2)[C@H](C)C(=O)N2CCCC2)c(OC)c1</smiles>

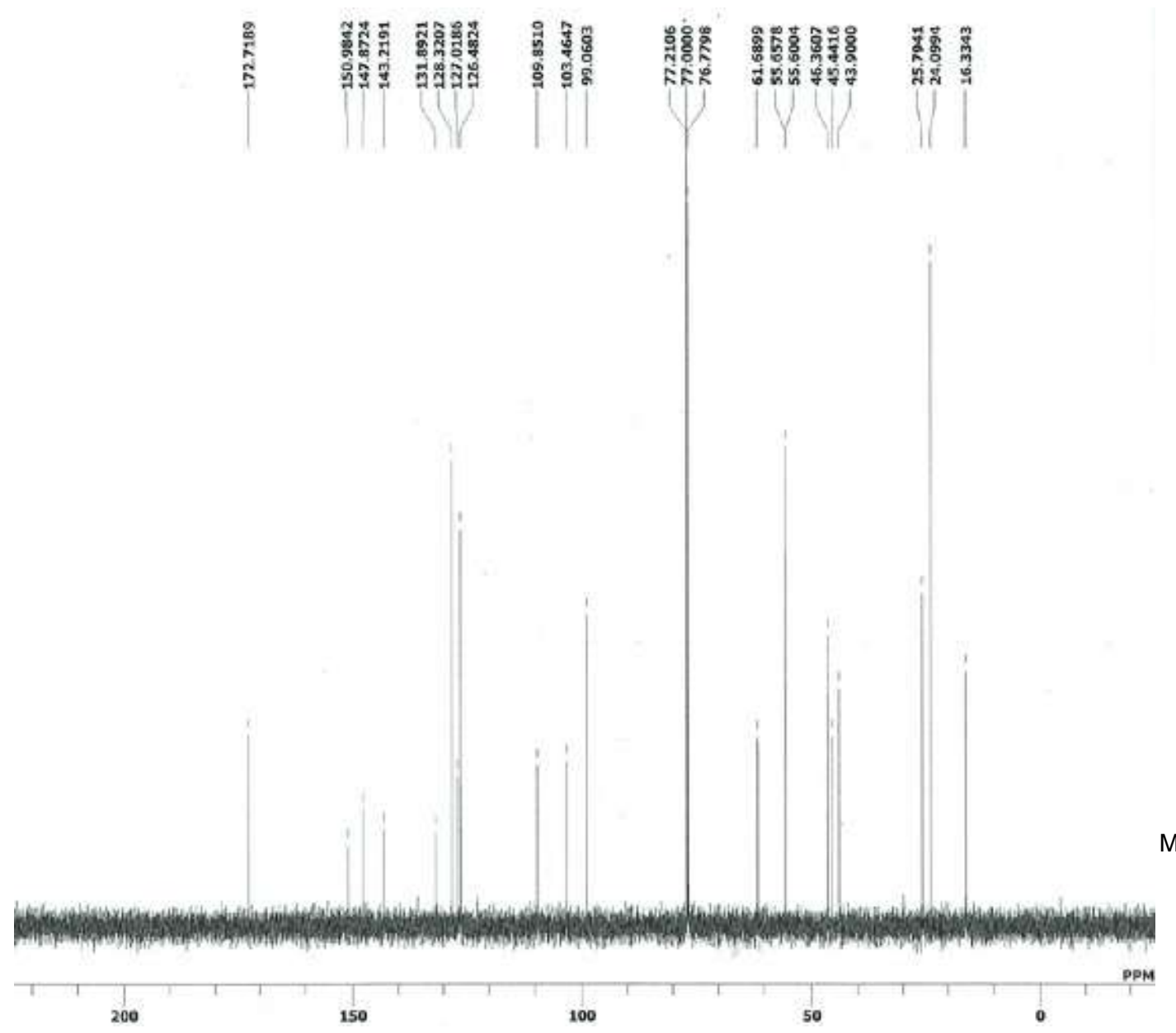<smiles>COc1ccc(N[C@H](c2ccccc2)[C@H](C)C(=O)N2CCCC2)c(OC)c1</smiles>

S107 

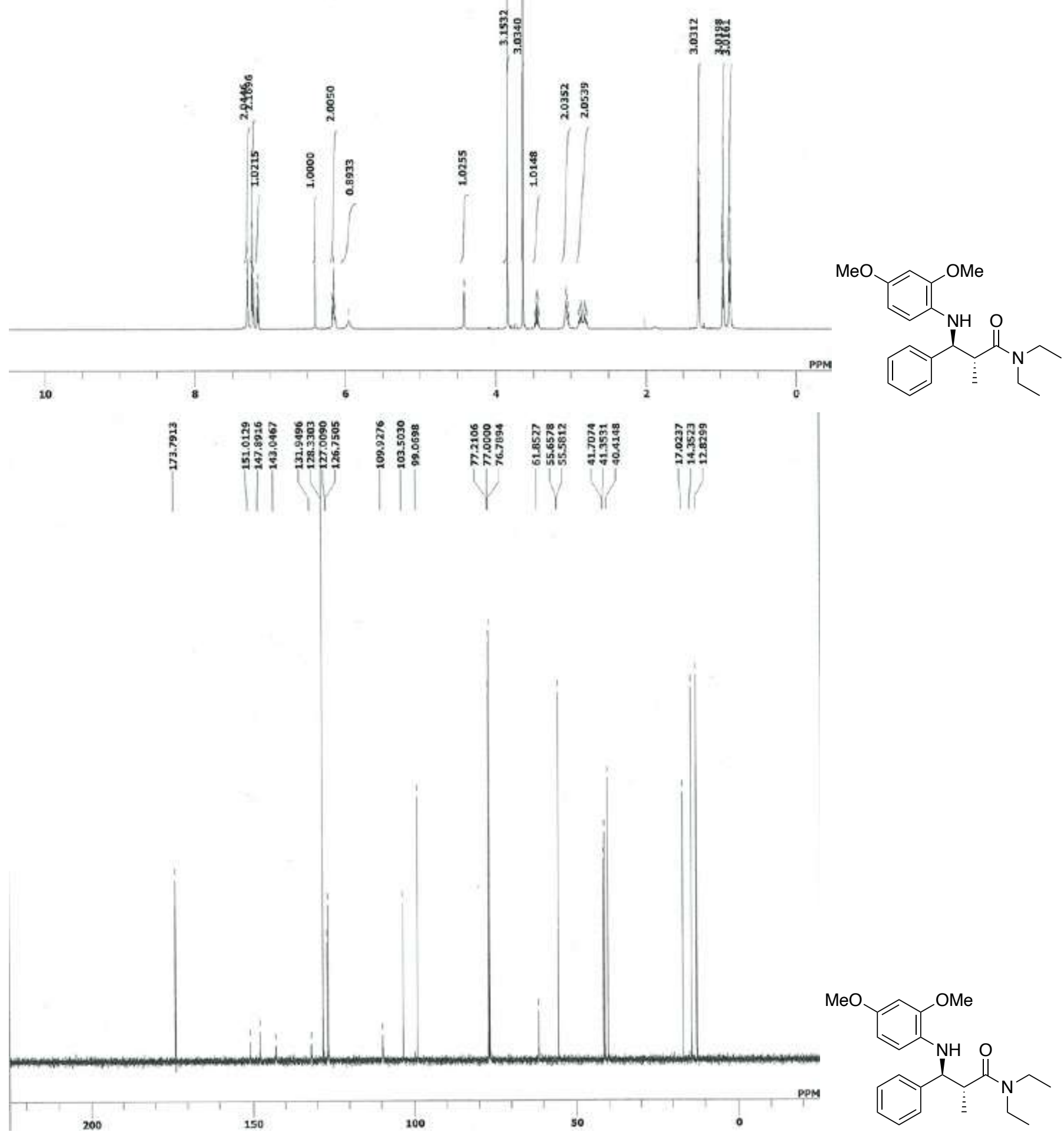


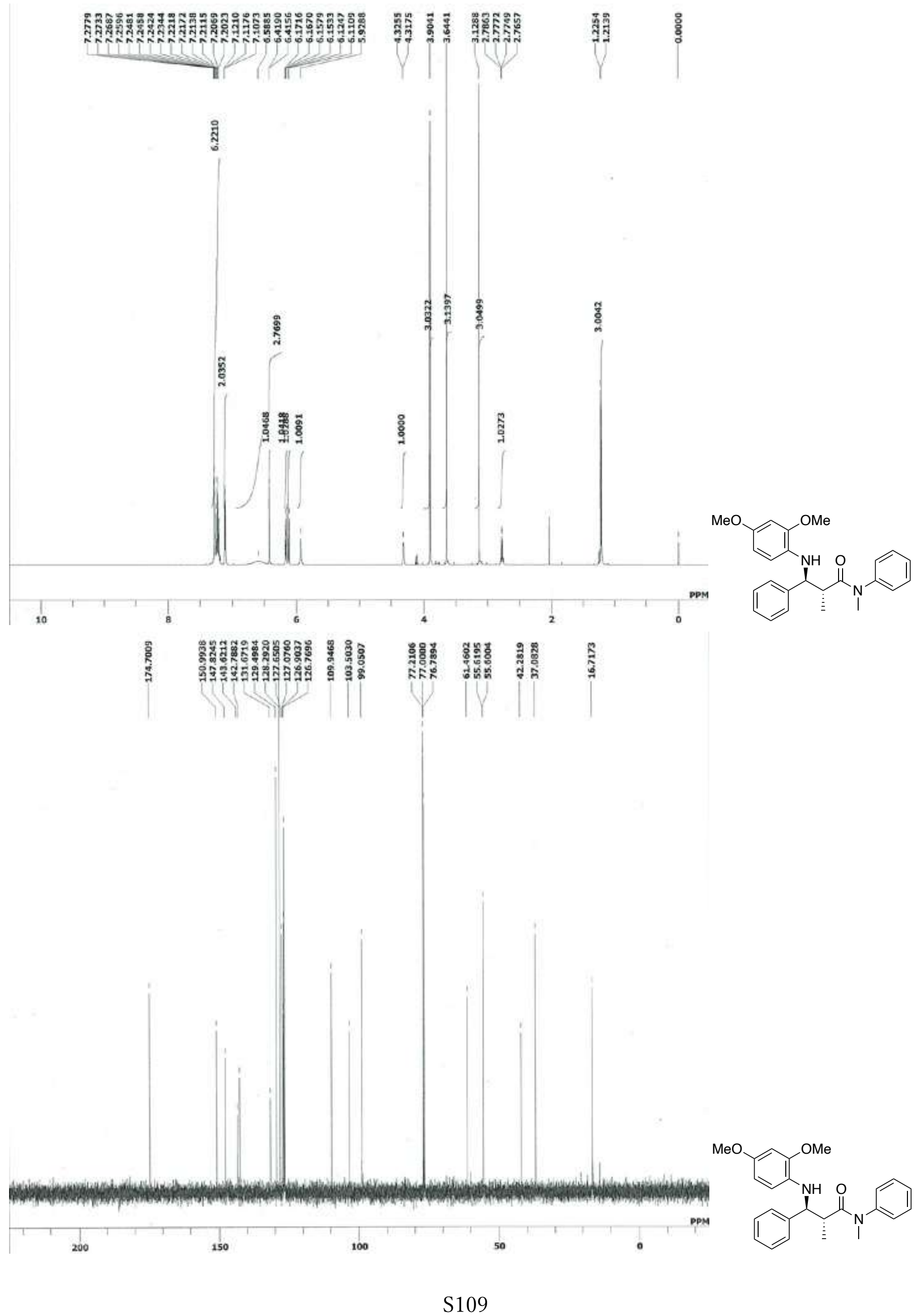




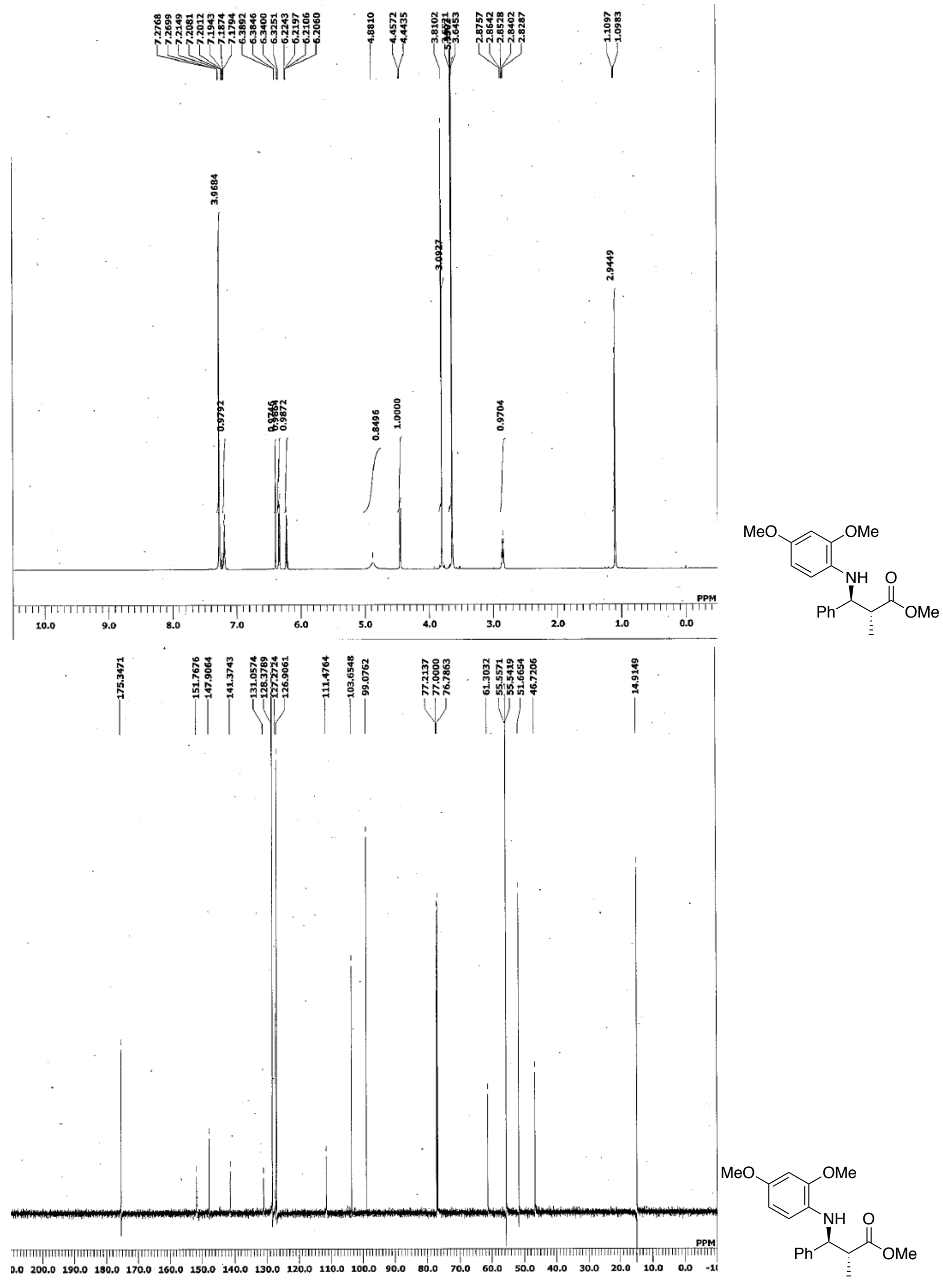




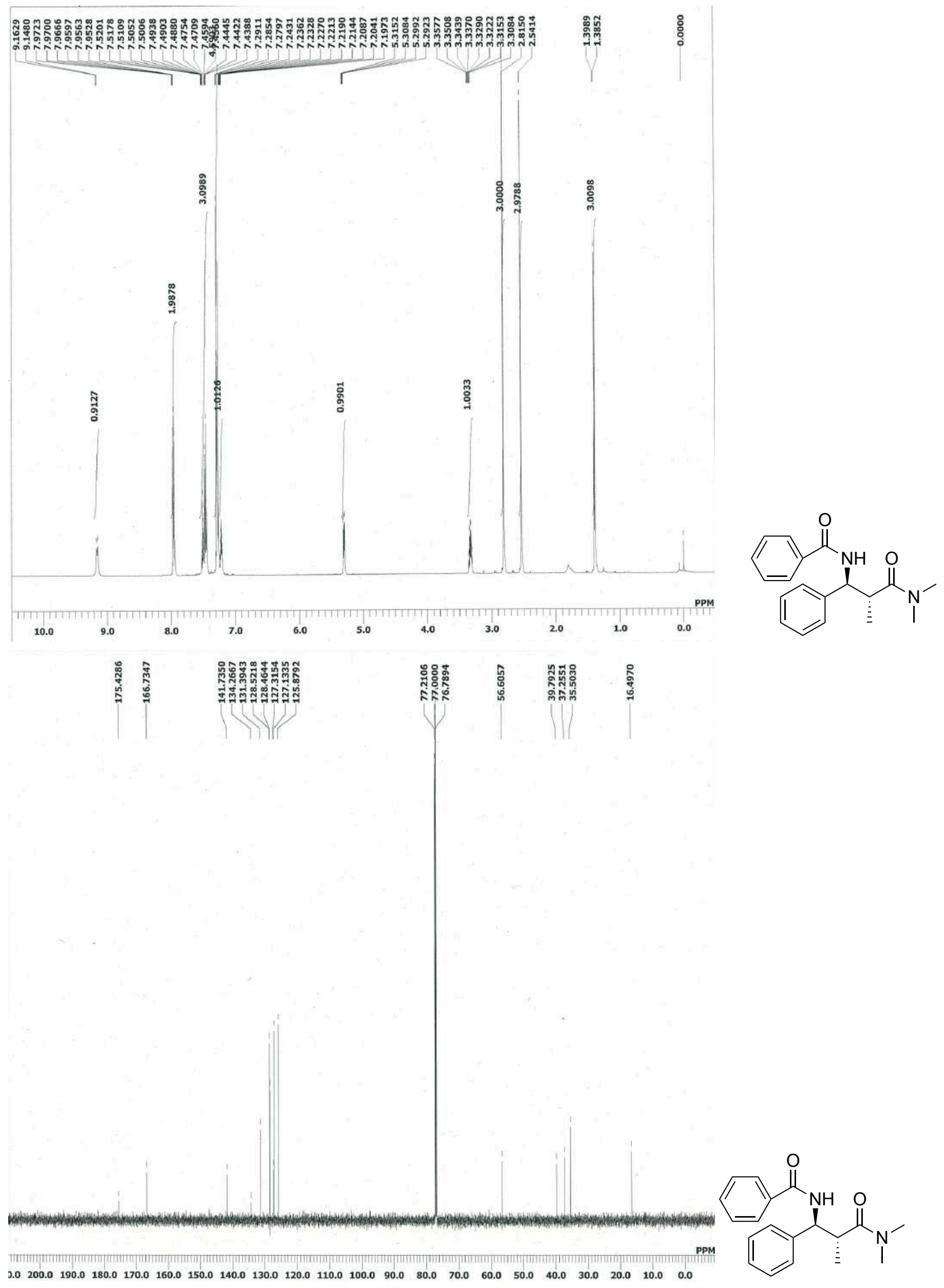




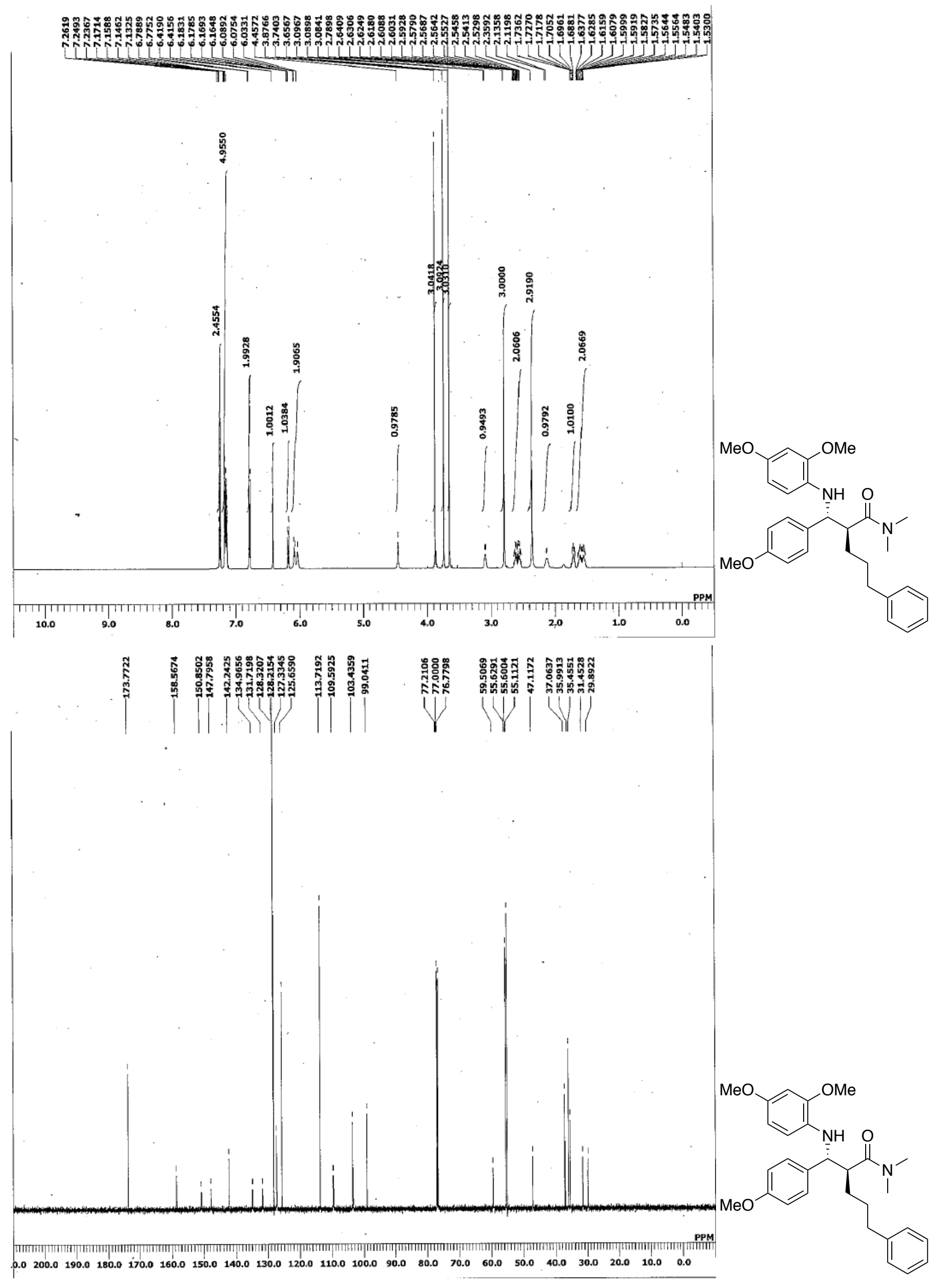




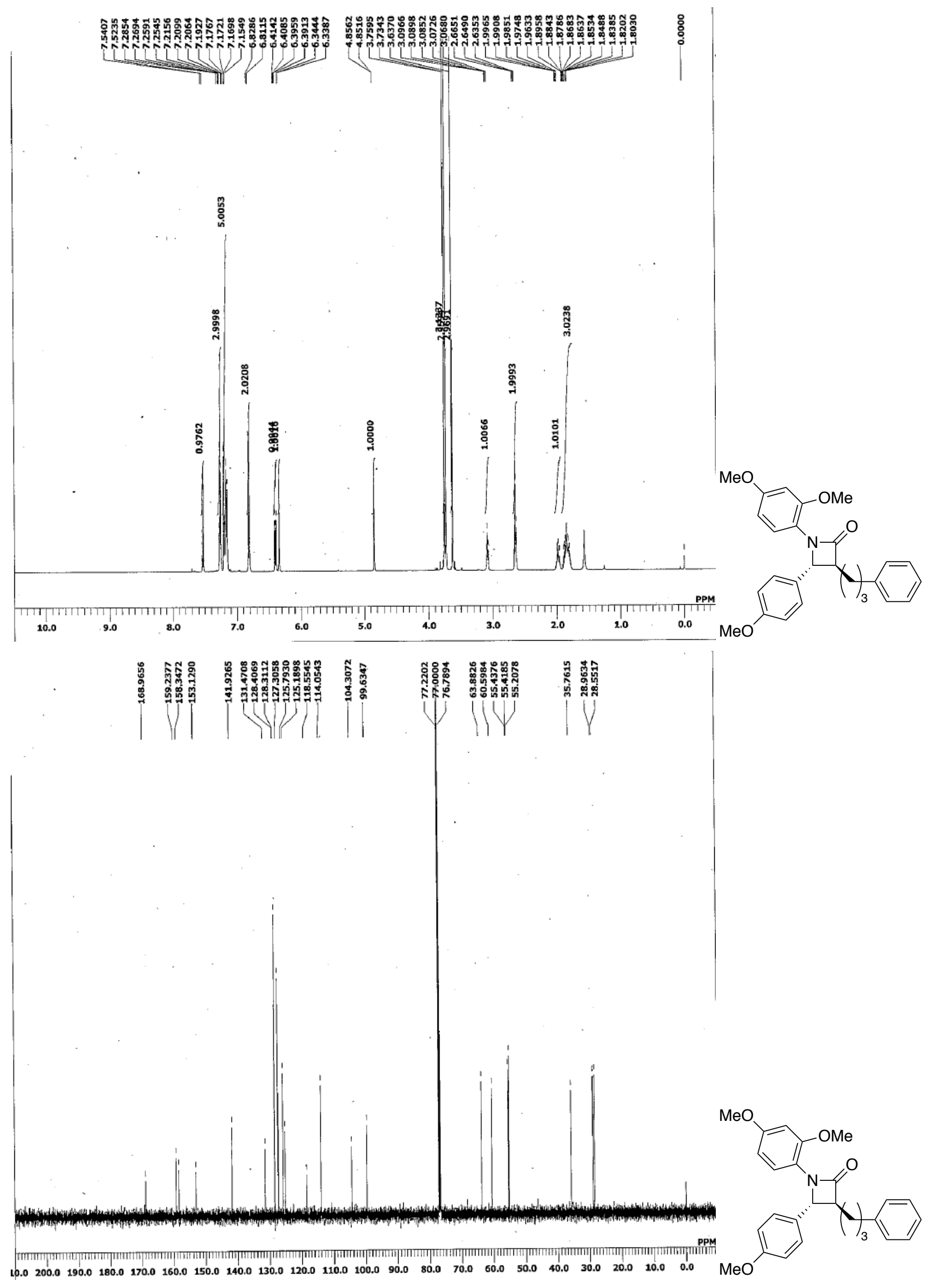




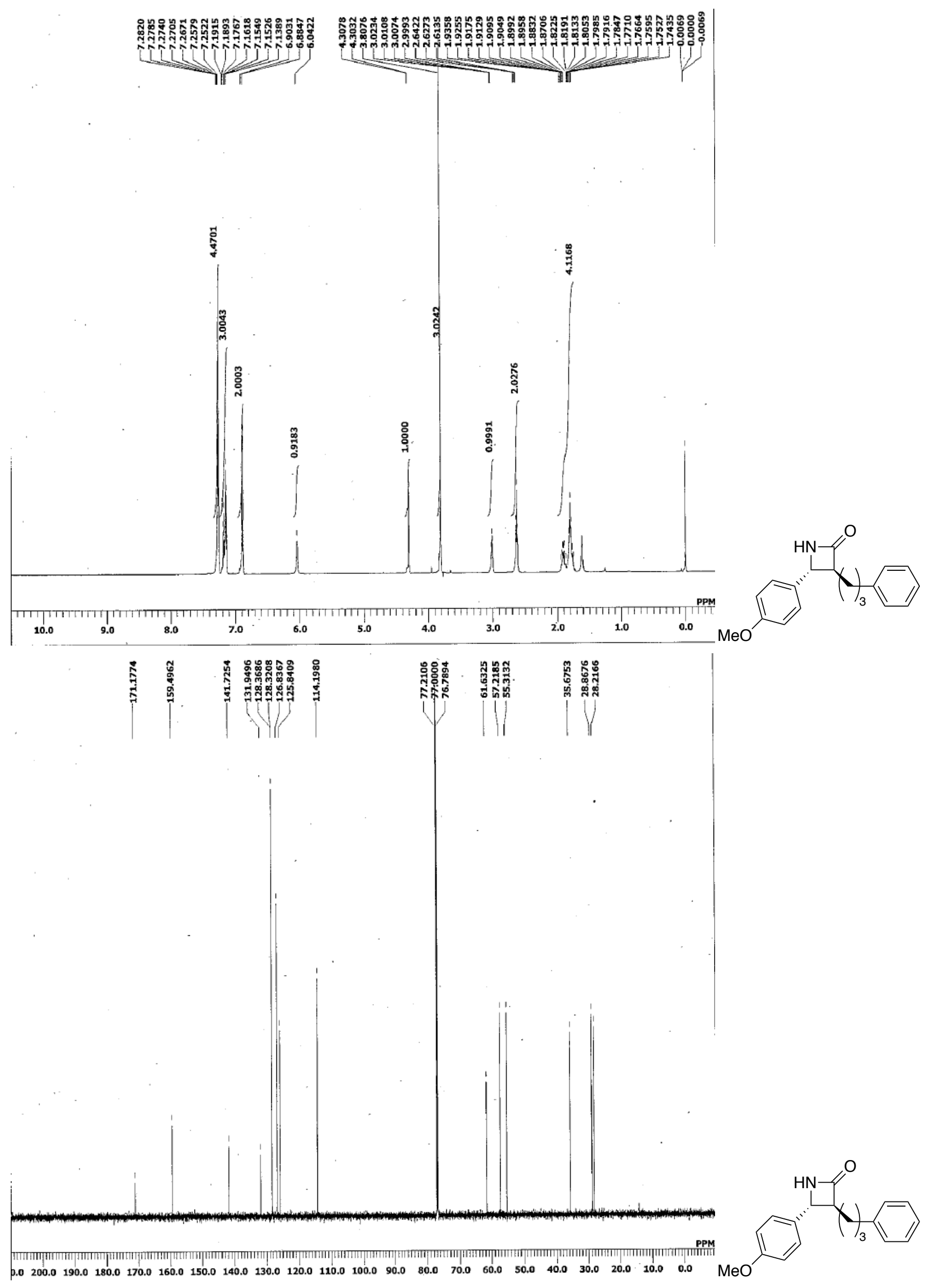




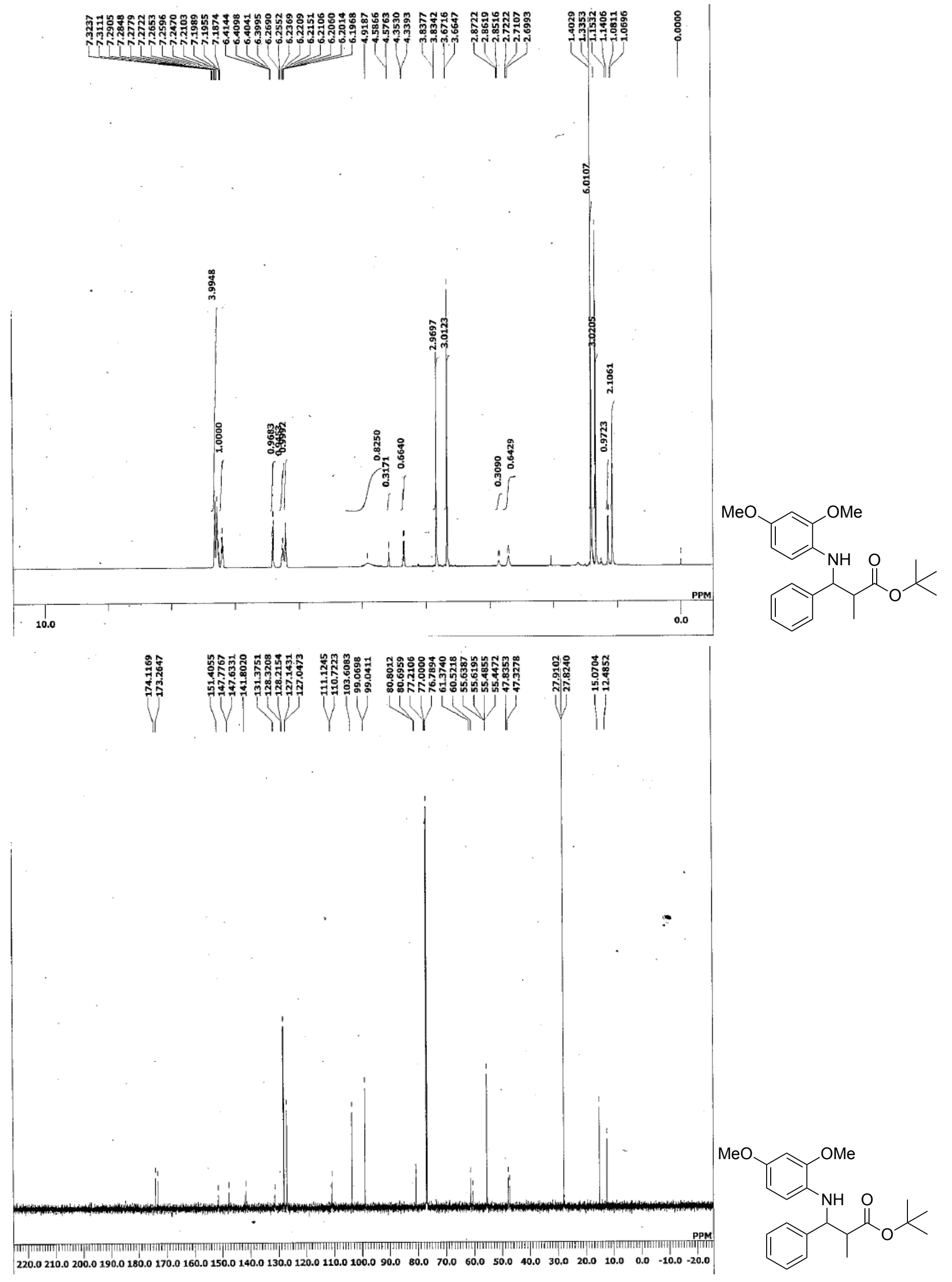




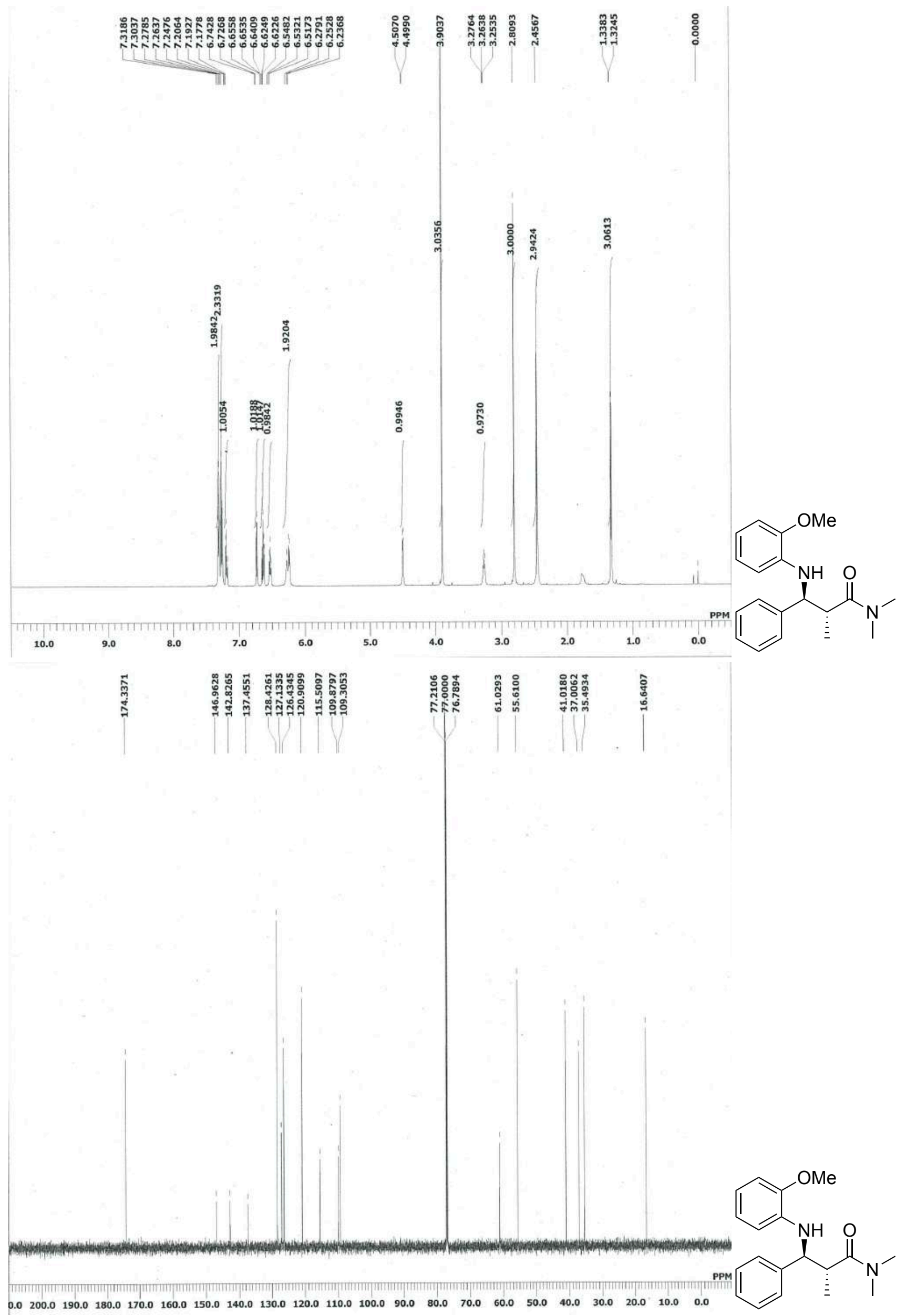



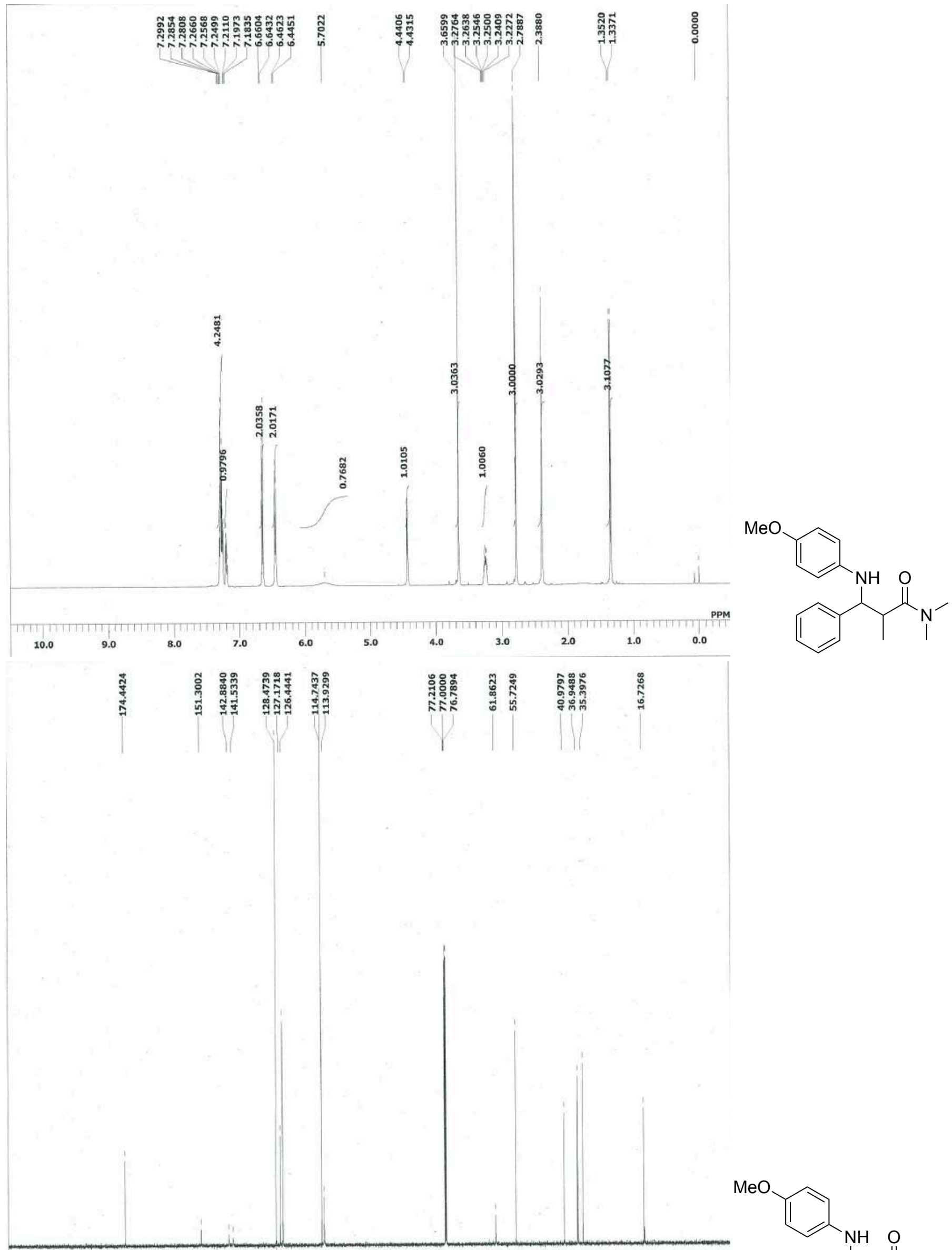

- PPM

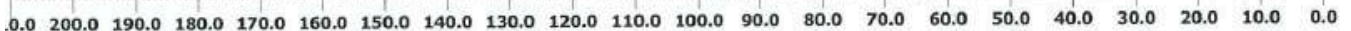

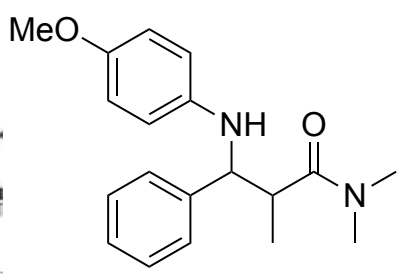



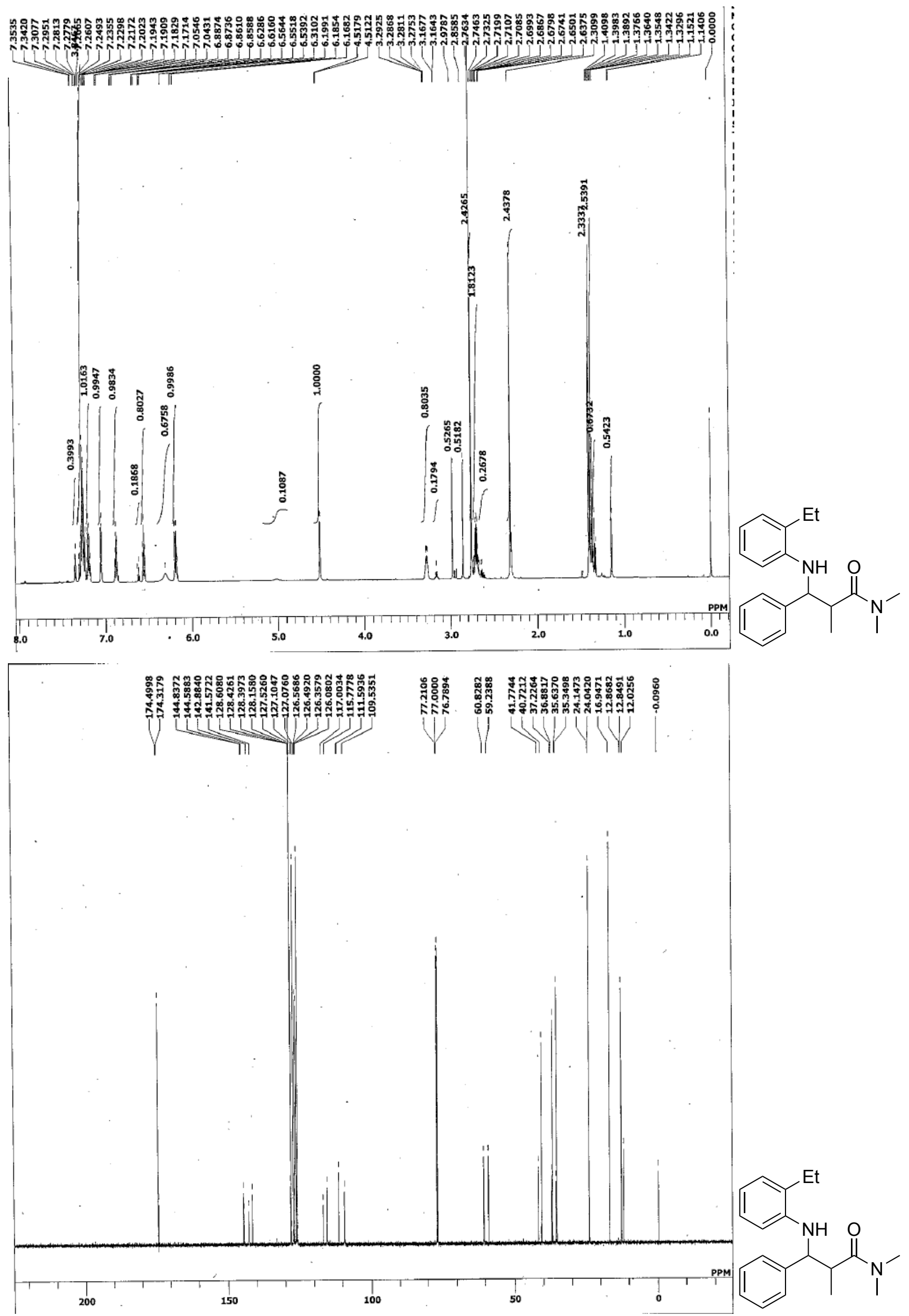


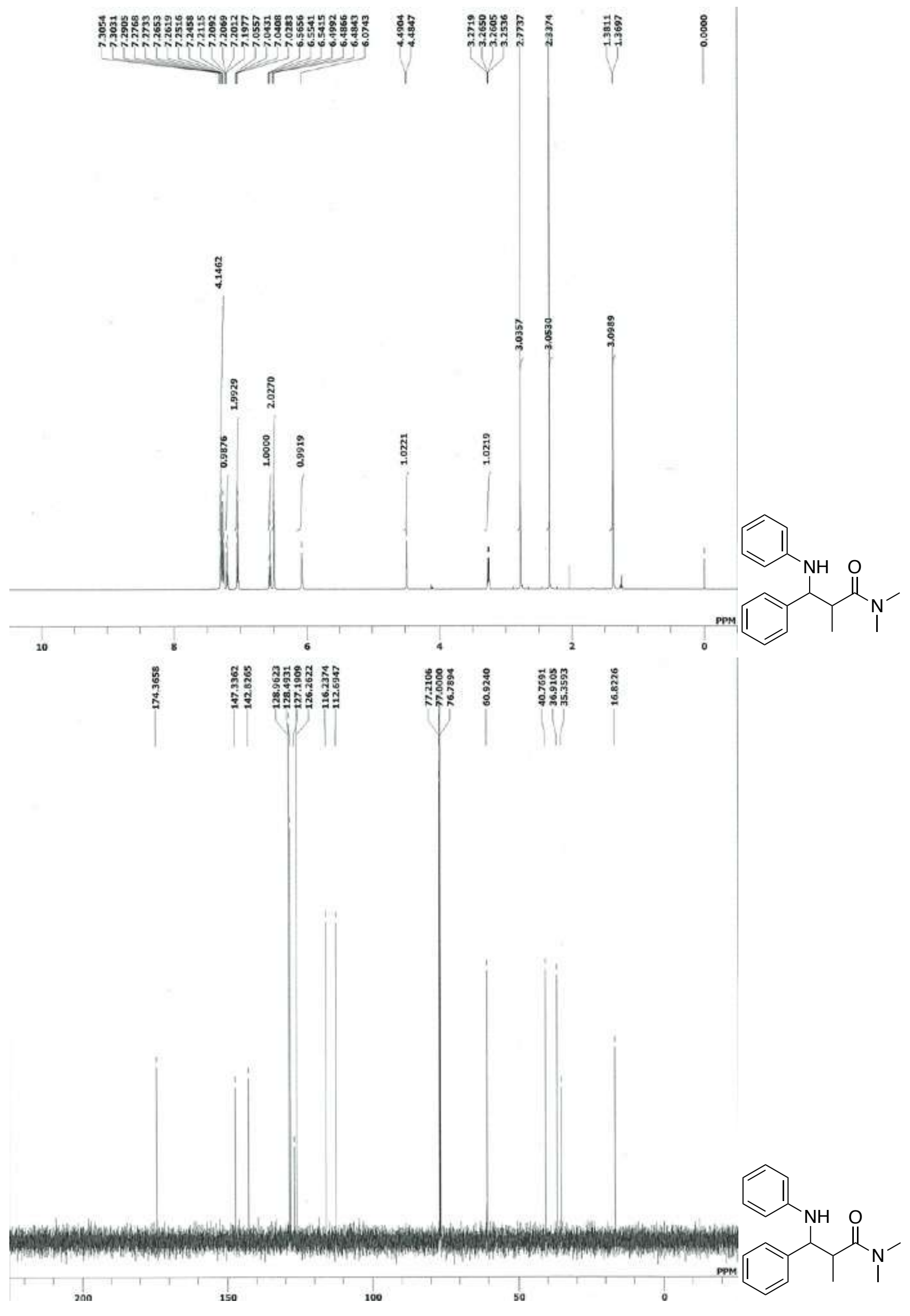




\section{HPLC charts of the products}

mAU
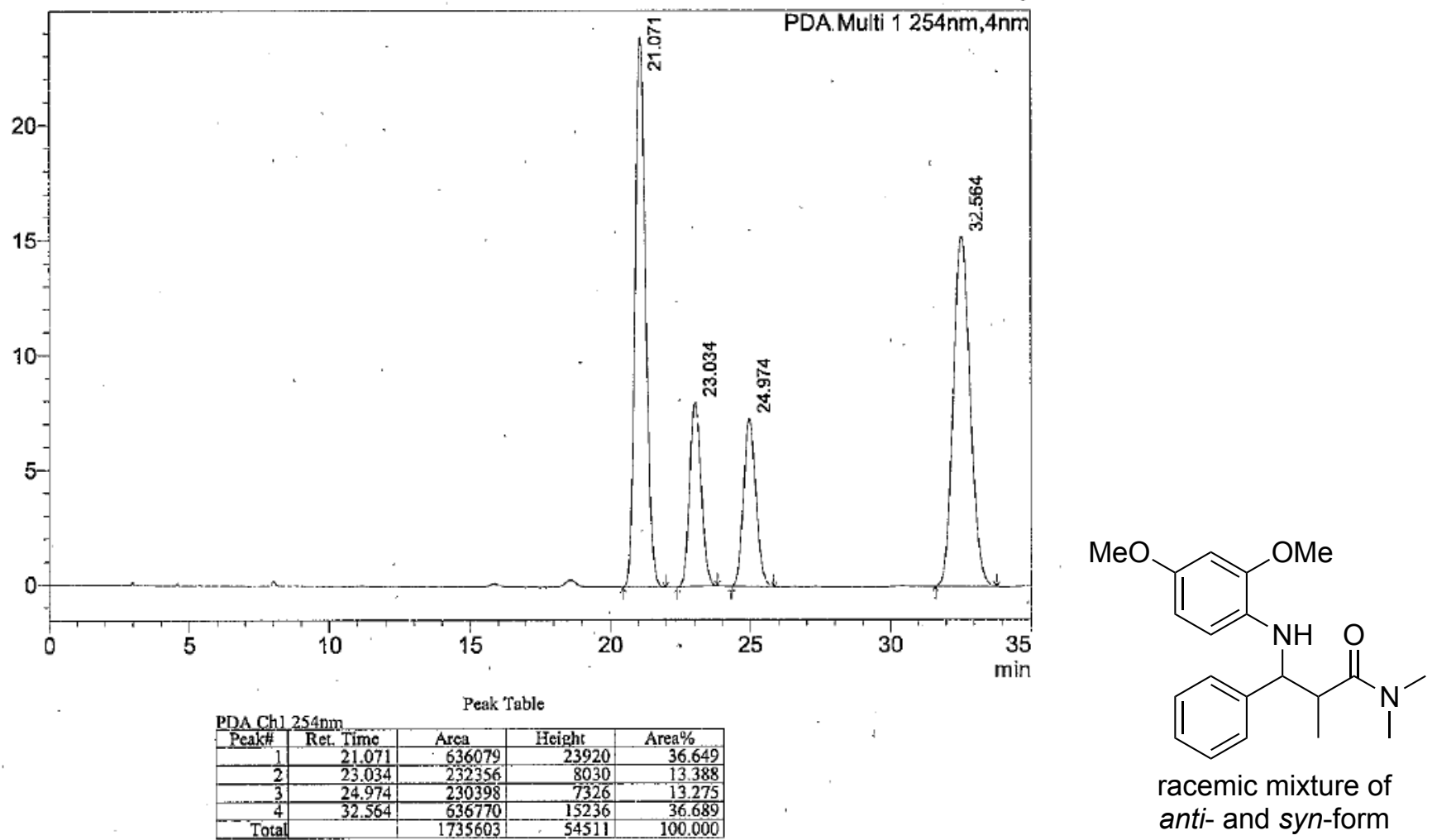

MAU

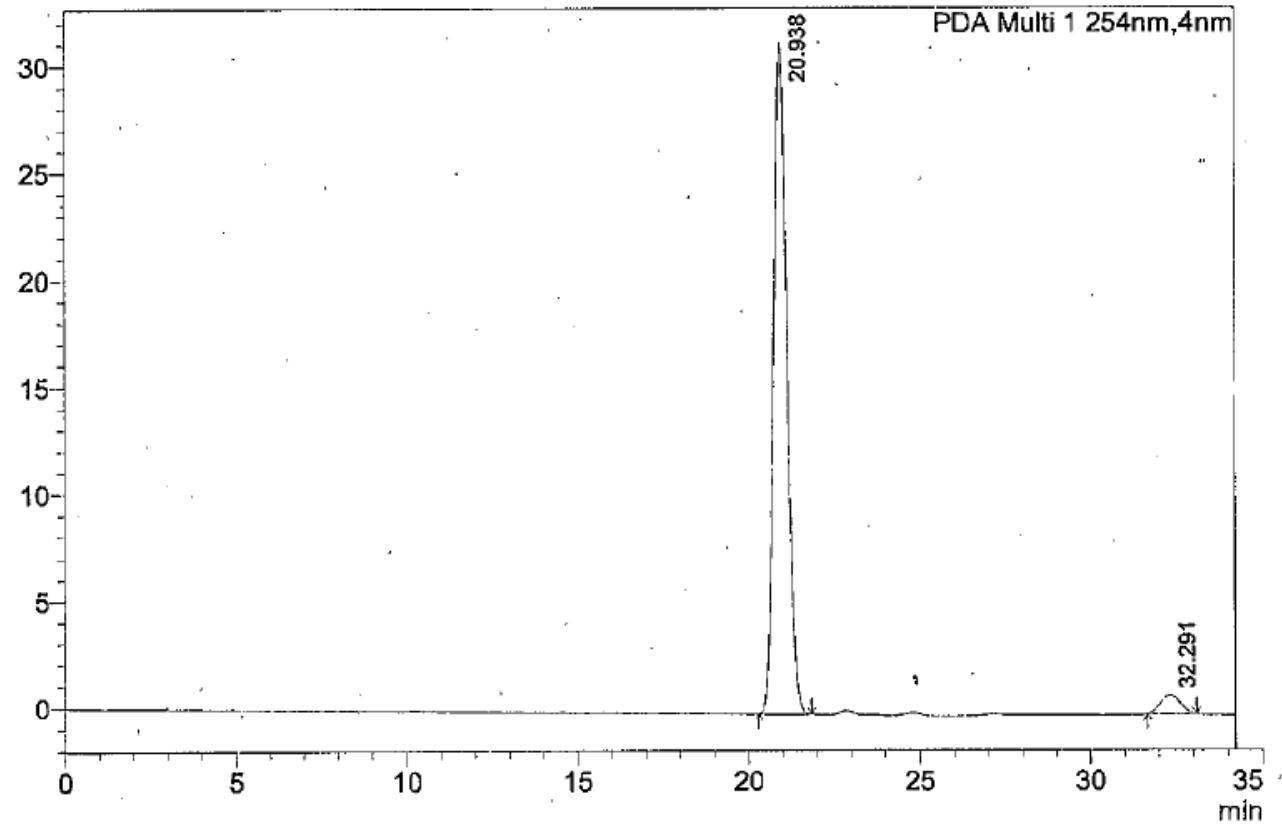<smiles>COc1ccc(N[C@H](c2ccccc2)[C@H](C)C(=O)N(C)C)c(OC)c1</smiles> 
mAU
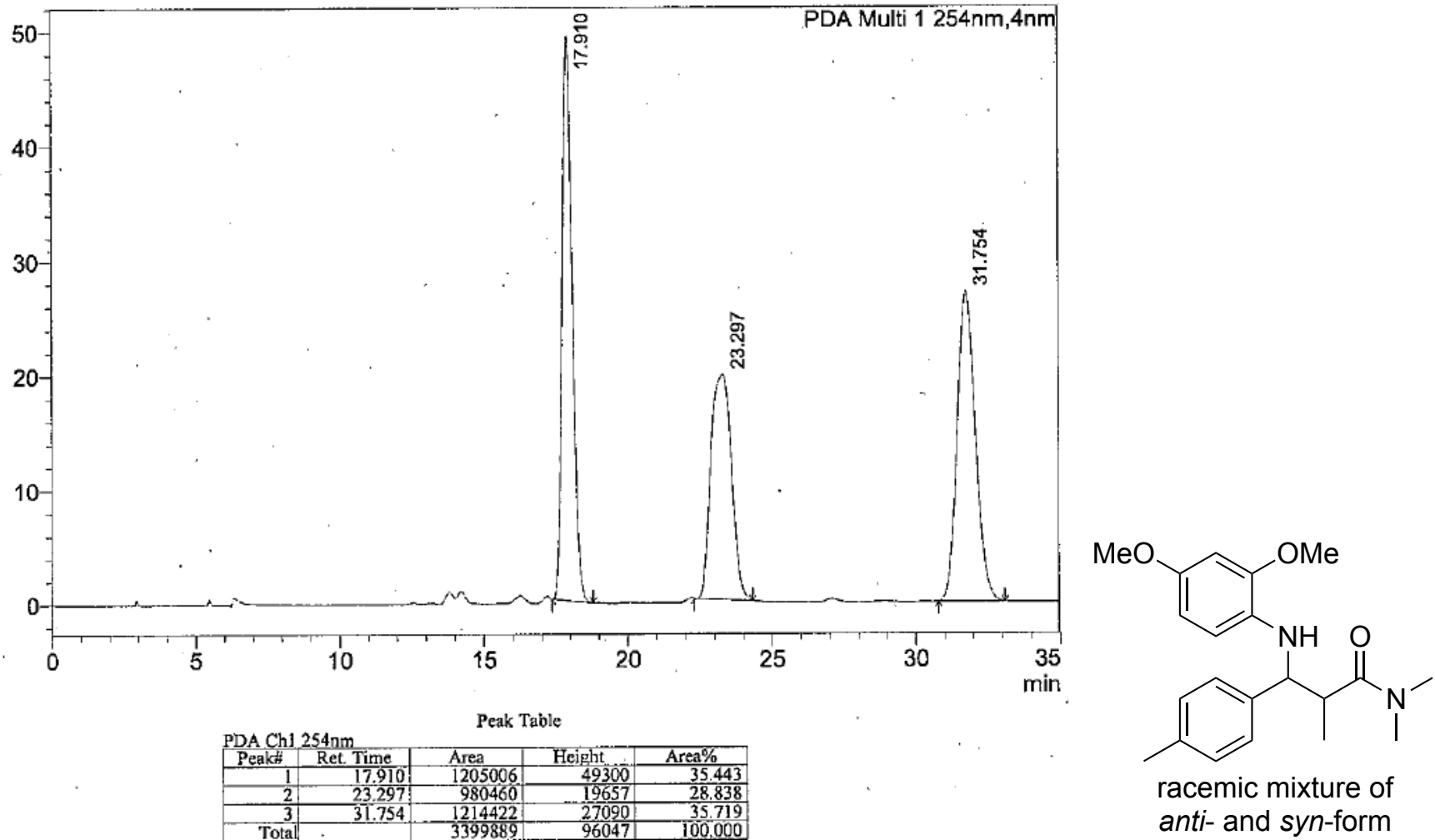

$\mathrm{mAU}$

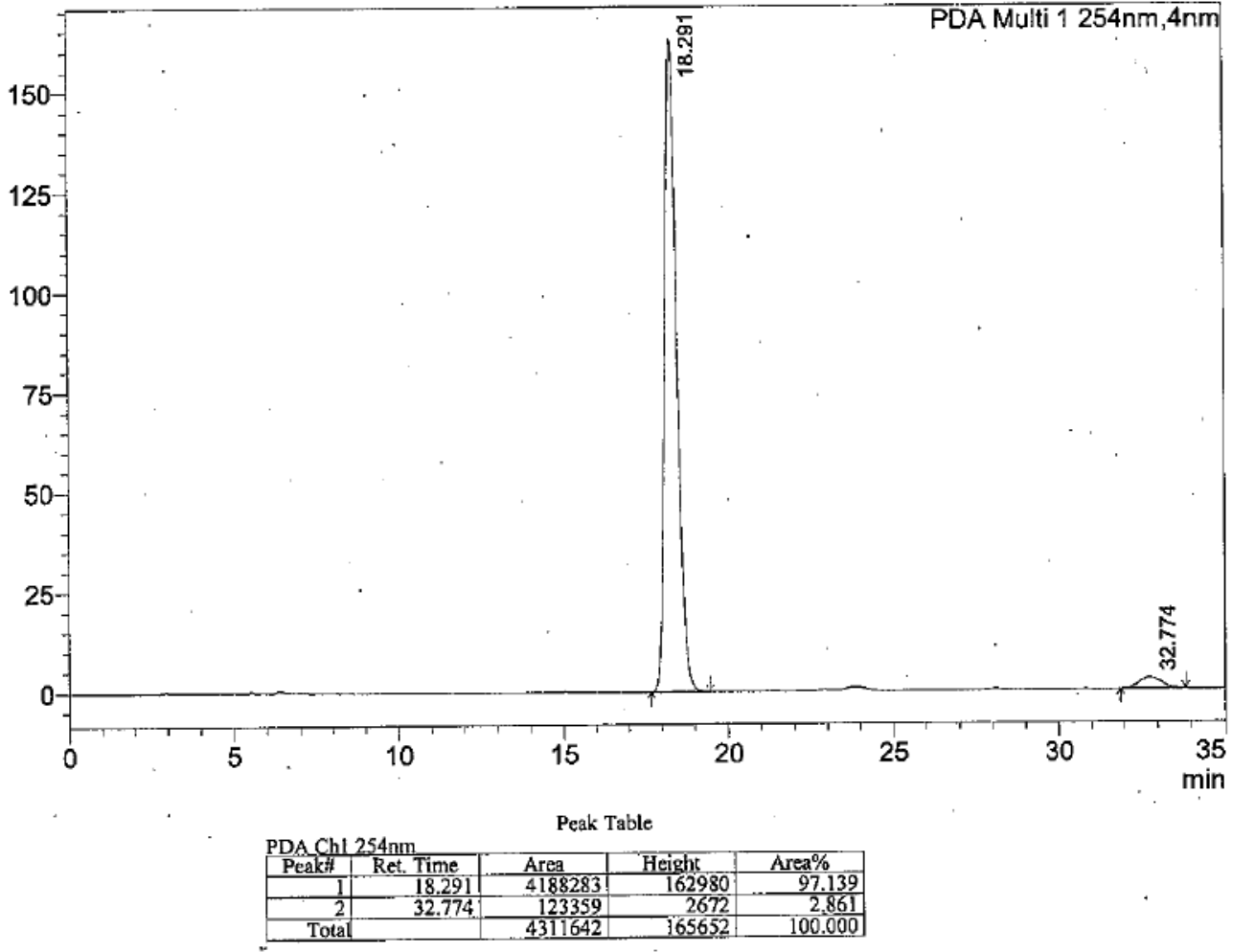

$\mathrm{MeO}$<smiles>COc1cc(C)ccc1N</smiles><smiles>C/C=C/N[C@H](c1ccc(C)cc1)[C@@H](C)C(=O)N(C)C</smiles> 
mAU

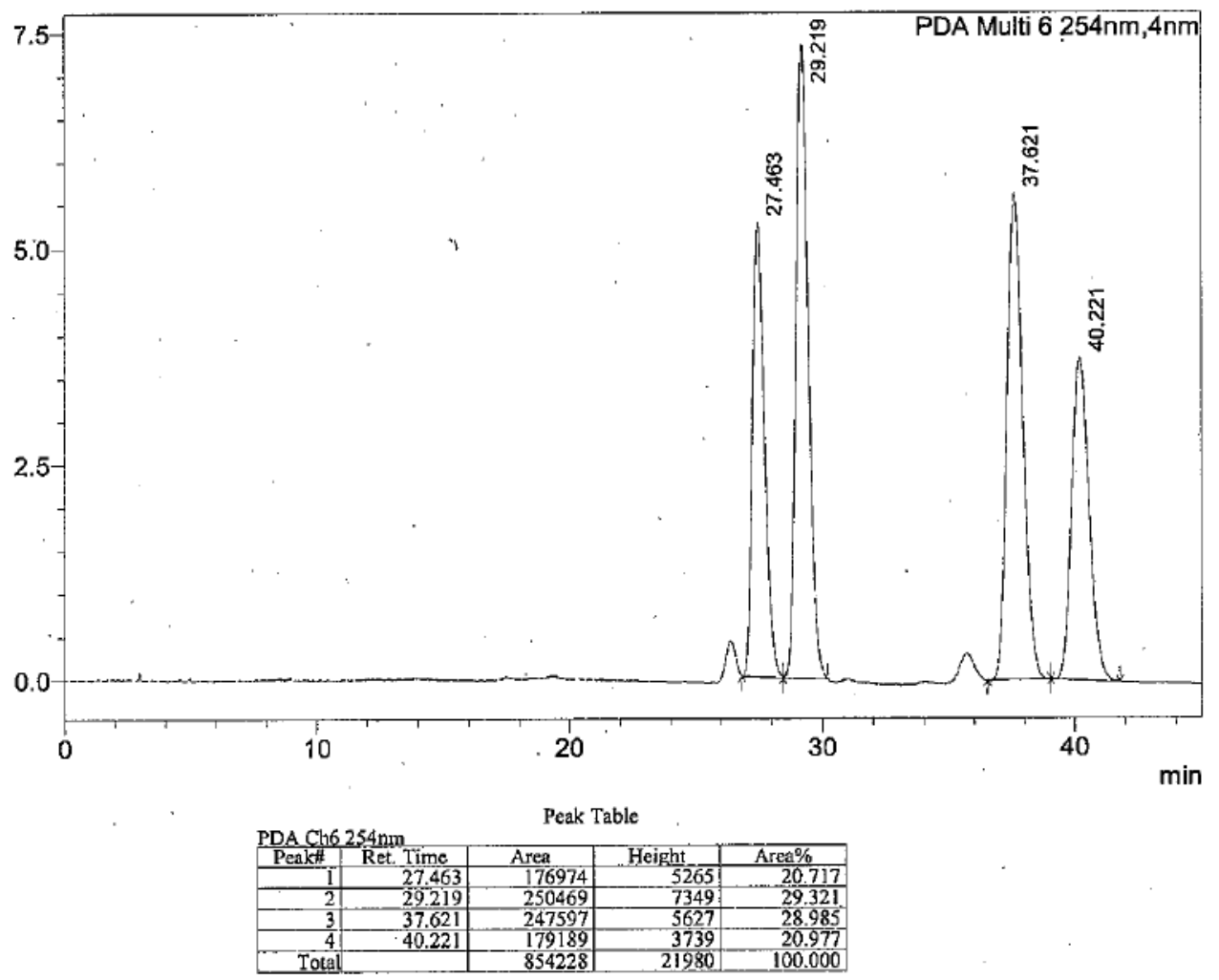<smiles>COc1ccc(NC(c2cccc(C)c2)C(C)C(=O)N(C)C)c(OC)c1</smiles>

racemic mixture of anti- and syn-form

mAU

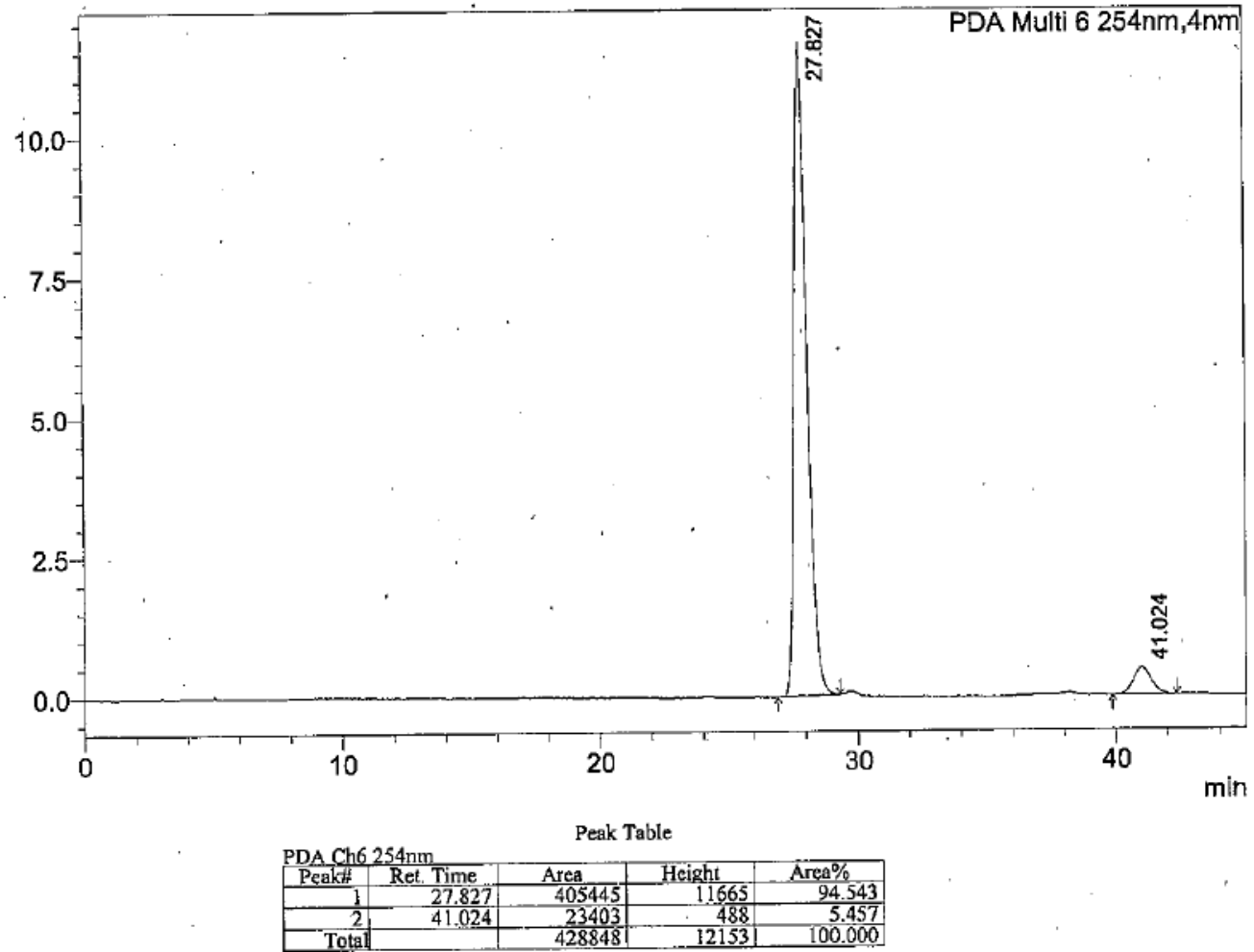<smiles>COc1ccc(N[C@H](c2cccc(C)c2)[C@H](C)C(=O)N(C)C)c(OC)c1</smiles> 
mAU

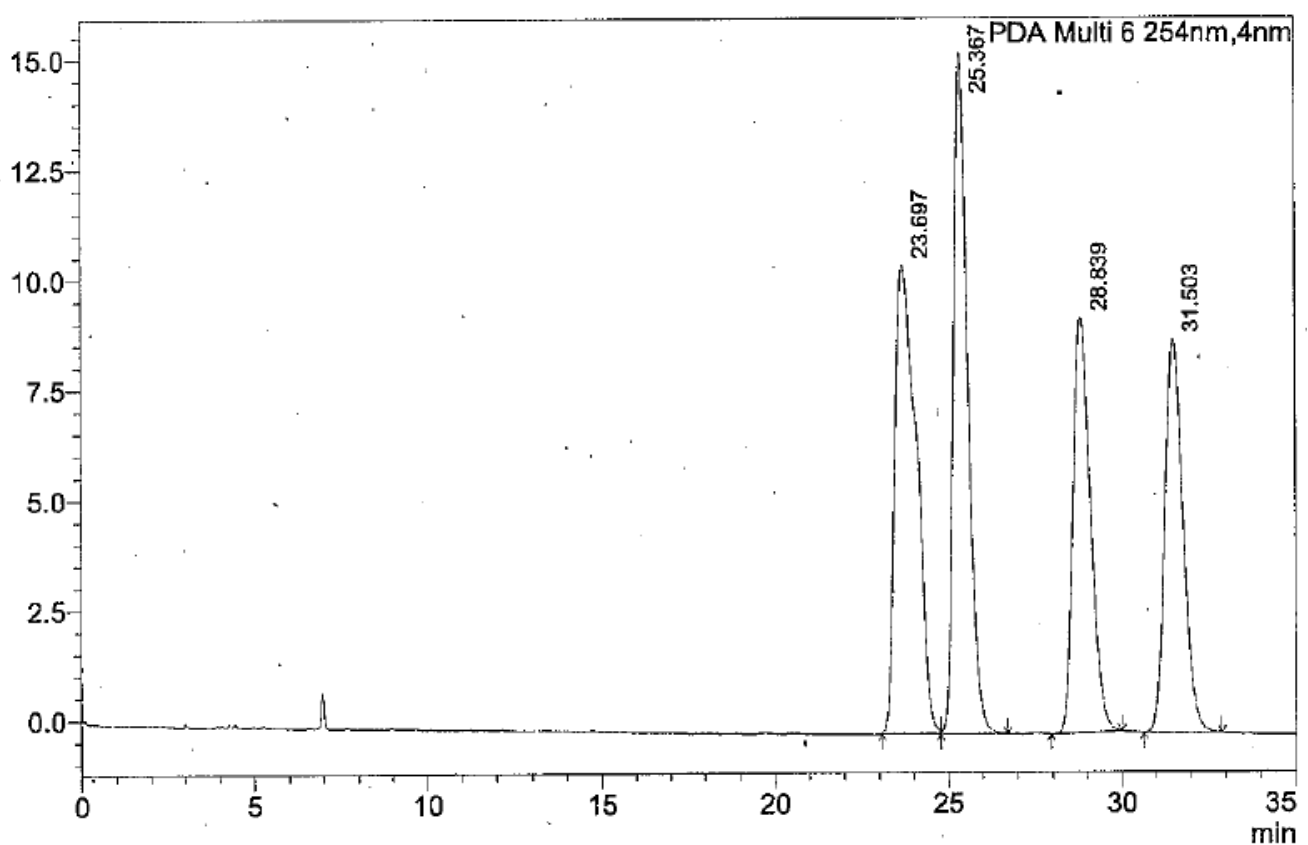<smiles>COc1ccc(NC(c2ccccc2C)C(C)C(=O)N(C)C)c(OC)c1</smiles>

racemic mixture of anti- and syn-form

mAU

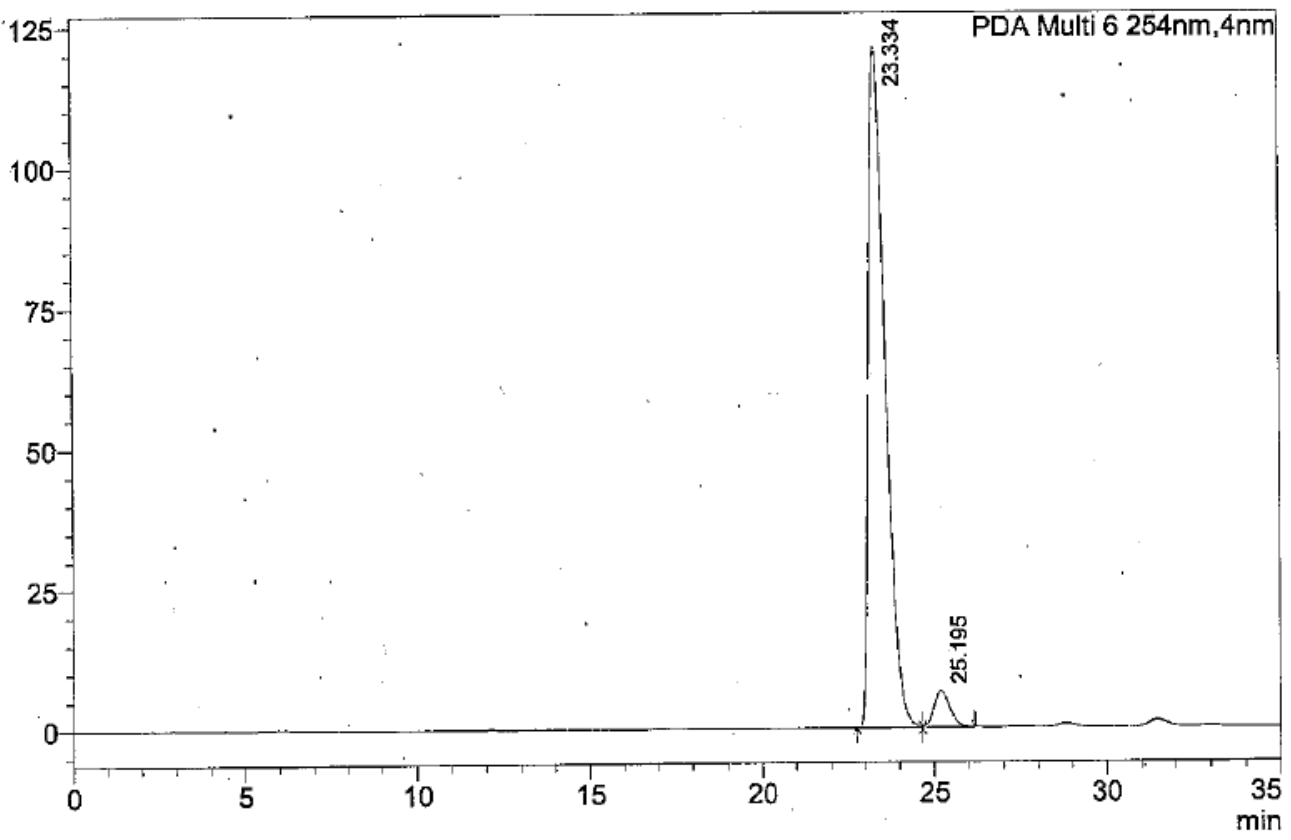

$\mathrm{MeO}$<smiles>COc1ccccc1N</smiles>

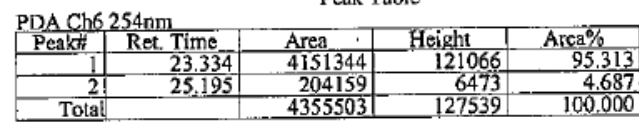<smiles>CCCN[C@H](c1ccccc1C)[C@H](C)C(=O)N(C)C</smiles> 
mAU
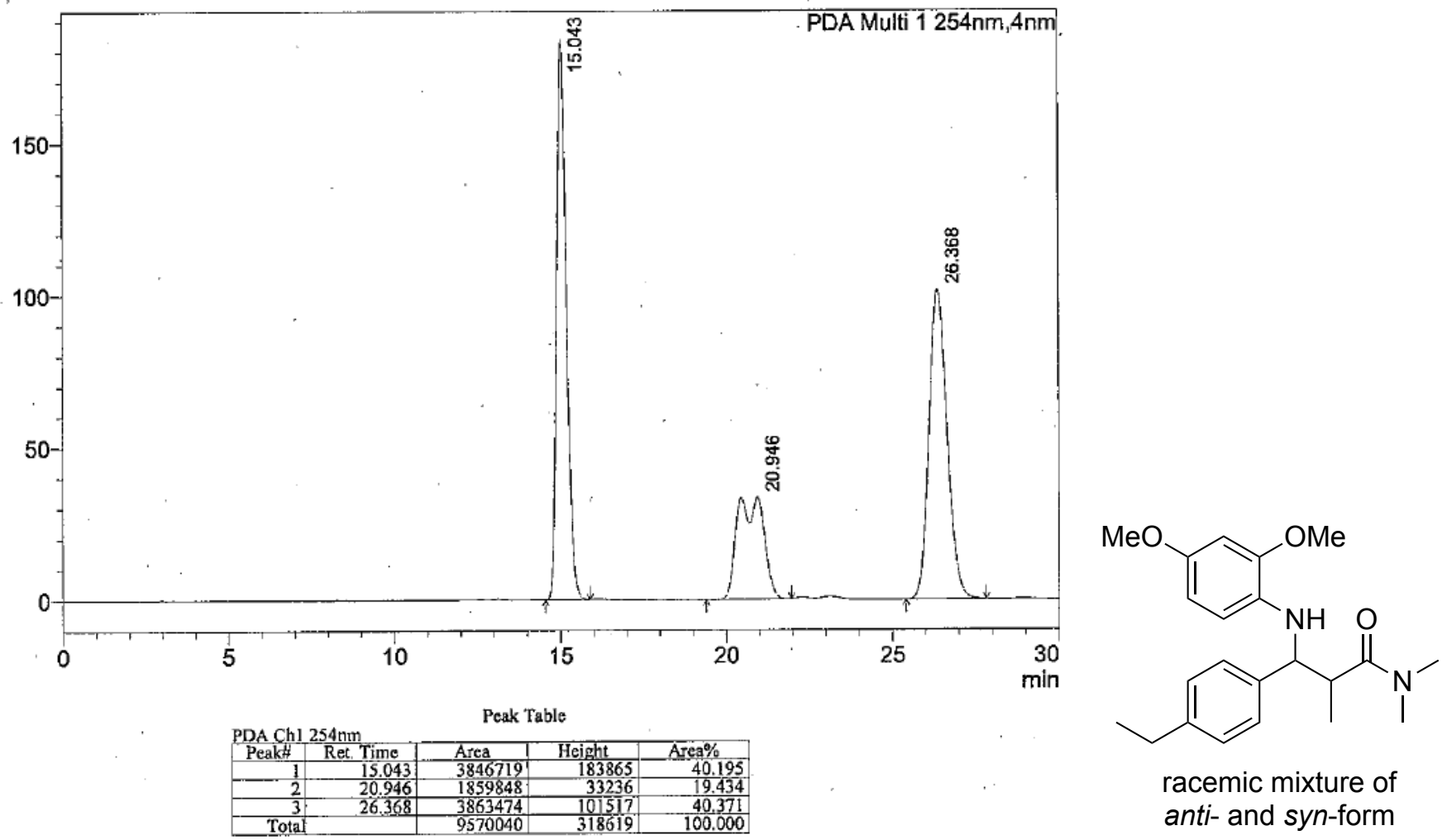

MAU

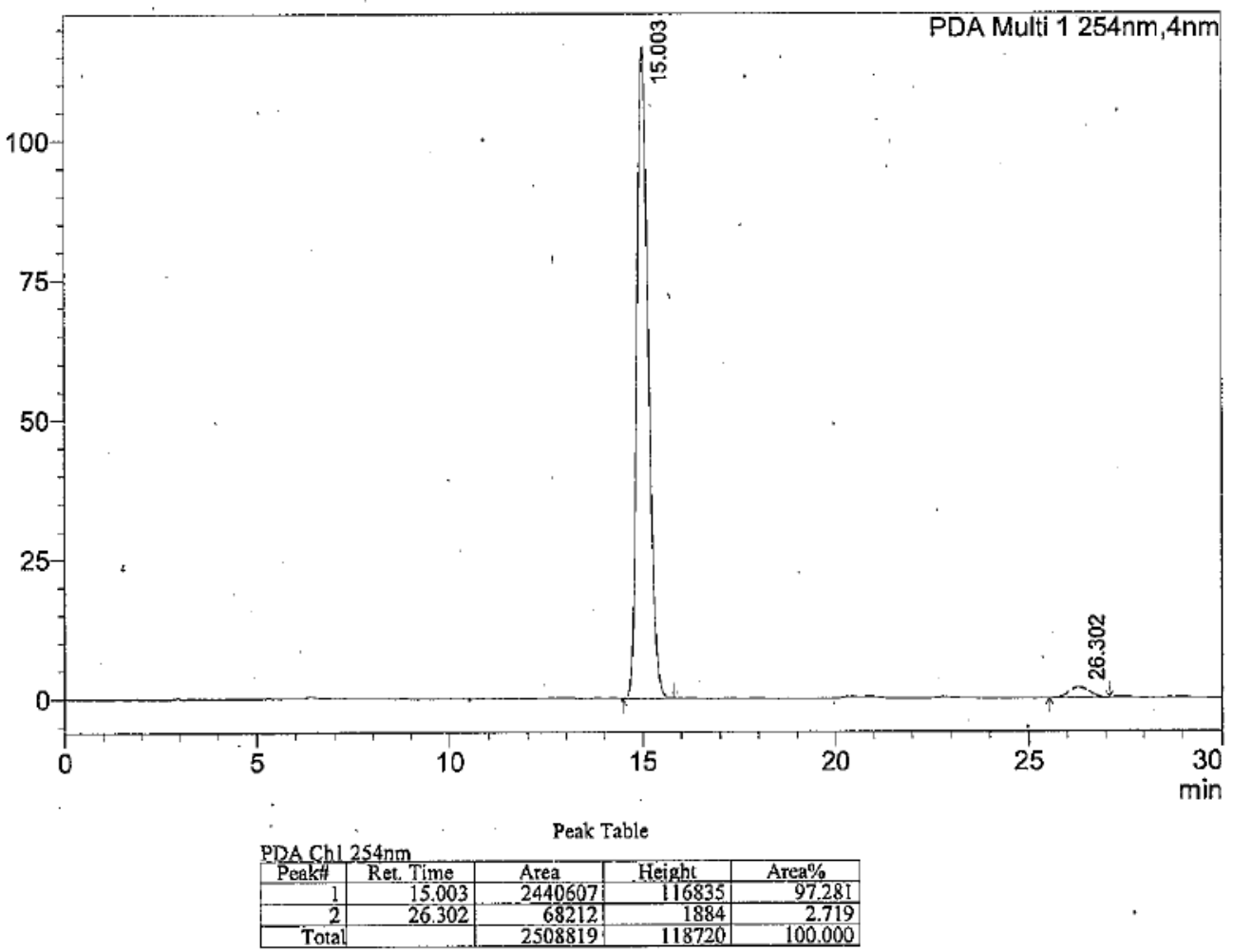<smiles>CCc1ccc([C@H](Nc2ccc(OC)cc2OC)[C@H](C)C(=O)N(C)C)cc1</smiles> 
mAU
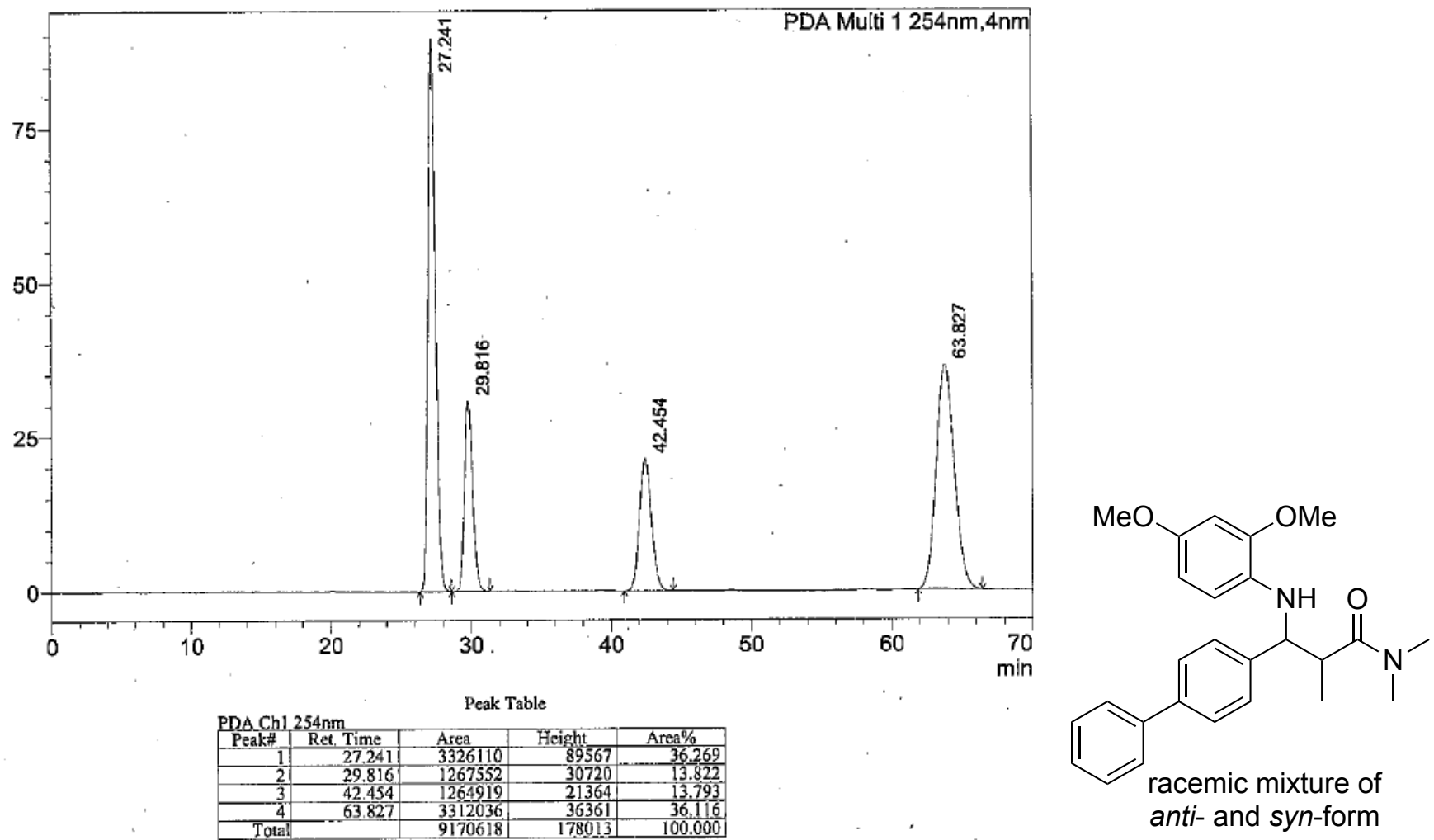

mAU

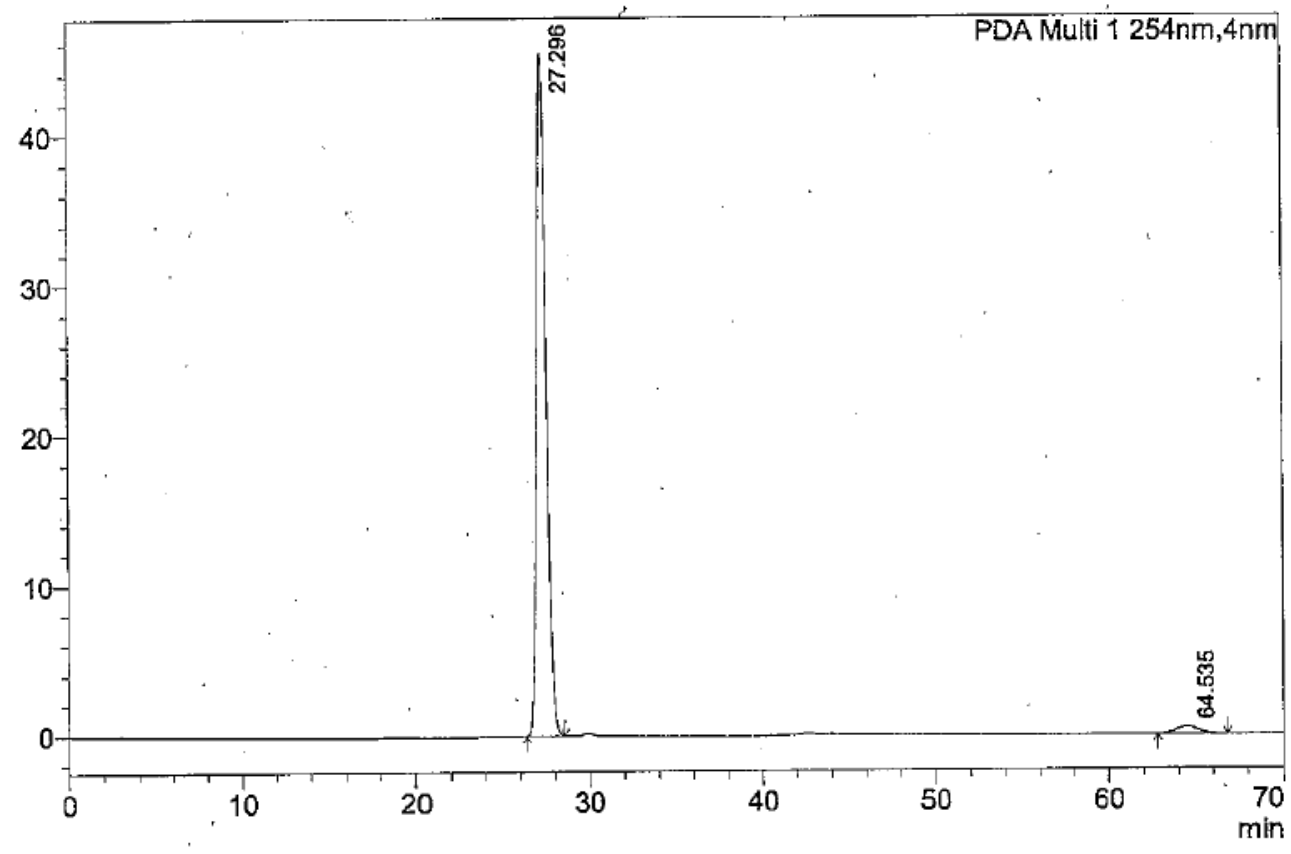<smiles>COc1ccc(N[C@H](c2ccc(-c3ccccc3)cc2)[C@H](C)C(=O)N(C)C)c(OC)c1</smiles> 

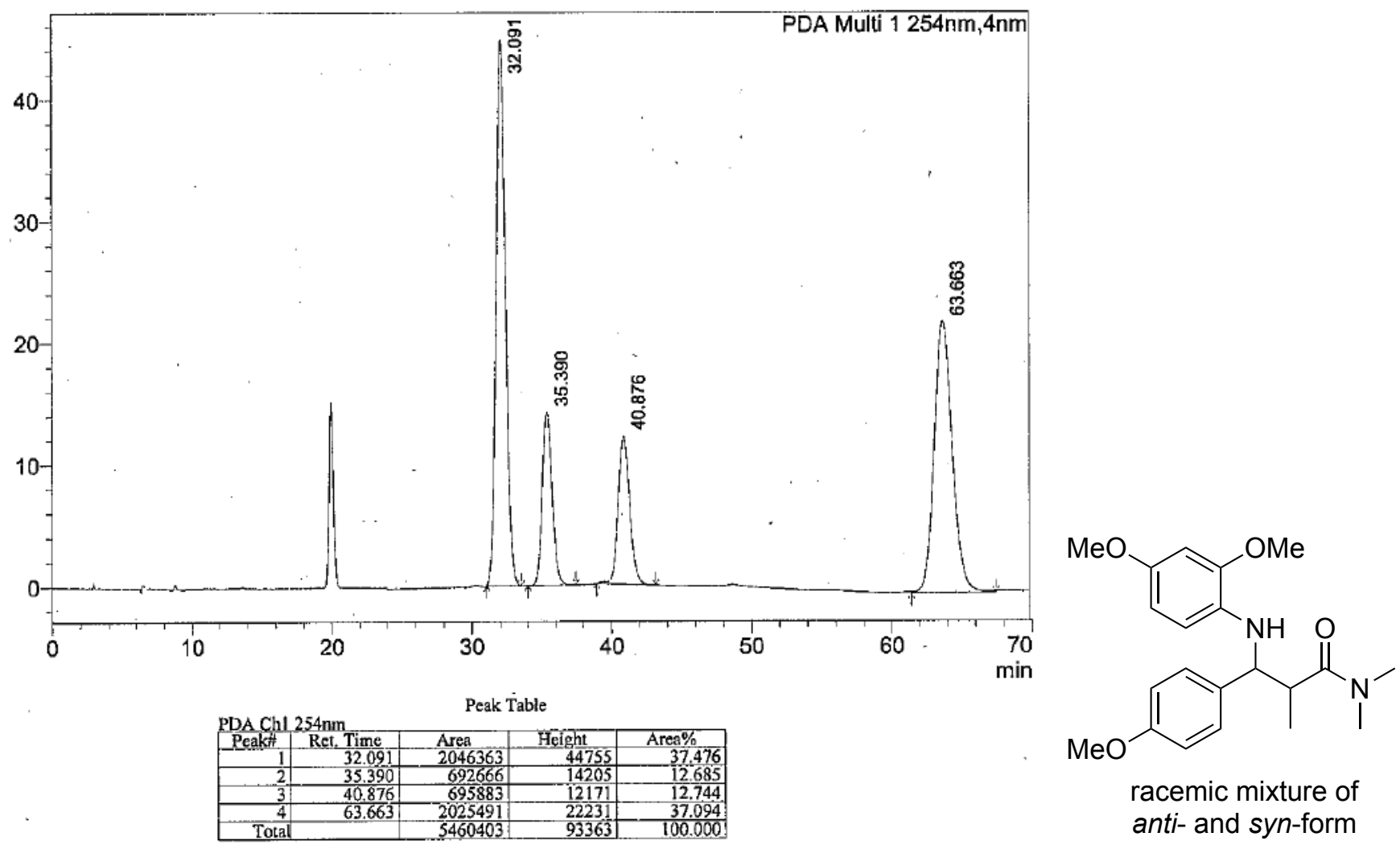

MAU

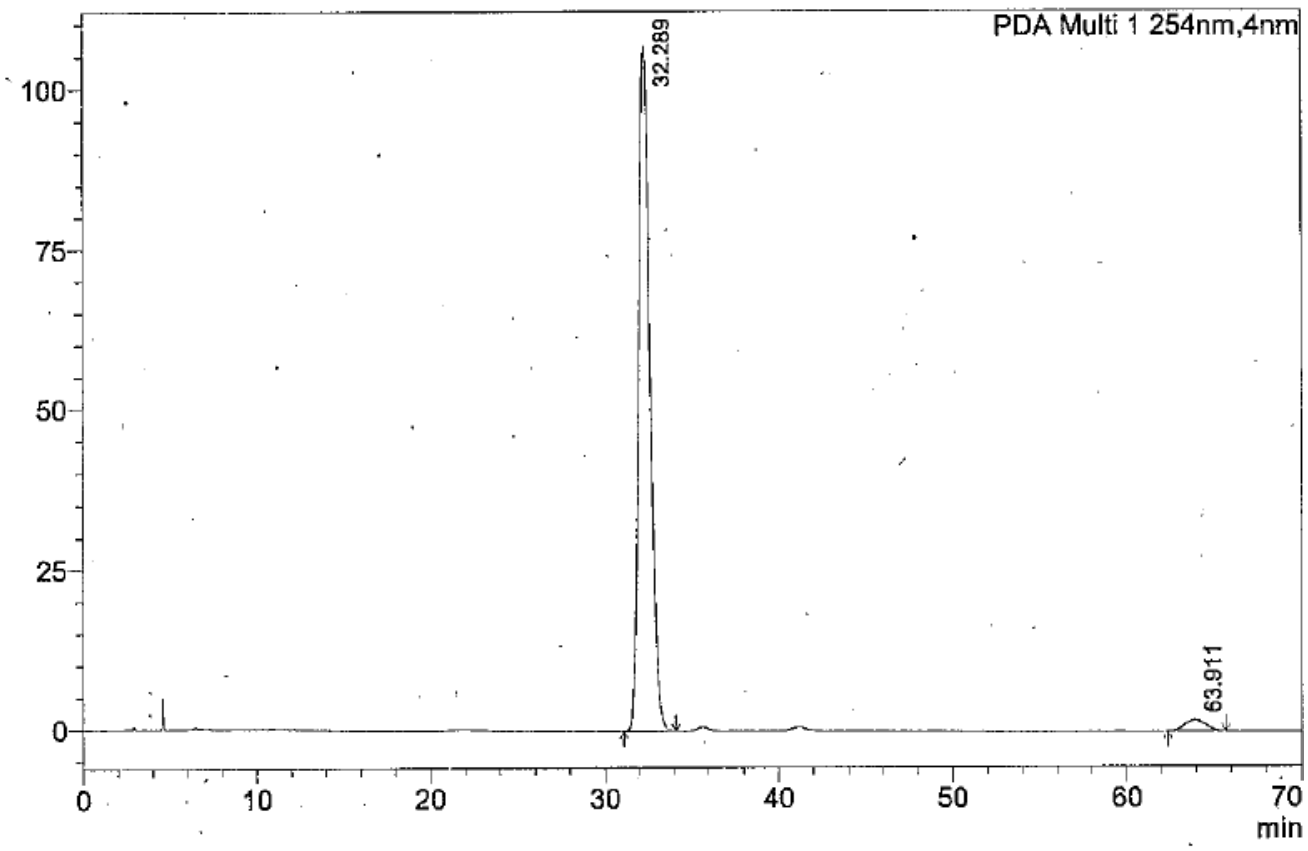<smiles>COc1ccc([C@H](Nc2ccc(OC)cc2OC)[C@H](C)C(=O)N(C)C)cc1</smiles> 
mAU

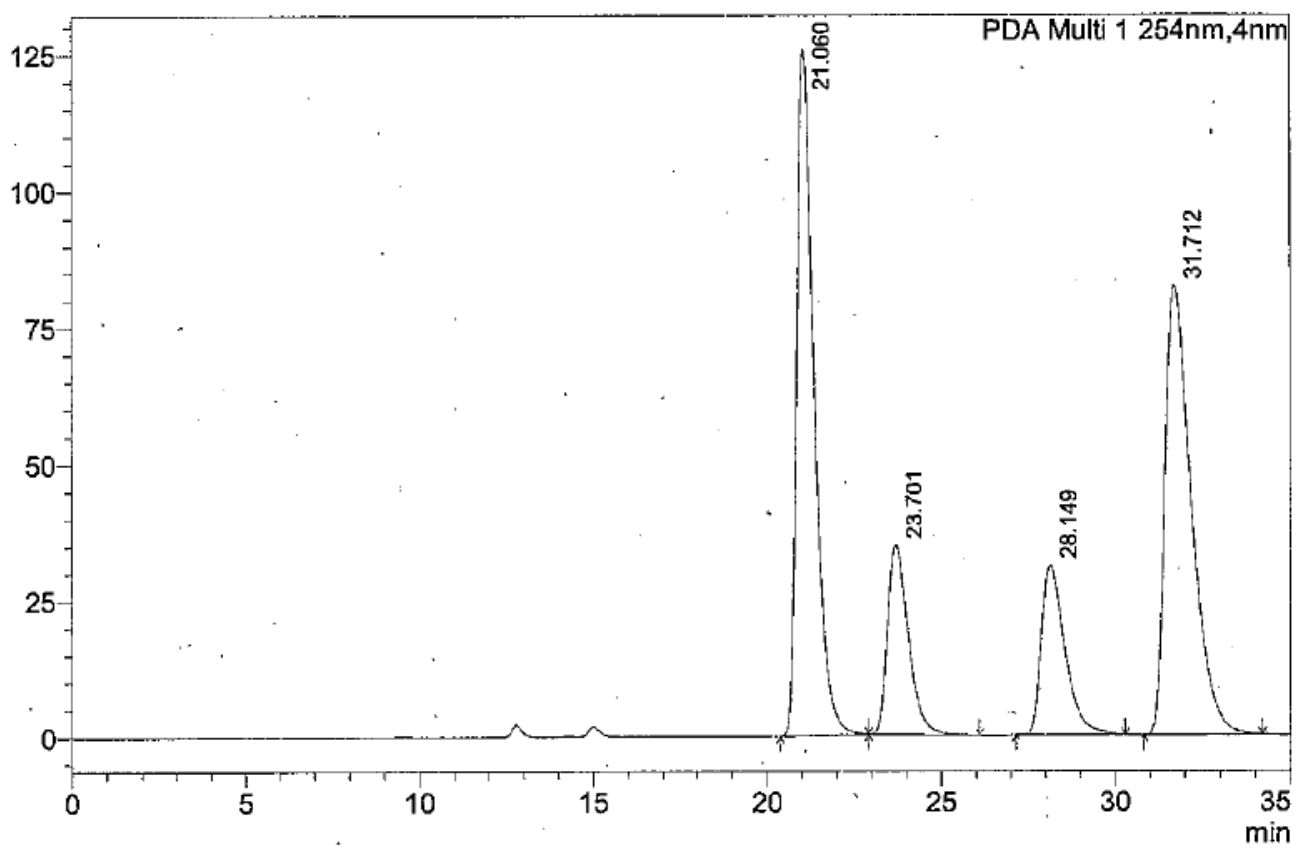

Peak Table

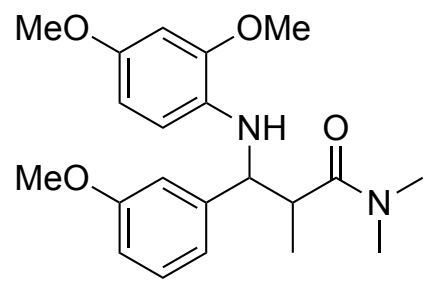

racemic mixture of anti- and syn-form

MAU

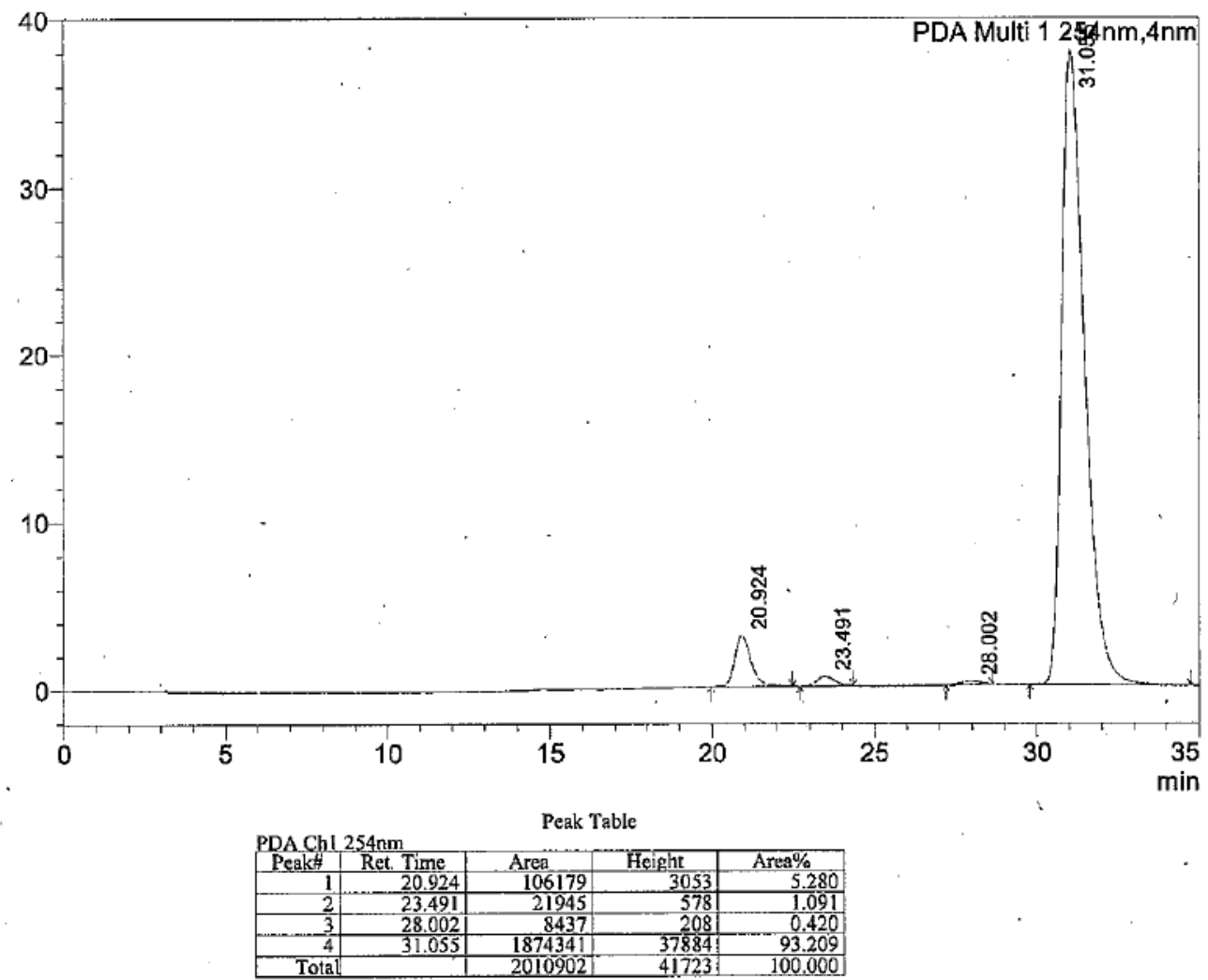<smiles>COc1cccc([C@H](Nc2ccc(OC)cc2OC)[C@H](C)C(=O)N(C)C)c1</smiles> 
mAU

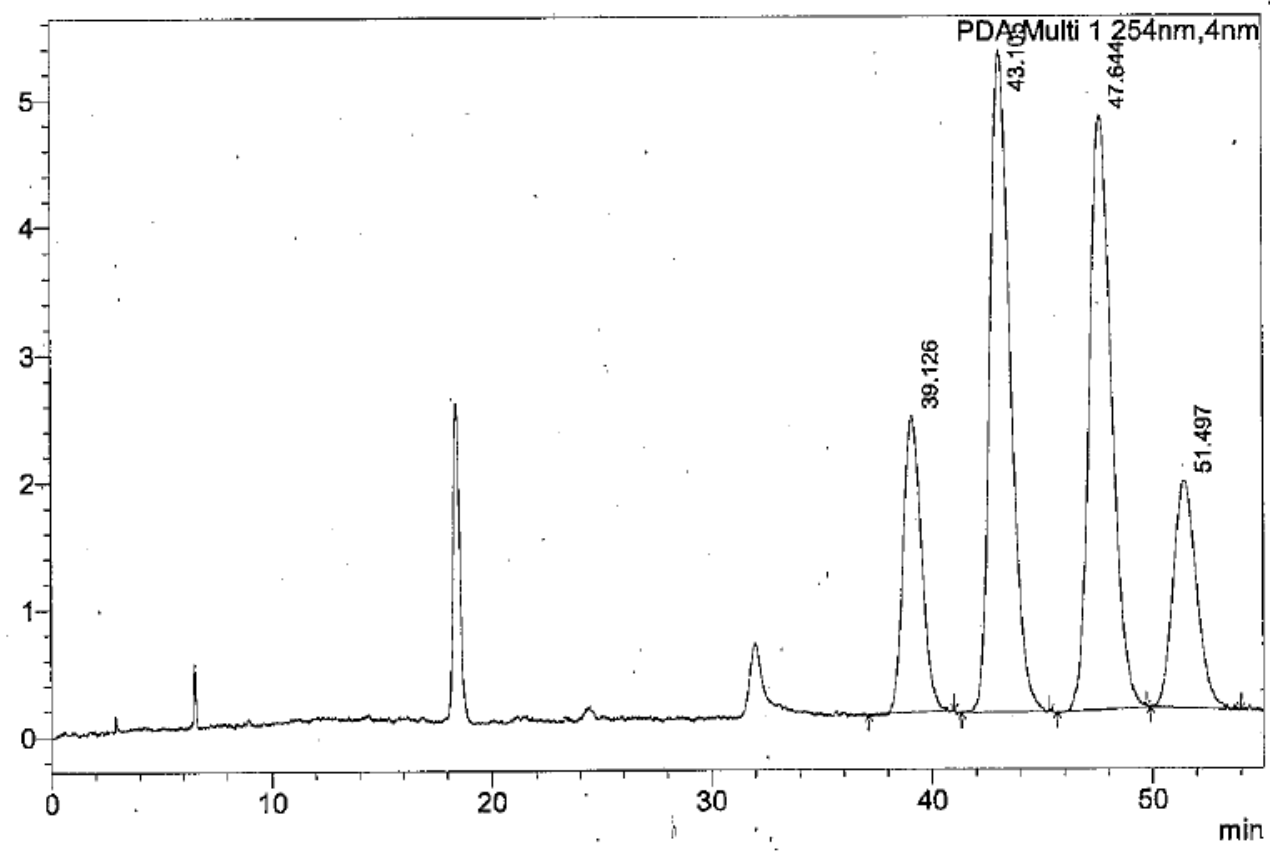

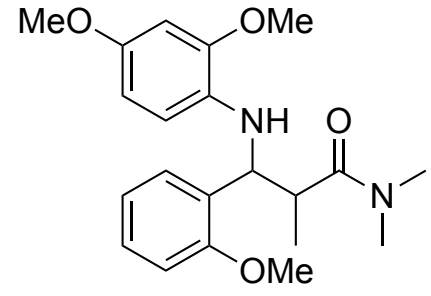

racemic mixture of anti- and syn-form

mAU

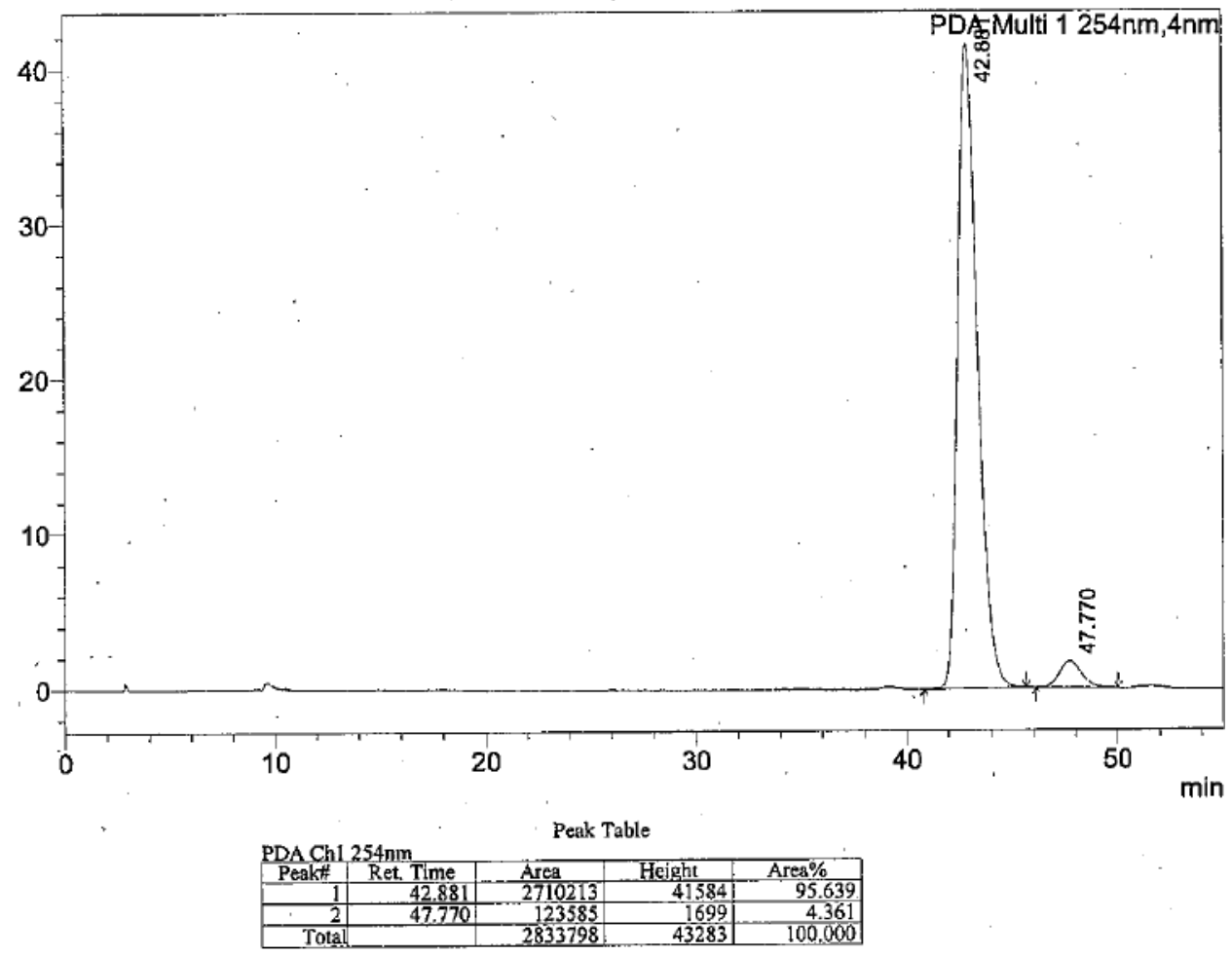

$\mathrm{MeO}$<smiles>COc1ccccc1N</smiles><smiles>C/C=C/N[C@H](c1ccccc1OC)[C@H](C)C(=O)N(C)C</smiles> 
mAU
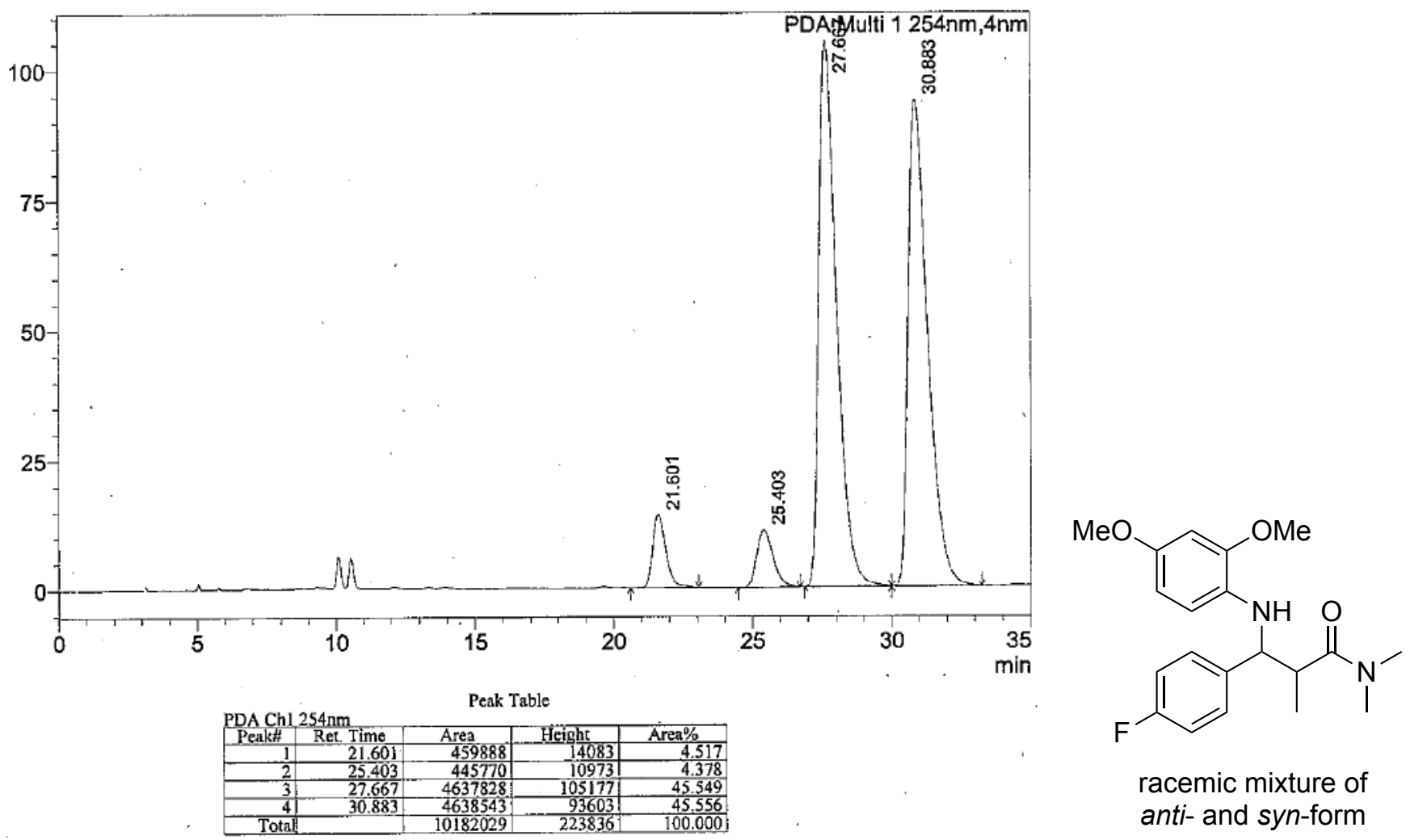

mAU

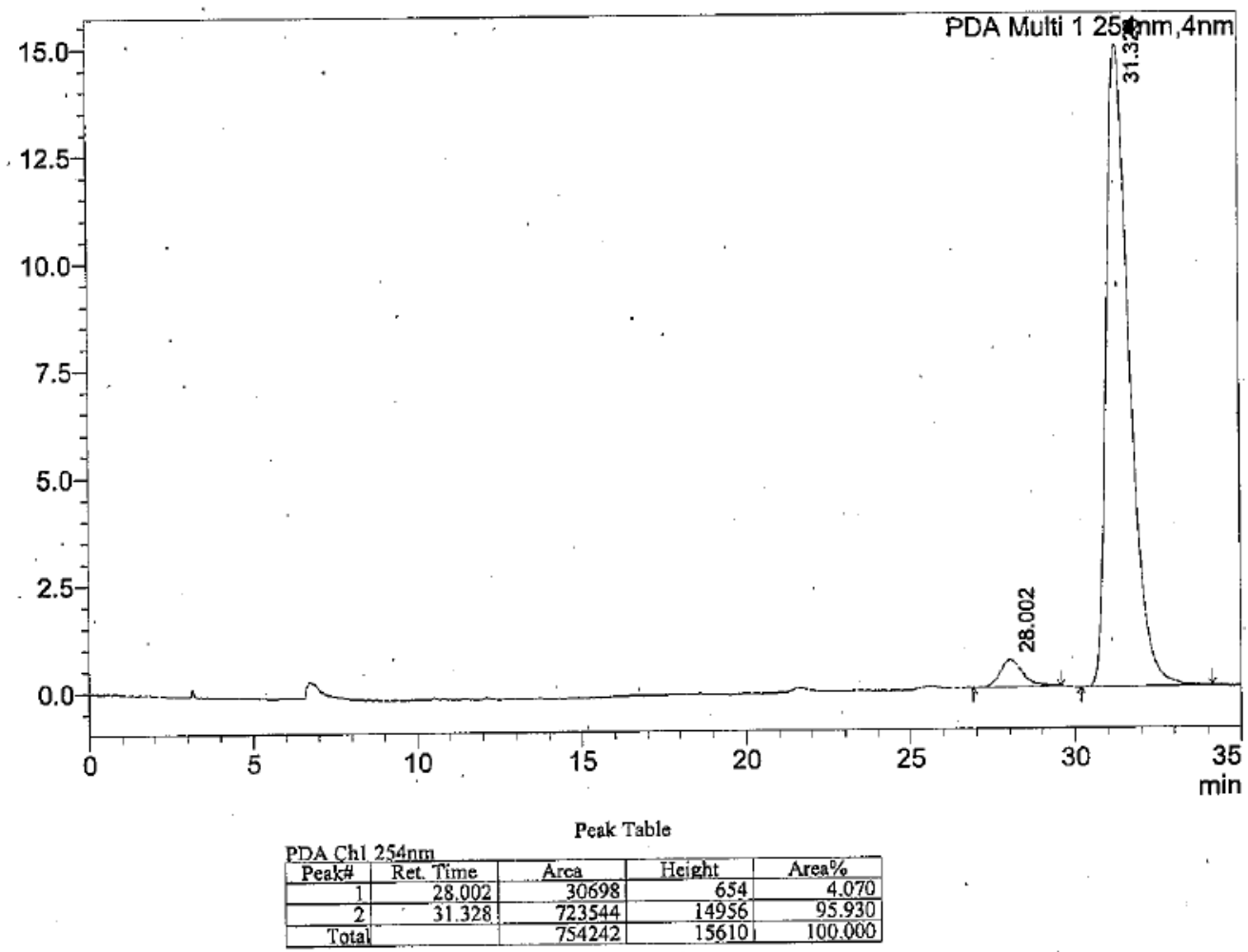<smiles>COc1ccc(N[C@H](c2ccc(F)cc2)[C@H](C)C(=O)N(C)C)c(OC)c1</smiles> 
mAU
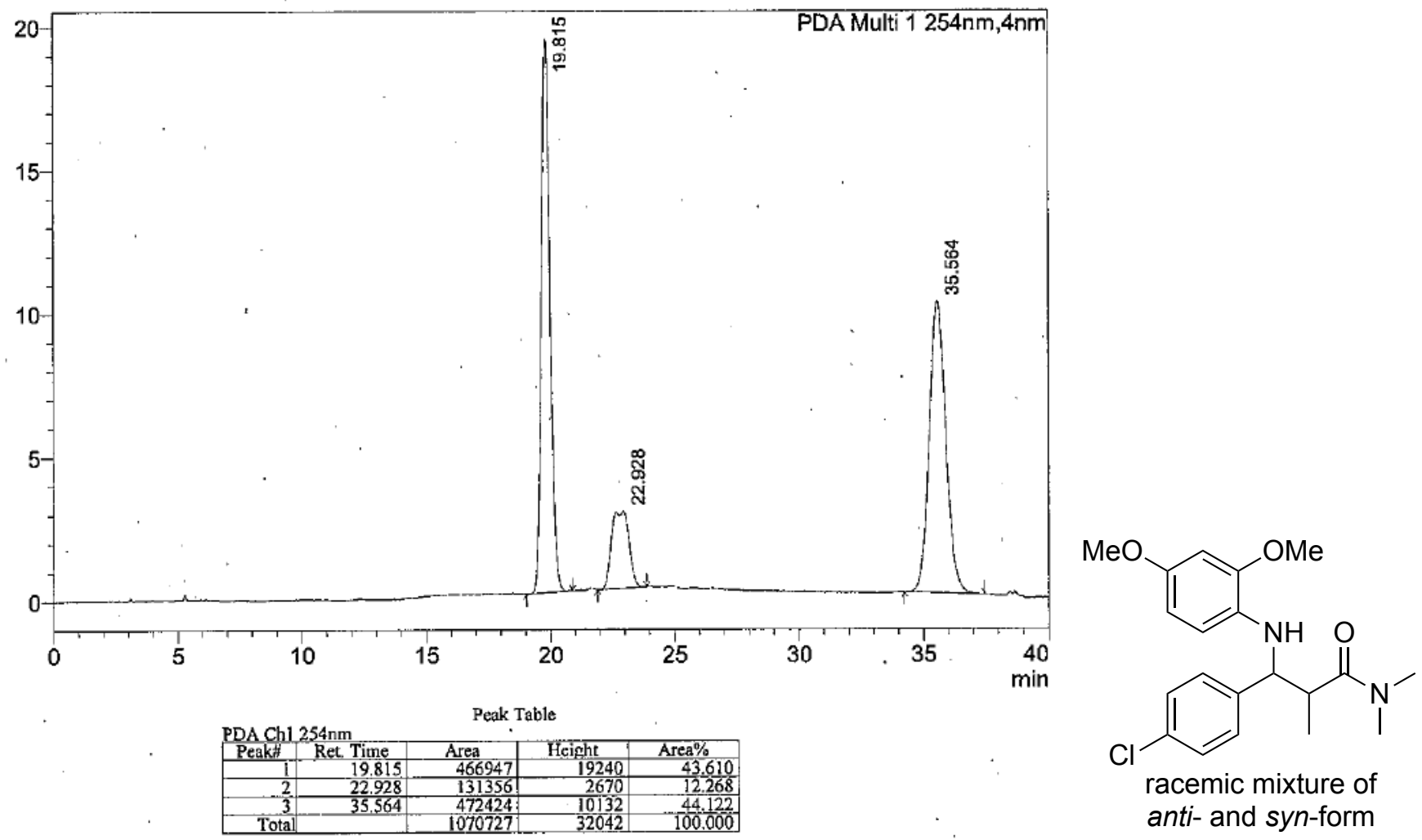

MAU

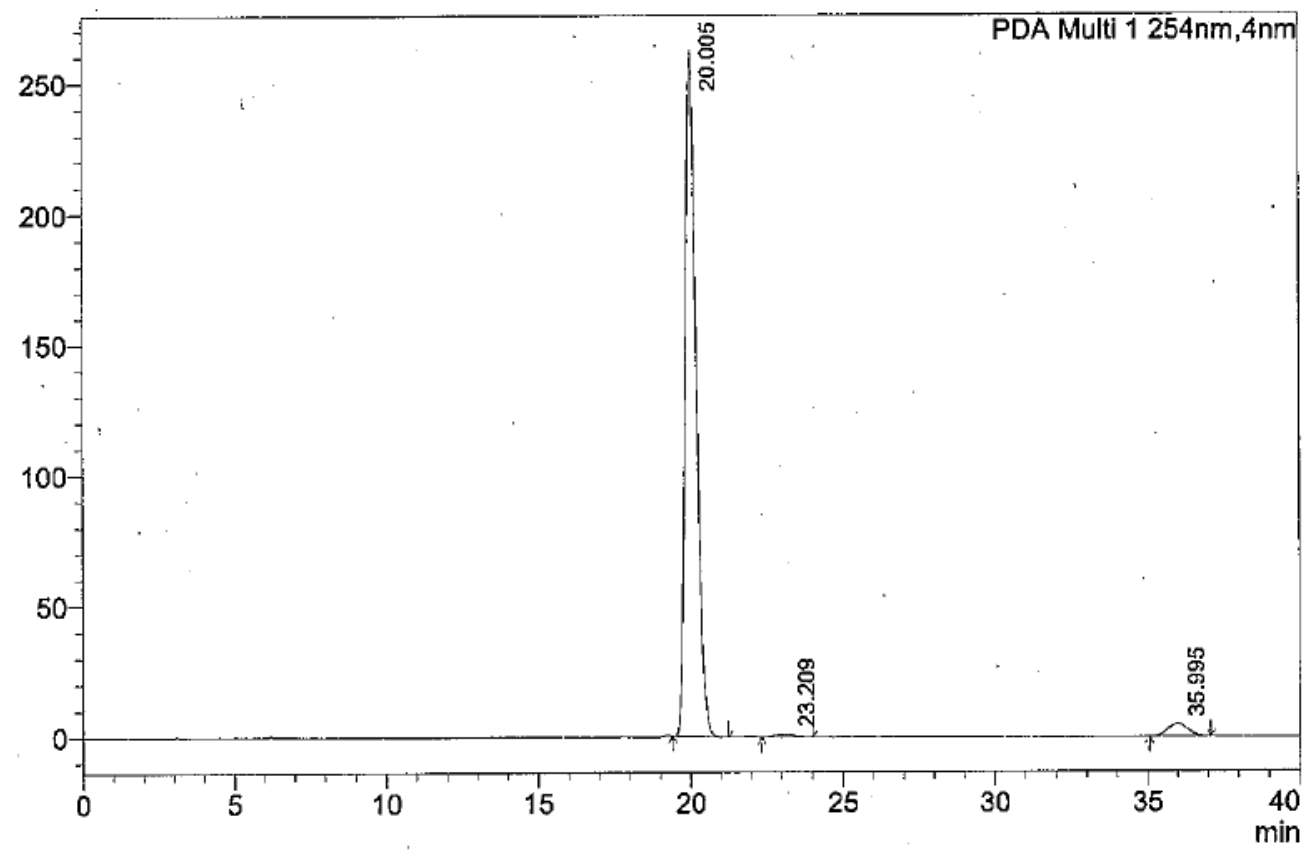<smiles>COc1ccc(N[C@H](c2ccc(Cl)cc2)[C@H](C)C(=O)N(C)C)c(OC)c1</smiles> 
mAU

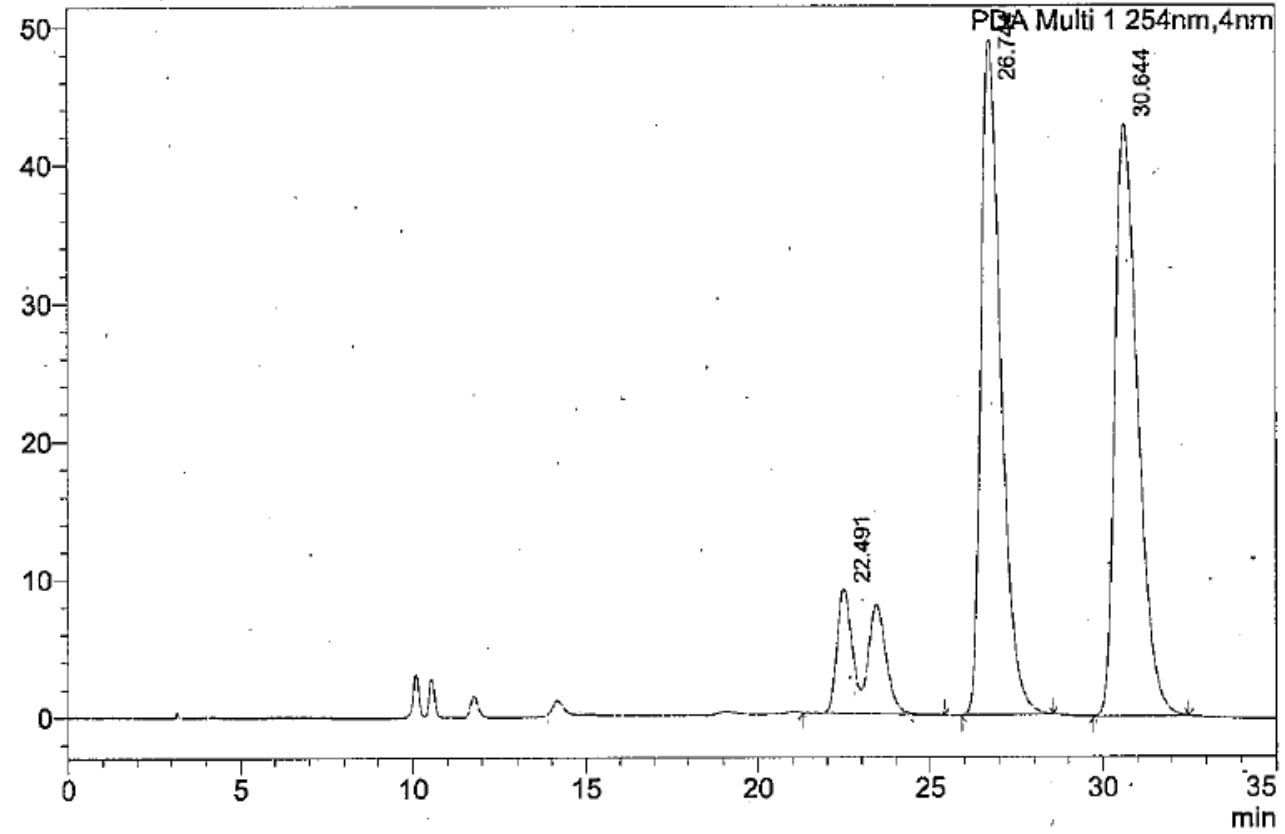

Peak Table
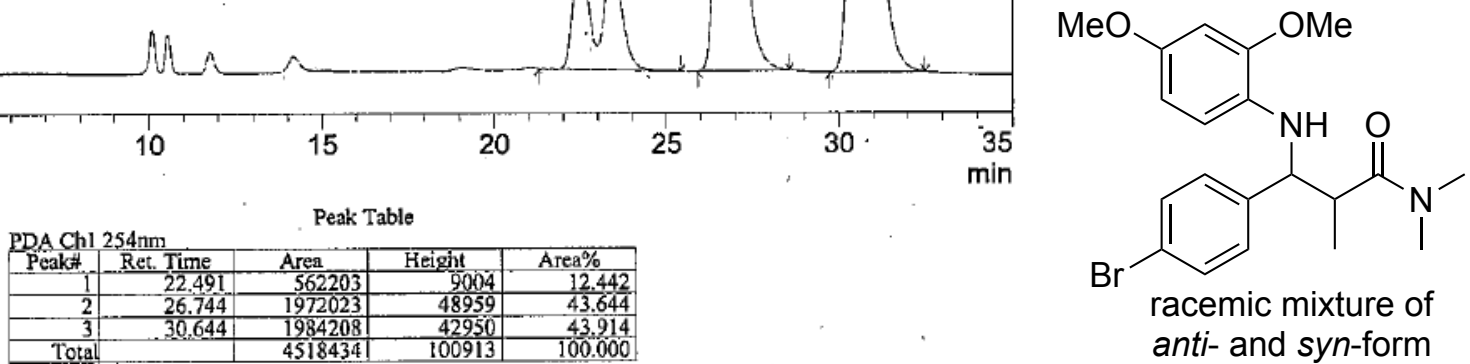

mAU

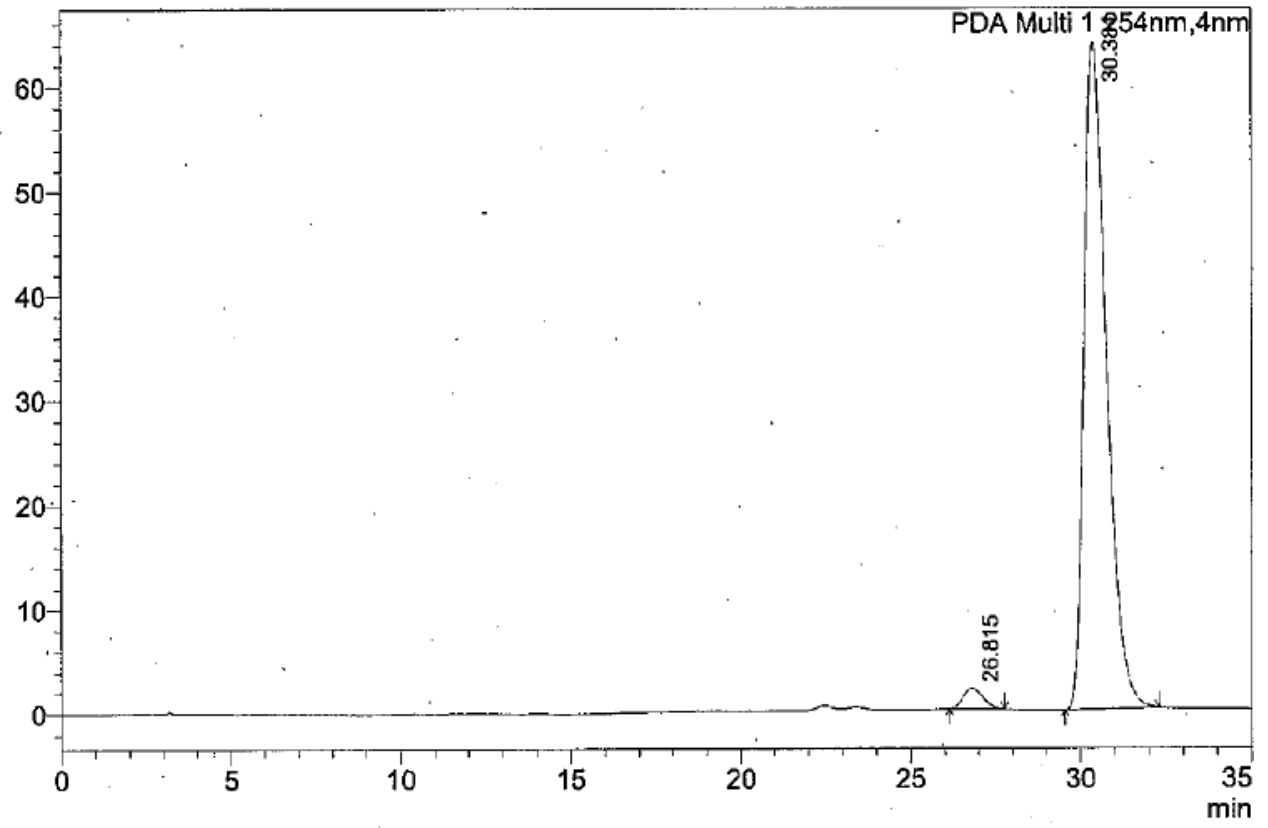<smiles>COc1ccc(N[C@H](c2ccc(Br)cc2)[C@H](C)C(=O)N(C)C)c(OC)c1</smiles> 
mAU
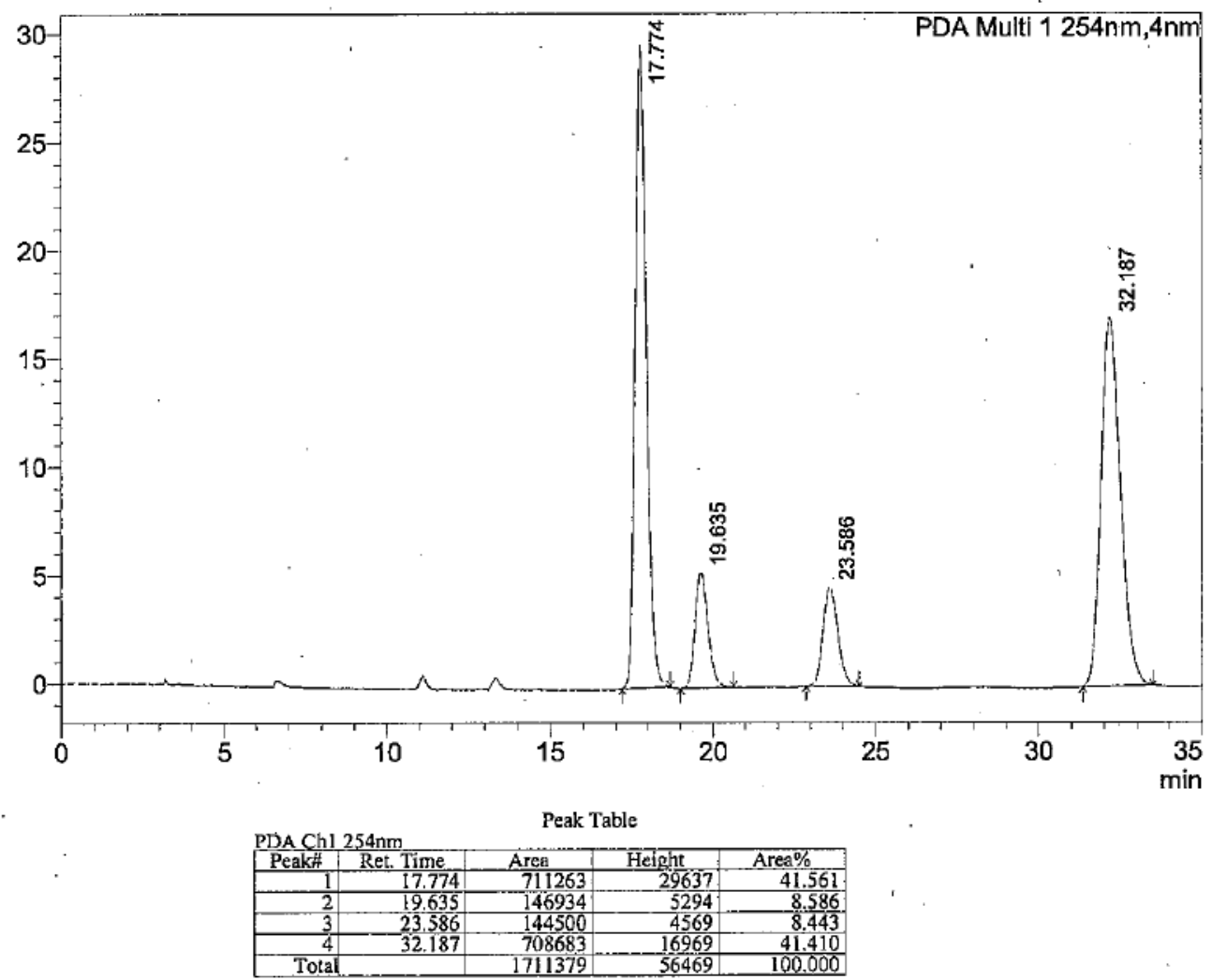

mAU

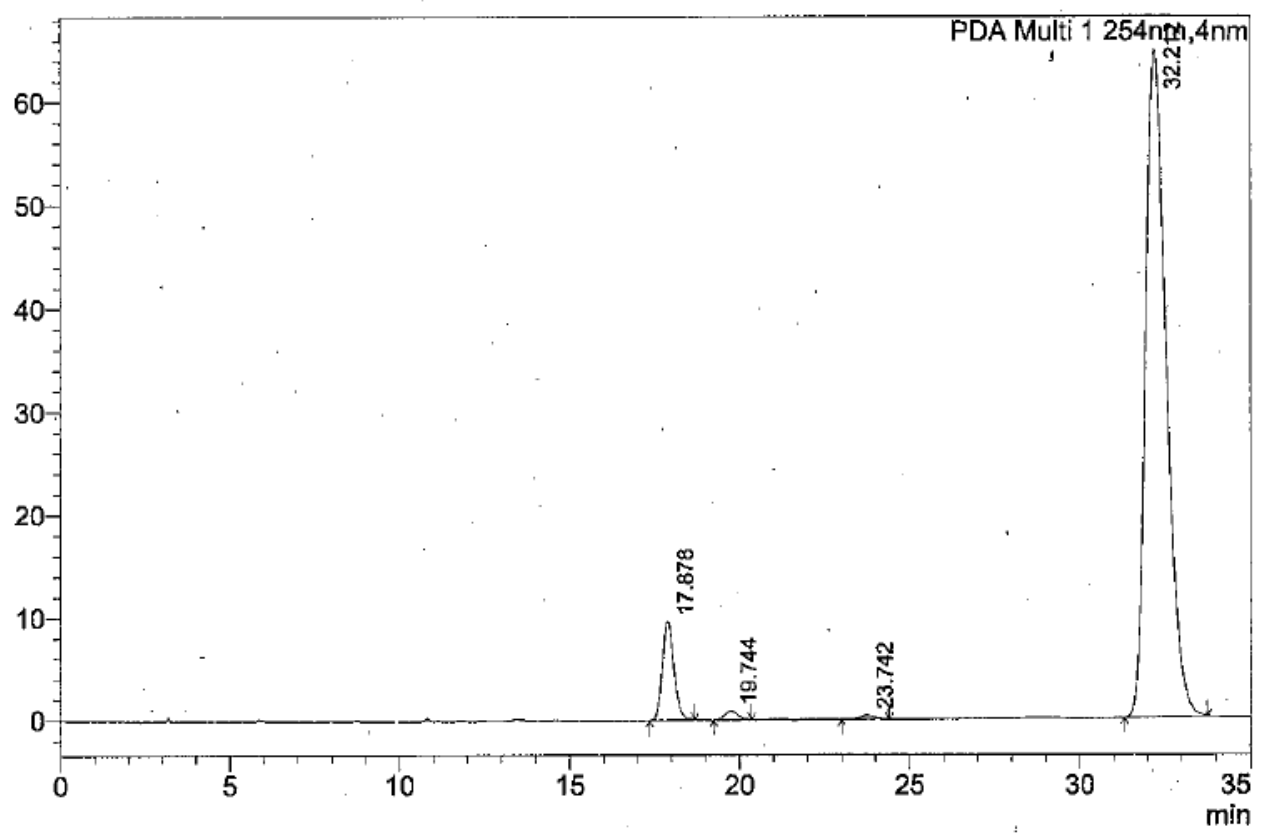<smiles>COc1ccc(N[C@H](c2cccc(Br)c2)[C@H](C)C(=O)N(C)C)c(OC)c1</smiles> 
mAU
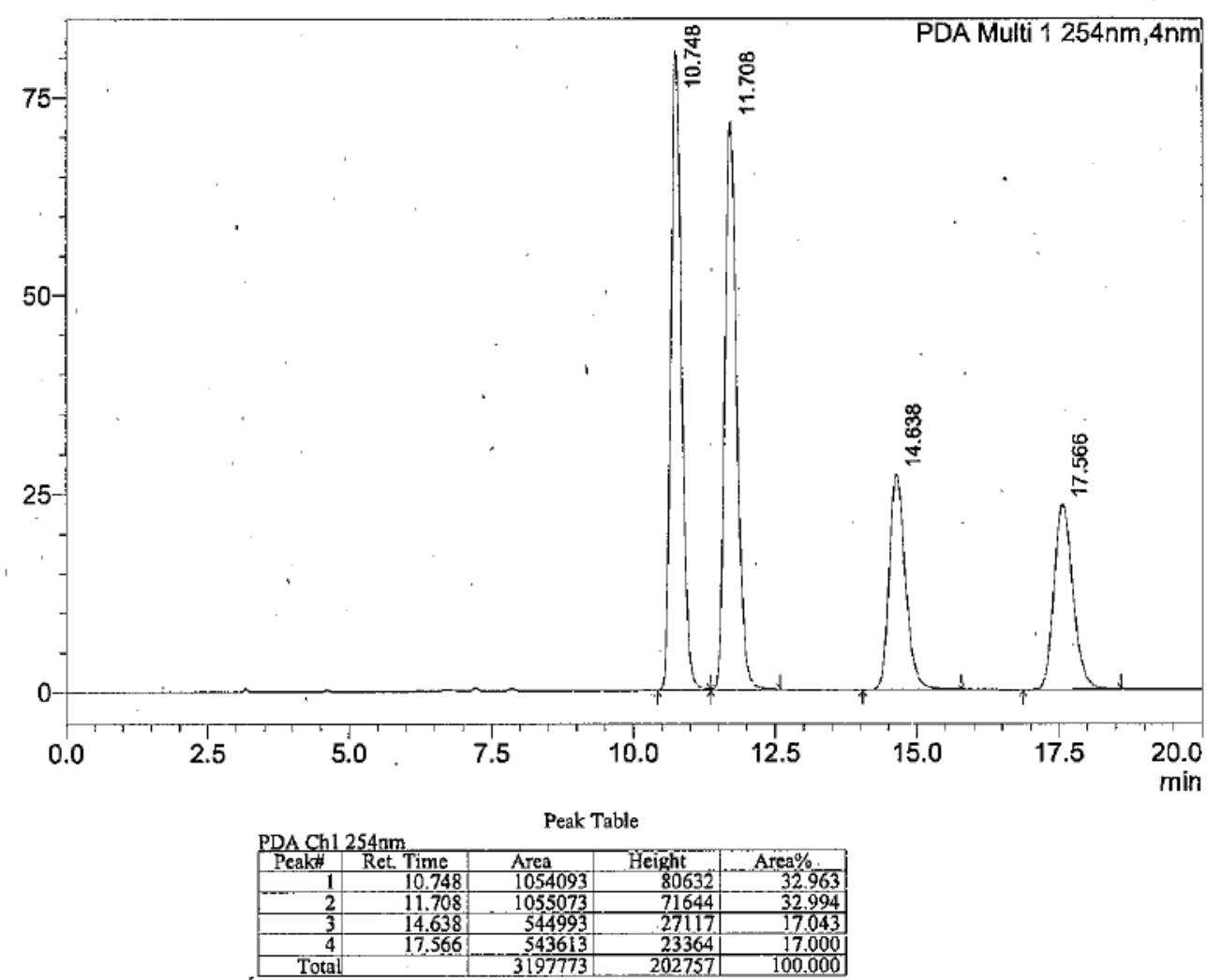

MAU

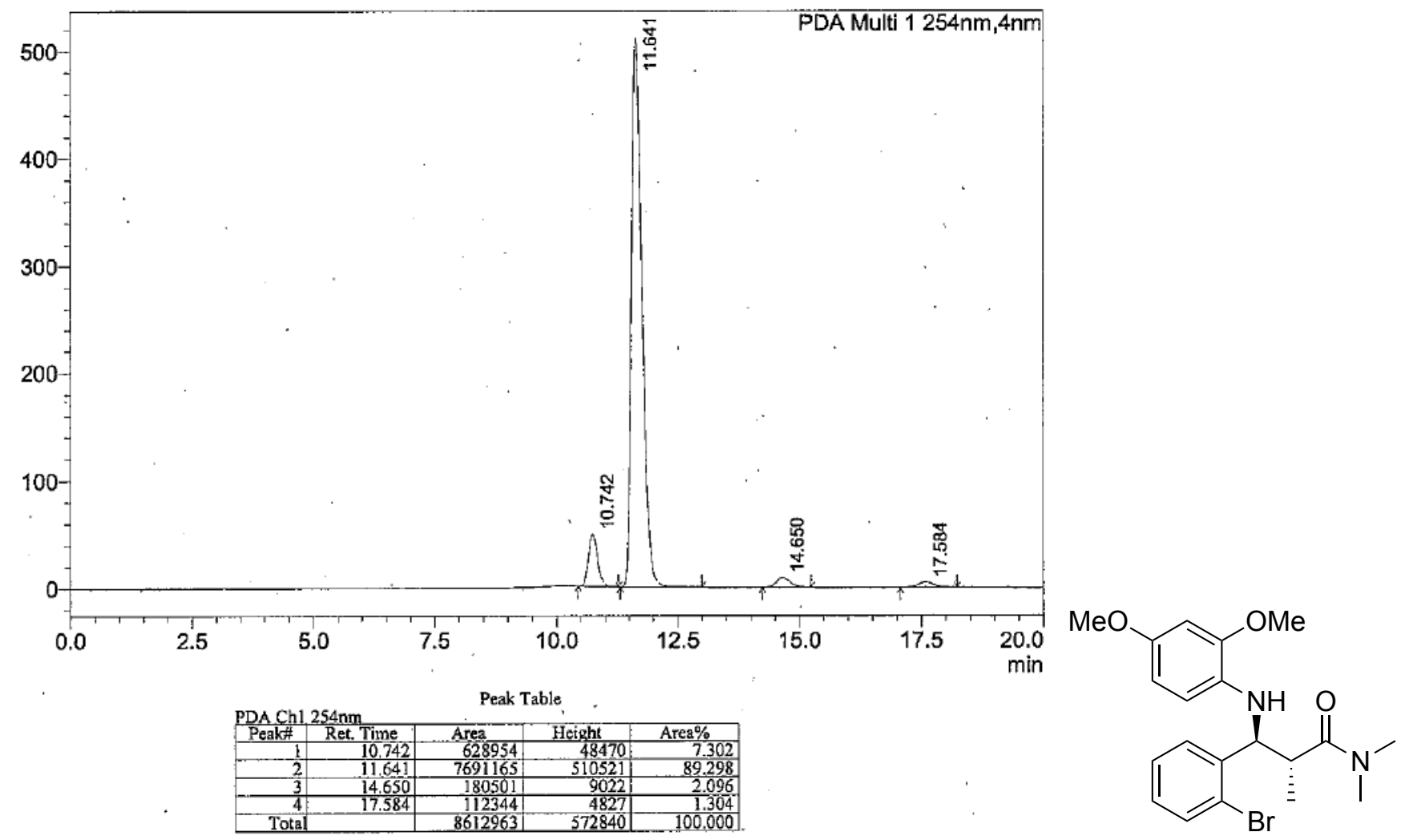


MAU
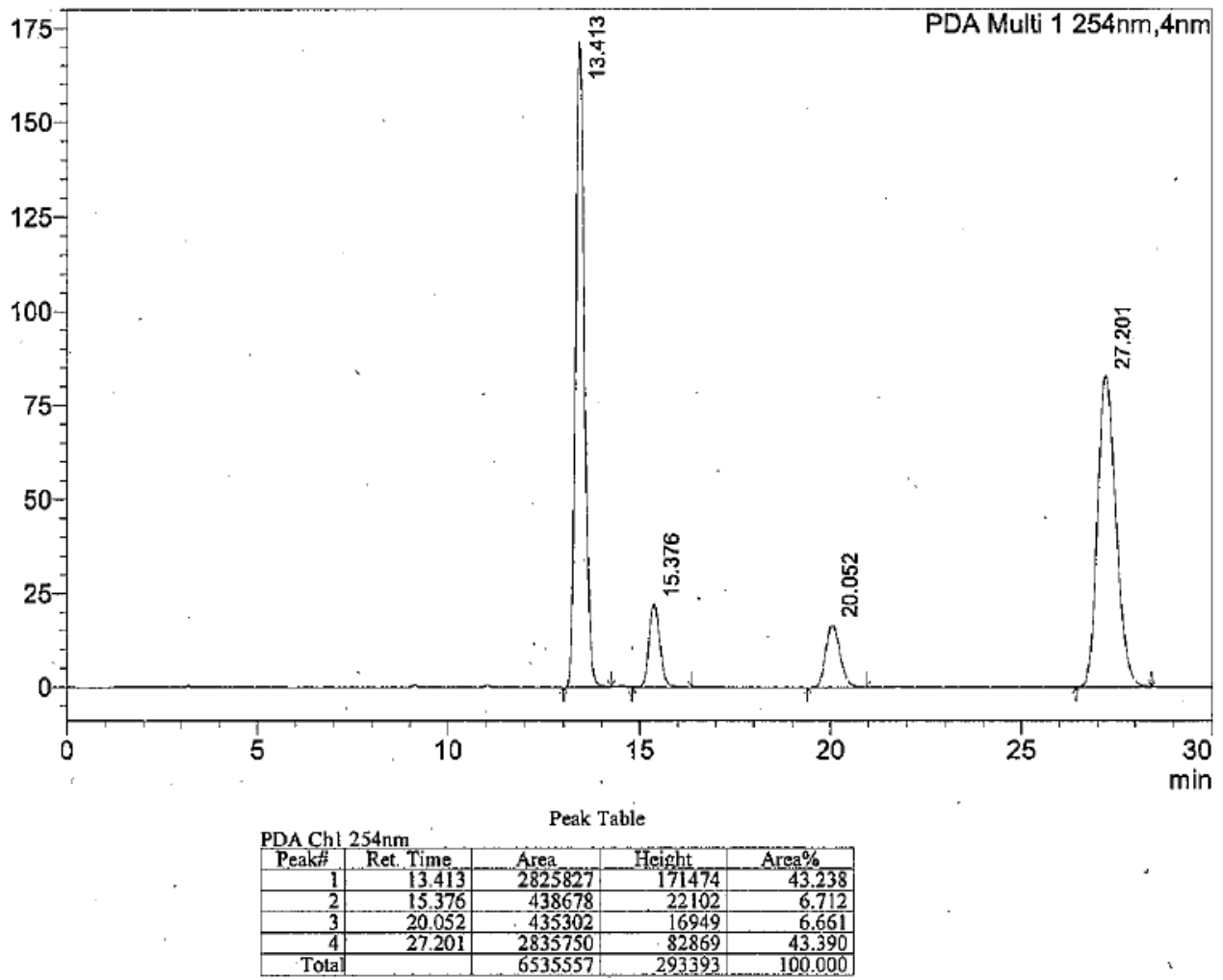

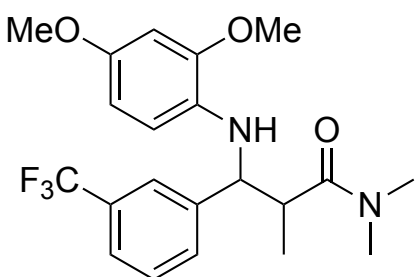

racemic mixture of anti- and syn-form

mAU

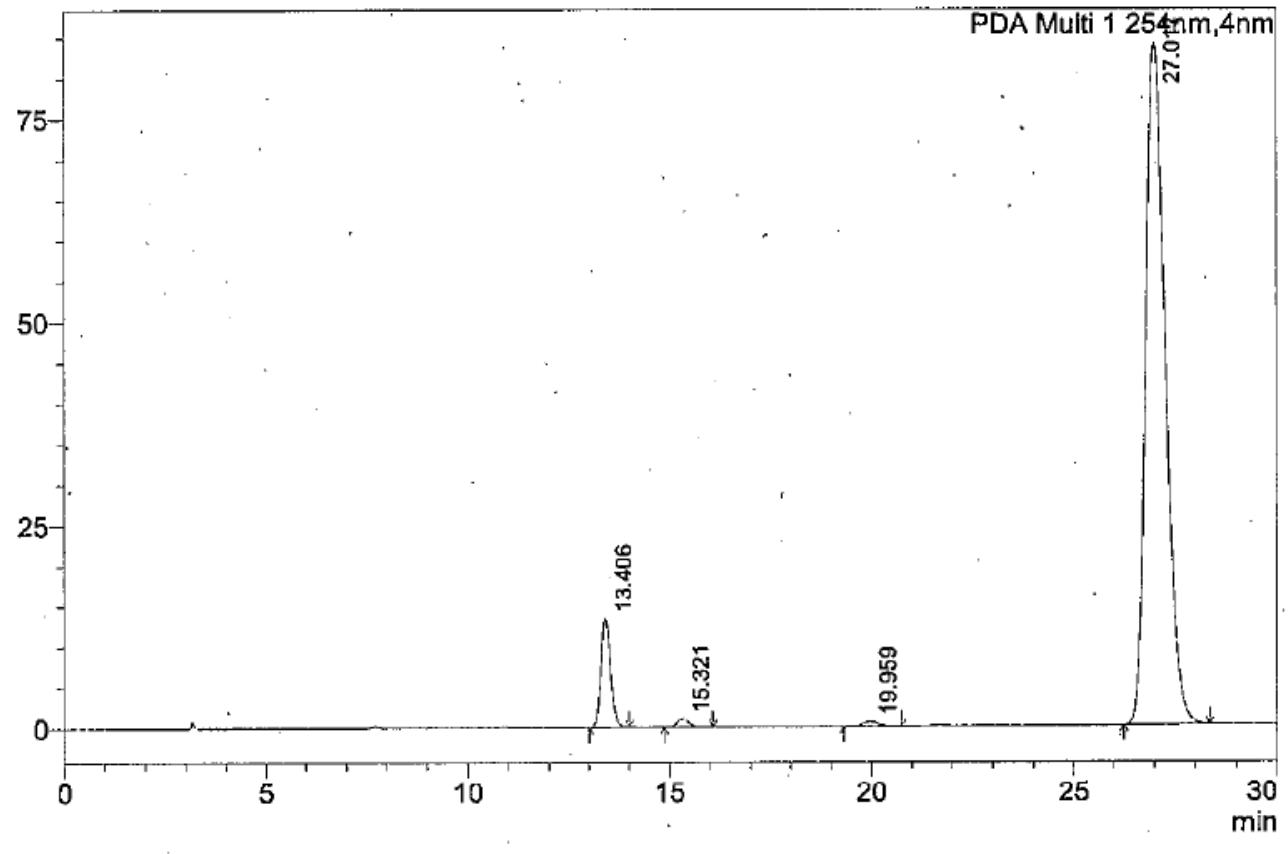<smiles>COc1ccc(N[C@H](c2cccc(C(F)(F)F)c2)[C@H](C)C(=O)N(C)C)c(OC)c1</smiles> 
mAU
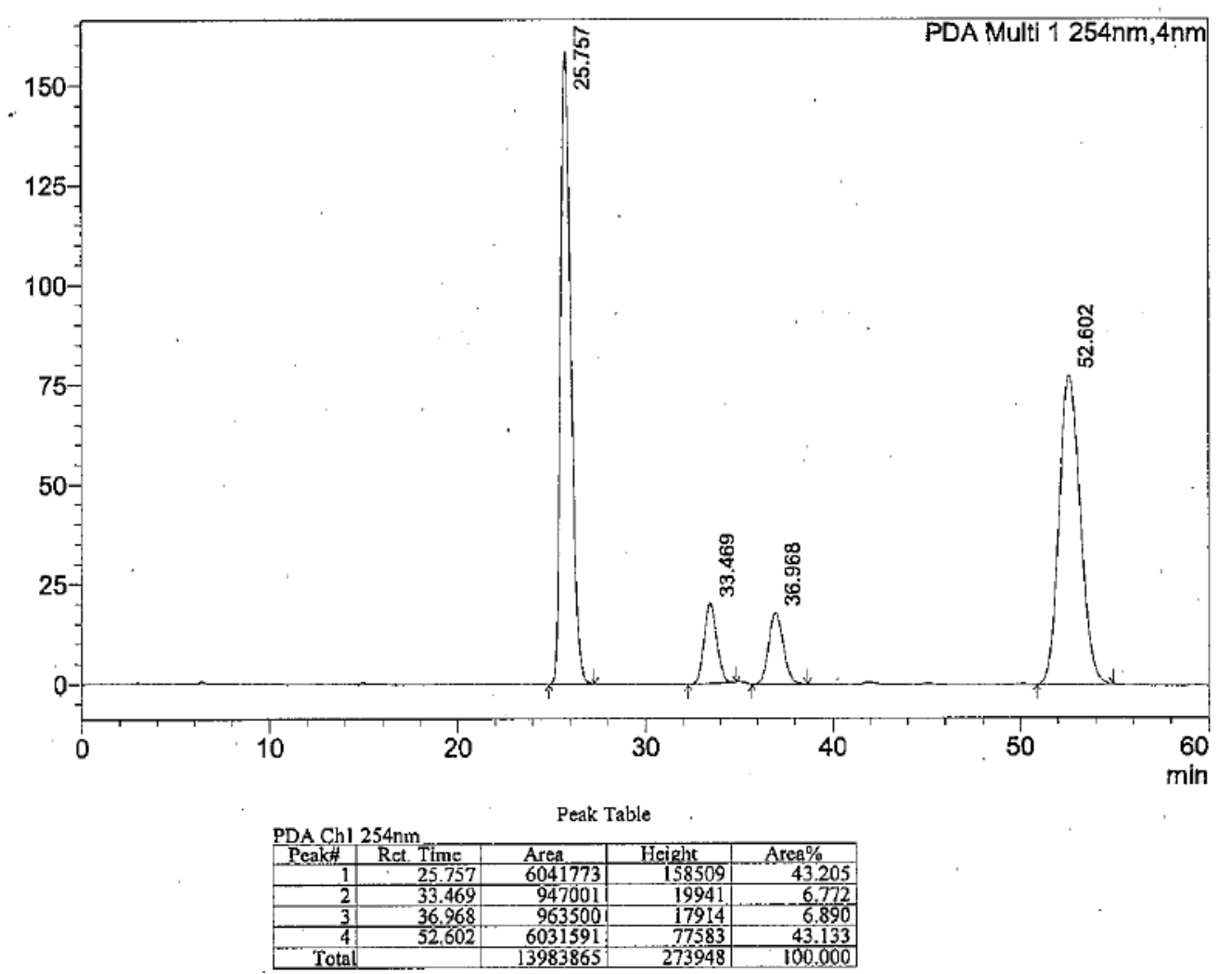

mAU

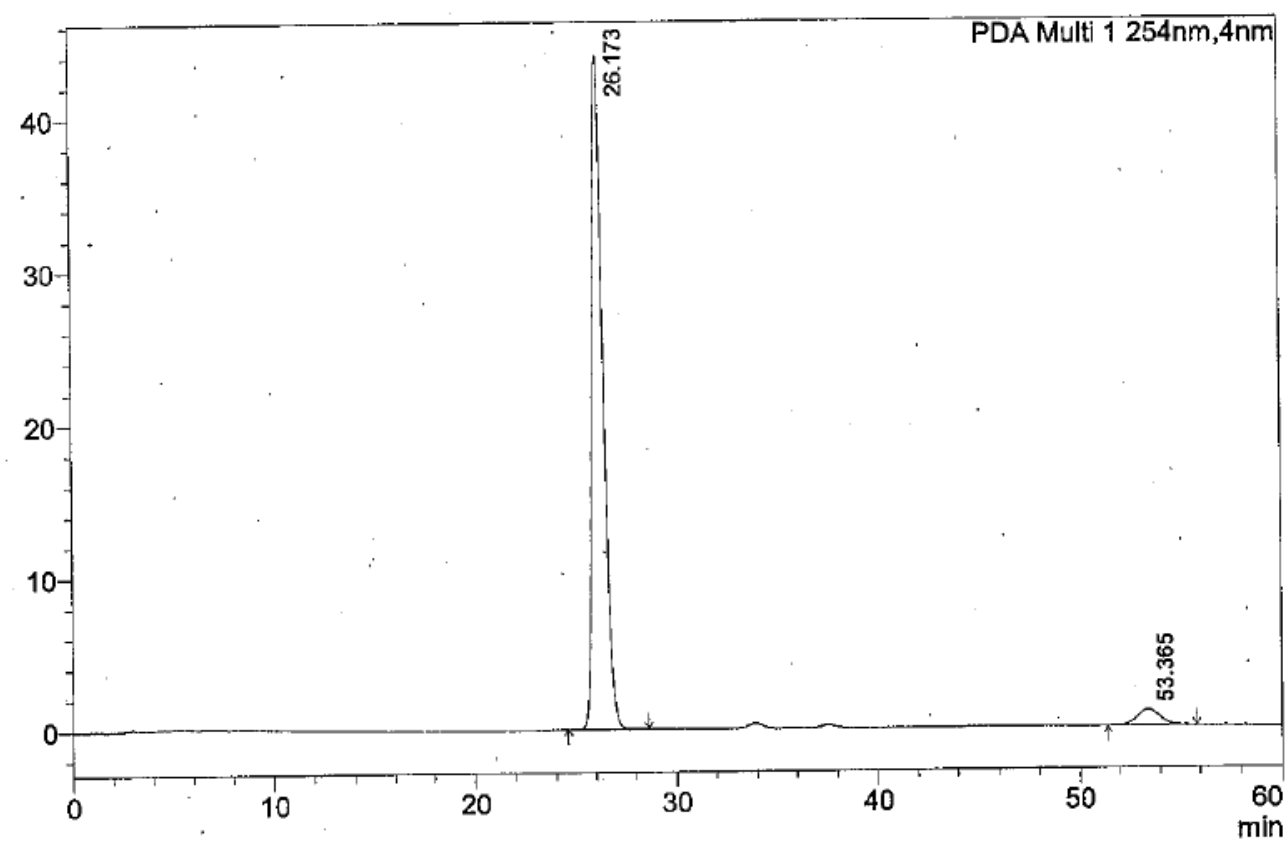<smiles>COc1ccc(NC(c2ccc3ccccc3c2)C(C)C(=O)N(C)C)c(OC)c1</smiles>

racemic mixture of anti- and syn-form

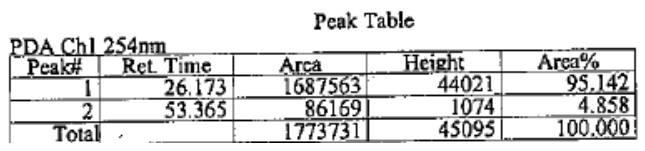<smiles>COc1ccc(N[C@H](c2ccc3ccccc3c2)[C@H](C)C(=O)N(C)C)c(OC)c1</smiles> 
mAU
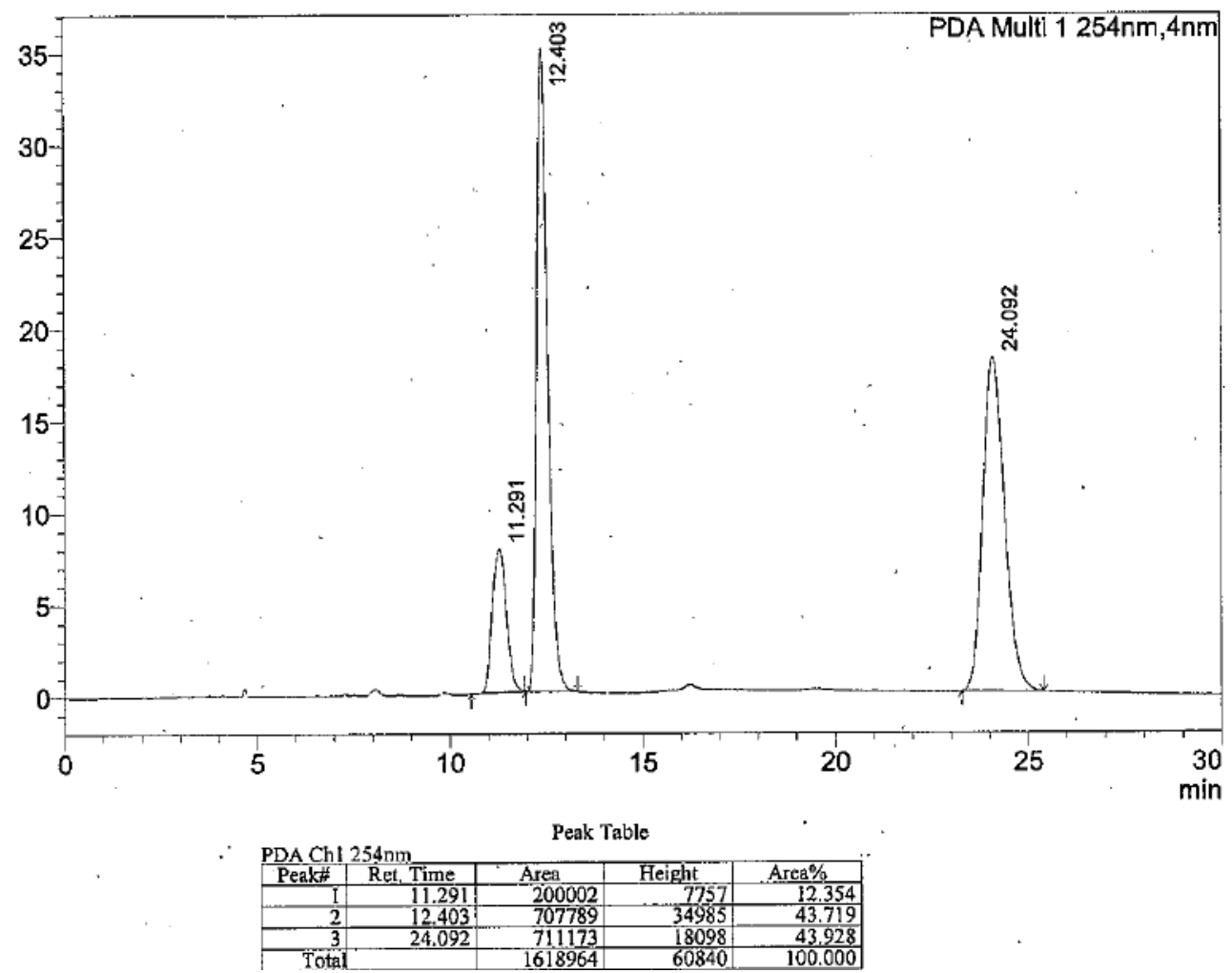<smiles>COc1ccc(NC(c2ccncc2)C(C)C(=O)N(C)C)c(OC)c1</smiles>

racemic mixture of anti- and syn-form
mAU

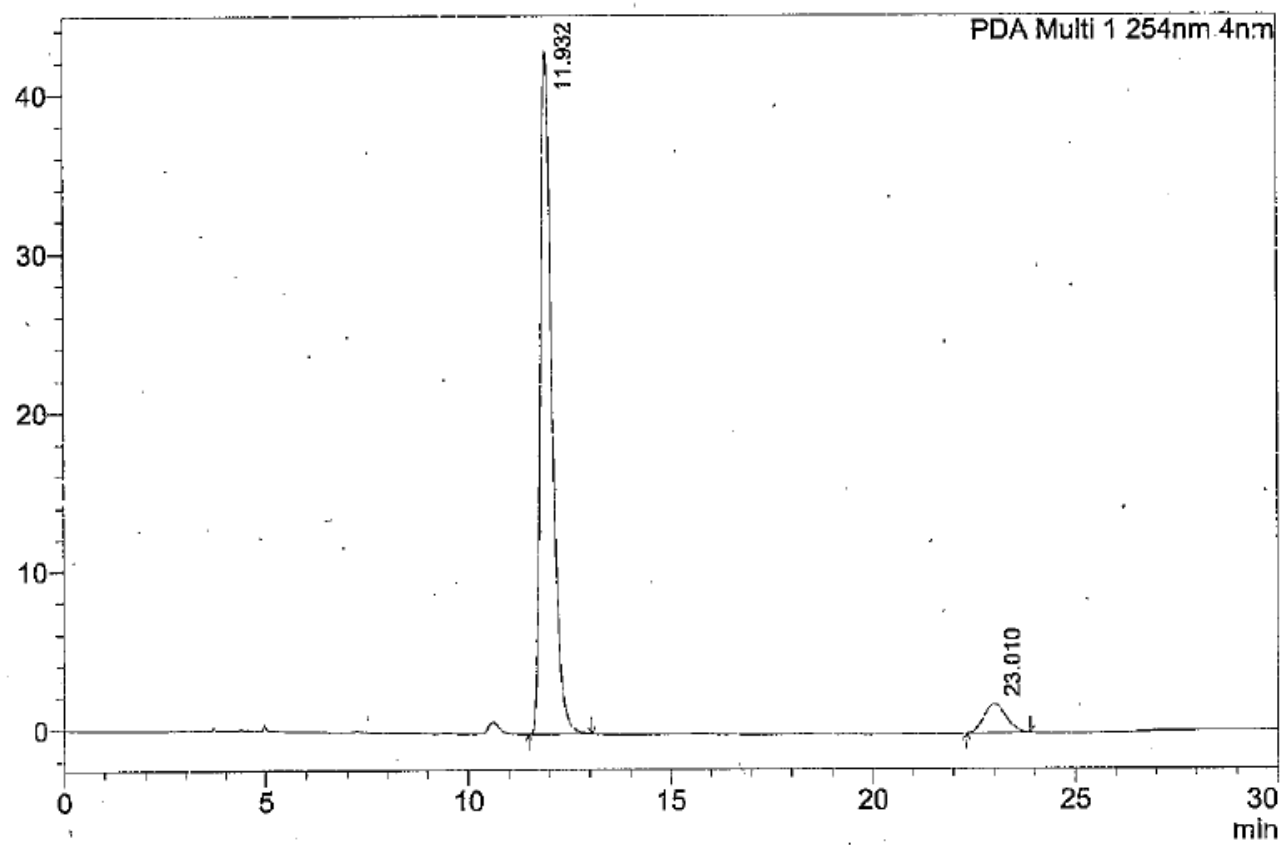

Peak Table<smiles>COc1ccc(N[C@H](c2ccncc2)[C@H](C)C(=O)N(C)C)c(OC)c1</smiles> 
UAU
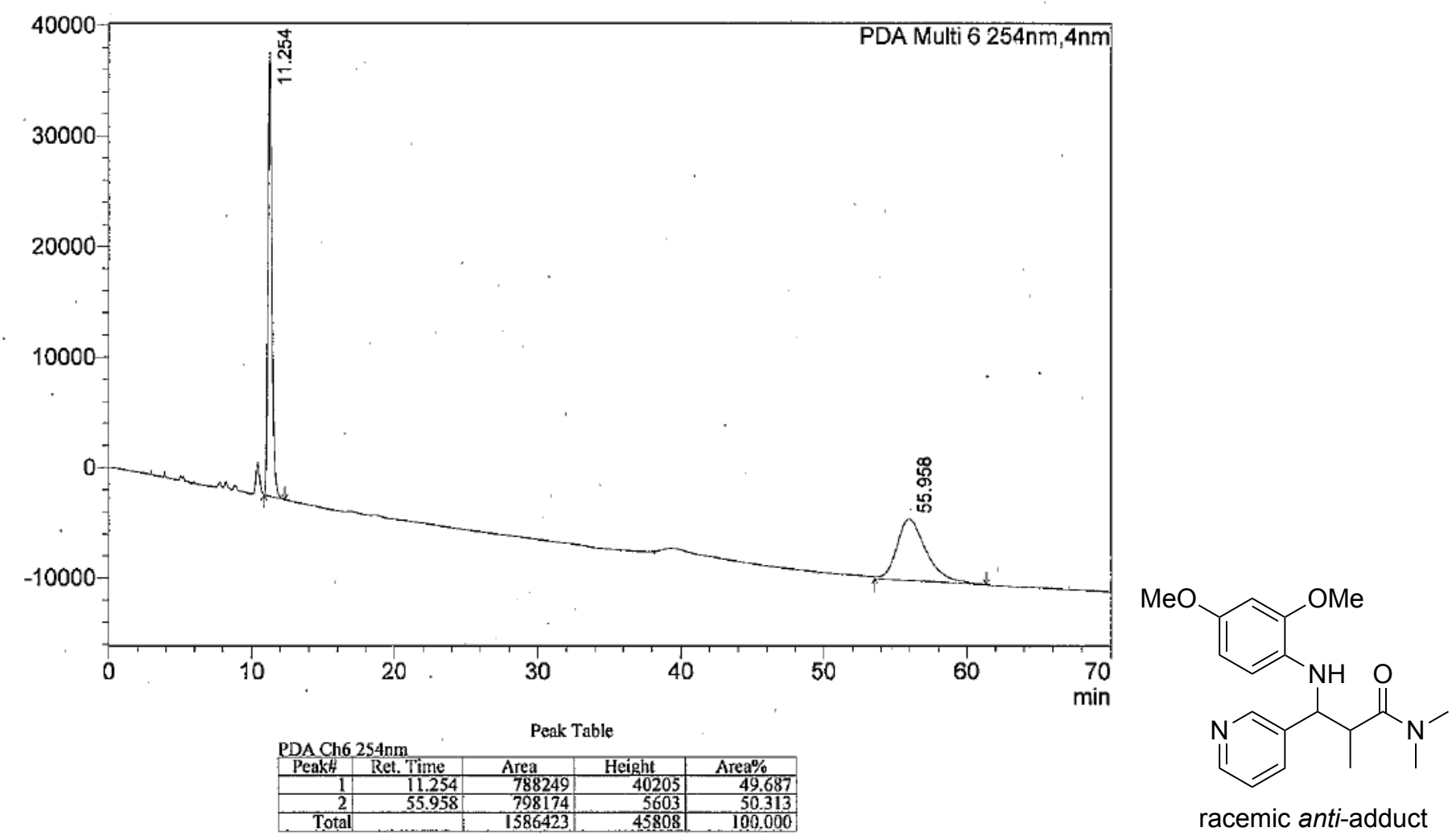

UAU

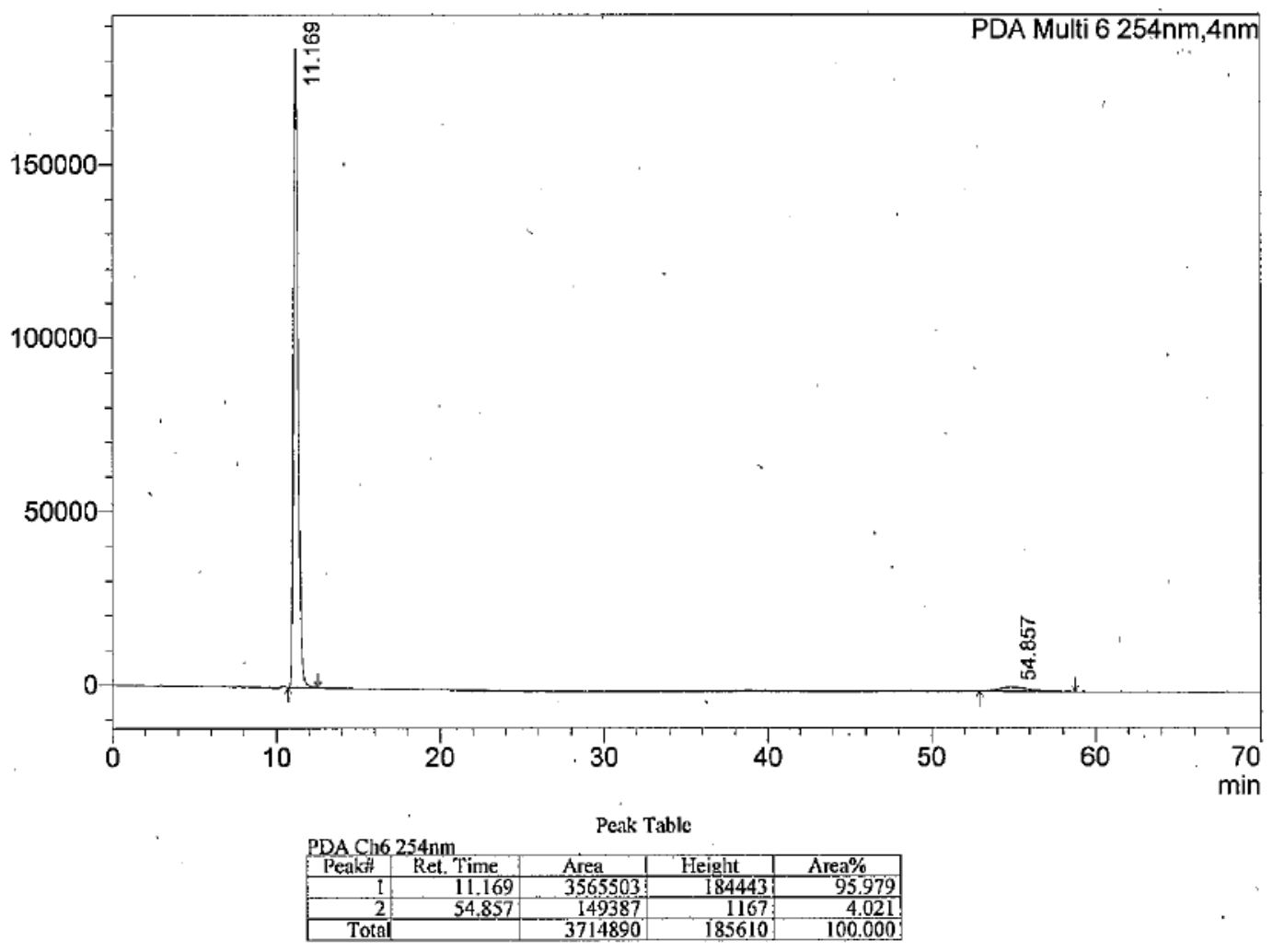

(OMe 
mAU
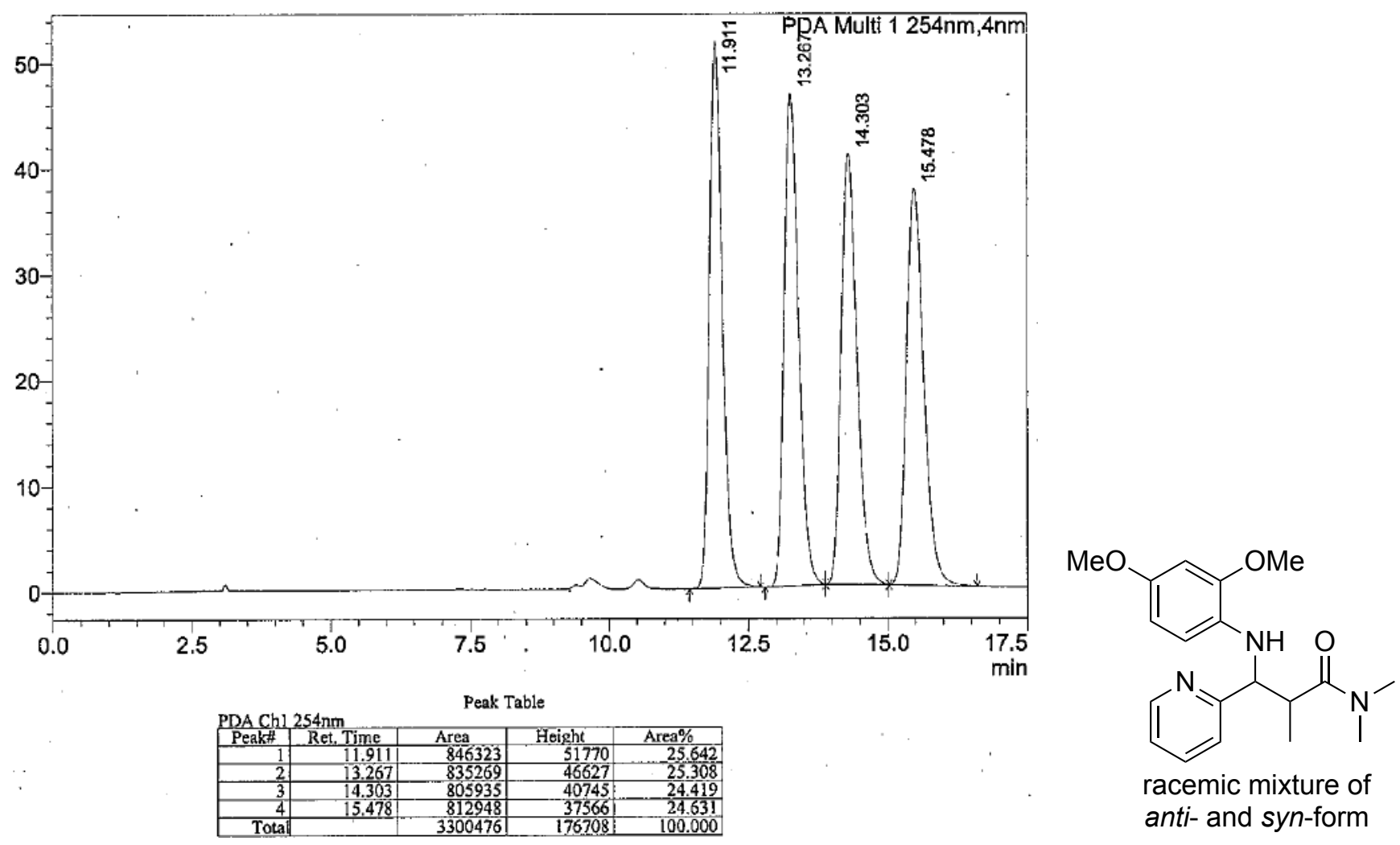

racemic mixture of anti- and syn-form

mAU

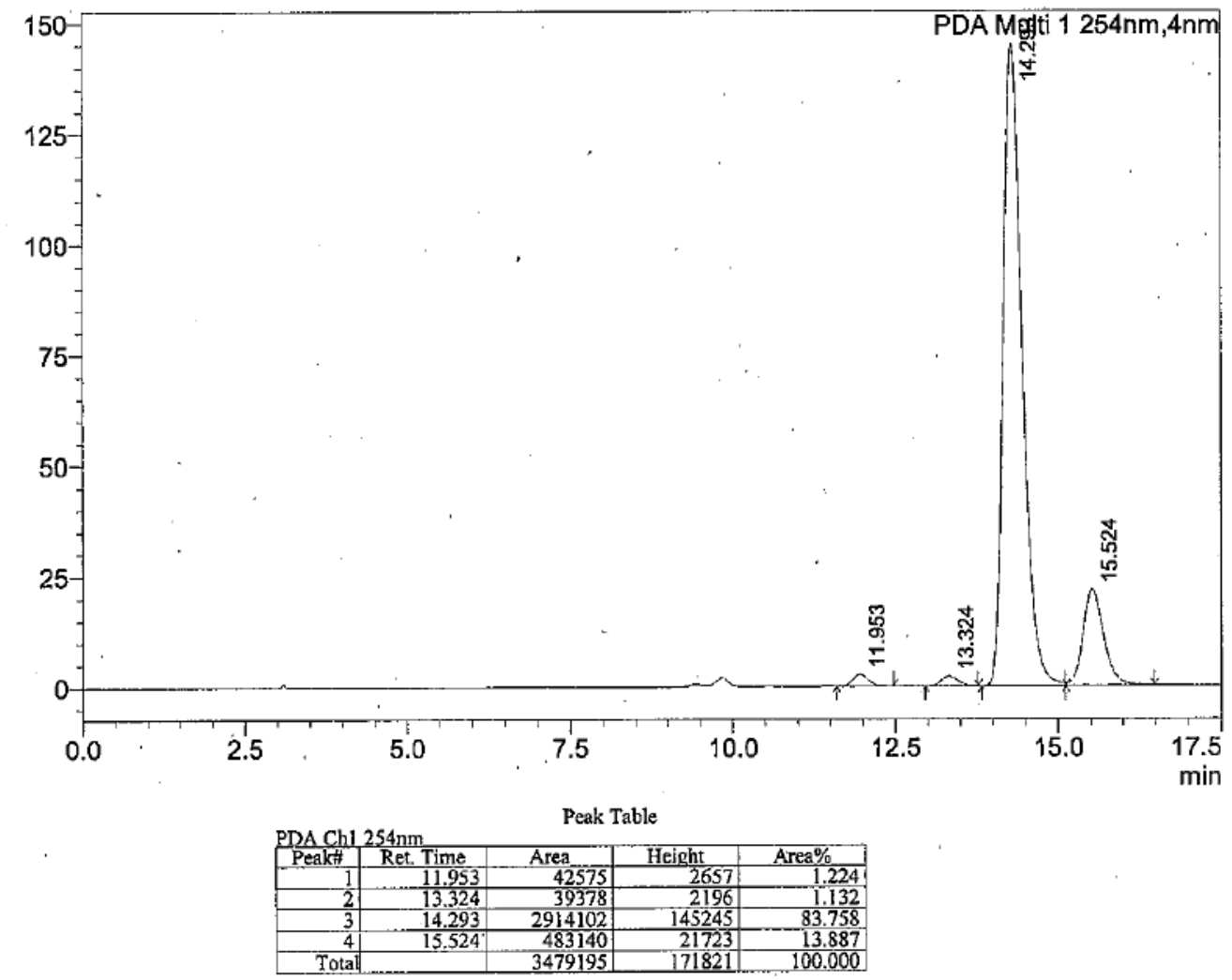<smiles>COc1ccc(N[C@H](c2ccccn2)[C@H](C)C(=O)N(C)C)c(OC)c1</smiles> 
mAU

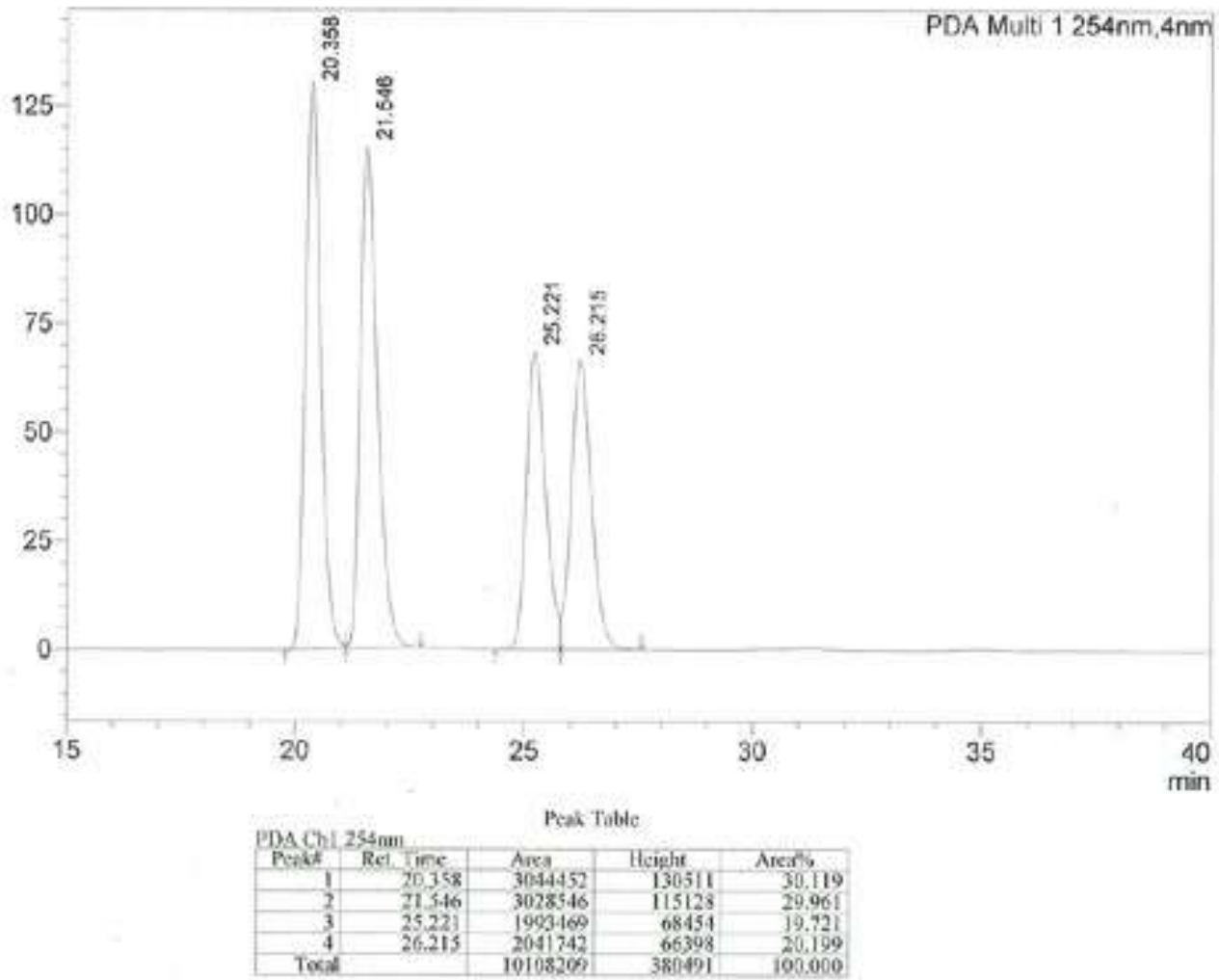

MAU

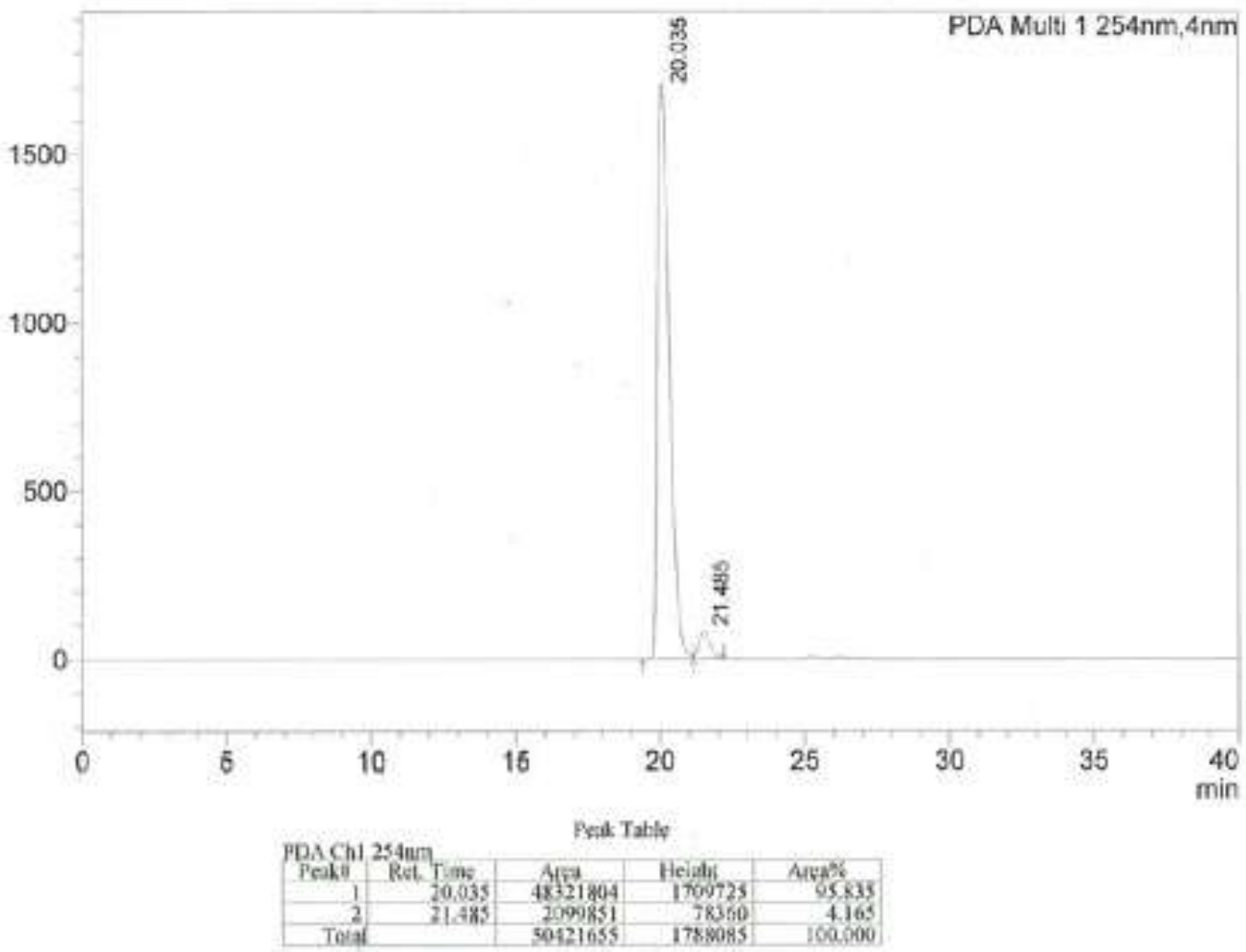

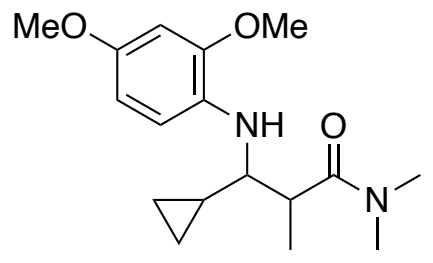

racemic mixture of anti- and syn-form
$\mathrm{MeO}$<smiles>COc1cc(I)ccc1N</smiles> 
MAU

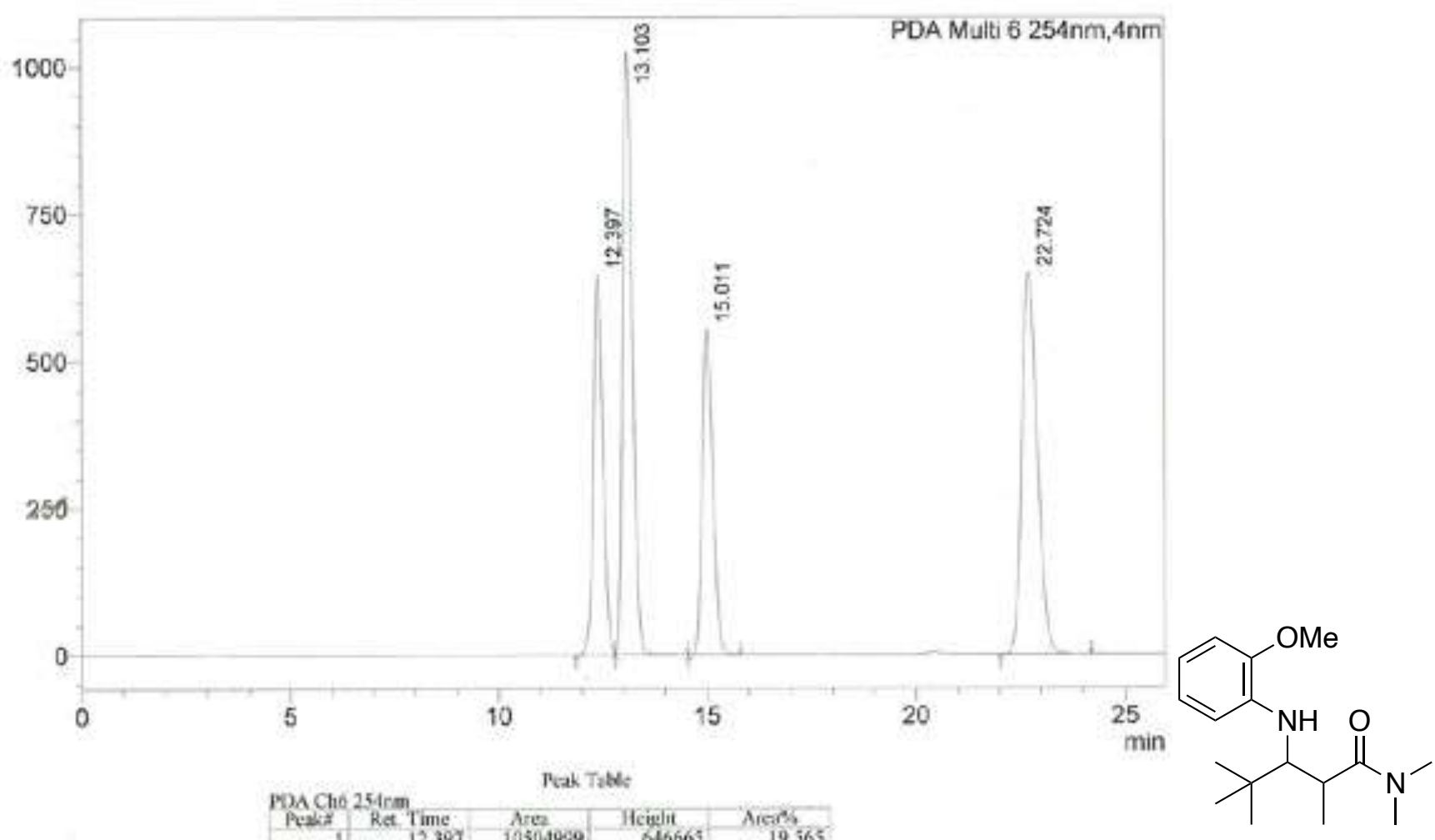

racemic mixture of anti- and syn-form

mAU

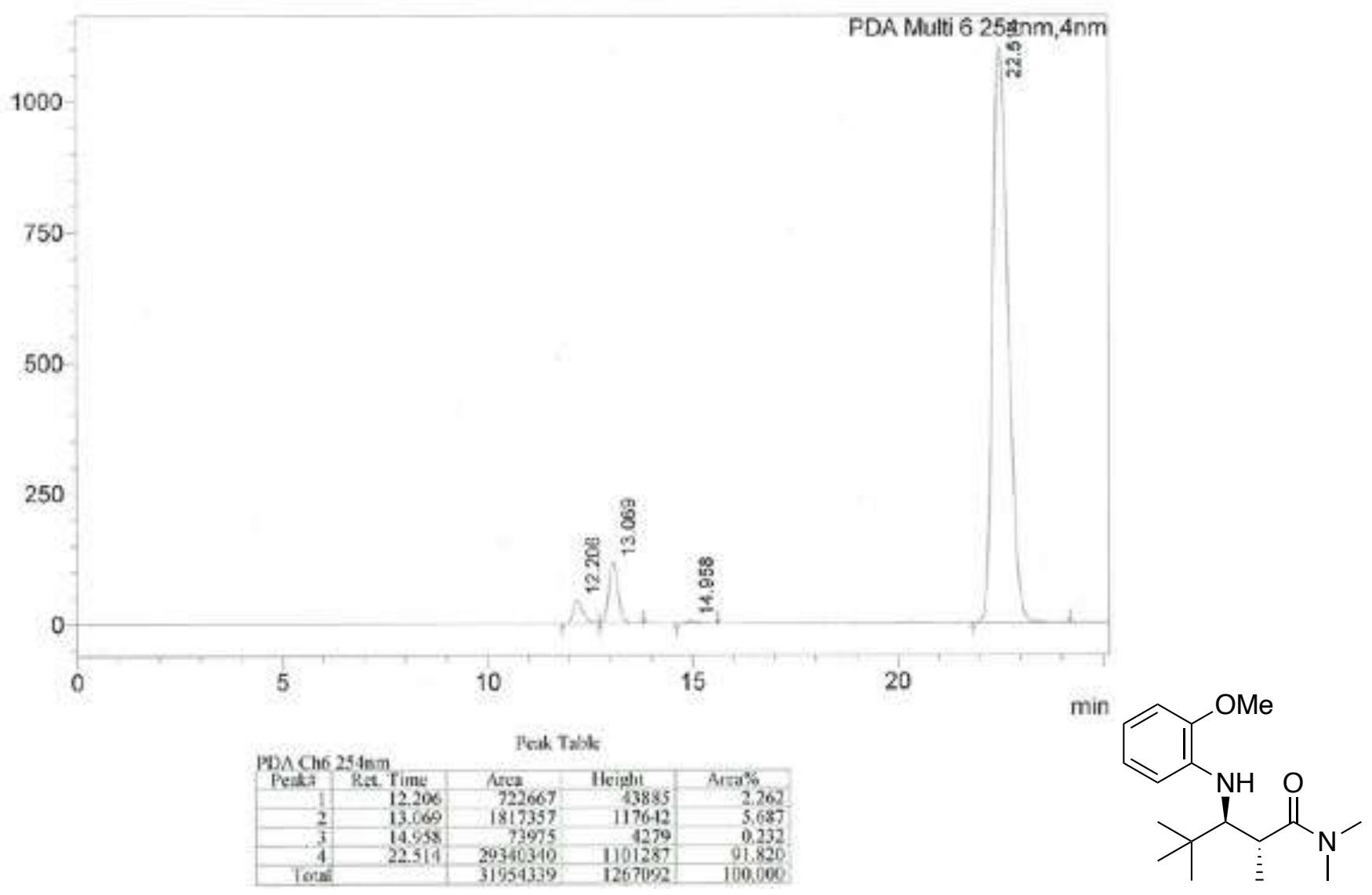


MAU
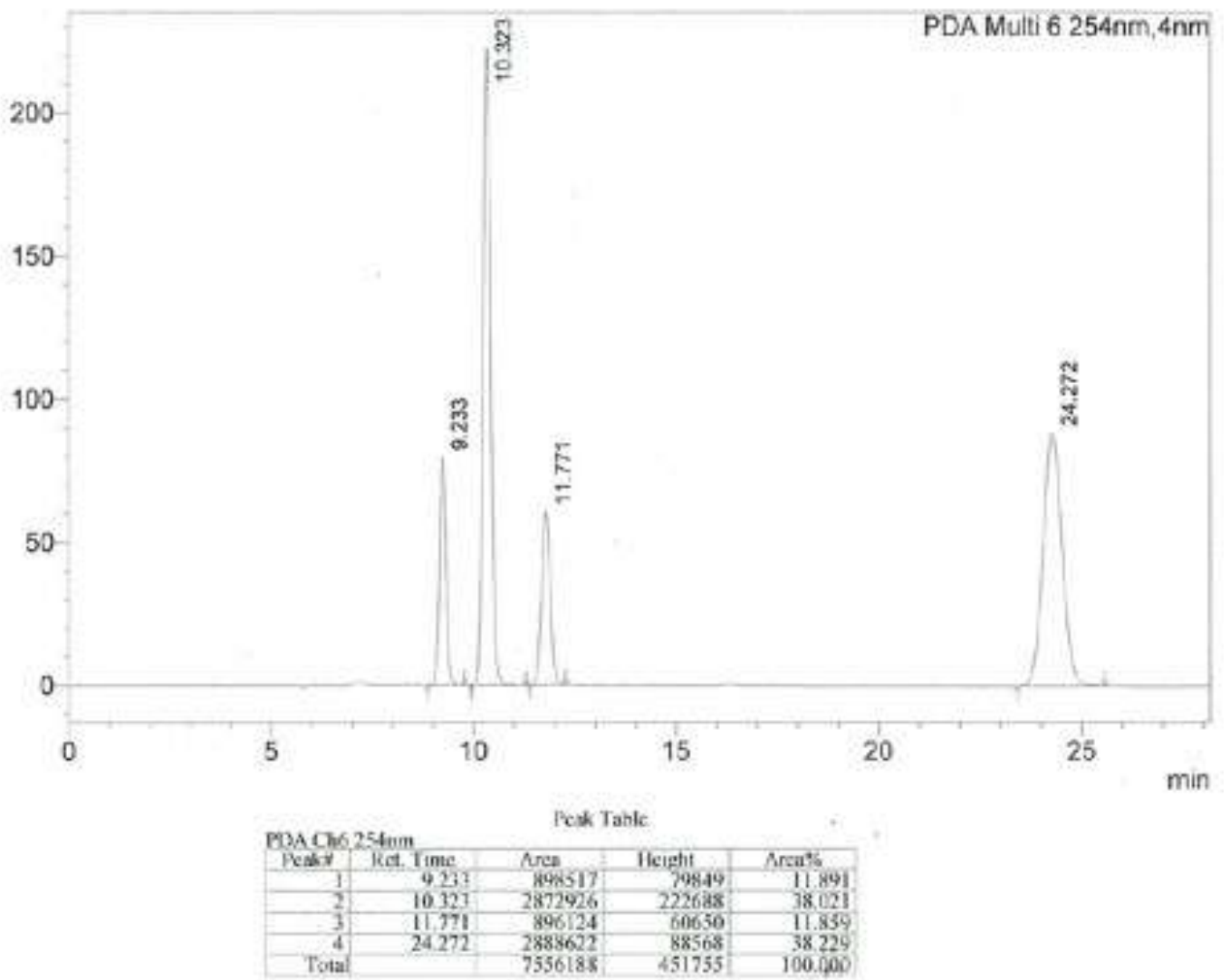

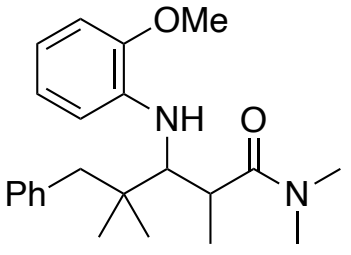

racemic mixture of anti- and syn-form

mAU

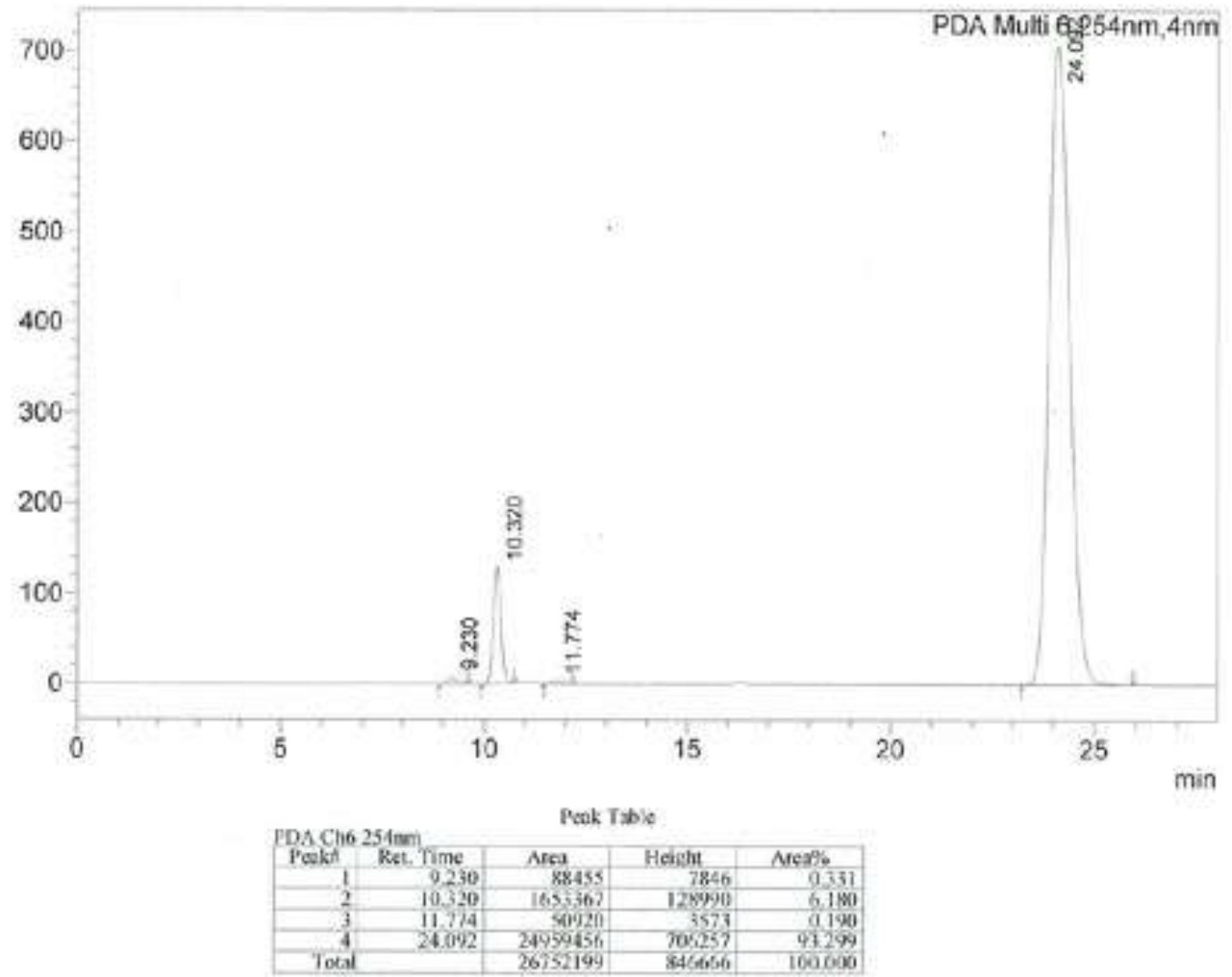<smiles>COc1ccccc1N[C@H](C(=O)N(C)C)C(C)(C)Cc1ccccc1</smiles> 
mAU

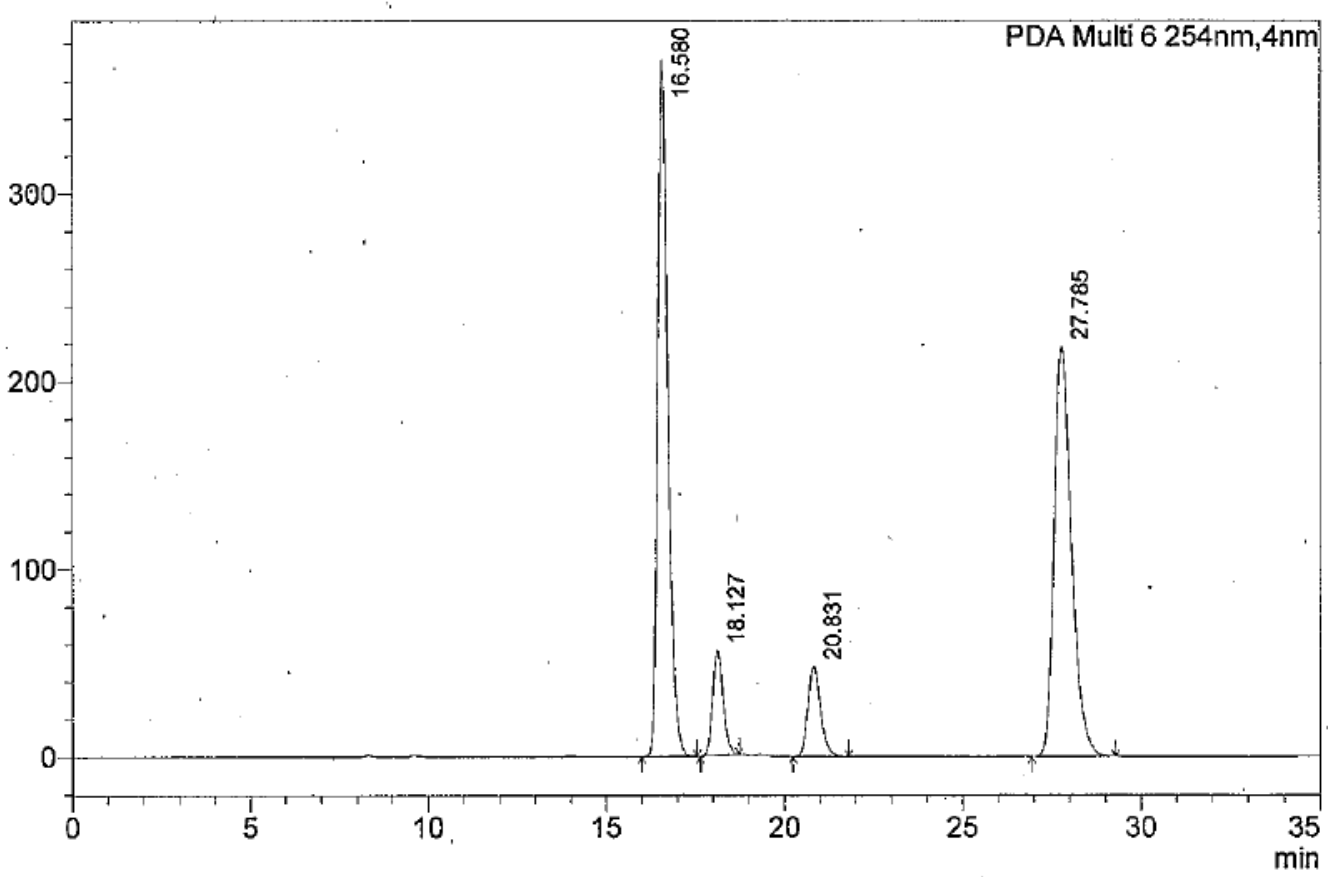

$\mathrm{MeO}$<smiles>CCC(C(=O)N(C)C)C(Nc1ccc(C)cc1OC)c1ccccc1</smiles>

racemic mixture of anti- and syn-form

mAU

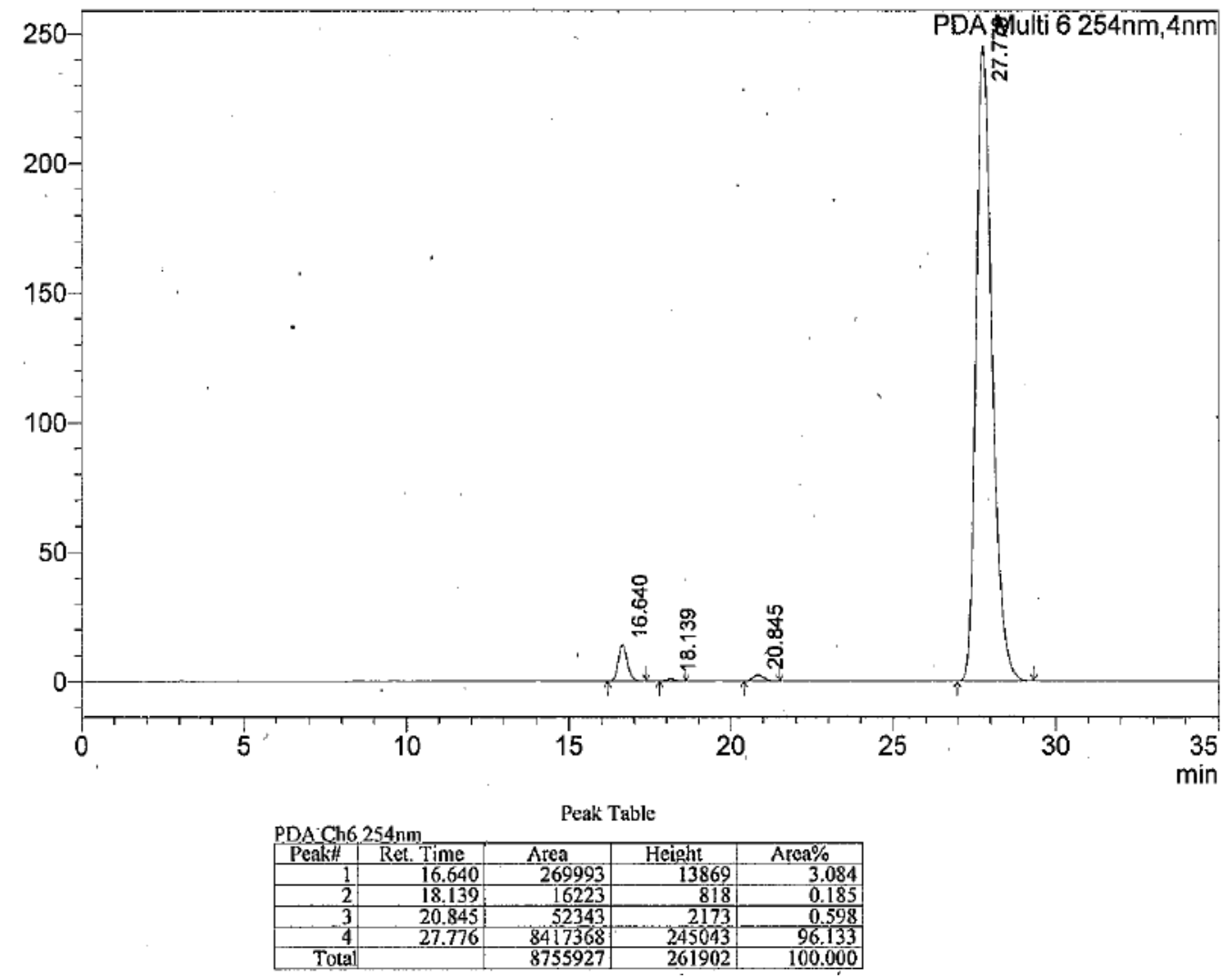

$\mathrm{MeO} \rightleftharpoons \mathrm{OMe}$<smiles>CC[C@H](C(=O)N(C)C)[C@H](Nc1ccc(C)cc1C)c1ccccc1</smiles> 

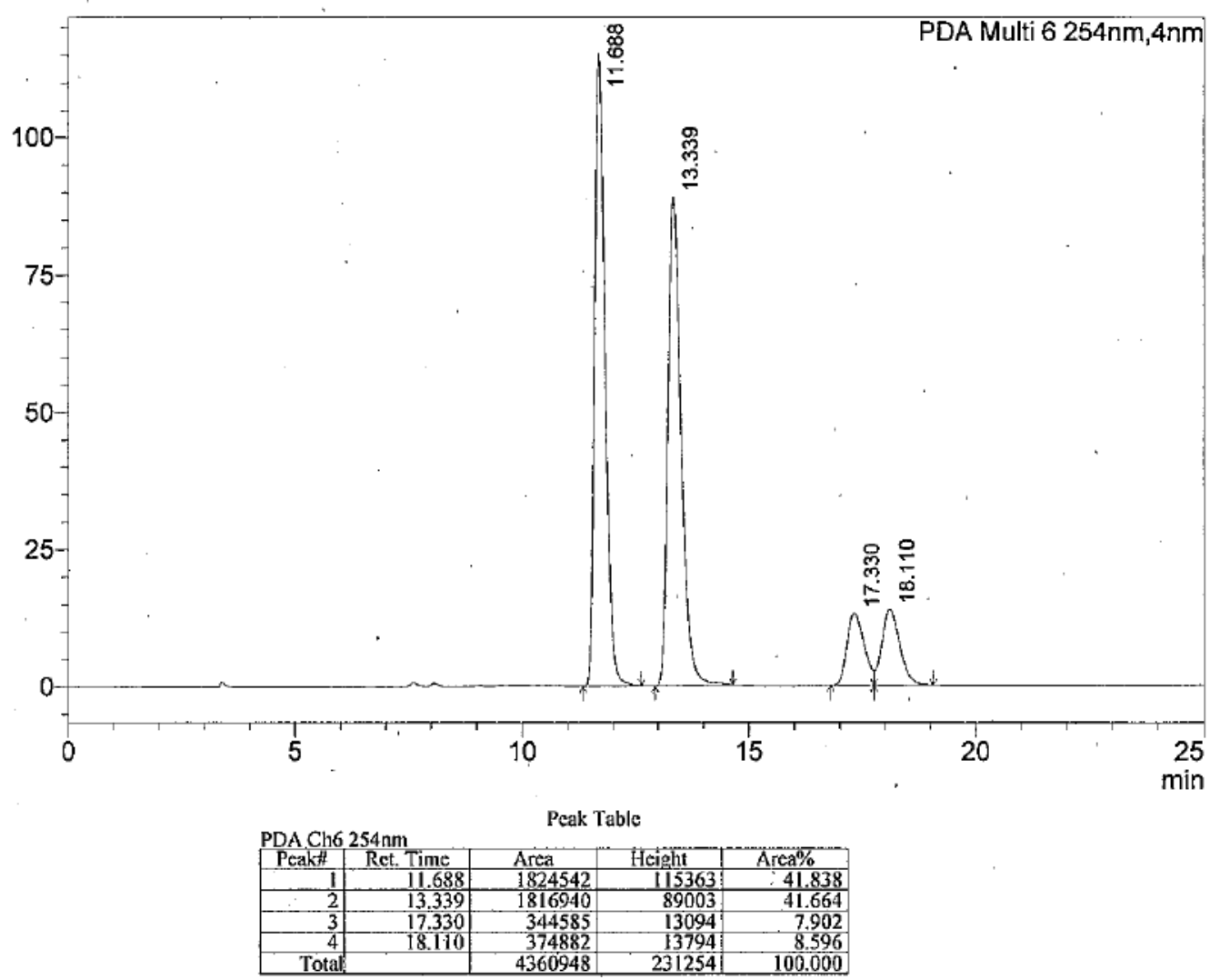

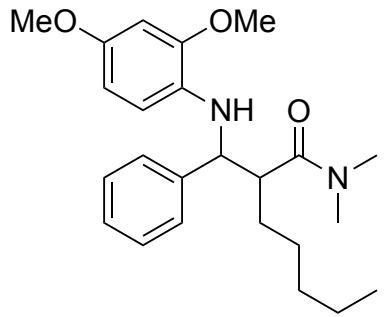

racemic mixture of anti- and syn-form

mAU

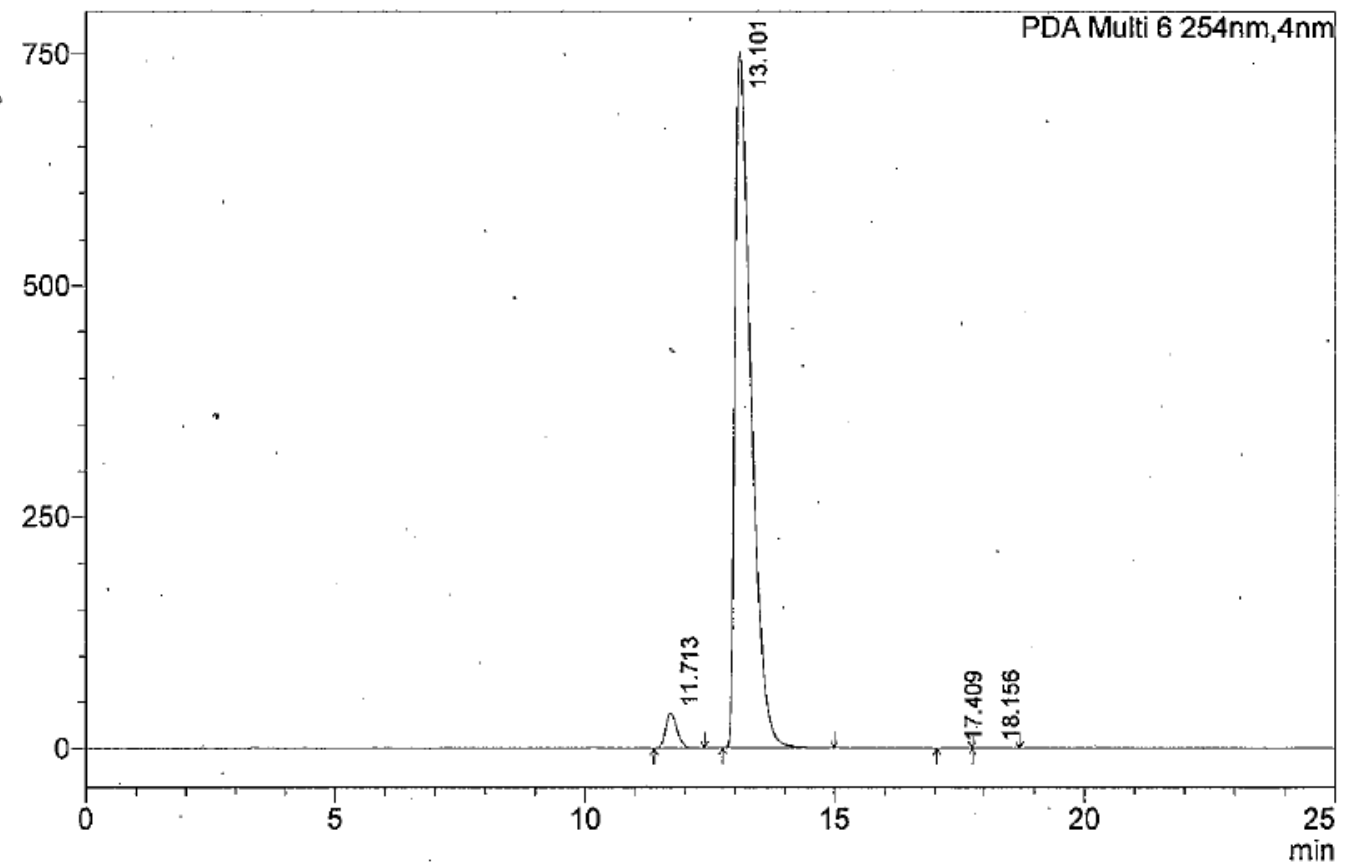

$\mathrm{MeO}$<smiles>COc1ccccc1N</smiles>

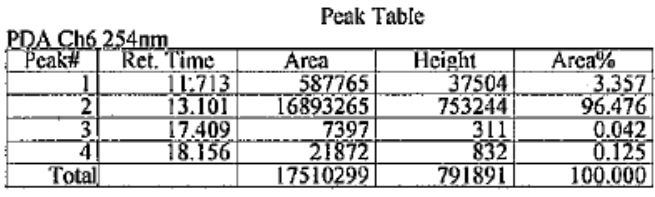



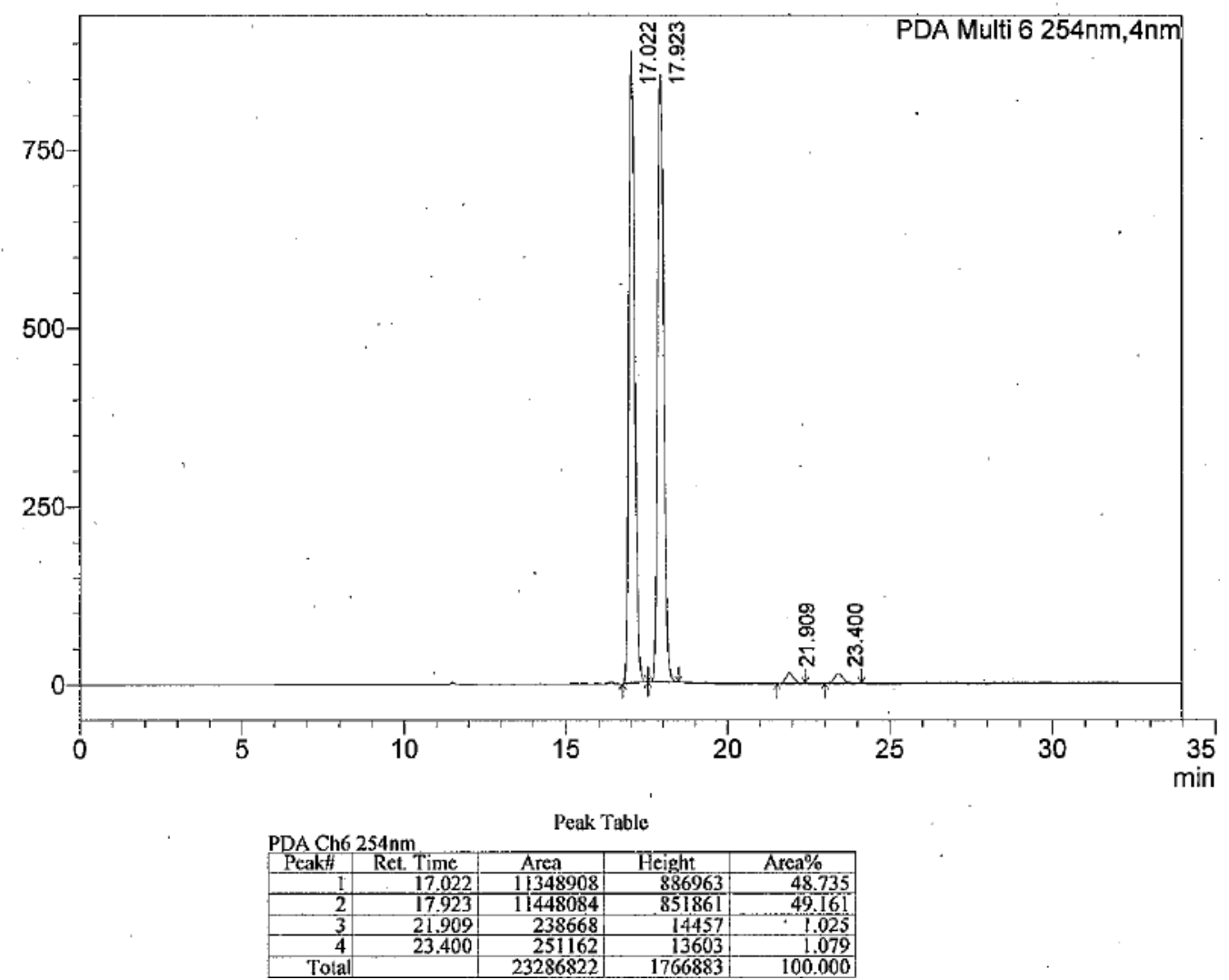

Peak Table

35 \begin{tabular}{rr|r}
13603 & 1.079 \\
\hline
\end{tabular}

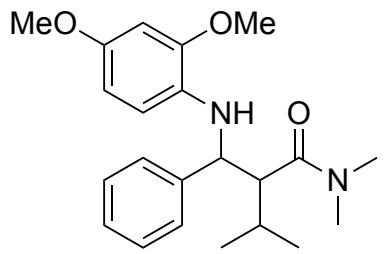

racemic mixture of anti- and syn-form

mAU

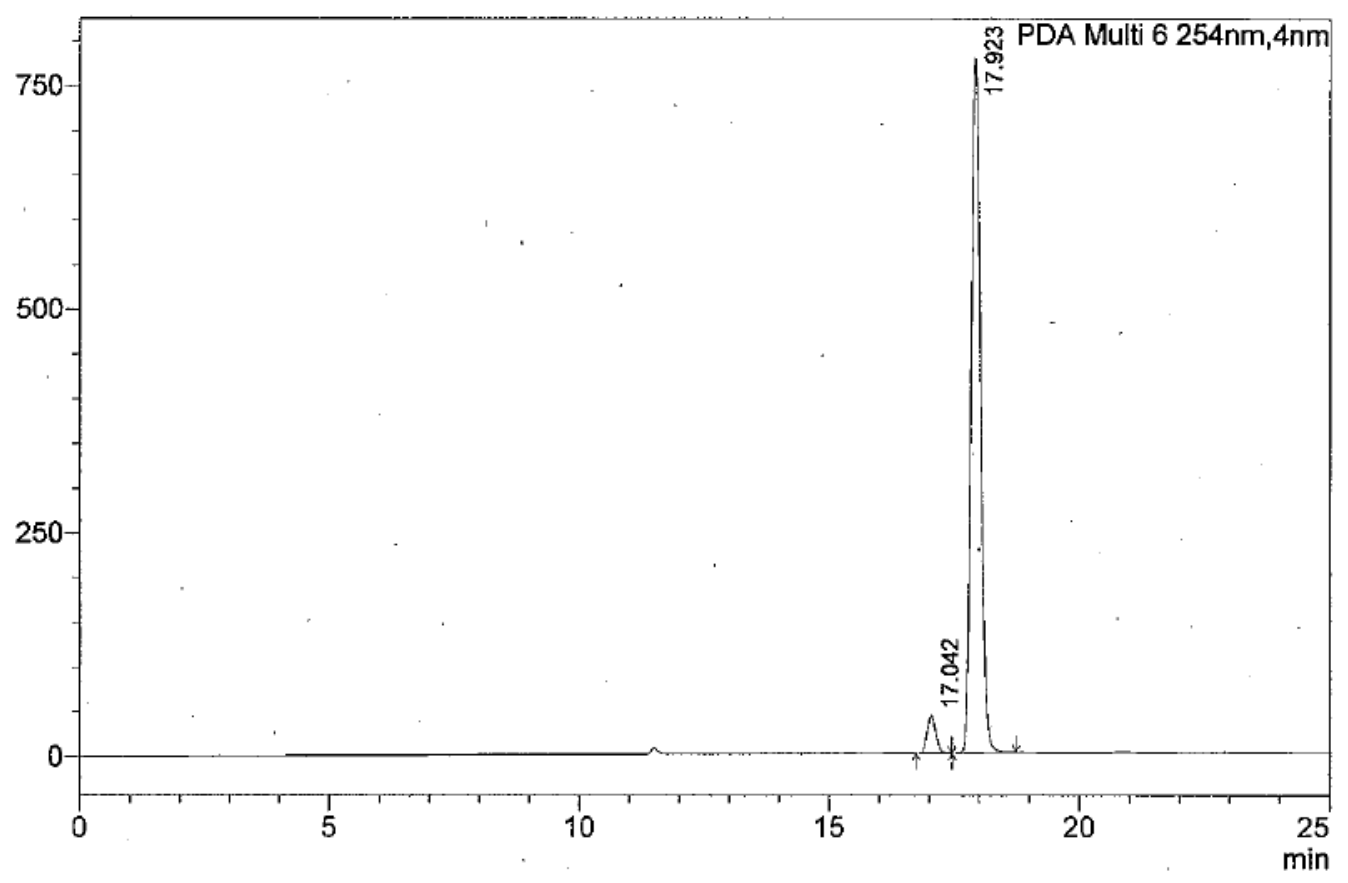<smiles>COc1ccc(N[C@H](c2ccccc2)[C@H](C(=O)N(C)C)C(C)C)c(OC)c1</smiles> 
mAU

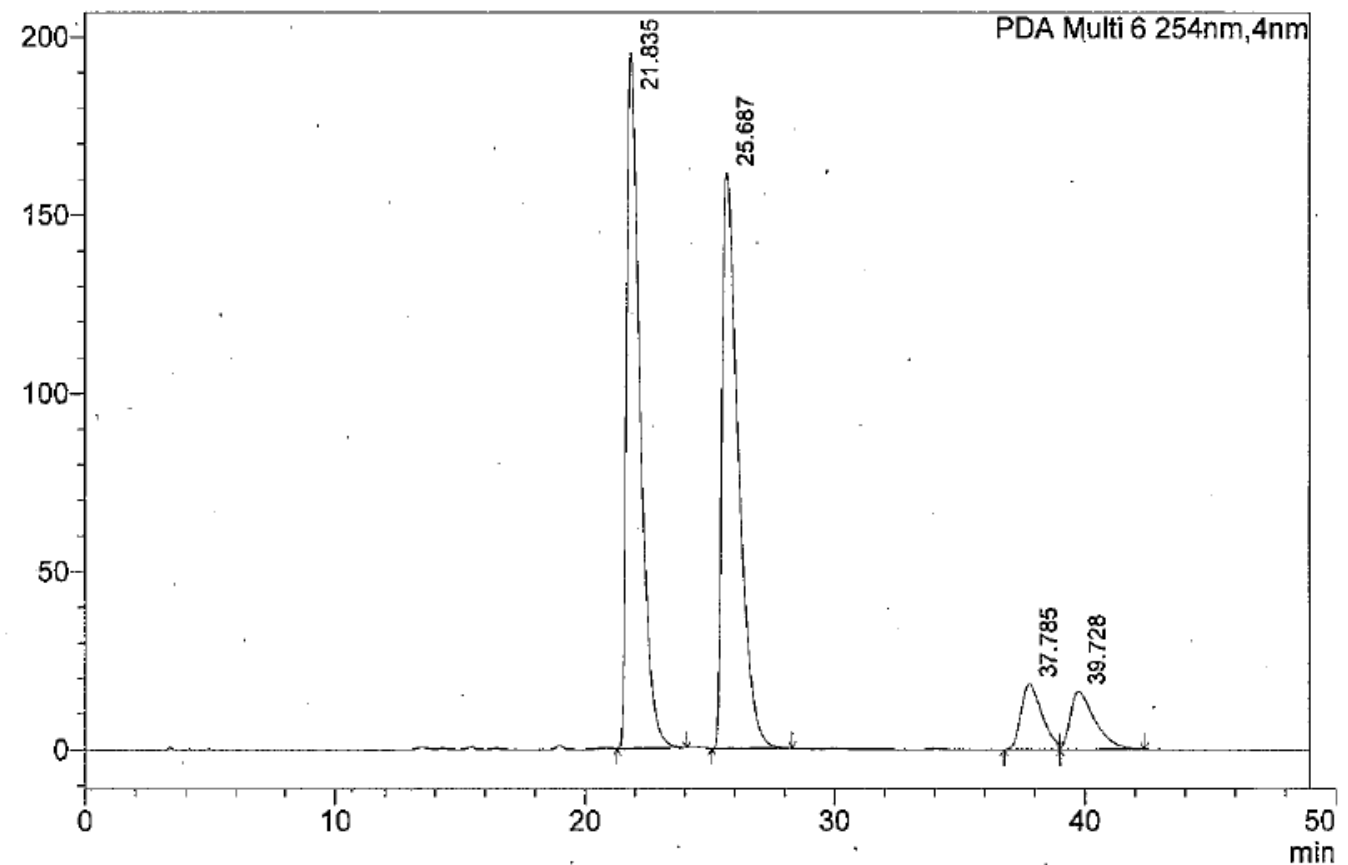

Peak Table

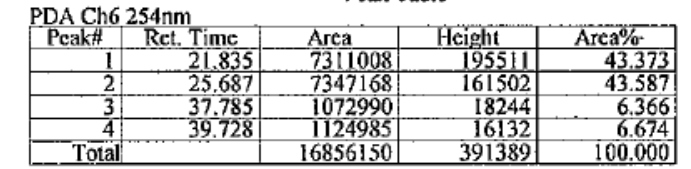

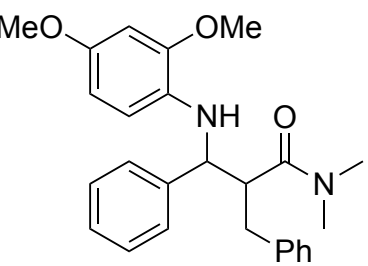

racemic mixture of anti- and syn-form

míu
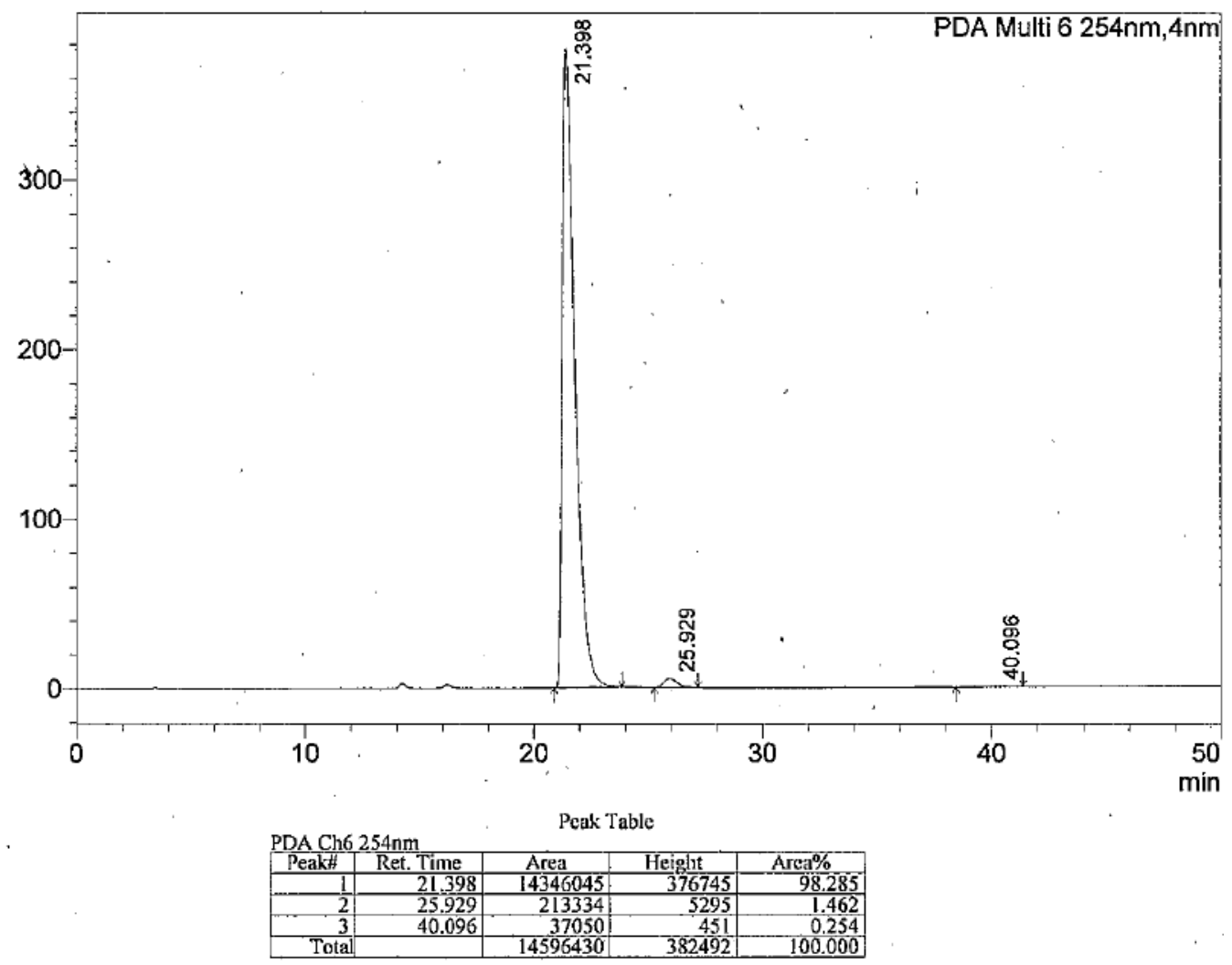

$\mathrm{MeO}$<smiles>COc1ccccc1N</smiles><smiles>C/C=C/N[C@H](c1ccccc1)[C@H](Cc1ccccc1)C(=O)N(C)C</smiles> 
mAU
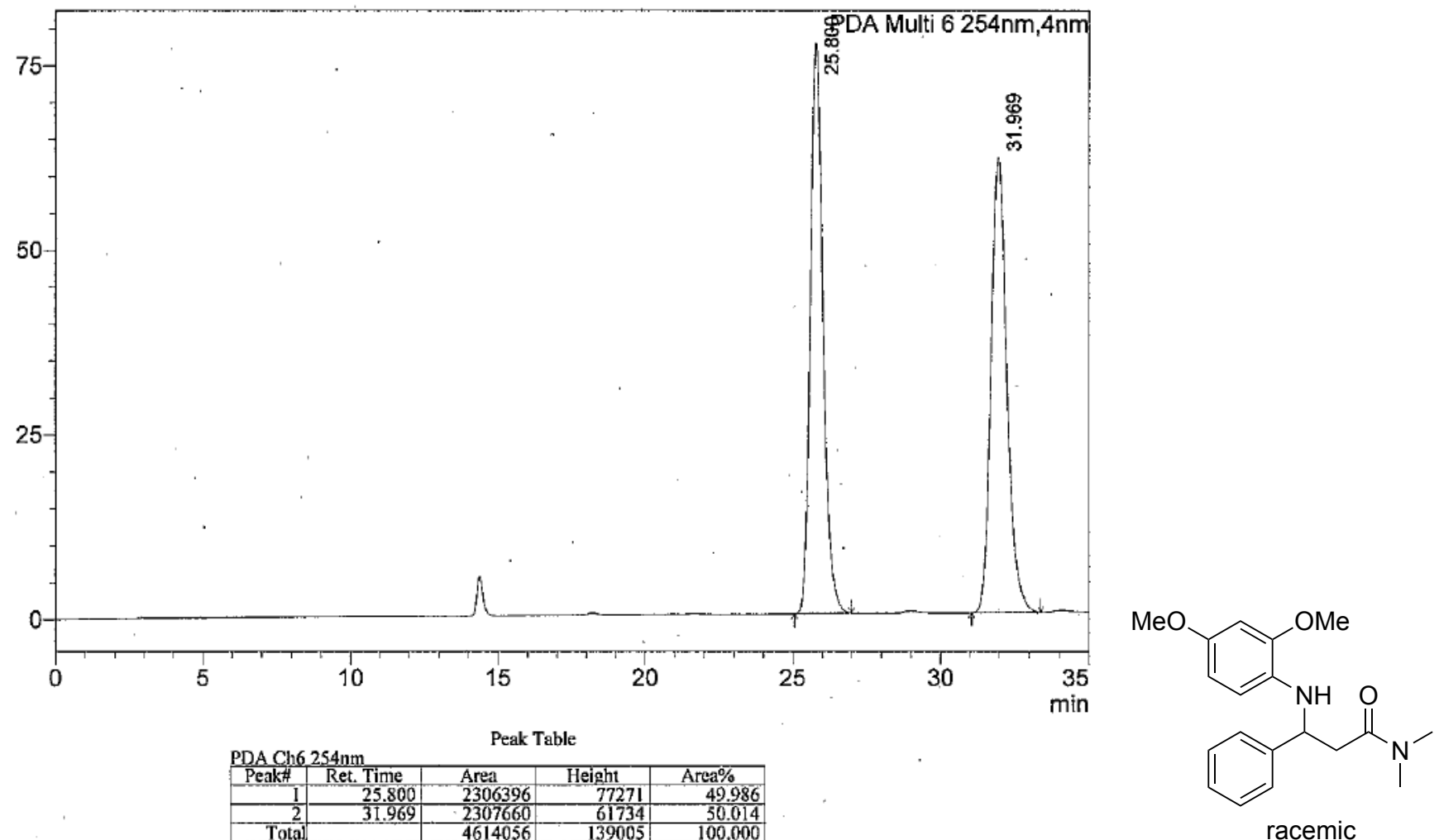

MAU

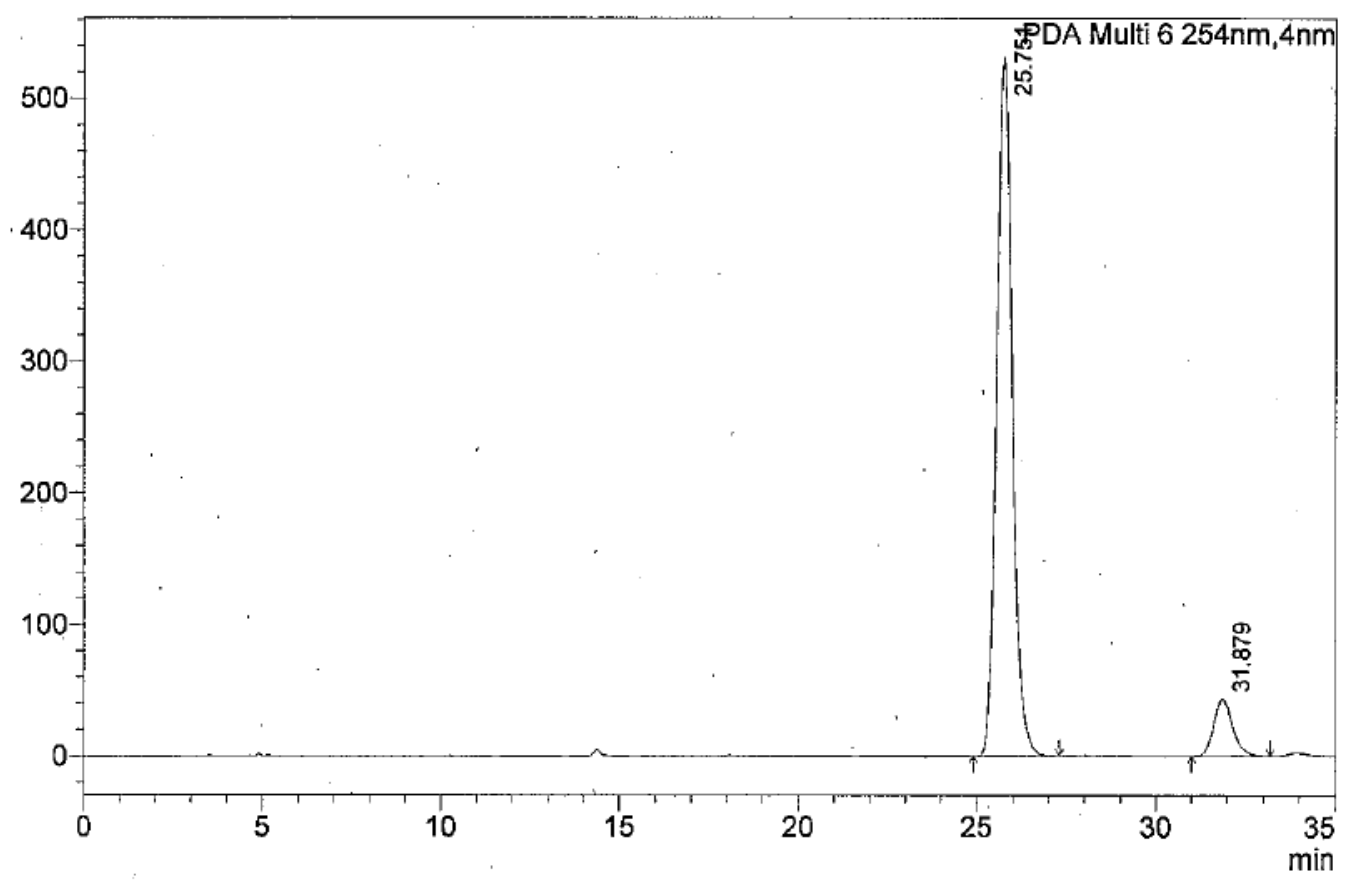

$\mathrm{MeO}$<smiles>COc1ccccc1N</smiles>

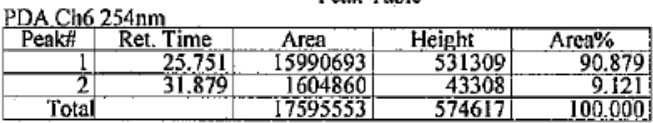<smiles>C/C=C\N[C@H](CC(=O)N(C)C)c1ccccc1</smiles> 
MAU

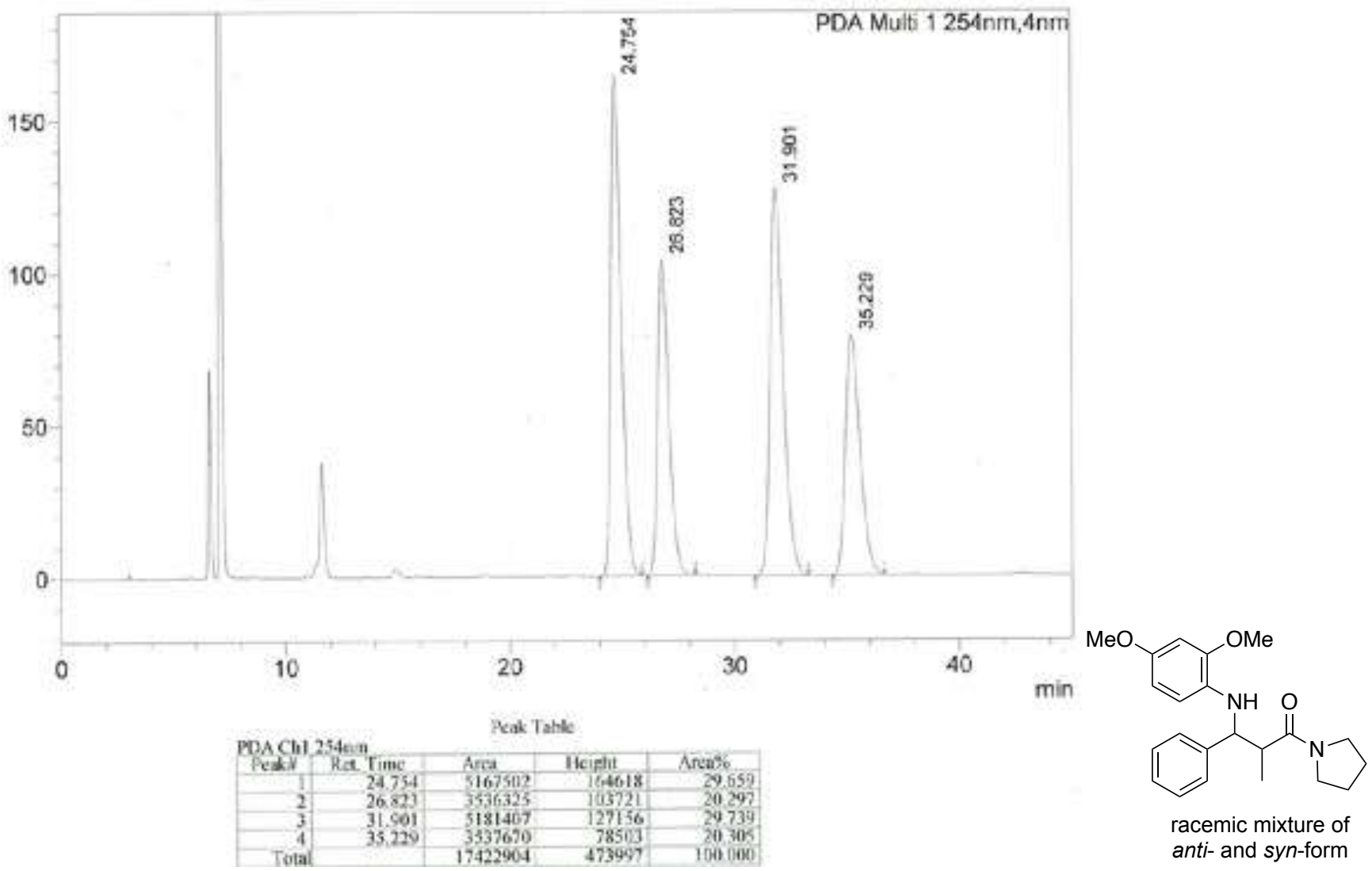

MAU

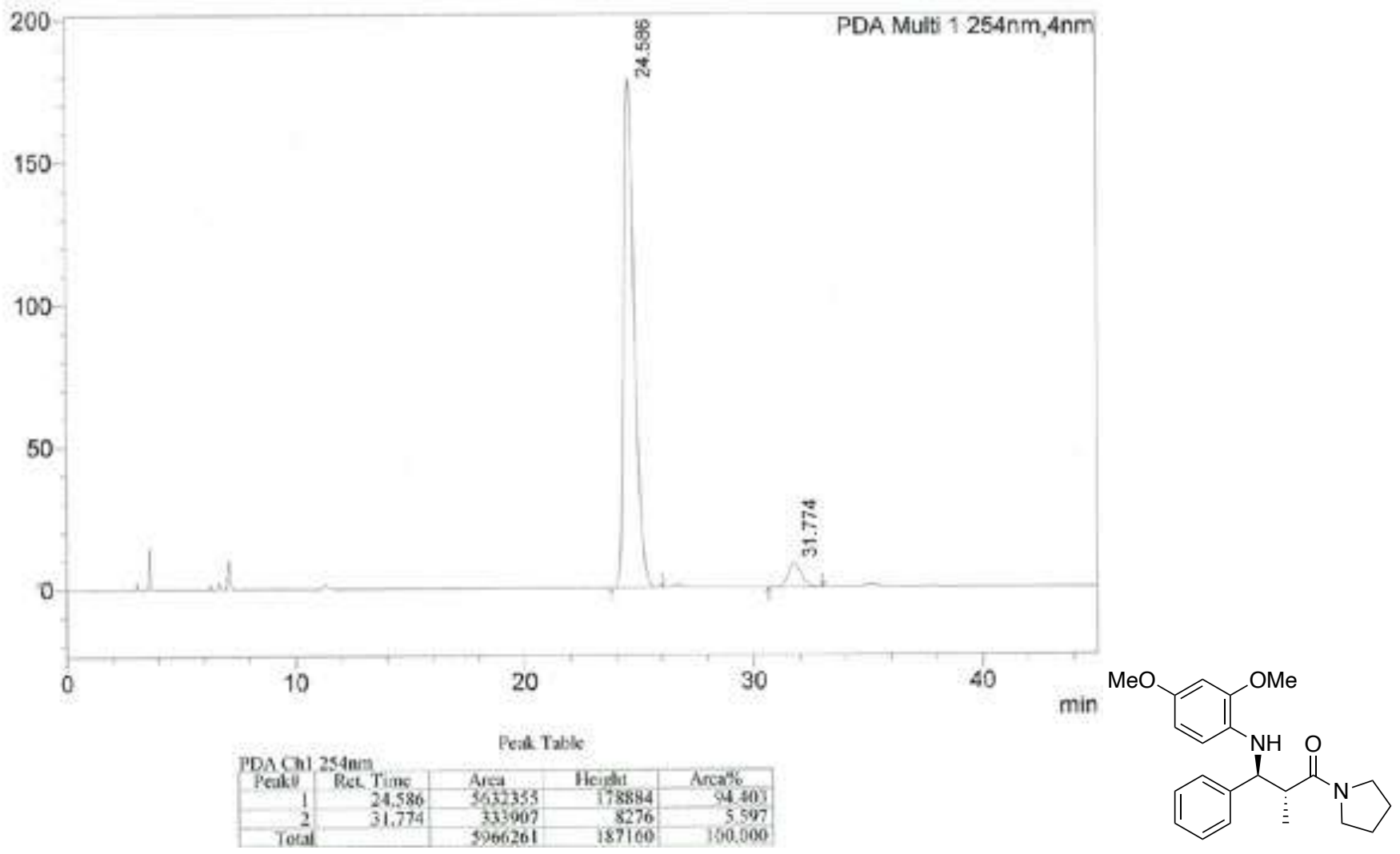


mAU

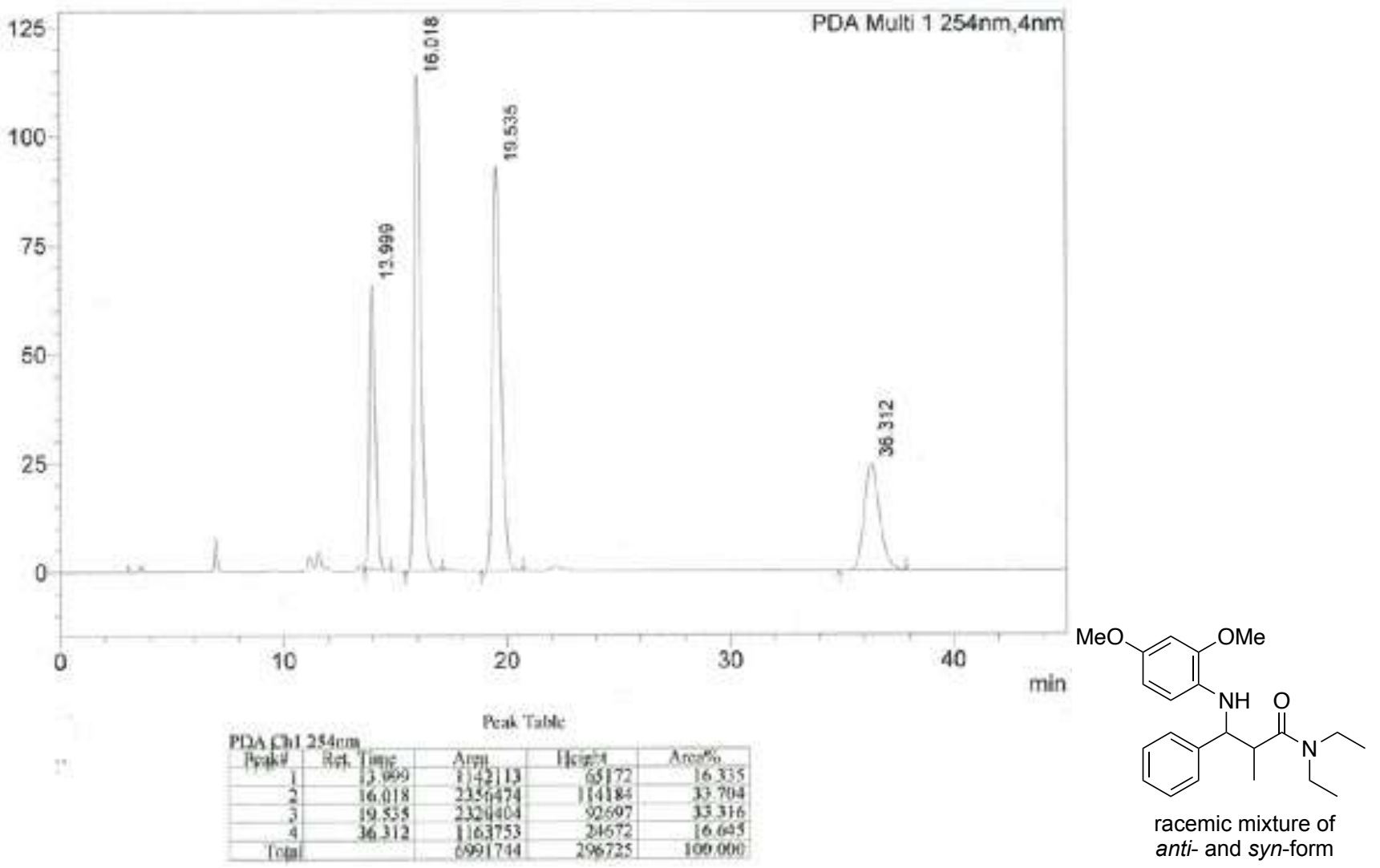

MAU

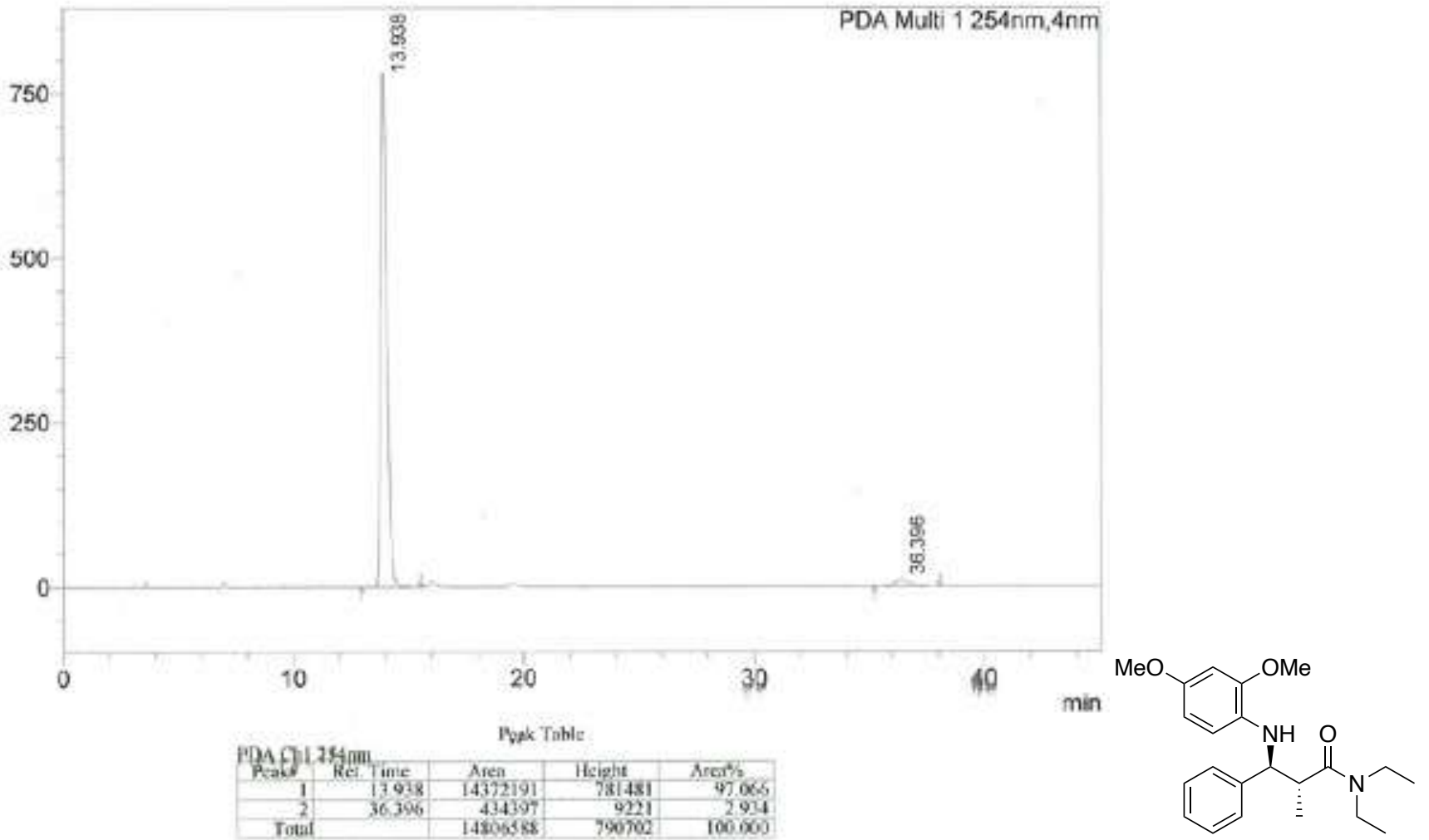


MAU

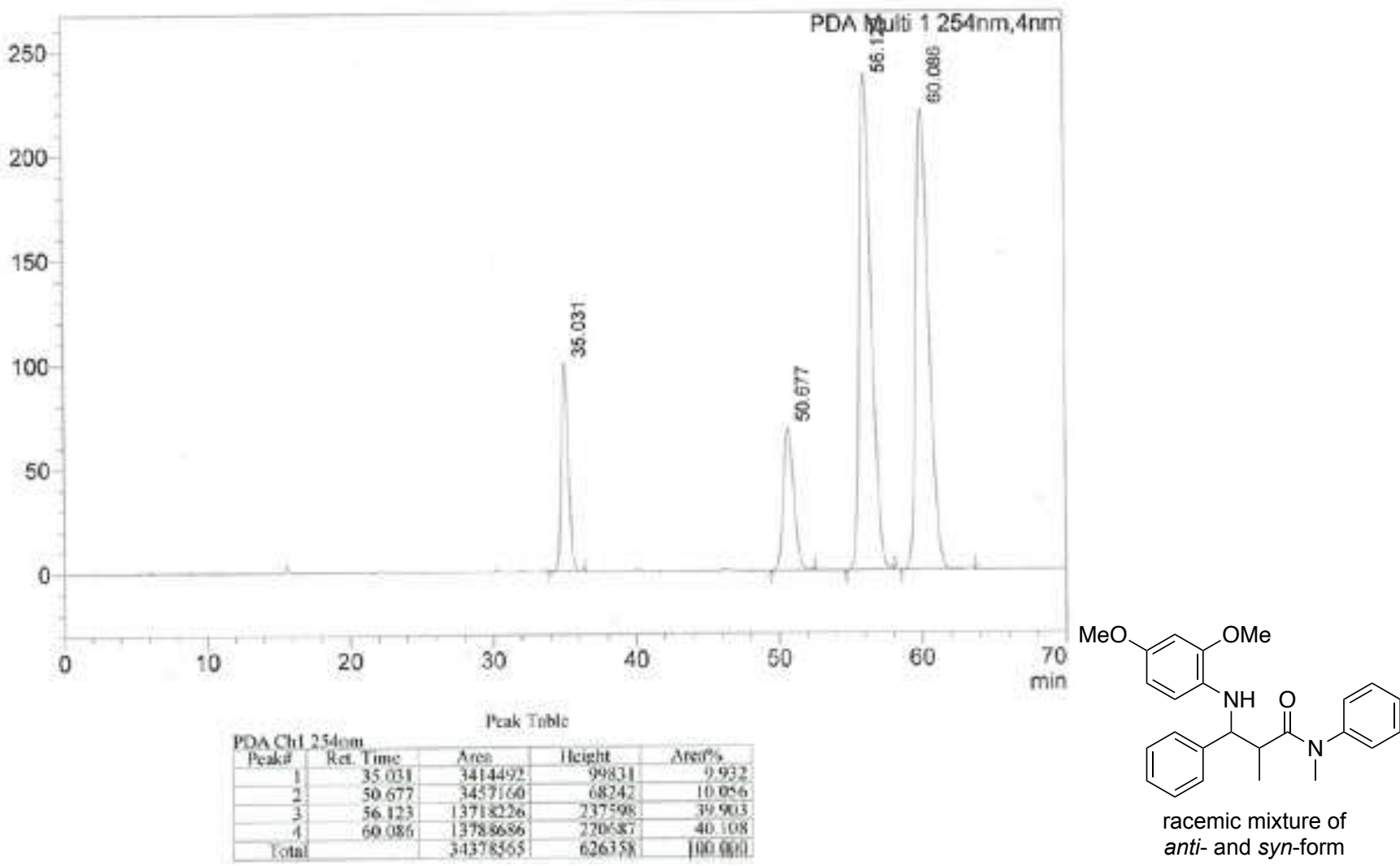

MAU

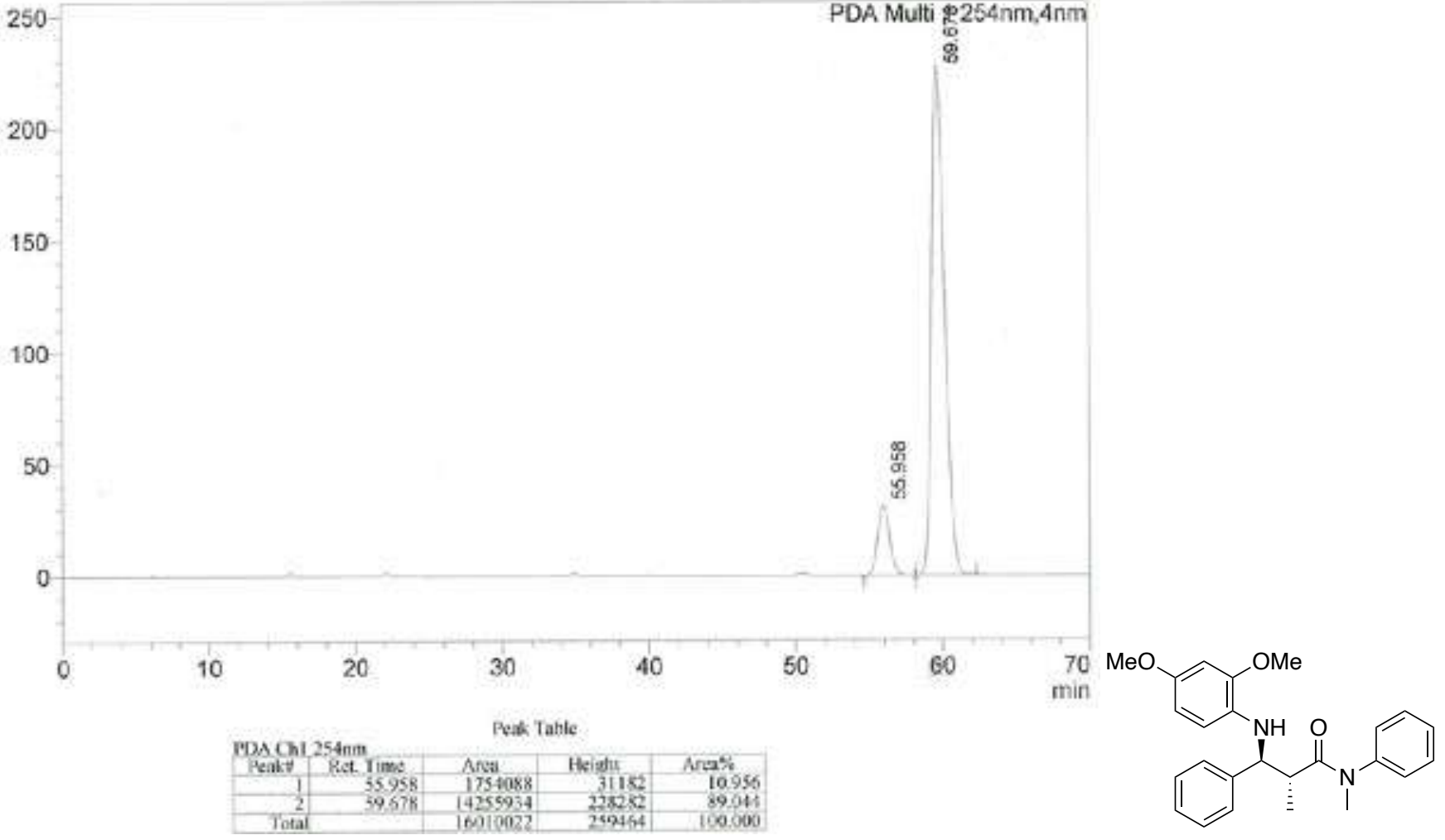


uAU

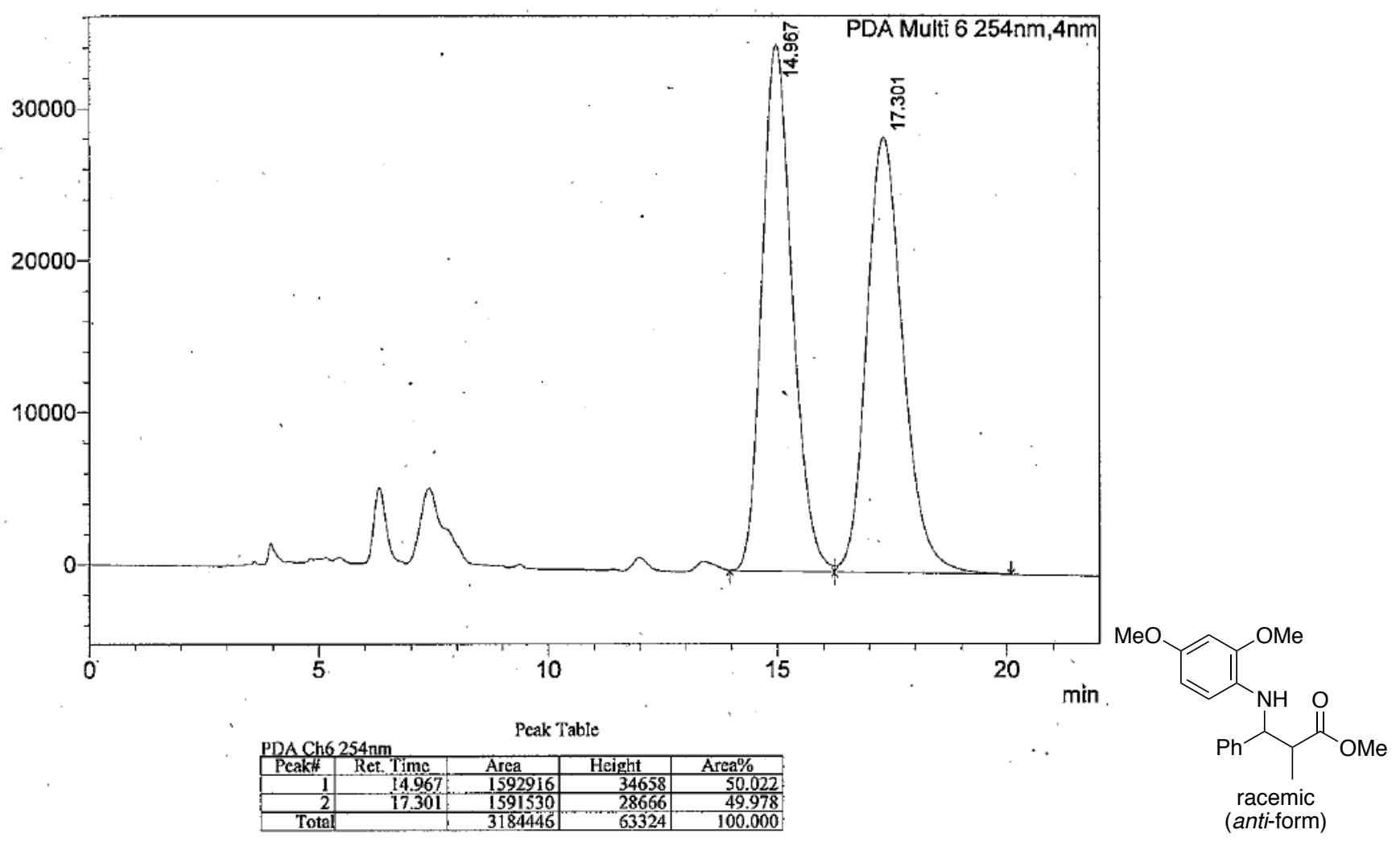

UAU

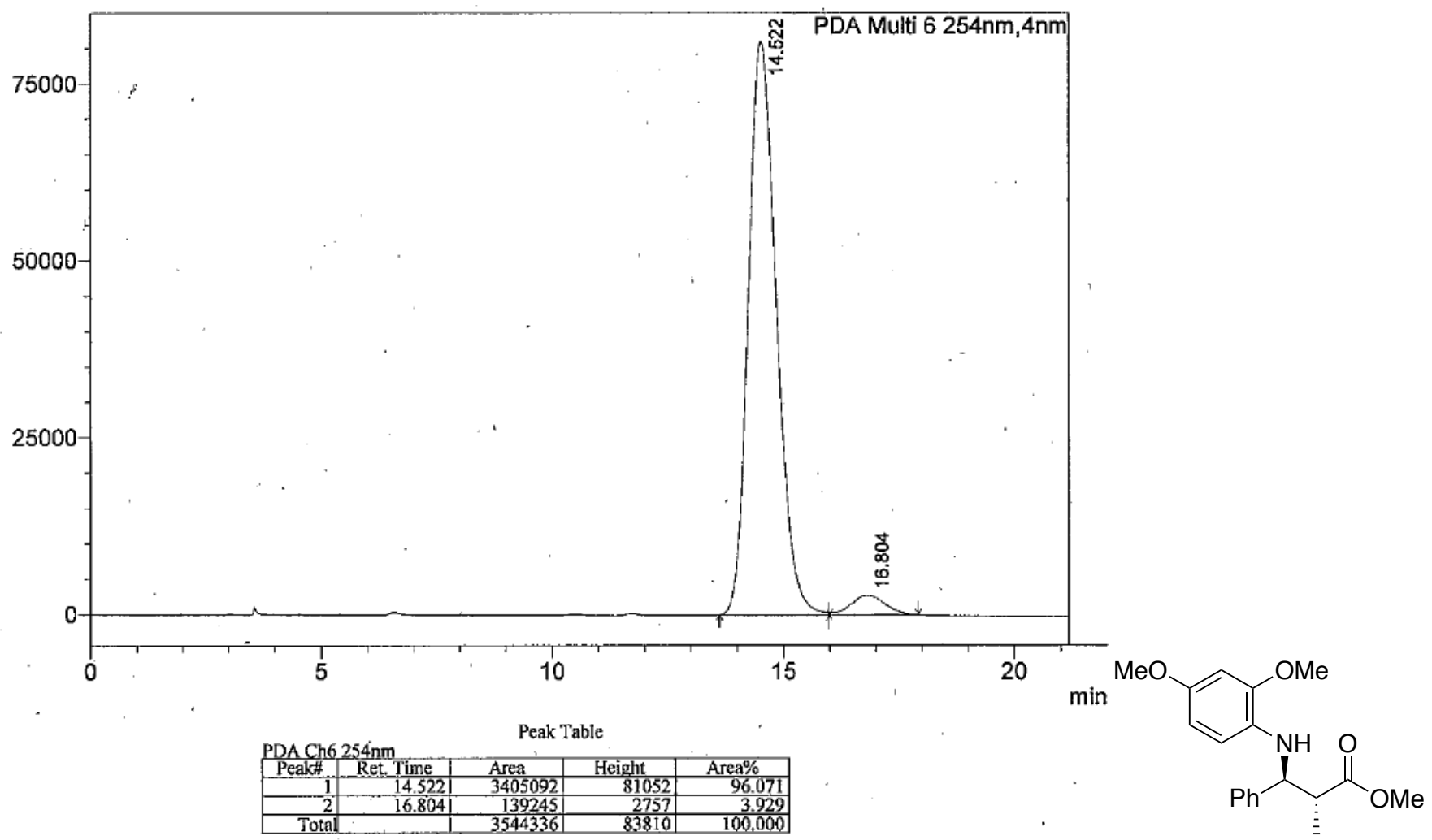


UAU
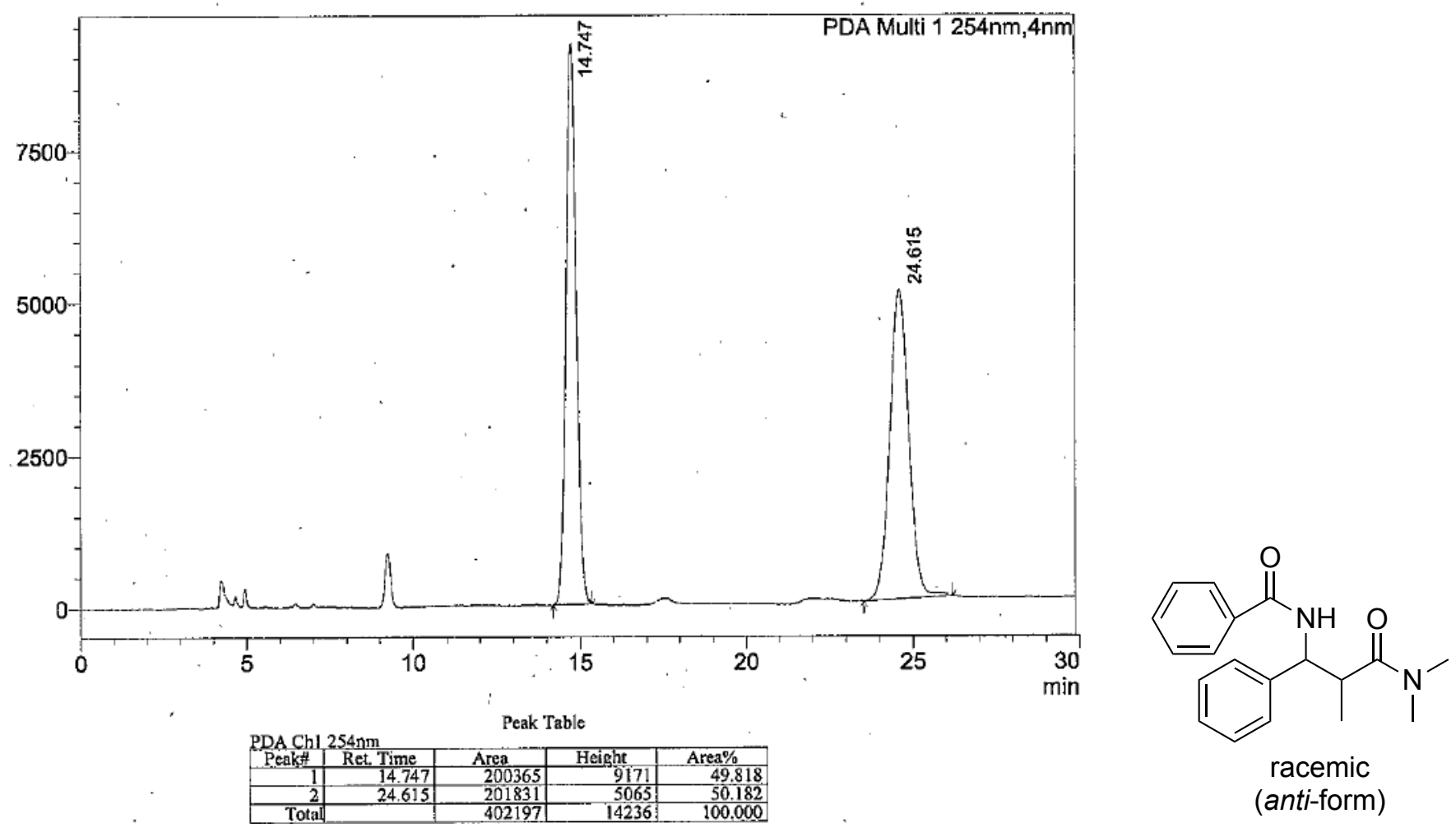

UAU

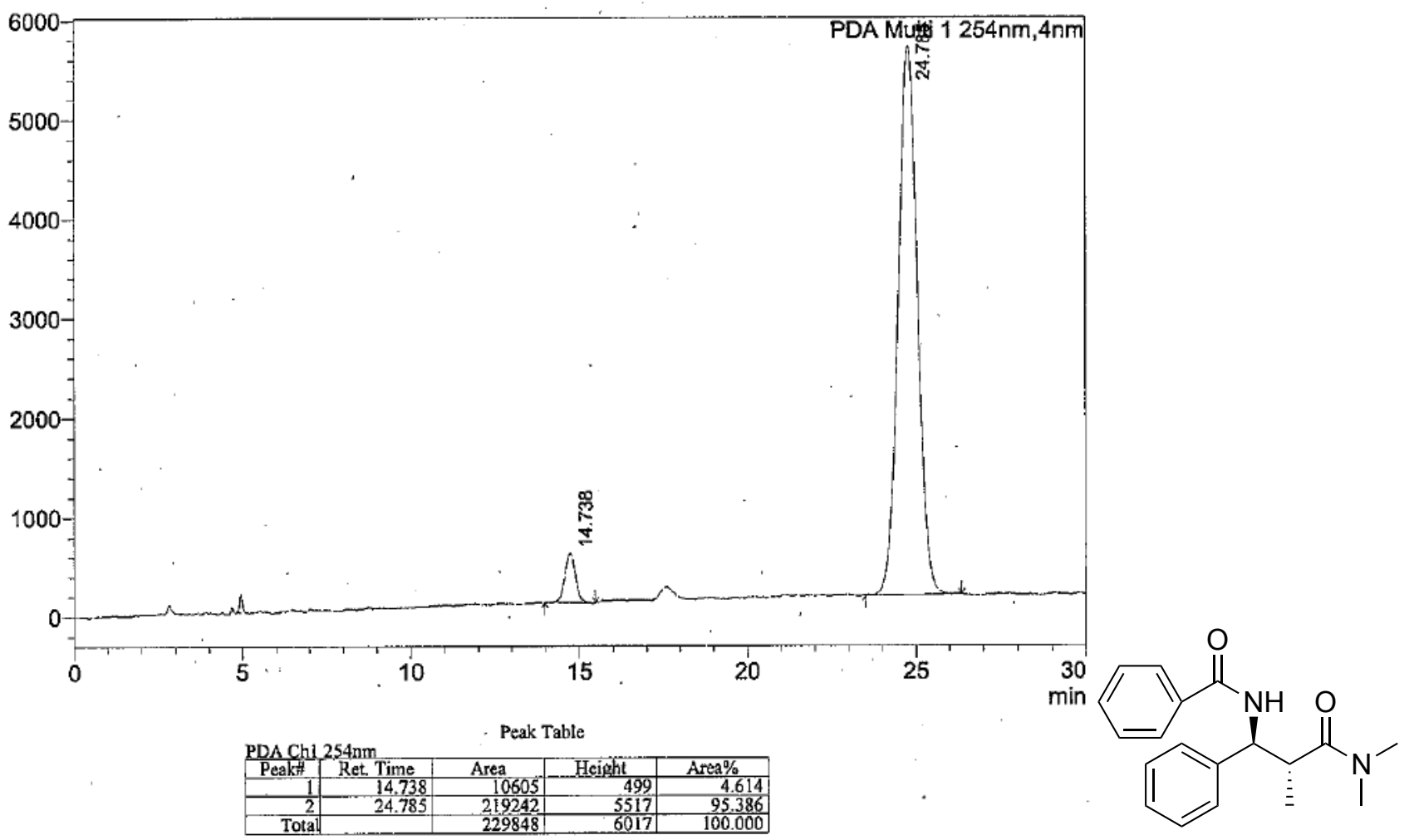



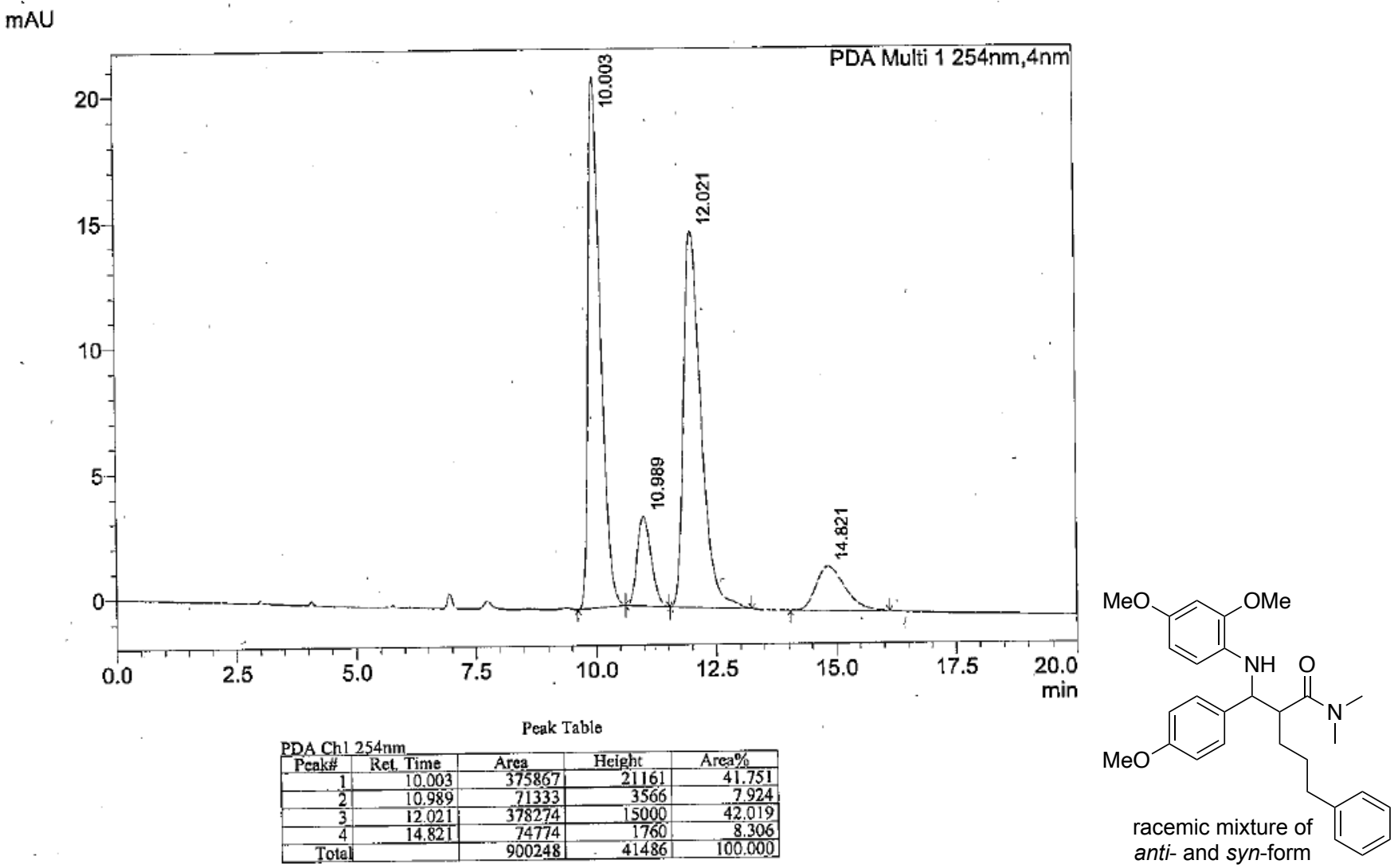

UAU

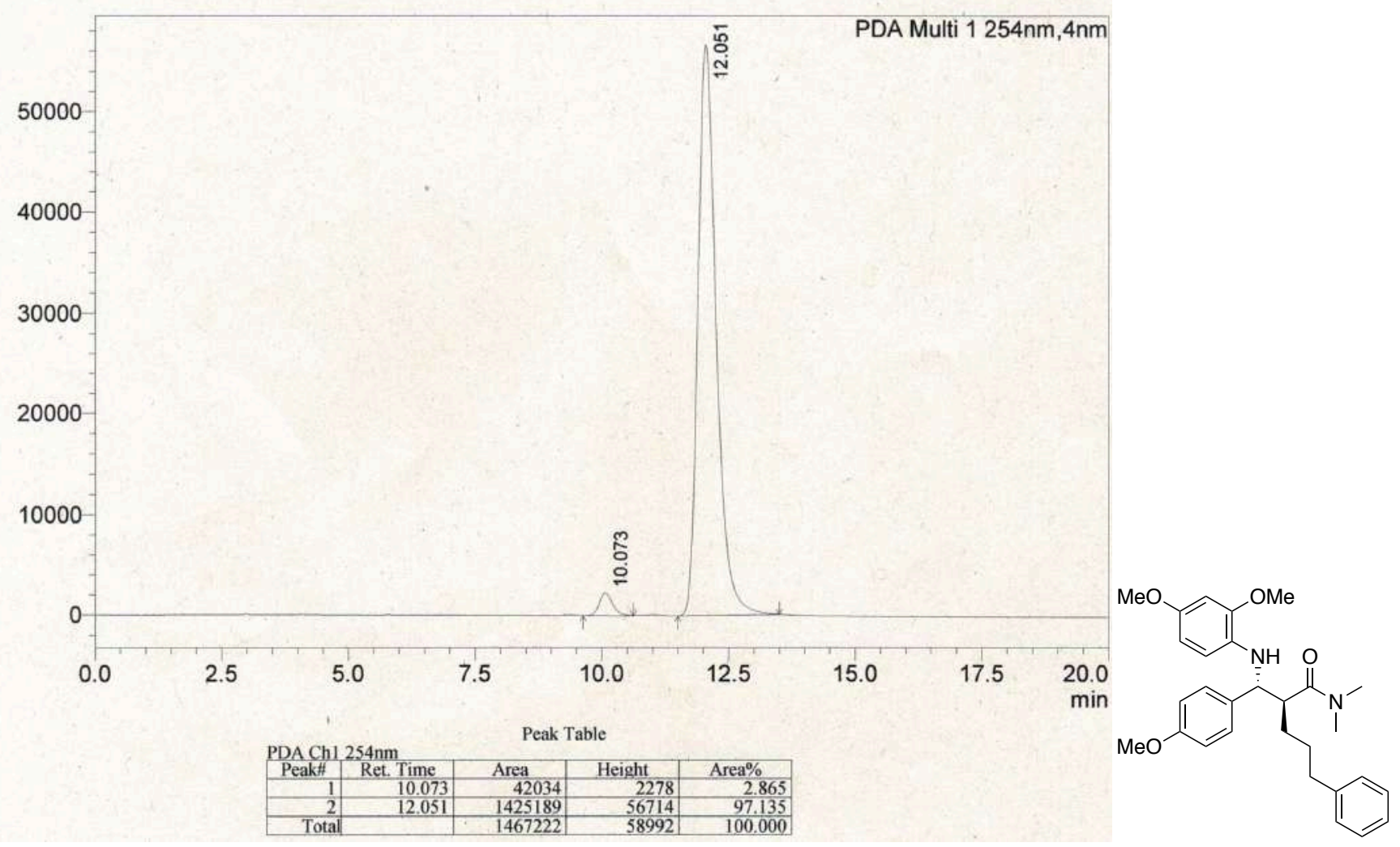


UAU

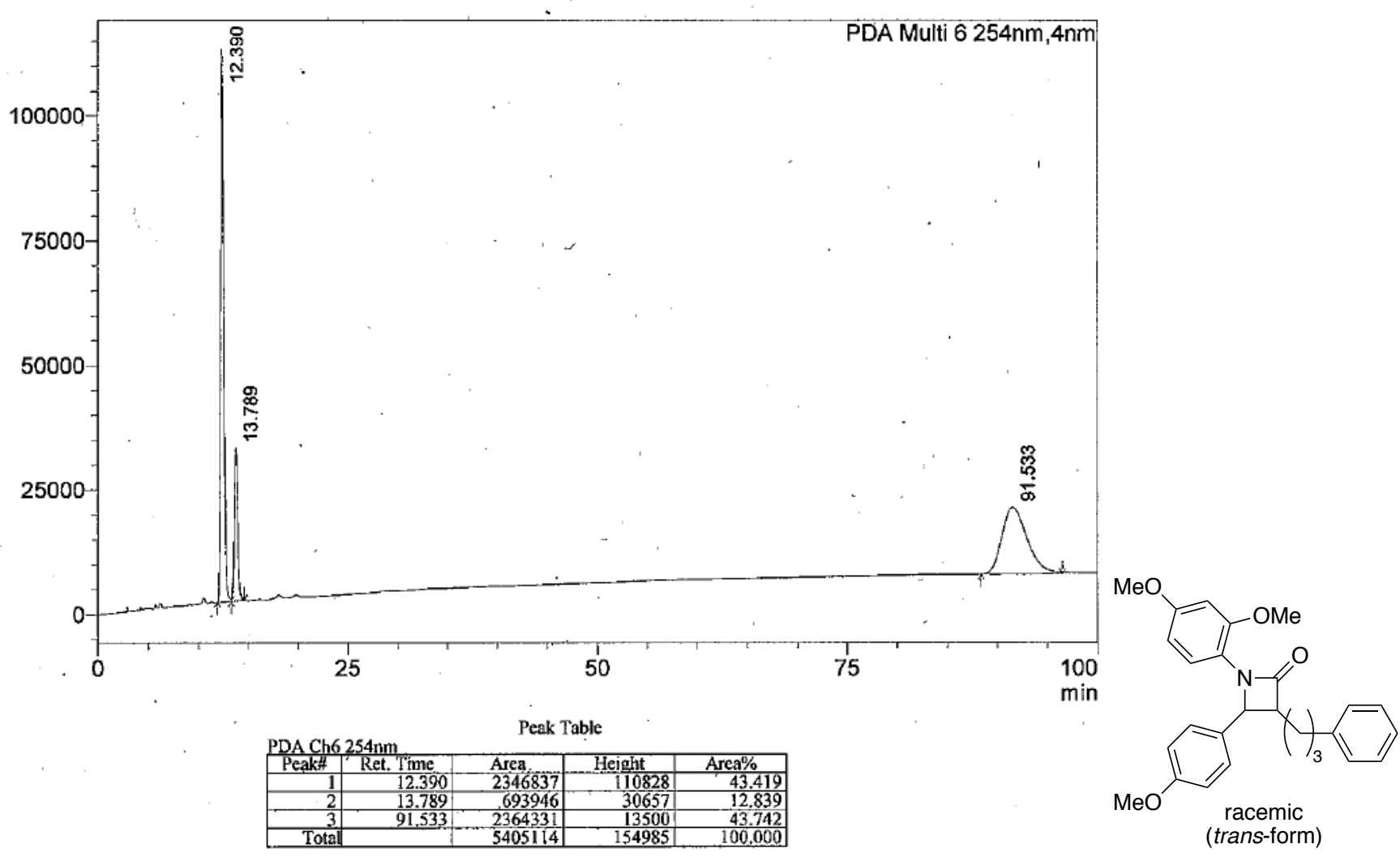

UAU

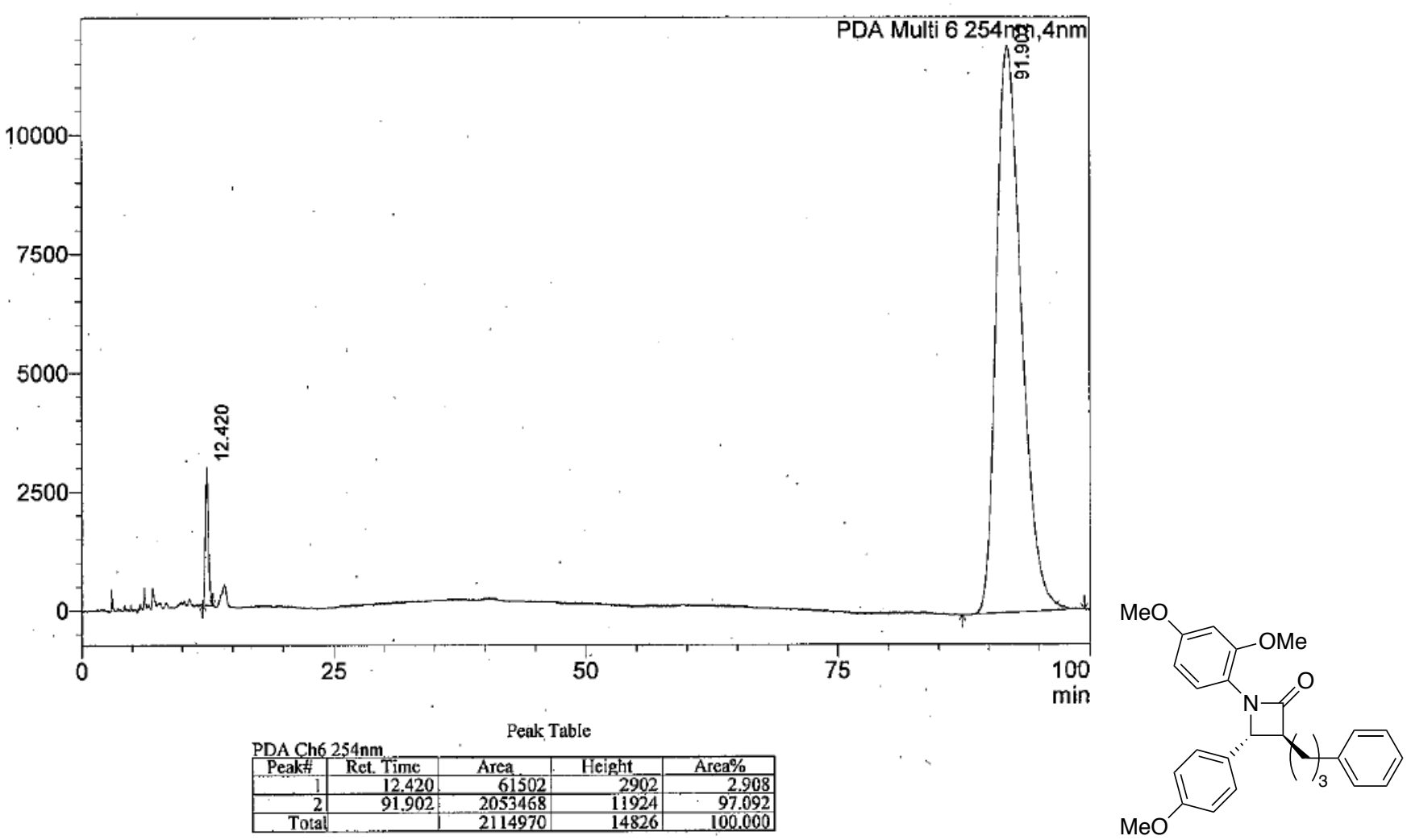


UAU

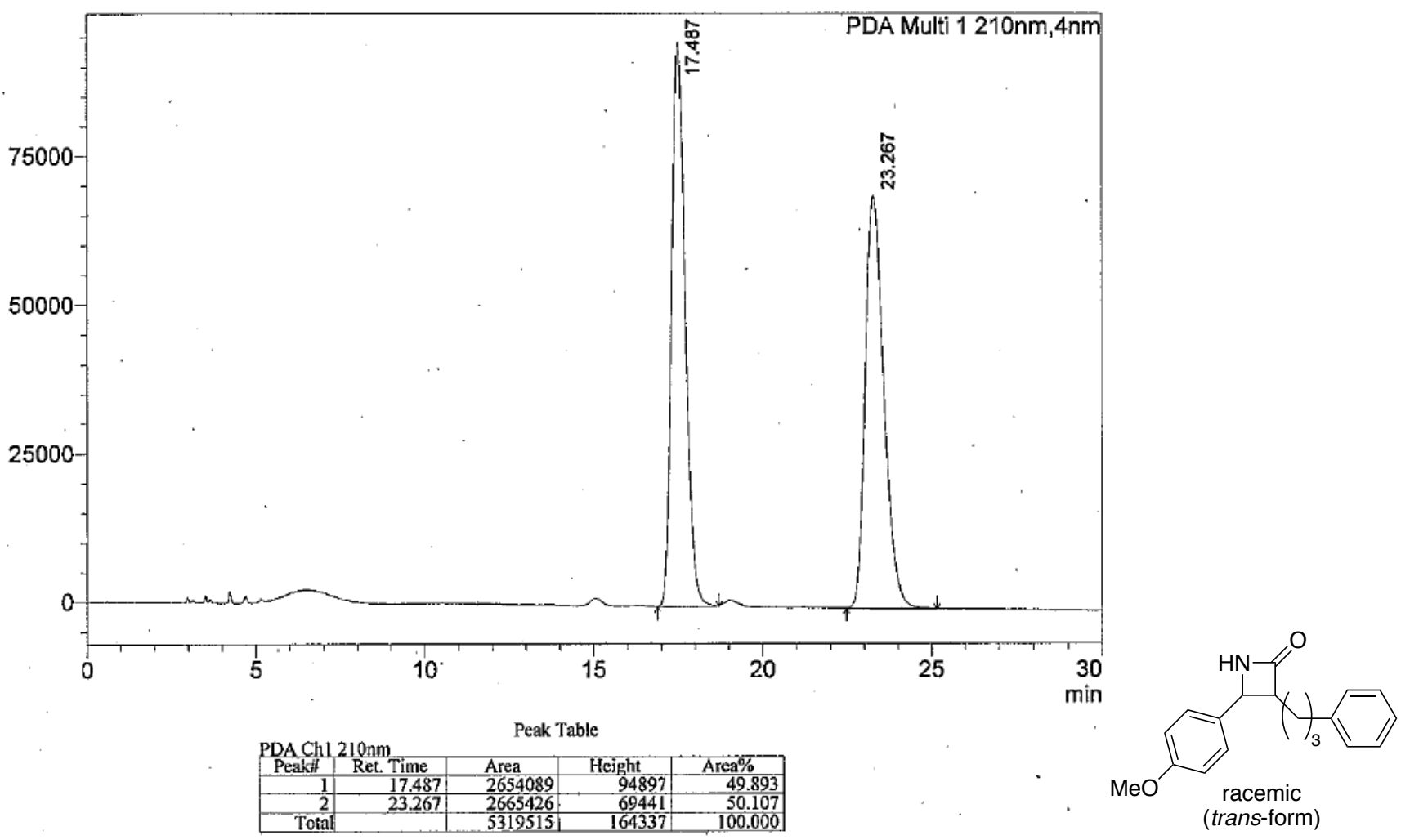

UAU

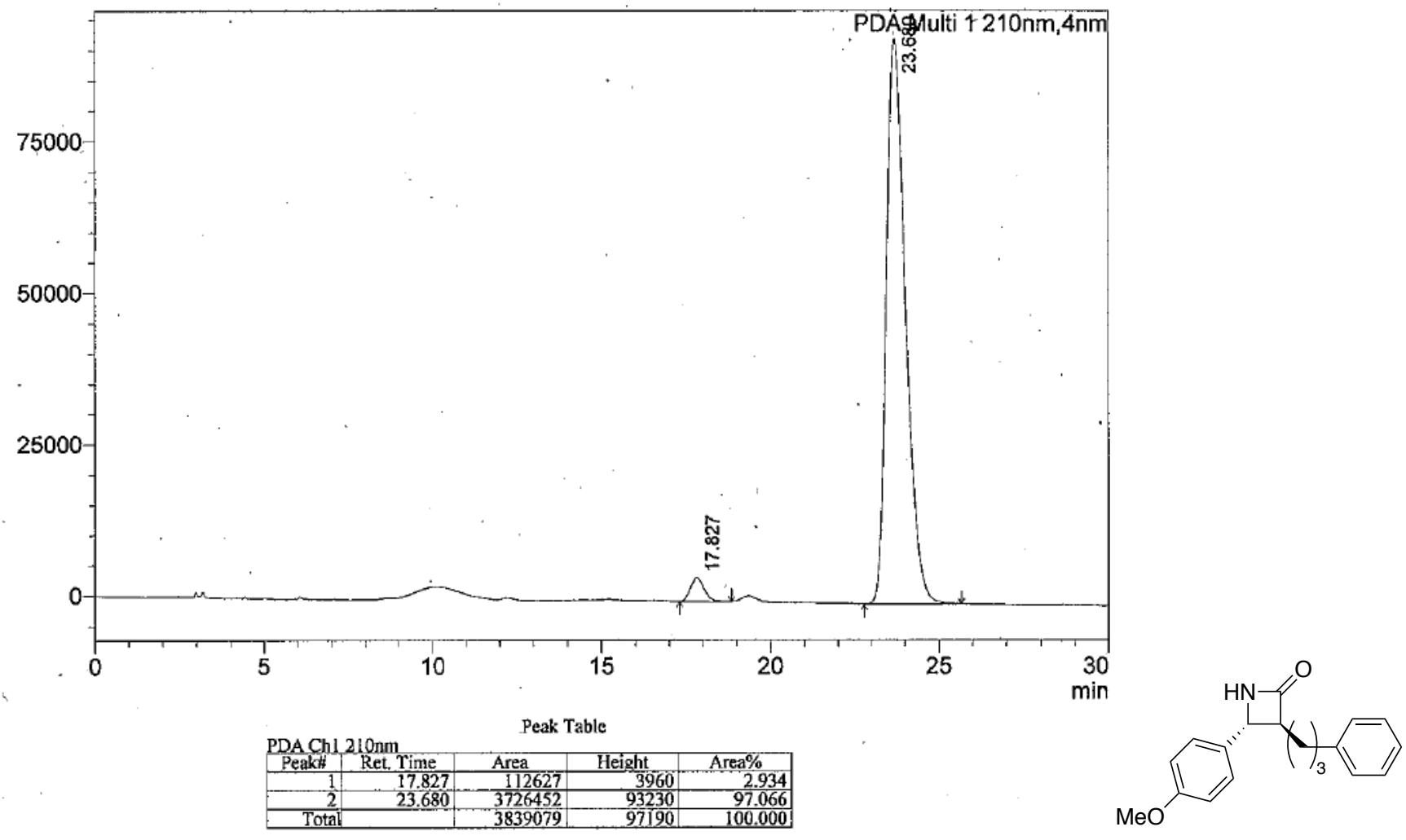


. UAU
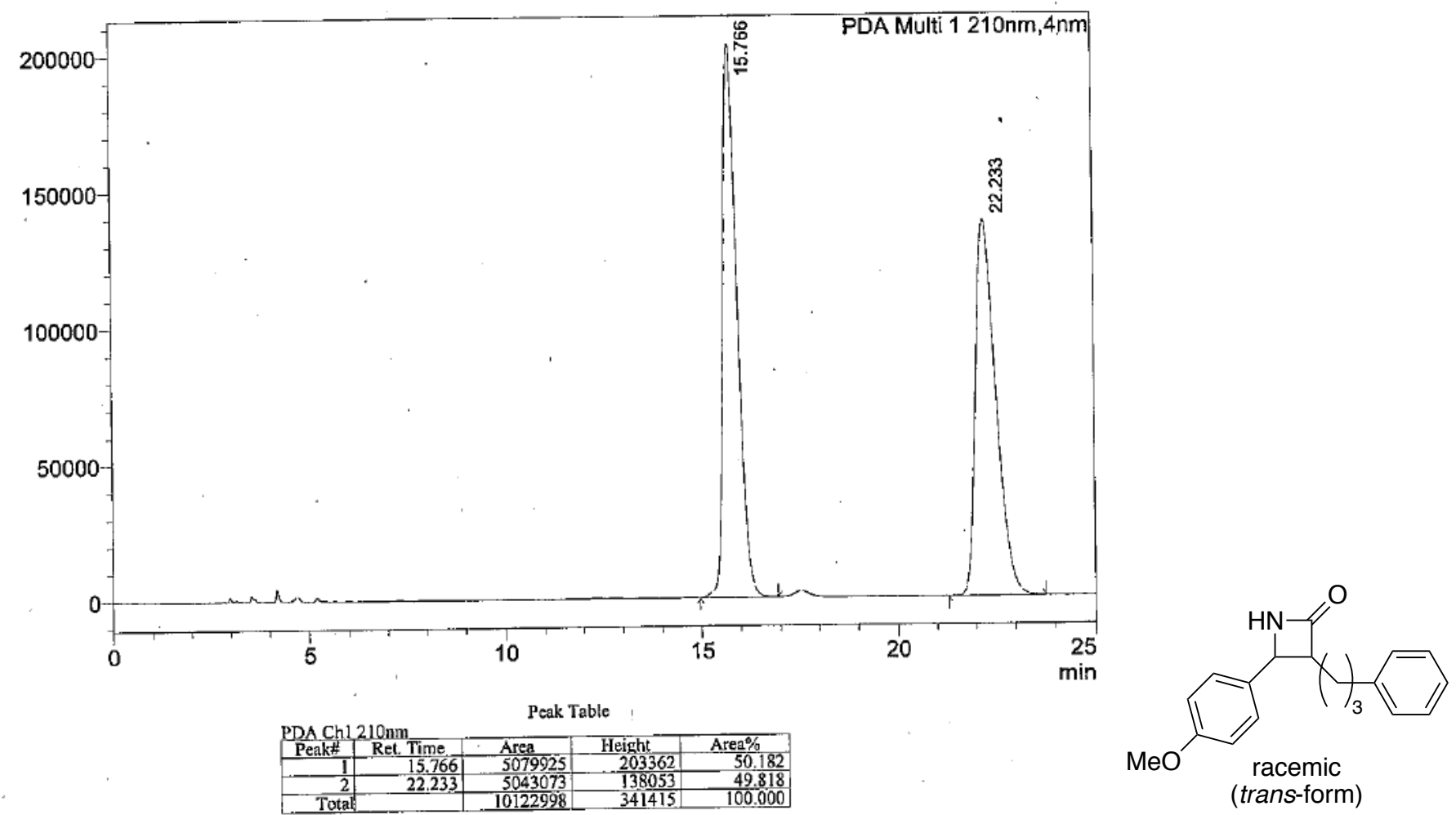

UAU
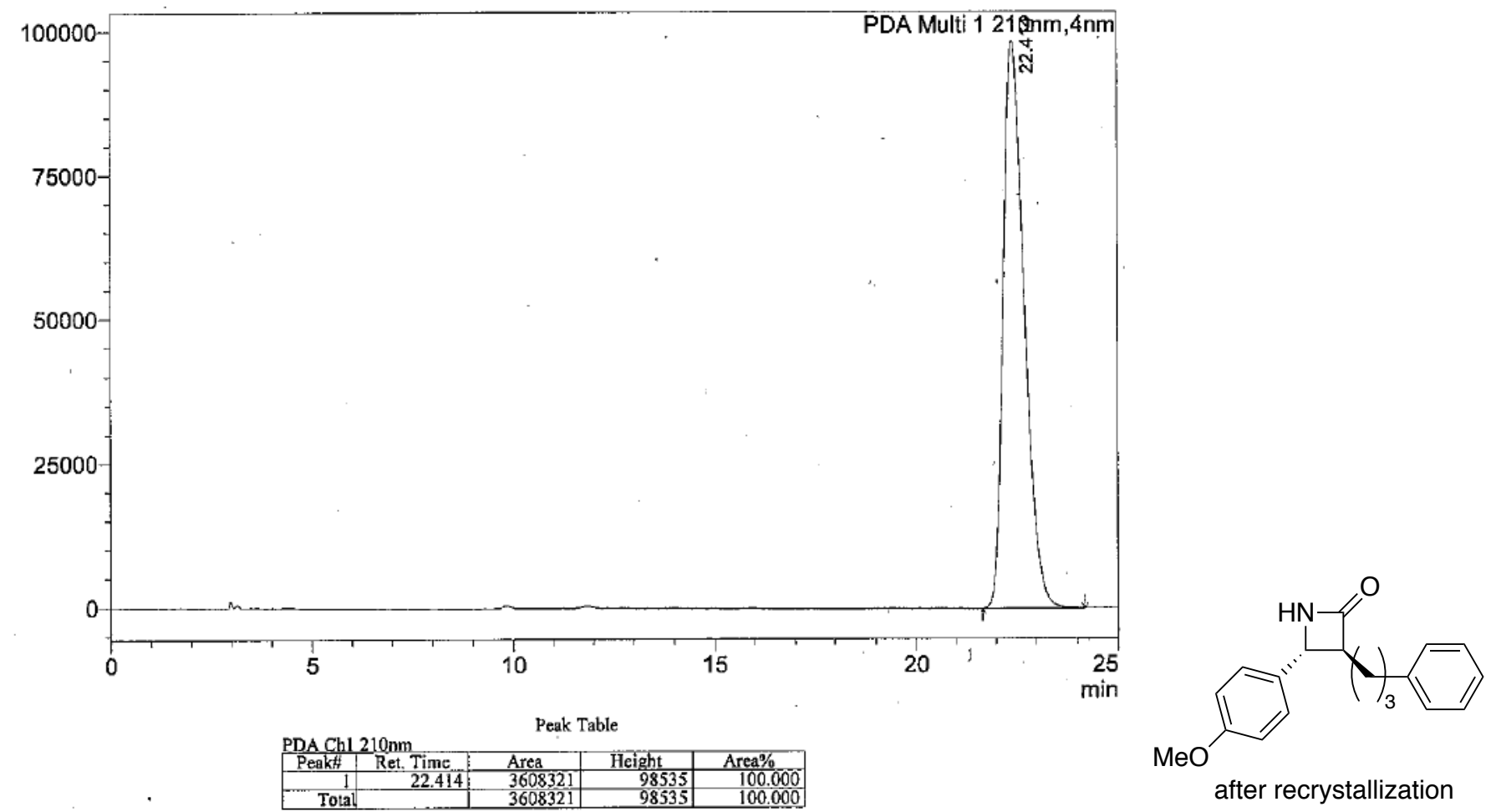
uAU
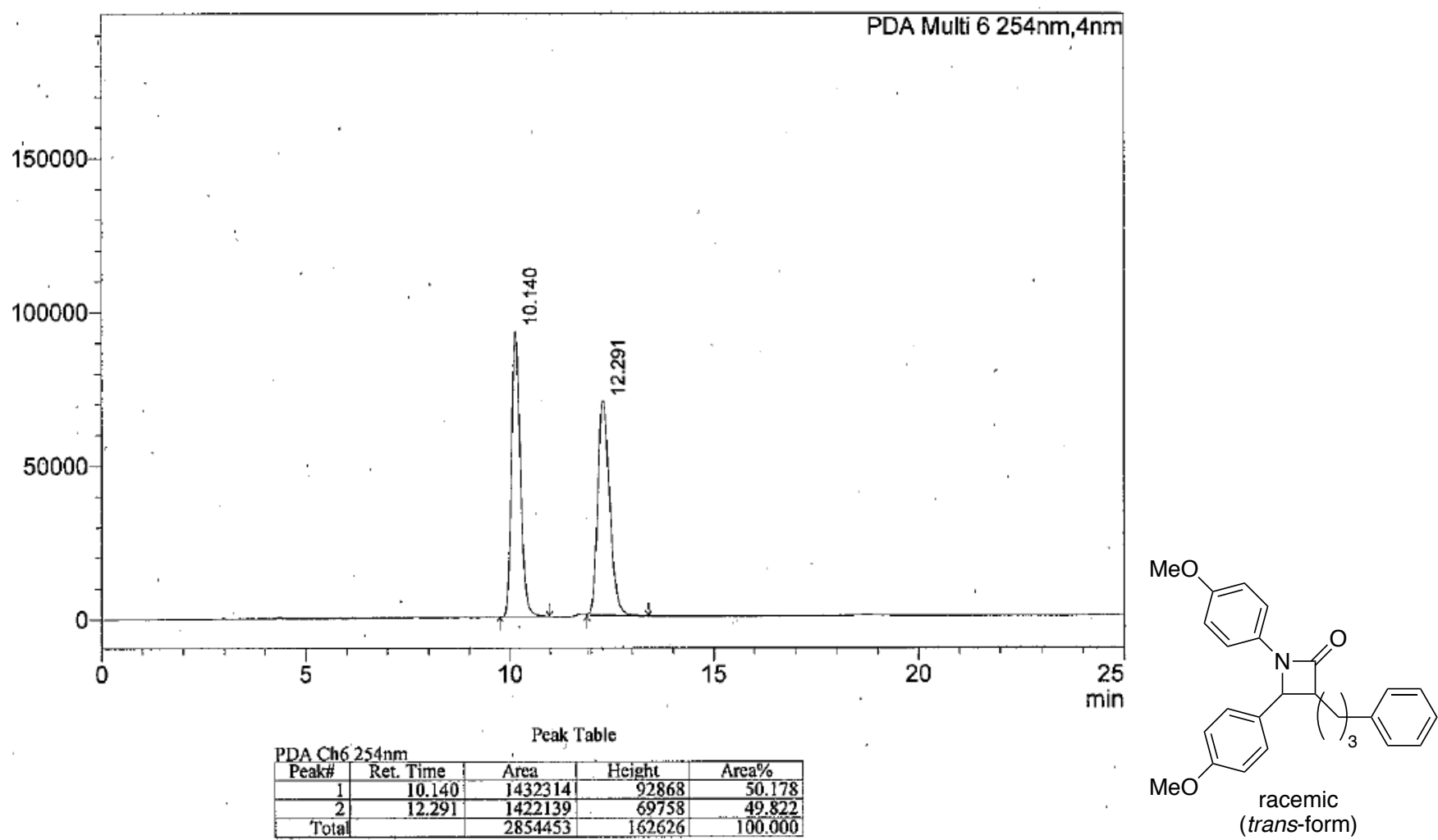

UAU
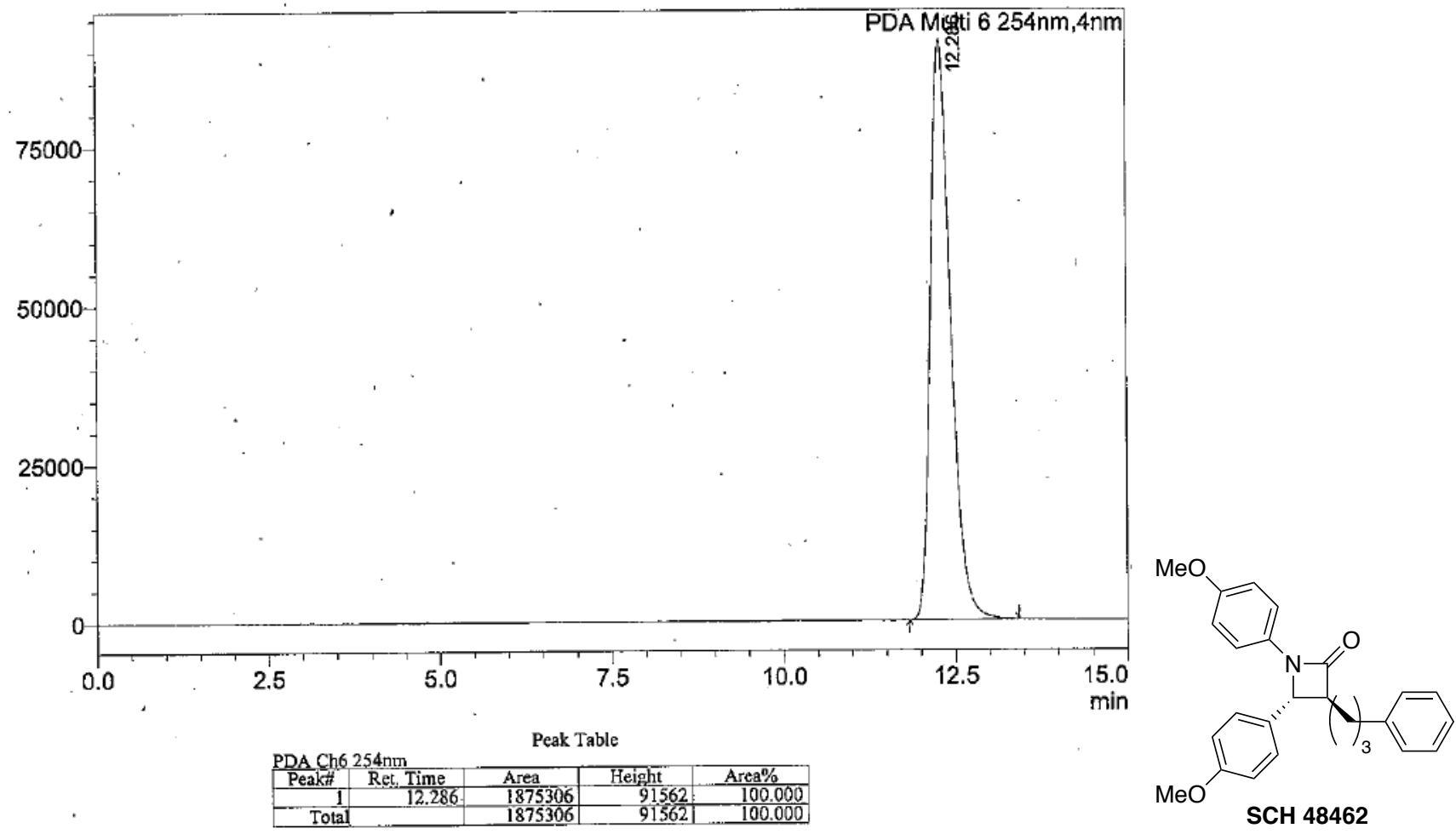
mAU

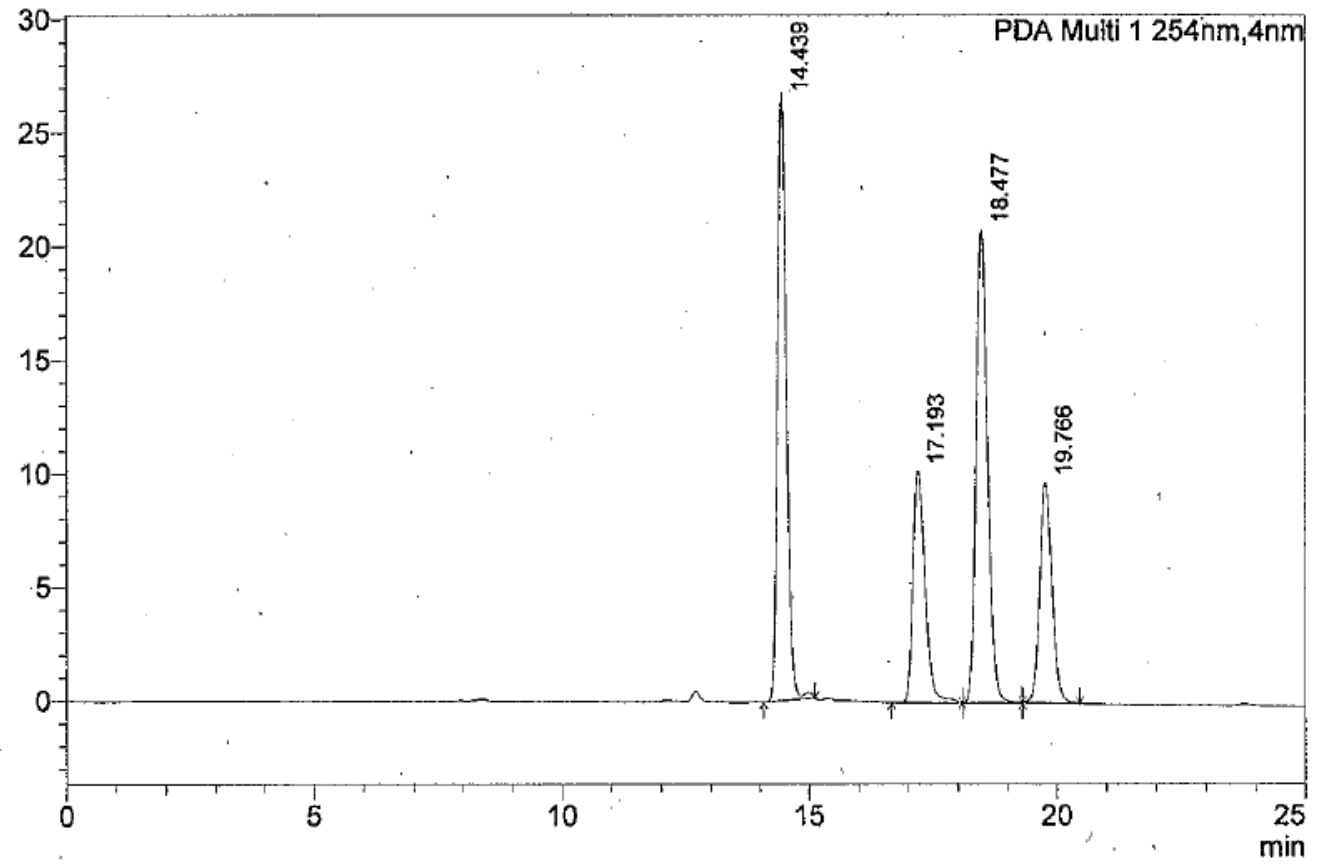

Peak Table
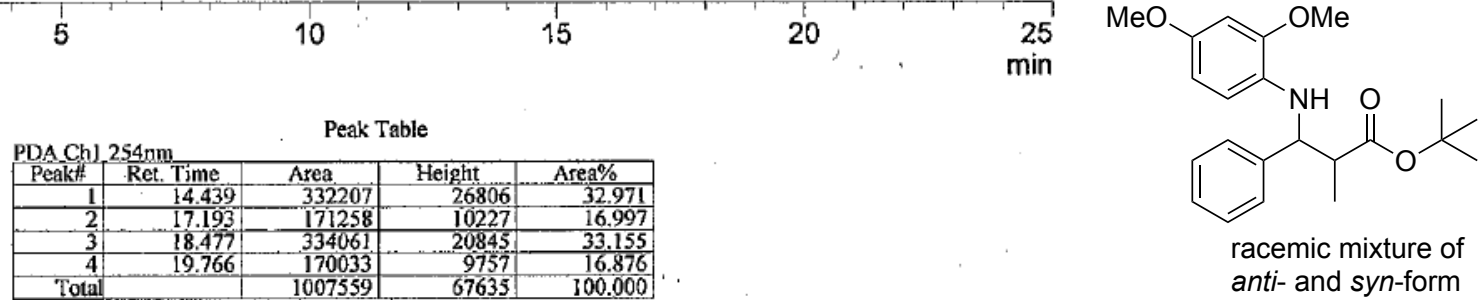

mAU
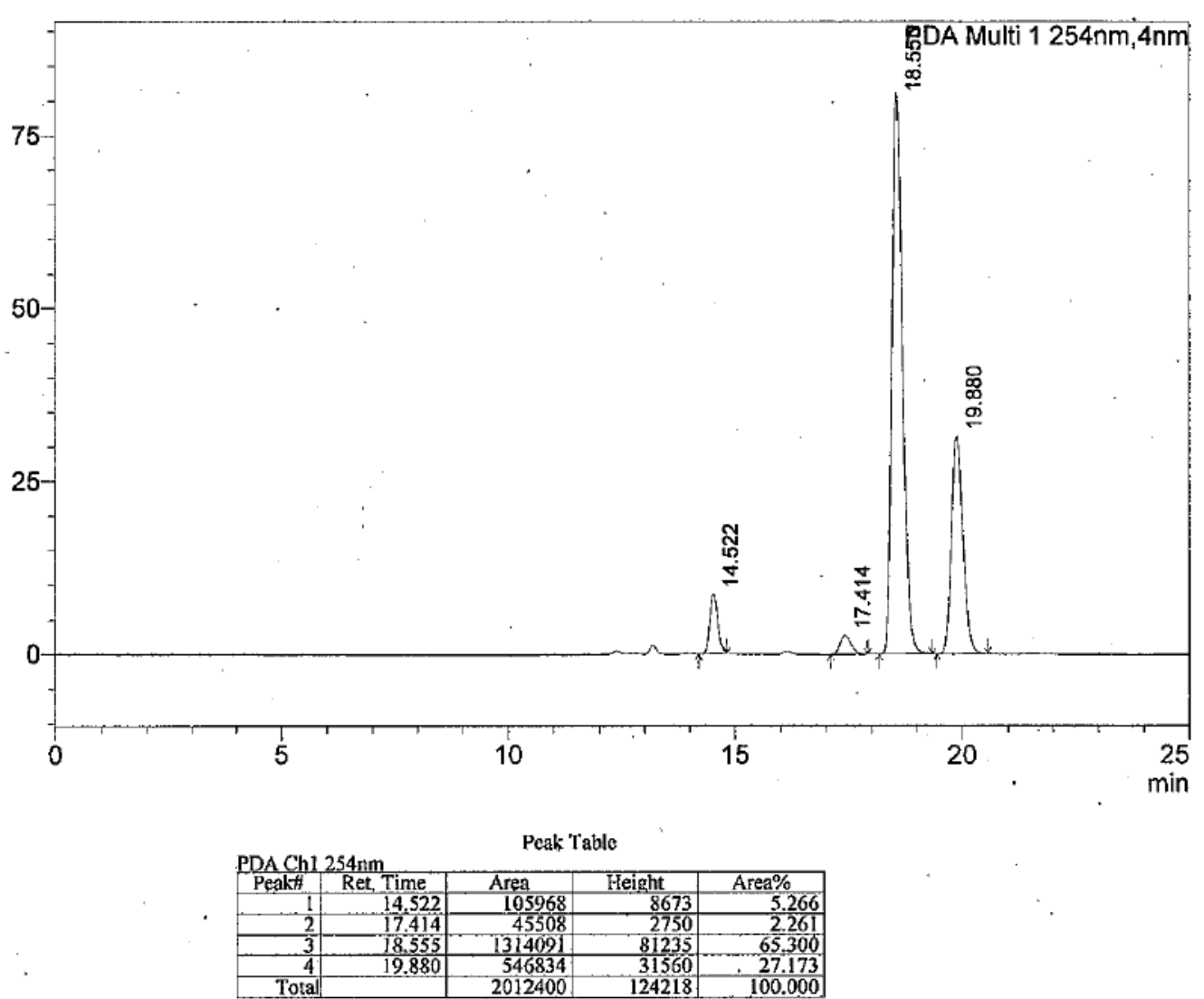

$\mathrm{MeO}$<smiles>COc1ccccc1N</smiles><smiles>C/C=C/N/C(=C(\C)C(=O)OC(C)(C)C)c1ccccc1</smiles> 
mAU
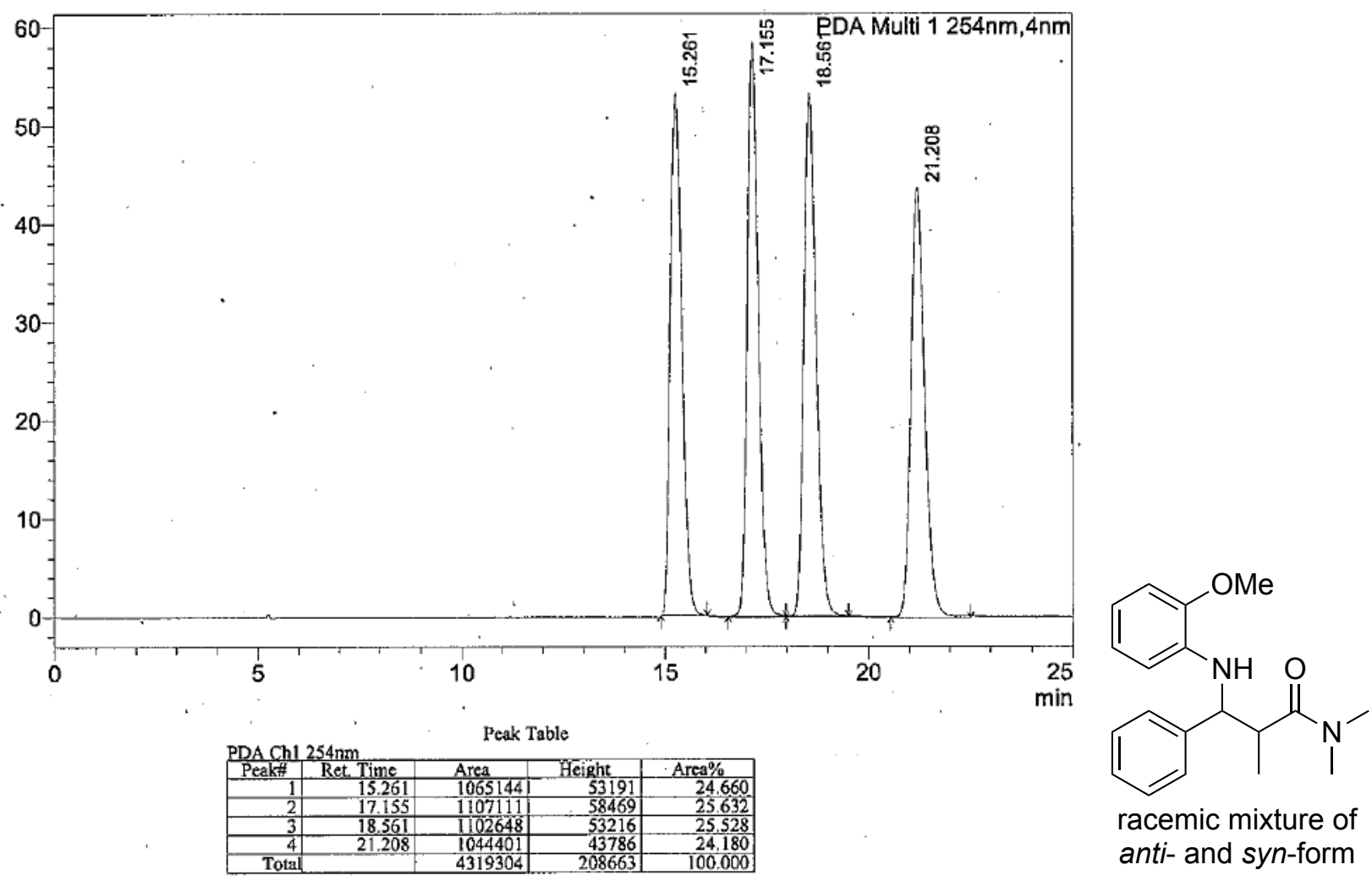

mAU

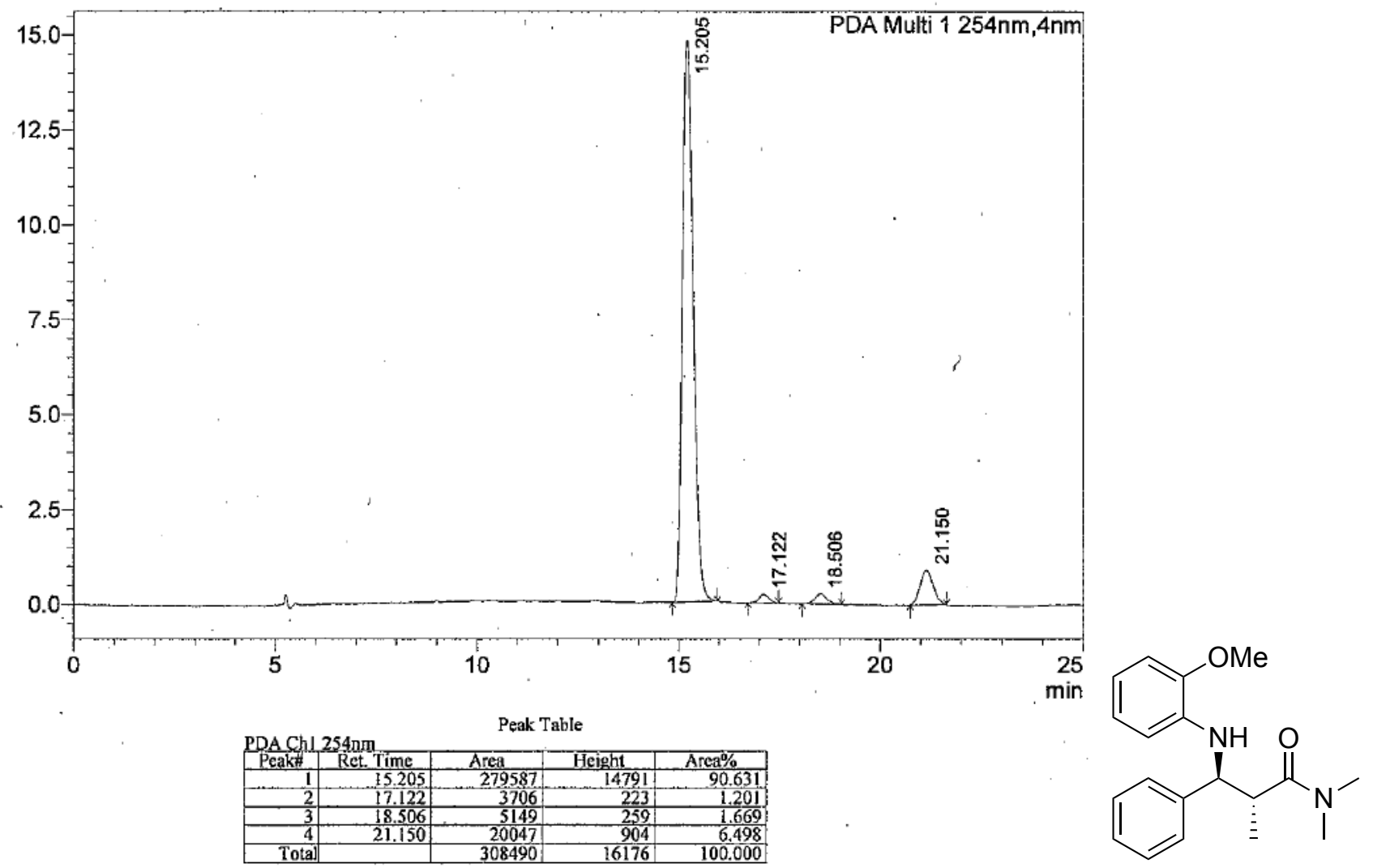


MAU

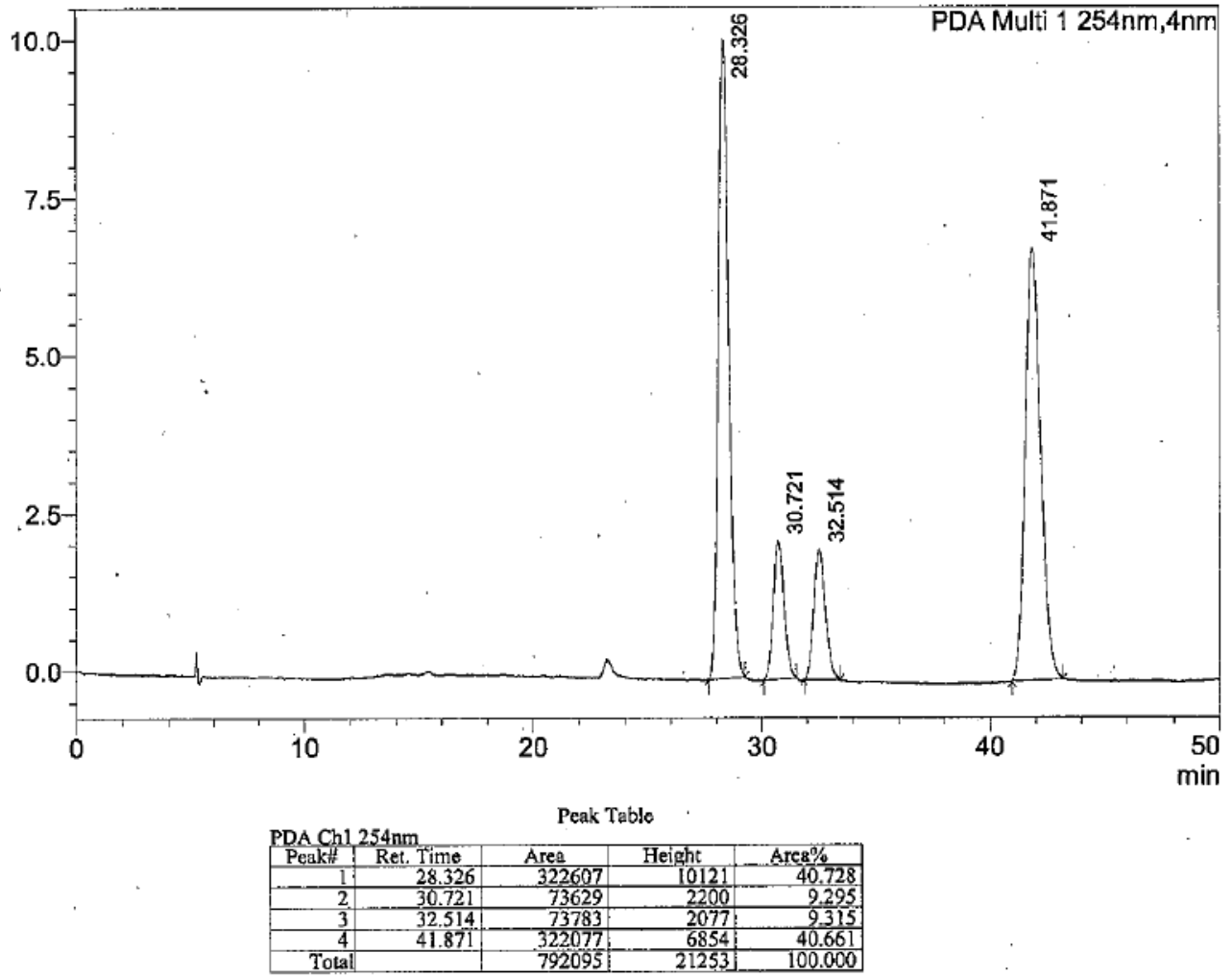<smiles>COc1ccc(NC(c2ccccc2)C(C)C(=O)N(C)C)cc1</smiles>

racemic mixture of anti- and syn-form

mAU

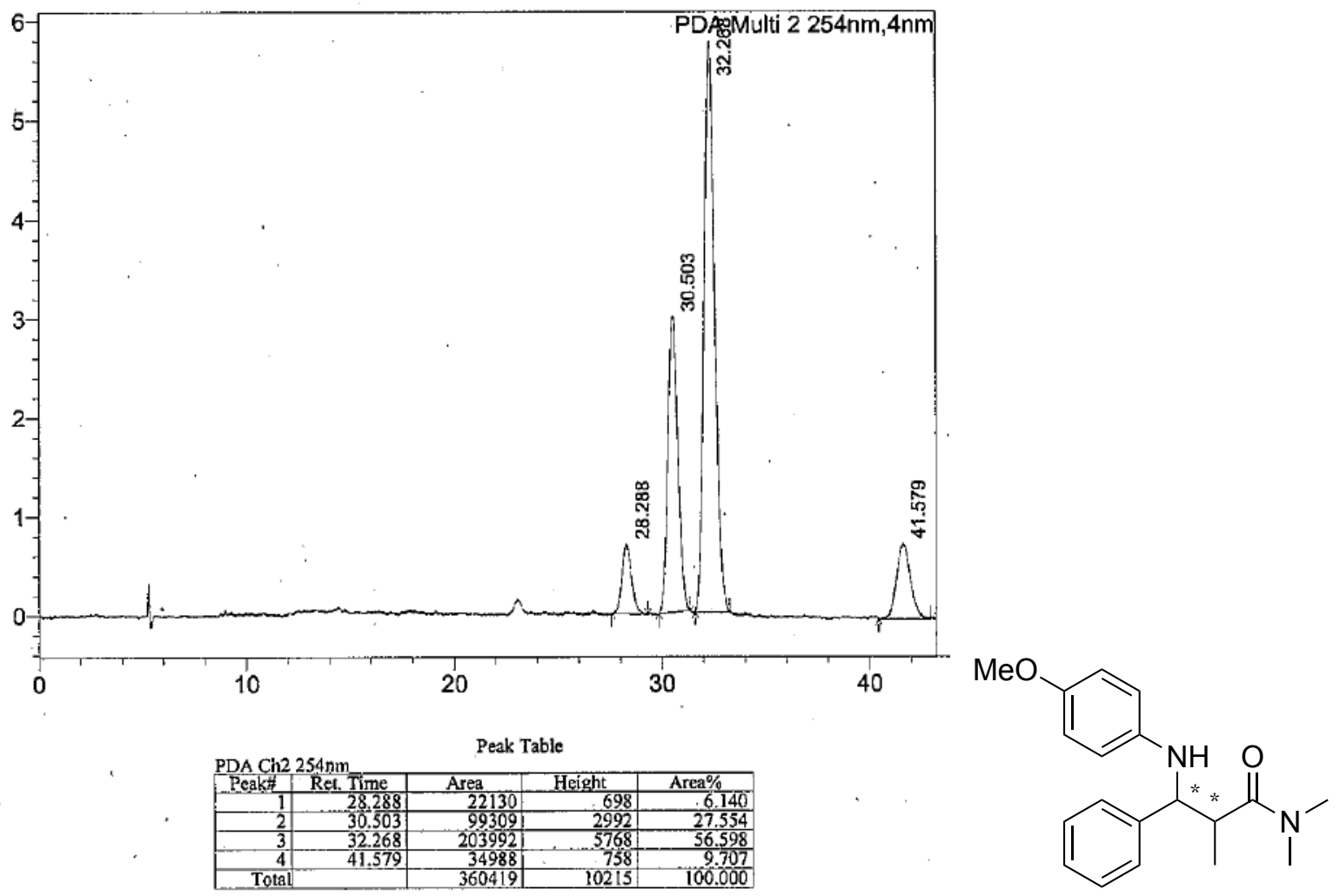



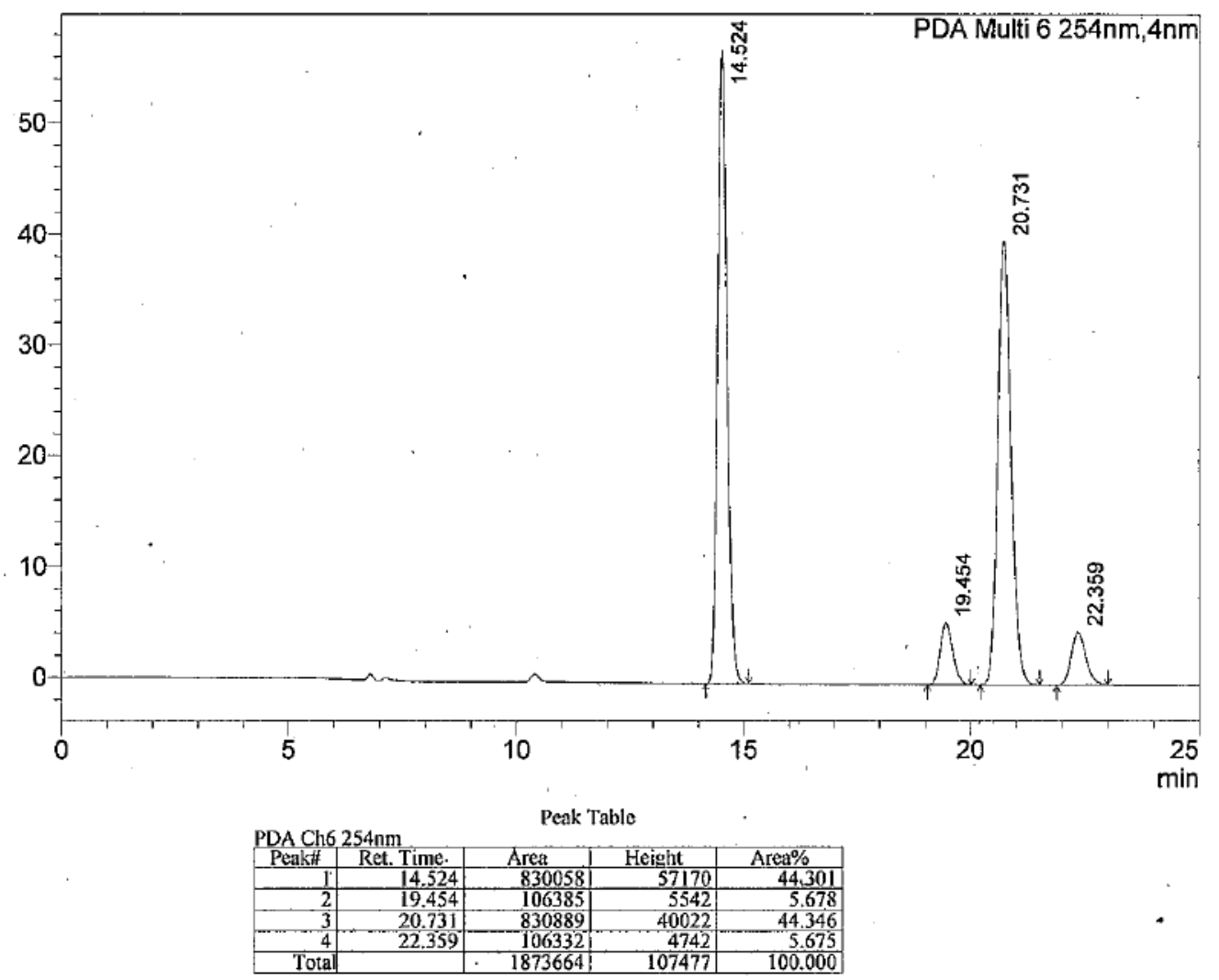

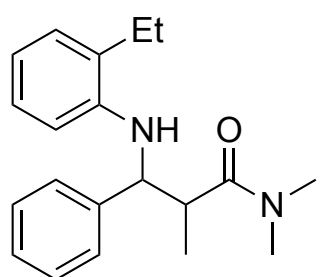

racemic mixture of anti- and syn-form

MAU

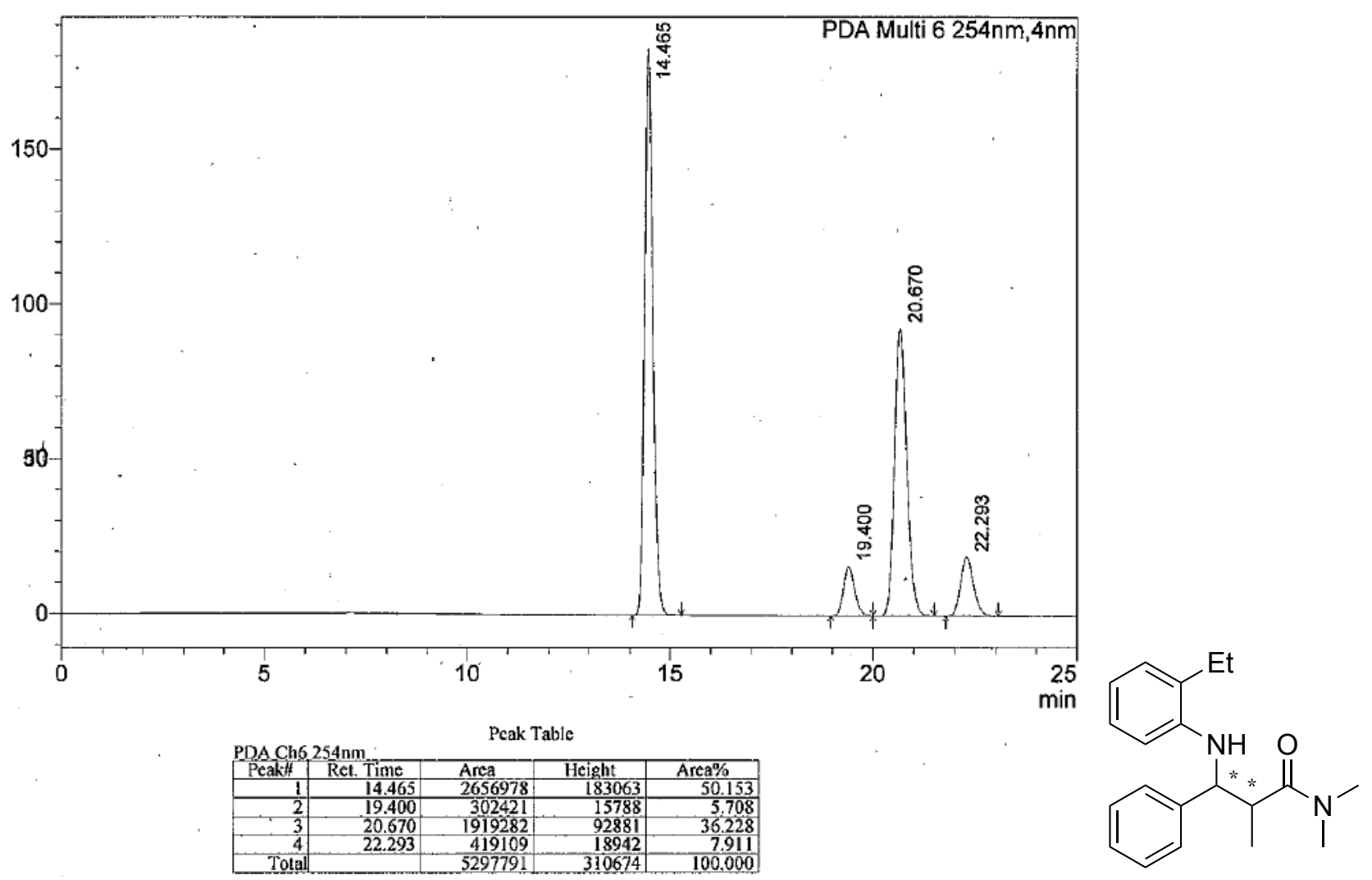


MAU

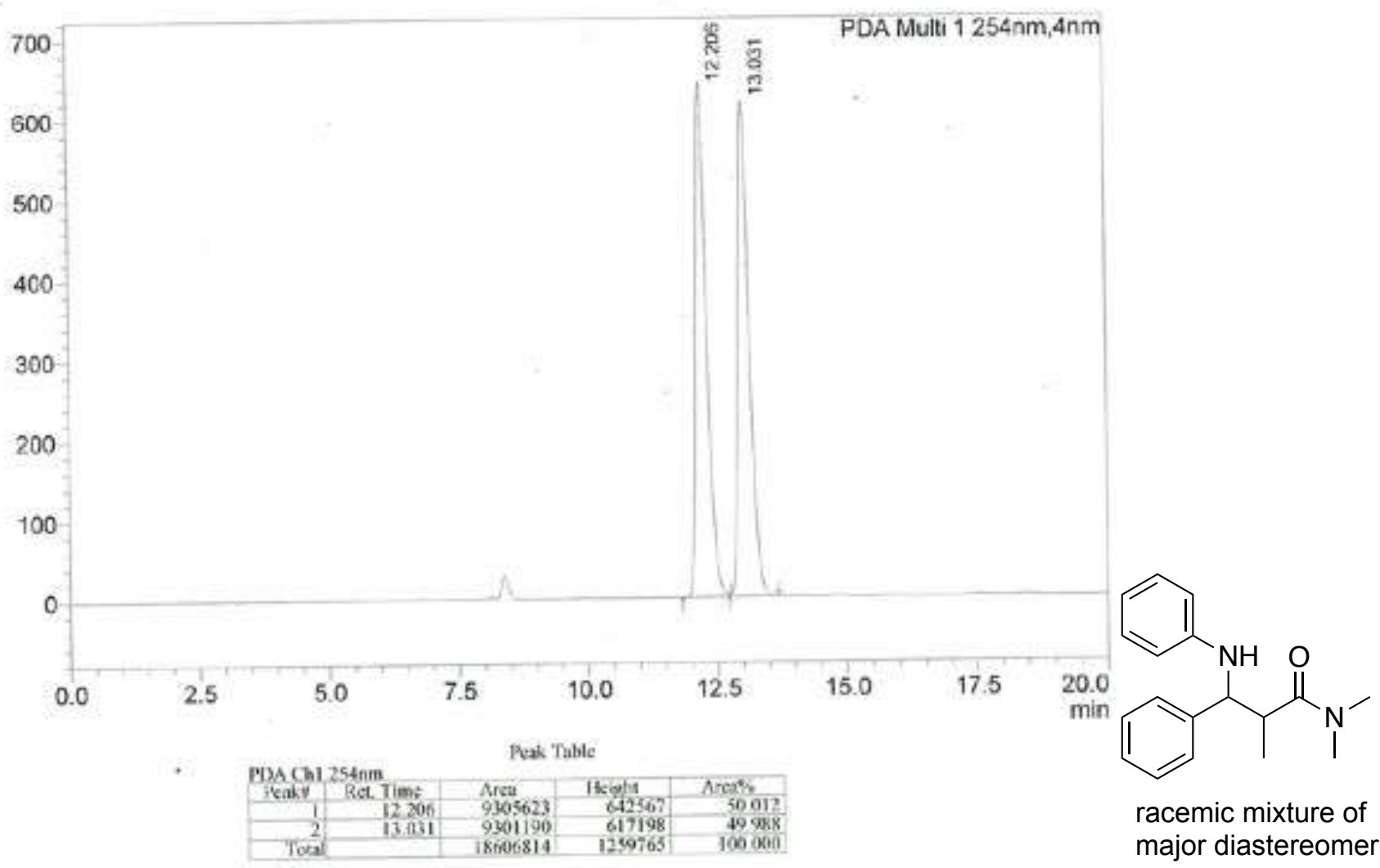

MAU

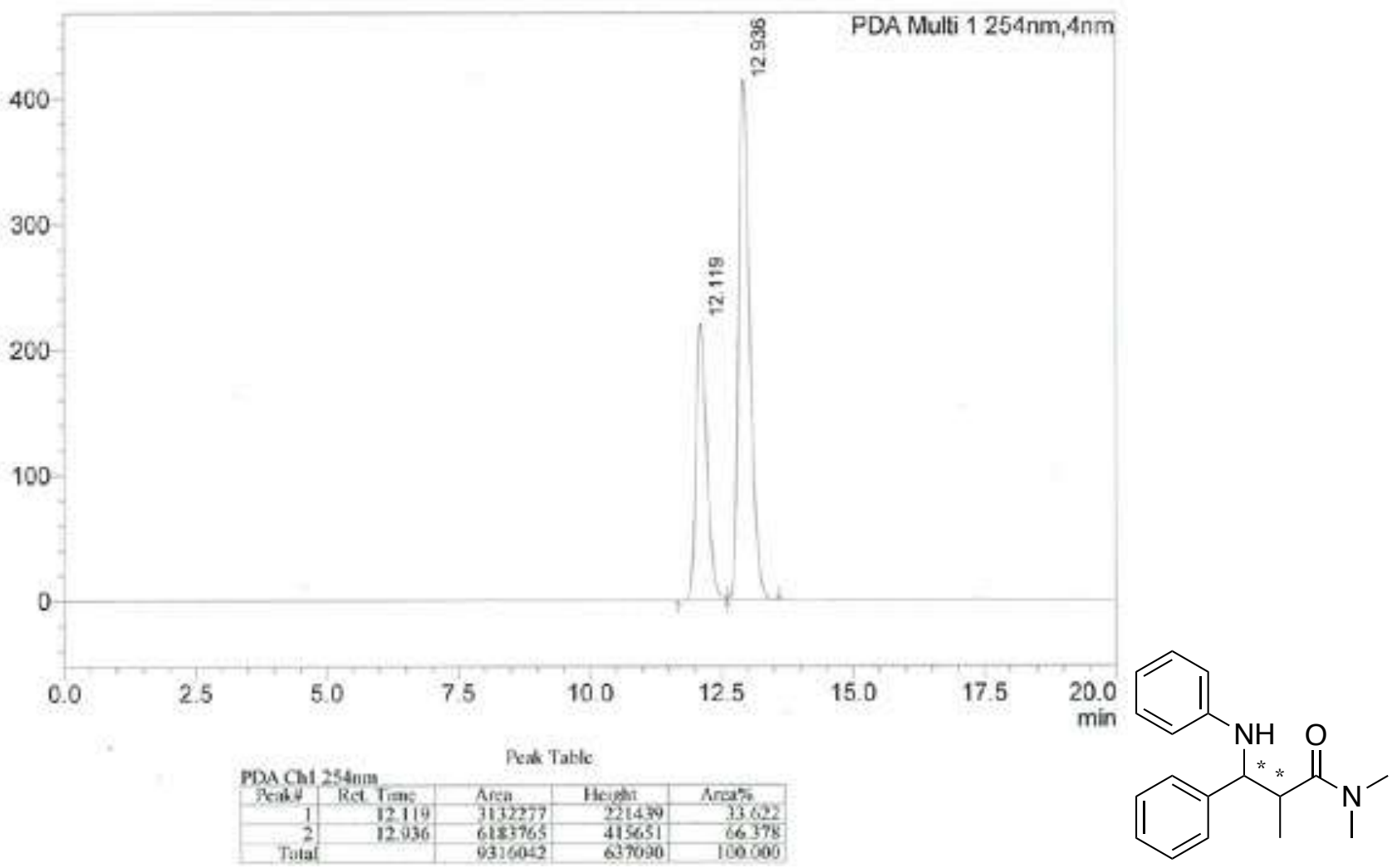

